PREMIO RICERCA «CITTÀ DI FIRENZE»

$-62-$ 
COLLANA PREMIO RICERCA «CITTÀ DI FIRENZE»

Commissione giudicatrice, anno 2017

\author{
Anna Dolfi (Presidente) \\ Maria Boddi \\ Andrea Bucelli \\ Roberto Casalbuoni \\ Roberto Ferrise \\ Marcello Garzaniti \\ Maria Cristina Grisolia \\ Patrizia Guarnieri \\ Roberta Lanfredini \\ Pierandrea Lo Nostro \\ Giovanni Mari \\ Alessandro Mariani \\ Paolo Maria Mariano \\ Simone Marinai \\ Rolando Minuti \\ Paolo Nanni \\ Giampiero Nigro \\ Angela Perulli
}


Stefano Malfatti

\section{Antonio da Borgonuovo. L'ascesa di un notaio a Trento fra Trecento e Quattrocento}


Antonio da Borgonuovo. L'ascesa di un notaio a Trento fra Trecento e Quattrocento / Stefano Malfatti. - Firenze : Firenze University Press, 2018.

(Premio Città di Firenze ; 62)

http://digital.casalini.it/97888645382I I

ISBN 978-88-6453-820-4 (print)

ISBN 978-88-6453-82I-I (online)

Progetto grafico di Alberto Pizarro Fernández, Pagina Maestra snc

Immagine di copertina: particolare di un documento rogato dal notaio Antonio da Borgonuovo (Archivio Diocesano Tridentino, Archivio Capitolare, capsa anniversari, rotoli medi/a, n. I I. Pubblicato con autorizzazione del 20/12/2018, prot. n. 2018-AR-AMM 1093).

Certificazione scientifica delle Opere

Tutti i volumi pubblicati sono soggetti ad un processo di referaggio esterno di cui sono responsabili il Consiglio editoriale della FUP e i Consigli scientifici delle singole collane. Le opere pubblicate nel catalogo della FUP sono valutate e approvate dal Consiglio editoriale della casa editrice. Per una descrizione più analitica del processo di referaggio si rimanda ai documenti ufficiali pubblicati sul catalogo on-line della casa editrice ( $w w w$.fupress.com).

Consiglio editoriale Firenze University Press

A. Dolfi (Presidente), M. Boddi, A. Bucelli, R. Casalbuoni, M. Garzaniti, M.C. Grisolia, P. Guarnieri, R. Lanfredini, A. Lenzi, P. Lo Nostro, G. Mari, A. Mariani, P.M. Mariano, S. Marinai, R. Minuti, P. Nanni, G. Nigro, A. Perulli, M.C.Torricelli.

La presente opera è rilasciata nei termini della licenza Creative Commons Attribution 4.0 International (CC BY 4.0: http://creativecommons.org/licenses/by/4.0/)

This book is printed on acid-free paper

CC 2018 Firenze University Press

Università degli Studi di Firenze

Firenze University Press

via Cittadella, 7, 50 I 44 Firenze, Italy

www.fupress.com

Printed in Italy 


\section{Sommario}

Prologo

\section{Capitolo 1}

Per una biografia di Antonio da Borgonuovo nel contesto politico trentino di inizio Quattrocento

1. La famiglia di Antonio: dalla Vallagarina al Borgonuovo

2. Fra matrimoni e alleanze politiche: la classe dirigente della Trento di inizio Quattrocento

3. I primi dati biografici su Antonio da Borgonuovo. Cenni sulle proprietà immobiliari

4. La presenza nella confraternita dei Battuti laici

5. Il ruolo di Antonio nelle vicende politiche di inizio Quattrocento

6. L'impegno politico di Antonio nel Comune di Trento

\section{Capitolo 2}

\section{La formazione di Antonio da Borgonuovo}

1. Sulla formazione di Antonio da Borgonuovo. Alcune considerazioni introduttive

2. Prima di Antonio. La formazione dei notai trentini

3. Dopo Antonio. Tappe formative dei notai trentini attraverso i verbali dell'Almo collegio dei dottori e notai della città (1459-1546)

\section{Capitolo 3}

\section{L'attività professionale di Antonio da Borgonuovo}

1. Fra notariato e cancelleria. Note sulla produzione documentaria vescovile fra la metà del Trecento e la metà del Quattrocento

2. L'attività al servizio del vicario vescovile

3. L'attività al servizio dell'Episcopato

4. L'archivio del Capitolo fino ad Antonio da Borgonuovo e l'opera decisiva e innovatrice di Pietro de Stanchariis

5. L'attività al servizio del Capitolo (1390-1435)

6. Note sulla produzione documentaria comunale all'inizio del Quattrocento

7. L'attività al servizio del Comune di Trento

8. Produzione e conservazione della documentazione: monasteri, conventi e confraternite cittadine fra XIV e XV secolo 


\section{Sommario}

9. L'attività al servizio di conventi, monasteri e confraternite 101

10. Gli altri clienti privati 104

11. Per un quadro di sintesi della clientela di Antonio da Borgonuovo 113

12. I ritmi di lavoro 113

13. Il 'tariffario' 120

14. Nomine di rettori di chiese, pievi e altari; nomine di canonici 126

\section{Capitolo 4}

Antonio da Borgonuovo al lavoro. Tracce di diplomatica notarile 131

1. Dalla 'scheda' all'instrumentum 131

2. I quaternelli 143

3. I prothocolli 144

$\begin{array}{ll}\text { 4. I quaterni o libri } & 147\end{array}$

5. Prothocolli e libri a confronto 148

6. Documenti in forma abbreviata redatti direttamente sui quaterni 157

7. Il registro Instrumenta capitularia 8 (1402-1434). Descrizione codicologica 166

8. Il registro Instrumenta capitularia 8bis (1423-1437). Descrizione codi$\begin{array}{ll}\text { cologica } & 176\end{array}$

9. La scrittura di Antonio da Borgonuovo 181

\section{Capitolo 5}

Il 'destino' delle carte 189

1. Tracce di trasmissione di documentazione notarile fra XIV e XV secolo 189

2. Antonio da Borgonuovo relevator di documentazione capitolare 191

3. La trasmissione della documentazione capitolare prodotta da Antonio da Borgonuovo

4. La trasmissione di altra documentazione prodotta da Antonio da Borgonuovo

Conclusioni

Appendice

Regesti

$\begin{array}{ll}\text { Abbreviazioni } & 241\end{array}$

$\begin{array}{ll}\text { Bibliografia } & 243\end{array}$

$\begin{array}{ll}\text { Indice dei nomi di persona } & 253\end{array}$

$\begin{array}{ll}\text { Indice dei nomi di luogo } & 269\end{array}$ 


\section{Prologo}

A Trento soltanto sul finire del Quattrocento si assiste al consolidamento di un iter professionale dei notai, grazie soprattutto alla definizione dei meccanismi di apprendistato, formazione, selezione e inserimento nel Collegio notarile. L'inizio del $\mathrm{XV}$ secolo, periodo nel quale si colloca il notaio Antonio da Borgonuovo, si configura quindi come una fase di piena transizione, in cui - dopo le rivolte cittadine contro il vescovo Georg Liechtenstein (1407-1409) e contro il nuovo presule Alessandro di Masovia (1435-1437) - le istituzioni comunali iniziano un lento e accidentato processo di «distanziamento» ${ }^{1}$ dal potere vescovile, con una maggiore definizione dei propri ruoli, pur in un contesto di forte dipendenza dall'Episcopato da cui, in ultima analisi, esse traevano ogni legittimità.

Proprio nei primi decenni del Quattrocento, all'interno dei nuovi statuti cittadini emanati dal vescovo Alessandro di Masovia, vengono promulgati gli statuti del Collegio notarile trentino (1425-1427) il quale, in una situazione di grande ritardo rispetto ad altre città dell'Italia centro-settentrionale, intraprese un percorso di consolidamento, cercando di adeguarsi ai modelli e alle modalità organizzative del notariato attivo nelle altre città nord-italiane.

La documentazione prodotta fra l'inizio e la metà del secolo consente di valutare la qualità di tale evoluzione: fonti come il Liber electionum officialium magnificae communitatis Tridenti, per parte comunale, e il codice 4272 (Registro delle immatricolazioni), per quanto riguarda il Collegio notarile, permettono di individuare da un lato l'organigramma delle istituzioni comunali e della matricola, dall'altro i meccanismi del loro funzionamento. I membri delle famiglie più in vista della città (dai Calepini ai de Fatis ai Negri, per citarne soltanto alcune) si spartiscono per buona parte del Quattrocento i seggi delle magistrature comunali e del Collegio notarile, a formare, nelle parole di Gian Maria Varanini, un' «oligarchia piuttosto chiusa»².

Se dal punto di vista dell'attività professionale notarile e del suo funzionamento, come si vedrà, nel corso del Quattrocento si vengono definendo criteri e «regole di funzionamento ${ }^{3}$, sotto il profilo della produzione e conservazione della documen-

1 Di «distanziamento» del Comune di Trento dal potere vescovile parla Franco Cagol in un recente saggio dedicato alla definizione degli spazi dell'identità comunale tra il XIII ed il XVI secolo: F. Cagol, Dal palatium episcopatus al palatium comunis. Spazi dell'identità comunale tra XIII e XVI secolo, in F. Cagol, S. Groff, S. Luzzi (a cura di), La torre di piazza nella storia di Trento, Atti della giornata di studio (Trento, 27 febbraio 2012), Società di Studi trentini di scienze storiche, Trento 2014 (Monografie. Nuova serie, 3), pp. 205-223.

2 G.M. Varanini, Il Collegio notarile di Trento nella seconda metà del Quattrocento, in A. Giorgi, S. Moscadelli, D. Quaglioni, G.M. Varanini (a cura di), Il notariato nell'arco alpino: produzione e conservazione delle carte notarili tra medioevo ed età moderna, Atti del convegno di studi (Trento, 24-26 febbraio 2011), Giuffrè, Milano 2014 (Studi storici sul notariato italiano, XVI), p. 488.

3 Ivi, p. 489. 


\section{Prologo}

tazione si rimane ben lontani dagli standard d'altre città; registrazioni, come quelle comunali sopra menzionate, si presentano infatti dal punto di vista diplomatistico assolutamente 'rudimentali' e vengono affidate - vista l'assenza di sistemi di produzione cancellereschi - alla redazione di notai diversificati. Lo stesso avviene, qualche decennio più tardi, con i verbali della matricola dei notai, pure caratterizzati da uno scarso livello formale oltre che dalla lacunosità delle registrazioni.

Sotto il profilo della conservazione della documentazione si deve attendere addirittura la piena età moderna (1595), prima di veder istituiti archivi dei notai vivi e defunti': prima di questa data ciò che rimane è costituito da un frammento dell'intera produzione documentaria dei professionisti trentini. Spesso, come nel caso dei due registri Instrumenta capitularia 8 e 8bis del notaio Antonio da Borgonuovo, ciò che si conserva è frutto della prestazione per enti, come Episcopato e Capitolo, la cui documentazione ha una prospettiva di sopravvivenza più favorevole, mentre la pur cospicua attività per enti minori o privati cittadini, affidata alla conservazione degli stessi notai o dei loro eredi, è destinata ad andare irrimediabilmente perduta dopo pochi decenni. Quanto rimane di questa variegata produzione per il lungo periodo che va dal XIII alla metà del XV secolo è così rappresentata da una manciata di registri ${ }^{5}$ oggi conservati, non a caso, negli archivi vescovile e capitolare.

Gli statuti masoviani avevano sancito la struttura del Collegio: erano previsti ad esempio un rettore (chiamato anche priore), nonché quattro consiglieri, oltre alle figure del massaro e dei bidelli: venivano per la prima volta definite le modalità di ingresso nella matricola, gli obblighi per i membri e le modalità di redazione della documentazione (dal punto di vista, ad esempio, delle tempistiche per il rilascio degli instrumenta ecc.). Il «punto d'arrivo» ${ }^{6}$ di questo processo, il cui 'impianto generale' come ricordato - è definito negli statuti masoviani degli anni Venti, va dunque collocato sul finire del Quattrocento, sebbene soltanto con i nuovi statuti di Bernardo Cles l'iter si possa dire pienamente concluso anche dal punto di vista normativo.

Una certa rilevanza, infine, ricoprono tutta una serie di norme che miravano a garantire un sufficiente livello qualitativo nella produzione documentaria. In un contesto evidentemente contrassegnato da scarsa professionalità ${ }^{7}$, vennero definite procedure per l'apprendistato e la formazione dei giovani notai. Dal 1461, ad esempio, l'esame per l'ammissione fu svolto pubblicamente «in generali Collegio». Il candidato, dopo aver ricevuto la nomina a notaio, generalmente per mezzo di un conte palatino, si presentava dinanzi al Collegio che valutava mediante specifico esame la conoscenza della materia. Si hanno così esempi di professionisti immediatamente 'immatricolati', perché giudicati «unanimiter et concorditer» idonei, mentre in altri casi i candidati furono sottoposti ad ulteriore esame previa frequenza di specifici percorsi formativi alla statio di notai più esperti (spesso lo stesso rettore del Collegio) o presso il pode-

4 A. Casetti, Il notariato trentino e l'istituzione dei più antichi archivi notarili in Trento: l'archivio (vecchio) dei morti e l'archivio (nuovo) dei vivi (1595-1607), «Studi trentini di scienze storiche», 31, 1952, pp. 242-286; F. Cagol, B. Brunelli, Archivio pretorio o archivi notarili? Primi risultati di un'indagine archivistica sulla documentazione giudiziaria della città di Trento, «Annali dell'Istituto storico italo-germanico in Trento», 28, 2002, pp. 691-700.

Per un'esaustiva descrizione della produzione documentaria in registro a Trento cfr. E. Curzel, Trento, CISAM, Spoleto 2013 (Il medioevo nelle città italiane, 5), con la bibliografia ivi citata.

Varanini, Il Collegio notarile di Trento, cit., p. 497.

7 Sovente la carica notarile fu attribuita dai conti palatini a giovani del tutto impreparati a svolgere la professione (cfr. ivi, p. 500). 
stà cittadino. In seguito, l'ammissione al Collegio poteva essere autorizzata ma con restrizioni nella produzione di talune tipologie documentarie.

La fase di transizione di inizio Quattrocento cui si è brevemente accennato in apertura può essere efficacemente indagata prendendo a riferimento l'esempio di Antonio di Bartolasio da Borgonuovo, notaio attivo a Trento fra la fine del Trecento e i primi decenni del Quattrocento. Il caso è interessante sia dal punto di vista dell'attività politico-istituzionale, in quanto membro della classe dirigente trentina di inizio secolo, sia per la professione esercitata, considerata la grande mole di documenti da lui prodotti ancora oggi conservati negli archivi trentini, e il lungo periodo (1386-1437) durante il quale prestò la propria opera di autenticatore della memoria documentaria di numerosi enti e privati. L'analisi sulla sua attività politico-sociale, sulla clientela e sulle modalità di lavoro nonché, in ultimo, sulla trasmissione documentaria delle sue carte, contribuiscono a fornire maggiori dettagli sul funzionamento del notariato trentino fra Tre- e Quattrocento, quale punto di partenza di un processo che, come ricordato, potrà considerarsi compiuto soltanto un secolo più tardi. 


\section{Prologo}

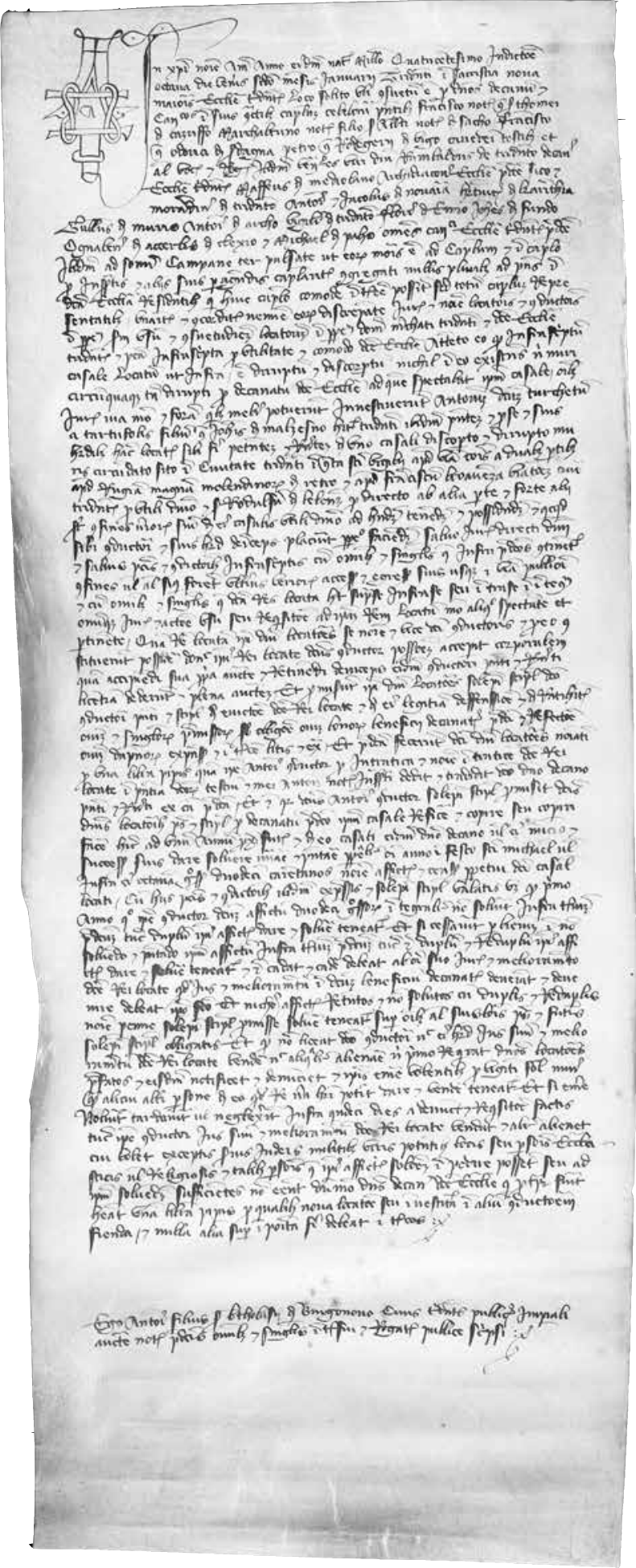

ADTn, ACap, capsa 1, n. 8. Redactio in mundum di Antonio da Borgonuovo (1400 gennaio 2). 


\section{Capitolo 1 \\ Per una biografia di Antonio da Borgonuovo nel contesto politico trentino di inizio Quattrocento}

\section{La famiglia di Antonio: dalla Vallagarina al Borgonuovo}

Si devono a Giangrisostomo Tovazzi e al suo Compendium diplomatum le prime notizie sulla famiglia di Antonio da Borgonuovo. Il nonno Cristiano proveniva da Mori, o forse dal vicino abitato di Piazzo ${ }^{1}$, piccola località nella pieve di Villa Lagarina situata pochi chilometri a sud di Pomarolo².

Anno incomperto, (saeculo XIV, ante 1367), quia initium membranae avulsum est, pretio et nomine pretii duodecim ducatorum, Anthonius filius ser Bonaventurae de Ravina diocesis Tridentinae vendidit ser Cristiano quondam ser Bertrami de villa Platii petiam unam terrae prativae positam in regula et pertinentiis Romagnani, in loco ubi dicitur ad Gardas, apud heredes Georgii de Romagnano et heredes magistri Gulielmi de Pomarollo, cum omnibus iuribus et actionibus etc. Ego Anthonius filius quondam magistri Nicholai sartoris de Nogaredo vallis Lagarinae diocesis Tridentinae imperiali auctoritate notarius publicus scripsi ${ }^{3}$.

La pergamena in questione, oggi deperdita, pervenne all'erudito francescano per mano dell'ex gesuita Alessandro Guarinoni. Il documento testimonia l'acquisto, da

Referenze fotografiche. Le figure 1 e 2 sono pubblicate con l'autorizzazione del Comune di Trento Servizio Biblioteca e Archivio storico (concessione del 23.11.2018, prot. n. C_L 378/RFS017/300360). La figura 4 è pubblicata con l'autorizzazione dell'Archivio di Stato di Verona (concessione n. 19/018 del 30.11.2018, prot. n. 4103 28.13.10/1).

1 La documentazione reperita menziona Bartolasio, padre del notaio Antonio, talvolta come figlio del fu ser Cristiano da Piazzo (pieve di Villa Lagarina), talaltra come figlio del fu ser Cristiano da Mori (nella pieve omonima); pur non potendo essere del tutto certi della corrispondenza, si ritiene, concordando con il Tovazzi, che in entrambi i casi si faccia riferimento allo stesso personaggio, non solo perché Piazzo e Mori sono due località poste a breve distanza l'una dall'altra, ma anche perché le fonti documentano un unico ser Bartolasio abitante nel quartiere di Borgonuovo, sempre menzionato come figlio del fu ser Cristiano. Fra le pergamene del convento delle Clarisse di San Michele si trovano, ad esempio, due documenti rogati rispettivamente nel 1356: G. Polli, Le Clarisse di San Michele a Trento. Ricostruzione dell'archivio ed edizione dei documenti (1193-1500), Società di Studi trentini di scienze storiche, Trento 2014 (Monografie. Nuova serie, 4), n. 111, e nel 1367: ivi, n. 114. In entrambi i casi si menziona Bartolasio ma, mentre nel documento del 1356, egli è ricordato come filius condam ser Crestiani de Murio, nell'atto del 1367 si dice che egli è filius condam ser Cristiani de Plazo plebis Lagarine.

2 Cfr. anche BFSB, ms. 26, la notizia riguardante 1'abitato di Piazzo (n. 163): «Platium, Piazzo, vicus parochiae Villanae inter Villam et Pomarolum ... In tabula anno 1339 dicitur Plazum sub gastaldia Castelnovi. In alia anno 1367 Plazum Vallis Lagarinae et Plazum plebis Lagari ... Anno 1367 vixit Bertholasius filius quondam ser Cristani de Plazo plebis Lagari habitator Tridenti».

3 BFSB, ms. 1, notizia n. 201. 
parte di ser Cristiano, di un prato situato nelle pertinenze di Romagnano di proprietà di Antonio di ser Bonaventura da Ravina ${ }^{4}$. Pochi sono i dati disponibili per permettere una datazione più circoscritta della compravendita. A tal proposito, il Tovazzi ricorda che «anno 1367 vixit quidam Bertholasius filius quondam Cristani ${ }^{5}$ de Plazo plebis Lagari habitator Tridenti. Ergo ante dictum 1367 scripta fuit haec charta» ${ }^{6}$. Un'imbreviatura sul registro del notaio Antonio da Pomarolo (1351-1357) consente, tuttavia, di retrodatare di oltre un decennio il terminus ante quem fissato da Tovazzi. Si tratta di un atto che documenta l'immissione in possesso di un terreno arativo venduto dai fratelli Iosio e Tommaso, figli di Giordano phisicus da Trento, ad Antonio del fu Filidussio da Gardumo ${ }^{7}$. Il documento, datato 31 maggio 1354, annovera fra i testimoni al negozio giuridico anche Bertolaxius condam ser Cristiani de Murio, il che porta a collocare la morte di ser Cristiano entro la fine di maggio del $1354^{8}$.

Nei pochi casi in cui le fonti menzionano il padre di Bartolasio è possibile osservare come non si riferiscano a lui come habitator Tridenti, né tantomeno come civis Tridentinus. Si deve pertanto supporre che soltanto la generazione successiva a Cristiano avesse scelto il capoluogo vescovile quale propria nuova residenza.

Numericamente maggiori sono le notizie sul figlio Bartolasio ${ }^{9}$, che la documentazione ricorda come portitor dal Borgonuovo di Trento. Il punto di partenza per ricostruire alcune note biografiche è il già citato documento del 31 maggio 1354 nel registro Instrumenta capitularia $4^{10}$. L'imbreviatura, che presenta Bartolasio fra i testimoni, costituisce la prima attestazione; da essa si deduce che nel 1354 il padre

4 Forse lo stesso Antonio citato, insieme a un fratello di nome Giacomo, il 18 marzo 1397 in ADTn, ACap, capsa 51, n. 8 .

5 Il nome corretto del nonno del notaio è Cristianus, come si riscontra anche nella documentazione di mano di Antonio da Borgonuovo. Cfr., ad esempio, i documenti in ADTn, ACap, Instrumenta capitularia 8, cc. 47r-v, n. 95: «ser Bartholasius condam ser Cristiani de Murio» e ivi, cc. $206 v, \mathrm{n}$. 413: «ser Bartholasius de Burgonovo de Tridento pater mei notarii infrascripti, filius condam ser Cristiani de Murio». La versione Cristanus si trova esclusivamente in alcune notizie del Tovazzi; nella citazione tratta dall'erudito francescano si mantiene questa seconda forma.

$6 \quad$ BFSB, ms. 1, notizia n. 201.

7 ADTn, ACap, Instrumenta capitularia 4, c. $77 v$, n. 133; edito in S. Mattivi, Il registro del notaio Antonio da Pomarolo (1351-1357). Economia e società a Trento alla metà del Trecento, tesi di laurea, Università degli Studi di Trento, a.a. 2009-2010, rel. E. Curzel, n. 143.

8 Due ulteriori documenti consentono di retrodatare la morte di ser Cristiano da Piazzo rispetto al 1367: un'imbreviatura del 24 gennaio 1356, veicolata ancora dal registro Instrumenta capitularia 4 del notaio Antonio da Pomarolo (ADTn, ACap, Instrumenta capitularia 4, c. 154v, n. 247, edito in Mattivi, Il registro del notaio Antonio da Pomarolo, cit., n. 261) e una redactio in mundum del 20 ottobre 1356, rogata dal notaio Rodolfo del fu ser Basino da Trento (APTn, Clarisse di San Michele, n. 45; edito in Polli, Le Clarisse di San Michele, cit., n. 111). In entrambi i casi Bartolasio da Borgonuovo è figlio quondam Cristiani.

9 Presentato spesso nelle fonti edite come Bertolasio dal Borgonuovo; tuttavia, nei rari casi in cui il notaio Antonio scrive per intero il nome del padre, lo cita come Bartolasio, unica dizione del nome che dunque sarà utilizzata in questa sede. Cfr., ad esempio, il documento in ADTn, ACap, Instrumenta capitularia 8, cc. 47r-v, n. 95: «... ser Bartholasius condam ser Cristiani de Murio civis et habitator Tridenti pater mei notarii infrascripti ...». Si noterà che sia Bartolasio sia il padre di costui, Cristiano, sono menzionati nelle fonti con il titolo di ser; si ricorda, a tal proposito, che tale appellativo è frequentemente attribuito nelle fonti trentine a personaggi che esercitano le professioni più varie $\mathrm{e}$ dunque non è sempre indicativo dello status professionale dell'individuo.

10 ADTn, ACap, Instrumenta capitularia 4, c. 77v, n. 133; edito in Mattivi, Il registro del notaio Antonio da Pomarolo, cit., n. 143. 


\section{Stefano Malfatti}

del notaio Antonio risiedeva già nel capoluogo vescovile ${ }^{11}$. Due anni più tardi, il 20 ottobre 1356, egli ricompare fra gli attori di un negozio giuridico a Trento ${ }^{12}$ : si tratta della riconsegna da parte del notaio Ognibene, figlio del fu magister Adelperio da Trento, del dominio utile su un appezzamento di terra vignata e arativa situato $a$ Man, località a sud-est della città, alla badessa delle Clarisse di San Michele. Nuovo affittuario del terreno è Bartolasio, il quale aveva acquistato dal notaio Ognibene il dominio utile sull'appezzamento per 36 lire di denari veronesi. Il documento rappresenta la prima traccia di una serie di attività economiche di Bartolasio.

Un ulteriore tassello è rappresentato dall'identificazione dell'abitazione del padre di ser Antonio a Trento. Due distinti instrumenta, rispettivamente del 1367 e del 1389, citano un'abitazione di Bartolasio ubicata nel quartiere di Borgonuovo; il primo documento $^{13}$ è la refuta da parte di Marco detto de la Zinella del dominio utile di una casa «cui coherent ab una parte versus meridiem et retro versus mane heredes quondam Iohannis quondam Nicolai de Mansatoribus de Tridento dicti Zoanelle, $\mathrm{ab}$ alia parte versus setentrionem Bertholasius filius quondam ser Cristiani de Plazo plebis Lagarine, habitator Tridenti, et de ante versus sero via et strata publica ${ }^{14}$. Il documento del $1389^{15}$, redatto da Antonio da Borgonuovo, è la riconsegna del dominio utile di un edificio da parte di Odorico a Sale del fu Nicolò da Ischia di Pergine. La casa, di cui si specificano le caratteristiche, «muris et lignamine edificata cum una canipa intus posita», si trova in Borgonuovo «apud Franceschinum aurificem quondam ser Pancerie de Levigo ab una parte et de retro apud ser Bartholasium portitorem de Burgonovo patrem mei notarii infrascripti ab alia parte pro utili dominio et iura dicti monasterii pro directo, apud viam comunis de ante» ${ }^{16}$.

Il Borgonuovo era il quartiere più meridionale della città sorto, a partire dal XII secolo, fuori dalla cinta muraria romana, nelle vicinanze del torrente Fersina; nella prima metà del Duecento venne incluso entro le nuove mura. Esso era costituito da formazioni edilizie in serie che si diramavano lungo strette vie, denominate androne ancora oggi ben individuabili - perpendicolari rispetto alla strada che, attraverso la porta di Santa Croce, conduceva a Verona ${ }^{17}$. Oltre ad ospitare numerose attività connesse con il commercio di transito, il quartiere costituiva il luogo di residenza di molte famiglie borghesi, nonché dei canonici del Capitolo della cattedrale ${ }^{18}$ (fig. 1).

11 Nella documentazione trentina di metà Trecento compaiono ulteriori nominativi che sembrerebbero avere, almeno dal punto di vista onomastico, qualche relazione con Cristiano e il figlio Bartolasio; fra i testimoni di una carta solutionis (ADTn, ACap, Instrumenta capitularia 4, c. 40r, n. 77b, 22 novembre 1352) vi sono, ad esempio, Francesco e Gislemberto, entrambi menzionati come figli del fu Cristiano detto Meiatus da Piazzo. Un testamento nel registro di Antonio da Pomarolo (ivi, c. $73 r$, n. 124, 15 marzo 1354) menziona Antonia figlia del fu Cristiano da Mori, famulla del testatore Marco del fu ser Hendricus da Molina di Mori; in entrambi i casi non si può tuttavia essere certi della corrispondenza con Cristiano padre di Bartolasio.

12 APTn, Clarisse di San Michele, n. 45; edito in Polli, Le Clarisse di San Michele, cit., n. 111.

Ivi, n. 47; edito ivi, n. 114.

14 Ibidem.

15 APTn, Clarisse di San Michele, n. 60a; edito in Polli, Le Clarisse di San Michele, cit., n. 128.

16 Ibidem.

17 Sulla struttura della città di Trento e, in particolare, sul quartiere di Borgonuovo cfr. R. Bocchi, Analisi dell'evoluzione della struttura urbana di Trento fino al secolo XVI, «Studi trentini di scienze storiche. Sezione seconda», 58, 1979, pp. 209-270; R. Bocchi, C. Oradini, Trento, Laterza, Roma-Bari 1983 (Le città nella storia d'Italia); R. Bocchi, C. Oradini, Immagine e struttura della città. Materiali per la storia urbana di Trento, Laterza, Roma-Bari 1983.

18 Sui luoghi di residenza dei canonici del Capitolo cattedrale di Trento cfr. E. Curzel, I canonici e il Capitolo della cattedrale di Trento dal XII al XV secolo, Edizioni Dehoniane, Bologna 2001 (Isti- 
Per una biografia di Antonio da Borgonuovo

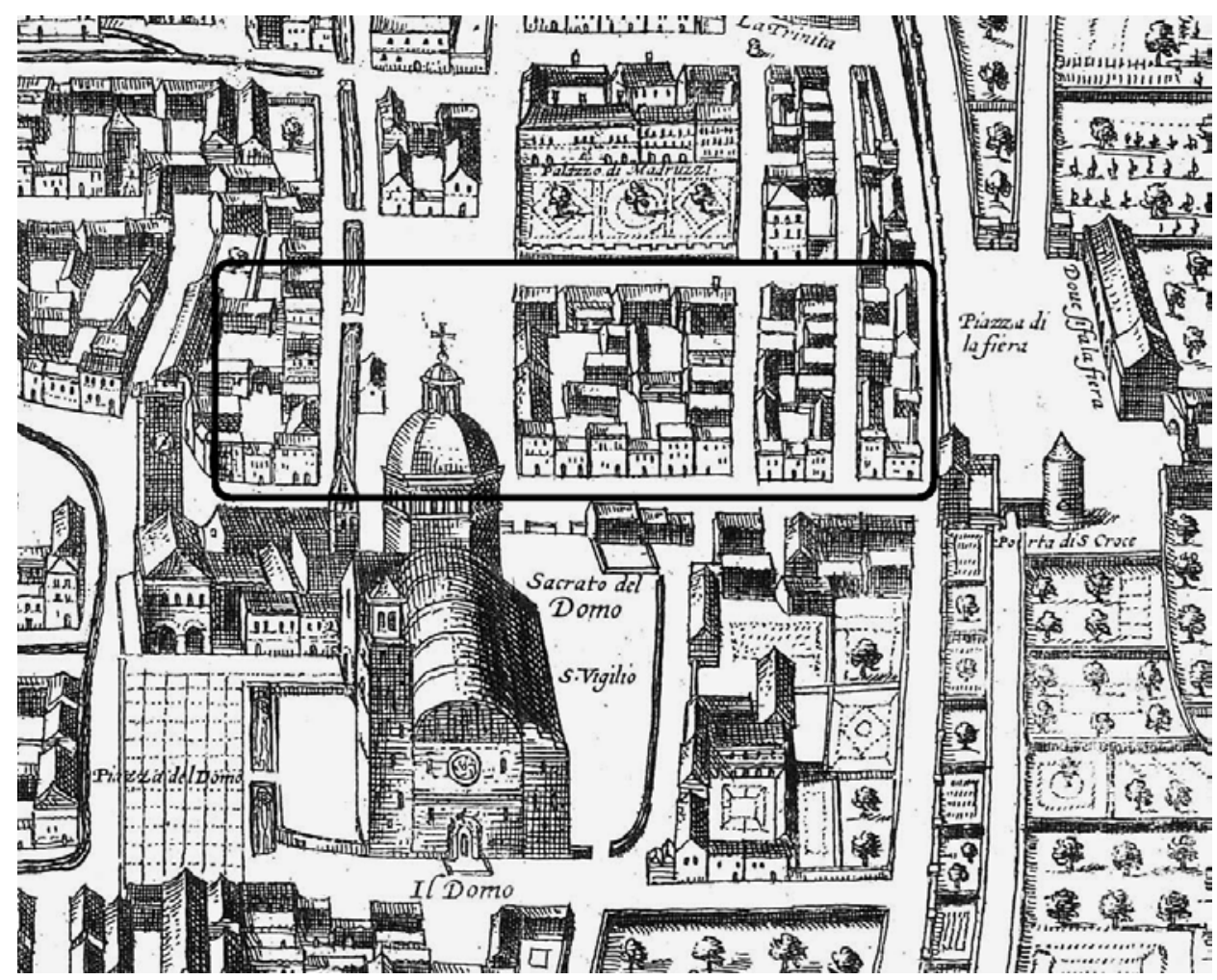

Fig. 1. Incisione su rame di Franz Hogenberg, Tridentum, Biblioteca comunale di Trento [1581]-1621 (particolare). Nella cornice l'area a sud-est della cattedrale in cui sorge il quartiere di Borgonuovo, definito talvolta nelle fonti 'contrada di San Vigilio'.

\subsection{Bartolasio da Borgonuovo. La professione e le attività economiche}

La prima attestazione di Bartolasio quale portitor è datata 24 gennaio $1356^{19}$, quando compare come testimone alla compravendita di un terreno arativo acquistato dal portitor Bartolomeo del fu Ognibene da Fadano di Brentonico; dei sette testimoni citati $^{20}$, tre sono espressamente qualificati come portitores. Il 21 luglio

tuto per le Scienze Religiose in Trento. Series maior, 8), pp. 310 e ss.; la maggior parte dei canonici aveva la propria residenza nel quartiere di Borgonuovo o nelle aree immediatamente adiacenti alla cattedrale. In particolare, alcune fra queste abitazioni erano collocate parallelamente al lato meridionale della cattedrale (attuale piazza d'Arogno), nella zona attigua al cimitero di San Vigilio.

19 ADTn, ACap, Instrumenta capitularia 4, c. $154 v$, n. 247, edito in Mattivi, Il registro del notaio Antonio da Pomarolo, cit., n. 261. Bartolomeo portitor del fu Ognibene da Fadano di Brentonico acquista da Francesco del fu Marco da Borgonuovo un arativo situato nelle pertinenze di Trento, nella località detta ad Rovredum. Le fonti a disposizione menzionano Bartolasio unicamente come portitor senza specificare se si trattasse di un semplice facchino o di un portitor vini. Le sue attività economiche, nonché il fatto che egli fu in grado di avviare il figlio Antonio alla carriera notarile fanno presumere che egli appartenesse alla categoria dei 'portatori di vino' piuttosto che ai semplici 'facchini'. Sui portitores vini cfr. L.K. Little, Indispensable Immigrants. The Wine Porters of Northern Italy and their Saints, 1200-1800, Manchester University Press, Manchester 2015, in particolare le pp. 35-63.

20 Si tratta di Trentino notaio detto a Sale del fu ser Bonaventura da Mori, Tura detto Turata portitor del fu Trentino a Ranto [?] da Trento, Florio portitor del fu Alberto da Pilcante, Bartolomeo 


\section{Stefano Malfatti}

1381, Bartolasio è tra i testimoni al testamento di Nicolò detto Rubeus ${ }^{21}$; anche in questo caso la maggior parte dei personaggi citati è costituita da portitores residenti a Trento, fra cui Bonaverio e Nicolò del fu Antonio da Isera, Bartolomeo del fu Belino da Bosentino e Bonafede del fu Giovanni da Lendinara, abitante a Pergine. La presenza di un nutrito gruppo di portitores alla registrazione delle ultime volontà di Nicolò, che il notaio rogante definisce genericamente laborator, ma che esercitava al pari degli altri testimoni la professione di portitor ${ }^{22}$, può essere considerata un elemento utile per attestare, a metà del XIV secolo, l'esistenza di una qualche forma di associazionismo fra quanti praticavano la medesima attività ${ }^{23}$. Confraternite e corporazioni medievali ${ }^{24}$ prevedevano di frequente nei propri statuti la presenza dei confratres al capezzale del malato ${ }^{25}$; sono proprio i testamenti la tipologia documentaria privilegiata per riscontrare tracce dell'esistenza di questo sodalizio nel corso del Trecento.

Il 25 maggio 1383 Bartolomeo portitor del fu Belino da Bosentino, abitante in Borgonuovo, detta le proprie disposizioni testamentarie al notaio Alberto del fu ser Negratus da $\mathrm{Sacco}^{26}$. Fra i vari lasciti, egli dispone che 10 lire di denari trentini siano lasciati al consortium portitorum di Trento «ex quibus decem libras ematur cirey pro salute anime sue et suorum mortuorum, secundum eorum consuetudinem $»^{27}$.

del fu Anselmo dal Borgonuovo, Pietro detto a Caseo del fu Martino da Campo Lomaso (Campo Iudicarie), Ognibene portitor del fu Pellegrino da Castellano e Bertoldo del fu Bonomo da Dro, tutti abitanti a Trento.

21 ADTn, ACap, capsa testamenti, rotoli lunghi/b, n. 10. Si tratta del testamento di Nicolò detto Rubeus del fu Giacomo da Folgaria laborator abitante a Trento. Cfr. l'edizione in L. Maino, 50 testamenti medievali nell'Archivio Capitolare di Trento, Liberty House, Ferrara 2001, n. 35.

22 Da quanto si ricava dalla documentazione, i portitores potevano essere talvolta definiti genericamente anche laboratores (ciò non significa che tutti i laboratores fossero dei portitores). Cfr., ad esempio, il documento in ADTn, ACap, Instrumenta Capitularia 6, c. 141r, n. 382; fra i testimoni ancora una volta, Bonaverio del fu Antonio da Isera abitante in Borgonuovo, Berto detto Cadella e Nicolò detto Rubeus, definiti laboratores dal Borgonuovo. Del primo, Bonaverio, è noto con certezza che esercitò la professione di portitor (cfr., ad esempio, ADTn, ACap, capsa testamenti, rotoli corti/a, n. 12).

23 Non mancano, difatti, altre circostanze in cui un semplice negozio giuridico diviene l'occasione per ritrovare riuniti, in qualità di testimoni, Bartolasio da Borgonuovo e altri portitores della città, ad esempio in occasione della promessa di pagamento datata 10 gennaio 1383 in $\mathrm{ADTn}$, ACap, Instrumenta capitularia 6, c. 194r, n. 517; fra i presenti, oltre a Bartolasio, ci sono Marcabruno del fu Belloto da Santorso (diocesi di Vicenza) e Bonafede del fu Giovanni da Pergine, tutti portitores abitanti a Trento.

24 Sul tema delle confratenite in Trentino in età medievale si rinvia al recente volume E. Curzel, M. Garbellotti, M.C. Rossi (a cura di), Confraternite in Trentino e a Riva del Garda, Cierre edizioni, Caselle di Sommacampagna (Vr) 2017 (Biblioteca dei Quaderni di storia religiosa, IX), con bibliografia ivi citata.

25 Cfr. L. Orioli, Le confraternite medievali e il problema della povertà: lo statuto della Compagnia di Santa Maria Vergine e di San Zenobio di Firenze, Edizioni di storia e letteratura, Roma 1985, p. 64 con la bibliografia ivi citata: «La disposizione deve essere intesa non tanto per la entità delle cifre in sé, quanto piuttosto per il valore del lascito come simbolo dell'unione che "doveva collegare anche post mortem i membri della confraternita"»; in particolare cfr. anche G. Mira, Primi sondaggi su taluni aspetti economico-finanziari delle confraternite dei disciplinati, in Risultati e prospettive della ricerca sul Movimento dei Disciplinati, Atti del convegno (Perugia, 5-7 dicembre 1969), Deputazione di storia patria per l'Umbria, Perugia 1972, p. 238, da cui Orioli trae la citazione, e G. De Sandre Gasparini, Statuti di confraternite religiose di Padova nel Medioevo. Testi, studio introduttivo e cenni storici, Istituto per la storia ecclesiastica padovana, Padova 1974, p. CXII.

26 ADTn, ACap, capsa testamenti, rotoli corti/a, n. 12; edito in Maino, 50 testamenti medievali, cit., n. 37, pp. 125-130.

27 Certamente dal XVI secolo, ma forse anche prima, i portitores avevano un proprio altare assegnato dal Capitolo della cattedrale nella chiesa di Santa Maria Maggiore, presso il quale venivano celebrate le funzioni religiose e le messe in suffragio dei defunti. 
Fra i sette testimoni, quattro sono portitores: Bonaverio del fu Antonio da Isera, Paolo del fu ser Aldrighetto da Brentonico, Michele del fu Andrea da Termeno e Luca del fu Matteo da Bolzano.

Qualche decennio più tardi, nel 1415, il notaio Antonio da Borgonuovo roga le ultime volontà di ser Nicolò a torcularibus dal Borgonuovo di Trento, figlio del fu Antonio da Isera $^{28}$. Fra gli otto testimoni chiamati a presenziare figurano cinque portitores: il padre di Antonio ser Bartolasio, Giovanni detto Rancagno del fu Nicolò da Sant'Ilario, Giovanni di Bartolasio del fu Giovanni de Alemania, Delaito del fu ser Benasuto a rotis da Trento e Matteo del fu Filippo da Sfruz. Fra i lasciti previsti dal testatore furono previste anche 20 lire da dare alla fratalia sive societas portitorum.

Se, fino alla metà del Trecento, le notizie riguardanti i portitores vini ${ }^{29}$ trentini sono limitate a sporadici nomi di personaggi che esercitavano il mestiere, con l'antico statuto dei sindici, databile alla prima metà del XIV secolo ${ }^{30}$, si fa esplicita menzione della professione. I capitoli statutari consentono infatti di chiarire con maggiore precisione le mansioni riservate ai 'portatori di vino' stabilendo, ad esempio, come sia di spettanza dei sindici il compito di sorvegliare quod vinum mensuretur ad rectam mensuram (cap. 60) e che, al fine di scongiurare ogni possibile controversia de falsis ponderibus et mensuris, questo pubblico ufficio sia sottoposto al rigido controllo del vicario vescovile. Un ulteriore capitolo (cap. 62) precisa inoltre che i sindici: «debeant constituere portitores, et quod inde recipiant iustum precium» ${ }^{31}$. Un'ulteriore norma relativa ai portitores trentini, tuttavia, doveva trovarsi già negli statuta antiqua della città se è vero, come si legge nella copia esemplata negli statuti roveretani del 1425 , che al capitolo 90, intitolato «De portatoribus tenentibus curere cum brentis ad ignem», si stabiliva che

quilibet portator teneatur continuo curere ad ignem cum brentis et aquam portare toto posse; et qui non cureret vel venire tardaret solvat domino $\mathrm{C}$ soldos pro quolibet, et si solvere non posset, stet in turrim per mensem, nisi in iusta causa remanserit ${ }^{32}$.

Nessuna traccia, invece, dei portatori nella legislazione degli anni Quaranta del Trecento, ovvero nei cosiddetti statuta nova ${ }^{33}$.

È da ricondurre verosimilmente alla seconda metà del Trecento lo sviluppo e la formazione di una vera e propria 'società' dei portitores della città di Trento; ne dà conferma, nel 1383, il già citato testamento del portitor Bartolomeo da Bosentino,

28 ADTn, ACap, capsa testamenti, rotoli medi/a, n. 16; edito in Maino, 50 testamenti medievali, cit., n. 43 , pp. $138-139$.

29 Sull'antica corporazione dei portitores a Trento cfr. G. Alberti, L'antica corporazione dei portatori di vino, «Tridentum», 2, 1899, pp. 49-90 e 149-165; S. Weber, La corporazione dei portitori, «L'Amico delle Famiglie», 22, 1914, pp. 110, 122-123; qualche dato anche in G.B. Zanella, S. Maria di Trento: cenni storici, G.B. Monauni, Trento 1879, pp. 85-86.

30 M. Welber, M. Stenico, Gli statuti dei sindici nella tradizione trentina, UTC, Trento 1997 (Collana di Storia), pp. 3-10. Il manoscritto si trova in ASCTn, Comune di Trento, Antico regime, ACT1-2545 (Libro vechio de statuti et designationi).

31 Welber, Stenico, Gli statuti dei sindici, cit., p. 9.

32 Statuti di Rovereto del 1425 con le aggiunte del 1434 e del 1538, a cura di F. Parcianello, introduzione di M. Bellabarba, G. Ortalli, D. Quaglioni, Comune di Rovereto, Biblioteca Civica - Accademia roveretana degli Agiati, Venezia 1991 (Corpus statutario delle Venezie, 9), p. 122. Come è noto, gli statuti roveretani del 1425 furono esemplati sul modello di quelli trecenteschi trentini.

33 Ivi, pp. 151-184. 
dove si fa esplicita menzione di un consortium portitorum. Si deve tuttavia attendere circa un trentennio, con la Carta edictorum et provisionum del 1407, prima che la corporazione sia nuovamente citata nelle norme statutarie cittadine. Le rivolte contro il vescovo Liechtenstein avevano avuto l'esito concreto di veder accordate alla cittadinanza nuove norme statutarie, successivamente confermate anche dal vescovo. La Carta sembra, tuttavia, limitare considerevolmente diritti e privilegi di cui da tempo i portitores potevano godere. Viene ad esempio stabilito «quod caratores et portitores civitatis Tridenti contribuere teneantur cum civibus Tridenti ad solutionem collectarum et ad contributionem quorumlibet onerarum realium et personalium, preterquam ad custodias portarum et murorum». Non devono trascorrere molti anni prima che, nel 1426, il nuovo vescovo Alessandro di Masovia ${ }^{34}$ riconfermi alla fratalia sive societas portitorum quegli antichi privilegi che, nel 1407, erano stati parzialmente aboliti. Il documento è di fondamentale importanza nella sanzione, anche legale, di questa corporazione.

Cum igitur portitores civitatis nostrae Tridenti ad portandum totum vinum nostrum pro curia nostra episcopali ad castrum Boni Consili sint astricti et lapides manuales ad proiecendum cum manibus super muros civitatis nostrae Tridenti pro ipsius defensione et aquam cum brentis ad extinguendum ignem quotiescumque aliquam domum in dicta nostra civitate contigit ardere, portare debeant et teneantur, nos, considerantes huiusmodi oneribus et servitiis quibus specialiter sunt obnoxi pariter et obbligati, ipsos a publicis functionibus realibus et personalibus, videlicet a collectis, custodiis, datiis et angariis quibuscumque nostrae civitatis praedictae Tridenti exemptos ac liberos et absolutos facimos et esse volumus. ... Statuentes et volentes quod in societate dictorum portitorum ultra viginti esse non debeant nec plures admittantur ${ }^{35}$.

L'esenzione dalle collette, ovvero dalle contribuzioni ordinarie e straordinarie, e la limitazione a venti unità del numero dei portitores procurava a questa categoria un indubitabile vantaggio economico; ciò fu probabilmente alla base della temporanea e parziale abolizione di questi benefici con la Carta del 1407. Un ulteriore privilegio, riconosciuto e confermato anche nella già citata provvisione, consisteva nell'esenzione ad custodias portarum et murorum. Si trattava di un beneficio di grande rilievo, tenuto conto dell'importanza allora attribuita alla sorveglianza delle porte e delle mura della città, da cui erano dispensate soltanto limitate categorie di cives, quali, ad esempio, i consoli. I vantaggi accordati ai portitores di vino furono concessi tenendo conto degli obblighi cui gli stessi erano sottoposti; primo fra tutti il recarsi «ad portandum totum vinum ... pro curia ... episcopali ad castrum ... Boni Consilii», cui si aggiungeva la responsabilità della difesa della città durante gli assedi e le custodias extraordinarias nei casi di imminente necessità; non ultimo per importanza, l'obbligo di accorrere portando l'acqua cum brentis per estinguere

34 Su Alessandro di Masovia cfr.: J.W. Woś, Alessandro di Masovia vescovo di Trento (14231444). Un profilo introduttivo, Trento 1990 («Civis». Supplemento, 6) e, più recenti, S. Vareschi, Profili biografici dei principi vescovi di Trento dal 1338 al 1444, «Studi trentini di scienze storiche. Sezione prima», 76, 1997, pp. 257-265; E. Curzel, I vescovi di Trento nel basso medioevo: profili personali, scelte di governo temporale e spirituale, in Storia del Trentino, III, L'età medievale, a cura di A. Castagnetti, G.M. Varanini, Il Mulino, Bologna 2004; A. Costa, I vescovi di Trento. Notizie, profili, Àncora, Milano 2017.

35 Zanella, Santa Maria di Trento, cit., pp. 85-86. 
gli incendi divampati entro le mura cittadine ${ }^{36}$. Le poche informazioni disponibili sui portitores vini trentini sono comunque di grande importanza nell'inquadramento della figura di ser Bartolasio da Borgonuovo. Come per molti altri nuovi habitatores, anche per il padre del notaio Antonio la città vescovile divenne luogo privilegiato per reperire i mezzi per una nuova fortuna economica e sociale.

È infatti nell'ambito di un progressivo accrescimento dello status familiare, economico e sociale di ser Bartolasio che si deve presumibilmente collocare l'assunzione della cittadinanza, attestata, per la prima volta, in un documento del $1413^{37}$. Per quanto concerne le norme relative alla concessione della cittadinanza a Trento, fino agli statuti del 1425 erano richiesti cinque anni di residenza, durante i quali era però prevista la completa esenzione fiscale; dagli statuti masoviani in poi (1425-1427) ${ }^{38}$, gli anni di residenza furono ridotti a tre, dopo i quali il semplice habitator veniva considerato civis Tridentinus a tutti gli effetti ed era quindi sottoposto a normale tassazione. Tali norme vanno lette alla luce della situazione economica di una città, la Trento di fine Trecento-inizio Quattrocento, in cui l'economia produttiva era praticamente assente; l'apertura nei confronti dell'elemento immigratorio (dentro e fuori i confini del Principato) va dunque interpretato come un tentativo, da parte dell'autorità vescovile, di attrarre nel capoluogo manodopera e personale di cui la città era sprovvista ${ }^{39}$.

Le notizie su ser Bartolasio da Borgonuovo, dal 1413 civis et habitator Tridenti, si susseguono - seppur frammentarie - nel corso dei primi anni del Quattrocento. Queste sporadiche attestazioni consentono di ricostruire alcune attività economiche svolte dal portitor del Borgonuovo: almeno tre sembrano infatti essere le proprietà di cui ser Bartolasio deteneva il dominio utile. La prima era un appezzamento di terra arativa situato nella contrada di Santa Croce, di cui egli vendette, nel 1406, il dominio utile a Kabriel laborator per 100 lire di denari trentini ${ }^{40}$; il notaio rogante informa inoltre che una metà del dominio utile era pervenuto a Bartolasio a titolo di eredità da parte del fu ser Pietro a Fossis dal Borgonuovo.

Il Quaternus bonorum civium Tridenti expulsorum ${ }^{41}$ del 1414 rappresenta un'ulteriore fonte di conoscenza delle attività economiche di ser Bartolasio; alla carta 7 di questo quaternus figura, fra gli affittuari di Rodolfo Belenzani, Bertolasius portitor

36 Si riprende qui quanto affermò l'Alberti sui «privilegiatissimi» portitores di vino: «quando erano liberi o avevano finito il loro compito potevano esercitare altri mestieri, poiché al loro nome si trova spesso aggiunta la privata professione di sartor, ferrar, tessadro, alla quale in particolare si erano prima dedicati senza poi trascurarla del tutto, non ostandovi alcuna disposizione speciale»: Alberti, L'antica corporazione dei portatori di vino, cit., p. 60, poi ripreso anche in Welber, Stenico, Gli statuti dei sindici, cit., p. LXXI. Lester Little ricorda, a tal proposito, come «the communes assigned other tasks to wine porters that were entirely incidental to their wine-transporting labours. The most important of these was to serve as fireman» (Little, Indispensable Immigrants, cit., p. 41).

37 ADTn, $A P$, Tomo III, n. 5.

38 Cfr. la più aggiornata edizione degli statuti di Trento in C. Bortoli, Per un'edizione dei testi statutari del Comune di Trento dei secoli $X I V-X V$, tesi di laurea, Università degli Studi di Trento, a.a. 2009-2010, rel. A. Giorgi, p. 260.

39 Sul tema dell'immigrazione a Trento nel tardo medioevo, con particolare attenzione agli arrivi soprattutto da centri esterni al Principato, cfr. S. Malfatti, Toscani a Trento nel tardo medioevo, «Studi trentini. Storia», 97, 2018, 2, pp. 409-448.

40 ADTn, ACap, Instrumenta capitularia 8, c. $206 v$, n. 413.

41 ASTn, $A P V$, Sezione latina, Codici, n. 16. 
de Burgonovo, il quale era tenuto a pagare annualmente 11 grossi di denari trentini per uno stabulum nel quartiere di residenza ${ }^{42}$.

Pochi anni più tardi, nel 1418, ser Bartolasio cedette titulo donationis inter vivos alla figlia Domenica ${ }^{43}$ e al genero Desiderato ${ }^{44}$ il dominio utile su un appezzamento di terra arativa e vignata situato a San Bartolomeo di Trento. L'atto in questione, rogato dal figlio Antonio da Borgonuovo, è seguito da una nota che specifica come ser Bartolasio avrebbe avuto donec ipse vixerit dai suddetti coniugi metà del vino ottenuto da quella vigna, pagando in loro vece al Capitolo la metà dell'affitto ${ }^{45}$.

$\mathrm{Si}$ è potuto fin qui constatare come le informazioni estratte dalla documentazione per ricostruire le linee biografiche di Bartolasio siano, tutto sommato, abbastanza consistenti. A tutto ciò è possibile aggiungere un'ulteriore notizia: il nome di Bartolasio figura, infatti, anche in un lungo elenco di benefattori che lasciarono dei beni alla confraternita dei Battuti. Il portitor compare fra i nominativi di molti altri personaggi della Trento del XIV e XV secolo, fra cui Giovanni, Antonio e Guglielmo Belenzani, Giovanni Malicie, il canonico Morandino, ser Marco de la Zinella, ser Bertoldo ab Oleo, il notaio Giacomo da Ravazzone, Lorenzo a Vaginis, Pietro Iacob, Guglielmo Gallo, Andrea Gallo e, fra i membri della famiglia Calepini, Bonaventura e i suoi figli Gottardo, Giovanni, Calepino e Donato, per citare soltanto alcuni dei

42 Alla c. $7 v$ si legge: «Item Bertolasius portitor de Burgonovo tenetur solvere de uno stabulo iacente in civitate Tridenti in contrata Burginovi apud viam publicam, apud heredes condam Gratatulle, apud ipsum Bertolasium et forte sunt confines veriores XI grossos denariorum Tridentinorum».

43 Definita soror mei in ADTn, ACap, Instrumenta capitularia 8bis, c. $100 \mathrm{v}, \mathrm{n}$. 277; viene citata un'abitazione posta nel quartiere del Borgonuovo e vicino alla casa di Domenica. Questa domus si trova sulla strada detta via regalis, per quam itur ad portam Sancte Crucis. Un'annotazione posta da ser Antonio sul dorso di una pergamena capitolare nel 1402 permette di conoscere un altro possibile membro della famiglia. L'atto registrato sul recto della medesima membrana rappresenta la locazione perpetua di una casa posta a Trento nella contrada detta Vadum Gislote a Phignonclus piliparius del fu Pietro da Rovereto, che ne aveva ricevuto il dominio utile in eredità dal magister Odorico da Pomarolo. Il notaio rogante appose, come di consueto, un breve regesto nel verso della pergamena, aggiungendo però un'ulteriore annotazione, «Nota quod simillem dedi Fignoclo cugnato meo gratis», dalla quale si deduce che potrebbe essere esistita un'altra sorella di Antonio, di cui non si conosce il nome, e che fu moglie di un certo Phignonclus (ADTn, ACap, capsa Fabbrica, rotoli lunghi, n. 8, edito in B. Tomasi, Le pergamene della capsa Fabricae dell'Archivio del Capitolo della cattedrale di Trento (1267-1674): edizione e commento, tesi di laurea, Università degli Studi di Trento, a.a. 2008-2009, rel. A. Giorgi, n. 33. Un esteso del documento si trova anche in ADTn, ACap, Instrumenta capitularia 8, c. 264r, n. 539).

44 Citati anche ivi, c. $86 v$, n. 245, 3 giugno 1427. Ysabeta moglie del fu ser Michele Approvini da Borgonuovo e Nicolò suo figlio vendono a Desiderato lanarolus del fu ser Francesco da Magrè (Schio) dal vicentino un affitto perpetuale di 3 lire di denari trentini che venivano pagati annualmente dallo stesso Desiderato a nome di Domenica sua moglie e figlia del fu Bartolasio dal Borgonuovo per una casa edificata in muratura e legno situata a Trento nel Borgonuovo, al prezzo di 60 lire di denari trentini. Desiderato lanarolus figlio del fu Francesco da Magré, al pari di ser Antonio, a partire dagli anni Venti del Quattrocento ricoprì una serie di cariche pubbliche negli uffici del comune cittadino: fu mensurator salis dal 5 febbraio 1420 al 9 febbraio 1421, ponderator pesarolli dal 9 ottobre 1429 al 2 febbraio 1430, console dall'8 ottobre 1440 al 6 novembre 1441, ancora stimator dal 6 novembre 1441 al 4 febbraio 1442 e giudice degli appelli dal maggio all'ottobre 1445; ricoprì inoltre un'ultima carica, quella di extimator possessionum, dal 12 ottobre 1449 all'8 febbraio 1450. Oltre a ciò, nel 1451, comparve come sindico della fratalia sive fraternitas di Santa Maria di Trento (per gli uffici ricoperti da Desiderato si rinvia a E. Valenti, $I l$ «liber electionum officialium magnificae communitatis Tridenti» [1415-1462]: edizione e studio introduttivo, tesi di laurea, Università degli Studi di Trento, a.a. 2003-2004, rel. G.M. Varanini; per quanto riguarda la carica di sindico della confraternita di Santa Maria cfr. BCTn, BCT3, capsa 28, mazzo 1, n. 111, 1451 dicembre 5).

45 Un ulteriore dato ricavabile da questa semplice refuta riguarda le confinazioni del terreno; esso è posto infatti a nord rispetto ad una proprietà del notaio Antonio da Borgonuovo. 
nomi più noti ${ }^{46}$ (fig. 2). È invece la documentazione del figlio Antonio a fornire gli elementi per circoscrivere la data di morte di ser Bartolasio; i dati ricavabili dalle sottoscrizioni apposte dal professionista in calce alle pergamene in mundum o agli estesi sui registri Instrumenta capitularia 8 e 8 bis consentono infatti di collocare questa data fra il 16 gennaio e il 18 febbraio del $1424^{47}$.

Merita infine un'ulteriore considerazione nella ricostruzione della struttura di questo gruppo familiare il tema dell'inurbamento. Si è rilevata la presenza in città del figlio di ser Cristiano nella seconda metà del Trecento, ma non sono note le motivazioni pratiche che spinsero Bartolasio a spostarsi dalla Vallagarina al capoluogo vescovile. Si può ipotizzare che lo spostamento della famiglia verso il centro politico e spirituale dell'episcopato, così come avvenne per molti altri nuovi habitatores in analogo scorcio d'anni, sia da ricondurre a un generalizzato flusso di inurbamento negli anni immediatamente successivi alla peste del $1348^{48}$. La Trento tardotrecentesca era una città strutturalmente debole sia dal punto di vista demografico sia dal punto di vista economico; tuttavia chi viveva in zone periferiche dell'episcopato o in aree limitrofe poteva vedere nel capoluogo vescovile una possibilità di migliorare la propria condizione economico-sociale ${ }^{49}$. Trento poté in questo modo divenire un luogo di «integrazione con le aree circostanti», soprattutto in virtù del flusso continuo di nobili rurali, mercanti, artigiani che seppero costruire e mantenere una trama di «contatti familiari, personali o clientelari» ${ }^{50}$ fra il capoluogo e il territorio da cui provenivano, incentivando, al contempo, l'arrivo di nuovi habitatores. Se da una parte la peste del 1348 ebbe probabilmente un ruolo di primo piano nel favorire questi processi, dall'altra la mobilità verso la città sembra essere collegata anche ad alcuni importanti snodi «dell'affermazione del potere vescovile», quali ad esempio «la compilazione degli statuti intorno al 1307, le Designationes communium civitatis Tridenti del 1339, la compilazione statutaria del $1340-1343 »{ }^{51}$ e, più in generale, la successiva riorganizzazione amministrativa della città per mano dei vescovi Alberto di Ortenburg e Georg Liechtenstein. Pur nell'impossibilità di trarre dalle fonti coeve dati qualitativamente e quantitativamente

46 BCTn, BCT2, n. 1083 (XV secolo).

47 Il documento del 16 gennaio 1424 è un atto matrimoniale cum dote e si trova in ADTn, ACap, Instrumenta capitularia 8bis, c. $6 v$, n. 16bis. La sottoscrizione del notaio recita: «Ego Antonius filius ser Bartholasii de Burgonovo de Tridento civis Tridentinus publicus imperiali auctoritate notarius predictis omnibus et singulis interfui et rogatus publice scripsi». Il documento datato 18 febbraio 1424 è un atto capitolare e si trova in ADTn, ACap, capsa anniversari, rotoli lunghi/d, n. 15. La sottoscrizione recita: «Ego Antonius condam ser Bartholasii de Burgonovo de Tridento civis Tridentinus publicus imperiali auctoritate notarius predictis omnibus et singulis interfui et rogatus scripsi». Pur non conoscendo la causa della morte di Bartolasio, si può ricordare che il 1424 fu un anno caratterizzato da epidemie di peste: K. Brandstätter, Vescovi, città e signori. Rivolte cittadine a Trento 1435-1437, Società di Studi trentini di scienze storiche, Trento 1995 (Monografie, 51), p. 72.

48 Cfr. E. Curzel, L. Pamato, G.M. Varanini, Giovanni da Parma, canonico della cattedrale di Trento, e la sua cronaca (1348-1377), «Studi trentini di scienze storiche. Sezione prima», 80, 2001, pp. 211-239.

49 Si questi temi cfr. anche M. Bettotti, La nobiltà trentina nel Medioevo (metà XII-metà XV secolo), Il Mulino, Bologna 2002 (Annali dell'Istituto storico italo-germanico. Monografie, 36), pp. 259-263 e M. Bellabarba, Tra la città e l'impero. Il principato vescovile di Trento nella prima età moderna, in G. Coppola, P. Schiera (a cura di), Lo spazio alpino: area di civiltà, regione cerniera, Liguori, Napoli 1991 (Europa Mediterranea. Quaderni, 5), pp. 147-164.

50 Ivi, pp. 154-155.

51 Bettotti, La nobiltà trentina nel medioevo, cit., p. 260. 


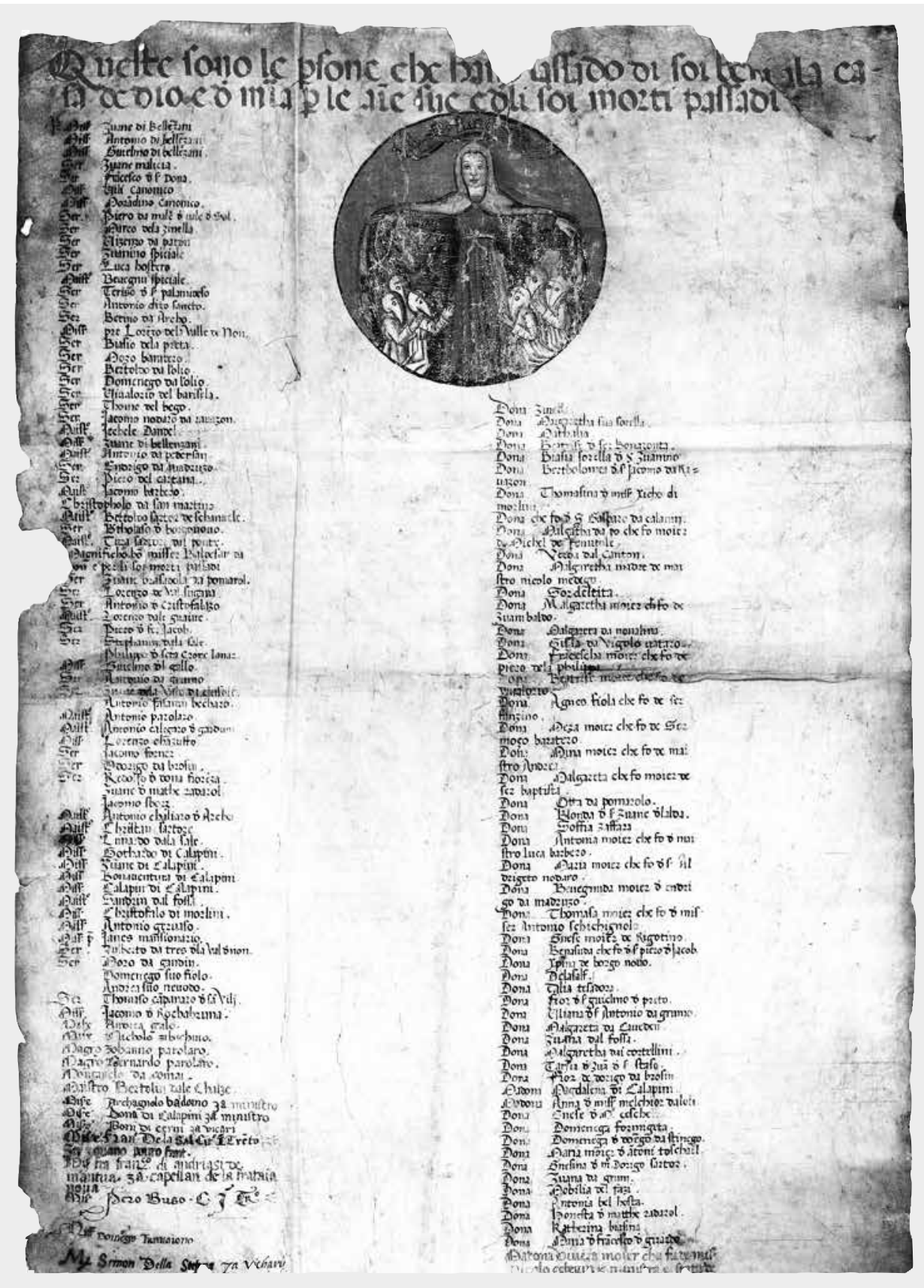

Fig. 2. BCTn, $B C T 2$, n. 1083 (particolare). Sezione superiore della pergamena del XV secolo (con aggiornamenti di XVI secolo) con l'elenco delle persone che lasciarono propri beni alla confraternita dei Battuti: «Queste sono le persone che han lassado di soi beni ala casa de Dio de misericodia per le anime sue e de li soi morti passadi». Nella colonna di sinistra, rigo 33: «Ser Bartholaso de Borgonovo», padre del notaio Antonio. 
sufficienti per stimare i valori dell'immigrazione verso il capoluogo atesino nella seconda metà del Trecento, le informazioni ricavabili dai protocolli dei notai Antonio da Pomarolo (1351-1357) ${ }^{52}$ e Alberto del fu Negratus da Sacco (1399-1402) $)^{53}$, consentono di fornire qualche stima approssimativa sui luoghi di provenienza e sulle professioni esercitate dai nuovi inurbati trecenteschi. Ne emerge una indubbia prevalenza di nuovi habitatores provenienti dai territori più prossimi al capoluogo, con un predominio di quanti giungono dalla Vallagarina ${ }^{54}$. Per quanto concerne invece le professioni svolte, prevalgono nettamente i notai, seguiti dagli artigiani (sarti, calzolai), da quanti vengono genericamente identificati come laboratores, dagli apotecarii e dai portitores.

I dati sull'immigrazione a Trento fra fine Trecento e inizio Quattrocento rendono manifesta un'indubbia importanza dei forestieri in una città dalle ridotte dimensioni, più volte falcidiata da ripetute ondate di epidemie, e in cui il ruolo economico della componente indigena fu sostanzialmente passivo, circoscritto a un'economia locale di mero consumo. Come più volte è stato messo in rilievo, l'inconsistenza delle attività manifatturiere, oltre al ruolo del tutto subordinato della città per quanto riguarda i commerci, avevano finito per relegare Trento al ruolo di «stazione di transito» ${ }^{55}$.

\section{Fra matrimoni e alleanze politiche: la classe dirigente della Trento di inizio Quattrocento}

Gli eventi che interessarono il capoluogo vescovile nei primi tre decenni del Quattrocento si rivelano di cruciale importanza nella definizione e nel consolidamento delle magistrature comunali, soprattutto per mettere in luce quel reticolo di rapporti che connetteva fra loro notai, ufficiali vescovili, mercanti che contribuirono al «rafforzamento identitario dell'istituzione comunitaria cittadina» ${ }^{56}$. Scorrendo la documentazione di inizio Quattrocento non è infatti insolito trovare iterati i nomi di famiglie e personaggi, variamente coinvolti nelle vicende del 1407-1409 e del 1435-1437, che ricoprirono con una certa regolarità ruoli di primo piano nelle magistrature urbane. L'analisi sulla classe dirigente trentina di inizio Quattrocento consentirà quindi di introdurre la figura di Antonio da Borgonuovo, le sue attività economiche, i suoi rapporti con l'élite cittadina e, in ultima analisi, la sua ascesa sociale.

Fonte imprescindibile per tentare di mettere in luce un profilo quanto più possibile definito della classe dirigente trentina è, per le vicende del primo decennio del XV secolo, il Quaternus bonorum civium Tridentinorum expulsorum de civitate Tridenti $^{57}$, che elenca i patrimoni sequestrati da Federico IV a quanti, dopo l'aprile del 1407, aderirono alla fazione capeggiata da Rodolfo Belenzani

52 Regestato in Mattivi, Il registro del notaio Antonio da Pomarolo, cit.

53 Edito in L. Zamboni, Economia e società in una piccola città alpina: Trento negli atti del notaio Alberto Negrati da Sacco (1399-1402). Con l'edizione o il regesto di 109 documenti, tesi di laurea, Università degli Studi di Trento, a.a. 1995-1996, rel. G.M. Varanini.

54 Ivi, capitolo IV (Aspetti della società urbana); Zamboni individua nel protocollo del notaio Alberto da Sacco habitatores provenienti da Lizzana, Isera, Sacco, Folàs, Nogaredo, Volano e Calliano.

55 G.M. Varanini, L'economia. Aspetti e problemi (XIII-XV secolo) in Storia del Trentino, III: L'età medievale, a cura di A. Castagnetti, G.M. Varanini, Il Mulino, Bologna 2004, p. 493.

56 F. Cagol, Dal palatium episcopatus al palatium comunis, cit., p. 207.

57 ASTn, $A P V$, Sezione latina, Codici, n. 16. 


\section{Stefano Malfatti}

contro il duca Federico. L'elenco riporta i nomi di ben ventotto cittadini variamente coinvolti negli episodi del 1407-1409. Fra i patrimoni confiscati spicca per consistenza proprio quello del referendarius Belenzani, il quale risulta proprietario di numerosi beni affittati in città e nel territorio dell'episcopato ${ }^{58}$. Ad integrazione di questa importante fonte, si farà riferimento alla documentazione d'archivio di inizio Quattrocento nonché al registro del notaio Alberto del fu ser Negratus da Sacco $^{59}$ che costituisce, per il periodo compreso fra il 1399 e il 1402, una fonte di grande rilevanza per ricostruire i rapporti fra alcuni esponenti dell'élite cittadina. Per quanto riguarda, invece, il periodo successivo, ossia gli anni compresi tra il 1402 e le rivolte antimasoviane del 1435-1437, sono di notevole importanza gli atti rogati dal notaio Antonio da Borgonuovo sui registri Instrumenta capitularia 8 e $8 \mathrm{bis}^{60}$, nonché altra documentazione reperita negli archivi trentini. Fonte primaria, accanto a quelle appena menzionate, è inoltre una frottola scritta in volgare probabilmente poco dopo gli eventi del $1435^{61}$. Si tratta di un componimento satirico composto da un autore anonimo dell'Italia settentrionale ${ }^{62}$, per il quale l'analisi linguistica rivela l'utilizzo di un volgare caratterizzato «da un ibridismo, proprio peraltro di tutti i componimenti non toscani del Quattrocento, che rende quasi impossibile una collocazione circoscritta del testo ${ }^{63}$. È pur vero tuttavia che la descrizione, talvolta dettagliata, degli episodi, dei personaggi e delle famiglie coinvolte nelle rivolte fa supporre che l'autore avesse vissuto in prima persona $i$ fatti narrati ${ }^{64}$. In poco meno di novecento versi l'anonimo rimatore elenca infatti tutti i traditori del vescovo: «una enumerazione feroce, nella quale il tradimento, la sollevazione formano come il substrato, e costituiscono la grande colpa, mentre di tutti quegli uomini che diressero il moto antivescovile, si ricercano i difetti, i vizi, spesse volte i delitti e le infamie» ${ }^{65}$.

L'erudito giudicariese Giuseppe Papaleoni, che per primo pubblicò le Rime nel 1889, ricondusse la composizione del testo agli anni compresi fra il 1435 e il 1438; tutta una serie di dati interni porta tuttavia a precisare ulteriormente il periodo di stesura del componimento satirico. Anzitutto l'anonimo fa riferimento ad Alberto d'Austria con il titolo di «dus» e non di re, il che induce a retrodatare la composizione rispetto alla data di incoronazione, il 18 marzo 1438. Un'ulteriore serie di elementi conduce addirittura a ritenere che le Rime siano state redatte in due fasi distinte: la prima parte sarebbe stata composta prima dell'arbitrato del duca Alberto d'Austria il 6 maggio del 1435, mentre la seconda potrebbe essere

58 B. Brunelli, F. Cagol (a cura di), Rodolfo Belenzani e la rivolta cittadina del 1407, Comune di Trento, Trento 2009 (Quaderni per la storia di Trento, 1), pp. 64-66.

59 BCT, BCT1, n. 1868.

60 ADTn, ACap, Instrumenta capitularia 8 e 8bis; il primo con documentazione compresa fra il 1402 e il 1434, il secondo fra il 1423 e il 1437.

61 G. Papaleoni, Rime di anonimo sulla sollevazione di Trento nel 1435, «Archivio Trentino», 8, 1889, pp. 167-207.

62 Sigismondo Antonio Manci nei suoi Annali (BCT, BCT1-1098, cc. 187-188) ipotizza che si possa trattare dello stesso Antonio da Molveno, già massaro vescovile e più tardi 'guida' della fazione filomasoviana. Per una trattazione più dettagliata sulle ipotesi di attribuzione delle rime cfr. C. Pegoretti, "Rime" di anonimo sulla sollevazione del 1435 a Trento, in E. Banfi, G. Bonfadini, P. Cordin, M. Iliescu (a cura di), Italia settentrionale: crocevia di idiomi romanzi, Atti del convegno internazionale di studi (Trento, 21-23 ottobre 1993), Max Niemeyer Verlag, Tübingen 1995, pp. 301-302.

63 Ivi, p. 304.

64 Papaleoni, Rime di anonimo, cit., p. 169.

65 Ivi, pp. 169-170. 
un'aggiunta più tarda ${ }^{66}$. Un dato, in particolare, permetterebbe di determinare con maggiore precisione la datazione di questa ipotetica seconda aggiunta; il notaio Antonio da Borgonuovo viene menzionato ai versi 723-736 del componimento ${ }^{67}$, ovvero nell'ultima parte della frottola. Di lui si dice che, a causa delle trame ordite contro il vescovo, trovò la morte durante la ribellione. Poiché l'ultimo documento disponibile redatto dal notaio risale al 24 febbraio $1437^{68}$, si deve ritenere successiva a questa data la seconda parte della frottola, mantenendo comunque come terminus ante quem l'incoronazione di Alberto d'Austria.

I nomi di quanti furono variamente coinvolti nelle rivolte del 1435-1437 si ripresentano inoltre nella breve memoria, probabilmente coeva ${ }^{69}$, che costituisce, assieme alle Rime, l'unica fonte diretta sulla sollevazione antimasoviana. Il documento descrive gli eventi occorsi il mattino del 15 febbraio del 1435, elencando di seguito i proditores del vescovo:

Et isti fuerunt illi proditores de quibus ipse dominus episcopus confidebat: primo quidem Michael a Muta, item Odoricus condam Federici de Paho, qui erat masarius et officialis predicti domini episcopi et suus vasalus; item Adelperius de Calapinis etiam vasalus domini predicti, Antoniolus a Turi dictus de Dona Mocina, magister Iohannes Luce et Lucas filius suus falsator monetarum publicus, Iacop de Archo, Melchior ab Oleo, Anthonius Bartolasii, Petrus Brusius de Sporo, Odoricus de Calapinis, Odoricus a fecibus, alias etiam derobator stratarum, Christoforus a Libeceltis, Franciscus et Iohannes Galli, Iohannes Benedictus de Vesentina, Adelpretus de Paho vasalus prelibati domini, Faustinus filius Mafei de Brixia, Nicolaus de Fundo.

L'elenco dei 'traditori' di Alessandro di Masovia è inoltre accompagnato da una lista dei cosiddetti satelites proditorum, con l'enumerazione di quanti, in vario modo, appoggiarono il partito antivescovile.

Item isti fuerunt satelites proditorum: primo Nicolaus ad Cantonum, Nicolaus Galefi, Iacobus de la Villa, Bonazonta aurifex, Magna carner, filii Odorici de Paho, Iorius magistri Iohanis Luce, filii Christofali ab hospitali, filius Ungari a Balistis et suus cognatus, Iacobus magistri Odorici fisici de Archo, filii Antonioli predicti de Mocina, Leo de Nigris sed fuit de principalibus ${ }^{70}$.

Fra le fonti utilizzate, va menzionato anche il manoscritto 3547 conservato presso l'Archivio storico del Comune di Trento e noto come Liber electionum officialium magnificae Communitatis Tridenti ${ }^{71}$. Esso documenta, in massima parte, l'attività deliberativa del Comune e le elezioni degli officiali dal 1415 al 1443 . Non mancano anche documenti di diversa tipologia, quali ad esempio locazioni, inventari ecc. ${ }^{72}$.

66 Brandstätter, Vescovi, città e signori, cit., pp. 239-240.

67 Papaleoni, Rime di anonimo, cit., p. 203.

68 ADTn, ACap, Instrumenta capitularia 8bis, c. $255 v$, nn. 665a-b.

69 L'analisi paleografica riconduce la stesura di questo scritto alla prima metà del Quattrocento.

70 ASTn, $A P V$, Sezione latina, capsa 4, n. 35.

71 ASCTn, Comune di Trento, Antico regime, Sezione antica, ACT1-3547; edito in Valenti, Il «liber electionum officialium», cit. Cfr. anche R. Fossali, Il più antico Liber actorum del Comune di Trento. Prime considerazioni per l'edizione, «Studi Trentini. Storia», 91, 2012, pp. 323-364.

72 Se ne veda una breve descrizione anche in G.M. Varanini, Gli uffici del Comune di Trento nel Quattrocento: spunti comparativi, in F. Cagol, S. Groff, S. Luzzi (a cura di), La torre di piazza nella 
Scorrendo le liste degli ufficiali addetti alle massime cariche comunitarie ${ }^{73}$ si nota come, per tutto il XV secolo, la gestione degli uffici cittadini fu nelle mani di un modesto gruppo di personaggi appartenenti a una ristretta e limitata cerchia di famiglie. Si tratta prevalentemente di giuristi e notai, ma non mancano commercianti e artigiani uniti dalla comune appartenenza a un'élite capace di esercitare un solido controllo sull'economia cittadina. Molti fra costoro erano giunti in città nella seconda metà del Trecento e nei primi anni del Quattrocento, richiamati dall'incremento delle attività commerciali e dalle riforme attuate dai vescovi Alberto di Ortenburg e Georg Liechtenstein: lo speciarius Melchiorre ab Oleo, il phisicus ser Bonadomano de Accerbis, il cirogicus Giovanni di ser Luca, l'oste Rigo da Francoforte, il daziere di Monaco di Baviera Michael Senftel, Antonio de Ceris, Francesco de Sichis, i notai Guglielmo Balzanini, Nicolò de Capris, Giovanni Conto de Fatis, Nicolò Approvini e molti altri. Essi poterono in breve tempo guadagnare posizioni di primo piano, assieme ai membri di alcune fra le più importanti famiglie dell'aristocrazia cittadina, i Belenzani, i Calepini, i Mezzasoma, i de Murlinis, i Mercadenti, i da Roccabruna, i da Molveno $^{74}$. Un ceto dirigente dalle notevoli possibilità economiche ${ }^{75}$ che, in un contesto di grande mobilità sociale, seppe aprirsi anche agli strati sociali più bassi e a homines novi ${ }^{76}$ ma, al contempo, un'élite urbana che spesso si dimostrò incapace di mantenere la necessaria compattezza di fronte ai tentativi vescovili di erodere quell'autonomia cittadina faticosamente costruita e perciò, «divisa in fazioni», si trovò ad oscillare «fra l'obbedienza al proprio signore ed i contatti con il principe del Tirolo» ${ }^{77}$.

Fra i parvenus che riuscirono in breve tempo a guadagnare posizioni di spicco nella società e nella politica cittadina, occupa un ruolo di primo piano il daziere Michael Senftel, fra i massimi esponenti del partito antivescovile durante le rivolte contro Alessandro di Masovia ${ }^{78}$. Giunto in città nei primissimi anni del Quattrocento, ricoprì già nel biennio 1412-1413 l'importante e remunerativa carica di mutarius, ufficiale del dazio, per conto del duca Federico IV di Tirolo. Forte della fiducia in lui riposta dal principe tirolese, Senftel ricevette in feudo un largo numero di proprietà che erano appartenute a Rodolfo Belenzani, fra cui alcuni immobili in città e il castello di Pietrapiana; egli inoltre rivestì cariche di prestigio, fra cui quella di massaro che, fino al maggio del 1422, era esercitata dal notaio Antonio da Molveno $^{79}$. Il prestigio economico cui Michael pervenne in breve tempo gli consentì di venire a contatto con una delle famiglie più antiche e rilevanti della città, i Calepini, che da tempo occupavano cariche urbane di rilievo. Certamente prima

storia di Trento, Atti della giornata di studio (Trento, 27 febbraio 2012), Società di Studi trentini di scienze storiche, Trento 2014 (Monografie. Nuova serie, 3), pp. 225-237.

73 Cfr., per le liste consolari, il sempre utile F. Ambrosi, Commentari della storia trentina, Artigianelli, Trento 1985 (prima ed. 1887), pp. 470 e ss.

74 Bettotti, La nobiltà trentina nel medioevo, cit., pp. 262-263.

75 Cfr., a titolo esemplificativo, fra i numerosi casi il documento in ADTn, ACap, Instrumenta capitularia 8bis, cc. $124 v-125 r$.

76 Per un confronto, per certi aspetti molto simile, con la realtà di Trento cfr. l'esempio descritto da Gian Maria Varanini per l'ambito roveretano: G.M. Varanini, La famiglia Del Bene di Rovereto nel Quattrocento: l'affermazione sociale e le attività economiche, in G.M. Varanini (a cura di), La famiglia Del Bene di Verona e Rovereto e la villa Del Bene di Volargne, Atti della giornata di studio (RoveretoVolargne, 30 settembre 1995), Accademia roveretana degli Agiati, Rovereto (Tn) 1996, pp. 9-34.

77 Bettotti, La nobiltà trentina nel medioevo, cit., p. 263.

78 Brandstätter, Vescovi, città e signori, cit., pp. 209-213.

79 TLA, Urk. I/1275; citato ivi, p. 91. 
del $1422^{80}$ egli infatti sposò Onesta, figlia del nobile ser Marco Calepini e sorella di Bonaventura, Adelperio, Odorico, Nascimbene e Calepino ${ }^{81}$. Soltanto pochi anni più tardi, nel 1428, il Senftel ricoprì per la prima volta la carica di console, ufficio che gli venne affidato consecutivamente anche negli anni 1433 e $1434^{82}$. In seguito alle sollevazioni contro il vescovo Masovia ottenne dal duca la torre Wanga, il possesso della quale gli fu confermato dal presule nel 1439 insieme al castello di Pietrapiana $^{83}$. Stante la totale assenza di notizie sul daziere negli anni seguenti si deve far risalire ad un periodo di poco successivo la data della morte ${ }^{84}$. La documentazione trasmessa dal registro Instrumenta capitularia 8bis consente di delineare con una certa precisione le attività economiche praticate dal daziere di Monaco; emerge così, negli anni compresi fra il 1423 e il 1437, il profilo di un personaggio con capacità economiche rilevanti, proprietario di numerosi immobili, affitti e terreni in città e nel territorio circostante ${ }^{85}$. Nel 1422 egli acquistò, ad esempio, per 167 ducati d'oro dal cognato Adelperio Calepini ben dodici affitti su case e terreni vignati posti a Trento e nelle vicinanze ${ }^{86}$. Degna di nota è anche la donazione che lo stesso Michael fece nel gennaio del $1429^{87}$ ai frati del convento di San Marco di Trento. Egli cedette infatti un vignale di circa 14 piovi situato a Preda Streta del ragguardevole valore di 80 ducati d'oro, in remissionem pecatorum suorum, insieme cum uno paramento fulcito da utilizzarsi per la celebrazione delle messe.

Nel tentativo di ricostruire, almeno in parte, la fitta trama dei rapporti fra $i$ membri della classe dirigente trentina, l'aiuto fornito dagli atti matrimoniali è di fondamentale importanza ${ }^{88}$. Ne è un esempio significativo il matrimonio celebrato a

80 Nel febbraio del 1422 egli risulta già sposato (ADTn, ACap, Instrumenta capitularia 8bis, c. $28 r$, n. 81$)$.

81 L'11 luglio 1439 Calepino Calepini, zio dell'omonimo noto giurisperito, detta testamento al notaio Graziadeo da Terlago. La data topica reca: «extra muros et portam Sancte Crucis de Tridento, in loco cubiculari domus habitacionis infrascripti testatoris, que domus est ser Odorici de Calapinis fratris ipsius testatoris et hedifficata in clausura ipsius ser Odorici». Calepino chiede di essere sepolto nel cimitero della chiesa di Santa Maria a Trento, lascia alla confraternita di Santa Maria dieci ducati d'oro pro anima sua; per il resto elegge quali suoi eredi universali i figli Marcadento, Pietro e Tommaso (BCTn, BCT3, capsa 28, mazzo 1, nn. 90, 91).

$82 \mathrm{Nel} 1434$ e nel 1435 ricopre anche la carica di sindaco e di gastaldo (Valenti, Il «liber electionum officialium», cit., pp. 543 e 547). Già nel 1429 egli aveva ricevuto in affitto il lago di Lidorno per un canone annuo di 4 lire di moneta trentina per volontà di Odorico Calepini, suo cognato e procuratore del Comune: «Nota che adì marti XI de zenar 1429 li consuli dela tera e my Odorigo di Chalapini si como procurador del comun cum lor e de so conseio per la utilità comuna a mostrar ch'el lago de Lidorno è del comun, afità lo dito lago in fina a dese agni a me cugnà Michel dala Muda pro affito de quatro livre al'an de moneda trentina e de questo fo rogado ser Antonius nodar quondam de ser Bertolas a farne carta et cetera» (ASCTn, Comune di Trento, Antico regime, Sezione antica, ACT13547, Liber electionum officialium (ed. in Valenti, Il «liber electionum officialium», cit.).

83 ASTn, $A P V$, Sezione latina, capsa 20, n. 27.

84 Brandstätter, Vescovi, città e signori, cit., p. 212.

85 Non è possibile, in questa sede, citare tutti i documenti attestanti l'attività economica messa in atto da Michael Senftel; fra i molti è importante ricordare che il 19 maggio del 1425 (ADT, ACap, Instrumenta capitularia 8bis, cc. 39v-40r, n. 110) egli aveva acquistato per la considerevole cifra di 200 ducati d'oro da Bartolomeo del fu Sicco, canonico di Trento, un appezzamento di terra arativa e vignata situata in località al Sale o ultra $l$ Sale.

86 Cfr. Appendice, regesti nn. 14-18, 24, 26, 27 (ADTn, ACap, Instrumenta capitularia 8bis, c. $40 v$, n. 112); si tratta di una serie di affitti su case e terreni vignati posti a Trento, Martignano, alle Laste e a Cognola.

87 Ivi, c. $118 r$, n. 322.

88 Per un'analisi dell'istituto matrimoniale in età medievale con un'edizione di buona parte degli atti matrimoniali contenuti in Instrumenta capitularia 8 bis cfr. M. Berlanda, Il matrimonio nel tardo 
castel Beseno l'11 febbraio 1400 fra il notaio e giurisperito Antonio da Molveno ${ }^{89}$ e Floridia figlia di Tommaso da Folgaria ${ }^{90}$. Come è già stato messo in evidenza da Gian Maria Varanini, il documento, redatto dal notaio Alberto del fu ser Negratus da Sacco, è particolarmente interessante per l'elenco dei testimoni presenti ${ }^{91}$. Vi figurano infatti numerosi personaggi che, di lì a pochi anni, saranno ricordati fra i protagonisti delle rivolte contro il vescovo Georg Liechtenstein: il magister Nicolò phisicus da Trento, Marco del fu Bonaventura Calepini, Rodolfo del fu Francesco Belenzani, Bartolomeo detto Cevoleta e il notaio Gioacchino Mezzasoma. Si tratta di nomi che compaiono in molti documenti rogati dal notaio Alberto da Sacco e che figureranno con grande frequenza anche nei registri di Antonio da Borgonuovo.

È infatti quest'ultimo a registrare, venticinque anni più tardi, il matrimonio fra il notaio Gioacchino Mezzasoma e Speranza, figlia del notaio ser Paolo da Trento ${ }^{92}$. Lo sposo ricevette contestualmente 350 ducati «in denariis et rebus mobilibus» dal fratello Giovanni Rauter ${ }^{93}$. Come nel caso esaminato in precedenza, la lista dei testimoni presenti risulta di grande interesse. Vengono infatti menzionati ser Pietro $\mathrm{Iacob}^{94}$, ser Bonadomano de Accerbis, ser Gianpietro da Feltre, ser Odorico Stratenperger, Adelpreto del fu ser Federico da Povo, Odorico Calepini, Palamidesio da Trento e il notaio ser Guglielmo detto Saraceno ${ }^{95}$. Anche in quest'occasione si è in presenza di cittadini che ricoprirono più volte importanti cariche comunitarie e furono variamente coinvolti nelle rivolte antimasoviane.

Soltanto tre giorni più tardi, il 30 gennaio del 1425, Antonio da Borgonuovo fu chiamato presso l'abitazione di ser Bonadomano de Accerbis ${ }^{96}$ per registrare il ma-

medioevo: studio preliminare ed edizione di documentazione notarile trentina (1424-1428), tesi di laurea, Università degli Studi di Trento, a.a. 2012-2013, rel. E. Curzel.

89 Cfr. anche Brandstätter, Vescovi, città e signori, cit., pp. 228-231 e, sul suo matrimonio, G.M.Varanini, Rodolfo Belenzani e il comune di Trento agli inizi del Quattrocento, in Brunelli, Cagol (a cura di), Rodolfo Belenzani e la rivolta cittadina del 1407, cit., pp. 9-20. Dati anche in R. Stenico, Notai che operarono nel Trentino dall'anno 845 ricavati soprattutto dal Notariale Tridentinum del P. Giangrisostomo Tovazzi, Trento 1999 (http://www.db.ofmtn.pen.net/ofmtn/files/biblioteca/Notai.pdf.; ultimo accesso $1^{\circ}$ dicembre 2018), p. 29. Sui da Molveno cfr. D. Reich, I castelli di Sporo e Belforte, Scotoni e Vitti, Trento 1901, pp. 122-130. La sorella di Antonio da Molveno era sposata con Giovanni Belenzani, un lontano parente del referendarius Rodolfo: ivi, p. 127 e C. de Festi, Memorie genealogiche sulla nobil famiglia de’ Belenzani, Pozzati, Verona 1896, p. 32.

90 Il documento è edito in Zamboni, Economia e società in una piccola città alpina, cit., n. 8.

91 Varanini, Rodolfo Belenzani e il comune di Trento agli inizi del Quattrocento, cit., p. 16.

92 Cfr. Appendice, regesti nn. 19-21 (ADTn, ACap, Instrumenta capitularia 8bis, c. 30r, n. 85a-b-c); il notaio ser Paolo da Trento ricoprì l'incarico di massaro della curia. Il matrimonio è datato 27 gennaio 1425. Il notaio Gioacchino Mezzasoma dettò il proprio testamento a Graziadeo da Terlago il 2 agosto 1439 (BCTn, BCT3, capsa 28, mazzo 1, n. 92), «in contrata Sancti Petri, in cubiculari camera domus habitacionis infrascripti testatoris». Chiese di essere sepolto nel cimitero della chiesa di Santa Maria in Trento lasciando 4 ducati alla suddetta chiesa perché vi si celebrasse l'anniversario. Dal testamento si deduce che egli ebbe una prima moglie di nome Maria. Lascia inoltre 25 lire alla confraternita di Santa Maria. Per il resto nomina propri eredi universali le figlie Giacoma e Agnese. Dona inoltre 10 lire di denari trentini alla fabbrica di San Vigilio.

93 Un altro fratello era Zambono, canonico del Capitolo della cattedrale di Trento: Curzel, I canonici e il Capitolo, cit., pp. 697-698.

94 Ricopre la carica di console nel 1418 e nel 1422: Ambrosi, Commentari della storia trentina, cit., p. 470; è a capo della confraternita dei Battuti laici tra il 1417 e il 1433: I. Dal Piaz, La confraternita dei battuti laici nella città di Trento fra il 1340 e il 1450, tesi di laurea, Università degli Studi di Verona, a.a. 1985-1986, rel. G. De Sandre Gasparini, p. 81 e ss.

95 Noto anche come de Facinis.

96 «Ricoprì più volte cariche cittadine, negli anni 1415, 1419, 1423, 1433, 1438 e 1443 nel magistrato consolare, dal giugno e quindi dall'ottobre 1434 come sindico e gastaldo, dal giugno 1439 
trimonio fra la figlia Andriota e il già citato Giovanni Rauter, fratello di Speranza'; il padre della sposa portò a Giovanni la considerevole dote di 400 ducati d'oro ${ }^{98}$; L'alleanza fra famiglie sancita dall'unione matrimoniale fu l'occasione per riunire presso la casa di ser Bonadomano alcuni fra i più illustri membri dell'élite cittadina. Fra i testimoni presenti si ricordano infatti il magister Odorico de Ruphalcatis da Arco, il magister Giovanni di Luca, i giurisperiti Antonio da Molveno e Antonio di ser Bonomo da Arco, Gioacchino Mezzasoma e ser Pietro Iacob.

Proseguendo il 'censimento' delle unioni matrimoniali registrate da ser Antonio, va menzionato, nell'anno $1424^{99}$, il matrimonio fra Aldrighetto del fu Giovanni Mezaoveta con Lucia, figlia del notaio Guglielmo Gallo. L'unione sanciva dunque l'alleanza fra la famiglia di Aldrighetto, il cui padre aveva ricoperto importanti cariche nel Comune di Trento ${ }^{100}$ e la famiglia di Lucia, il cui padre Guglielmo era pure un personaggio di spicco della classe dirigente trentina ${ }^{101}$. Fra i testimoni figurano: il già più volte citato Antonio da Molveno, il notaio Gioacchino Mezzasoma, ser Gianpietro da Feltre, i fratelli Adelperio e Odorico Calepini, Nicolò Mercadenti, Antonio de Castro e Odorico a fecibus.

Il fatto che questi documenti trovino spazio fra le carte del registro di Antonio da Borgonuovo manifesta, probabilmente, il rapporto di fiducia instaurato fra $\mathrm{i}$ membri di queste famiglie e il notaio. Fra i matrimoni registrati si hanno, inoltre, quello di Battista da Bologna ${ }^{102}$ e di Giovanni Alde ${ }^{103}$, nonché le carte dotali di Bartolomea, nipote di ser Pietro Iacob, che aveva sposato Matteo de Murlinis ${ }^{104}$, e di Clara, figlia di ser Giovanni Layner da Termeno, che aveva sposato il gestore dell'osteria alla Corona Rigo da Francoforte ${ }^{105}$.

nuovamente come sindaco e infine dal febbraio 1440 come gastaldo»: Brandstätter, Vescovi, città e signori, cit., p. 227 e nota 109.

97 Cfr. Appendice, regesti nn. 22, 23 (ADTn, ACap, Instrumenta capitularia 8bis, c. 30v, n. 86a-b-c).

98 Come sottolinea Marco Bettotti, le doti messe in campo dalle famiglie del patriziato urbano non sono neppur minimamente paragonabili a quelle rese disponibili dall'alta nobiltà trentina, che superavano non di rado i 1000 ducati e potevano raggiungere anche i 2000. Tuttavia, osserva lo studioso, cifre di 400-500 ducati erano certamente considerevoli e tali da «mettere in difficoltà le finanze di famiglie del patriziato urbano»: Bettotti, La nobiltà trentina nel medioevo, cit., p. 179.

99 ADTn, ACap, Instrumenta capitularia 8bis, c. $16 r$, n. 47, edito in Berlanda, Il matrimonio nel tardo medioevo, cit., n. 5; il matrimonio è datato 18 giugno 1424. La dote consegnata ad Aldrighetto da Guglielmo Gallo è pari a 200 ducati d'oro.

${ }^{100}$ Oltre ad essere implicato nelle vicende del 1407-1409 (Brunelli, Cagol, a cura di, Rodolfo Belenzani e la rivolta cittadina del 1407, cit., p. 48), Giovanni Mezaoveta fu eletto console per il biennio 1415-1416: Ambrosi, Commentari della storia trentina, cit., p. 470).

101 Se ne vedano le cariche ricoperte in Brandstätter, Vescovi, città e signori, cit., p. 219, nota 59.

102 Cfr. Appendice, regesti nn. 12, 13 (ADTn, ACap, Instrumenta capitularia 8bis, c. $27 r$, nn. $79[\mathrm{a}], 79[\mathrm{~b}]$ ); il matrimonio è datato 8 gennaio 1425. Battista del fu Silvestro da Bologna sposa Francesca del fu Antonio Zagagnini a clavis. La dote ammonta a 210 ducati d'oro. Battista ricoprì più volte la carica di console tra il 1418 e il 1433, nel 1435 fu scelto quale sindaco del Comune e, l'anno successivo, quale gastaldo.

103 Cfr. Appendice, regesto n. 36 (ADTn, ACap, Instrumenta capitularia 8bis, c. 88v, n. 250); il documento è datato 22 luglio 1427. Giovanni Alde sposa Beatrice sua concubina.

104 Cfr. Appendice, regesti nn. 48, 49 (ADTn, ACap, Instrumenta capitularia 8bis, c. 228r, nn. 598a-b), datato 24 febbraio 1434; Matteo de Murlinis dichiara di aver ricevuto da ser Iacob, speziale in Trento e figlio di ser Pietro Iacob, una dote di 300 ducati d'oro versati per la nipote Bartolomea.

105 Cfr. Appendice, regesto n. 37 (ADTn, ACap, Instrumenta capitularia 8bis, c. 100r, n. 276), 28 gennaio 1428; Rigo da Francoforte, hosterius a Corona, ricopre la carica consolare in ben quattro occasioni, nel 1426, nel 1430, nel 1434 e nel 1439; fu inoltre nominato iudex venditionis nel 1429 e nel 1436: Brandstätter, Vescovi, città e signori, cit., pp. 226-227. Sul matrimonio fra Rigo e Klara Layner 


\section{Stefano Malfatti}

Parlando di alleanze fra famiglie di spicco nella Trento di inizio Quattrocento, si dovrà necessariamente anticipare qualche dato sulla biografia di Antonio da Borgonuovo. Risultano infatti di grande rilevanza per chiarire almeno in parte le relazioni politico-sociali fra i membri del ceto dirigente cittadino anche i matrimoni di due figlie del notaio con i fratelli Adelperio e Bonaventura Calepini. Nel $1430^{106}$ ser Antonio registra sul volume Instrumenta capitularia 8bis un documento che egli definisce, nella consueta rubrica, Carta sive littera traditionis. Si tratta di una memoria scritta con cui il professionista ricorda la dote versata per una delle figlie ${ }^{107}$, Maddalena, ad Adelperio, «gener meus» e al fratello di costui Bonavenura, «etiam gener meus».

Universis et singulis hanc litteram inspecturis facio manifestum ego Antonius notarius condam ser Bartholasii de Burgonovo de Tridento civis Tridentinus quod cum Adelperius condam ser Marchi de Calapinis gener meus, una cum Bonaventura de Calapinis eius fratre et etiam genero meo, confessus fuerit habuisse et recepisse a me ducatos quatuorcentum boni auri et iusti ponderis in dotem et nomine dotis Magdalene filie mee et uxoris dicti Bonaventure et ipse se in solidum obligando cum dicto Bonaventura solempniter sub obligatione bonorum suorum et refectione dampnorum promisit dictas dotes reddere et restituire in omnem casum et eventum dotis restituende ${ }^{108}$.

I matrimoni cui s'è fatto cenno in precedenza, non ultimi per importanza quelli delle figlie del notaio Antonio, possono essere interpretati come vere e proprie alleanze fra famiglie che condividevano interessi politici ed economici comuni, una delle strategie messe in atto dalla borghesia cittadina per conservare stabilmente la propria presenza ai vertici degli uffici cittadini ${ }^{109}$. Non è un caso che i membri di queste famiglie - i Calepini, i Mezzasoma ${ }^{110}$, i da Molveno, gli ab Oleo, i Balzanini, gli a Sale, per citarne solo alcune - ricoprano, almeno fino a fine Quattrocento, le maggiori cariche in seno al magistrato consolare ${ }^{111}$. Lo status politico e la condizione economica divenivano così i criteri che guidavano le famiglie dell'élite cittadina nella scelta del coniuge, laddove invece le titolature nobiliari non sembravano costituire un discrimine così forte, tanto da permettere a homines novi, si è visto l'esempio di Michael Senftel, di legarsi alle più importanti famiglie della città.

Fra le famiglie cui si è accennato, i Calepini rivestono indubbiamente un ruolo di primo piano. Giunti a Trento nel corso del XIII secolo dal piccolo centro rurale

cfr. anche S. Luzzi, Stranieri in città. Presenza tedesca e società urbana a Trento (secoli XV-XVIII), Il Mulino, Bologna 2003 (Annali dell'Istituto storico italo-germanico. Monografie, 38), p. 68.

106 Il documento non è datato ma si trova fra due documenti del 1430; tenendo conto che, usualmente, i documenti nel registro sono disposti in ordine cronologico, la scrittura di questa carta sive littera traditionis potrebbe essere ricondotta al 1430 o comunque agli anni immediatamente precedenti o successivi.

${ }_{107}$ Non è noto il nome dell'altra figlia di Antonio da Borgonuovo.

108 Cfr. Appendice, regesto n. 42 (ADTn, ACap, Instrumenta capitularia 8bis, c. 145v, n. 380). Il notaio ricorda anche l'acquisto, da Adelperio, della metà pro indiviso di un affitto per un manso di 20 piovi.

109 Sulla questione cfr. anche L. Fabbri, Alleanza matrimoniale e patriziato nella Firenze del '400: studio sulla famiglia Strozzi, Olschki, Firenze 1991, pp. 195-196.

${ }^{110} \mathrm{Si}$ ricorda, a tal proposito, che nell'aprile del 1429 (ADTn, ACap, Instrumenta capitularia 8bis, cc. 124v-125r, nn. 335-336) Gioacchino Mezzasoma investì Adelperio Calepini di un mulino $a$ duabus rotis molentibus et una sega cum eius molendini et sege fulcimentis et cum aqueductibus et cavalibus suis situato nelle pertinenze di Trento nel luogo detto a la Vis, per il quale si pagava un affitto annuo di 20 ducati d'oro.

111 Ambrosi, Commentari della storia trentina, cit., pp. 470-473. 
di Fiavé nel Lomaso, essi poterono in breve tempo accumulare case e affitti in città, terreni e feudi nel circondario, fino ad assurgere alle massime cariche comunali tra la fine del Trecento e l'inizio del Quattrocento ${ }^{112}$. Fra i primi a stabilirsi definitivamente nel capoluogo vescovile c'è Bonaventura di Adelperio il quale, nel 1385, ricoprì anche il ruolo di tutore di Rodolfo Belenzani ${ }^{113}$. Il figlio di Bonaventura, Marco, risulta già nel 1395 proprietario di un mulino posto in corrispondenza della roggia grande, nella zona immediatamente ad est della cattedrale e, pochi anni più tardi, figurerà fra i cittadini espulsi dal duca tirolese Federico IV nel corso delle rivolte del 1407-1409114. È proprio con i figli di Marco - Bonaventura, Odorico, Nascimbene, Adelperio e Calepino - che la partecipazione alla vita politica della città si fa più intensa. L'anonimo autore delle Rime non risparmiò parole di disprezzo nei confronti della famiglia:

\author{
La Ca' de' Calepini \\ fo boni citadini; \\ or fatti è robatori, \\ ribaldi e traditori, \\ che non teme vergogna. \\ Li s'han tirato rogna \\ paramixina a dosso, \\ e rodasse questo osso; \\ che li sparisse i denti! \\ Tutti li soi parenti \\ se posson gloriare, \\ ancora molto exaltare \\ de sì fatte figure, \\ che li usano le usure, \\ specialmente Adelpero. \\ Credi non digi el vero, \\ che io do qual dà moline \\ che avete pase e fine ${ }^{115}$ \\ e più avanti, continua riferendosi ancora ad Adelperio
}

112 Sui Calepini cfr. G. Costisella, N. Rasmo, Il palazzo Calepini a Trento, Istituto di Credito Fondiario della Regione Tridentina, Trento 1996 e L. de Finis, L. Borrelli, M. Lupo (a cura di), Palazzo Calepini a Trento in cinque secoli di storia, Fondazione Cassa di risparmio di Trento e Rovereto, Trento 2010. Brevi cenni anche in L. Santarelli, Un giurista nel Quattrocento trentino: Calepino de Calepini, «Studi trentini di scienze storiche. Sezione prima», 75, 1996, pp. 245-265.

113 BFSB, ms. 42, n. 18: «Anno 1385. Sapiens vir dominus Bonaventura iuris utriusque peritus filius quondam domini Adelperii iudicis de Calapinis de Tridento, tutor Rodulphi pupilli filii et heredis quondam Francisci ser Balenzani de Balenzanis de Tridento, feudatarii ecclesiae Tridentinae».

114 ASTn, APV, Sezione latina, Codici, n. 16. Nell'elenco delle case confiscate al Belenzani sono presenti anche alcune proprietà poste nel quartiere del Borgonuovo, che vanno segnalate per la vicinanza con immobili di proprietà di Marco Calepini e dei da Molveno: L. de Finis, Palazzo Calepini a Trento in cinque secoli di storia, in de Finis, Borrelli, Lupo (a cura di), Palazzo Calepini a Trento, cit., p. 18.

115 Papaleoni, Rime di anonimo, cit., p. 183. Nell'edizione di Cristina Pegoretti: «La cha di Calapini, / fo boni citadinj, / or fatti è robadori / ribaldi e traditori / che non teme vergogna. / Li fan tirato rogna / paramixina adosso / e rodasse questo osso / chi li spavisse i denti. / Tutti li soi parenti / se posson gloriare / anchora molto exaltare / de sì fatte figure / che li usano le usure, / sp(ecia)lmente Adelpero. / Credi non digi el vero / che io de qual da moline, / che havete pase et fine»: C. Pegoretti, Le rime sulla sollevazione di Trento del 1435. Esame linguistico del manoscritto della biblioteca di San Bernardino in Trento, tesi di laurea, Università degli Studi di Trento, a.a. 1992-1993, rel. P. Cordin, p. 61. 


\section{Stefano Malfatti}

Questo fè far el boia

d'Adelper Calepino

a cui piase el quatrino

vegna donde se vole ${ }^{116}$.

Le accuse più pesanti sembrano essere dunque rivolte proprio ad Adelperio, duramente criticato per l'attività di prestito ad usura ${ }^{117}$. L'affermazione dell'anonimo rimatore non sembra essere del tutto infondata, se è vero che, nel luglio del 1429, Antonio Prevedonus promise ad Adelperio i 53 ducati d'oro che questi aveva dato in prestito a Domenica, vedova del magister Bonomo da Arco. Costei, non essendo in grado di restituire il denaro ricevuto, su consiglio dello stesso Adelperio vendette un suo terreno ad Antonio in modo che questi potesse costituirsi principale debitore nei confronti del Calepini ${ }^{118}$. E ancora nel 1435, Adelperio vendette, ricavandone 100 ducati d'oro, un casa con altre proprietà annesse (fra cui un orto, un prato, un mulino e un terreno arativo) che gli erano state cedute, per ordine del giudice vescovile, da Giovanazzo del fu ser Giacomo da Terlago come pagamento dei debiti che quest'ultimo aveva nei suoi confronti ${ }^{119}$.

Già feudatario del vescovo, fu eletto agli uffici comunali negli anni 1419, 1424, 1429, 1433 e 1436 $6^{120}$; nel 1425 fu chiamato in qualità di giurisperito, insieme a Bartolomeo, figlio del canonico Sicco ${ }^{121}$, ad avviare l'elaborazione dei nuovi statuti cittadini, poi confermati da Alessandro di Masovia nel $1427^{122}$. Nell'aprile del 1434 fu membro, insieme a Luca de Lippi, della delegazione inviata al vescovo presso il concilio di Basilea ${ }^{123}$. Per quanto riguarda la sua attività di iurisperitus, sembra utile citare un documento registrato dal notaio Antonio da Borgonuovo. Nel 1426 Adelperio prestò a Morandino, figlio del magister Giovanni Bono da Trento, studente in diritto civile, alcuni libri legali, fra cui «unum pulcrum Digestum Novum et unum Digestum Vetus, Decretale, Tres libros codicis et Institutioni sive Institutionis [...]

116 Ivi, p. 197. Nell'edizione di Pegoretti si legge: «Questo fe' far el boia / d'Adelper Calapino / a chui piase el quatrino / vegna donda se vole»: ivi, p. 69 .

117 Adelperio Calepini non fu certamente l'unico in città a praticare il prestito ad usura; si può ricordare infatti anche il nome di ser Gianpietro da Grigno (pure noto come «da Feltre»: Grigno, in bassa Valsugana, faceva parte della diocesi feltrina). Costui ottenne, per un breve periodo, la croce «argenteam deauratam oblatam in dicta ecclesia per reverendum in Christo patrem et dominum dominum Georgium Dei gratia olim episcopum Tridentinum» che i canonici, «ex gravi necessitate» avevano dovuto impegnare a ser Gianpietro: E. Curzel, La croce del vescovo Giorgio Liechtenstein, in Id. (a cura di), In factis mysterium legere: miscellanea di studi in onore di Iginio Rogger in occasione del suo ottantesimo compleanno, Edizioni Dehoniane, Bologna 1999 (Istituto trentino di cultura. Pubblicazioni dell'Istituto di Scienze religiose in Trento. Series maior, 6), p. 58. A testimonianza dell'attività di prestito praticata da ser Gianpietro cfr. il documento in ADT, ACap, Instrumenta capitularia 8bis, cc. $112 v-113 r$, n. 310.

${ }_{118}$ Cfr. Appendice, regesti nn. 40, 41 (ADT, ACap, Instrumenta capitularia 8bis, c. 132v, nn. 354, 355).

119 ADT, ACap, Instrumenta capitularia 8bis, c. $249 r$, n. 647.

120 Brandstätter, Vescovi, città e signori, cit., p. 214.

121 Il 9 luglio 1433 Adelperio Calepini riceve molti beni in donazione da parte di Bartolomeo domini Siconis, fra cui case, mulini e terreni posti in città e nelle vicinanze. Registrano l'atto i notai Antonio da Borgonuovo e Graziadeo da Terlago: cfr. Appendice, regesto n. 47 (ADTn, ACap, Instrumenta capitularia 8bis, cc. $218 v-219 r$, n. 579) e ASTn, APV, Sezione latina, capsa 3, n. 52.

122 «Petitionem et instantiam honorabilis viri Adelpreti de Calapinis de Tridento ad presens sindici una cum Bartholomeo domini Siconis et in dicto officio sindicorum Tridenti presidentis qui ea statuta fieri fecit pro ea communitate et de propriis denaris lucratis ex dicto officio sindicari et in ipso officio ad imperpetuum (sic) mansura»: ASCTn, Comune di Trento, Antico regime, Sezione antica, ACT1-2467.

${ }_{123}$ Cfr. Appendice, regesti nn. 50, 51 (ADTn, ACap, Instrumenta capitularia 8bis, cc. 230v-231r, nn. 604a-b). 
nomine extimationis et pro extimatione ipsorum librorum comuni concordio ibidem facta ducatorum centum et viginti quinque boni auri» ${ }^{124}$.

Il fratello di Adelperio, Odorico, esercitò le funzioni di Politmeister ${ }^{125}$ per il duca Federico IV Tascavuota fino al $1424^{126}$, mentre nel 1427 , nel 1432 e nel 1439 ricoprì la carica di console. Fra i numerosi uffici occupati si ricordano, fra il 1435 e il 1439, quelli di giudice delle tutele, sindaco, gastaldo e giudice delle appellazioni $^{127}$. Prima del 1424 sposò Sofia, figlia del notaio Pietro da Isera ${ }^{128}$.

Fra i figli di Marco Calepini vanno menzionati anche Nascimbene, canonico del Capitolo della cattedrale di Trento ${ }^{129}$ e Bonaventura. Costui, al pari degli altri fratelli, ricoprì la carica consolare in diverse occasioni: una prima volta nel 1437 e, successivamente, nel 1442, nel 1445, nel 1450, nel 1454 e infine nel $1458^{130}$. La figura di Bonaventura Calepini è rilevante non soltanto nell'ambito degli uffici comunali ma anche nei sodalizi confraternali ${ }^{131}$; già nel 1441 poté infatti esercitare incarichi di dirigenza nella confraternita di Santa Maria della Misericordia, alla quale aveva donato un calice del valore di un ducato ${ }^{132}$. Fra l'altro, pare degno di nota il fatto che quell'anno furono eletti a rezimento della Compagnia della Fradaia de Santa Maria i cittadini Bonadomano de Accerbis in qualità di ministro, Giovanni Rauter come massaro mentre, fra i consiglieri, furono scelti, oltre al già citato Bonaventura, il padre Marco, il fratello Odorico, nonché Nicolò Mercadenti ${ }^{133}$. Fra il 1446 e il 1452 egli ricoprì inoltre la carica di massaro della

124 Cfr. Appendice, regesto n. 32 (ADTn, ACap, Instrumenta capitularia 8bis, c. 71v, n. 203).

125 Si tratta, con ogni probabilità, della carica di magister bullettarum, l'ufficiale di nomina vescovile che rilasciava ai forestieri in transito per Trento la relativa 'bolletta' dietro pagamento di una proporzionata tariffa (a seconda, ad esempio, che il transito avvenisse a piedi, a cavallo, con merci ecc.). La statio bullettarum era sita presso il palazzo vescovile e, sebbene essa si trovi spesso indicata come data topica di documenti relativi alle magistrature comunali, non rientrava fra le prerogative del Comune ma vescovili (la carica non è infatti presente fra quelle di spettanza comunale registrate in ACT1-3547). Odorico Calepini rivestì infatti quell'ufficio in nome del duca Federico. A titolo esemplificativo, anche se redatto durante l'episcopato del vescovo Iohannes Hinderbach, si veda un frammento di registro del 1474, con l'elenco giornaliero delle 'bollette' rilasciate dal magister bullettarum per il transito attraverso la città di Trento (BCTn, BCT1-335). L'unità archivistica proviene in origine dall'archivio vescovile. Il registro Instrumenta capitularia 8bis permette di conoscere il nome di alcuni bullettarii in servizio fra gli anni Venti e Trenta del Quattrocento; fra il gennaio e il marzo del 1434 si menziona Cristoforo Capuzio figlio di ser Tommaso Capuzio da Como; fra il 1426 ed il 1427 viene citato il notaio Marco domine Zinele figlio di Valentino dal Borgonuovo di Trento; nel febbraio del 1429 è magister bullettarum il nobilis vir Uborch familiaris del vescovo Alessandro di Masovia; nel 1430 il notaio Giacomo del fu Cariolo dalle Giudicarie, nel 1431 e nel 1433 Antonio della Berlina del fu magister Giacomo sarto da Volano; dal dicembre del 1433 sembra ricoprire analoga carica il notaio Giovanni Conto del fu ser Paolo de Fatis da Terlago; nel luglio del 1434 Bonacordo, probabilmente figlio dello stazonerius Maffeo da Brescia.

126 TLA, Cod. 133, c. 82, citato in Brandstätter, Vescovi, città e signori, cit., p. 214.

127 Valenti, Il «liber electionum officialium», cit.

128 ADTn, ACap, Instrumenta capitularia 8bis, c. $20 r$, n. 58.

129 Cfr. la scheda biografica in Curzel, I canonici e il Capitolo, cit., p. 631.

130 Ambrosi, Commentari della storia trentina, cit., pp. 471-472. Su Bonaventura Calepini cfr. anche I. Dal Piaz, Il movimento francescano e la confraternita trentina dei Battuti in un documento del 1452, «Le Venezie francescane», 4, 1987, n.1, pp. 105-117.

131 Ivi, p. 106.

132 BFSB, ms. 14, notizia n. 329. «Anno 1441 miser Bonadoman di Acerbi Ministro della Compagnia. Odorigo, et ser Marcho de Calapini. Bonaventura de Calapini. Il detto Bonaventura ha donato alla Compagnia un caleso pagato un ducato d'oro Zuan Rauter massaro».

133 Ivi, notizia n. 353: "Anno 1441 misser Bonadiman de Ascerbi Ministro de la Compagnia, mi Zohan Rautter massaro. Odorigo et ser Marco de Calapini, Bonaventura de Calapini, Odorico de le fesse, 
confraternita dei Battuti laici nell'ambito della quale, insieme al sindaco Vricius a Vaginis, donò ai frati minori di Trento un terreno per l'edificazione di un immobile, che sarebbe diventato sede di un convento dell'osservanza francescana, poi dedicato a San Bernardino ${ }^{134}$.

Successivamente al matrimonio con Maddalena, Bonaventura spostò la propria residenza dal quartiere di San Benedetto ${ }^{135}$ al Borgonuovo, nella casa che appartenne alla famiglia Calepini sino alla fine del XVI secolo ${ }^{136}$. Dall'unione con la figlia del notaio Antonio nacquero quattro figli: Calepino ${ }^{137}$, forse il più noto giureconsulto del Quattrocento trentino, che fu in seguito anche massaro per il vescovo Iohannes Hinderbach ${ }^{138}$; il canonico e giureconsulto Gottardo ${ }^{139}$, decano della cattedrale dal 1465 e poi vicario in spiritualibus; Giovanni, che rivestì più volte la carica consolare fra il 1465 e il $1475^{140}$; e Donato che, al pari del padre, esercitò dal 1511 la carica di ministro della confraternita dei Battuti ${ }^{141}$. La moglie Maddalena, che nel frattempo era divenuta terziaria dell'ordine francescano ${ }^{142}$, fu sepolta nella chiesa del convento di San Bernardino come si deduce dalla lastra tombale che il francescano Giangrisostomo Tovazzi trascrisse nel 1795:

Magdalena iacens hic Bartolasia quondam | tertia Francisci castra secuta fidi | quae Bonaventurae Calapini ... decreti | coniunx pro meritis . . . ministra sui | Donatus filius dilectae matri dicavit MCCCCLXXXXVIIII | (stemma duplex) ${ }^{143}$.

Nicolò de Marchadento, Maistro Antonio parolar, Isepo Passera, maistro Biasio fornaser et ser Andrea compagni, honorevoli homeni eleti in rezimento della Compagnia della Fradaia de Santa Maria».

134 Edizione in Dal Piaz, Il movimento francescano, cit., pp. 115-117. L'atto di donazione avvenne soltanto tredici anni dopo la visita, a Trento, del vicario dei minori Giovanni da Capistrano, che fu in città almeno fino al 1439. Non si conosce nel dettaglio il ruolo rivestito da quest'ultimo nella fondazione del convento intitolato a San Bernardino, ma sembrano degni di nota i contatti avuti con Bonaventura Calepini, ministro della confraternita dei Battuti nel periodo in cui avvenne la donazione del terreno. Bonaventura fu infatti destinatario di una lettera del Capestrano (Zanella, S. Maria di Trento, cit., pp. 52-53) con la quale il vicario dei minori concedeva la «partecipazione ai beni spirituali del suo ordine»: Dal Piaz, Il movimento francescano, cit., p 112. Una lettera analoga fu inviata, nello stesso periodo, anche al massaro dell'ospedale di Santa Maria della Misericordia, Melchiorre $a b$ Oleo (BFSB, ms. 12-13, p. 9).

135 ASTn, $A P V$, Sezione latina, capsa 64, nn. 222 e 223.

136 Costisella, Rasmo, Il palazzo Calepini a Trento, cit., p. 8.

137 Santarelli, Un giurista nel Quattrocento Trentino: Calepino Calepini, cit.

138 BCTn, BCT1, n. 335; contiene una serie di registri cartacei (in formato 'vacheta') con le rese di conto del massaro vescovile Calepino Calepini, inerenti agli anni fra il 1478 ed il 1483. Interessante l'annotazione relativa all'uscita destinata "pro salario massariatus pro dictis tribus annis cum dimidio» ammontante a «m(archas) 87 libras $5 »$.

139 Il testamento è conservato in ADTn, ACap, capsa testamenti, rotoli lunghi/b, n. 17 ed è datato 4 gennaio 1465. Egli dispone che il suo corpo venga seppellito nel cimitero della cattedrale in molimento $[$ sic] in cui è sepolto il padre Bonaventura; lascia inoltre alla fabbrica della chiesa di San Bernardino, luogo di sepoltura della madre Maddalena, 25 lire da pagare in un'unica soluzione.

140 Ambrosi, Commentari della storia trentina, cit., p. 473.

141 Costisella, Rasmo, Il palazzo Calepini a Trento, cit., p. 331.

142 BFSB, ms. 30, n. 49.

143 G. Tovazzi, Variae inscriptiones Tridentinae, a cura di R. Stenico, con saggi di L. Borrelli e F. Leonardelli, Biblioteca Padri Francescani, Trento 1994, pp. 342-343. L'erudito trentino informa inoltre che la lastra tombale presentava, oltre all'iscrizione, anche gli stemmi della famiglia Calepini e dei Bortolasi. L'iscrizione funeraria rimase nella chiesa di San Bernardino fino al 1695, quando fu trasferita nell'odierna chiesa di San Francesco. Lì fu trascritta dal Tovazzi a fine XVIII secolo. Nel 1913 è stata coperta dal nuovo pavimento marmoreo. Sull'identificazione dello stemma della famiglia di Maddalena cfr. Costisella, Rasmo, Il palazzo Calepini a Trento, cit., p. 12; M. Lupo, scheda n. 7 
Le notizie relative alla famiglia Calepini permettono di collocare questi personaggi nell'alveo del 'partito antivescovile' e, di fatto, essi ricoprirono un ruolo non secondario nelle rivolte del 1435-1437 contro Alessandro di Masovia.

L'opposizione di queste famiglie nei confronti della politica del presule e dei suoi ufficiali non riguardò però, da quanto si può ricavare dalle fonti, i primi anni di governo del vescovo Alessandro; nel 1425, ad esempio, i fratelli Adelperio, Calepino e Odorico «attendentes eciam sincerum affectum quem predicti fratres ad nos et ecclesiam nostram gerere comprobantur» ottennero dal vescovo numerosi feudi a Terlago ${ }^{144}$, il che dimostra, quanto meno, un certo rapporto di fiducia da parte di Alessandro nei confronti della famiglia. Allo stesso modo, anche Antonio da Borgonuovo, seppur piuttosto sporadicamente come si vedrà, prestò la propria opera di pubblico notaio ad Alessandro di Masovia e ciò avvenne almeno fino all'anno $1430^{145}$. Pertanto, pur se la divergenza andò crescendo nel corso degli anni, soltanto nel periodo immediatamente precedente alla rivolta si arrivò all'aperta separazione fra quanti continuarono a sostenere il vescovo e quanti, coagulando attorno a sé il malcontento generale, si ribellarono al potere vescovile cercando l'appoggio del duca d'Austria Federico IV; fra questi ultimi si annoverano certamente anche i membri della famiglia Calepini. Non per nulla, sia l'anonimo autore delle Rime sia la memoria del 1435 non mancheranno di additare gli artefici della rivolta come proditores del vescovo, rimarcando ancor più il tradimento di quel probabile rapporto di fiducia che il presule aveva instaurato con molti di loro dopo il suo arrivo a Trento (fig. 3).

\section{I primi dati biografici su Antonio da Borgonuovo. Cenni sulle proprietà immobiliari}

Relativamente abbondanti, dato il periodo, sono le informazioni a disposizione per ricostruire la biografia del notaio Antonio da Borgonuovo. La prima notizia sul professionista corrisponde alla sua sottoscrizione in calce a un documento redatto

(Monumento funebre di Calepino Calepini), in E. Castelnuovo et alii (a cura di), Il duomo di Trento, Temi, Trento 1992, pp. 100-101 e L. Borrelli, Lo stemma dei Calepini, in de Finis, Borrelli, Lupo (a cura di), Palazzo Calepini a Trento, cit., p. 65. Nel duomo di Trento si trova il sarcofago del giurisperito Calepino Calepini $(\uparrow 1485)$, il nipote di Antonio da Borgonuovo; esso presenta, frontalmente, due putti che reggono altrettanti stemmi. A destra lo scudo della famiglia Calepini, a sinistra lo stemma che Nicolò Rasmo ha attribuito alla famiglia di Maddalena Bortolasi (o Bartolasi); Luciano Borrelli osserva invece che potrebbe trattarsi dello stemma della moglie di Calepino, il cui nome non è però noto, ma aggiunge che «è consuetudine che lo stemma della moglie sia collocato alla sinistra di quello del marito (la destra per chi guarda) ma nel monumento di Calepino le posizioni sono invertite». Permane dunque l'incertezza dell'attribuzione, stante l'impossibilità di verificare la corrispondenza di questo stemma 'anonimo' con quello visto dal Tovazzi nel 1795 nella chiesa dei francescani. Sulla questione dello stemma di famiglia di Maddalena Bortolasi è importante l'affermazione di Tovazzi che, trascrivendo la lapide funeraria della stessa Maddalena, ricorda: «Donatus Magdalenae Bartolasiae quondam Tertiariae sancti Francisci, Bonaventurae Calapini coniugi, ac matri suae dilectae, anno 1499 sepulchrum posuit in ecclesia Sancti Bernardini Fratrum Minorum Observantium apud Tridentum, cum duplici stemmate, scilicet calapiniano leonato, et bartolasiano. Lapis nunc est in ecclesia nova sancti Bernardini Fratrum Minorum Reformatorum apud Tridentum, non longe a porta principe» (BFSB, ms. 30, p. 138), il che sembra dimostrare, comunque, l'esistenza di uno stemma di famiglia anche per la figlia del notaio Antonio da Borgonuovo.

144 ASTn, $A P V$, Sezione latina, capsa 59, n. 152.

145 ADTn, ACap, Instrumenta capitularia 8bis, c. $153 r-v$, n. 397. 
Stefano Malfatti

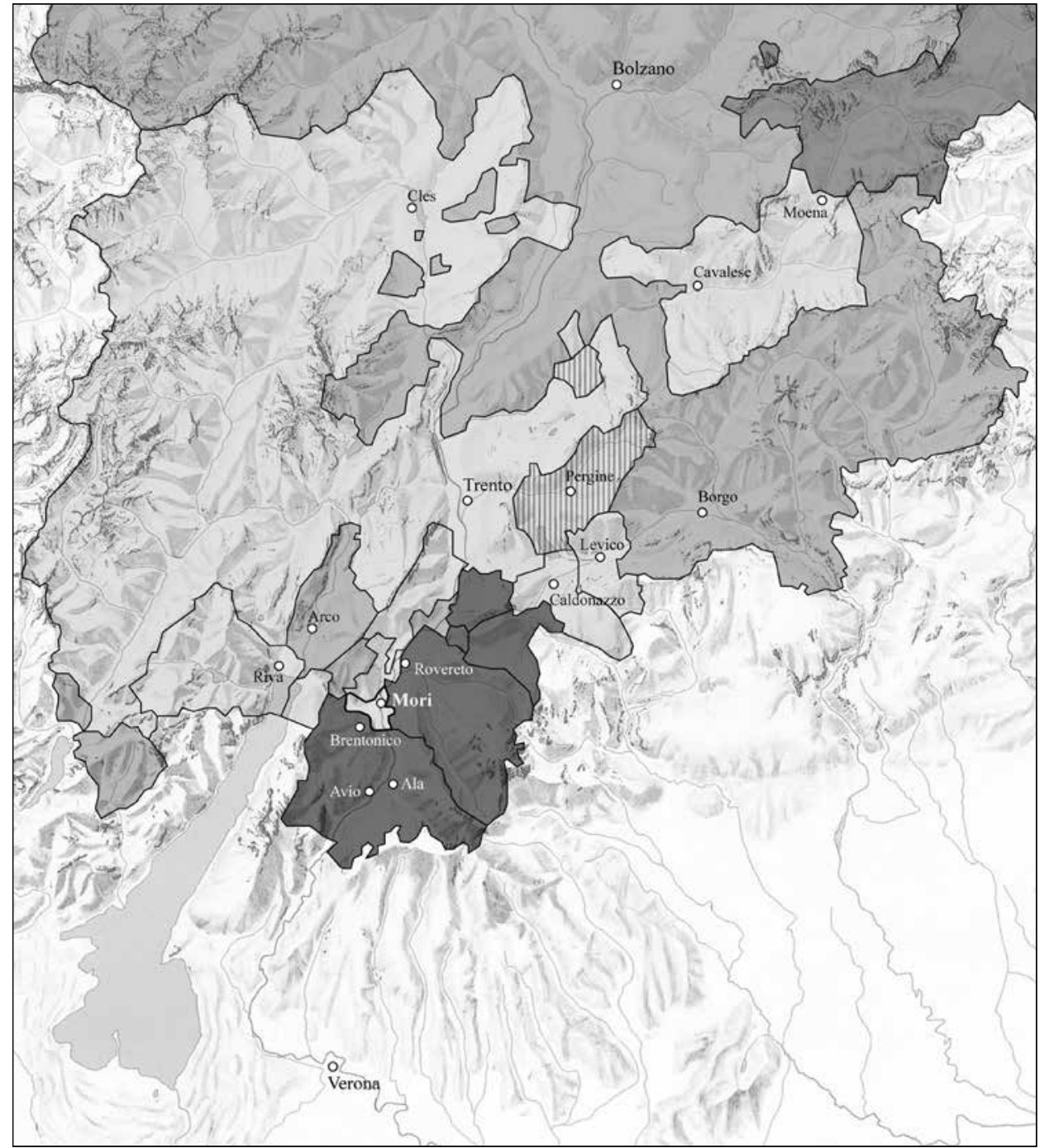

Fig. 3. Il territorio trentino-tirolese intorno al 1430 (elaborazione grafica di G. Weber).

\section{Legenda:}

$\square$ Giurisdizioni del vescovo di Trento

Giurisdizioni tirolesi

$\square$ Giurisdizioni del vescovo di Bressanone

Giurisdizioni del vescovo di Trento occupate da Venezia all'inizio del XV secolo

IIII Giurisdizioni del vescovo di Trento date in feudo ai conti del Tirolo 
il 13 febbraio del $1386^{146}$, una sentenza arbitrale emessa in episcopali palatio dai notai Vigilio da Seregnano, figlio del fu Guglielmo da Roccabruna, Alberto del fu ser Negratus da Sacco e Federico figlio di ser Ognibene da Povo, arbitri scelti per dirimere la controversia fra il canonico Morandino e Loisio detto dal Dosso. Nulla è noto prima di quella data; il suo nome non compare nelle fonti reperite né in qualità di testimone né tantomeno in qualità di notaio rogatario.

A partire dalla metà degli anni Ottanta, e con frequenza sempre maggiore dagli anni Novanta del Trecento, il nome del professionista si presenta nelle sottoscrizioni di un largo numero di documenti, prevalentemente rogati per il Capitolo della cattedrale, ma anche per privati cittadini, per l'autorità vescovile e per il Comune di Trento. Mettendo, per il momento, da parte il tema della clientela del notaio trentino, che sarà oggetto di trattazione in un capitolo specifico ${ }^{147}$, si analizzeranno ora alcune fasi nella vita del professionista che possono contribuire a mettere in luce la fitta trama di rapporti politici, economici e sociali fra i membri del ceto dirigente cittadino.

Lasciata la casa paterna, Antonio si stabilì a poca distanza dal padre Bartolasio, nel quartiere del Borgonuovo. Un paio di documenti, in particolare, consentono di individuare con una certa precisione la posizione della casa del professionista. Il primo è la riconsegna del dominio utile di uno stabulum da parte di Antonio in favore di Ognibene del fu Azzio da Calliano. Lo stabulum è posto nella contrada del Borgonuovo «apud heredes condam Bonifacii de Clusolis ab una parte versus sero, apud me Antonium ab alia parte versus mane, apud patrem meum ser Bartholasium $\mathrm{ab}$ alia parte de retro versus septentrionem et apud viam comunis de ante versus meridiem $\rangle^{148}$. Un ulteriore documento consente di delimitare con precisione ancora maggiore la probabile residenza del notaio: Bella, moglie del fu Pietro da Sant'Orsola, vende al magister Giovanni detto Darvino da Como il dominio utile su una casa situata a Trento «in contrata ecclesie kathedralis predicte ex opposito dicte sacrestie medietate via comunis, apud Michaelem Fenutoli de Sporo ab una parte et partim de retro, apud heredes condam Mucii baraterii de Tridento ab alia parte, apud me Antonium notarium infrascriptum partim de retro et apud dictam viam comunis de ante» ${ }^{149}$. Il massaro del Capitolo Bartolomeo Bonetti (fine XVI secolo) specifica ulteriormente la posizione della casa oggetto di refuta in un breve regesto posto sul verso della pergamena, affermando che essa si trova «ex opposito ecclesie Sancti Iohannis et Blasii». Le confinazioni permettono quindi di individuare nel gruppo di case poste ad oriente della cattedrale, nei pressi del cimitero di San Vigilio, l'abitazione del notaio Antonio. La sacrestia del duomo citata nel documento, oggi non più esistente, corrispondeva infatti a una struttura posta in prossimità dell'abside maggiore della basilica wangiana e accostata alla cappella dei Santi Giovanni e Biagio, la cui parte superiore è oggi nota con il nome di Castelletto ${ }^{150}$. Immediatamente a ridosso del complesso trovava posto il cimitero di San Vigilio, che si estendeva lungo le mura meridionali e orientali della cattedrale ${ }^{151}$.

${ }^{146}$ Cfr. Appendice, regesto n. 3 (ASTn, APV, Sezione latina, capsa miscellanea I, n. 126).

147 Cfr. infra, capitolo 3.

148 Cfr. Appendice, regesto n. 7 (ADTn, ACap, Instrumenta capitularia 8, c. 181v, n. 362).

149 ADTn, ACap, Instrumenta capitularia 8bis, c. 184v, n. 369a e in ADTn, ACap, capsa 1, n. 10.

150 Cfr. Curzel, I canonici e il Capitolo, cit., p. 312 e, in particolare, la nota 238.

151 Cfr. W. Landi, Il palatium episcopatus di Trento fra XI e XIII secolo. Dato documentario ed evidenze architettoniche, in Cagol, Groff, Luzzi (a cura di), La torre di piazza, cit., pp. 141-203. 


\section{Stefano Malfatti}

Si tratta di un gruppo di abitazioni situato nei pressi della Roggia Grande ${ }^{152}$, le cui evidenze documentarie attestano la presenza di numerose proprietà capitolari e vescovili. L'abitazione del notaio si trovava a brevissima distanza da quella della famiglia Calepini e, per la precisione, da quel palazzo in cui, almeno dal $1430^{153}$ in poi, trovarono stabilmente residenza la figlia Maddalena e Bonaventura Calepini ${ }^{154}$.

Per quanto concerne la struttura dell'abitazione del professionista, le poche informazioni disponibili, ricavabili prevalentemente dalle datazioni topiche dei documenti redatti, attestano la presenza di un broilo, ossia un piccolo giardino probabilmente recintato e situato nei pressi dell'abitazione ${ }^{155}$, e di scale in pietra che, presumibilmente, potevano condurre ai piani superiori ${ }^{156}$. Molti fra gli atti stesi per i privati risultano inoltre rogati in una stupa parva o vetia ${ }^{157}$ da contrapporre, con ogni probabilità, a una stupa nova che fa la sua comparsa nella documentazione del notaio a partire dal novembre del $1427^{158}$.

Quasi nulla, invece, è noto sulla moglie di ser Antonio; un unico documento, reperito sul registro Instrumenta capitularia 8, permette comunque di formulare qualche ipotesi al riguardo. L'atto in questione è la locazione di una casa di proprietà del Capitolo al notaio Gioacchino Mezzasoma, che ne aveva ereditato il dominio utile da Francesco di ser Endrico da Albiano ${ }^{159}$. Nell'usuale rubrica che ser Antonio appone in margine all'atto egli ricorda che la casa fu «condam soceri sui». Non si conosce con certezza chi sia il suocero di Antonio da Borgonuovo, tuttavia il documento informa che sull'abitazione insisteva un affitto di 5 lire di denari trentini da versare annualmente al Capitolo per la celebrazione dell'anniversario della morte di Nicolò Goseto dal Borgonuovo che ne aveva lasciato il dominio eminente ai canonici. Le possibilità sono dunque due: il notaio potrebbe aver sposato la figlia di Francesco di ser Endrico da Albiano, che deteneva il dominio utile sulla casa in Borgonuovo prima di Gioacchino Mezzasoma, oppure la figlia di Nicolò Goseto da Albiano. Si tratta indubbiamente di un'ipotesi, ma non sembra del tutto casuale il fatto che fra i testimoni al testamento di quest'ultimo personaggio, il 10 agosto del 1399, figuri in prima posizione proprio il padre del notaio Antonio, Bartolasio del fu Cristiano da Mori ${ }^{160}$. Questo secondo indizio porterebbe, dunque, a individuare nella figlia di Nicolò Goseto la moglie di Antonio da Borgonuovo.

Un tema altrettanto importante nella ricostruzione della biografia di ser Antonio è quello relativo alle sue attività economiche, ricostruibili attraverso esili tracce documentarie che attestano le sue scelte in tema di acquisto e locazione di case e

152 Corrispondente all'attuale via Carlo Dordi.

153 Anno dal quale è certamente attestato il matrimonio fra la figlia Maddalena e Bonaventura Calepini. Cfr. Appendice, regesto n. 42.

${ }^{154}$ In particolare, si osservi come l'edificio, anche oggi noto come palazzo Calepini, avesse annesso, già in età basso medievale, un orto (broilo), un pozzo, magazzini per lo stoccaggio dei rifornimenti agricoli e stabula, che si trovano menzionati in molti documenti coevi: cfr. Costisella, Rasmo, Il palazzo Calepini a Trento, cit., p. 329.

155 ADTn, ACap, Instrumenta capitularia 8bis, n. 166.

156 BCT, BCT3, capsa 4, mazzo 1, p. 2, con data topica «Tridentum, in contrata Sancti Vigilii, super via publica, iuxta scalas lapideas domus habitationis mei Antonii notarii infrascripti».

157 Cfr., fra i molti esempi, ADTn, ACap, Instrumenta capitularia 8bis, nn. 71, 102, 241.

158 Ivi, n. 260.

159 ADTn, ACap, Instrumenta capitularia 8, n. 403a.

160 ADTn, ACap, capsa testamenti, rotoli lunghi/a, n. 11

161 Nel 1396 Antonio da Borgonuovo è citato nelle confinazioni di un terreno vignato e arativo situato a San Bartolomeo, ad Planum (il terreno si trova «apud heredes ... condam Guillielmi Ropreti 
terreni. La prima notizia ${ }^{161}$ di una sua attività in questo senso risale al 18 marzo $1401^{162}$, quando acquistò per 50 ducati d'oro da Giovanni detto Zibechino il dominio utile su una casa posta nella contrada di San Marco a Trento, per la quale si pagava al Capitolo della cattedrale un affitto annuo di 40 denari $^{163}$; non passarono, tuttavia, molti mesi prima che, il 17 febbraio del $1402^{164}$, il notaio cedesse, ancora per 50 ducati, a Francesco di Giovanni detto dela Massa il dominio utile sulla casa in San Marco. Pochi mesi più tardi, il 5 maggio $1402^{165}$ il notaio riconsegnò al Capitolo il dominio utile su una clausura vignata situata in località a Brusa Laste, per 60 ducati d'oro in favore di Giovanni detto Feragu, figlio del professor gramatice Stefano da $\mathrm{Cles}^{166}$.

Nessun altro documento consente di seguire le attività economiche di ser Antonio fino al $1420^{167}$, quando egli stesso registra sul volume Instrumenta capitularia 8 la già citata refuta, per 18 ducati e mezzo, dello stabulum situato in Borgonuovo in favore di Ognibene del fu Azzio da Calliano ${ }^{168}$.

Il notaio doveva intrattenere rapporti economici anche con i membri della famiglia Calepini, come si deduce dal documento che attesta la dote versata per la figlia Maddalena ${ }^{169}$. Oltre a ricordare i 400 ducati d'oro consegnati ad Adelperio e Bonaventura Calepini a titolo di dote per la figlia, egli annota infatti l'acquisto dal genero Adelperio della metà pro indiviso di un affitto perpetuo di 24 staia di cereali che venivano pagate annualmente dai fratelli Antonio Sumptag e Federico per un manso di 20 piovi. Nel 1425 ser Antonio aveva inoltre acquistato per 16 ducati d'oro da Nicolò de Capris il dominio utile su un appezzamento di terra coltivata ad orto posta nella contrada del ponte dell'Adige, il cui dominio eminente apparteneva al monastero di San Lorenzo ${ }^{170}$.

Ciò che si conosce dell'attività economica di Antonio da Borgonuovo è quindi solo un esiguo frammento di un possibile più vasto insieme di proprietà e affitti

versus setentrionem, apud Anthonium Bertholasii versus meridiem, apud viam consortallem de subtus, apud Petrum de Burmo habitatorem in Burgonovo et apud ipsa affictalinam»: Codex Wangianus. I cartulari della Chiesa trentina (secoli XIII-XIV), a cura di E. Curzel, G.M. Varanini, con la collaborazione di D. Frioli, Il Mulino, Bologna 2007 (Annali dell'Istituto storico italo-germanico in Trento. Fonti, 5), doc. n. 249.

${ }_{162}$ Cfr. Appendice, regesto n. 4 (BCT, BCT1, n. 1868, cc. 119-120).

${ }_{163} \mathrm{Si}$ tratta probabilmente di una cifra puramente simbolica, visto il valore reale del bene.

$164 \mathrm{BCT}, B C T 1$, n. 1868, cc. 173-175; regesto in Zamboni, Economia e società in una piccola città alpina, cit., doc. n. 96.

165 ADTn, ACap, capsa 23, n. 117; il documento è rogato dal notaio Melchiorre figlio del magister Leonardo sarto da Trento. Nel verso della pergamena si legge: «Nunc magister Iohannes barberius de Arimino habitator Tridenti tenet dictam clausuram $\mid$ et bene solvit monete Tridentine libras IX, vide in Bertolasio folio CCLXXXXIX ${ }^{\circ}$ et alibi est in libro dicto Bertholasio folio $\mathrm{XXVI}^{\circ}$. In carnerio non inveni instrumentum nove locationis facte dicto magistro Iohanni manu ser Antonii Bertholasii de anno Domini $\mathrm{M}^{\circ} \mathrm{CCCC}^{\circ} \mathrm{XXXII}{ }^{\circ}$ quia hec instrumenta insimul colligata diversis temporibus et per diversos notarios scripta idem significant».

${ }^{166}$ La cifra di 60 ducati d'oro non è irrilevante, soprattutto se si tiene conto che, soltanto cinque anni più tardi, nel 1406, lo stesso Giovanni Feragu rivendette al padre Stefano il dominio utile su quel terreno per 'soli' 10 ducati.

167 Cfr. Appendice, regesto n. 6 (ADTn, ACap, Instrumenta capitularia 8, c. 47r-v, n. 95, 1418 dicembre 7); fra le confinazioni di una proprietà a San Bartolamé che il padre Bartolasio dona alla figlia Domenica e al genero Desiderato, si annovera anche un bosco (nemus) di proprietà del notaio Antonio.

168 Il documento, datato 15 luglio 1420, si trova in appendice, regesto n. 7 (ADTn, ACap, Instrumenta capitularia 8, c. $181 v$, n. 362).

${ }_{169}$ Cfr. Appendice, regesto n. 42 (ADTn, ACap, Instrumenta capitularia 8bis, c. 145v, n. 380).

170 ADTn, $A P$, Tomo II, n. 68.2. 


\section{Stefano Malfatti}

che il professionista dovette accumulare nell'arco dei circa cinquant'anni di attività. È noto, infatti, che l'acquisto di rendite immobiliari era un mezzo consueto di investimento; le poche tracce dell'attività economica di Antonio sembrano confermare questo modus operandi ${ }^{171}$. Le posizioni di prim'ordine che, come si vedrà, egli poté ricoprire in città nei primi decenni del Quattrocento, i matrimoni delle figlie con due membri della famiglia Calepini, nonché l'esercizio ininterrotto della professione notarile per i più importanti enti dell'episcopato e per privati cittadini gli consentirono indubbiamente di consolidare nel corso degli anni la propria condizione economica ${ }^{172}$.

\section{La presenza nella confraternita dei Battuti laici}

A partire dalla metà degli anni Ottanta del Trecento, come ricordato, il nome del notaio ricorre in numerosi instrumenta; fra il 1387 e il 1393 egli compare, ad esempio, in qualità di testimone in diverse investiture feudali dei vescovi Alberto di Ortenburg, redatte dal notaio e scriba vescovile Marco di ser Odorico da Trento ${ }^{173}$, e Georg Liechtenstein ${ }^{174}$.

Il suo cursus honorum prende avvio nel 1395 quando, sotto la reggenza di Giovannino Girardi ${ }^{175}$, ricopre il ruolo di massaro della confraternita dei Battuti laici ${ }^{176}$ insieme al magister Francesco barberius.

Die XXV iulii $\mathrm{M}^{\circ}$ IIIC LXXXXV creavimus masarios domus nostre Antonium notarium Bartolaxii et magistrum Francischum barberium qui receperunt a predecesoribus suis $\mathrm{XXXIIII}^{\circ}$ solidos Veronensium, videlicet ab Antonio notario condam ser Pauli et a magistro Antonio sartore de Pederxano olim massarii domus suprascripte ${ }^{177}$.

Un confronto fra i nomi di quanti ricoprirono cariche entro il sodalizio e quanti, nella prima metà del Quattrocento, rivestirono incarichi pubblici negli uffici cittadini, sembra infatti portare alla luce una serie di corrispondenze interessanti ${ }^{178}$; emerge infatti una sovrapposizione non trascurabile fra gli officiali del Comune e i

171 In riferimento ad un personaggio attivo circa un secolo prima, cfr. E. Curzel, Delaito da Noarna, notaio e "civis Tridentinus" († 1323), in F. Leonardelli, G. Rossi (a cura di), Officina humanitatis. Studi in onore di Lia de Finis, Società di Studi trentini di scienze storiche, Trento 2010, pp. 345-356.

172 Il probabile affidamento della registrazione di molti di questi contratti ad altri notai di cui oggi non si conservano più $\mathrm{i}$ rogiti non permette di seguire nel dettaglio le scelte economiche del notaio Antonio da Borgonuovo.

173 Stenico, Notai che operarono nel Trentino, cit., p. 225, sub voce.

174 Cfr. Codicis Clesiani archivi episcopalis regesta, a cura di M. Morizzo, D. Reich, Comitato diocesano tridentino, Trento [s.d.], pp. 233, 234, 236, 240, 244.

175 Costui fu nominato ministro della confraternita il 25 ottobre 1394 e conservò l'incarico almeno fino al marzo del 1414: Dal Piaz, La confraternita dei Battuti laici, cit., p. 67.

176 Ivi e, più recentemente, D. Ressegotti, Gli antichi statuti della confraternita dei Battuti, «Studi trentini. Storia», 92, 2013, pp. 65-96.

177 BCT, BCT1, n. 2387, Quaderno dei lasciti concessi alla casa della Misericordia.

178 Lorenza Pamato evidenzia una considerevole presenza di gruppi familiari ai vertici delle confraternite laiche italiane; in molti casi queste famiglie esercitarono funzioni di rilievo anche nella vita pubblica della città: cfr. L. Pamato, Le confraternite medievali. Studi e tendenze storiografiche, in G. De Sandre Gasparini, G.G. Merlo, A. Rigon (a cura di), Il buon fedele. Le confraternite tra medioevo e prima età moderna, Cierre, Sommacampagna (Vr) 1998 («Quaderni di storia religiosa», V), p. 27, citato in Ressegotti, Gli antichi statuti della confraternita dei Battuti, cit., p. 70. 
membri della confraternita, una relazione «fra $l$ 'ambiente politico-civile e $i$ gruppi istituzionalizzati di penitenti laici» ${ }^{179}$.

Alla luce delle considerazioni svolte sulle rivolte antimasoviane del 1435, non può passare inosservato il fatto che molti fra i membri del partito filoducale ricoprissero cariche di rilievo nella confraternita dei Battuti. Con l'esclusione del solo Antonio da Molveno, che sembrò parteggiare per il Masovia, molti dei confratelli risultano infatti fra i proditores che, nel febbraio del 1435, occuparono la città. È noto come spesso le confraternite furono «luoghi di costruzione e di consolidamento degli schieramenti politici» ${ }^{180}$; a Trento non vi sono però prove dirette che consentano di considerare il sodalizio dei Battuti il luogo attorno al quale si coagularono i motivi di scontento di una parte dei cives nei confronti del proprio vescovo. $\mathrm{Si}$ trattava, piuttosto, dell'immagine riflessa di un ceto cittadino, quello trentino di inizio Quattrocento, che pur manifestando evidenti e precise necessità di rinnovamento e autocoscienza, non si dimostrò sufficientemente coeso e autonomo per porsi in maniera univoca di fronte al vescovo e ai suoi ufficiali ${ }^{181}$.

Dopo aver ricoperto la carica di massaro della confraternita nel 1395, Antonio da Borgonuovo rivestì il ruolo di console nel 1415, nel 1418, nel 1422 e nel 1427. Giovanni Alde, già massaro dei Battuti nel $1404^{182} \mathrm{e}$ in seguito più volte sindaco e procuratore della confraternita, esercitò la carica di console in due occasioni, nel 1423 e nel 1427. Nicolò a Sale, massaro dal 1404 e poi nel 1418, nel 1425 e nel 1434, risulta contemporaneamente nella cerchia dei consules et provisores del Comune di Trento nel 1425. Il ministro della confraternita ser Pietro Iacob, in carica sicuramente dal 1417 al 1433, esercitò pure numerosi uffici comunitari, fra cui quello di console nel 1418 e nel 1422 e quello di giudice degli appelli nel 1417. Battista da Bologna, già vicario del ministro dei Battuti nel 1428, fu console in ben cinque occasioni: nel 1418, nel 1422, nel 1427, nel 1430 e nel 1433. Fra i personaggi degni di nota vanno inoltre ricordati Francesco del fu Adelperio de Sichis, il quale ricoprì la carica di ministro della confraternita per l'anno 1437 , e fu console nel 1417, nel 1430, nel 1435 e nel 1442; il notaio Nicolò de Capris, che nel 1441 prese il posto di Battista da Bologna quale nuovo vicario dei Battuti, esercitò la carica consolare per tre volte, negli anni 1428, 1436 e 1443, mentre fra il 1435 e il 1438 fu massaro dei sindici, giudice degli appelli, procuratore e giudice delle tutele ${ }^{183}$. Anche il ministro Bonaventura Calepini, attivo nella confraternita laica dal 1446, venne nominato console nel 1454 e nel 1458, mentre Antonio da Molveno, che ricoprì per i Battuti la carica di sindaco in una sola occasione, nel 1433, fu console già nel 1426, nel 1429 e nello stesso 1433.

179 Dal Piaz, La confraternita dei Battuti laici, cit., p. 111.

180 Cfr. ad esempio quanto rilevato da M. Berengo, L'Europa delle città. Il volto della società urbana europea tra Medioevo ed Età moderna, Einaudi, Torino 1999 (Biblioteca di cultura storica, 224), pp. 858-866.

181 Come sottolineato da Ida Dal Piaz, «le esigenze 'nuove' tipiche di questo gruppo sono dunque riconducibili non tanto ad un'opposizione di fondo alla struttura politica tradizionale, quanto piuttosto al bisogno di affermazione e di prestigio che solo la partecipazione attiva alla vita civile poteva conferire»: Dal Piaz, La confraternita dei Battuti laici, cit., p. 123.

182 Dal Piaz, La confraternita dei Battuti laici, cit., p. 66.

183 Brandstätter, Vescovi, città e signori, cit., p. 225. 


\section{Il ruolo di Antonio nelle vicende politiche di inizio Quattrocento}

Sul ruolo di alcuni fra i più importanti membri della classe dirigente trentina nelle note rivolte anti-vescovili di inizio Quattrocento si è già in parte fatto cenno; rimane ora da verificare quale parte vi rivestì il notaio Antonio da Borgonuovo, tenuto conto del fatto che, a conclusione delle vicende, l'anonimo rimatore filovescovile lo annoverò fra i proditores di Alessandro di Masovia.

Che il notaio avesse allacciato già da tempo rapporti con i più influenti membri dell'élite cittadina è cosa nota; meno noto è il ruolo che questi ricoprì già durante le rivolte degli anni Dieci contro Georg Liechtenstein. Il primo dato importante da rilevare è che in seguito a tali eventi il Comune di Trento iniziò a dotarsi di una struttura amministrativa meglio organizzata, con un organigramma maggiormente definito; seppur lentamente e con gradualità si venivano dunque consolidando le prerogative delle magistrature cittadine, non senza attriti con l'autorità vescovile: il vescovo era stato costretto, seppur temporaneamente, a riconoscere alla città alcuni privilegi, raccolti in un documento noto come Carta edictorum et provisionum ${ }^{184}$, che poi furono confermati da Federico IV Tascavuota, in seguito al rafforzamento dell'autorità tirolese sul capoluogo vescovile; la Carta aveva frattanto profondamente mutato il tessuto istituzionale della città, alla quale veniva, ad esempio, confermato il diritto a scegliere il proprio vicario, la massima autorità giudiziaria, previo riconoscimento del vescovo. A tal ufficio, che doveva essere affidato ad un forestiero esperto in diritto, competevano inoltre i casi di diritto civile e criminale $^{185}$.

L'assenza del vescovo Liechtenstein, che dopo le rivolte era stato allontanato dalla città, lasciò il Principato nelle mani del duca d'Austria Federico, il quale poté consolidare i propri rapporti con una parte della cittadinanza; una politica tutto sommato conciliante nei confronti degli interessi e dell'autonomia del capoluogo vescovile da parte del principe tirolese, il riconoscimento e il peso attribuito al Comune con le convocazioni alle varie assemblee della Landschaft ${ }^{186}$, e una serie di disposizioni in materia economica contribuirono a far sì che una parte dell'élite cittadina guardasse con benevolenza l'advocatus tirolese. Nelle complicate vicende trentine del primo decennio del Quattrocento ricoprì un ruolo non del tutto marginale anche ser Antonio.

Con lo scoppio della rivolta infatti, Georg Liechtenstein era stato costretto a riconoscere alla città, oltre ai privilegi cui si è fatto cenno, anche alcuni castelli di spettanza vescovile, fra cui quelli di Selva, Tenno e Stenico. Quest'ultimo fu occupato, su incarico del Comune, da Negro de Negri da San Pietro, membro di spicco di una famiglia della città, che lo prese in consegna da Erasmo Thun, capitano di

${ }^{184}$ Si tratta dei privilegi concessi alla città dal vescovo Liechtenstein e poi confermati dal duca Federico IV. Cfr. l'edizione in Welber, Stenico, Gli statuti dei sindici nella tradizione trentina, cit., pp. 45-54.

${ }^{185}$ Il testo è pubblicato parzialmente in H. von Voltelini, Gli antichi statuti di Trento, Accademia roveretana degli Agiati, Rovereto 1989 (ed. orig. Die ältesten Statuten von Trient und ihre Überlieferung, Wien 1902), p. 114 e in M. Bellabarba, Legislazione statutaria cittadina e rurale nel Principato vescovile di Trento (sec. XV), in P. Schiera (a cura di), 1948-1988. L'autonomia trentina. Origini ed evoluzioni fra storia e diritto, Consiglio della Provincia autonoma di Trento, Trento 1988, p. 29.

${ }^{186}$ Per un'interpretazione del termine tedesco Landschaft, di difficile resa in lingua italiana, cfr. Brandstätter, Vescovi, città e signori, cit., p. 27, nota contrassegnata da asterisco. 
quel castello almeno dal $1406^{187}$. Il nobile trentino ne mantenne il controllo fino al febbraio del 1408, quando il duca Federico IV, ormai in rotta con il Belenzani, ne ordinò la riconsegna ${ }^{188}$. Di fronte al rifiuto di Negro di restituire il castello, Federico fece arrestare alcuni cittadini di Trento, prendendo in ostaggio «specialiter qui erant de consilio civium ipsius civitatis Tridenti», e li rinchiuse «in quadam rocha Tirolenssi cum aliquibus aliis civibus» in modo che «facilius posse recuperari per intercessionem, solertiam et operationes infrascriptorum detentorum». Si tratta del medico Odorico da Arco, di Marco Calepini, di Francesco da Campo, dello speziale ser Giovanni e di Nicolò di Benvenuto da Coredo.

Il capitano Wilhelm von Matsch, luogotenente e capitano di Trento, e i suoi consiglieri fissarono inoltre il termine della successiva domenica delle Palme (8 aprile) del 1408 per la consegna del castello da parte di Negro al duca Federico. Nel caso la trattativa avesse avuto esito positivo i cittadini prigionieri sarebbero stati rimessi in libertà, in caso contrario essi avrebbero dovuto riconsegnarsi nelle mani del duca d'Austria. Per ciascuno dei prigionieri si costituirono inoltre fideiussori «sponte et precibus amicorum dictorum detentorum» alcuni cittadini di Trento, i cui nomi si ritroveranno nelle vicende politiche trentine di inizio Quattrocento. Costoro, in caso di mancata restituzione del castello al duca Federico, avrebbero dovuto presentarsi presso il Buonconsiglio alla scadenza stabilita consegnando al luogotenente la considerevole somma di 4.000 ducati d'oro per ciascun ostaggio. Antonio di Bartolasio da Borgonuovo, insieme al miles Giacomo da Roccabruna, al massaro vescovile Antonio da Molveno, al magister ciroycus Giovanni di Luca, all'apotecarius Gabriele del fu Ivano, ai fratelli Bonomo e Giacomo da Arco, e a Franceschino a candelis prestarono garanzia. Non è noto con precisione come si svolsero in seguito i fatti, tuttavia, pochi mesi più tardi, il castello pervenne nelle mani del duca Federico. Ad inizio Quattrocento, dunque, ser Antonio sembrava avere a disposizione un capitale finanziario piuttosto consolidato e aver acquisito una certa posizione in seno alla società trentina, posizione che si tradurrà negli anni a seguire, come si è visto, nell'esercizio di importanti cariche nel Comune di Trento.

\subsection{La 'parentesi' veronese di Antonio da Borgonuovo}

Se è vero, come si avrà modo di verificare nel dettaglio, che ser Antonio mantenne sempre un forte radicamento cittadino, soprattutto dal punto di vista dell'attività notarile, risulta quanto meno singolare il suo spostamento, proprio dal 1408 in poi, da Trento a Verona (fig. 4). I verbali del Consiglio cittadino della città scaligera registrano infatti come, il 25 gennaio di quell'anno ${ }^{189}$, ser Antonio venne ammesso fra i nuovi cives di Verona, insieme a un nutrito gruppo di stranieri

187 C. Ausserer, Il castello di Stenico nelle Giudicarie coi suoi signori e capitani, Scotoni e Vitti, Trento 1911, pp. 51-56 (ed. orig. Schloss Stenico in Judicarien [Süd Tirol], seine Herren und seine Hauptleute, «Jahrbuch der heraldischen Gesellschaft 'Adler' in Wien», 18, 1908), p. 50.

${ }_{188}$ Cfr. Appendice, regesto n. 5 (ASTn, $A P V$, Sezione latina, capsa 8, n. 11). La presa in possesso del castello di Stenico da parte di Negro venne sostenuta dall'intero consiglio dei sapientes del Comune di Trento. Infatti, nel 1414, gli otto rappresentanti del Comune che si erano schierati contro il duca Federico furono puniti attraverso il sequestro dei beni. Si tratta di Odorico de Ruphalcatis da Arco, di Marco Calepini, di Francesco da Castel Campo, del notaio Paolo de Fatis da Terlago, dello speziale Nicolò di Benvenuto da Coredo, di Odorico a fecibus, di Bartolomeo detto Toscanelo, di Giovanni Mezaoveta e di Bonifacio da Chiusole (cfr. Brunelli, Cagol, Rodolfo Belenzani e la rivolta cittadina del 1407, cit., p. 48).

189 ASVr, Antico archivio del Comune, Atti del consiglio, n. 56, c. $97 r$. 
fra cui anche il trentino Melchiorre del fu Domenico ab Oleo. Sulle motivazioni che spinsero ser Antonio a chiedere la cittadinanza veronese non si possono che formulare ipotesi. Un certo peso possono aver avuto ragioni di tipo economico, tuttavia, la concomitanza fra la concessione della cittadinanza veronese al notaio e il culmine delle rivolte antivescovili a Trento potrebbe far pensare che il notaio, insieme ad altri cittadini di Trento, avesse voluto assicurarsi un 'riparo' più sicuro nella città veneta. È proprio il cronista veronese Bartolomeo Lando ad annotare, in analogo scorcio d'anni, sul Liber dierum iuridicorum del Comune di Verona, come la città ospitò «misere mulieres cum parvulis» che erano fuggiti da Trento. Ricorda il cronista che i soldati tirolesi «omnes Tridentinos et Italicos quoscumque invenerunt sevientes gladio trucidarunt, civitatem diebus sequentibus depredabantur, maximam eius partem incendio consumpserunt ${ }^{190}$; non menziona tuttavia la presenza di esuli 'politici'.

Sulle attività veronesi di ser Antonio poco si può dire. Le fonti veronesi consultate, fra cui le verbalizzazioni del Comune, gli estimi cittadini, le anagrafi di epoca veneziana e, infine, i documenti notarili copiati sui volumi dell'Ufficio del Registro a partire dal $1408^{191}$, non forniscono molte informazioni. Ne emerge così che Antonio fu registrato una prima volta nell'estimo del 1409, residente nella centralissima contrada di San Giovanni in Foro, nelle immediate vicinanze dell'attuale piazza delle Erbe ${ }^{192}$, avendo come suo responsalis il notaio veronese Giacomo de Bonalinis. Due dati si possono ricavare dalla registrazione: il fatto che, nel 1409 , gli venisse affiancato un responsalis può dipendere da un lato dalla sua recente acquisizione della cittadinanza veronese, dall'altro dal fatto che in realtà ser Antonio non fosse stabilmente residente in città, e dunque avesse bisogno di un garante che rispondesse fiscalmente di fronte all'amministrazione cittadina del suo patrimonio; tipicamente - non si può dire con certezza se questo è il caso - il responsalis era un socio in affari ma, allo stato attuale, non si conosce il rapporto fra il notaio trentino e il veronese. Il secondo, importante dato che si deduce dall'allibramento del 1409 è la cifra d'estimo che viene associata a ser Antonio: 1 lira. Si tratta, come si ricava dagli studi di Amelio Tagliaferri ${ }^{193}$, di un coefficiente di buon livello, raggiunto, nel 1409 , da poco più del $9 \%$ degli estimati veronesi. Gli viene poi confermato, nell'estimo del 1418, il medesimo livello contributivo ${ }^{194}$, mentre risulta

190 Varanini, Rodolfo Belenzani e il comune di Trento agli inizi del Quattrocento, cit.; in particolare, le note sulla cronaca veronese del Lando, in G.M. Varanini, Le annotazioni cronistiche del notaio Bartolomeo Lando sul Liber dierum iuridicorum del comune di Verona (1405-1412), in A. Castagnetti, A. Ciaralli, G.M. Varanini, Medioevo. Studi e documenti, II, Libreria Universitaria Editrice, Verona 2007, pp. 551-604.

191 Come noto, nel 1723 un rogo distrusse quasi completamente l'archivio del Collegio dei notai di Verona; ciò che rimane della documentazione notarile veronese è quanto, in copia autentica, venne registrato nei volumi dell'Ufficio del Registro: ciò che nei volumi oggi si legge è tuttavia una parte di quanto venne in origine rogato dai notai veronesi, per di più copiato senza rispettare un preciso ordine cronologico. Sul tema cfr. G. Sancassani, Il Collegio dei Notai di Verona, in G. Sancassani, M. Carrara, L. Magagnato (a cura di), Il notariato veronese attraverso i secoli. Catalogo della mostra in Castelvecchio, Collegio Notarile di Verona, Verona 1966, p. 19. Sull'Ufficio del Registro cfr. A. Vitaliani, L'organizzazione e il funzionamento dell'Ufficio del Registro in Verona nei primi decenni del sec. XV, «Atti dell'Accademia di agricoltura, scienze e lettere di Verona», s. V, 16, 1938, pp. 199-218 e G. Sancassani, L'archivio dell'Antico Ufficio del Registro di Verona, «Vita veronese», 10, 1957, pp. 481-490.

192 ASVr, Antico archivio del Comune, Estimi, reg. 249, c. $99 r$.

193 A. Tagliaferri, L'economia veronese secondo gli estimi dal 1409 al 1635, Giuffrè, Milano 1966, p. 64.

194 ASVr, Antico archivio del Comune, Estimi, reg. 250, c. 52v. 
Per una biografia di Antonio da Borgonuovo

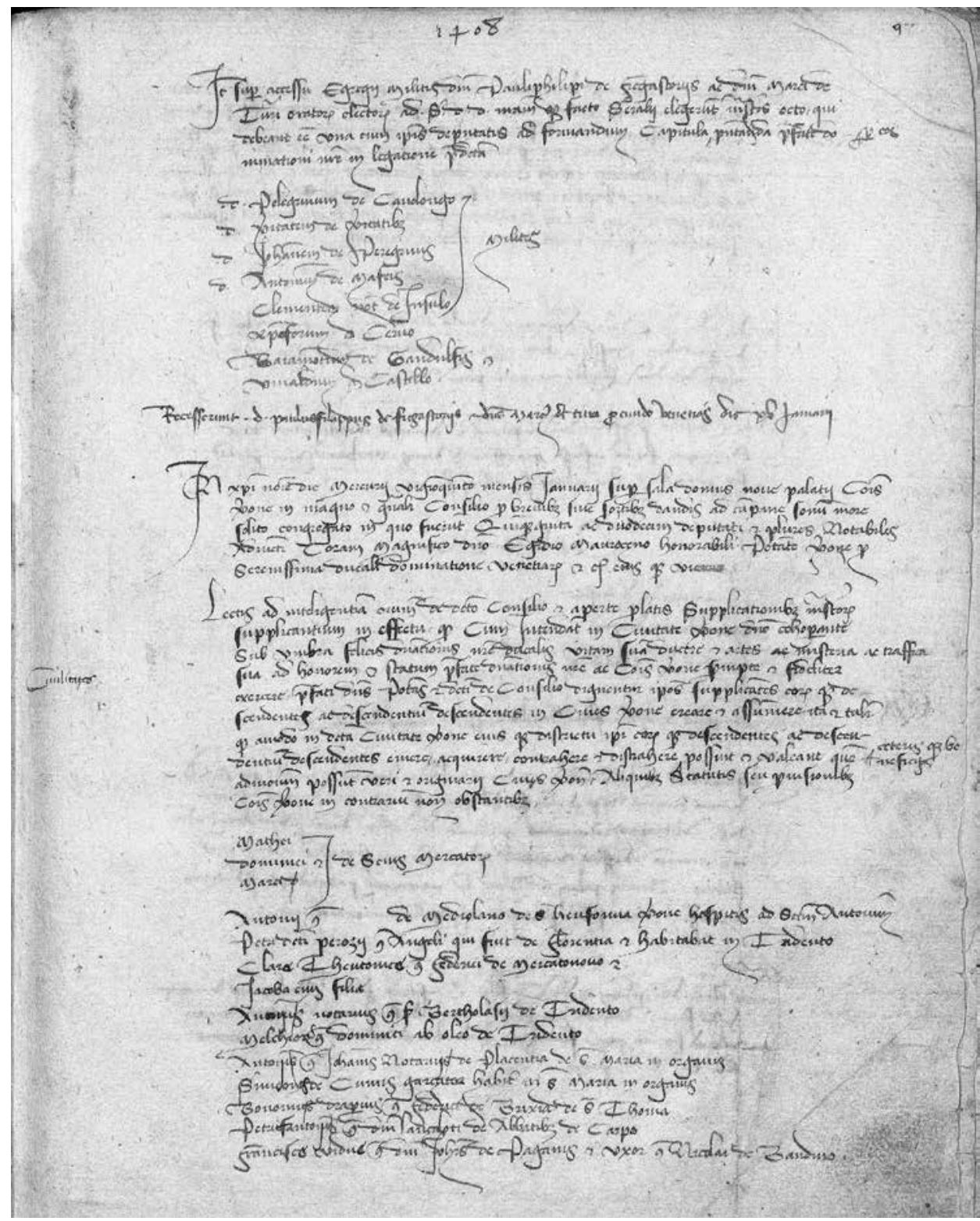

Fig. 4. ASVr, Antico archivio del Comune, Atti del Consiglio, reg. 56, c. 97r, 1408 gennaio 25. Concessione della cittadinanza veronese al notaio Antonio da Borgonuovo (settima riga dal basso della c. 97r). Ricevono la cittadinanza anche: Matteo, Domenico e Marco da Siena, mercanti; Antonio del fu *** da Milano da Sant'Eufemia di Verona hospes a Sant'Antonio; Pietro detto Perozzo del fu Angelo qui fuit da Firenze, abitante a Trento; Clara theutonica del fu Federico de Mercatonovo e Giacoma sua figlia; Melchiorre del fu Domenico ab Oleo da Trento; Antonio del fu Giovanni notaio da Piacenza da Santa Maria in Organo; Simone da Como garzator abitante in Santa Maria in Organo; Bonomo drappiere del fu Federico da Brescia da San Tommaso; Pietro Antonio del fu $d$. Panzalotus de Abbatibus de Carpo; Francesca vedova del fu $d$. Giovanni de Paganis e moglie del fu Nicolò da Bandino. 


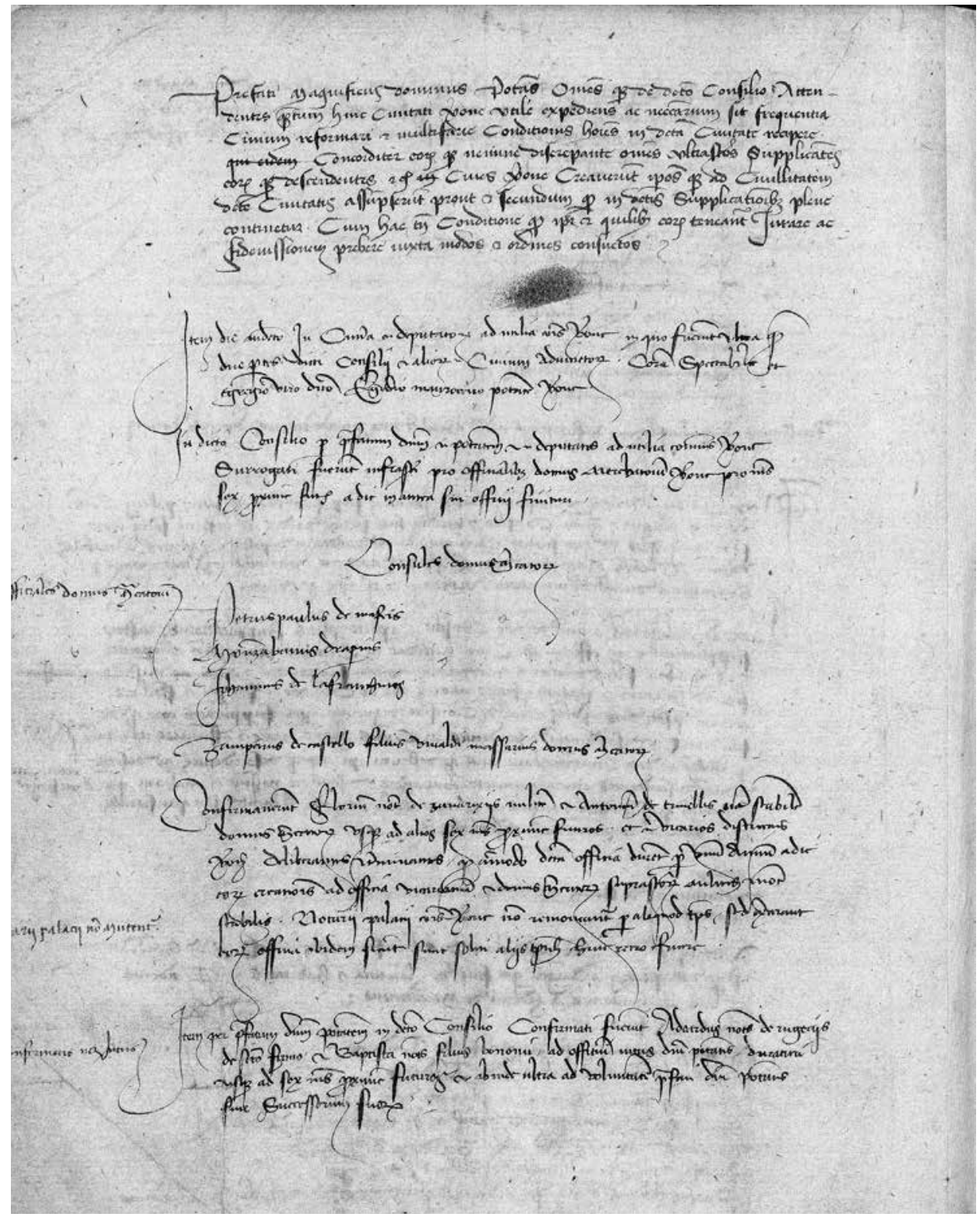

Fig. 4. ASVr, Antico archivio del Comune, Atti del Consiglio, reg. 56, c. $97 v$.

ancora residente nella contrada di San Giovanni in Foro. La registrazione nell'estimo del 1418 è l'ultima notizia che testimonia la presenza del notaio Antonio da Borgonuovo in Verona; un unico documento, risalente ancora al 5 settembre 1409, lo vede nominato, insieme al suo responsalis Giacomo de Bonalinis, curatore di un certo Melchiorre del fu Domenico da Sasso di Vigolo Baselga ${ }^{195}$.

195 ASVr, Ufficio del Registro, Istrumenti, reg. 24, c. $1220 v$. 


\section{L'impegno politico di Antonio nel Comune di Trento}

All'indomani delle rivolte contro il vescovo Georg Liechtenstein, il Comune aveva iniziato a dotarsi di una propria struttura organizzativa abbastanza definita e stabile $^{196}$ che si riflesse, a partire dal 1415 , nella prima serie di registrazioni degli officiales eletti alle massime cariche comunali. Si tratta del già citato manoscritto 3547 , anche noto come Liber electionum officialium magnificae communitatis Tridenti ${ }^{197}$.

La prima carta del codice trasmette il verbale di elezione degli addetti alle massime cariche istituzionali per l'anno 1415; fra i nove sapientes et provisores eletti «per homines sapientes et communes conscilium civitatis Tridenti» ${ }^{198}$ figura, in seconda posizione, proprio Antonio da Borgonuovo. Insieme al notaio vengono menzionati Bonadomano de Accerbis, Bartolomeo da Roccabruna, Antonio de Ceris da Pergine, Bernardo de Bernardis, il notaio Guglielmo Balzanini, l'apothecarius Maffeo da Brescia, Giovanni Mezaoveta e il magister sarto Ianes. Tre anni più tardi, nell'ottobre del 1418, Antonio da Borgonuovo sarà nuovamente eletto console, carica che ricoprirà per un anno insieme a ser Pietro Iacob, ser Gianpietro da Feltre, Bartolomeo figlio di Sicco, Battista da Bologna, Bartolomeo Cevoleta, Odorico da Povo e Melchiorre ab Oleo. Analogo incarico verrà esercitato una terza volta, nel 1422, insieme al magister Odorico phisicus, ser Pietro Iacob, Antonio da Roccabruna, Nicolò Mercadenti, Bernardo de Bernardis, Melchiorre ab Oleo e Battista da Bologna e, nel 1427, insieme ad Antonio da Roccabruna, Battista da Bologna, Odorico Calepini, Giacomo Fanzini, Giovanni Alde e al magister Michele piliparius. È in occasione di quest'ultimo incarico consolare che, il 28 giugno del 1428, ser Antonio, insieme agli altri consoli in carica e al procuratore Nicolò Mercadenti, nominano Antonio da Molveno, ser Odorico detto Stratenperger e ser Francesco de Sichis ambasciatori e procuratori della città per recarsi a Bolzano a manifestare la ferma opposizione della città «super taxa imposita et imponenda ad obiurandum et debelandum» gli ussiti ${ }^{199}$.

L'elenco dei cives chiamati annualmente a ricoprire la massima carica istituzionale del Comune consente di riconoscere una parte di quel già citato gruppo di maggiorenti che, nel corso del Quattrocento, si spartì i più importanti uffici cittadini, lasciando invece a «cives di estrazione molto varia ${ }^{200}$ le cariche di minore rilevanza. Fra i ruoli assunti da Antonio all'interno del Comune va ricordato anche quello di sindaco, l'ufficio giudiziario più antico, ricoperto nel 1418 insieme a Calepino Calepini, nel 1424 insieme a Vigilio Morzati da Pergine e nel $1429^{201}$ insieme a Bartolomeo da Bologna. Nello stesso anno il notaio ricoprì inoltre, con Antonio da Molveno, la carica di procurator ad causas ad

196 Sulla questione cfr. Cagol, Dal palatium episcopatus al palatium comunis, cit., pp. 205-223 e Varanini, Gli uffici del Comune di Trento nel Quattrocento, cit., pp. 225-237.

197 ASCTn, Comune di Trento, Antico regime, Sezione antica, ACT1-3547; Valenti, Il «liber electionum officialium», cit.

198 Ivi, p. 435.

199 Cfr. Appendice, regesto n. 38 (ADTn, ACap, Instrumenta capitularia 8bis, n. 294). Il $1^{\circ}$ settembre successivo i cittadini di Trento, insieme ai rappresentanti delle valli e delle comunità di Riva e Tenno, si rivolsero al principe elettore Federico di Brandeburgo per ottenere l'esonero dal pagamento del contributo; cfr. l'analoga reazione da parte della città di Toul e dell'arcivescovo di Riga: Brandstätter, Vescovi, città e signori, cit., p. 72, note 88-91.

200 Varanini, Gli uffici del comune di Trento nel Quattrocento, cit., p. 228.

201 ASCTn, Comune di Trento, Antico regime, Sezione antica, ACT1-3547, c. $227 v$. 
agendum et defendendum ${ }^{202}$. A tal proposito si deve ricordare che, già il 10 ottobre 1422, tre giorni dopo essere stato eletto console, Antonio da Borgonuovo esercitò, ancora insieme ad Antonio da Molveno, la funzione di sindaco e procuratore della città in occasione della sentenza emanata da Wilhelm von Matsch, capitano generale all'Adige, nell'ambito della vertenza fra il Comune di Trento e ser Negro de Negri da San Pietro ${ }^{203}$. Quattro anni più tardi, nel 1426, ser Antonio ricoprì analoga funzione, insieme al già citato Antonio da Molveno e ai notai Giacomo da Arco e ser Vigilio Saraceno, ricevendo dal consiglio generale il mandato per agire legalmente nella causa mossa dalla città di Trento contro le comunità delle pievi situate oltre il Bus de Vela e citra Attacem, che non avevano corrisposto le dovute contribuzioni e oneri alla città ${ }^{204}$. Meno di un anno dopo, nel marzo del 1427, Antonio appare nuovamente impegnato quale procuratore e sindicus della città nella causa mossa contro le comunità «subiecte iurisdictioni et curie Tridenti»» ${ }^{205}$, affinché queste pagassero le spese occorrenti per la difesa e il mantenimento della città.

Nel 1418, nel 1424 e nel 1429, dopo aver ricoperto la carica di sindicus del Comune, esercitò il ruolo di gastaldo, l'ufficiale chiamato a presenziare alla tortura giudiziaria comminata dal vicario vescovile.

Nel 1421, nel 1426, nel 1432 e nel 1433 svolse l'incarico di giudice degli appelli. Gli officiali addetti a questa magistratura restavano in carica per la durata di quattro mesi e avevano il compito di valutare in appello le cause civili e criminali emesse dai sindici. Se la causa non avesse trovato soluzione, i cittadini avrebbero potuto ulteriormente appellarsi ai consoli e ai provveditori della città.

Fra le cariche pubbliche ricoperte da Antonio va ricordata anche quella di giudice delle tutele, che egli esercitò in ben cinque occasioni nel 1423, nel 1426, nel 1429, nel 1433 e nel 1434. Analogamente all'ufficio del giudice degli appelli, anche lo iudex tutellarum restava in carica per quattro mesi ed era incaricato di raccogliere le notifiche di morte del padre o della madre nelle famiglie di tutta la pretura, procedendo successivamente all'affidamento degli orfani ai tutori scelti ${ }^{206}$.

Il ruolo esercitato da Antonio negli uffici cittadini, tale da renderlo uno fra i più titolati membri della classe dirigente di inizio Quattrocento, l'abile politica matrimoniale messa in atto nei primi decenni del secolo e l'attività di notaio al servizio di molti fra i cives che, in vario modo, si assicuravano il controllo sugli uffici comunali, sono dati che consentono l'inclusione del notaio in quella trama di rapporti familiari, politici ed economici gradualmente intessuta dai membri della classe dirigente trentina ${ }^{207}$.

Alla luce di queste considerazioni, non può essere casuale la presenza del suo nome fra i proditores che, nel febbraio del 1435, si ribellarono al vescovo Alessandro di Masovia ${ }^{208}$. Ecco come l'anonimo descrive Antonio:

\footnotetext{
202 Ivi, ACT1-3547.

203 Cfr. Appendice, regesto n. 8 (ASCTn, Comune di Trento, Antico regime, Sezione antica, ACT1-3263).

204 ASCTn, Comune di Trento, Antico regime, Sezione antica, ACT1-3069.

205 Ivi, ACT1-3359.

206 Statuto clesiano, libro I del Civile, capitolo 10; alcuni casi di affidamento a tutore vengono registrati dal notaio Antonio da Borgonuovo nel registro Instrumenta capitularia 8bis. Cfr., a titolo esemplificativo, il documento n. 386, c. $147 v$.

207 Non può, ad esempio, passare inosservato il fatto che la maggior parte dei matrimoni cui si è fatto cenno nel paragrafo precedente siano stati registrati proprio da Antonio da Borgonuovo.

208 ASTn, $A P V$, Sezione latina, capsa 4, n. 35.
} 
Volite pur che tasa?

Io ve conterazo, ma pur ve contarazo

de Antonio Bertolaso,

lo qual mai n'ebe paso

se non pensando male;

el s'asirò le spale

cum tuta la persona;

e certo el se rasona

che el fo per li conseli

che el dete ali cativelj

di zendri e delli Galli

che intrase in questi ballj,

e sì morì con quella ${ }^{209}$.

David Ressegotti fornisce un'interpretazione del passaggio:

Vi racconterò anche di Antonio Bertolaso, che mai non ebbe pace se non tramando intrighi: gli si piegarono le spalle con tutto il corpo - si dice che ciò avvenne a causa dei cattivi consigli che diede a quei meschini degli zendri e dei Galli, affinché entrassero anche loro nel ballo - e di conseguenza ne mori ${ }^{210}$.

Non si conosce con certezza il contributo del notaio alle sollevazioni contro Alessandro di Masovia, tuttavia le poche fonti a disposizione per ricostruire gli eventi del biennio 1435-1437, ovverosia le Rime e la memoria conservata nell' $A r-$ chivio principesco vescovile, sono concordi nell'attribuire ad Antonio un ruolo di primo piano nelle vicende. Oltre a ciò, il fatto che sia la memoria sia le Rime si riferiscano a lui come un traditore, «lo qual mai n'ebe paso / se non pensando male», non contrasta con la posizione di fiducia che certamente il professionista si vide accordare in qualità di notaio del Capitolo $^{211} \mathrm{e}$, seppur in misura minoritaria, del vescovo Alessandro di Masovia ${ }^{212}$; questo almeno fino agli eventi del 1435.

Null'altro è noto sulla partecipazione di Antonio alle rivolte antivescovili, ma l'anonimo rimatore gli attribuìsce il coinvolgimento nelle sollevazioni dei non meglio noti zendri, e dei Galli ${ }^{213}$. A tal proposito, mentre dei Galli è noto che si

209 Papaleoni, Rime di anonimo, cit., pp. 167-207. Cfr. anche D. Ressegotti, «Spala de portadoro»: una nota quattrocentesca in volgare trentino, «Studi trentini. Storia», 91, 2012, pp. 191-202.

${ }^{210}$ Ivi, p. 196.

211 In molte occasioni i canonici del Capitolo nominarono Antonio sindico e procuratore; cfr., ad esempio, ADTn, ACap, Instrumenta capitularia 8, n. 675b. Il notaio Antonio, procuratore del Capitolo, agisce contro Bartolomeo del fu Sicco, olim canonico di Trento, a motivo di un affitto di 4 ducati d'oro non corrisposto al Capitolo. Cfr. anche ADTn, ACap, capsa 32, n. 241.1 (novembre 1412).

212 Tuttavia il fatto che, appena pochi mesi prima delle rivolte, nel maggio del 1434 (ADTn, ACap, Instrumenta capitularia 8bis, n. 617) il notaio avesse prestato la propria opera al vescovo Alessandro, induce a ritenere che le massime istituzioni ecclesiastiche del principato avessero riposto una certa fiducia nel notaio; si spiega alla luce di queste considerazioni il fatto che in seguito il notaio sia definito 'traditore'.

${ }^{213}$ Si tratta, con ogni probabilità, di Francesco e Giovanni Gallo, che le Rime descrivono in termini negativi a causa delle spregiudicate attività commerciali: Papaleoni, Rime di anonimo, cit., p. 192, vv. 344-357. Del primo non si conosce nulla, mentre Giovanni fu nominato iudex venditionis nel 1431, massaro dei Battuti nel 1440 (Dal Piaz, La confraternita dei Battuti laici, cit., p. 100) e console nel 1455 (Brandstätter, Vescovi, città e signori, cit., p. 219). 


\section{Stefano Malfatti}

trattava di notai attivi in città nel corso del XV secolo, sugli zendri (che si sceglie pertanto di scrivere con la lettera minuscola), nulla è noto, a meno che l'anonimo rimatore non si riferisse, impiegando un termine dialettale ancora in uso $^{214}$, ai generi di ser Antonio, ovvero i due Calepini, Bonaventura e Adelperio, che - come noto dovettero ricoprire un certo ruolo nelle rivolte. Se tale interpretazione si rivelasse corretta, il rimatore attribuirebbe al notaio il ruolo di artefice di primo piano della rivolta. Anche i versi 729-730 («el s'asirò le spale / cum tuta la persona») sono interessanti nella definizione delle responsabilità del notaio nelle vicende degli anni Trenta. In questo senso, si ripropone in questa sede l'interpretazione che recentemente ne ha dato David Ressegotti:

il particolare delle spalle curve attira l'attenzione: si potrebbe persino intendere il passo immaginando un'effettiva deformità che si acuì all'incirca intorno al 1435 , portando così il notaio alla morte in quello stesso giro d'anni. Tuttavia, considerato anche il tono molto pungente e canzonatorio dell'intero componimento..., la menzione delle spalle curve potrebbe essere piuttosto un riferimento all'umile professione che viene attribuita al padre di Antonio, ser Bertolasius portitor; in questo senso l'allusione potrebbe indicare che la persona tutta s'incurvò (sotto il peso di una deformità 'morale') come curve erano già le spalle. Uno scherno, dunque, lanciato alla condizione di parvenu di ser Antonio, la cui famiglia aveva nelle passate generazioni svolto un lavoro assai umile ${ }^{215}$.

Al di là delle possibili interpretazioni dei versi, le fonti disponibili documentano, proprio per il biennio 1435-1437 216 , un radicale decremento dell'attività scrittoria del notaio, fino alla totale battuta d'arresto dal febbraio del $1437^{217}$. L'assenza di ulteriore documentazione rogata da ser Antonio dopo questa data induce a collocarne la morte poco dopo. Due anni prima, il 14 aprile 1435, l'estensore aveva chiuso un rogito con la seguente sottoscrizione:

Ego Antonius condam ser Bartholasii de Burgonovo de Tridento civis Tridentinus, publicus imperiali auctoritate notarius ... una cum dictis testibus interfui, vidi et sic audivi. Et quia ego rogatus et requisitus infirmitate oppressus predictam in formam publicam ad presens scribere non valui, alteri scribendum comissi et in fidem et verum testimonium premissorum me hic subscripsi et signum mei tabellionatus consuetum hic apposui et signavi ${ }^{218}$

dichiarando dunque che la sua condizione fisica era talmente compromessa da impedirgli di estrarre il documento in publicam formam.

214 Cfr. la voce «zendro» in G. Azzolini, Vocabolario vernacolo-italiano pei distretti roveretano e trentino, Manfrini, Trento 1976 (edizione del manoscritto, Lizzana 1836), p. 1131.

215 Ressegotti, "Spala de portadoro», cit., p. 196.

216 Ma già in precedenza, il 22 agosto del 1432, ad esempio, il notaio si dichiara infirmatus: ADTn, ACap, Instrumenta capitularia 8bis, n. 538.

217 Ivi, c. $244 v$, n. 665. A partire dal documento n. 661 (di data 16 gennaio 1436), la scrittura del rogatario diviene meno posata e non sono presenti registrazioni fra il 22 febbraio (doc. n. 663) e il 15 dicembre 1436 (doc. n. 664), in coincidenza con gli aspri contrasti che opponevano il vescovo Alessandro di Masovia al gruppo di cittadini che sostenevano il duca tirolese Federico.

218 ADTn, ACap, Instrumenta capitularia 8bis, c. 248/1, n. 646. 
Per una biografia di Antonio da Borgonuovo

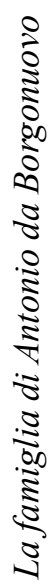

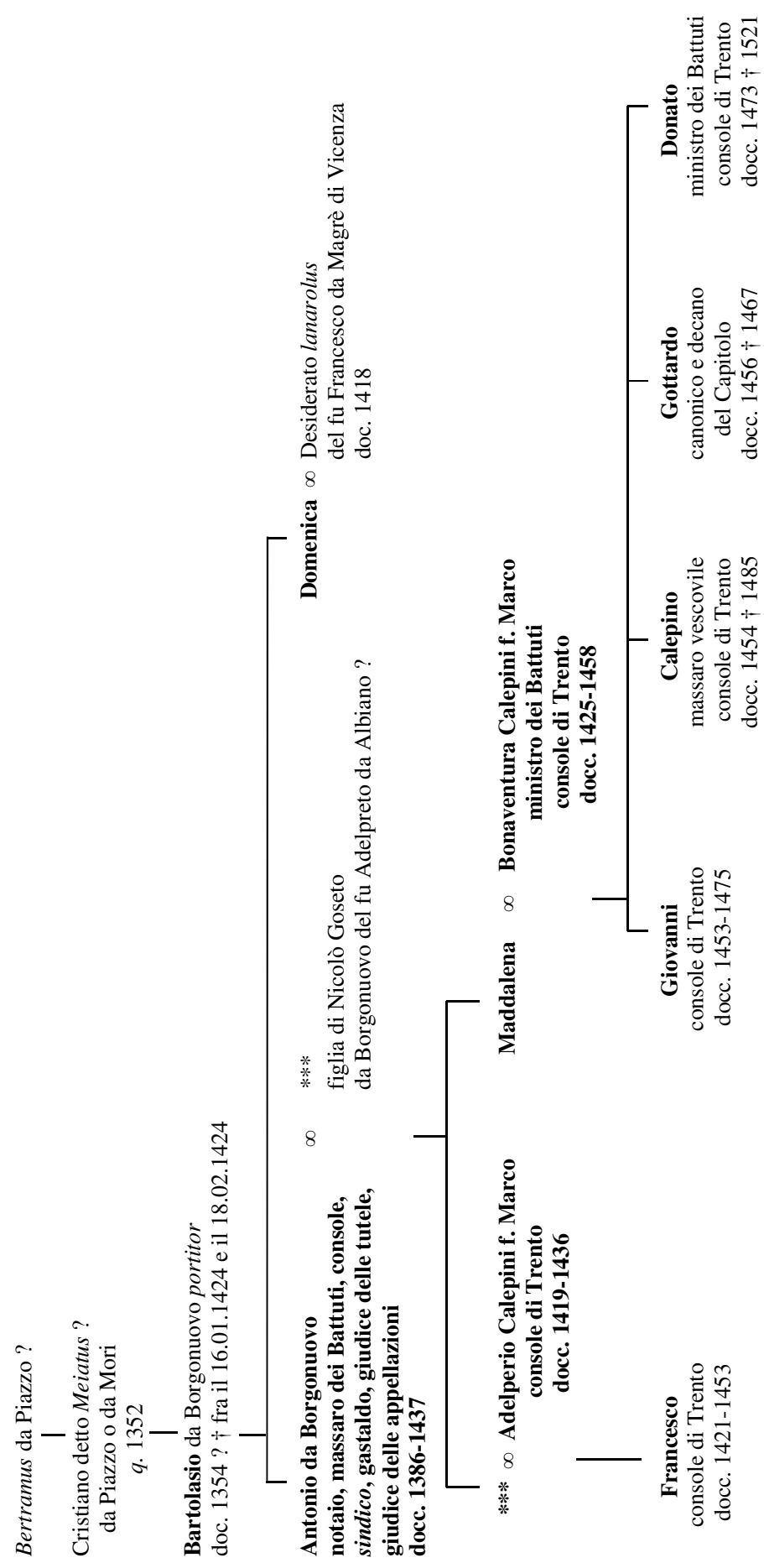




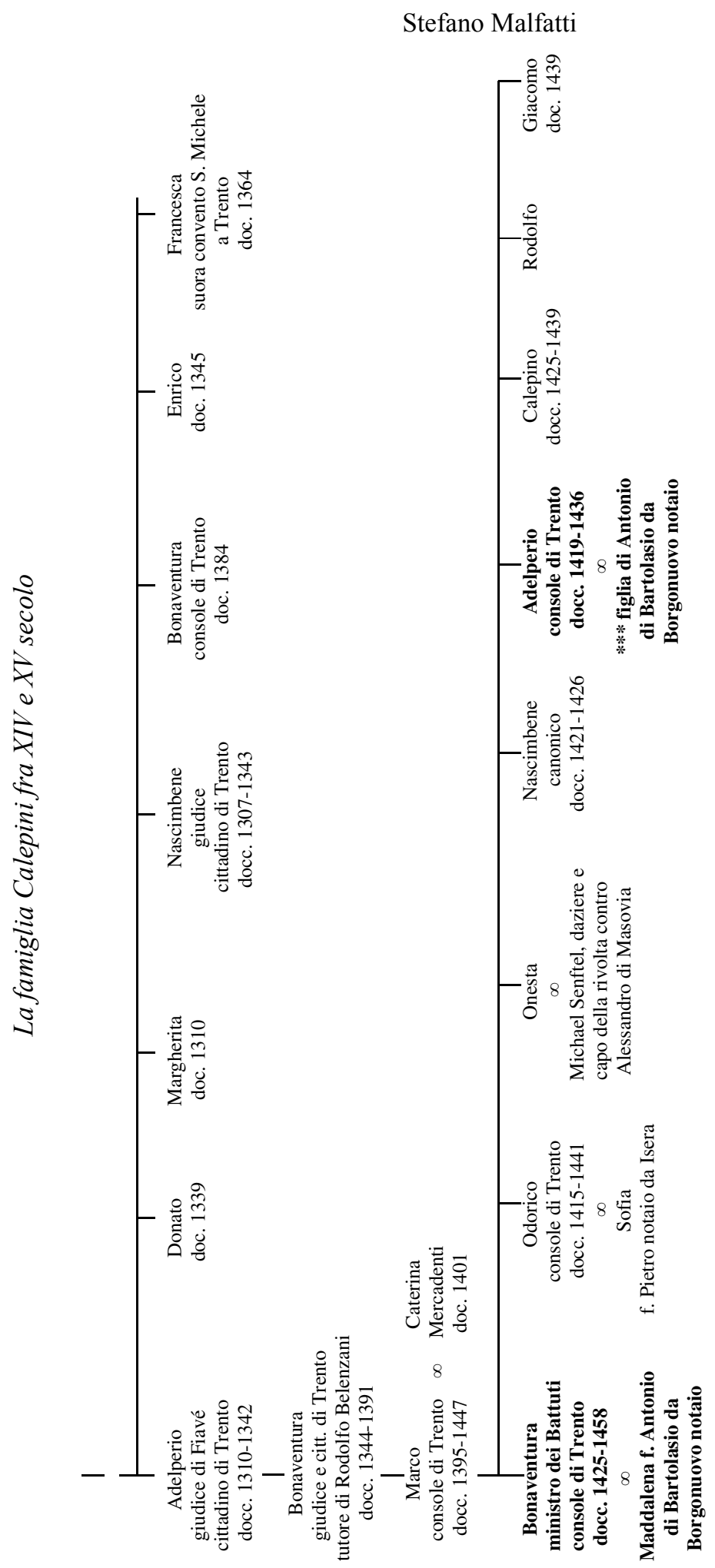





\section{Capitolo 2}

\section{La formazione di Antonio da Borgonuovo}

\section{Sulla formazione di Antonio da Borgonuovo. Alcune considerazioni introduttive}

Fino alla metà degli anni Venti del Quattrocento manca a Trento una matricola dei notai e si deve attendere almeno fino alla metà del secolo prima di avere a disposizione le verbalizzazioni del Collegio notarile; considerate queste premesse, in assenza di altre fonti specifiche (ad esempio elenchi con i nomi degli ammessi alla matricola), non è possibile allo stato attuale della ricerca conoscere tempi e modalità in cui ser Antonio divenne notaio.

Quanto è noto della formazione del professionista si può dunque ricavare unicamente dall'analisi dei suoi documenti. Da questo punto di vista egli non sembra introdurre nel formulario impiegato nelle diverse tipologie documentarie alcun elemento di novità; non vi sono variazioni che possano lasciar intravedere modalità operative diverse rispetto a quelle dei colleghi. Per ciascuna tipologia documentaria, dalla locazione, alla refuta, fino alla compravendita e al testamento i documenti rogati da Antonio sono conformi a modelli ben consolidati. Se variazioni nelle formule e nei termini utilizzati possono talvolta emergere, si tratta invero di fluttuazioni lievissime, riscontrabili anche nella documentazione redatta dallo stesso notaio. Gli instrumenta dei notai trentini presentano infatti una struttura uniforme, con un formulario che subisce un'evoluzione, dovuta ad esempio all'inserimento di specifiche clausole, soltanto sul lungo periodo, tendendo ad arricchirsi scendendo lungo i secoli.

Anche il notariato trentino mostrava di conoscere le formule raccolte nel XIII secolo dai giuristi e notai bolognesi, in primis Rolandino Passeggeri, tanto da poterne riscontrare talvolta un impiego letterale nelle forme e nella loro successione. Che le opere del notaio bolognese non fossero sconosciute in area trentina lo prova, peraltro, la presenza nel fondo Manoscritti della biblioteca comunale di Trento $^{1}$ di

1 BCT, BCT1, ms. 2892; cfr. M.A. Casagrande Mazzoli et alii (a cura di), I manoscritti datati della provincia di Trento, Sismel, Firenze 1996 (Manoscritti datati d'Italia, 1), p. 50, n. 46. La Summa di Rolandino continuò a diffondersi, non solo in Italia, per tutto il medioevo e per buona parte dell'età moderna; la sua fortuna accrebbe ancor più dopo l'introduzione della stampa a fine Quattrocento. L'editio princeps della Rolandina fu stampata a Modena nel 1476: cfr. F.C. von Savigny, Geschichte des Römischen Rechts im Mittelalter, Scientia, Aalen 1986, V, pp. 544-545; II, p. 513 e, più recentemente, sulla diffusione dell'opera di Rolandino: G. Tamba (a cura di), Rolandino e l'ars notaria da Bologna all'Europa, Atti del convegno internazionale di studi storici sulla figura e l'opera di Rolandino (Bologna 9-10 ottobre 2000), Giuffrè, Milano 2002 (Per una storia del notariato nella civiltà europea, 5). Non mancano, anche nelle biblioteche trentine, copie a stampa della Rolandina, la cui edizione più antica è datata 1485 . 
una copia databile al 1457 del Flos ultimarum voluntatum di Rolandino². È anche su questi testi che probabilmente i giovani aspiranti notai trentini si formarono a partire dalla fine del Duecento, è a partire da questi exempla che i professionisti improntarono i loro formulari, traendone formule specifiche per ciascuna tipologia documentaria da adattare alle particolari necessità del luogo. Gli esempi raccolti nella Summa rolandiniana finirono così, col tempo, per «regionalizzarsi»» ${ }^{3}$, armonizzandosi con il sistema giuridico locale.

L'adesione alle formule della scuola notarile bolognese può essere efficacemente rilevata mettendo a confronto il formulario adottato da ser Antonio per redigere, ad esempio, una semplice compravendita con quello utilizzato da altri colleghi trentini, fra cui ad esempio Venturino de Trechis ${ }^{4}$, notaio del Capitolo fra gli anni Venti e Quaranta del Trecento, e Alberto da Sacco ${ }^{5}$, attivo alla fine del XIV secolo. I testi delle compravendite ricalcano, praticamente de verbo ad verbum, il formulario della venditio simplex contenuta nella Summa di Rolandino Passeggeri ${ }^{6}$, tanto da far ipotizzare che, in tutti i casi, i notai abbiano avuto davanti agli occhi proprio il formulario del notaio bolognese nel momento in cui redassero tali negozi.

Un secondo importante dato che emerge dall'esame sulla documentazione prodotta da Antonio è il fatto che egli non si definisce mai, né in sottoscrizione né altrove, doctor o iuris peritus, né lo fanno gli altri notai a lui coevi o successivi, quando menzionano il professionista del Borgonuovo. Antonio è semplicemente publicus imperiali auctoritate notarius, come buona parte dei colleghi del tempo. Se è vero che non molti tabellioni dell'epoca ricordano in sottoscrizione il titolo acquisito presso una qualsiasi università dell'epoca, va detto comunque che costoro, o i loro colleghi, non scordano di farne menzione altrove, fuori dall'escatocollo. È il caso, ad esempio, del notaio Gioacchino Mezzasoma, che in sottoscrizione si chiama imperiali auctoritate notarius ${ }^{7}$, ma che dalla documentazione coeva sappiamo essere uno iuris peritus ${ }^{8}$. Queste considerazioni relative alla sottoscrizione e alle 'definizioni' attribuite a ser Antonio, unitamente alle informazioni tratte dal suo formulario contribuiscono a rendere plausibile, seppur non dimostrabile con certezza, una formazione locale del notaio trentino.

L'affermazione, come detto, non può che rimanere al livello della semplice ipotesi; il notariato trentino di fine Trecento e inizio Quattrocento è ben lontano dalle

2 Il manoscritto presenta inoltre la particolarità di recare, sul primo foglio di guarda, una serie di citazioni tratte dal De amicitia di Cicerone, vergate da una mano databile alla metà del Quattrocento. Il fondo diplomatico (BCT2) della Biblioteca comunale di Trento conserva inoltre alcuni frammenti di testi scolastici e di diritto con glosse (BCTn, BCT2, n. 1087, testo di diritto con glossa del XIV-XV secolo, frammento di 2 cc.; ivi, n. 1088, testo scolastico con glossa del XIV-XV secolo, frammento di 1 c.; ivi, n. 1085, frammento pergamenaceo di coperta che reca l'iscrizione, solo parzialmente decifrabile, Formulare instrumen(torum) (...) Stilus Romane curie. | De arte notariatus (...).

3 L. Sinisi, Formulari e cultura giuridica notarile nell'età moderna: l'esperienza genovese, Giuffrè, Milano 1997 (Fonti e strumenti per la storia del notariato italiano, 8), p. 176.

4 S. Malfatti, Il registro del notaio Venturino de Trechis nell'archivio del Capitolo della cattedrale di Trento - Instrumenta capitularia 3 (1324-1347). Edizione e commento, tesi di laurea, Università degli Studi di Trento, a.a. 2011-2012, rel. A. Giorgi, n. 178.

5 Zamboni, Economia e società in una piccola città alpina, cit., n. 40.

6 Rolandini Passaggerii Summa totius artis notariae, Forni, Sala Bolognese (Bo) 1977 (rist. anastatica a cura del Consiglio nazionale del notariato).

7 Cfr., ad esempio, BCTn, BCT3, capsa 29, mazzo 1, n. 26.

8 Cfr., ad esempio, ADTn, ACap, Instrumenta capitularia 8bis, c. 62r, n. 168. 


\section{Stefano Malfatti}

forme organizzative dei collegi notarili di altre città dell'Italia centro-settentrionale; come recentemente ha avuto modo di sottolineare Gian Maria Varanini, infatti,

quello del Collegio notarile trentino è ... semplicemente l'esito in ritardo di un processo di consolidamento e di affermazione che risaliva a due secoli prima: l'associazione professionale si consolida e si assesta in ritardo, in armonia con lo sviluppo lento e contrastato delle istituzioni comunali, e in condizioni di ineliminabile subalternità rispetto al potere vescovile, che resta la fonte di legittimità tanto per l'amministrazione cittadina quanto per la publica fides dei notai ${ }^{9}$.

Questa «subalternità» non poteva che rispecchiarsi nella mancanza di norme ben definite per la regolazione dell'accesso alla professione, sulle quali si deve attendere ben oltre la metà del secolo per avere notizie un po' più precise. Prima non si possono che formulare ipotesi, il che dimostra - anche in questo settore - come Trento si trovasse ad inizio Quattrocento in una fase ancora pienamente transitoria e dai contorni non ben definiti.

\section{Prima di Antonio. La formazione dei notai trentini}

Piuttosto limitate sono le informazioni relative alla formazione dei notai trentini in età medievale; soltanto con gli statuti emanati dal vescovo Alessandro di Masovia nel 1425-1427 e, dal 1459, con l'inizio delle verbalizzazioni delle sedute del Collegio dei notai e giudici di Trento ${ }^{10}$ si inizia ad avere qualche dato più preciso in merito alle modalità di accesso alla matricola. Per quanto riguarda i secoli precedenti, ci si deve basare essenzialmente sul confronto con quanto avvenuto altrove e sull'esame della documentazione prodotta dagli stessi notai. Se infatti si verifica l'esistenza di norme in merito all'accesso alla professione notarile negli statuti roveretani del 1425 che, come noto, furono redatti ricalcando sostanzialmente gli statuti trentini (antiqua e vetera) del XIV secolo ${ }^{11}$, anche la redazione normativa del borgo lagarino tace sulla materia, pur dedicando specifici capitoli ai notai.

Questa analisi inizierà quindi dagli statuti masoviani del 1425-1427 i quali, al Liber I, De civilibus, presentano gli statuti del Collegio dei notai di Trento; tre norme, in particolare, regolavano l'ammissione alla matricola:

Nullus advocatus, procurator seu tabellio possit scribere, advocare seu procurare in civitate Tridenti nisi fuerit descriptus in collegio predicto, sub pena decem librarum pro quolibet et qualibet vice.

Item quod nullus qui non sit descriptus in collegio seu matricula collegii predicti possit vel audeat scribere instrumenta vel ultimas voluntates vel acta aliqua iuditialia, vel offi(t)ium notarie aut procuratoris vel advocati exercere aliqualiter in civitate

$9 \quad$ Varanini, Il Collegio notarile di Trento, p. 489.

10 M.V. Ceraolo, Il collegio notarile di Trento nella seconda metà del Quattrocento, tesi di laurea, Università degli Studi di Trento, a.a. 2001-2002, rel. G.M. Varanini e Varanini, Il Collegio notarile di Trento, cit., pp. 483-514.

11 Statuti di Rovereto del 1425, cit. In particolare, sull'aderenza degli statuti roveretani alle redazioni statutarie di Trento del XIV secolo, cfr. i saggi di M. Bellabarba, Rovereto castrobarcense, veneziana, asburgica: identità ed equilibri istituzionali, ivi, p. 18 e G. Ortalli, Percorsi statutari trentini, ivi, p. 35. 


\section{La formazione di Antonio da Borgonuovo}

Tridenti et aliis locis pertinentibus ad curiam Tridentinam, sub pena decem librarum denariorum Tridentinorum pro quolibet et qualibet vice.

Et nichilominus instrumenta et ultime voluntates et acta scripta et notata per eos vel aliquod eorum careat iuris effectu ${ }^{12}$.

Il divieto ai notai di esercitare l'arte se non precedentemente iscritti al Collegio di Trento sembra riprendere un precedente capitolo degli statuti trecenteschi che oggi si legge attraverso la redazione roveretana del 1425, pur leggermente modificata rispetto all'originale. In particolare il capitolo 42 degli Statuta nova, intitolato «De tabellionibus forensibus instrumenta scribere non debentibus» prescriveva

quod aliquis notarius forensis, qui non fuerit naccione vel habitacione terre vel districtu Roveredi, non audeat vel prosumat seu debeat scribere in civitate vel districtu Roveredi aliqua instrumenta publica vel ultimas voluntates, nisi illud instrumentum publicum fuerit conceptum in presencia boni et legalis notarii nati et habitacionis in terra et districtu Roveredi et per eum publice subscriptum. Quod si secus factum fuerit per aliquem notarium forensem, illud vel illa quod seu que scripta fuerint sine suprascriptis solemnitatibus in civitate et districtu Roveredi «corretto su Tridenti parzialmente eraso> non valeat ipso iure, nisi de comissione et licencia data dicto notario forensi per nos vel successores nostros ${ }^{13}$.

Tali disposizioni, assenti negli statuti antichi (di inizio XIV secolo) di Trento, vietano dunque ai notai forestieri nati e abitanti nell'episcopato di redigere instrumenta, a meno che questo non avvenisse in presenza d'altro notaio del luogo. Si specifica, tuttavia, alla fine del capitolo, che la norma può essere superata attraverso specifica licenza conferita al notaio forestiero, sottointeso, almeno per quanto riguarda Trento, dall'autorità vescovile.

Ciò avvenne, ad esempio, il 20 aprile 1390 quando il vescovo Georg Liechtenstein concesse al notaio Martino di Giovanni Ceraduce dal Tesino la licenza di rogare a Trento «non obstantibus legibus et statuto municipalibus ... ut valeat in civitate Tridentina et diocesi tabellionatus sive notarie officium publice exercere ${ }^{14}$.

Una seconda disposizione, inserita negli statuti masoviani, riguardava invece l'ammissione al Collegio notarile di Trento, normando il procedimento che regolava l'ingresso nella matricola:

Item quod nullus admittatur in matricula et collegio prefato nisi fuerit examinatus et approbatus pro sufficienti per examinatores dicti collegii deputatos ${ }^{15}$.

Vita onesta e buona fama costituivano poi ulteriori prerequisiti richiesti ai candidati che, superato l'esame dinanzi alla commissione presieduta dal rettore del Collegio, procedevano al giuramento e al pagamento della tassa d'ingresso, fissata

12 Cfr. B. Chemotti, La legislazione statutaria nel Principato vescovile di Trento: gli Statuti alessandrini (1425), tesi di laurea, Università degli Studi di Trento, a.a. 1989-1990, rel. D. Quaglioni, pp. 192-203 e, più recentemente, Bortoli, Per un'edizione dei testi statutari del Comune di Trento, cit.

13 Statuti di Rovereto del 1425, cit., p. 170. Roveredi corretta su un precedente Tridenti è ulteriore prova del fatto che gli statuti roveretani vennero esemplati, de verbo ad verbum, sul modello trecentesco trentino.

14 Codicis clesiani, cit. p. 176 (II, c. 227b); documento redatto dal notaio Marco del fu Odorico da Trento.

15 Chemotti, La legislazione statutaria nel Principato vescovile di Trento, cit., p. 198. 
nel 1425 in dieci lire di denari trentini ${ }^{16}$. Si tratta di norme che si ritroveranno, sostanzialmente immutate, anche nelle successive redazioni statutarie e che saranno messe in pratica dal Collegio notarile di Trento, come si legge nei verbali della matricola della seconda metà del XV secolo.

Per quanto concerne, invece, le 'tappe' precedenti che l'aspirante notaio trentino doveva affrontare, come per le altre città dell' Italia centro-settentrionale ${ }^{17}$, anche a Trento per esercitare la professione era necessaria anzitutto l'investitura da parte dell'imperatore o di un suo delegato, il conte palatino. Fra XII e XIII secolo tuttavia a Trento anche $\mathrm{i}$ vescovi si arrogarono la prerogativa di nominare notai ${ }^{18}$; tale pratica fu in seguito scarsamente praticata, tanto più che se è vero che alcuni vescovi (Adelpreto, Salomone, Alberto da Campo e Corrado da Beseno) crearono notai, essi scelsero poi «coscientemente e costantemente di non servirsene, ... come se la legittimazione che un "notarius N. episcopi" poteva garantire alla documentazione non fosse del tutto adeguata»" 19 .

Un qualche interesse desta invece la facoltà di creare notai rilasciata «ex speciali gratia», nel 1432, ad Alessandro di Masovia da parte dell'imperatore Sigismondo di Lussemburgo. Il vescovo ottenne l'autorizzazione a creare venti nuovi tabellioni «ac $\mathrm{ab}$ iis nomine Romano regio recipere fidelitatis iuramentum ${ }^{20}$; la concessione al principe e vescovo di Trento, definito nel diploma 'consigliere' dell'imperatore, può essere letta alla luce del particolare rapporto di fiducia fra Alessandro e Sigismondo; ma si tratta, come le fonti coeve sembrano dimostrare, di un caso piuttosto isolato considerato che, per gran parte del medioevo, anche nell'episcopato tridentino la via più usuale per diventare notai fu quella della nomina da parte dei conti palatini che agivano per conto dell'autorità imperiale ${ }^{21}$. Ne danno testimonianza un paio di documenti registrati rispettivamente nel $1426^{22}$ e nel $1427^{23}$ da Antonio da Borgonuovo, che consentono di ricostruire l'intero iter procedurale.

Il 10 novembre 1426, presso il convento degli Eremitani di San Marco in Trento, fu letto pubblicamente un privilegio, attraverso il quale Antonio da Molveno, e insieme a lui tutti i suoi discendenti maschi, veniva nominato conte palatino dall'imperatore Sigismondo:

\footnotetext{
16 L'intratica fu successivamente aumentata a 20 lire e, nel XVI secolo, fu portata a 8 fiorini renani.

17 G. Tamba, Formazione professionale del notaio, Relazione tenuta presso il Centro G. Costamagna (Genova, 18 aprile 2007) (http://www.centrostudicostamagna.it/testi/GiorgioTAMBAGenova163KB.pdf; ultimo accesso $1^{\circ}$ dicembre 2018), senza dimenticare i riferimenti contenuti nel 'classico' P. Torelli, Studi e Ricerche di Diplomatica Comunale, Consiglio Nazionale del Notariato, Roma 1980 (Studi storici sul notariato italiano, 5), su cui D. Puncuh, La diplomatica comunale in Italia dal saggio del Torelli ai nostri giorni, in W. Prevenier, Th. De Hemptinne (a cura di), La diplomatique urbaine en Europe au moyen âge, Actes du congrès de la Commission internationale de Diplomatique (Gand, 25-29 août 1998), Garant, Leuvein-Apeldoom 2000, pp. 383-406.

18 Cfr. E. Curzel, Notai di nomina vescovile a Trento tra XII e XIII secolo, in Giorgi, Moscadelli, Quaglioni, Varanini (a cura di), Il notariato nell'arco alpino, cit., pp. 461-482.

19 Ivi, p. 474.

20 ASTn, $A P V$, Sezione latina, capsa 39, n. 28 (il documento è datato Parma, 1432 aprile 7).

21 Nel 1459 il vescovo Iohannes Hinderbach fu creato conte del sacro palazzo lateranense con la facoltà di creare notai imperiali auctoritate: ivi, n. 36.

22 ADTn, ACap, Instrumenta capitularia 8bis, c. $74 r$, n. 210.

23 Ivi, c. $73 v$, n. 209; si prenderà ad esempio il documento del 1426 poiché la nomina del 1427 è stata registrata solo nella sua forma abbreviata.
} 


\section{La formazione di Antonio da Borgonuovo}

Te, filios ac liberos tuos masculos ex te iam natos et in futurum ex te et filiis tuis nascituros in perpetuum ex tunc descendentes, animo deliberato, sano principum, baronum et procerum nostrorum et sacri Romani imperii accedente conscilio, de certa nostra scientia et de plenitudine Romane regie potestatis ac motu proprio sacri Lateranensis palacii ac aule nostre et imperialis concistorii comittes facimus, citamus, exigimus, nobilitamus, attollimus et gratiosius insignimus cum omnibus honoribus, dignitatibus, iurisdictionibus, iuribus, privilegiis, gratiis, libertatibus, officiis et utilitatibus universis, nec non omnibus emolumentis et comodis que et quas ceteri nostri et sacri Romani imperii comittes palatini obtinere, percipere et habere noscuntur quoslibet de consuetudine vel de iure te et heredes tuos predictos ipsorum palatinorum comittum numero et consortio de certa nostra scientia et auguste potestatis plenitudine ac motu proprio aggregantes.

Quale notaio dotato del titolo di conte palatino, Antonio ottenne quindi la facoltà di nominare nuovi notai:

dantes et concedentes tibi et eisdem tuis filiis et heredibus masculini sexus eadem regia auctoritate plenam omnimodam de certa nostra scientia ac motu proprio potestatem creandi tabeliones, notarios publicos et iudices ordinarios ubicumque locorum et per sacrum Romanum imperium qui ydoney sint et in literatura sufficienter experti cum plenitudine potestatis ad tabellionatus et iudicatus officium pertinente ${ }^{24}$.

L'autorità imperiale raccomandava dunque che si investissero all'esercizio dell'ufficio notarile individui ritenuti idonei e sufficientemente preparati in materia. Il che, come si vedrà successivamente, ha una certa rilevanza ai fini della ricostruzione dell'iter formativo dell'aspirante tabellione.

Pochi giorni dopo aver ricevuto pubblicamente l'investitura imperiale e aver prestato giuramento dinanzi ai testimoni e al notaio Antonio da Borgonuovo, il nuovo conte palatino iniziò ad esercitare la facoltà di creare tabellioni. Le prime nomine verbalizzate nel registro Instrumenta capitularia 8bis sono piuttosto significative perché, a quanto consta, rappresentano i primi esempi in area trentina di investiture da parte di un conte palatino di cui si abbia notizia ${ }^{25}$.

Il 19 novembre 1427 si presentò dinanzi ad Antonio da Molveno il giovane Giovanni, figlio di Ermanno da Mori e, inginocchiatosi dinanzi al conte, «nobis humiliter supplicaverit quatenus de predictis officiis notariatus et ordinarii iudicatus gratiam sibi faciendo ipsum investire dignaremus». Dopo aver udito tale supplica e dopo aver ricevuto giuramento il conte procedette all'investitura

dantes et largientes eidem Iohanni auctoritate predicta plenam licentiam et liberam potestatem ex nunc in antea ubique locorum et per totum sacrum Romanum imperium prenotata officia in iudicio et extra iudicium, recte, pure, fideliter et legaliter exercendi litteras, instrumenta, acta, prothocolla, testes et ultimas voluntates notandi,

24 Ivi, cc.72v-73r, n. 208. Sono presenti quali testimoni magister Giovanni di Luca cirogicus da Trento, magister Thomeus phisicus dal Tesino, Gioacchino Mezzasoma, ser Gianpietro e Agostino, fratelli e figli di ser Corradino da Feltre, ser Pietro Iacob, ser Bonadomano de Accerbis, Adelperio Calepini, Francisco de Sichis, ser Bartolomeo da Bologna, Guglielmo notaio detto Saraceno, Martino notaio da Volano, Giacomo figlio del magister Maffeo cirogicus de Metallis de Gargnano.

25 Albino Casetti dà notizia di una nomina a notaio da parte del conte palatino Giovanni Antonio Pona nel 1518 (ASTn, Atti dei notai, Rogiti di Simone Patton di Trento, cc. 33-36): A. Casetti, Il notariato trentino, cit., pp. 245-247. 


\section{Stefano Malfatti}

conficiendi, copiandi, exemplandi, emancipandi, manumittendi et adoptandi tutores et curatores, dandi et costituendi alimenta pupilis, viduis et orfanis decernendi, testes recipiendi, examinandi, scribendi, publicandi confessiones super quibuscumque contractibus audiendi, recipiendi, scribendi et insinuandi, decreta interponendi et omnia alia et singula faciendi que ad publicum imperialem notarium et iudicem ordinarium pertinere noscuntur ${ }^{26}$.

L'8 maggio dell'anno seguente Giovanni de Fraudental, già scriba presso il castello del Buonconsiglio e cappellano della cattedrale, come recita la rubrica in margine al documento rogato da Antonio da Borgonuovo, dopo aver prestato giuramento fu creato «notarium publicum et iudicem ordinarium per pennam et calamarium $\gg{ }^{27}$.

Questa, dunque, doveva essere una delle tappe nell'ideale percorso formativo dell'aspirante notaio. Tornando all'investitura a conte palatino rilasciata ad Antonio da Molveno, si legge che fondamentale per l'accesso alla professione doveva essere la conoscenza «in literatura», ovvero si deve presumere - in mancanza di ulteriori specificazioni - nella grammatica, nella lingua latina, come avveniva un po' ovunque nei collegi notarili dell'epoca.

Dove si formassero con precisione i notai trentini non è dato sapere con certezza; si ritiene comunque plausibile, come avveniva anche altrove, che i primi rudimenti di latino, se non una solida conoscenza della materia, venissero acquisiti presso $\mathrm{i}$ numerosi professores in gramaticalibus che la città ospitava almeno dal XIII secolo ${ }^{28}$. La documentazione trentina di XIV e XV secolo non manca infatti di ricordare fra gli attori di qualche negozio giuridico, ma più spesso fra $\mathrm{i}$ testimoni chiamati a presenziare ai rogiti, i nomi di quanti in città esercitavano la professione di magistri, magistri a scolis, professores artis gramatice, doctores artis gramatice, loice ac retorice o, addirittura, rectores scollarum in gramaticalibus ${ }^{29}$. Fra questi, all'inizio del Trecento, si ricordano il magister Pace, denominato nelle fonti scolastico nel 1306, maestro di grammatica nel 1320, professore dell'arte grammaticale in diverse occasioni fra il 1326 e il 1333. Dei suoi tre figli, Giacomino detto a Cantono, Andrea e Bonifacio, i primi due esercitarono l'arte notarile, il terzo è menzionato come apothecarius ${ }^{30}$.

26 Il documento (ADTn, ACap, Instrumenta capitularia 8bis, c. 74r, n. 210) presenta la nomina a notaio di Giovanni da Mori ma Antonio da Borgonuovo, nella usuale rubrica che affianca l'atto, scrive: «Carta sive privilegium qualiter dominus Antonius de Molveno comes palatinus etc superius nominatus creavit Iohannem filium Hermani de Murio et Laurencium Antonii notarii de Castro notarios et iudices ordinarios cum pena et calamario eos investiendo de officiis predictis, recepto prius ab eis corporali debito sacramento quod erunt fideles etc et fideliter scribent etc ut infra in utriusque eorum privilegio sibi concesso et facto describitur». Di seguito il notaio trascrive il privilegio al nuovo notaio Giovanni da Mori ma manca quello di Lorenzo de Castro.

27 Ivi, c. $73 v$, n. 209.

28 Sul tema dei maestri di grammatica a Trento nel medioevo cfr. S. Weber, I maestri di grammatica a Trento fino alla venuta dei pp. Gesuiti, «Studi trentini di scienze storiche», 1, 1920, pp. 193-200, e, più recentemente, L. de Finis, Dai maestri di grammatica al liceo di via S. Trinità in Trento, Società di Studi trentini di scienze storiche, Trento 1987 (Monografie, 44) e Q. Antonelli, Storia della scuola trentina, dall'umanesimo al fascismo, Il Margine, Trento 2013, in particolare le pp. 25-32.

29 De Finis, Dai maestri di grammatica, cit., p. 26.

30 Weber, I maestri di grammatica a Trento, cit., p. 199. Nella prima metà del Trecento si annoverano anche il magister Rainaldo, citato nel 1339 e, secondo Simone Weber, dovette insegnare a Trento anche Manfredo da Verona: ivi, p. 200. 


\section{La formazione di Antonio da Borgonuovo}

Fra le figure di una certa rilevanza nella Trento di metà Trecento, c'è anche il notaio Nicolino da Mechel che le fonti ricordano come magister olim rector scollarum Tridenti in gramaticalibus ${ }^{31}$; il termine rector permette di ipotizzare l'esistenza di forme di insegnamento 'primario' più organizzate, con la presenza di una figura, il rettore per l'appunto, che poteva ricoprire una funzione coordinativa rispetto alle singole figure di maestri che, individualmente, impartivano lezioni di latino. Secondo Simone Weber, Nicolino (o Nicolò) da Mechel esercitò contemporaneamente la professione notarile ${ }^{32}$, il che non è inusuale visto lo stretto legame che connetteva la grammatica all'arte notarile ${ }^{33}$.

Dopo circa un decennio compare nella documentazione il nome di un'altra figura di spicco del Trecento trentino, il doctor artis gramatice Stefano da Cles, figlio del magister Trentino. Il personaggio si rivela di una certa importanza nel tentativo di ricostruire le tappe formative dei notai trentini poiché, il 9 gennaio del 1373, costui si era formalmente impegnato a tenere un corso d'istruzione di quattro anni a un gruppo di diciotto giovani, ricevendo a tal scopo uno stipendio di 18 marche:

magister Stefanus de Clex, artis gramatice doctor, solemni stipulatione promisit et convenit cum Henrico quondam domini Petri de castro Clex, Guillelmo et Nicolao fratribus notariis filiis quondam ser Otonis notarii de Clex, Ropreto quondam Venturini de Clex, Benevenuto quondam Degeleguardi de Dresio et mihi Bartholomeo notario infrascripto dicto Thomeo, bene et legaliter docere decem et octo scolares ... ad quatuor annos proximos per dictos ser Henricum, Guillelmum, Nicolaum, Ropretum, Benevenutum et me Bartholameum notarium dictum Thomeum infrascriptum, in scholis dicti magistri Stefani eligendos ${ }^{34}$.

Dopo aver specificato che l'impegno sarebbe stato valido se si fosse raggiunto il numero di diciotto scholares («si in dictis scolis suis fuerunt ad numerum dictorum XVIII scolarium in predicto numero dictorum XVIII scolarium computandorum filios dictorum ser Henrici, Guillelmi, Nicolai, Ropreti, Benevenuti et mei Bartholomei dicti Thomei notarii infrascripti»), il documento informa che Enrico, Nicolò e Benvenuto si impegnavano a pagare ciascuno trenta lire di denari piccoli veronesi, mentre Guglielmo e Roberto avrebbero pagato trentacinque lire ciascuno; venti lire sarebbero inoltre state versate dal notaio rogante Bartolomeo, per un totale di 180 lire veronesi, ossia 18 marche. Oltre al prezzo pattuito per i quattro anni di lezioni in gramaticalibus l'istrumento rivela qualcosa di molto importante sui 'destinatari' di questo corso scolastico: si è infatti in presenza dei figli di alcuni notai. Dei sei domini citati, infatti, tre (i fratelli Guglielmo e Nicolò e il notaio

31 Ibidem e de Finis, Dai maestri di grammatica, cit., p. 33, in particolare alla nota 41. Per l'attestazione di Nicolò da Mechel quale rector schollarum cfr. il documento in ADTn, ACap, capsa anniversari, rotoli lunghi/b, n. 10, di data 1362 maggio 13.

32 Weber, I maestri di grammatica a Trento, cit., p. 200.

33 Gherardo Ortalli presenta una serie di esempi di professores in gramaticalibus contemporaneamente impegnati nel notariato: cfr. G. Ortalli, Scuole e maestri fra medioevo e rinascimento: il caso veneziano, Il Mulino, Bologna 1996, in particolare le pp. 112-120.

34 V. Inama, Una scuola di grammatica in Cles nel secolo XIV, «Archivio trentino», 13, 18961897, pp. 231-234. L'Inama dichiara di aver visto una delle due copie (datate rispettivamente 1373 e 1375) di un documento contenuto in un registro di instrumenta notarili appartenente all'archivio di Castel Thun e che si trovava presso l'archivio dei conti Thun di Děčín in Boemia. Si tratta del registro del notaio Bartolameo detto Tomeo da Tuenno (Quaternum imbreviaturarum scriptarum per me Bartholomeum dictum Thomeum de Tuyeno. Anno MCCCLXII, indictione X). 


\section{Stefano Malfatti}

rogante Bartolomeo) sono espressamente qualificati come notai. Non è inverosimile, dunque, pensare che costoro avessero intenzione di far intraprendere anche ai propri figli la carriera notarile, iniziando proprio dall'insegnamento primario. Come accade altrove $^{35}$, non si conosce nel dettaglio il programma di queste scole; si deve tuttavia ritenere plausibile il fatto che il percorso di studi servisse anzitutto ad acquisire una certa padronanza della lingua latina, propedeutica e necessaria per l'accesso alla professione notarile.

A partire dal $1378^{36}$ il nome di Stefano da Cles si ripresenta nella documentazione rogata a Trento; egli presenzia infatti alla tonsura di Francesco figlio di Bartolomeo da borgo San Martino, detto «Polenton», parente del più noto umanista Sicco. Più tardi, il 22 giugno 1391, dichiara di dover saldare al Capitolo un debito di 7 lire di denari piccoli veronesi per l'affitto di una casa nel quartiere del Borgonuovo ${ }^{37}$. Muore prima del 12 ottobre 1406 lasciando un figlio di nome Giovanni, detto Feragu, e una figlia di nome Gasperina che nel frattempo era andata in sposa ad Antonio da Seregnano. La moglie Pellegrina è ancora viva nel $1415^{38}$. Parlando di Stefano da Cles, Simone Weber ricorda che, nello stesso periodo in cui il professor di origini anaune teneva scuola in città, erano attestati nel capoluogo anche un certo magister Giovanni «repetitor in gramaticalibus puerorum» ${ }^{39} \mathrm{e}$, in precedenza, un magister Matteo da Riva, pure professor artis gramatice, che compare come testimone in un documento del 29 ottobre $1378^{40}$, il che potrebbe far presumere che vi fosse la necessità di un numero maggiore di maestri di fronte ad un aumentato numero di studenti ${ }^{41}$.

Solo negli anni Venti del Quattrocento si trova nuovamente nelle fonti un professor a scolis; è il caso del magister Hendricus, detto per l'appunto a scolis, originario di Mori che, oltre a comparire in un rilevante numero di documenti, spesso in qualità di testimone ${ }^{42}$, viene chiamato il 18 ottobre 1424 dal Comune di Trento a redigere una responsio alla lettera che il vescovo Alessandro di Masovia aveva spedito ai rappresentanti della città pochi giorni prima ${ }^{43}$.

Parlando di formazione non si può dimenticare come, almeno fino al XII secolo, essa fu affidata prevalentemente, se non esclusivamente, alle scuole che sorsero presso

35 Cfr., fra i molti esempi, il caso di Como: M.L. Mangini, Il notariato a Como: «Liber matricule notariorum civitatis et episcopatus Cumarum» (1427-1605), Insubria University Press, Varese 2007, pp. 48 e ss.

36 De Finis, Dai maestri di grammatica, cit., p. 43. Desiderio Reich, ma senza alcun documento che attesti con sicurezza il fatto, ritenne plausibile che Rodolfo Belenzani, formatosi certamente a Trento fra il 1380 e il 1390, fosse stato allievo proprio di Stefano da Cles: cfr. D. Reich, Rodolfo de' Belenzani e le rivoluzioni trentine (1407-1409). Tradizione e storia, «Tridentum», 10, 1907, pp. 1-38, p. 18.

37 Weber, I maestri di grammatica a Trento, cit., p. 201.

38 ADTn, ACap, Instrumenta capitularia 8, c. $228 r-v$, n. 454.

39 Codicis clesiani, cit., p. 223 (III, c. 51).

40 ADTn, ACap, capsa anniversari, rotoli corti/a, n. 8.

41 De Finis, Dai maestri di grammatica, cit., pp. 43-44.

42 Fra i molti: ADTn, ACap, capsa anniversari, rotoli lunghi/e, n. 13; ADT, ACap, Instrumenta capitularia 8bis, c. $21 r$-v, n. 63; cfr. Appendice, regesto n. 22 (ADTn, ACap, Instrumenta capitularia 8bis, c. $30 v$, n. 86 e 86a); ADTn, ACap, Instrumenta capitularia 8 bis, c. $113 v$, n. 312; ADTn, ACap, Instrumenta capitularia 8, cc. 245v-246r, n. 494; ADTn, ACap, Instrumenta capitularia 8, c. 301r-v, n. 614.

43 ADTn, ACap, Instrumenta capitularia 8bis, c. $21 r-v$, n. 63. Non è irrilevante il fatto che la città avesse affidato proprio a un esperto in grammatica e retorica la stesura della lettera al vescovo, soprattutto considerando il tenore della missiva vescovile. 
monasteri e scuole cattedrali; esse rappresentano, infatti, il luogo privilegiato per la formazione non soltanto dei futuri ecclesiastici, ma anche dei laici che intendevano acquisire un'istruzione di base ${ }^{44}$. Ciò avvenne, con ogni probabilità, anche a Trento ove, tuttavia, pur ritenendo verosimile la presenza di una scuola cattedrale, non si hanno attestazioni certe né di scolastici né di una vera e propria scuola prima della metà del XIII secolo, ovvero prima del IV Concilio lateranense ${ }^{45}$, che aveva introdotto due gradi di insegnamento distinti: il primo, elementare e gratuito, era presente in tutte le chiese, comprese le cattedrali, e veniva finanziato dal Capitolo; un secondo grado, superiore, era invece riservato ai futuri ecclesiastici e prevedeva insegnamenti di carattere teologico e biblico. Ad impartire le lezioni era chiamato un teologo, spesso scelto fra gli stessi canonici, e denominato nelle fonti scolasticus.

L'analisi svolta da Emanuele Curzel sulle fonti capitolari trentine mostra, almeno fino alla metà del XV secolo, un'assoluta carenza di informazioni relativamente a questo secondo grado di istruzione; nessuno fra coloro che ricoprirono la carica di scolastico, infatti, lasciò tracce significative, né dal punto di vista documentario né dal punto di vista culturale. Mancano, al contempo, anche scolares - termine che in area trentina designa quanti avevano a che fare, in vario modo, con la scuola, e dunque sia i magistri veri e propri sia gli studenti ${ }^{46}$ - che dichiarino esplicitamente la loro appartenenza alla scuola cattedrale, con la sola eccezione di Giacomo da Cadore $^{47}$. Nemmeno è possibile verificare in alcun modo l'esistenza di rapporti fra i numerosi magistri in gramaticalibus attivi in città nel corso del XIV secolo, e ancor prima, con la scola cathedralis, rispetto alla quale essi potevano esercitare l'attività in assoluta autonomia.

In conclusione, se da un lato la basilica di San Vigilio non sembra mostrare, per buona parte del basso medioevo, alcuna significativa traccia di un insegnamento superiore, tale da rendere a Trento - per usare le parole di Emanuele Curzel - «a dir poco evanescente ${ }^{48}$ il secondo livello di istruzione previsto dal IV Concilio lateranense, dall'altro non si può negare la presenza in cattedrale di personaggi (canonici, cappellani ecc.) che senza dubbio portavano con sé un importante bagaglio culturale, derivante da una formazione che, in molti casi, avvenne presso le maggiori scole europee dell'epoca.

Non si può dunque escludere a priori qualsiasi attività di insegnamento e formazione rivolta agli aspiranti tabellioni che, come si deduce dalla documentazione capitolare, affollavano pressoché quotidianamente le navate della basilica wangiana, soprattutto considerando che alcuni fra questi notai, fra cui ad esempio Venturino de Trechis, Alberto de Floriis e Alberto de Stanchariis, erano essi stessi canonici oppure ricoprivano cariche all'interno del Capitolo.

44 Sul tema generale delle scuole cattedrali in Italia in età precomunale cfr. D.A. Bullough, Le scuole cattedrali e la cultura dell'Italia settentrionale prima dei comuni, in Vescovi e diocesi in Italia nel medioevo (sec. IX-XIII), Atti del II convegno di storia della Chiesa in Italia (Roma, 5-9 settembre 1961), Antenore, Padova 1964, pp. 111-143. In merito alla presenza, anche a Trento, di una scuola cattedrale cfr. E. Curzel, Scolastici e scolares nella cattedrale di Trento (secoli XII-XV), «Annali di storia dell'educazione e delle istituzioni scolastiche», 9, 2002, pp. 191-204, ampiamente utilizzato per la redazione di questa parte dedicata alle scuole cattedrali.

45 Ivi, p. 193.

46 Ivi, p. 202.

47 Questi, nel 1377, dichiarò di essere stato pagato dal massaro del Capitolo per il servizio prestato ut scolaris presso la cattedrale: ivi, p. 200.

48 Ivi, p. 203. 


\section{Dopo Antonio. Tappe formative dei notai trentini attraverso $i$ verbali dell'Almo collegio dei dottori e notai della città (1459-1546)}

In assenza di ulteriori informazioni che consentano di chiarire il processo formativo dei giovani aspiranti notai trentini si deve attendere, come ricordato, la metà del Quattrocento per avere a disposizione qualche dato più preciso. Sebbene il Collegio notarile di Trento sia attestato nelle fonti dal $1425^{49}$ e, in particolare, l'intero capitolo 91 degli statuti masoviani riporti le norme statutarie della matrico$1 \mathrm{a}^{50}$, soltanto dalla metà del XV secolo sono a disposizione le verbalizzazioni delle sedute del Collegio dei notai e dei giudici di Trento, contenute nel manoscritto 4272 dell'Archivio storico del Comune di Trento ${ }^{51}$.

La lunga serie di norme e verbalizzazioni concernenti le procedure di ammissione al Collegio risultano di grande interesse per dedurre le modalità di apprendistato e formazione degli aspiranti notai. Va tuttavia sottolineato come ci si trovi ormai a quasi un secolo dagli anni in cui, presumibilmente, si formò Antonio da Borgonuovo; sarebbe dunque scorretto applicare il modus operandi in uso nella seconda metà del Quattrocento a quello, oggi sconosciuto, della seconda metà del Trecento. Nonostante ciò, vista la quasi totale assenza di informazioni per il secolo precedente, si è costretti a prendere in considerazione le fonti del pieno Quattrocento, allorquando la documentazione disponibile rende manifesti una serie di percorsi che, al di là di specifiche norme, sostanzialmente non sembrano discostarsi da quelli in uso, già nel secolo precedente, presso altre città dell'Italia centro-settentrionale.

Il 17 dicembre 1459 il giurisperito e canonico trentino Gottardo Calepini, nipote di Antonio da Borgonuovo, si presentò in episcopali palacio, al banchum del vice rettore del Collegio notarile, Cristoforo da Molveno, chiedendo di essere immatricolato

a prefato domino Christoforo vice rectore ... in collegio notariorum civitatis Tridenti, offerens se stare examini et statuta collegii ... observare et manutenere et solvere libras viginti bone monete pro intratica dicti collegii ... et facere omnia que de iure facere tenetur verssus dictum collegium ${ }^{52}$.

Udita la richiesta, il vice rettore affidò, con il consenso dei presenti, a se stesso $\mathrm{o}$ ad altro notaio il compito di sottoporre il candidato ad esame. Due giorni più tardi, il 19 dicembre, gli esaminatori incaricati di interrogare Gottardo, «unanimiter et concorditer», lo giudicarono idoneo a svolgere l'attività di pubblico notaio. Il Calepini procedette dunque al giuramento, promettendo di rispettare gli statuti del Collegio, il suo rettore e di pagare la tassa dovuta per l'ingresso nella matricola.

49 ADTn, ACap, Instrumenta capitularia 8bis, c. 53r, n. 142.

50 Liber I. De civilibus, capitolo 91; vi erano riportati gli Statuta collegii notariorum Tridenti. Edizione in Chemotti, La legislazione statutaria, cit., pp. 192-203 e, più recentemente, Bortoli, Per un'edizione dei testi statutari del Comune di Trento, cit.

51 ASCTn, Comune di Trento, Antico regime, Sezione antica, ACT1-4272, Almo collegio dei dottori e notai della città di Trento, Registri delle immatricolazioni; edito parzialmente e regestato integralmente in Ceraolo, Il collegio notarile di Trento, cit.; cfr., più recentemente, lo studio di Gian Maria Varanini basato sul suddetto registro: Varanini, Il Collegio notarile di Trento, cit. Come sottolinea Gian Maria Varanini, il Collegio dei notai di Trento non produsse un unico registro veicolante sia gli statuti, sia la matricola, cosicché ciò che si trova fra le carte del manoscritto 4272 è un alternarsi continuo di verbali per l'ammissione al Collegio, atti di ordinaria amministrazione e provvedimenti normativi (Ivi, p. 504).

52 ASCTn, Comune di Trento, Antico regime, Sezione antica, ACT1-4272, c. $4 r-v$. 


\section{La formazione di Antonio da Borgonuovo}

Anche il Collegio notarile di Trento, dunque, non diversamente dai collegi di molte altre città, ammetteva i nuovi membri soltanto dopo uno specifico esame che era previsto già negli statuti alessandrini del 1425-1427. Su cosa esattamente vertesse questo examen si dirà a breve.

Il 26 gennaio 1470 tre candidati, Antonio da Grigno, Andrea Gallo e Giovanni da Calavino fecero richiesta di ammissione «in cetum notariorum». Di essi il verbale specifica che, dopo aver mostrato i privilegi di notariato - che dunque il candidato alla matricola doveva già possedere al momento della richiesta di ammissione - furono sottoposti ad esame e pronunciarono il giuramento. La commissione esaminatrice stabilì tuttavia

quod hinc ad unum annum proxime venturum non valeant neque audeant scribere acta iudicialia seu processus a XXV libris superius, neque alia instrumenta nisi instrumenta procurationis et creditus, cum consilio tamen massarii ... collegii vel alterius notarii intelligentis et pratici sub pena privationis officio ${ }^{53}$.

I tre furono dunque ammessi alla matricola, ma con riserva: non potevano redigere atti giudiziari o processi il cui valore eccedesse le 25 lire, né altre tipologie di instrumenta, con l'eccezione delle procure e dei crediti alla presenza, tuttavia, del massaro del Collegio o di un altro notaio più esperto, pena la privazione dell'ufficio. Norme così rigorose avevano senza dubbio lo scopo di impedire a notai non sufficientemente esperti di rogare documentazione di una certa complessità, tutelando al contempo la professionalità di quanti già esercitavano l'arte ${ }^{54}$ ed evitando in questo modo il progressivo degrado del notariato pubblico. La commissione aggiunse infatti che i candidati avrebbero dovuto «scholas visitare et audire Notariam saltim per annum vel Institutam a domino potestate vel ab alio lectore iuxta posse suum» ${ }^{55}$. Le schole qui menzionate non si riferiscono necessariamente a vere e proprie scuole di notariato, sullo stile di quella istituita presso lo Studium bolognese; pur non essendovene certezza, potrebbe trattarsi di quelle già menzionate scuole laiche, a pagamento, in cui magistri e professores impartivano ai giovani aspiranti notai una conoscenza quanto più possibile solida della lingua latina oppure, più semplicemente, una sorta di «attività di formazione per opera di un notaio nella propria statio, nei confronti di allievi, suoi coadiutori e futuri colleghi» ${ }^{56}$. D'altro canto, non si può comunque escludere del tutto la presenza di corsi di ars notarie specificamente dedicati ai giovani apprendisti notai considerato che, anche nella vicina città di Verona, nel 1462 era stato affidato a un dottore del Collegio dei giudici e

53 Ivi, c. $15 r$.

54 Varanini, Il Collegio notarile di Trento, cit., p. 506. Alla luce di queste considerazioni va letta la disposizione del 1464 quando il Collegio presieduto dal rettore Calepino Calepini alzò a venticinque anni l'età minima per accedere alla matricola; la seduta fu inoltre l'occasione per ammettere due nuovi membri, Federico figlio del nobile Giovanni di Michele de Ceris da Pergine e Graziadeo del fu Nicolò Galego; non prima, tuttavia, di averli esaminati e dopo aver ricordato agli stessi l'importanza di mantenere un comportamento adeguato «ne scandalum eis incurat propter eorum iuventutem»: ASCTn, Comune di Trento, Antico regime, Sezione antica, ACT1-4272, c. 14r. Per un confronto con l'età richiesta negli altri collegi notarili, G. Tamba, E. Tavilla (a cura di), Nella città e per la città. I notai a Modena dal IX al XX secolo, Atti del convegno di studi (Modena, 16 ottobre 2010), Giuffrè, Milano 2013 (Collana del dipartimento di scienze giuridiche e della facoltà di giurisprudenza dell'Università di Modena e Reggio Emilia. Nuova serie, 89), p. 94, nota 60.

55 ASCTn, Comune di Trento, Antico regime, Sezione antica, ACT1-4272, c. $15 r$.

56 Tamba, Formazione professionale del notaio, cit., p. 1276. 


\section{Stefano Malfatti}

degli avvocati il compito di leggere pubblicamente e commentare le Istitutiones di Giustiniano, che forse corrispondono a quell'Institutam cui si riferisce la commissione esaminatrice trentina, la Summa di Rolandino e l'Aurora, due trattati di ars notarie, ovvero quanto necessario a praticare la professione notarile ${ }^{57}$. Ciò che qui è utile notare è che queste 'lezioni' furono affidate al podestà, che era per statuto uno iurisperitus, o ad altro lettore che avesse pari conoscenze («iuxta posse suum») e non a un semplice notaio ${ }^{58}$.

Il 21 gennaio 1459 il celebre notaio trentino Approvino Approvini chiese al Collegio di immatricolare il fratello Stefano, il quale aveva ricevuto l'investitura a tabellione dal conte palatino Giacomo Cariolo; avendolo tuttavia giudicato «nimis iuvenis», Antonio de Fatis, nuovo rettore del Collegio, decise di accogliere il candidato nella propria casa per metterlo alla prova, «quod interim ipse dominus rector vollebat eum examinare utrum esset sufficiens» ${ }^{59}$. Dopo un congruo periodo di apprendistato - poco meno di quattro mesi - ad aprile l'Approvini si presentò nuovamente dinanzi alla commissione che, dopo averlo giudicato «idoneum et bonum gramaticum», lo ammise nella matricola seppur con alcuni limiti nella redazione di instrumenta e testamenti. Un anno più tardi, il 20 febbraio 1460, dopo aver sostenuto un «examen circa confectionem instrumentorum» ${ }^{60}$ ottenne l'autorizzazione a redigere qualsiasi tipologia documentaria pur sotto il controllo, per un ulteriore periodo di dodici mesi, di almeno un membro del Collegio:

Item idem dominus rector ... cui comissum fuit examen infrascripti Stefani notarii ... dixit et approbavit dictum Stefanum sufficientem et ydoneum notarium ad exercendum artem notarie quo ad scribendum acta, sed quo ad instrumenta conficiendum videtur sibi quod non audeat reddigere in formam instrumenta aliqua hinc ad unum annum, nisi prius sint visa et lecta per ser Christoforum notarium de Molveno, ser Leonardum notarium a Sale, ser Antonium notarium de Brezio et ser Iesamantum notarium de Archo vel per unum eorum ${ }^{61}$.

L'attenzione, da parte del Collegio notarile trentino, per la conoscenza della grammatica, fra i principali temi dell'examen cui venivano sottoposti i giovani candidati, si presenta in molteplici occasioni; essa potrebbe nascondere la volontà e l'esigenza di migliorare la qualità di un ceto, quello notarile, forse non all'altezza dei gravosi compiti che gli venivano affidati ${ }^{62}$. Così si trova, ad esempio, che

57 G. Sancassani, M. Carrara, L. Magagnato (a cura di), Il notariato veronese attraverso i secoli. Catalogo della mostra in Castelvecchio, Collegio Notarile di Verona, Verona 1966, pp. 16-17. Lorenzo Sinisi (Formulari e cultura giuridica notarile, cit., p. 171, nota 11) sottolinea «la significativa connessione fra l'insegnamento della «Notaria» e quello relativo alle Institutiones [...] attestata anche nell'ambito degli «studia generalia».

58 Come sottolinea Marco Bellabarba, infatti, il podestà trentino «era un giurista a tutto tondo, un tecnico del diritto preparatosi negli studia universitari e cresciuto nell'applicazione rigorosa della dottrina romano-canonica; per occupare il tribunale vescovile, secondo quanto stabilivano le rubriche statutarie degli Alessandrini, il grado dottorale era un requisito indispensabile»: Bellabarba, Rovereto castrobarcense, cit., p. 21. Diversamente da Trento, nella Rovereto veneziana del Quattrocento, il tribunale cittadino non era presieduto da un legum doctor ma da un semplice funzionario delle magistrature veneziane, sostanzialmente privo di qualsiasi rudimento in materia.

59 ASCTn, Comune di Trento, Antico regime, Sezione antica, ACT1-4272, cc. $1 v-2 r$.

60 Ivi, c. $5 v$.

61 Ivi, c. $6 v$.

62 Gian Maria Varanini sottolinea come «la definizione e l'applicazione delle regole, nella estenuante ripetitività documentata dal registro, è essa stessa una circostanza rilevante; così come è rilevante 
vanni Battista de Murlinis fu «ydoneus et bonus scolaris in gramaticalibus» ${ }^{63}$, mentre Francesco del fu ser Vittore da Grigno fu giudicato «unanimiter et concorditer ... sufficientem in gramaticha» ma non ancora idoneo a redigere «instrumenta [et] ultimas voluntates usque ad dicta festa natalicia», mentre, come al solito, non gli sono preclusi gli atti giudiziari ${ }^{64}$. Per altri, è il caso del notaio Giovanni Caligaroti, l'ammissione alla matricola fu vincolata «sub pactis et conditionibus quod dictus Iohannes debeat perserverare scolam et adissere $\rangle^{65}$.

In qualche modo, come rileva anche Gian Maria Varanini, per ogni caso «l'avvio alla professione è guidato in modo personalizzato e attento» ${ }^{66} \mathrm{e}$, sembra di dover aggiungere, non si individua un unico iter formativo che accomuni tutti i candidati. Se è vero, infatti, che il privilegio rilasciato per conto dell'autorità imperiale e una solida conoscenza della lingua latina dovevano costituire una base di partenza comune per tutti i giovani candidati, poco si può dire in merito alla formazione tecnico-pratica, e dunque più propriamente notarile, di questi individui. Certo, non si può fare a meno di soffermarsi su quel «perseverare scolam», che può avere il duplice significato di perseverare nello studio e nell'impegno, ma anche nella frequenza di una scuola, o di un corso, per la formazione dei notai. Forse quello stesso 'corso' che sembra essere affidato, come già ricordato, al podestà cittadino, oppure veri e propri 'corsi' attivi presso i notai più esperti.

Da un lato si trovano candidati come Gottardo Calepini e suo fratello Calepino, già doctores iurisperiti, con in mano un bagaglio culturale nettamente superiore rispetto a quello di un semplice notaio; costoro, infatti, non sembrano mostrare alcuna difficoltà nel superare l'esame del Collegio, tanto che gli esaminatori li accolgono immediatamente «unanimiter et concorditer». Si tratta, tuttavia, di casi isolati. Soltanto una minima parte della cittadinanza, spesso i membri delle famiglie più in vista, poté recarsi al di fuori dell'episcopato, presso uno degli Studia più noti dell'epoca (Padova e Bologna per ricordarne un paio), per compiere specifici studi in diritto ${ }^{67}$. Per tutti gli altri la formazione doveva necessariamente avvenire in loco, tanto più considerando il fatto che la cultura del notaio, sostanzialmente di natura tecnico-pratica, non richiedeva la conoscenza di letteratura giuridica specifica. I formulari più noti, fra cui appunto quello di Rolandino, e una conoscenza di massima dei negozi giuridici in essi trattati, unitamente alla conoscenza della grammatica, sembravano infatti sufficienti. In mancanza di uno specifico corso poi,

la modifica alle procedure d'esame, che a partire dal 1461 avvengono «publice in generali collegio» anziché, come in precedenza, a cura di una commissione ristretta»: Varanini, Il Collegio notarile di Trento, cit., p. 508. Non mancano, tuttavia, le contraddizioni di un sistema che a Trento, come altrove, affidava a conti palatini, spesso incapaci di valutare criticamente la qualità dei giovani candidati, le nomine a notaio. Nella seconda metà del Trecento troviamo a Trento, residente nel quartiere di Borgonuovo, il conte palatino Francesco de Patheriis da Parma (anno 1375): cfr. ADTn, ACap, Instrumenta Capitularia 6, c. $29 r$, n. 56.

63 ASCTn, Comune di Trento, Antico regime, Sezione antica, ACT1-4272, c. $6 r$.

64 Ivi, c. $8 r$.

65 Ivi, c. $32 v$.

66 Varanini, Il Collegio notarile di Trento, cit., p. 507.

67 Esiguo è il numero dei trentini attestati presso lo Studium bolognese fra Duecento e Quattrocento: cfr. A. Bertoluzza (a cura di), Studenti trentini all'università di Bologna (dal 1200 al 1700), Centro culturale "Fratelli Bronzetti", Trento 1989. Cfr. anche l'elenco degli studenti trentini elaborato nel 1885 da Cesare Festi e relativo alle università di Bologna, Padova, Ferrara, Parma, Pavia, Perugia, Pisa, Siena e Roma: C. De Festi, Studenti trentini alle università italiane, «Archivio storico per Trieste, l'Istria e il Trentino», 4, 1885, pp. 36-63. 
era l'apprendistato presso un notaio più anziano ed esperto, magari parente dello stesso aspirante professionista, a rivestire un ruolo centrale, ma spesso insufficiente, per l'acquisizione delle conoscenze tecniche e pratiche utili all'esercizio della professione.

Non si è dunque di fronte a un modello formativo standardizzato, unitario, quanto piuttosto - lo si ribadisce - costruito volta per volta a seconda delle necessità del candidato. Nella varietà delle modalità applicate pare comunque di scorgere tutti quegli elementi che, pur variamente disposti, la maggior parte dei collegi notarili basso medievali applicarono. Dalle normative statutarie ai prerequisiti necessari per fare domanda alla matricola, nulla sembra distinguere nettamente il Collegio trentino dagli altri collegi notarili italiani, se non un'applicazione tardiva delle stesse modalità. 



\section{Capitolo 3 \\ L'attività professionale di Antonio da Borgonuovo}

Il primo documento noto redatto da Antonio da Borgonuovo è una sentenza arbitrale pronunciata in episcopali palatio il 13 febbraio $1386^{1}$. Per qualche tempo, la documentazione conservata prodotta dal professionista è ancora piuttosto irregolare e sporadica, mentre tende ad infittirsi sul finire del XIV secolo allorquando, divenuto notaio di fiducia del Capitolo della cattedrale, i rogiti conservati si fanno via via più frequenti e abbondanti, tali da poterlo considerare fra i più 'prolifici' e dinamici professionisti della città.

Come si vedrà nel dettaglio, la documentazione redatta da ser Antonio, soprattutto se messa a confronto con quella dei colleghi trentini di fine Trecento-inizio Quattrocento, si caratterizza sia per la quantità, in parte da imputare alla conservazione di documenti prodotti per enti - quali, ad esempio, il Capitolo - che hanno sin da allora una Überlieferungschance fisiologica, sia per la qualità, ovvero per l'estrema varietà dei negozi giuridici trattati, in un panorama documentario che si contraddistingue solitamente per la massiccia presenza di locazioni e refute.

Non meno importante, rispetto all'attività svolta da ser Antonio per il Capitolo e per altri enti cittadini, è l'impegno del professionista per una clientela privata più 'occasionale', ma non meno interessante e per lui professionalmente complementare, della quale rimangono tracce soprattutto attraverso il registro Instrumenta capitularia 8bis, oltre che per mezzo delle extensiones sopravvissute che egli affidò ai suoi clienti.

Nelle pagine che seguono si prenderà pertanto in considerazione l'attività professionale di Antonio da Borgonuovo per le più importanti istituzioni della città ma anche per la clientela privata; sarà, a tal proposito, necessario individuare, per ciascun ente preso in esame, le caratteristiche dei sistemi di 'organizzazione della memoria', onde poterne distinguere con maggiore chiarezza il ruolo svolto dai notai, in particolare da ser Antonio. Ciò da un lato permette di restituire il dinamismo della professionalità del notaio, dall'altro consente di contestualizzarne l'operato nell'ambito di una più ampia riflessione sull'evoluzione dei sistemi di produzione, conservazione e trasmissione della documentazione a Trento fra Trecento e Quattrocento, mettendo, ove possibile, a confronto il suo operato con quello di altri colleghi attivi in analogo scorcio d'anni (fig. 5).

Referenze fotografiche. Le figg. 6-10 sono pubblicate con l'autorizzazione dell'Archivio di Stato di Trento (concessione n. 6/2018 del 21.11.2018, prot. 2124 del Ministero per i beni e le attività culturali).

1 Cfr. Appendice, regesto n. 3; ASTn, APV, Sezione latina, capsa Miscellanea I, n. 126. 
L'attività professionale di Antonio da Borgonuovo

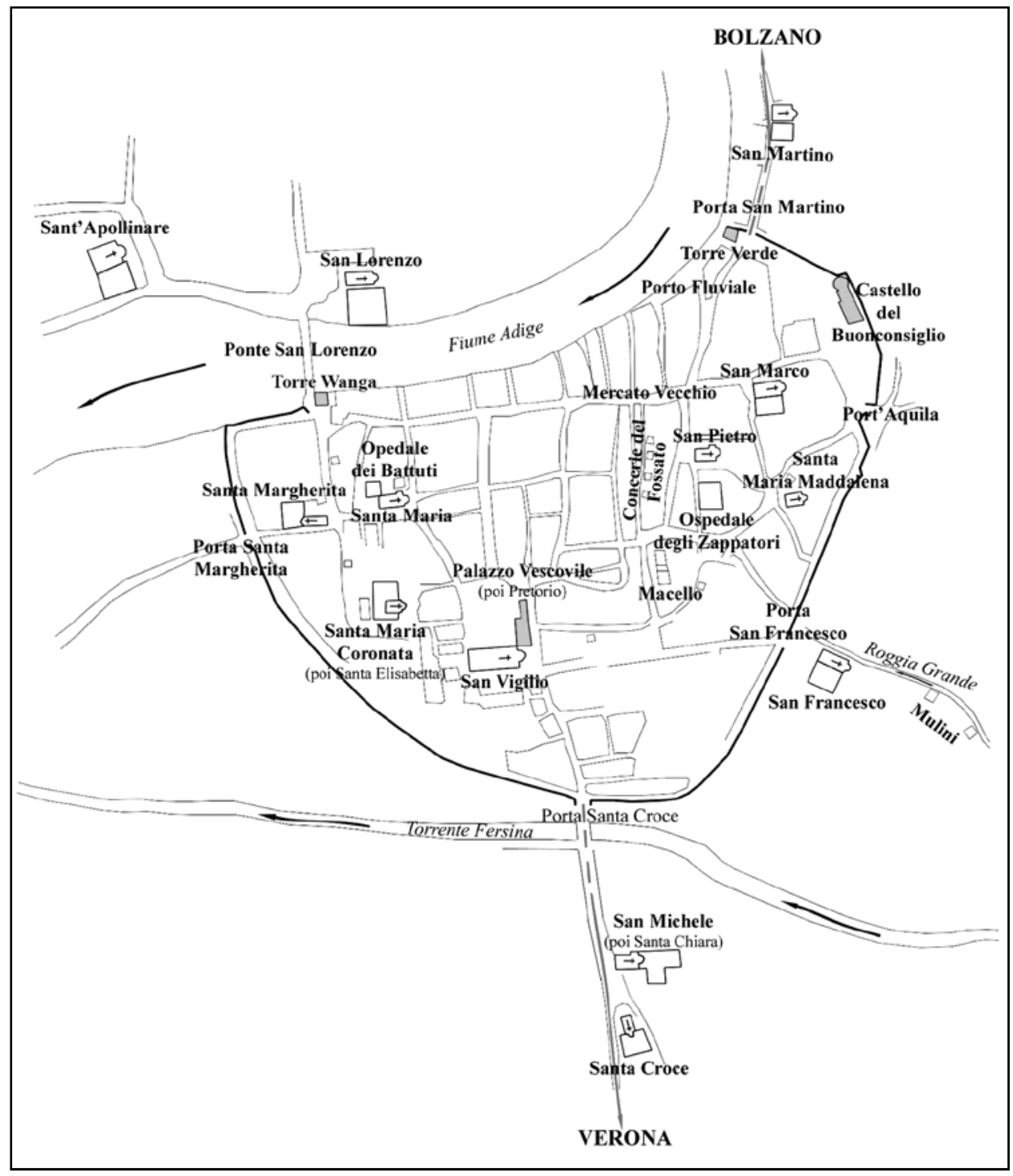

Fig. 5. Edifici religiosi, spazi del potere civile e della vita economica a Trento in età medievale (tratto da: G. Albertoni, G.M. Varanini [a cura di], Il territorio trentino nella storia europea, II: L'età medievale, FBK Press, Trento 2011, p. 158. Elaborazione grafica di G. Weber). 


\section{Fra notariato e cancelleria. Note sulla produzione documentaria vescovile fra la metà del Trecento e la metà del Quattrocento}

Antonio da Borgonuovo inizia a lavorare nella Trento degli anni Ottanta quando lentamente una cancelleria episcopale si stava strutturando e diventava un punto di riferimento per un giovane, intraprendente notaio che voleva fare carriera. Erano trascorsi oltre sessant'anni da quando, ai primi del Trecento, Bongiovanni di Bonandrea aveva dato nuovo impulso ai sistemi di produzione e conservazione della documentazione vescovile ${ }^{2}$. L'opera innovatrice del bolognese proseguì nei decenni successivi, con i notai Trentino di Zuccolino da Tuenno e, dal 1333, Enrico da Landsberg, che si sottoscrivevano utilizzando la qualifica di scriba episcopi, in quanto chiamati a svolgere una funzione di 'supervisione' e 'coordinamento' sui «prodotti documentari di altri notai» ${ }^{3}$.

Alla metà del Trecento, a più di un secolo dalla redazione, su richiesta del vescovo Federico Vanga, del Liber Sancti Vigilii (o Codex Wangianus Minor), venne portato a compimento il Codex Wangianus Maior ${ }^{4}$. Con esso furono riproposti, senza mutazioni di rilievo, gli atti contenuti nel codice duecentesco cui, tuttavia, fu aggiunta una ulteriore serie di documenti, di tipologia eterogenea, legati prevalentemente al periodo in cui il nuovo presule boemo Nicolò da Brno promosse quest'iniziativa $^{5}$. Un'attenta analisi codicologica e testuale del Codex Maior permette di rilevare come il codice trecentesco fu redatto quasi per intero dalla mano del notaio transalpino «Conradus natus Friderici Greusseri civis de Monte Kuctis imperiali auctoritate notarius $\rangle^{6}$; nella sottoscrizione di ogni documento da lui esemplato, Corrado elenca dettagliatamente i propri dati 'biografici' e l'auctoritas imperiale che l'ha investito di publica fides, chiarendo inoltre quale sia il suo status professionale nella confezione del Codex, ovvero qualificandosi quale scriba domi-

2 Cfr. D. Rando, M. Motter, Il «Quaternus rogacionum» del notaio Bongiovanni di Bonandrea (1308-1320), Il Mulino, Bologna 1997 (Storia del Trentino. Serie II. Fonti e testi, 1).

3 Ivi, p. 65. Enrico da Landsberg, «imperiali auctoritate notarius et ... domini episcopi scriba» (ASTn, $A P V$, Sezione latina, capsa 61, n. 65), è attivo almeno dal 1333. Questi roga per il vescovo anche fuori della città di Trento; lo si ritrova, ad esempio, in molte occasioni a sottoscrivere documentazione datata a Bolzano fra il gennaio e il febbraio del 1339 (cfr., ad esempio, ASTn, APV, Sezione latina, capsa 57, nn. 3, 72, 73; capsa 61 , nn. 67, 68; capsa 59, n. 118; capsa 57, nn. 44, 75) e, nel dicembre dello stesso anno, al seguito del vescovo Nicolò da Brno presso il castello di Tenno, ove redige l'investitura feudale di Riprando da Campo (ivi, capsa 68, n. 149). L'attività di Enrico da Landsberg, che dal 1336 è citato anche quale canonico della cattedrale (Curzel, I canonici e il Capitolo, cit., p. 514), prosegue almeno fino al 1343 (lo si ritrova, in episcopali castro Boni Conscilii, a sottoscrivere un documento insieme ai notai Bonaventura del fu Pellegrino da Riva, Nicolò da Trento e Nicolò del fu Bonaventura da Volano [1343 gennaio 22]. È definito olim scriba il 14 gennaio 1344: ivi, p. 514).

4 D. Frioli, La "costruzione" di un registro vescovile: Nicolò da Brno, vescovo di Trento (13381347) e il Codex Wangianus Maior, in G.G. Merlo (a cura di), Vescovi Medievali, Biblioteca francescana, Milano 2003, p. 208; le datazioni esplicite riportate nel Codex non si riferiscono all'esemplazione del corpus documentario, ma alla sola prassi di autenticazione del materiale stesso.

5 Ivi, p. 209.

6 Precendenti al suo arrivo in area trentina sono i rapporti fra Corrado e il vescovo Nicolò; Corrado infatti è attestato quale notaio di Giovanni Volek, vescovo di Olmütz e, negli stessi anni, decano del Capitolo cattedrale è Nicolò da Brno. Corrado appare al fianco di Carlo, il futuro imperatore Carlo IV, e dunque dello stesso Nicolò, in occasione del viaggio che, nel 1336, vide il conte in Tirolo per difendere gli interessi del fratello Giovanni Enrico. Sulla base dei rapporti già esistenti, il notaio transalpino può dunque ricondursi a quella familia episcopale che, abbandonati i luoghi di origine, si ricostituì nell'episcopio tridentino. 


\section{L'attività professionale di Antonio da Borgonuovo}

ni episcopi ${ }^{7}$. Ed è proprio la diversa facies che caratterizza i due cartulari, quello duecentesco dalla struttura grafica e codicologica più variegata, e quello trecentesco più unitario, a rendere palesi le tracce di un'evoluzione documentaria legata alla progressiva specializzazione e fidelizzazione dei notai attivi per l'episcopio. Federico Vanga ricorse alle prestazioni di vari notai, i cui nomi ricorrono numerose volte, replicati, fra i fascicoli del Codex Minor, tuttavia nessun vincolo sembrava legare quei professionisti a Federico o all'episcopato. Un secolo più tardi, la prestazione prioritaria di Corrado Greusser nel Codex Maior è indice di maggiore stabilità nei rapporti tra i notai di curia e il vescovo di Trento. Ciò rappresenta indubbiamente un primo tentativo da parte dell'episcopato di stringere un legame più saldo con i notai attivi nella propria curia; un gruppo di professionisti che, tuttavia, risulta ancora scarsamente articolato ${ }^{8}$.

Le dinamiche connesse con la produzione e la conservazione delle carte vescovili fra Tre e Quattrocento necessitano di ricerche più approfondite ${ }^{9}$, che possono essere svolte soltanto attraverso lo spoglio e lo studio sistematico della documentazione relativa a quegli anni. Tuttavia, pur senza trarre conclusioni definitive, è possibile individuare alcune linee di tendenza per il periodo che intercorre fra gli episcopati di Alberto di Ortenburg (1360-1390) e Alessandro di Masovia (1423-1444).

Proprio a partire dall'episcopato di Alberto di Ortenburg fanno la comparsa documenti che, sotto il profilo diplomatistico, sono pienamente ascrivibili alla categoria del documento notarile, redatti transversa charta, con la datazione topica e cronica in protocollo, il signum e la sottoscrizione del notaio rogante. Tuttavia, queste pergamene presentano la particolare caratteristica di essere dotate di sigillo cereo pendente. Si tratta di documentazione rogata prevalentemente presso il Buonconsiglio, spesso «in stupa parva superiori», ove il vescovo agisce quale auctor dell'azione giuridica (fig. 6).

Il 2 febbraio 1368 il vescovo Alberto fa redigere una investitura di alcune decime esatte presso la villa di Stenico; roga il documento Gioacchino Mezzasoma, «imperiali auctoritate notarius» il quale, tuttavia, prima di sottoscrivere il rogito scrive: «in quorum omnium testimonium et ad maius robur presentes fieri fecit et sui sigilli appensione muniri mandavit», annunciando così il sigillo vescovile che si trova infatti appeso alla plica ${ }^{10}$. In altri casi, pure caratterizzati dalla presenza del

7 Sulla qualifica di scribae episcopalis curiae cfr. G. Chittolini, "Episcopalis curiae notarius". Cenni sui notai di curie vescovili nell'Italia centro-settentrionale alla fine del Medioevo, in Società, istituzioni, spiritualità. Studi in onore di Cinzio Violante, CISAM, Spoleto 1994, pp. 221-232.

8 F. Cagol, Il ruolo dei notai nella produzione e conservazione degli atti delle cancellerie giudiziarie della città di Trento (secoli XIII-XVI), in A. Giorgi, S. Moscadelli, C. Zarrilli (a cura di), La documentazione degli organi giudiziari nell'Italia tardo-medievale e moderna, Atti del convegno (Siena, 15-17 settembre 2008), Ministero per i beni culturali e ambientali, Roma 2012 (Pubblicazioni degli Archivi di Stato. Saggi, 109), I, p. 178.

$9 \quad \grave{E}$ attualmente in corso una specifica ricerca condotta da Rossella Ioppi destinata a ricostruire la fisionomia dell'archivio vescovile fra medioevo ed età moderna, inerente anche alle caratteristiche della cancelleria vescovile. Quanto si riassume in questo paragrafo, dunque, non può che avere natura provvisoria, essendo frutto di un primo censimento sulla documentazione episcopale Tre- e Quattrocentesca. Le ricerche di Ioppi consentiranno senza dubbio di chiarire al meglio l'argomento.

10 ASTn, $A P V$, Sezione latina, capsa 62, n. 98. Per un caso analogo cfr. ivi, capsa 59, n. 132: si tratta di un documento notarile redatto da Sicherio del fu Michele da Vezzano, vergato tuttavia transversa charta, ove però manca il sigillo sebbene prima della sottoscrizione si legga: «In cuius rey testimonium hec publicum instrumentum quod fieri et conscribi fecit per me Sicherium notarium 


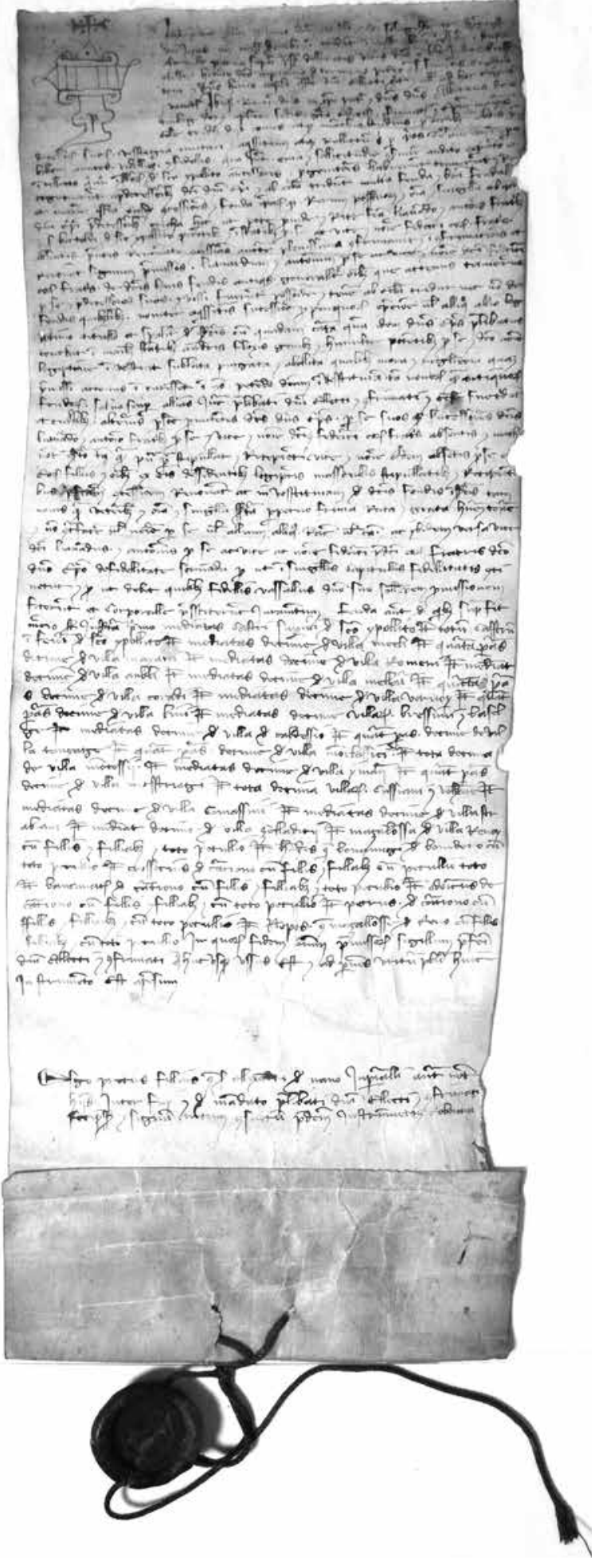

Fig. 6. ASTn, $A P V$, Sezione latina, capsa 58, n. 40 (1365 dicembre 4). Il vescovo Alberto di Ortenburg rinnova l'investitura a Lianardo e Antonio figli del fu ser Bertoldo da Sant'Ippolito. Documento notarile sottoscritto da Pietro del fu Alessandro da Nanno, notaio per autorità imperiale, ulteriormente convalidato da sigillo cereo del presule trentino, annunciato in escatocollo dalla formula: «in quorum fidem omnium premissorum sigillum prefati domini ellecti et confirmati, quo huc usque ussus est et ad presens utitur publice huic in instrumento est apensum». La data topica, in protocollo, recita: «Tridenti, in castro Boni Conscilii, in stupa a fornello parva superiori». 


\section{L'attività professionale di Antonio da Borgonuovo}

vescovo quale auctor, si hanno invece semplici documenti notarili, privi di sigillo e di relativa corroborazione ${ }^{11}$.

Un'ulteriore tipologia che, in analogo scorcio d'anni, viene prodotta per conto di Alberto di Ortenburg è quella del documento redatto recta charta, privo in apertura della invocazione tipica del rogito notarile e con il datum in escatocollo (fig. 7). Apre il protocollo l'intitulatio «Nos Albertus de Ortenburg electus et confirmatus», auctor dell'azione giuridica, cui fa seguito un dettato redatto in forma soggettiva, senza l'intermediazione della mano di un pubblico notaio. L'investitura registrata nel documento è valida in quanto emessa dall'autorità vescovile, lì rappresentata dal sigillo cereo pendente ${ }^{12}$ (fig. 8).

Tipologie documentarie eterogenee, analoghe a quelle poc'anzi descritte, continuano ad essere impiegate durante l'episcopato di Alberto di Ortenburg il quale, pur privilegiando il documento sigillato, con o senza l'intermediazione notarile, fa anche sporadico utilizzo del tradizionale documento redatto da pubblici professionisti ${ }^{13}$.

Esclusivamente notarile è invece la documentazione redatta su commissione dei procuratori del vescovo, i quali si servono di un ristretto gruppo di notai, probabilmente legati da un qualche rapporto di fiducia con l'autorità vescovile, per rogare $i$ propri documenti. Così, ad esempio, il procuratore vescovile Francesco da Molveno, egli stesso un notaio, che agì in rappresentanza di Alberto di Ortenburg dal $1367^{14}$ al $1383^{15}$, si servì nel tempo di un certo numero di notai: Guglielmo ab Equabus, Rodolfo del fu ser Basino da Trento, Antonio del fu Michele da Ranzo, Antonio da Pomarolo, Giacomo del fu Bartolomeo da Ravazzone, Giovanni del fu Enrico da Viarago, Pietro Mezzasoma, Francesco del fu Pietro da Isera, Alberto del fu ser Negratus da Sacco, Marco del fu Odorico del fu Giacomo da Spormaggiore. Costoro non sembrano tuttavia esplicitare, attraverso la sottoscrizione, alcun particolare rapporto con il procuratore vescovile, dichiarandosi costantemente e semplicemente notai d'autorità imperiale. Per quanto concerne, invece, la documentazione in cui il vescovo figura come auctor, nei casi in cui tali documenti furono redatti dalla mano di professionisti, si rileva la presenza di un ristretto gruppo di tabellioni che, in alcuni casi, esplicitano la loro qualifica di «notarius et domini episcopi scriba».

Nel 1368, ad esempio, il notaio di origini lagarine Francesco del fu magister Martino da Volano, si firma in sottoscrizione «publicus imperiali auctoritate notarius et officialis curie Tridentine ${ }^{16}$ sottolineando un rapporto di tipo burocratico-funzionariale con la curia vescovile. Come del resto è già stato ben messo in rilievo da Daniela Rando per l'inizio del Trecento, anche con l'episcopato di Alberto di Ortenburg la figura dello scriba episcopi si configura come preminente rispetto a un gruppo più ampio di notai che, più o meno saltuariamente, prestano servizio per l'episcopato.

infrascriptum sigili hereditatis sue quo utitur de presenti idem dominus episcopus appensione muniri mandavit et absque ipsius sigili apensione nulam roboris firmitatem habere voluit imo nulius fore momenti et eficacie».

11 ASTn, $A P V$, Sezione latina, capsa 59, n. 131 (1364 gennaio 7).

12 Ivi, capsa 58, n. 50 (1364 gennaio 26). Per un caso analogo si veda, ad esempio, ivi, capsa 10, n. 41 (1364 febbraio 2).

13 Ivi, capsa 37, n. 41 (1364 febbraio 4), redatto dal notaio Antonio del fu Filippo da Mori.

14 Ivi, capsa 64, n. 190 (1367 ottobre 31).

15 Ivi, capsa 64, n. 228 (1383 dicembre 6).

16 Codicis Clesiani, cit., pp. 192-193 (II, cc. 36-37). 


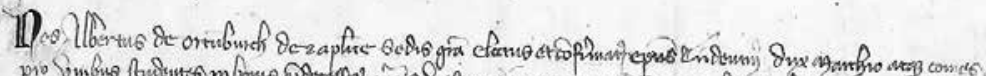

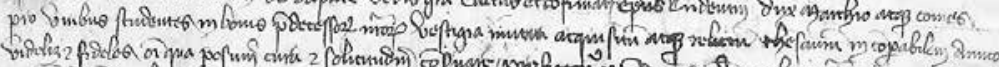

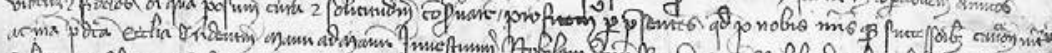

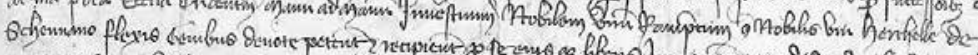

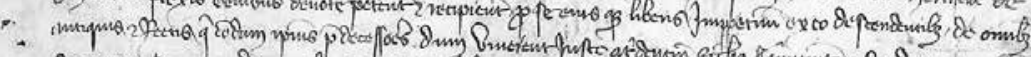

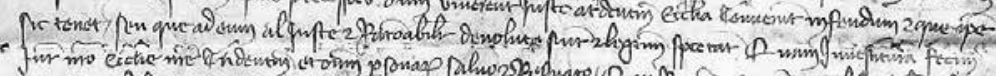

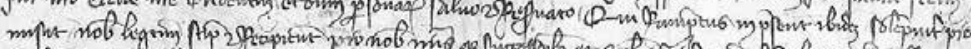

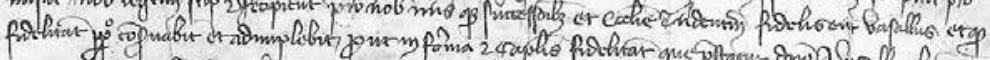

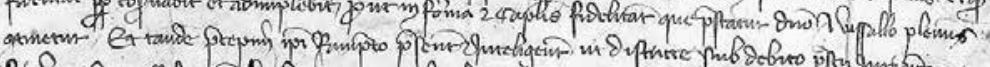

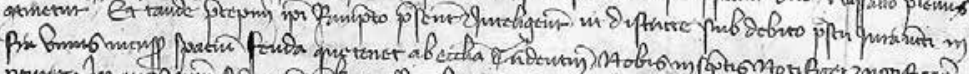

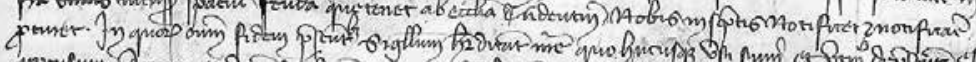

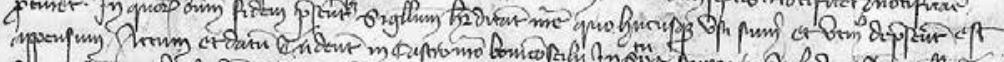

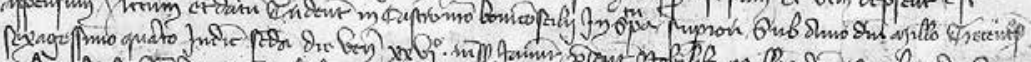

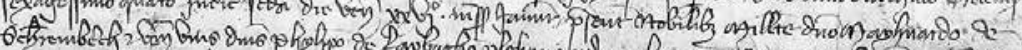

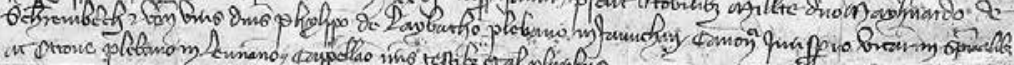
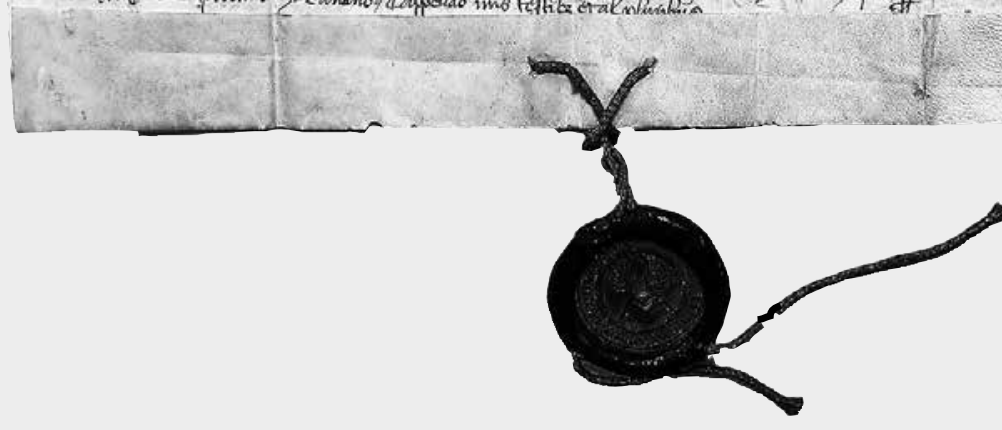

Fig. 7. ASTn, $A P V$, Sezione latina, capsa 58, n. 50 (1364 gennaio 26). Il vescovo Alberto di Ortenburg investe il nobile Ramperto de Heithele da Scenna dei feudi che già anticamente la sua famiglia teneva dall'episcopato. In escatocollo, dopo l'annuncio del sigillo, l'actum e il datum («in castro nostro Boniconscilii, in stupa superiori»), seguiti dall'elenco dei testimoni. Sigillo cereo del vescovo Alberto.

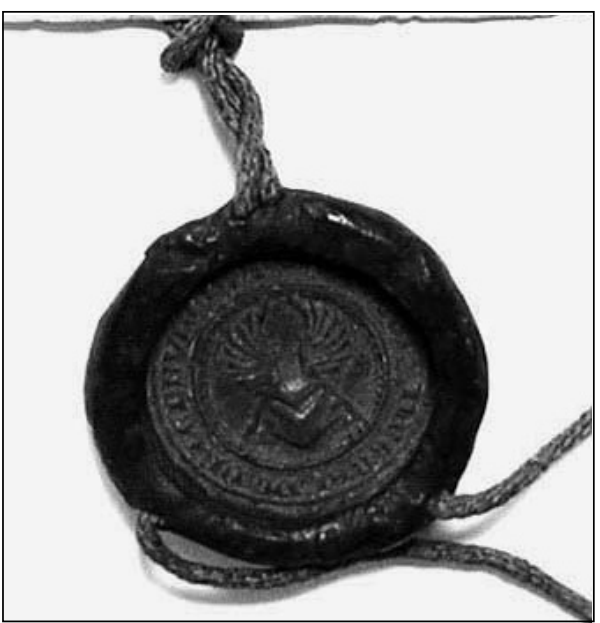

Fig. 8. ASTn, $A P V$, Sezione latina, capsa 58, n. 50 (particolare). 


\section{L'attività professionale di Antonio da Borgonuovo}

Fra i notai che possono vantare il titolo di scriba e officialis di curia si annovera anche Pietro Paolo figlio di ser Giovanni detto Ianes che ricoprirà analoga carica anche per l'abate di San Lorenzo a partire dal $1374^{17}$. Di costui si conserva presso l'archivio vescovile un registro di imbreviature con documentazione rogata per un buon numero di enti, prevalentemente ecclesiastici ${ }^{18}$.

Sul finire degli anni Settanta del Trecento, anche Giovanni del fu Corrado da Novacella si sottoscrive «imperiali auctoritate notarius et scriba publicus domini episcopi» in una serie di investiture redatte per conto del vescovo Ortenburg ${ }^{19}$. È il caso, per fare ancora un paio di esempi, di Giacomo del fu Federico de Rupprechtstorff «clericus Pataviensis diocesis et scriba episcopi Tridentini», attivo per Alberto di Ortenburg sul finire degli anni Sessanta del Trecento ${ }^{20}$; la medesima qualifica viene richiamata in sottoscrizione dal notaio Marco del fu Odorico da Trento del fu Giacomo da Spormaggiore, che roga per il vescovo fra il 1383 e il 1388, sottoscrivendosi «notarius et officialis domini episcopi» ${ }^{21}$ o «notarius atque scriba et officialis domini episcopi» ${ }^{22}$. Questi, nel 1383, fu anche il redattore di un «Liber instrumentorum spectantium ad dominum episcopum Tridentinum et ad episcopatum et ecclesiam Tridentinam $\rangle^{23}$, con negozi quali locazioni, refute, rinnovi di locazione e immissioni in possesso ${ }^{24}$, stesi secondo modalità che avvicinano questo registro a simili esempi di ambito capitolare ${ }^{25}$.

Fra i notai che, a partire dagli ultimi anni Settanta del Trecento, produssero documentazione per conto del vescovo figura Alberto di ser Negratus da Sacco; questi produsse un buon numero di investiture senza tuttavia esplicitare particolari relazioni con l'autorità vescovile, ma dichiarandosi semplicemente «imperiali auctoritate notarius» (fig. 9). Molti fra i rogiti prodotti per il vescovo si presentano nella forma del consueto documento notarile ma in alcuni casi la sottoscrizione viene fatta precedere da formule del tipo «in quorum fidem et maius robur predictorum predictus dominus fieri mandavit hoc publicum instrumentum et sigilli sui consueti appensione muniri», preannunciando dunque l'apposizione del sigillo vescovile. Nel febbraio $1387^{26}$, l'Ortenburg aveva nominato Alberto da Sacco proprio procuratore, cosicché, a partire da quella data, il notaio figura quale auctor di numerose investiture, vedendosi riconfermare nella carica anche da Georg Liechtenstein. Analogamente a chi lo aveva preceduto nella carica, il già citato notaio Francesco da Molveno, Alberto poté servirsi di un ristretto numero di notai attivi sulla piazza di Trento; fra questi si possono ricordare, ad esempio, il figlio Marcabruno, Antonio di ser Paolo Peverada da Milano, Giacomino di ser Albertino da Posena.

17 R. Ioppi, I registri del monastero di San Lorenzo di Trento. Regesto degli atti (1369-1430), tesi di laurea, Università degli Studi di Trento, a.a. 2013-2014, rel. A. Giorgi, p. 44.

18 ASTn, $A P V$, Sezione latina, capsa 29, n. 8. L'intestazione recita: «Quaternus instrumentorum sub breviate notatorum per me Petrum Paulum notarium infrascriptum». Il protocollo è stato studiato in I. Ricci, Aspetti della società e della chiesa trentina nella seconda metà del Trecento, dal protocollo del notaio Pietro Paolo (1376), tesi di laurea, Università degli Studi di Trento, a.a. 1991-1992, rel. G.M. Varanini.

19 Si vedano i molti esempi in Codicis Clesiani, cit., p. 138 (II, ad esempio alle cc. 128-129).

20 Cfr, ad esempio, Codicis Clesiani, cit., pp. 95-96 (II, cc. 29b-30b).

21 ASTn, $A P V$, Sezione latina, capsa 62, n. 103 (1386 maggio 27).

22 Ivi, capsa 59, n. 139 (1383 gennaio 23).

23 Ivi, capsa 22, n. 2.

24 Per una descrizione del registro cfr. Ioppi, I registri del monastero di San Lorenzo, cit., p. 40.

25 Cfr., per il Capitolo, quanto riportato infra, al paragrafo 4 del presente capitolo.

26 Codicis Clesiani, cit., p. 109 (II, c. 57). 


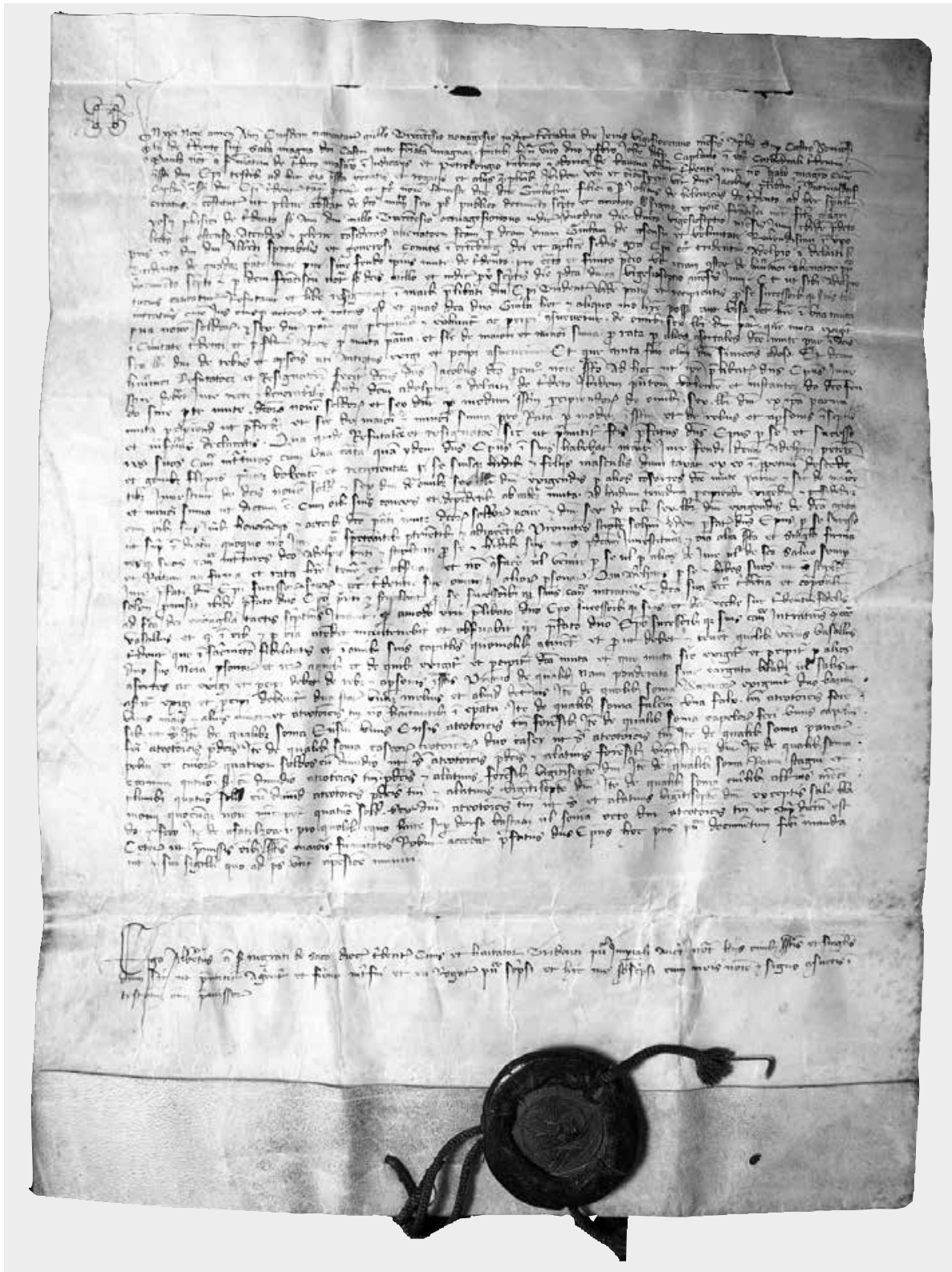

Fig. 9. ASTn, $A P V$, Sezione latina, capsa 3, n. 44 (1390 aprile 28). Guglielma, figlia del fu ser Giovanni Belenzani, refuta nelle mani del vescovo Alberto di Ortenburg una muta parva in favore di Adelperio del fu Delaito da Trento che ne viene investito. Documento notarile sottoscritto da Alberto del fu ser Negratus da Sacco, notaio per autorità imperiale, ulteriormente convalidato da sigillo cereo del presule trentino, annunciato in escatocollo dalla formula: «omnibus suprascriptis maioris firmitatis robur accedat prefatus dominus episcopus hoc presens publicum documentum fieri mandavit et sui sigilli quo ad presens utitur appensione muniri». La data topica, in protocollo, recita: «super castro Boniconsilii de Tridento, super sala magna dicti castri, ante feriatam magnam». 


\section{L'attività professionale di Antonio da Borgonuovo}

Tra i notai chiamati a redigere documentazione per conto del vescovo Liechtenstein, va ricordato anche Paolo del fu ser Martino da Trento. Sebbene costui produca un buon numero di investiture non menziona mai, in sottoscrizione, eventuali rapporti di tipo funzionariale con la curia o il vescovo, dichiarandosi costantemente «imperiali auctoritate notarius». Le pergamene redatte da ser Paolo da Trento per conto dello stesso vescovo, tuttavia, presentano sovente il sigillo vescovile e recano in escatocollo la seguente corroboratio: «Ut autem predictis omnibus maioris robur firmitatis accedat prefatus dominus episcopus voluit et mandavit fieri hoc presens publicum instrumentum per me Paulum notarium infrascriptum et sigilli sui appensione muniri» ${ }^{27}$. Analoga considerazione vale, nello stesso scorcio d'anni, per Thomas da Friesach detto Vasthel e per Paolo del fu Giovanni de Fatis da Terlago i quali, «de mandato domini episcopi», rogano molteplici investiture all'inizio degli anni Novanta. Il primo era un chierico e proveniva da Friesach, località della Carinzia a breve distanza da Ortenburg, città natale del vescovo Alberto. Paolo de Fatis, trasferitosi a Trento negli anni Ottanta, apparteneva invece all'importante consorteria dei da Terlago, il cui capostipite era il nonno Fato ${ }^{28}$.

Mentre rimane sostanzialmente invariato il modus operandi dei procuratori vescovili che, anche nei primi anni del Quattrocento, continuano a servirsi di notai attivi sulla piazza di Trento per redigere la propria documentazione, sembrano invece aumentare numericamente $\mathrm{i}$ documenti con sigillo redatti presso il Buonconsiglio senza alcuna intermediazione notarile. In essi il nome del vescovo campeggia in prima posizione nel protocollo («Georgius Dei gratia episcopus Tridentinus»), seguito dalla inscriptio e, in escatocollo, dalla data topica e cronica con l'elenco dei testimoni. La plica nel margine inferiore regge inoltre il sigillo vescovile poco prima annunciato nella corroboratio. Si tratta, tuttavia, di esempi che non sembrano costituire la regola, essendo ancora prevalenti i documenti in forma di instrumentum notarile.

Una maggior discontinuità, rispetto a quanto descritto finora, sembra emergere soltanto all'indomani dell'arrivo a Trento del nuovo vescovo Alessandro di Masovia (1424-1444), allorquando diventano prevalenti i documenti sigillati redatti in forma di littera (fig. 10).

Il primo agosto 1424 Alessandro rinnova l'investitura di alcuni feudi a Giacomo da Rumo; la pergamena, redatta transversa charta, presenta il nome del vescovo in intitulatio, cui fanno seguito i nomi dei destinatari e il dispositivo in forma soggettiva. In ultimo dopo le varie clausole, il datum che recita: «Datum Tridenti, in castro nostro Boniconsili ... per manus venerabilis Stanislay de Sobnaw artium liberalium magistri Magne Polonie et nostri cancellarii» ${ }^{29}$ : Stanislao Sobniowski canonico, poi preposito dal $1425^{30}$.

La struttura deputata alla produzione della documentazione episcopale mostrava dunque, in età masoviana, un'organizzazione maggiormente articolata, con a capo anche un personaggio di rilievo; i procuratori vescovili continuavano invece a servirsi di notai pubblici per la redazione della documentazione prodotta su loro

27 Si veda, ad esempio, ASTn, $A P V$, Sezione latina, capsa 64, n. 243.

28 Paolo intraprese un'importante carriera come funzionario vescovile (cfr. Bettotti, La nobiltà trentina nel medioevo, cit., p. 261); nel 1432 ottenne dal re Sigismondo la dignità nobiliare insieme ad Antonio e a Giovanni Conto. Nel 1433 la dignità fu confermata dal vescovo Alessandro. Il re aveva contestualmente riconosciuto la famigliarità a Nicolò Mercadenti (cfr. ivi, p. 124).

29 ASTn, $A P V$, Sezione latina, capsa 60, n. 52.

30 Cfr., per una scheda biografica del canonico e preposito, Curzel, I canonici e il Capitolo, pp. 671-672. 


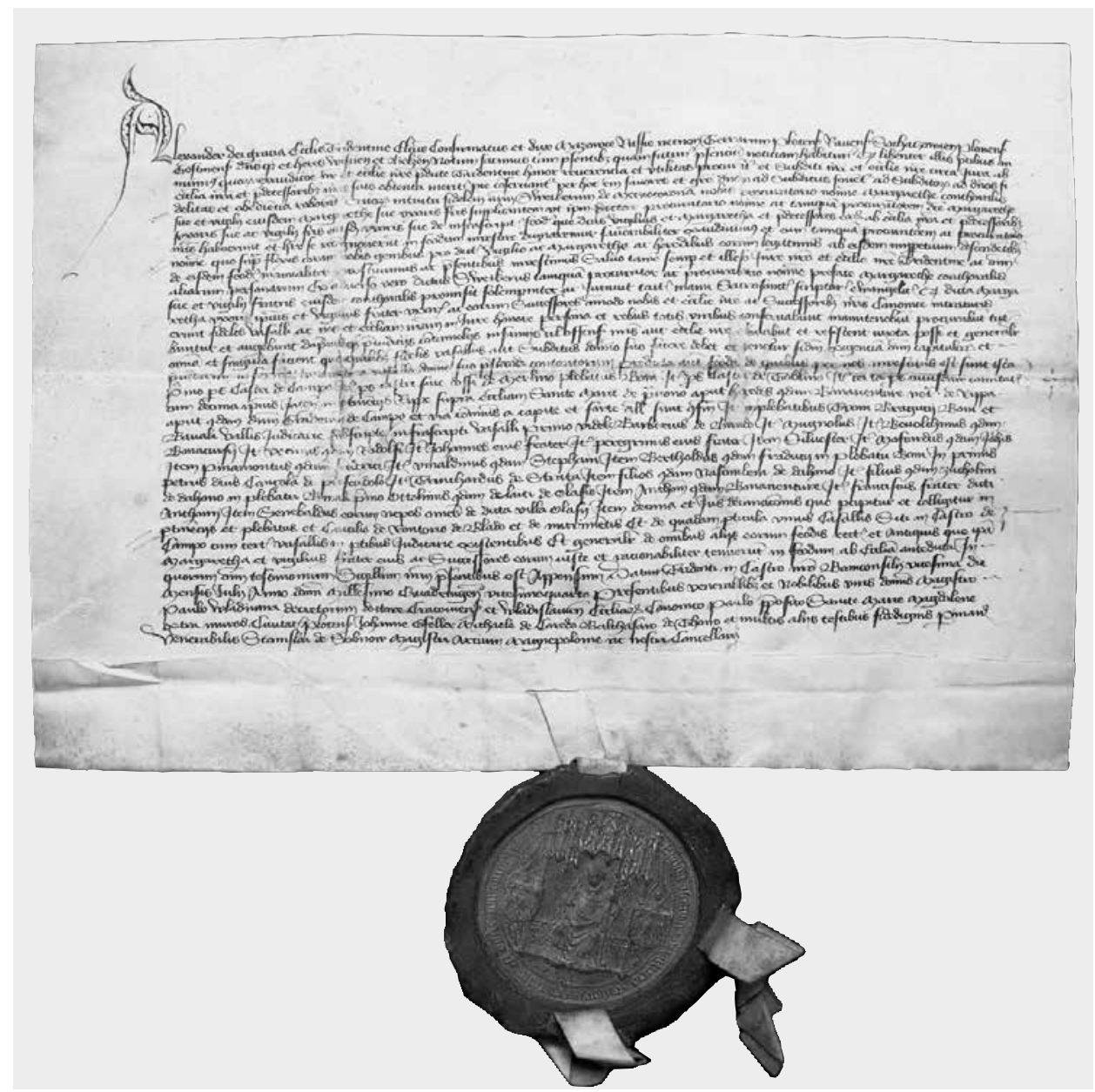

Fig. 10. ASTn, $A P V$, Sezione latina, capsa 58, n. 30 (1424 luglio 20). Il vescovo Alessandro di Masovia investe Sweicherium da Mezzocorona, procuratore di Margherita sua moglie e di Vigilio suo cognato, di parte del castello seu Dossus di Merlino nella pieve di Bono, di parte di castel Toblino e di altre decime situate a Riva del Garda, nelle pievi di Tione, Breguzzo, Bono e Banale nelle Giudicarie. In escatocollo, l'annuncio del sigillo «in quorum omnium testimonium sigillum nostrum presentibus est appensum». Segue il datum, «Datum Tridenti, in castro nostro Boniconsilii ... per manus venerabilis Stanislai de Sobnow magistri artium Magne Polonie ac nostri cancellarii».

mandato. Graziadeo del fu Antonio da Terlago divenne così notaio quasi esclusivo, allorquando il procuratore episcopale Matteo de Murlinis ${ }^{31}$ lo chiamò a rogare una serie di negozi giuridici (per la maggior parte locazioni o rinnovi) fra gli anni Trenta e Quaranta del Quattrocento.

Pur minoritari non mancano, tuttavia, anche durante l'episcopato di Alessandro di Masovia, documenti notarili che presentano come auctor il vescovo; il percorso

31 Cfr., fra i molti esempi, ASTn, APV, Sezione latina, capsa 64, nn. 256-262. 
verso forme di produzione della documentazione pienamente cancelleresche, tali da rendere superflua l'intermediazione della fides notarile, non sembra dunque ancora pienamente compiuto. Se da un lato, tuttavia, si deve constatare una diminuzione della documentazione rogata dai notai trentini su richiesta dei vescovi (in particolare Alessandro di Masovia), dall'altro la documentazione redatta per conto dei procuratori vescovili continua a essere prodotta esclusivamente dai notai attivi sulla piazza di Trento; una buona porzione della produzione documentaria vescovile rogata da ser Antonio, come si vedrà, è redatta infatti per conto dei procuratori del vescovo.

È all'interno di questo contesto che va collocata la figura del giovane Antonio da Borgonuovo. Sebbene la documentazione superstite prodotta dal notaio sia piuttosto esigua (poche unità per gli anni Ottanta e Novanta del Trecento), occorre piuttosto fare attenzione alla qualità e alla tipologia di quei documenti. Le dinamiche e gli incidenti conservativi possono infatti distorcere in modo sostanziale il profilo professionale di un notaio trentino, proprio perché il notariato locale è debole, talvolta casuale, privo di coscienza professionale o di gruppo, e spesso legato alle mutevoli politiche documentarie dei vescovi. Ne è tangibile dimostrazione l'attività di Antonio che, sin da giovanissimo - a fine anni Ottanta doveva avere non più di 25-30 anni -, appare impegnato come notaio al servizio del vicario vescovile presso il palatium episcopi.

\section{L'attività al servizio del vicario vescovile}

Fra la documentazione di Antonio da Borgonuovo una certa rilevanza, soprattutto sotto il profilo degli argomenti trattati, assumono le sentenze e gli atti giudiziari, rogati prevalentemente presso il palatium episcopi, già residenza vescovile, luogo deputato all'amministrazione della giustizia nel capoluogo.

Anche a Trento l'attività scrittoria in giudizio era affidata ai notai, che registravano sui propri protocolli gli atti relativi alle sedute giudiziarie; con l'emanazione dei nuovi statuti cittadini da parte del vescovo Alessandro di Masovia nel 1425-1427, tale compito divenne prerogativa esclusiva degli iscritti al Collegio dei giudici e notai della città di Trento ${ }^{32}$.

Come accennato, il primo documento sopravvissuto è proprio una sentenza, pronunciata nel palazzo vescovile nel $1386^{33}$ dai notai Vigilio da Seregnano, figlio di Guglielmo da Roccabruna, Alberto di Negratus da Sacco e Federico figlio di ser Ognibene da Povo, arbitri scelti per dirimere la controversia fra il canonico Morandino $^{34}$ e Loisio detto dal Dosso ${ }^{35}$.

32 Cfr. Cagol, Il ruolo dei notai, cit., in particolare alle pp. 174-179.

33 Cfr. Appendice, regesto n. 3 (ASTn, APV, Sezione latina, capsa Miscellanea I, n. 126).

34 Sul canonico Morandino da Trento cfr. la scheda biografica in Curzel, I canonici e il Capitolo, cit., pp. 629-631.

35 I notai condannano il canonico Morandino «ad dandum et solvendum dicto Aloysio pro resto affictuum dicti Aloysii per dictos decem annos non exactorum, ut apparet in quaterno rationis receptorum dicti domini Morandini, infrascriptas bladorum quantitates aut sibi Aloysio consignare inquilinos obligatos secundum inventarium datum et consignatum dicto Aloysio per dictum dominum Morandinum, videlicet ...; item pro resto affictuum denariorum non exactorum octo ducatos auri aut sibi consignare ut supra; item dictus dominus Morandinus debeat restituere dicto Aloysio omnes suos libros, cartas et instrumenta quelibet spectantia ad dictum Aloysium, cum omnibus rebus mobilibus, ...». 


\section{Stefano Malfatti}

Negli anni successivi non mancano altri esempi di sentenze con la sottoscrizione del professionista. Il 13 febbraio 1388, ad esempio, il notaio venne chiamato a registrare una serie di acta al palazzo vescovile, «apud banchum iuris maleficiorum», dove il vicario del vescovo Alberto di Ortenburg presiedeva la causa per i diritti di pascolo e di malga sul monte Bondone ${ }^{36}$. I notai che si alternavano nella verbalizzazione delle sedute giudiziarie registravano gli atti sui propri protocolli, seguendo un sostanziale ordine cronologico per cui la documentazione giudiziaria era alternata a rogiti d'altro argomento. Oggi, vista la scarsa conservazione di registri notarili per buona parte del Trecento e del primo Quattrocento nella città di Trento, molta documentazione di natura giudiziaria risulta irrimediabilmente perduta, per cui ciò che rimane è rappresentato in gran parte dalle pergamene in mundum che furono estratte a partire dalle 'matrici' sui cartulari notarili perduti.

Ciò è vero anche per ser Antonio da Borgonuovo, del quale - come ricordato - si hanno alcune pergamene relative a sentenze emesse in episcopali palatio, conservate in ottica tesaurizzante prevalentemente nell'archivio vescovile; a partire dalla metà degli anni Venti del Quattrocento, tuttavia, con l'inizio delle registrazioni su Instrumenta capitularia 8bis, si hanno a disposizione otto scritture inerenti a processi e sentenze discusse dinanzi al vicario vescovile ${ }^{37}$.

Fra queste, si prenderà in esame quella datata fra il 26 e il 28 giugno $1426^{38}$, relativa al processo contro i ferarinos della val di Sole. L'atto non si svolge presso il palazzo del vicario ma al castello del Buonconsiglio, dove Alessandro di Masovia aveva spostato la discussione di alcune sedute giudiziarie da lui stesso presiedute o affidate a propri delegati ${ }^{39}$. Rappresenta il vescovo dinanzi ai giudici Giovanni da Isny e Antonio de Civolis da Ledro il giurisperito Antonio da Molveno che presenta istanza per conto del presule contro i minatori della val di Sole, assistiti dal procuratore Guglielmo Saraceno. Oggetto del contenzioso è il mancato versamento della decima dovuta al vescovo di Trento per l'estrazione del ferro dal tempo della morte di Georg Liechtenstein. Il notaio rogatario Antonio da Borgonuovo registrò, consecutivamente, tutto il procedimento giudiziario, fino alla sentenza, emessa il 28 giugno, con la quale i minatori furono condannati a pagare la decima per il periodo in cui non avevano versato quanto dovuto, ovvero dal 1419.

Il documento brevemente riassunto permette di ricostruire, almeno in parte, $\mathrm{i}$ processi di produzione documentaria del tribunale vescovile. Come accennato in precedenza, il sistema di produzione e conservazione delle scritture giudiziarie era affidato unicamente ai notai, i quali raccoglievano i verbali delle sedute dinanzi al vicario sui propri protocolli e registri; non è dunque casuale il fatto che l'intero procedimento processuale si trovi, oltre che nella redactio in mundum presso l'Archivio vescovile, anche nel registro del notaio Antonio da Borgonuovo. Una nota dello stesso professionista informa inoltre che il testo del processo fu rilevato «ex quibusdam foliis ubi notatus erat per me». Si deve pertanto presumere che Antonio avesse inizialmente steso l'intero verbale della causa su fogli sciolti o su piccoli

36 ASCTn, Comune di Trento, Antico regime, Sezione antica, ACT1-3347.

37 ADTn, ACap, Instrumenta capitularia 8bis, nn. 142, 213, 404a, 404b, 405, 351, 356a, 438. Del n. 213 si ha a disposizione anche la redactio in mundum in ASTn, APV, Sezione latina, capsa 66, n. 2 .

38 ADTn, ACap, Instrumenta capitularia 8bis, n. 213 e ASTn, APV, Sezione latina, capsa 66, n. 2.

39 Cagol, Il ruolo dei notai, cit., p. 178. 
fascicoli per procedere poi alla trascrizione sul proprio registro degli estesi, ovvero in Instrumenta capitularia 8 bis $^{40}$.

Analogo procedimento sembrò seguire il notaio quando, nel 1430, registrò la sentenza emessa «in episcopali palacio, ad banchum ubi ius civile redditur consuete» dal giudice arbitro Vigilio de Trahugis da $\mathrm{Povo}^{41}$. Senza entrare nel merito del contenuto della causa, si può constatare come, anche in quell'occasione, Antonio da Borgonuovo pose in margine al testo steso nel suo registro una nota relativa a una precedente redazione della sentenza: «Relevata ex foliis ubi primitus fuit notata».

Fra le attività riconducibili al palazzo vescovile c'era anche quella del giudice delle tutele che, come ricordato, aveva il compito di affidare gli orfani a dei tutori, dopo aver ricevuto notifica della morte dei genitori. Il 7 maggio $1430^{42}$ Antonio da Borgonuovo, che aveva ricoperto analoga carica nel 1423 e nel 1426, fu chiamato dal giudice delle tutele, il notaio Martino del fu Francesco da Volano, a registrare la nomina di Marco da Lusiana, quale tutore di Guglielma, figlia del fu Nicolò Sardagnole, che era morto nelle Giudicarie durante la guerra fra Paride Lodron e Alessandro di Masovia. Marco procedette quindi al giuramento dinanzi al giudice e al notaio impegnandosi anche a redigere un inventario dei beni dell'orfana. Il 18 settembre successivo ${ }^{43}$ il tutore presentò l'inventario al vicario vescovile Gioacchino Mezzasoma e Antonio da Borgonuovo fu chiamato a metterlo per iscritto ${ }^{44}$.

Fra gli incarichi svolti dal professionista presso il palazzo vescovile va ricordato anche quella di procuratore, che aveva il compito di presentare davanti al giudice le istanze di una delle parti in causa. Tale mansione è documentata in modo preciso e dettagliato anche attraverso un piccolo registro cartaceo, composto da diversi fascicoli di provenienza eterogenea ${ }^{45}$, il primo dei quali trasmette le più antiche registrazioni di cause in giudizio relative agli anni 1414-1417, con estratti di verbali redatti dalla mano del notaio Nicolò da Arco. Si tratta di un vero e proprio registro di acta, dove un unico notaio predispose in ordine cronologico i verbali di più procedimenti; il codice 2544 rappresenta pertanto un unicum, poiché dalla lettura di questi fascicoli è talvolta possibile ricostruire l'intero procedimento giudiziario, altrimenti frammentato fra le carte - in larga misura oggi disperse - dei notai chiamati di volta in volta a prenderne nota.

Ampiamente attestata è l'attività di ser Antonio come procuratore al servizio del Capitolo della cattedrale ${ }^{46}$; con le costituzioni sinodali emanate nel 1336 dal

40 La rubrica di mano del notaio che precede il documento recita: Processus factus per dominum nostrum contra ferarinos in valis Solis pretextu decime petite cum sententia ex inde lata ut infra, relevatus ex quibusdam foliis ubi notatus erat per me etc. La rubrica è preceduta dalla nota relativa all'estrazione del mundum: Facta extra cum sententia in uno rodulo et data in castro etc. Il documento su pergamena presso l'Archivio del Principato vescovile non è di mano del notaio Antonio da Borgonuovo; si tratta di copia non autenticata di mano coeva che reca pure la sottoscrizione di Antonio (ma non di sua mano) con due sigilli pendenti da fili serici. L'inventario di Ippoliti e Zatelli presenta alla Capsa 66 in $A P V$, Sezione latina (mineralia) anche un documento n. 3 uguale al precedente n. 2 ma oggi mancante; poteva trattarsi dell'originale del notaio Antonio da Borgonuovo.

41 ADTn, ACap, Instrumenta capitularia 8bis, cc. 167v-168r, n. 438.

42 Ivi, n. 386.

43 Ivi, n. 387.

44 Il 9 marzo 1431 il notaio fu chiamato inoltre a stendere un'additio inventarii (ivi, n. 387a).

45 BCTn, BCT1, n. 2544.

46 Fra i molti esempi, cfr. ADTn, ACap, Instrumenta capitularia 8, n. 675b (senza data): di fronte a Giovanni Anhang in iure canonicus peritus, canonico e vicario in spiritualibus di Alessandro vescovo di Trento e duca di Masovia, il notaio Antonio di ser Bartolasio procuratore del Capitolo chiede di 
vescovo Enrico di Metz, infatti, il Capitolo fu invitato a scegliere dei procuratori, salariati, «ad petendum, exigendum et recipiendum et bona possessiones et iura Capituli perdita, alienata illicite et distracta recuperandum cum clausolis opportunis $\rangle^{47}$; tale funzione, che veniva affidata a uomini di fiducia, comportava quindi un ulteriore guadagno per il procuratore, che aveva il compito di rappresentare l'ente presso il tribunale vescovile.

\section{L'attività al servizio dell'Episcopato}

Più marginale appare l'attività di Antonio per i vescovi di Trento; poco più di dieci documenti, di cui un paio redatti per conto di Georg Liechtenstein e undici per conto del suo successore Alessandro di Masovia, si sono conservati a testimoniare la collaborazione occasionale del notaio con tali enti.

Il primo documento redatto da ser Antonio per il Liechtenstein è una donazione datata 3 agosto 1405 nota unicamente grazie a una notizia sul Codice clesiano ${ }^{48}$ che, come noto, trasmette in larga misura investiture e atti negoziali promossi dall'autorità vescovile ${ }^{49}$. Null'altro si è conservato relativamente a quel periodo. Se infatti si considera che l'attuale conformazione dell'Archivio principesco vescovile di Trento risulta depauperata di buona parte della documentazione prodotta fra medioevo ed età moderna, oltre al fatto che molti documenti furono trasferiti ad Innsbruck già in occasione del sacco subito dalla città dalle truppe tirolesi nel 1409, e che per gli anni precedenti al 1423-1424 non si hanno a disposizione i registri su cui il notaio potrebbe aver steso documentazione commissionata dal vescovo, si deve ipotizzare che quanto rimasto per gli anni dell'episcopato Liechtenstein non sia che una minima traccia della sua attività per l'ente. Scorrendo le carte del Codice clesiano si nota infatti come Antonio fu presente, come testimone, alla rogazione di un certo numero di documenti del vescovo fra il 1388 e il $1392^{50}$. Il registro Instrumenta capitularia 8 trasmette inoltre un ulteriore documento redatto per conto del vescovo il 14 gennaio $1407^{51}$. Pochi giorni prima i canonici avevano esaminato

costringere Bartolomeo del fu Sicco olim canonico cittadino di Trento di rispondere se è erede di suo padre e se completamente o in parte, e se in parte per quale parte, e se per testamento o intestato, in quanto intende agire contro gli eredi e i beni del fu Siccone a motivo di un affitto di 4 ducati d'oro non pagato da molti anni. Cfr. anche ADTn, ACap, Instrumenta capitularia 8, n. 689b; ADTn, ACap, capsa 32, n. 241.1 (1413 novembre 27); ASTn, $A C D$, n. 546, documento rogato dal notaio Graziadeo del fu ser Antonio fu ser Biagio da Castel Terlago (1414 aprile 27); ivi, nn. 564.1 e 564.2 e 751 procuratore del Capitolo e del canonico Giovanni Anhang.

47 L. Santifaller (a cura di), Urkunden und Forschungen zur Geschichte des Trientner Domkapitels im Mittelalter, I (1147-1500), Universum, Wien 1948 (Veröffentlichungen des Instituts für Österreichischer Geschichtforschung, 6), p. 166.

48 Codicis Clesiani, cit., pp. 346-347 (IV, cc. 247v-248v).

49 Il secondo volume contiene i documenti prodotti fra il 1363 e il 1390 su iniziativa del vescovo Alberto di Ortenburg; il terzo volume i documenti prodotti sempre su iniziativa del vescovo Alberto di Ortenburg fra il 1380 ed il 1388; il IV volume i documenti prodotti fra il 1390 e il 1419 dal vescovo Georg Liechtenstein; il V volume i documenti prodotti su iniziativa del vescovo Alessandro di Masovia fra il 1424 e il 1444.

50 Codicis Clesiani, cit., p. 233 (III, c. 72, 1388 dicembre 28; c. 73, 1387 agosto 4; c. 76, 1387 maggio 23; c. 80, 1388 gennaio 15; c. 86, 1388 febbraio 13; c. 94, 1388 maggio 20. Vol. IV, cc. $98 r$ - ; cc. $182 r-v ;$ cc. $206 r-207 v$ ).

${ }^{51}$ ADTn, ACap, Instrumenta capitularia 8, n. 268b. 
i quaderni degli introiti e delle spese della Fabbrica della cattedrale, amministrata per diciotto anni dal canonico Rambaldo da Trento. Avendo giudicato buona la sua amministrazione, il Capitolo rilasciò quietanza al canonico ${ }^{52}$, liberandolo da ogni obbligo nei confronti della Fabbrica. L'atto fu ratificato anche da Georg Liechtenstein che chiamò presso il castello del Buonconsiglio proprio il notaio Antonio da Borgonuovo, che successivamente stese il documento sul registro del Capitolo.

Null'altro si ha a disposizione fino al 1425, quando il nuovo vescovo Alessandro di Masovia incaricò Antonio di redigere una serie di documenti, di cui rimane traccia in un paio di pergamene reperite fra le capsae dell'Archivio principesco vescovile e, soprattutto, fra le carte del registro Instrumenta capitularia 8bis.

Il primo documento, redatto nell'aprile del 1425 è di un certo rilievo non soltanto dal punto di vista contenutistico ${ }^{53}$, ma anche per il fatto che furono chiamati dal vescovo a redigerlo ben due notai: il canonico Artuico di Enrico chierico di Passau e Antonio da Borgonuovo. La pergamena venne però redatta unicamente dal primo notaio, pur essendo espressamente nominato nella rogatio anche il secondo, del quale, tuttavia, mancano sottoscrizione e signum. Fra le carte del registro Instrumenta capitularia 8bis Antonio predispose invece la sola rubrica relativa all'atto in questione, senza redigere il documento in esteso.

Un secondo rogito redatto il 10 ottobre $1425^{54}$, denominato dal notaio «Prepositura et unio facta», rappresenta l'atto costitutivo della Prepositura, dignità capitolare che incamerò i beni e i redditi del monastero di San Lorenzo. Con essa il vescovo dispose anche l'elezione di un preposito, nella persona di Stanislao di Giovanni Sobniowski, artium liberalium magister, canonico di Trento e suo protocancelliere, che prese immediatamente possesso della carica.

Fra il 1427 e il 1434 Antonio da Borgonuovo fu chiamato a redigere ulteriori nove documenti, che trovarono concretizzazione scritta soprattutto nel suo registro personale (Instrumenta capitularia 8bis) e, in soli due casi, in un paio di pergamene oggi conservate presso l'Archivio vescovile ${ }^{55}$. Si tratta di atti che documentano prevalentemente locazioni, refute e permute attuate da Alessandro di Masovia attraverso suoi procuratori.

Per quanto riguarda i luoghi di rogazione, le date topiche dei documenti vescovili rinviano al castello del Buonconsiglio, in particolare a stanze poste al suo interno: «super sala et ad feratam a capella» ${ }^{56}$, oppure «in canzelaria de prope capellam sita» ${ }^{57}$. Il documento relativo all'erezione della Prepositura fu invece rogato in cattedrale, all'interno della sacrestia magna, probabilmente per il fatto che la nuova dignità dipendeva direttamente dal Capitolo. Da rilevare, invece, come la maggior parte dei documenti attestanti le refute, le locazioni e le permute furono rogati presso la stazione delle bollette, in platea comunis.

52 Ivi, n. 267b.

53 Il vescovo Alessandro intima ai rappresentanti del Comune di Trento di pagare la somma di 700 ducati d'oro agli eredi di Erasmo Thun, quale rimanenza della spesa di 800 ducati che costoro avevano dovuto versare per le spese di fortificazione del castello di Stenico, nel periodo in cui Negro de Negri da San Pietro era stato inviato dalla città come nuovo capitano del maniero.

${ }_{54}$ Cfr. Appendice, regesto n. 28 (ADTn, ACap, Instrumenta capitularia 8bis, n. 131).

55 Si tratta dei documenti con segnatura ASTn, $A P V$, Sezione latina, capsa 53, n. 10; capsa 64, n. 264; ADTn, ACap, Instrumenta capitularia 8bis, nn. 284, 383, 385, 389, 395, 397, 395b, 617.

56 Cfr. Appendice, regesto n. 25 (ASCTn, Comune di Trento, Antico regime, sezione antica, ACT1-3328).

57 ADTn, ACap, Instrumenta capitularia 8bis, n. 395. 


\section{L'archivio del Capitolo fino ad Antonio da Borgonuovo e l'opera decisiva e innovatrice di Pietro de Stanchariis}

I primi decenni del XIV secolo, sia per l'Episcopato sia per il Capitolo della cattedrale, costituiscono una fase di transizione verso nuove forme documentarie che prevedono, come rilevato, non più soltanto la mera conservazione di instrumenta in forma sciolta o la copiatura di una selezione degli stessi in appositi cartolari, ma anche una stabile e continuativa registrazione della documentazione in specifici volumi che, attraverso la normale sedimentazione archivistica, porteranno alla nascita di vere e proprie serie; si passa cioè da quello che si è soliti definire archivio thesaurus ${ }^{58}$, a quello che la moderna teoria archivistica chiama archivio sedimento.

Certamente anche il Capitolo della cattedrale non fu estraneo alla rinnovata attività scrittoria e alle novità in campo documentario in seno all'Episcopio; anche il secondo ente della diocesi, infatti, iniziò a conservare in maniera abbastanza stabile dagli anni Venti del XIV secolo una serie di registri con documentazione di natura prevalentemente, anche se non esclusivamente, patrimoniale rogata da professionisti attivi nel capoluogo vescovile. Si tratta della serie oggi nota con il nome di Instrumenta capitularia ${ }^{59}$.

Un'evoluzione nei sistemi di produzione e conservazione della documentazione del Capitolo si manifestò non soltanto nella progressiva adozione della forma-libro, ma anche nella graduale fidelizzazione di un sempre più ristretto numero di notai chiamati a dare concretizzazione scritta ai negozi giuridici dell'istituzione. Fino agli inizi del Trecento la documentazione capitolare - in prevalente forma sciolta - non rivela alcun tentativo di fidelizzazione dei notai estensori da parte del Capitolo ${ }^{60}$; a

58 Per una qualifica della locuzione 'archivio thesaurus' cfr. A. Giorgi, S. Moscadelli, Ut ipsa acta illesa serventur. Produzione documentaria e archivi di comunità nell'alta e media Italia tra medioevo ed età moderna, in A. Bartoli Langeli, A. Giorgi, S. Moscadelli (a cura di), Archivi e comunità tra medioevo ed età moderna, Ministero per i beni culturali e ambientali - Università degli Studi di Trento, Roma-Trento 2009 (Pubblicazioni degli Archivi di Stato, Saggi, 92; Labirinti, 114), p. 32; si tratta di «un deposito generalmente avulso dal contesto amministrativo corrente, costituito di documenti per lo più in pergamena sciolta, ma anche [...] di statuti o libri iurium, pervenuti o fatti produrre da notai collegati a vario titolo al comune: documenti conservati gelosamente in archivio a memoria di diritti patrimoniali e giurisdizionali, raccolti in casse o armadi». In questo senso, il thesaurus, come rileva Attilio Bartoli Langeli, rappresenta il senso più etimologico del lemma 'archivio', inteso come 'arca', 'arcano', ovvero il luogo deputato alla conservazione degli oggetti più preziosi: cfr. A. Bartoli Langeli, E. Irace, Gli archivi, in G. Pugliese Carratelli (a cura di), La città e la parola scritta, Scheiwiller, Milano 1997 («Civitas europaea»), p. 402.

59 Per una descrizione completa dei volumi del XIV fino alla metà del XV secolo cfr. E. Curzel, Per la storia del Capitolo della Cattedrale di Trento nel Trecento e nel Quattrocento: la serie degli Instrumenta Capitularia, «Studi trentini di scienze storiche. Sezione prima», 71, 1992, pp. 223-260; più recentemente cfr. B. Tomasi, L'archivio del Capitolo della Cattedrale di Trento: produzione e conservazione documentaria (secoli XIII-XX). Con un'edizione delle più antiche pergamene (1147-1250), tesi di dottorato di ricerca, Università degli Studi di Trento, XXV ciclo, a.a. 2011-2012, pp. 45-55. Le origini della serie Instrumenta capitularia vanno probabilmente ricondotte alle disposizioni normative del vescovo Enrico di Metz, contenute negli statuti sinodali del 1336 (Santifaller [a cura di], Urkunden und Forschungen, cit., pp. 155-182). Con esse i canonici erano tenuti a far redigere in un liber sive quaternus, da un notaio di fiducia, i negozi giuridici trattati, quale memoria presente e futura degli affari. Ogni volume risulta infatti vergato quasi esclusivamente da un'unica mano che vi esemplava, per lo più in ordine cronologico, documenti in forma di imbreviatura ma, più frequentemente, di esteso.

60 I canonici sceglievano cioè di volta in volta i professionisti cui affidare la redazione dei propri documenti, senza stabilire con essi alcun legame specifico, come sembrano dimostrare le variegate sottoscrizioni poste in calce ai documenti dagli stessi rogatari. Nessuno fra essi dichiara rapporti di 
partire dall'inizio del XIV secolo si assiste al progressivo affidamento a notai di fiducia, in alcuni casi scelti fra i canonici stessi, della redazione di instrumenta e registri.

Il primo e più antico registro oggi conservato è quello attribuibile alla mano del notaio, già mansionario della cattedrale ${ }^{61}$, Venturino del fu Antonio de Trechis da Mantova, con documentazione - in larga maggioranza locazioni e refute - rogata per i canonici fra il 1324 e il $1347^{62}$. Si tratta di un volume, di cui non è possibile attestare $a b$ origine la presenza nell'archivio capitolare, frutto dell'assemblaggio di più fascicoli cartacei di dimensioni simili ma non identiche. In nessuna sottoscrizione Venturino pone in risalto un rapporto fiduciario instaurato con l'ente capitolare, continuando a definirsi semplicemente imperiali auctoritate notarius ma, circa un secolo più tardi, Antonio da Borgonuovo, ricevendo l'autorizzazione ad estrarre dai rogiti di Venturino, qualificò il notaio defunto come «publicus imperiali auctoritate notarius ac scriba et notarius publicus capituli ecclesie Tridentine ${ }^{63}$.

All'inizio degli anni Trenta del Trecento Venturino fu affiancato nella redazione della documentazione capitolare dal notaio Alberto de Floriis, pure di origini mantovane (si definisce in sottoscrizione civis Mantuanus), già cappellano della cattedrale di Trento dal 1327 e poi canonico dal $1354^{64}$. Costui produsse decine di instrumenta per il Capitolo, cui prestò la propria opera almeno fino al 1356; parallelamente dovette redigere un certo numero di instrumenta anche per privati cittadini, come sembrano attestare alcuni testamenti ancora oggi conservati. Non mancano, anche per questo professionista, gli esempi di documentazione in registro recante atti di interesse capitolare, segno che, al pari di Venturino, anche Alberto predispose appositi fascicoli in cui registrare su richiesta dei canonici i vari negozi giuridici. Ciò che oggi rimane dei 'registri' di Alberto è un fascicolo cartaceo allegato al volume Instrumenta capitularia 5 di Pietro de Stanchariis e un bifolio cartaceo intruso nel corso dei secoli fra le carte del registro di Venturino de Trechis.

Più unitario dal punto di vista codicologico, ma variegato dal punto di vista della committenza, è il secondo volume della serie, noto con il nome di Instrumenta capitularia 4 (1351-1357) ${ }^{65}$; prodotto dal notaio Antonio da Pomarolo, il registro conserva 372 documenti in extenso rogati per enti e privati della città fra cui, pur in minima parte, anche per il Capitolo. L'analisi codicologica e l'esame delle note

collaborazione con l'autorità capitolare, che rappresenta uno fra i molti clienti cui essi prestavano la propria opera (cfr., a titolo esemplificativo, l'indice dei notai rogatari degli instrumenta capitolari in E. Curzel, I documenti del Capitolo della cattedrale di Trento. Regesti 1147-1303, Società di Studi trentini di scienze storiche, Trento 2000, Rerum Tridentinarum Fontes, 6, pp. 531-535). Barbara Tomasi, curando l'edizione delle più antiche pergamene del Capitolo comprese nell'arco temporale 1147-1250 (si tratta di poco più di cento documenti), ha rilevato l'impiego di ben quarantacinque notai diversi (cfr. Tomasi, L'archivio del Capitolo, cit., p. 56). Si tratta, in molti casi, di professionisti attivi, in analogo scorcio d'anni, anche per l'episcopato).

61 Compito precipuo del mansionario era, secondo quanto stabilito dallo Statuto del 1336, quello di amministrare i sacramenti e occuparsi della cura delle anime: Santifaller, Urkunden und Forschungen, cit., n. 143 al capitolo V (De officio mansionariorum). Venturino ricoprì tale carica insieme a Martino da Novara e appare in coppia con quest'ultimo nell'elenco dei presenti al Sinodo del 1336: ivi, n. 143, pp. 156 e 179 .

62 Si tratta del registro Instrumenta capitularia 3; edizione in Malfatti, Il registro del notaio Venturino de Trechis, cit.

63 ADTn, ACap, capsa 44, n. 5.

64 Per la scheda biografica di Martino da Novara cfr. Curzel, I canonici e il Capitolo, cit., pp. 459-460.

65 Mattivi, Il registro del notaio Antonio da Pomarolo, cit. Sono soltanto diciotto i documenti rogati su committenza dei canonici del Capitolo. 
marginali dimostrerebbe, anche per questo volume di metà Trecento, una presenza nell'archivio capitolare soltanto in una seconda fase. Anche per il Capitolo, come per l'Episcopato, si deve ipotizzare come fosse nel pieno interesse dell'istituzione avere a completa disposizione la documentazione attestante il proprio patrimonio; i canonici potrebbero pertanto aver acquisito il registro dagli eredi o dai successori del notaio Antonio nel corso della seconda metà del Trecento o in epoca ancora più tarda ${ }^{66}$.

Il volume successivo della serie, identificato dalla segnatura $n$. 5, è caratterizzato da fascicoli di natura composita; esso risulta infatti redatto, per la maggior parte, dalla mano del notaio (e canonico) Pietro de Stanchariis da Teglie di Brescia. La prima sezione del registro potrebbe invece essere ricondotta ad Alberto de Floriis da Mantova, canonico e notaio negli anni centrali del Trecento ${ }^{67}$.

Ma i registri che segnano il vero mutamento nella politica documentaria e conservativa del Capitolo della cattedrale sono quelli oggi noti come Instrumenta capitularia 6 e $7^{68}$. Essi risultano redatti per intero dal già citato Pietro de Stanchariis, nella sua funzione di notaio capitolare, fra il 1370 e il 1390-1391.

Instrumenta capitularia 6 trasmette prevalentemente «quietanze e promesse di pagamento, nomine di canonici e collazioni di prebende, procure, collazioni di altari e chiese, promozioni agli ordini sacri» ${ }^{69}$ disposte in ordine cronologico. Il registro successivo ha invece una scansione che, in un certo senso, potrebbe qualificarlo ulteriormente rispetto ai volumi precedenti; il codice cartaceo, infatti, al pari di Instrumenta capitularia 8 di Antonio da Borgonuovo, risulta suddiviso in quattro sezioni: la prima è riservata prevalentemente alle locazioni per le prebende individuali, la seconda alle locationes altarium, la terza alle locationes comunitas Capituli, e la quarta alle locationes anniversariorum. Ognuna di esse presenta atti ordinati cronologicamente dall'anno 1370. Dal punto di vista della organizzazione della produzione scritta capitolare il canonico-notaio Pietro de Stanchariis rappresenterebbe dunque un modello per l'attività dei notai successivi, in particolare per Antonio da Borgonuovo che, con il registro Instrumenta capitularia 8 proseguiva in un certo senso l'opera innovatrice del de Stanchariis $^{70}$.

Nonostante Pietro non espliciti mai nelle sottoscrizioni il suo rapporto con l'istituzione, l'assenza di qualsiasi altro documento, per quanto è oggi noto, da lui rogato per altri committenti, fa di costui un notaio al servizio esclusivo del Capitolo. Alla carta $1 r$ del suo registro Instrumenta capitularia $7^{71}$ egli ricorda infatti che, nell'anno 1375, fu designato dal Capitolo della cattedrale di Trento come «scriba

66 Fu il notaio Pietro Mezzasoma, nel 1376, ad ereditare le carte di Antonio da Pomarolo (cfr. ADTn, ACap, capsa testamenti, rotoli lunghi/a, n. 7); ciò, tuttavia, non chiarisce né il modo né il tempo in cui il registro entrò a far parte dell'archivio capitolare.

67 L'ipotesi è in Curzel, Per la storia del Capitolo, cit., p. 234; il confronto grafico fra i documenti in Instrumenta capitularia 5 e alcuni munda sottoscritti da Alberto da Mantova 5 sembrerebbero confermare questa ipotesi. Barbara Tomasi evidenzia, tuttavia, come non sia del tutto escluso che si possa trattare dello stesso Pietro de Stanchariis, il quale avrebbe compiuto operazioni di riordino, recupero e censimento della documentazione capitolare (cfr. Tomasi, L'archivio del Capitolo, cit., p. 50).

68 Il primo si trova in ADTn, ACap, Instrumenta capitularia 6; il secondo in ASTn, ACD, n. 1398 (ex Instrumenta capitularia 7).

69 Tomasi, L'archivio del Capitolo, cit., p. 50.

70 Si rinvia, infra, al capitolo 4, paragrafo 7.2 per una descrizione dell'organizzazione del registro Instrumenta capitularia 8.

71 ASTn, $A C D$, n. 1398-01 (ex Instrumenta capitularia 7). 
et notarius» al servizio dell'ente, ricevendo «in conficiendo instrumenta Capituli et alia undecumque» la somma di 100 lire di moneta di Merano; in seguito, per il periodo compreso fra il 1376 e il 1391, Pietro annotò con precisione l'emolumento versatogli annualmente dai canonici, ottenendo complessivamente per i quindici anni di servizio una somma superiore a 615 ducati d'oro ${ }^{72}$.

Nel $1392^{73}$ Giacomo del fu Pietro da Revò prese il posto per alcuni anni del defunto de Stanchariis, producendo nello stesso periodo un certo numero di documenti anche per il monastero di San Lorenzo ${ }^{74}$. Di costui si hanno attualmente a disposizione poche decine di instrumenta redatti su commissione dei canonici fra il $1390^{75}$ e il $1398^{76}$, mentre non sembrano essere sopravvissute registrazioni in fascicoli o volumi. Nel 1413 Antonio da Borgonuovo, riferendosi al notaio Giacomo da Revò, lo qualificò come «tunc scriba et notarius Capituli»» ${ }^{77}$

Un secondo notaio attivo per il Capitolo fra gli anni Ottanta del XIV e i primissimi anni del XV secolo è Alberto figlio del fu ser Negratus da Sacco. Costui rogò per l'ente un buon numero di instrumenta, registrando una parte degli atti anche su suoi protocolli personali. Nessun registro di mano del professionista sembra essere stato conservato nell'archivio capitolare, dove si trovano soltanto redactiones in mundum. Alberto da Sacco fu attivo, al pari di altri notai impegnati per il Capitolo, per un gran numero di istituzioni cittadine: dall'episcopato, al convento delle Clarisse e per numerosi privati. Per il vescovo Alberto di Ortenburg, in particolare, egli ricoprì la carica di procuratore «pro investituris faciendis» ${ }^{78}$. La sua eterogenea attività di registrazione è oggi documentata anche attraverso un protocollo di esigue dimensioni, attualmente conservato presso il fondo Manoscritti (BCT1) della Biblioteca Comunale di Trento ${ }^{79}$. Il registro trasmette 109 documenti,

72 L'annotazione recita: «Noverint universi presentem pagenda inspecturi pro millesimo $\mathrm{CCC}^{\circ}$ LXXV quo anno fui ellectus per Capitulum Tridentinum scriba et notarius ipsius Capituli lucratus fui illo anno centum libras bone monete in conficiendo instrumenta Capituli et alia undecumque et aliis singulis annis infrascriptis infra lucratus fui infrascriptam peccunie quantitatem scripsi. | Item millesimo $\mathrm{CCC}^{\circ}$ LXXVI lucratus fui circa XL ducatos auri. | Item millesimo CCC $^{\circ}$ LXXVII lucratus fui XLV ducatos. | Item millesimo $\mathrm{CCC}^{\circ}$ LXXVIII lucratus fui XXXVIII ducatos. | Item millesimo $\mathrm{CCC}^{\circ}$ LXXVIIII lucratus fui circa LX ducatos. | Item millesimo $\mathrm{CCC}^{\circ} \mathrm{LXXX}$ lucratus fui XXII ducatos (..). | Item millesimo $\mathrm{CCC}^{\circ}$ LXXXI lucratus fui LII ducatos. | Item millesimo $\mathrm{CCC}^{\circ}$ LXXXII lucratus fui XXXIII ducatos. | Item millesimo $\mathrm{CCC}^{\circ}$ LXXXIII fui lucratus circa XXX ducatos. | Item millesimo $\mathrm{CCC}^{\circ}$ LXXXIIII lucratus fui XVIII ducatos. | Item millesimo $\mathrm{CCC}^{\circ} \mathrm{LXXXV}$ lucratus fui XVIIII ${ }^{\text {or }}$ ducatos vel paulo plus. | Item millesimo $\mathrm{CCC}^{\circ}$ LXXXVI lucratus fui XXXIII ducatos. | Item millesimo $\mathrm{CCC}^{\circ}$ LXXXVII lucratus fui XXIIII ducatos. | Item millesimo $\mathrm{CCC}^{\circ} \mathrm{LXXXVIII} \mathrm{lucratus} \mathrm{fui} \mathrm{XLI} \mathrm{ducatos} \mathrm{prout} \mathrm{in} \mathrm{zedula} \mathrm{continetur.}$ | Item millesimo $\mathrm{CCC}^{\circ}$ LXXXVIIII lucratus fui XXXII ducatos et $\mathrm{IIII}^{\text {or }}$ grossos prout in zedula iacet. | Item millesimo $\mathrm{CCC}^{\circ} \mathrm{LXXXX}$ lucratus fui XXXVI ducatos et I grossus prout patet in zedula. | Item millesimo $\mathrm{CCC}^{\circ} \mathrm{LXXXXI}$ lucratus fui XXXIIII ducatos prout in zedula apparet».

73 Cfr. Curzel, I canonici e il Capitolo, cit., pp. 657-658.

74 ADTn, AP, Registri, reg. n. 2, doc. n. 6 (in quest'occasione Giacomo si sottoscrive come «apostolica et imperiali auctoritate notarius et scriba domini abbatis») e ADTn, $A P$, Tomo II, n. 78.

75 ADTn, ACap, capsa 39, n. 23.

76 ASTn, $A C D$, n. 1140.

77 ADTn, ACap, Instrumenta capitularia 8, cc. $160 r-161 r$, n. 315.

78 ASTn, APV, Sezione latina, capsa 22, n. 2, cc. 57v-58r (1387 febbraio 4): Liber feudorum seu investiturarum domini Alberti episcopi Tridenti, ab anno 1383 ad annum 1388, rogato dal notaio Marco figlio di Odorico di Trento del fu ser Giacomo di Spormaggiore. Cfr. anche Codicis Clesiani, cit., vol. III.

$79 \mathrm{BCTn}, B C T 1$, n. 1868; edizione in Zamboni, Economia e società in una piccola città alpina, cit. Il protocollo presenta un titolo vergato da mano settecentesca: Matrici dei rogiti del notaio Alberto quondam Negrati di Sacco. Diversae investiturae et locationes de anno 1400 circiter. 
fra imbreviature ed estesi, redatti fra il 1399 ed il 1402: locazioni, compravendite, testamenti e prestiti rappresentano le tipologie documentarie prevalenti, anche se non mancano atti diversi come quello, già citato, attestante il matrimonio fra il notaio e giurisperito Antonio da Molveno e Floridia figlia di Tommaso da Folgaria ${ }^{80}$. Fra i circa 100 rogiti raccolti nel protocollo di Alberto da Sacco si trovano 32 documenti capitolari. La sua produzione documentaria risulta pertanto quanto mai varia per la qualità dei committenti e per la quantità degli atti prodotti, tale da renderlo fra i notai più prolifici della fine del XIV secolo.

Alla morte di Alberto, probabilmente non molto tempo dopo il $1403^{81}$, Antonio da Borgonuovo era già attivo per il Capitolo da qualche anno ${ }^{82}$, precisamente dal 1390. Sono gli anni in cui è ancora in attività Pietro de Stanchariis e, dopo di lui Giacomo da Revò. Verificando infatti la produzione documentaria capitolare sul finire del Trecento, si nota come i documenti redatti da ser Antonio siano ancora sporadici, mentre tendono ad aumentare numericamente soltanto all'inizio del 1400, in concomitanza con il calo d'attività (si presume dovuto all'età avanzata) del collega Alberto da Sacco. Non desta meraviglia, dunque, che proprio a partire dal 1403, quando l'attività di ser Alberto si interrompe del tutto, il numero di instrumenta prodotti da Antonio da Borgonuovo aumenti considerevolmente; dal 1402 ser Antonio diede inizio alla redazione del registro Instrumenta capitularia 8, il quale attesta l'attività continuativa per l'ente. A tal proposito, è lecito supporre che i canonici lo avessero scelto sulla base della sua preparazione tecnica, riponendo in lui un rapporto di fiducia che non derivava, come nel caso di Venturino de Trechis, Alberto de Floriis o Pietro de Stanchariis, dall'essere mansionario o, addirittura, canonico del Capitolo. Nel caso di Alberto da Sacco e Antonio da Borgonuovo furono dunque altri parametri ad entrare in gioco.

\section{L'attività al servizio del Capitolo (1390-1435)}

La carriera notarile di Antonio da Borgonuovo fra gli anni Ottanta e Novanta del Trecento non sembra rivelare particolari elementi che possano distinguere la sua attività rispetto a quella di altri colleghi del periodo; ciò almeno fino ai primissimi anni del Quattrocento, quando il notaio - già occasionalmente attivo come notaio per il canonici - comincia a produrre consistenti quantitativi di documentazione per il Capitolo, ente per cui, come si vedrà, fungerà da notaio quasi esclusivo per oltre trent'anni, ricoprendo spesso la funzione di procuratore dei canonici e producendo al contempo un voluminoso registro di instrumenta (il n. 8 della serie Instrumenta capitularia) contenente esclusivamente documentazione di interesse capitolare.

Il numero dei documenti prodotti da Antonio da Borgonuovo per conto del Capitolo è, come detto, piuttosto elevato, tanto da farne uno fra i più importanti committenti del notaio, se non addirittura il più importante. Fra i circa 2.000 rogiti di sua mano presi in esame, il primo documento prodotto per i canonici è datato

80 Cfr. Zamboni, Economia e società in una piccola città alpina, cit., doc. n. 8 .

81 L'ultimo documento redatto da Alberto da Sacco è datato 1403 marzo 30 (cfr. ivi, p. 27, in particolare la nota 24)

82 Il primo documento rogato da ser Antonio per i canonici è datato 1390 agosto 13: ASTn, APV, Sezione latina, capsa 44, n. 15. 


\section{L'attività professionale di Antonio da Borgonuovo}

13 agosto $1390^{83}$ ed è attualmente conservato presso l'Archivio del Principato vescovile di Trento, alla capsa 44 (denominata Capitulum) della sezione latina. Si tratta di un appello da presentarsi presso la curia romana redatto dal notaio su commissione dei canonici. L'occasione è data dalle lettere contenenti «monitiones et mandata multum gravia» per il Capitolo e il clero diocesano, presentate ai canonici da Cosma Migliorati, legato pontificio in partibus Lombardie. L'appello rivolto al pontefice Bonifacio IX aveva pertanto lo scopo di esporre le motivazioni che impedivano al Capitolo di pagare quanto richiesto. Anzitutto si fa presente che gli ufficiali del duca d'Austria si erano impossessati con la violenza di quasi tutto il reddito del clero diocesano, vendendo le decime e le proprietà, fra cui i redditi solitamente spettanti al Capitolo per un valore di 800 ducati, per recuperare i quali i canonici spesero ben 1.000 ducati non senza impegnare parte delle decime e dei beni sub usuris $^{84}$. L'importanza dell'appello è sottolineata dalla presenza, insieme ai canonici, dei rappresentanti dei più importanti ordini monastici e conventuali della città, fra cui fra Francesco da Ferrara priore del monastero di San Lorenzo dell'ordine dei Predicatori, fra Nicolò da Mantova guardiano del convento di San Francesco dell'ordine dei Minori, fra Basilio da Treviso priore del convento degli Eremitani di San Marco, il notaio ser Francesco del fu ser Martino da Volano, in qualità di sindaco del Capitolo, il notaio ser Simone del fu Azzone da Campo, pure sindaco del Capitolo e dei frati alemanni, fra Bartolomeo da Camerino priore dell'ospedale di Santa Croce in Trento, e il notaio ser Alberto del fu ser Negratus da Sacco, sindaco del convento delle suore dell'ordine di Santa Chiara. Si tratta di un atto di grande rilevanza per i maggiori rappresentanti della chiesa tridentina, a ragione delle implicazioni economiche sottese; risulta quindi alquanto singolare il fatto che la redazione di questo testo sia stata affidata proprio ad Antonio da Borgonuovo che nel 1390 aveva intrapreso da pochi anni la carriera come tabellione.

A partire dal 1390, dunque, i documenti prodotti per il Capitolo della cattedrale si presentano fra le carte di Antonio con frequenza sempre maggiore, con una produzione che si attesta su livelli di una certa importanza a partire dal 1402, anno in cui il professionista dà avvio al registro Instrumenta capitularia 8.

\begin{tabular}{lcc}
\hline anno & n. documenti reperiti per anno & n. documenti prodotti per il Capitolo \\
\hline 1386 & 1 & 0 \\
1387 & 0 & 0 \\
1388 & 3 & 0 \\
1389 & 4 & 0 \\
1390 & 2 & 1 \\
1391 & 5 & 2 \\
1392 & 2 & 1 \\
1393 & 2 & 0 \\
1394 & 13 & 0 \\
1395 & 5 & 0 \\
1396 & 0 & 0 \\
1397 & 2 & 1 \\
\hline
\end{tabular}

83 ASTn, $A P V$, Sezione latina, capsa 44, n. 15.

84 Cfr., sull'argomento, Curzel, I canonici e il Capitolo, cit., pp. 173-174. 
Stefano Malfatti

\begin{tabular}{|c|c|c|}
\hline anno & n. documenti reperiti per anno & n. documenti prodotti per il Capitolo \\
\hline 1398 & 5 & 3 \\
\hline 1399 & 10 & 2 \\
\hline 1400 & 20 & 15 \\
\hline 1401 & 6 & 4 \\
\hline 1402 & 19 & 15 \\
\hline 1403 & 29 & 27 \\
\hline 1404 & 53 & 52 \\
\hline 1405 & 66 & 59 \\
\hline 1406 & 58 & 53 \\
\hline 1407 & 57 & 48 \\
\hline 1408 & 63 & 62 \\
\hline 1409 & 20 & 20 \\
\hline 1410 & 10 & 10 \\
\hline 1411 & 30 & 27 \\
\hline 1412 & 28 & 26 \\
\hline 1413 & 26 & 25 \\
\hline 1414 & 33 & 23 \\
\hline 1415 & 65 & 46 \\
\hline 1416 & 42 & 36 \\
\hline 1417 & 30 & 22 \\
\hline 1418 & 35 & 24 \\
\hline 1419 & 23 & 18 \\
\hline 1420 & 37 & 37 \\
\hline 1421 & 58 & 57 \\
\hline 1422 & 66 & 59 \\
\hline 1423 & 51 & 33 \\
\hline 1424 & 106 & 22 \\
\hline 1425 & 146 & 47 \\
\hline 1426 & 137 & 44 \\
\hline 1427 & 133 & 55 \\
\hline 1428 & 88 & 23 \\
\hline 1429 & 110 & 35 \\
\hline 1430 & 113 & 28 \\
\hline 1431 & 139 & 52 \\
\hline 1432 & 71 & 38 \\
\hline 1433 & 83 & 35 \\
\hline 1434 & 72 & 32 \\
\hline 1435 & 27 & 12 \\
\hline 1436 & 8 & 0 \\
\hline 1437 & 2 & 0 \\
\hline
\end{tabular}

Tab. 1. Documenti di Antonio reperiti per ciascun anno; documenti rogati per il Capitolo. 


\section{L'attività professionale di Antonio da Borgonuovo}

Come si evince dalla tabella allegata (tab. 1) la documentazione redatta per il Capitolo della cattedrale è piuttosto esigua, se non nulla, fino al biennio 1400-1402 quando, con l'avvio del registro Instrumenta capitularia 8, tende ad aumentare considerevolmente tanto da costituire per molti anni, e almeno fino al 1424, la fetta più consistente di quanto conservato. Soltanto con il 1424, quando prende avvio il registro Instrumenta capitularia 8bis, gli atti redatti per i canonici sembrano diminuire, costituendo circa un terzo di quanto oggi si conserva della produzione annua di Antonio. Un calo piuttosto evidente nel numero dei documenti conservati si ha per il biennio 1409-1410, con un trend che sostanzialmente tende a mantenersi piuttosto stabile almeno fino agli ultimi anni quando, dal 1435 in poi, non si hanno più documenti capitolari di sua mano. L'ultimo rogito commissionato ad Antonio dai canonici è datato 12 agosto $1435^{85}$ ed è il conferimento (collatio) dell'altare dei Santi Innocenti al prete Bertoldo da Trento e il successivo giuramento da questi prestato nelle mani del canonico senior Zambono.

Individuati gli estremi cronologici (1390-1435) dell'impegno di Antonio nei confronti del Capitolo, rimane da indagare il tema delle tipologie documentarie che i canonici richiesero al professionista. La maggioranza dei documenti rogati è rappresentata da locazioni, o rinnovi di locazioni, refute e permute. Ben attestate, anche se in numero non consistente, sono le tipologie documentarie che riguardano la cura d'anime, come le nomine a rettore di chiese, pievi e altari, seguite dalle nomine a canonico ${ }^{86}$. Dal punto di vista della consistenza, refute e locazioni rappresentano una percentuale compresa fra l'80 ed il 90\% dell'intera produzione documentaria del notaio per questo ente. Seguono le nomine a vicario di pieve o a rettore di chiesa, le elezioni a canonico e le collazioni d'altare. Non mancano, seppur in misura piuttosto ridotta, ulteriori tipologie documentarie, quali ad esempio le permute ${ }^{87}$, le procure $^{88}$ o le nomine a procuratore ${ }^{89}$. Più rari, come è solito nella documentazione degli enti ecclesiastici, sono i negozi giuridici che prevedevano l'alienazione dei beni immobiliari, quali ad esempio le compravendite ${ }^{90}$. Non vi sono periodi in cui si riscontrano maggiormente alcune tipologie documentarie rispetto ad altre; refute e locazioni sono infatti frequenti in tutto il periodo in cui Antonio da Borgonuovo roga per il Capitolo. Una maggiore concentrazione delle nomine a canonico, a rettore di chiesa o di pieve e delle collazioni d'altare è invece rilevabile soprattutto negli anni Venti-Trenta del Quattrocento, ma ciò è dovuto in larga misura al fatto che questi documenti trovarono spazio quasi esclusivamente fra le carte del registro Instrumenta capitularia 8bis (1423-1437) e ciò, come si vedrà nel dettaglio nel capitolo dedicato al modus operandi del notaio ${ }^{91}$, non avvenne casualmente. Tali tipologie documentarie, infatti, furono probabilmente commissionate ad Antonio già dall'inizio del XV secolo, o forse anche prima, come sembrano dimostrare alcuni esempi su pergamena ${ }^{92}$.

85 ADTn, ACap, Instrumenta capitularia 8bis, c. 252v, n. 657.

86 Per un elenco dei documenti rogati da Antonio inerenti a nomine a rettore di chiese, pievi e altari e a nomine a canonico, si rinvia infra al paragrafo 14: «Nomine di rettori di chiese, pievi e altari; nomine di canonici», pp. 126-129.

87 Cfr., ad esempio, ADTn, ACap, Instrumenta capitularia 8, nn. 340a, 477, 580, 606, 622 e passim.

88 Cfr., ad esempio, ADTn, ACap, capsa 22, n. 3.1; Instrumenta capitularia 8, n. 104 e passim.

89 Cfr., ad esempio, ivi, n. $628 \mathrm{~b}$.

90 Cfr., ad esempio, ivi, n. 508.

91 Cfr., infra, capitolo 4.

92 ADTn, ACap, capsa 44, n. 12. 
Esaminando le date topiche dei documenti per il Capitolo si ricava come il luogo privilegiato, se non esclusivo, per le riunioni dei canonici ${ }^{93}$, e dunque anche per la rogazione, sia la cattedrale. Salvo rare eccezioni, dunque, i negozi giuridici si svolgono in ecclesia Tridentina. All'interno dell'edificio sacro i luoghi usualmente utilizzati sono la sacrestia, che sovente viene definita magna, il podiolum (o podioletum) dell'altare del Corpo di Cristo, del quale spesso il notaio specifica anche la posizione, post chorum posito ${ }^{94}$. Più rare sono le attestazioni di un banchum dei canonici ${ }^{95}$, probabilmente adiacente alla canipa, dove venivano raccolti gli affitti e ammassate le derrate alimentari, da distinguere comunque dal banchum ubi ius spirituale redditur consuete che aveva sede nella cattedrale e fungeva probabilmente da tribunale per dirimere questioni inerenti alla sfera spirituale ${ }^{96}$. Fra i luoghi di rogazione più frequenti entro la cattedrale si hanno anche le zone circostanti ad alcuni altari, fra i quali: l'altare di Sant'Agata, l'altare del Santo Sepolcro, l'altare delle Sante Gertrude e Dorotea, dei Santi Pietro e Paolo, della Santa Trinità, dei Santi Sisinio, Martirio e Alessandro, l'altare maggiore ${ }^{97}$.

\section{Note sulla produzione documentaria comunale all'inizio del Quattrocento}

Soltanto nella prima metà del Quattrocento, dopo le rivolte cittadine contro il vescovo Georg Liechtenstein (1407-1409), la produzione documentaria del Comune di Trento diviene meno frammentata, pur in un contesto ancora caratterizzato dalla presenza di documenti conservati in ottica eminentemente tesaurizzante (privilegi, statuti e documentazione in forma sciolta) e da una produzione sporadica di registri ${ }^{98}$. Se infatti fra il 1415 e il 1466 viene prodotto il noto Liber electionum officialium, contenente $\mathrm{i}$ verbali di elezione alle massime cariche comunali, intervallate da locazioni, urbari e registrazioni contabili, si deve attendere la seconda metà del secolo prima di vedere altri 'prodotti archivistici' in forma di libro: fra il 1458 e il 1517 si ha ad esempio il registro delle locazioni perpetuali ${ }^{99}$, fra il 1466 e il 1542 il registro delle locazioni temporali ${ }^{100}$, mentre viene inaugurata soltanto nel 1469 la serie dei Libri actorum ${ }^{101}$, caratterizzata da lacune e «prassi di registrazione diversificate» ${ }^{102}$ fino al 1518 .

La modestia sia qualitativa - sotto il profilo diplomatistico - che quantitativa dei registri del Comune di Trento del Quattrocento non permette certamente di parlare di documentazione in forma seriale; si tratta infatti dei primi timidi tentativi da

pp. 310-316.

93 Cfr., relativamente ai luoghi di riunione dei canonici, Curzel, I canonici e il Capitolo, cit.,

94 Sulla struttura e sulla posizione del podiolum cfr. ivi, p. 313.

95 Cfr., ad esempio, ADTn, ACap, Instrumenta capitularia 8bis, nn. 85, 595c.

96 ADTn, ACap, capsa 41, corte, n. 7.

97 Ivi, capsa 45, rotoli, n. 2 (Sant'Agata); ADTn, ACap, Instrumenta capitularia 8bis, n. 649 (Santo Sepolcro); ivi, n. 74 (Sante Gertrude e Dorotea); ivi, n. 95 (Santi Pietro e Paolo); ADTn, ACap, Instrumenta capitularia 8, n. 168 (Santa Trinità); ADTn, ACap, Instrumenta capitularia 8bis, n. 200 (Santi Sisinio, Martirio e Alessandro); ADTn, ACap, Instrumenta capitularia 8, n. 178 (l'altare maggiore).

98 Per la redazione di questa breve introduzione ci si è serviti degli studi di Fossali, Il più antico Liber actorum, cit.; Varanini, Gli uffici del Comune di Trento nel Quattrocento, cit.

99 ASCTn, Comune di Trento, Antico regime, sezione antica, ACT1-3804.

100 Ivi, ACT1-2645.

101 Cfr. Fossali, Il più antico Liber actorum, cit.

102 Ivi, p. 328. 
parte della magistratura comunale di organizzare la propria memoria documentaria attraverso soluzioni che, tuttavia, sono ben lontane dagli esempi di altri comuni dell'Italia centro-settentrionale.

All'interno di questo contesto un ruolo di primo piano rivestono un certo numero di notai che si alternarono nella redazione della documentazione comunale. Il Liber electionum, ad esempio, fu prodotto da notai che spesso appaiono contemporaneamente eletti fra gli officiali del Comune; ciò, tuttavia, come ha avuto modo di sottolineare Roberta Fossali, «non è significativo di un rapporto privilegiato con il Comune ${ }^{103}$. Se infatti fanno eccezione i casi dei notai Gioacchino Mezzasoma, Giovanni Conto de Fatis e Nicolò de Capris, i quali sembrano essere stati investiti, almeno temporaneamente, dell'incarico di redigere gli atti comunali ${ }^{104}$, non sembra di poter rintracciare nella documentazione del Comune veri e propri rapporti di fidelizzazione con determinati notai. Si nota piuttosto una preferenza per un ristretto numero di professionisti, i cui nomi ricorrono con maggiore frequenza nelle sottoscrizioni dei rogiti comunali. Fra questi: Guglielmo Balzanini, Antonio da Nogaredo, Gioacchino Mezzasoma, Antonio de Castro, Nicolò de Capris, Giovanni Conto de Fatis e, non ultimo per importanza, Antonio da Borgonuovo.

\section{L'attività al servizio del Comune di Trento}

La documentazione prodotta da Antonio da Borgonuovo per il Comune di Trento non è certo abbondante, ma l'intera produzione documentaria comunale del primo Quattrocento, come visto, è lontana dai numeri dell'Episcopato e del Capitolo della cattedrale ${ }^{105}$, che pure, se paragonati agli 'standard' d'altre realtà dell'Italia centro-settentrionale, mostravano inequivocabili elementi di ritardo. Il Comune di Trento non fu in grado, almeno fino all'età moderna, di raggiungere un grado di maturazione istituzionale che lo rendesse in qualche misura autonomo dal potere vescovile e che gli consentisse di produrre e organizzare documentazione pienamente pubblica ${ }^{106}$.

Se dunque si mette da parte la quantità dei documenti e si considera il contenuto degli stessi, si nota come la documentazione redatta da Antonio per la magistratura comunale rivesta in realtà una grande importanza nella storia delle istituzioni cittadine. A ciò si aggiunge il fatto, non irrilevante, che Antonio fu nel Comune

103 Ivi, p. 331.

104 Ivi, p. 332: «del Mezzasoma si dice ... che "per unum annum fuit canzelarius comunitatis Tridenti"».

105 Fra la vasta produzione dedicata all'argomento da Franco Cagol cfr. F. Cagol, A. Mura, Trento e Bolzano: due città a confronto. Modi e forme di produzione documentaria nei due Comuni tra Quattro e Cinquecento, in L. de Finis (a cura di), La proclamazione imperiale di Massimiliano I d'Asburgo (4 febbraio 1508), Atti del convegno (Trento, 9 maggio 2008), supplemento a «Studi Trentini di Scienze Storiche», 87, 2008; F. Cagol, L'Archivio del comune di Trento di antico regime: ordinamenti e strumenti repertoriali, «Studi trentini di scienze storiche. Sezione prima», 79, 2000, pp. 749-827; Id., Il Comune di Trento in antico regime, in M. Hausberger (a cura di), "Volendo questo illustrissimo magistrato consolare”: trecento anni di editoria pubblica a Trento, Provincia autonoma di Trento, Soprintendenza per i beni librari e archivistici, Trento 2005 (Quaderni, 6), pp. IX-XLVII. Sull'argomento cfr. anche Id., Dal palatium episcopatus al palatium comunis, cit., pp. 205-223; Varanini, Gli uffici del Comune di Trento nel Quattrocento, cit. Sui notai al servizio del Comune di Trento ad inizio Quattrocento cfr. Fossali, Il più antico Liber actorum, cit.

106 Varanini, Gli uffici del Comune di Trento nel Quattrocento, p. 227. 


\section{Stefano Malfatti}

di Trento al contempo notaio, pubblico autenticatore della memoria documentaria dell'ente e ufficiale eletto alle massime cariche comunali fra il 1415 e il 1434.

Il documento in originale più antico redatto da ser Antonio su richiesta del Comune di Trento è datato 24 ottobre 1395 ${ }^{107}$; un altro importante cliente si era dunque servito del notaio in una fase assai precoce della sua carriera. Si tratta di una refuta con nuova locazione di un appezzamento di terra arativa posto fuori dalla porta di Santa Croce, in contrata ubi dicitur in Campo Marzio (l'attuale piazza Fiera). Rappresentano il Comune di Trento i notai Pietro del fu Gioacchino Mezzasoma e Pietro de Capris. Il documento più recente, datato 14 marzo 1434, si trova invece nel registro Instrumenta capitularia 8bis ed è la nomina a sindaci e procuratori di Luca di Giovanni e di Adelperio Calepini. Costoro furono infatti inviati, quali rappresentanti del Comune, dinanzi al vescovo Alessandro di Masovia che si trovava al concilio di Basilea ${ }^{108}$. I due documenti aprono e chiudono un periodo di circa quarant'anni, fra il 1395 e il 1434, durante il quale Antonio fu chiamato a redigere documentazione per $\mathrm{i}$ vari uffici comunali; anche in questo caso, tuttavia, ciò che rimane non è che un frammento di una probabile originaria produzione: circa 35 documenti su pergamena o in registro piuttosto diversificati per la tipologia del negozio trattato e per argomento. Fra i negozi giuridici maggiormente rappresentati si hanno le locazioni (perpetue o temporali) e le refute, rogate fra il 1395 e il $1429^{109}$. Nel 1415 Antonio fu chiamato insieme ai notai Marco del fu ser Valentino da Borgonuovo, Giacomo Fanzini figlio del fu ser Bertoldo a Ture e Nicolò figlio di ser Ognibene da Borgonuovo, ad autenticare l'unico documento allora esistente relativo alla livellazione dei mulini posti a Trento lungo la Roggia Grande $^{110}$. Dal documento si ricava come quell'unico instrumentum in originale sopravvissuto al sachomannum del 1410 era conservato proprio presso la statio di Antonio da Borgonuovo; il vicario in temporalibus Lorenzo de Gauslinis da Feltre aveva pertanto autorizzato il notaio a trarre da quell'originale, oggi deperdito, una nuova redazione da inserire «super libro statutorum et designationum sindicorum et comunitatis Tridenti», dove ancora oggi si trova.

L'11 novembre 1415 Antonio fu invece chiamato a rogare un consulto che aveva lo scopo di dirimere la controversia fra il Comune di Trento e un gruppo di cittadini proprietari di immobili nell'area di Campo Trentino, nelle pertinenze di Gardolo. Il Comune aveva a tal scopo istituito una commissione arbitramentale con il compito di valutare i danni causati ai beni comunali e alla strada pubblica dalle rogge e dai canali che lì scorrevano. L'esame svolto dalla commissione, così come le decisioni da essa assunte, furono registrate da Antonio da Borgonuovo in due esemplari in originale ${ }^{111}$.

107 ASCTn, Comune di Trento, Antico regime, sezione antica, ACT1-3036. Il documento è seguito dall'immissione in possesso datata 28 ottobre 1395 .

108 Cfr. Appendice, regesti nn. 50, 51 (ADTn, ACap, Instrumenta capitularia 8bis, nn. 604a-b).

109 Alle segnature: ASCTn, Comune di Trento, Antico regime, sezione antica, ACT1-3036; ACT1.13056; ACT1.1-3002; cfr. Appendice, regesti nn. 29, 33 (ADTn, ACap, Instrumenta capitularia 8bis, nn. 132, 132b) e regesto n. 35 (ADTn, ACap, Instrumenta capitularia 8bis, nn. 253a-b-c); n. 255; n. $298 ;$ n. 316 ; n. 339.

110 ASCTn, Comune di Trento, Antico regime, sezione antica, ACT1-2545, cc. 35v-37r. La copia autentica è interamente redatta dalla mano di Antonio ed è chiusa dalle sottoscrizioni di tutti e quattro $i$ notai.

111 ASCTn, Comune di Trento, Antico regime, sezione antica, ACT1-327.1-2. 
Fra i documenti redatti dal notaio per il Comune cittadino, grande rilevanza ricoprono le verbalizzazioni delle elezioni degli officiali chiamati ad esercitare gli uffici comunali, raccolte nel Liber electionis officialium magnificae communitatis Tri$d e n t i^{112}$. Come già accennato, Antonio vi appare sia come ufficiale eletto in numerose occasioni sia come notaio chiamato a redigervi alcuni verbali: una prima volta nel febbraio del $1423^{113}$, poi nel febbraio ${ }^{114}$, nel giugno ${ }^{115}$ e nell'ottobre ${ }^{116}$ del 1428. Sempre all'interno del codice 3547 si trova inoltre una copia semplice di mano di Antonio di una lettera inviata dal Comune al duca d'Austria Federico IV il 20 novembre $1427^{117}$. Due ulteriori documenti, entrambi raccolti fra le carte di Instrumenta capitularia 8bis, furono probabilmente commissionati al notaio dal Comune di Trento ${ }^{118}$ : il primo, datato 25 ottobre 1425, è denominato dallo stesso Antonio, nella rubrica posta in margine, «Comissio cride super vino» ${ }^{119}$. Ser Francesco de Sichis, Giacomo de Ruphalcatis da Arco, il notaio Iosio de Iosiis, Antonio de Castro, Antonio del fu Bommartino da Trento, Adelpreto del fu Federico da Povo e Nicolò del fu ser Odorico a Sale, consoli e provvisori del Comune, si erano presentati dinanzi ad Alessandro di Masovia insieme a Rigo da Valeggio di Verona, procuratore generale della città, presentando una norma a suo tempo già emanata dal duca Leopoldo d'Austria e dal vescovo Alberto di Ortenburg; con essa si intendeva vietare il commercio e la conduzione in città del vino «natum a Gardulis superius et a lapide stricto ultra Cugnola posito ulterius et a Novalina ulterius et a Matarelo inferius et a Romagnano inferius et a Bocha de Vella superius». Udita la richiesta il vescovo confermò la norma ordinando che il decreto venisse posto nel libro degli editti della città e fosse reso pubblico. Un secondo documento, datato 28 febbraio 1429 , è invece denominato "Comissio cride facto statere communitatis Tridenti» ${ }^{120}$. Il testo informa che Odorico Calepini si presentò in qualità di procuratore del Comune dinanzi al vicario vescovile Ludovico de Calvis per chiedere che venisse aggiunto al libro degli statuti della città l'editto con il quale si vietava a qualunque persona di pesare, vendere o comprare merce con peso superiore alle 100 libbre né altra mercanzia di spettanza dell'ufficio della stadera senza la licenza dell'ufficiale addetto. Udita la richiesta, il vicario vescovile ordinò che l'editto fosse reso noto e diede ad Antonio il compito di redigerlo nel libro degli statuti.

Il professionista registrò inoltre in forma di copia semplice all'interno del proprio registro (Instrumenta capitularia 8bis) una serie di documenti di grande rilevanza per le istituzioni cittadine. Per alcuni di questi atti si ha dunque a disposizione sia la redazione in originale presso l'Archivio storico del Comune, sia la copia nel registro di Antonio; fra questi c'è ad esempio una lettera inviata alla città dal vescovo Alessandro di Masovia 1'11 ottobre $1424^{121}$ in risposta a una serie di capitoli

112 Ivi, ACT1-3547; cfr. l'edizione in Valenti, Il «Liber electionum officialium», cit.

113 ASCTn, Comune di Trento, Antico regime, sezione antica, ACT1-3547, c. $27 r-v$.

114 Ivi, c. $38 r-v$.

115 Ivi, c. $39 r$.

116 Ivi, c. $39 v$.

117 Ivi, cc. $225 r-266 r$.

118 I due casi qui citati andrebbero, a rigor di logica, inseriti fra la documentazione redatta su commissione del vicario vescovile; si citano comunque in questa sede poiché si tratta comunque di documentazione relativa al Comune di Trento.

119 ADTn, ACap, Instrumenta capitularia 8bis, cc. 49v-50r, n. 134.

120 Ivi, c. $121 v$, n. 327.

121 L'originale si trova in ASCTn, Comune di Trento, Antico regime, sezione antica, ACT1-1664; la copia di Antonio da Borgonuovo si trova in ADTn, ACap, Instrumenta capitularia 8bis, c. $21 r$, n. 62. 


\section{Stefano Malfatti}

trasmessi dai rappresentanti della città ${ }^{122}$. Il notaio registrò inoltre anche la copia della lettera di risposta (responsio) inviata dal Comune pochi giorni dopo al vescovo ${ }^{123}$.

Nel Libro vechio de Statuti e dessignationi de beni et confini della Città di Trento si trova un primo verbale del consiglio generale della città nel quale il vicario vescovile, i sapientes e i due sindici deliberano alcuni capitoli atti a regolamentare il mercato del pesce in città; un secondo verbale riguarda invece la delibera dei capitoli inerenti ai salari dei laboratores ${ }^{124}$. Nella sottoscrizione il notaio dichiarò di aver esemplato i due documenti su mandato del vicario del vescovo Alessandro e dei sindaci del Comune.

Osservando i documenti redatti da Antonio per il Comune di Trento, si nota una concentrazione di atti rogati negli anni compresi fra il 1415 e il 1434: da un lato, infatti, soltanto dopo il 1415 il Comune iniziò la registrazione dei verbali di elezione delle proprie cariche, cui pure ser Antonio diede il proprio contributo; dall'altro, numerosi documenti comunali da lui prodotti sono raccolti fra le carte del registro Instrumenta capitularia 8bis che comprende la documentazione rogata dopo il 1423-1424.

Parlando della definizione degli spazi dell'identità comunale tra il XIII e il XVI secolo ${ }^{125}$, Franco Cagol ha evidenziato i faticosi tentativi, da parte delle magistrature comunali, di «distanziarsi» dal potere vescovile. Se, infatti, è riconoscibile un tentativo da parte delle istituzioni cittadine di esercitare prerogative politiche fino ad allora saldamente in mano al potere episcopale, ancora fra gli anni Dieci e gli anni Trenta del XV secolo non è possibile individuare luoghi specificamente dedicati alle riunioni dei consigli cittadini che in qualche modo facciano presagire un allontanamento, anche fisico, dal luogo tradizionalmente deputato alle assemblee del consilium: il palatium episcopi, sede del vicario episcopale. Analizzando le date topiche dei documenti redatti da Antonio da Borgonuovo si rileva infatti come gran parte degli atti sia stato rogato in varie sale all'interno del palazzo vescovile. Tutti i verbali di elezione alle cariche comunali redatti da Antonio fra il 1423 e il 1428 furono ad esempio rogati in episcopali palacio, in sala ubi ius civile reditur et fit conscilium generale. Non mancano, tuttavia, alcuni documenti la cui data topica rimanda a luoghi diversi, ma non lontani, dal palazzo episcopale; nel 1430, ad esempio, il vicario in temporalibus Gioacchino Mezzasoma, insieme a Giovanni di Luca, Adelperio Calepini e Cristoforo a Libeceltis, consoli della città, si riunirono nell'attigua stazione delle bollette ${ }^{126}$, per commissionare al notaio Antonio la reda-

122 La minuta dei capitoli si trova in ASCTn, Comune di Trento, Antico regime, sezione antica, ACT1-1322.

123 ADTn, ACap, Instrumenta capitularia 8bis, c. $21 r-v$, n. 63.

124 ASCTn, Comune di Trento, Antico regime, sezione antica, ACT1-2545, c. 33r-v. I capitoli citati verranno inseriti nello statuto del 4 agosto 1427, ai capitoli 77 e 78 (ACT1-3467) e nello statuto masoviano del 1427 edito verso il 1434 (ASTn, APV, Codici, ms. 1, libro sindicale, capitoli 79-80).

125 Cagol, Dal palatium episcopatus al palatium comunis, cit., pp. 205-223.

126 Le date topiche dei documenti stipulati ad stationem buletarum, consentono di delimitare e circoscrivere con maggiore precisione la posizione della 'stazione delle bollette'; anzitutto molte delle date riconducono al portico della suddetta stazione. In alcune occasioni si specifica inoltre che essa si trova iuxta scalas palacii, con riferimento alle scale che conducevano al palazzo del vicario vescovile. In altri casi ancora si menziona la vicinanza ad una fons situata nei pressi del già citato portico nella piazza del Comune. Un esempio, in particolare, permette di delimitare con maggiore precisione la statio: un documento del 1429 (ADTn, ACap, Instrumenta capitularia 8bis, n. 331) ha come data topica la piazza comune, «sub porticu arengerie, intra stazionem buletarum et puteum». Nel 1430 un'ulteriore 
zione dell'esenzione concessa al magister Guglielmo da Roncegno dal pagamento di ogni tassa dovuta al Comune per un periodo di tre anni quale ricompensa per non aver mai abbandonato la città durante l'epidemia di peste allora in corso ${ }^{127}$. Proprio l'ufficio, o stazione, delle bollette divenne in quegli anni luogo privilegiato, o per lo meno alternativo al palazzo vescovile, per la stipulazione di locazioni, «per la riscossione del dazio, per la misurazione del sale e di altre merci o per concludere i contratti di appalto dei pubblici servizi» ${ }^{128}$; due refute, stipulate nel 1429, presentano infatti quale data topica la statio buletarum posta nella piazza del Comune ${ }^{129}$.

\section{Produzione e conservazione della documentazione: monasteri, conventi e confraternite cittadine fra XIV e XV secolo}

La documentazione superstite di monasteri, conventi e confraternite cittadine, per quanto riguarda il XII e il XIII secolo, rivela modalità organizzative del tutto assimilabili a quelle dei maggiori enti della città: si hanno cioè pergamene sciolte conservate da ciascun ente a garanzia dei propri diritti; i monaci di San Lorenzo, così come le Clarisse di San Michele e gli Agostiniani di San Marco si rivolgono ai notai attivi sulla piazza di Trento, servendosi prevalentemente di professionisti già impegnati per il vescovo e il Capitolo ${ }^{130}$. Non esiste, anche in questo caso, alcun elemento che lasci intravedere un pur minimo rapporto di fidelizzazione fra gli enti e i notai chiamati, di volta in volta, a dare formalizzazione scritta ai loro negozi giuridici.

La fase più antica di questi enti, dunque, sembra essere attestata soltanto attraverso redactiones in mundum, nemmeno numericamente molto consistenti. Per trovare forme di registrazione diverse dalla pergamena sciolta si deve attendere la seconda metà del XIV secolo, allorquando - ma non ovunque, come si vedrà cominciano ad essere attestati, direttamente o indirettamente, anche dei registri.

Ciò accade, ad esempio, dal 1369 nel monastero di San Lorenzo, allorquando inizia la compilazione di una serie di registri pergamenacei (1369-1430) prevalentemente con documentazione di natura patrimoniale ${ }^{131}$ la cui redazione fu affidata ad un gruppo piuttosto ristretto di notai. Continuava frattanto, seppur forse in misura meno rilevante, una parallela produzione di munda accanto alla registrazione nei fascicoli pergamenacei. Fra i tredici notai ${ }^{132}$ al servizio dell'ente è utile notare come,

data topica informa inoltre che, nella piazza del Comune, presso il portico della stazione esisteva, oltre alla già nota 'stazione delle bollette', anche un «officium duorum sindicorum» (ivi, n. 394).

127 Ivi, n. 400.

128 Cagol, Dal palatium episcopatus al palatium comunis, cit., p. 213.

129 ADTn, ACap, Instrumenta capitularia 8bis, n. 316 e ASCTn, Comune di Trento, Antico regime, sezione antica, ACT1-3002.

130 E. Curzel, S. Gentilini, G.M. Varanini (a cura di), Le pergamene dell'archivio della Prepositura di Trento (1154-1297), Il Mulino, Bologna 2004 (Annali dell'Istituto storico italo-germanico in Trento. Fonti, 2, p. 53).

131 Prima di questa data si ha a disposizione un solo frammento (si tratta di un bifolio pergamenaceo) che una mano non identificabile del secolo XIV ha definito "Quaternus iurium ecclesie Sancti Nicolay»: Curzel, Gentilini, Varanini (a cura di), Le pergamene dell'Archivio della Prepositura, cit., p. 56. Esso è conservato fra le carte dell'ospedale di San Nicolò, poi confluite nell'archivio di San Lorenzo e della Prepositura, e attesterebbe la presenza di forme di registrazione diversificate rispetto alle pergamene sciolte in un periodo molto risalente.

${ }_{132}$ Si tratta di Pietro Paolo di ser Giovanni detto Ianes macellaio da Trento, Francesco di ser Tomeo de Cazuffo, Giacomo di Pietro da Revò, Francesco del fu Martino da Volano, Martino di ser 
in alcuni casi circoscritti, vi furono quanti in sottoscrizione qualificarono il proprio rapporto con l'autorità, definendosi «scriba dicti domini abbatis»" ${ }^{133}$.

Il percorso evolutivo verso forme documentarie simili, se non analoghe, a quelle descritte ad esempio per il Capitolo, si compirà per la Prepositura soltanto nella seconda metà del XV secolo con la compilazione di registri di investiture notarili ${ }^{134}$ «contenenti documentazione notarile relativa all'amministrazione dei beni e delle rendite ${ }^{135}$ disposta generalmente in ordine cronologico e diplomaticamente affine agli Instrumenta capitularia.

Più complicata è la ricostruzione delle vicende documentarie del convento degli Eremitani di San Marco ${ }^{136}$. Anche in questo caso, soltanto dalla metà del XIV secolo iniziano a comparire alcuni notai impiegati in più di un'occasione. Fra questi Dionisio del fu Pietro becarius da Trento e il più noto Guglielmo del fu Antonio $a b$ Equabus, attivo per il convento fra gli anni Cinquanta e Sessanta del secolo ${ }^{137}$. Negli anni Novanta del Trecento fa invece la sua comparsa Antonio da Borgonuovo.

Alcuni atti in Instrumenta capitularia 8bis fatti redigere al notaio trentino dai frati di San Marco recano marginalmente delle note di mano dello stesso ser Antonio; si tratta di annotazioni relative all'estrazione di un'ulteriore redazione dello stesso documento, che oltre ad essere esemplato sul registro personale del notaio, ed eventualmente anche in publicam formam su pergamena sciolta, venne pure redatto su un non meglio noto registro di proprietà dello stesso convento. Si trovano così note del tipo, «Posita continua super libro auctentico instrumentorum infrascripti monasterii cum meis signo et subscriptione» ${ }^{138}$, «Posita continua cum meis signo et subscriptione super libro instrumentorum dicti monasterii $\rangle^{139}$, «Posita continua super libro auctentico dicti monasterii cum signo et subscriptione meis; item etiam

Francesco notaio da Volano, Antonio di ser Bartolasio da Borgonuovo, Giovanni del fu ser Guglielmo ab Equabus, Guglielmo del fu ser Guglielmo de Balzaninis da Verona, Graziadeo del fu ser Antonio del fu ser Biagio da Castel Terlago, Antonio del fu ser Giovanni da Fai, Nicolò del fu Ognibene da Trento, Nicolò del fu Pietro Baldovino de Capris da Maderno e Antonio del fu ser Bonifacio da Nogaredo: Ioppi, I registri del monastero di San Lorenzo, cit., p. 54. Per brevi biografie relative a questi professionisti cfr. ivi, pp. 55-63.

${ }_{133}$ ADTn, $A P$, reg. 1 , doc. n. 22 (1374 febbraio 26): si tratta del già noto Pietro Paolo di Ianes, già scriba della curia vescovile.

${ }^{134}$ Ioppi, I registri del monastero di San Lorenzo, cit., pp. 63-64.

135 Ivi, p. 63. L'archivio prepositurale ospita in tutto diciannove registri per il periodo che copre gli anni fra il 1472 ed il 1818 .

136 Quanto oggi rimane dell'antico archivio degli Eremitani di San Marco è ricostruibile virtualmente assemblando la documentazione conservata presso il fondo BCT3 della Biblioteca comunale di Trento e presso il fondo Capitolo del Duomo dell'Archivio di Stato. Si hanno così a disposizione poco meno di 60 documenti, tutti in forma sciolta, redatti fra il 1275 (BCTn, BCT3, capsa 1, mazzo 1, n. 63) e il 1498 (ASTn, $A C D$, n. 1211). La documentazione superstite, infatti, risulta alquanto lacunosa soprattutto per quanto riguarda il XIII secolo, di cui rimangono soltanto tre pergamene (BCTn, BCT3, capsa 1, mazzo 1, nn. 63, 69, 90); soltanto a partire dagli anni Quaranta del Trecento si assiste a un progressivo aumento degli instrumenta, sebbene sempre su livelli assolutamente non consistenti. Oltre a ciò va ricordato che la conformazione dell'archivio degli Eremitani, così come oggi si presenta, deriva solo in parte dalla conservazione di carte prodotte su richiesta dei frati Agostiniani; in molti casi, infatti, si è in presenza di pergamene da essi conservate per dimostrare e tenere memoria dei diritti acquisiti. Si ha dunque un buon numero di testamenti (14), seguiti dalle donazioni e dalle compravendite. Si tratta pertanto, anche in questo caso, di un archivio thesaurus, molto simile per conformazione a quello degli altri enti presi in considerazione.

137 Cfr., ad esempio, BCTn, BCT3, capsa 2, mazzo 2, nn. 16, 25; capsa 2, mazzo 1, n. 83.

138 ADTn, ACap, Instrumenta capitularia 8bis, c. $105 v$, n. 290.

139 Ivi, c. $120 r$, n. 324. 


\section{L'attività professionale di Antonio da Borgonuovo}

facta extra distincte dicto conductori ${ }^{140}$, in margine a documenti datati alla fine degli anni Venti del Quattrocento. Il notaio, dunque, sembrerebbe far riferimento a quaterni contenenti instrumenta dotati, come affermato dallo stesso professionista, di ogni elemento di convalida, signum e sottoscrizione compresi. Il documento è inoltre redatto per esteso (continua). Un archivio, quello degli Eremitani, che dunque pur nella sua attuale conformazione, lacunosa e quanto mai depauperata, sembrerebbe rivelare forme organizzative del tutto assimilabili a quello dei benedettini di San Lorenzo.

Diversa, per certi aspetti, è la situazione riscontrabile nell'archivio delle Clarisse di San Michele, per le quali si deve constatare l'assenza di qualsiasi forma di registrazione in libro ${ }^{141}$. Qualche dato, semmai, si può aggiungere sul tema dei notai al servizio del convento; a partire dagli anni Settanta del XIV secolo, infatti, il già più volte citato notaio Alberto figlio di Negratus da Sacco divenne redattore quasi esclusivo del convento delle Clarisse. Per il periodo compreso fra il 1379 e il $1394^{142}$ si hanno a disposizione 21 redactiones in mundum e ben 18 presentano in escatocollo la sottoscrizione di Alberto da Sacco. L'attività del notaio proseguì con ogni probabilità fino alla fine del secolo, così come era avvenuto per il Capitolo della cattedrale, tuttavia, già sul finire degli anni Ottanta, le suore cominciarono a servirsi anche di Antonio da Borgonuovo. A partire dal 1431, invece, fece la sua comparsa Graziadeo figlio del fu ser Antonio da Castel Terlago, il quale produsse un buon numero di instrumenta fra il 1431 ed il 1452. Questi fu prima affiancato, poi definitivamente sostituito dal notaio e conte palatino Cristoforo da Molveno, figlio del noto giusperito Antonio, che venne chiamato a rogare dalle Clarisse almeno fino agli anni Sessanta del Quattrocento.

Un'ultima considerazione potrà essere svolta sulla documentazione della confraternita dei Battuti di Trento; anche i disciplinati, per i quali si conserva documentazione a partire dalla seconda metà del Trecento ${ }^{143}$, si servirono dei notai

140 Ivi, c. $128 r-v$, n. 345.

141 L'assenza di un sistema documentario organizzato non deve tuttavia stupire se è vero che, come evidenzia Attilio Bartoli Langeli, «i mendicanti in quanto tali non potevano sviluppare una organica strategia documentaria. Lo fanno intravedere quelle fondazioni che, essendo proprietarie, "usarono" la documentazione in funzione della salvaguardia e dello sviluppo delle proprie risorse patrimoniali. [...] Il documento è strumento e fonte di iura; perciò esso pretende un uso elementare e minimale, ossia di esser conservato» (A. Bartoli Langeli, N. D’Acunto, I documenti degli Ordini mendicanti, in G. Avarucci, R.M. Borraccini Verducci, G. Borri (a cura di), Libro, scrittura, documento della civiltà monastica e conventuale nel basso Medioevo, Atti del convegno della Associazione Italiana dei Paleografi e dei Diplomatisti [Fermo, settembre 1997], CISAM, Spoleto 1999 [Studi e ricerche. Centro italiano di studi sull'Alto Medioevo, 1], p. 390). Nessun cartulario, dunque, né tantomeno alcun registro sembra emergere dall'archivio di San Michele; ciò non significa, ovviamente, che non possano essere esistite forme di registrazione differenti rispetto alle pergamene sciolte. La Biblioteca di San Bernardino conserva infatti una sorta di 'libro di conti' cartaceo del convento relativo agli anni 1476-1477, inerente ai beni e agli affitti di beni situati fra Trento, Civezzano e Piné (FSBN, ms. 324, in originale. Edito in Polli, Le Clarisse di San Michele, cit., appendice, n. 15). Si tratta di cinque fogli cartacei, appartenenti con ogni probabilità a un originario fascicolo unitario, su cui due mani diverse, una anonima, l'altra attribuibile alla badessa suor Fina da Folgaria, vergarono in lingua volgare due distinti elenchi: la colonna sinistra contiene gli affitti dovuti al convento, la colonna destra i pagamenti effettuati.

142 Fra il 1394 e il 1400 si assiste ad un calo considerevole della documentazione conservata.

143 Dal 1350, fino alla fine del XV secolo, si sono rinvenuti circa 70 instrumenta riconducibili all'archivio dei Battuti, oggi per intero conservati presso il fondo BCT3 della Biblioteca comunale di Trento. 
attivi in città, privilegiando sin da subito pochi professionisti con i quali sembra di poter intravvedere un qualche elemento di fidelizzazione: Giovannino figlio del fu Marchetto detto de la Porta da Trento, attivo negli anni Settanta del XIV secolo, Pietro figlio del fu Gioacchino Mezzasoma negli anni Ottanta e, a partire dagli anni Novanta del secolo, Antonio di Bartolasio da Borgonuovo. L'attività di ser Antonio al servizio dell'ente proseguì almeno fino al $1433^{144}$ ma non mancano, nello stesso periodo, documenti fatti redigere ad altri notai cittadini; 'collaborano' infatti con i disciplinati anche Nicolò figlio del fu magister Ognibene sarto dal Borgonuovo di Trento, Martino figlio del fu ser Francesco da Volano, Antonio del fu ser Bonifacio da Nogaredo e, soprattutto dagli anni Trenta del Quattrocento, Graziadeo da Terlago, Nicolò de Capris, Antonio del fu Guglielmo Gallo e Cristoforo da Molveno. La Casa della Misericordia sembra dunque aver fatto affidamento su un ristretto numero di professionisti, seguendo modalità operative già in uso presso le principali istituzioni; peraltro, non si può fare a meno di notare il ripetersi di nomi più volte menzionati quali 'redattori di fiducia' per l'Episcopato, il Capitolo, la Prepositura. Si tratta, è bene ribadirlo, di notai di primo livello nel panorama del notariato cittadino che, probabilmente in virtù delle capacità tecniche, ma anche grazie alle posizioni di rilievo che occuparono nella società trentina, furono oggetto di preferenza da parte delle varie istituzioni.

\section{L'attività al servizio di conventi, monasteri e confraternite}

Una porzione della clientela di ser Antonio è rappresentata da conventi, monasteri e confraternite cittadine che si servirono del professionista per dare forma scritta a documentazione prevalentemente di natura patrimoniale, quali refute e locazioni.

Fra gli enti conventuali più rappresentati vi sono in prima posizione le Clarisse di San Michele presso Trento, il monastero di San Lorenzo, poi Prepositura dal 1425, gli Eremitani di San Marco, il convento di San Francesco e il convento di Santa Croce.

Per le Clarisse di San Michele ser Antonio produsse un certo numero di rogiti che oggi sono noti in parte attraverso le relative redactiones in mundum del fondo Clarisse di San Michele, dove sono stati reperiti quattro documenti ${ }^{145}$, tre dei quali datati 1389 e l'ultimo 1412, e in parte attraverso il registro Instrumenta capitularia 8bis, fra le cui carte si trovano cinque documenti compresi fra il 1423 e il 1429; a questi atti si aggiungono inoltre le notizie di documenti oggi deperditi tradite attraverso la Collectio diplomatum ${ }^{146}$ di Giangrisostomo Tovazzi. Dal codice settecentesco emergono ulteriori dieci notizie di documenti rogati da Antonio per le monache fra il 1402 e il 1422. Pur trattandosi, presumibilmente, di un frammento dell'intera attività scrittoria del notaio per le Clarisse, ciò che rimane permette di delimitare il periodo di attività per questo ente fra il 1389 e il 1429; il dato non è comunque insignificante perché rivela un precoce impegno di ser Antonio per il convento: già nei primissimi anni di attività, dunque, poteva vantare fra $\mathrm{i}$ suoi clienti enti di una certa rilevanza.

144 ADTn, ACap, Instrumenta capitularia 8bis, n. 590a-b.

145 APTn, Clarisse di San Michele, nn. 59, 60a, 60b, 73.

146 BFSB, ms. 6, cc. 323-324; cc. 341-343; cc. 329-330; c. 328; cc. 320-321; cc. 333-334; cc. 331332,403 ; c. 324 ; cc. $336-338$. 


\section{L'attività professionale di Antonio da Borgonuovo}

Si tratta di documenti prevalentemente di tipo negoziale, quali locazioni (perpetue o temporali) e refute; tali negozi sono poi sovente affiancati da altre tipologie documentarie che avevano lo scopo di concludere o perfezionare il negozio, quali ad esempio le immissioni in possesso di immobili concessi in affitto. Si hanno inoltre una sola compravendita ${ }^{147}$ e una permuta ${ }^{148}$.

Luogo esclusivo per la rogazione dei documenti è il convento di San Michele, di cui si individua anzitutto la posizione rispetto alla città di Trento, attraverso locuzioni del tipo: in pertinenciis Tridenti, in monasterio Sancti Michaelis seu Sancte Clare de prope Tridentum ${ }^{149}$, oppure in Burgo Sancte Crucis de prope Tridentum ${ }^{150}$.

L'attività di Antonio per il monastero di San Lorenzo e, dal 1425, per la Prepositura è testimoniata da circa 30 documenti rogati su commissione dell'ente negli anni compresi fra il 1407 e il 1430. Il primo atto rogato dal notaio per il monastero di San Lorenzo è datato 26 novembre 1407 e trasmette una refuta con nuova locazione $^{151}$. L'ultimo documento risale invece al 21 gennaio 1430, si trova fra le carte del registro Instrumenta capitularia 8bis e rappresenta un rinnovo di locazione. La documentazione richiesta ad Antonio è in larga parte nota grazie alle registrazioni sui registri pergamenacei della Prepositura ${ }^{152}$, dove il notaio redasse quindici documenti e, dopo il 1424, nel registro Instrumenta capitularia 8bis; qui, come ricordato parlando della documentazione commissionata dal vescovo, trova spazio anche l'atto di erezione della dignità capitolare della Prepositura ${ }^{153}$. Un solo documento, datato 27 gennaio 1419, è stato trasmesso attraverso la sua redactio in mundum ${ }^{154}$.

Fra i luoghi di rogazione privilegiati si ha l'abbazia di San Lorenzo, della quale talvolta il notaio specifica la posizione rispetto alla città (de prope Tridentum); con la soppressione del monastero nel 1425, su volere del vescovo Alessandro di Masovia ${ }^{155}$, nuovo luogo scelto per la rogazione ai notai fu il quartiere di Borgonuovo, dove aveva sede la casa del canonico Stanislao Sobniowski, primo preposito eletto dal vescovo Alessandro.

Un ulteriore ente monastico-conventuale per cui saltuariamente ser Antonio presta la propria opera è l'ordine degli Eremitani del convento di San Marco in Trento. Per gli Agostiniani si hanno a disposizione una dozzina di documenti redatti da ser Antonio, compresi fra il 1394 ed il 1429, traditi attraverso alcune pergamene sciolte rinvenute nelle capsae del fondo Congregazione di Carità - BCT3 della Biblioteca comunale di Trento, nel fondo Capitolo del Duomo presso l'Archivio di Stato di Trento e, in misura ancor più rilevante, fra le carte del registro Instrumenta capitularia 8bis. Nulla si può aggiungere, rispetto a quanto detto per gli altri enti fin qui menzionati, in merito alle tipologie documentarie, le solite refute e locazioni; per quanto concerne invece i luoghi di rogazione, luogo esclusivo scelto dagli Agostiniani di San Marco è il convento stesso.

147 Ivi, ms. 6, cc. 320-321.

148 ADTn, ACap, Instrumenta capitularia 8bis, n. 4.

149 Cfr., ad esempio, APTn, Clarisse di San Michele, n. 59.

150 ADTn, ACap, Instrumenta capitularia 8bis, n. 50.

151 ADTn, $A P$, reg. II, n. 90.

152 Sui registri pergamenacei del monastero di San Lorenzo, poi Prepositura, dove si è potuto riscontrare un buon numero di documenti redatti da Antonio in forma di esteso, cfr. Ioppi, I registri del monastero di San Lorenzo, cit.

${ }^{153}$ Cfr. Appendice, regesto n. 28 (ADTn, ACap, Instrumenta capitularia 8bis, n. 131).

154 ADTn, $A P$, Tomo III, n. 10

155 Cfr. Appendice, regesto n. 28 (ADTn, ACap, Instrumenta capitularia 8bis, n. 131). 
Fra le carte redatte da Antonio da Borgonuovo non mancano tracce di documenti redatti per conto di altri enti conventuali cittadini; si tratta rispettivamente del convento di San Francesco e di Santa Croce. Per il primo si hanno due soli documenti redatti il 28 novembre $1423^{156}$ e il 17 febbraio $1424^{157}$ : una permuta fra il frate Giovanni da Verona, predicatore dell'ordine dei minori di san Francesco, agente in qualità di rettore del convento, e ser Pietro Iacob, seguito, a distanza di pochi mesi, dalla ratifica dei frati non presenti al momento della stipula. Vista l'esiguità della documentazione conservata, poco si può aggiungere sull'impegno di Antonio nei confronti dell'ente francescano. Per quanto riguarda, invece, il luogo scelto per la rogazione, la data topica rinvia - in entrambi i casi - alla chiesa del convento situato de prope Tridentum.

Ancora meno si ricava dall'unico documento redatto da Antonio per il convento di Santa Croce, situato a sud della città, a poca distanza dal convento delle Clarisse di San Michele. Si tratta di un rinnovo di locazione rogato il 13 dicembre $1424^{158}$. Se il notaio avesse registrato ulteriore documentazione per questo ente in anni precedenti non è dato saperlo; l'archivio dei Crociferi risulta infatti in gran parte disperso. Il convento e ospedale di Santa Croce furono allora rappresentati dal frate Antonio da Venezia, priore e rettore il quale - «vestigia predecessorum suorum imitando» - concesse a Francesco magister a scutelis da Padova una porzione di terra arativa con vigne e prato situata proprio vicino all'ospedale. La rogatio si svolse in una stupa presso lo stesso monastero.

Certamente più consistente è la documentazione redatta da ser Antonio per la confraternita dei Battuti laici di Trento ${ }^{159}$, per la quale sono stati rinvenuti circa quaranta rogiti compresi fra il $1393^{160}$ ed il $1433^{161}$. L'impegno per la confraternita sembra dunque iniziare abbastanza precocemente per concludersi negli ultimi anni di attività. Del resto - lo si è evidenziato parlando della sua biografia - Antonio da Borgonuovo ricoprì per i Battuti la carica di massaro già nel 1395, mentre il padre Bartolasio aveva lasciato un lascito alla fratalia fra le sue disposizioni testamentarie.

Gli atti, reperiti in parte nelle capsae del fondo Congregrazione di Carità$B C T 3$, in parte fra le carte del registro Instrumenta capitularia 8bis, sono distribuiti piuttosto uniformemente negli anni compresi fra il 1393 ed il 1433, con una media di due-tre documenti all'anno. Per quanto riguarda le tipologie documentarie rogate, anche la confraternita dei Battuti non sembra discostarsi dagli esempi fin qui proposti; locazioni e refute costituiscono infatti la maggioranza dei documenti, intervallate da una sola permuta ${ }^{162}$.

Dal punto di vista dei luoghi di rogazione, l'analisi sulle date topiche riconduce a spazi piuttosto diversificati nella città; anzitutto si nota come il primo documento del 1393 sia rogato proprio presso la sede della confraternita, «in contrata Sancte

156 ADTn, ACap, Instrumenta capitularia 8bis, n 6.

157 Ivi, n. $7 \mathrm{~b}$.

158 Ivi, n. 72.

159 Del tutto assente sembra essere il suo impegno per la confraternita alemanna degli Zappatori; lo spoglio delle pergamene del fondo Congregazione di Carità - BCT3, dove trova spazio la maggior parte della documentazione relativa a questo ente, ha infatti portato alla luce un certo numero di documenti redatti da Antonio; non si tratta tuttavia, in nessun caso, di documentazione commissionata dagli Zappatori.

160 BCTn, BCT3, capsa 21, mazzo 1, n. 39.

161 ADTn, ACap, Instrumenta capitularia 8bis, n. 590a-b.

162 Ivi, n. 225. 


\section{L'attività professionale di Antonio da Borgonuovo}

Marie Maioris, in domo fratalie Batutorum laycorum de Tridento, apud banchum magistri dicte fratalie» ${ }^{163}$; una «domus Misericordie et hospitalis Batutorum Tridenti» viene citata anche nella datazione di due permute del $1427^{164}$. Altrove si menzionano invece luoghi come la casa dell'apothecarius Giovannino Girardi, che ricopriva in quell'occasione il ruolo di ministro e rettore della fratalia $^{165}$. Altri negozi vengono rogati nella piazza del Comune, «ad cambium domus stazionis speciarie et habitationis Melchioris condam ser Dominici ab Oleo de Tridento» ${ }^{166} \mathrm{o}$, addirittura, presso l'abitazione del notaio Antonio ${ }^{167}$.

Un'altra serie di documenti furono prodotti da ser Antonio negli anni Venti del Quattrocento per alcuni enti cittadini; si tratta prevalentemente di documentazione di natura patrimoniale (refute e locazioni) redatte su richiesta dell'Ordine teutonico (con sede presso la chiesa di Santa Elisabetta) fra il 1424 e il $1426^{168}$, e per gli ospedali di San Martino ${ }^{169}$, San Pietro ${ }^{170}$, San Tommaso presso Romeno ${ }^{171}$ e Santa Maria Maddalena ${ }^{172}$.

\section{Gli altri clienti privati}

Non meno importante, rispetto agli enti fin qui menzionati, è la clientela privata che scelse Antonio da Borgonuovo per registrare molteplici tipologie documentarie. Per citarne solo alcune, fra le più frequenti: le compravendite, le locazioni e le refute, le immissioni in possesso, i testamenti, i matrimoni e le doti, le carte di debito (o di credito), le quietanze liberatorie, le procure, le permute, le cancellazioni di debito, le donazioni, le cessioni, le dichiarazioni (confessiones), i patti societari, gli scioglimenti di società, le convenzioni, le nomine a notaio e giudice ordinario, le divisioni di beni ed eredità, le rinunce a diritti, gli inventari ${ }^{173}$.

Si tratta di documentazione commissionata da un vasto numero di clienti che in questa sede non è opportuno elencare nel dettaglio; si citeranno pertanto soltanto quei clienti che, fra il 1386 ed il 1437, si rivolsero ad Antonio con maggiore frequenza. Per costoro, si cercherà di individuare le tipologie documentarie mag-

163 BCTn, BCT3, capsa 21, mazzo 1, n. 39.

164 ADTn, ACap, Instrumenta capitularia 8bis, n. 225; BCTn, BCT3, capsa 4, mazzo 1, p. 45.

165 Ivi, capsa 28, mazzo 1, nn. 12, 13.

166 Cfr., ad esempio, BCTn, BCT3, capsa 28, mazzo 1, n. 40.

167 Cfr., ad esempio, ADTn, ACap, Instrumenta capitularia 8bis, n. 444.

168 Ivi, nn. 44, 114, 151

169 Ivi, nn. 193, 223, 223a, 231, 238, 281, 367. Agisce, quale rettore e priore dell'ospedale il canonico, nonché pievano di Cembra, Giovanni Zeiss da Bopfingen.

170 Ivi, n. 67. Sindaco e provisor dell'ospedale di San Pietro è Floriano Malicie, figlio del fu Francesco da Trento.

171 Ivi, n. 279. L'atto fu rogato presso l'abitazione del notaio dal presbitero Marco, pievano a Livo e cappellano della cattedrale, che agiva quale priore e rettore pro tempore dell'ospedale; la presenza, fra i committenti di ser Antonio, di un'istituzione ecclesiastica non avente sede a Trento è senza dubbio insolita, visto il forte radicamento cittadino del notaio, e va probabilmente ricondotta alla residenza in città del suo rettore.

172 Ivi, n. 241.

173 Si tratta prevalentemente di documentazione tradita attraverso il registro Instrumenta capitularia 8bis e, in misura meno rilevante, attraverso redactiones in mundum su pergamena reperite in archivi privati e di famiglia. 
giormente richieste, gli estremi cronologici e, non ultimi per importanza, i luoghi scelti per la rogazione.

Fra i clienti privati maggiormente rappresentati fra le carte del notaio Antonio da Borgonuovo, spiccano per quantità di documentazione richiesta i membri della famiglia Calepini: Adelperio, in prima posizione e, con un numero di rogiti nettamente inferiori, i fratelli Odorico e Bonaventura; la frequenza con cui i Calepini si rivolsero al professionista non deve destare meraviglia, considerati gli stretti rapporti di parentela fra le due famiglie. Si sono a tal proposito potuti individuare circa tredici documenti rogati direttamente per Adelperio fra il 1424 e il 1435, tutti trasmessi dal registro Instrumenta capitularia 8 bis $^{174}$; si tratta, in larga misura, di refute, locazioni e compravendite che palesano indubbiamente l'intensa attività speculativa messa in atto dal giurisperito genero del notaio. Non mancano, tuttavia, anche documenti particolari quali, ad esempio, il già citato prestito di libri legali concesso nel 1426 dal giurisperito Adelperio a Morandino, figlio del magister Giovanni Bonus da Trento, iurisperitus e studente in diritto civile, il quale ricevette un Digestum Novum e uno Vetus, i Tres libri, le Institutiones e le Decretali, stimati di comune accordo ben 125 ducati d'oro. Meno attestata è invece l'attività economica dei fratelli Odorico e Bonaventura, i quali commissionarono complessivamente ad Antonio quattro locazioni fra il 1424 ed il $1430^{175}$.

Se piuttosto rilevante fu l'attività del notaio nei confronti dei membri della famiglia Calepini, ancor più rappresentata e stabile nel tempo fu la documentazione richiesta da Gioacchino Mezzasoma. Il giurisperito ricorre quale cliente di Antonio in ben venticinque occasioni; refute, locazioni e permute rappresentano le tipologie documentarie commissionate con maggiore frequenza fra il 1424 ed il 1435'76; in particolare, negli anni compresi fra il 1427 e il 1429, Gioacchino Mezzasoma fece registrare da Antonio una serie di nove vendite di proprietà immobiliari e affitti ${ }^{177}$. Ma certamente, fra tutti i rogiti fin qui elencati, quello che sembra dimostrare il rapporto di fiducia del giurisperito nei confronti del notaio è il matrimonio, celebrato il 27 gennaio 1425178, con Speranza, la figlia del notaio Paolo da Trento e sorella di Giovanni Rauter. Quest'ultimo, non a caso, figura, al pari dei personaggi fin qui citati, fra $\mathrm{i}$ clienti più rappresentati nella documentazione di Antonio; fra il 1428 e il 1433, infatti, si rivolse al professionista in sei occasioni per registrare quattro compravendite $^{179}$, una quietanza ${ }^{180}$ e una promessa di pagamento ${ }^{181}$. Oltre a ciò, tre giorni dopo la sorella Speranza, precisamente il 30 gennaio del $1425^{182}$, Giovanni si sposò con Andriota, la figlia di ser Bonadomano de Accerbis e, ancora una volta, fu il notaio Antonio da Borgonuovo a registrare il contratto nuziale.

174 ADTn, ACap, Instrumenta capitularia 8bis, nn. 32, 80, 195, 196, 203, 256, 260, 336, 508, 512, 568, 639a-b, 647 .

175 Ivi, n. 58, locazione da parte di Odorico Calepini; ivi, nn. 167, 321, 377, locazioni da parte di Bonaventura Calepini.

${ }^{176}$ Ivi, nn. 53, 85, 137, 168, 169, 187, 194, 198, 202c, 233, 252, 295, 296, 304, 305, 306, 308, $329,331,335 \mathrm{a}, 335 \mathrm{~b}, 505,572 \mathrm{~b}, 621 \mathrm{a}, 642$.

177 Ivi, nn. 233, 295, 296, 304, 305, 306, 329, 331.

178 Ivi, n. 85.

179 Ivi, nn. 303, 456; inoltre, ivi, nn. 391, definita dal notaio permutatio, venditio et insolutum traditio e ivi, nn. 476, definita in rubrica venditio et insolutum traditio.

${ }^{180}$ Ivi, n. $303 \mathrm{c}$.

181 Ivi, n. 590b.

182 Ivi, n. 86. 


\section{L'attività professionale di Antonio da Borgonuovo}

Fra i principali clienti del notaio si ricordano anche il giurisperito e conte palatino Antonio da Molveno, per il quale si sono reperiti otto documenti rogati fra il 1424 ed il $1431^{183}$. Non mancano, anche in questo caso, refute, locazioni e compravendite, che testimoniano altresì l'intensa attività speculativa messa in atto da alcuni fra i più importanti membri del ceto dirigente cittadino; tuttavia, nel caso di Antonio da Molveno si devono annoverare nuove tipologie documentarie, altrimenti poco rappresentate negli archivi trentini: le nomine a notaio. Subito dopo l'elezione a conte palatino da parte dell'imperatore Sigismondo ${ }^{184}$, come ricordato, Antonio da Molveno nominò infatti notai e giudici ordinari Giovanni figlio di Ermanno da Mori e Lorenzo del notaio Antonio de Castro $^{185}$. Pochi mesi più tardi fu la volta di Giovanni de Fraudental, già scriba presso il castello e cappellano in cattedrale ${ }^{186}$, mentre, il 5 marzo 1431, venne scelto quale nuovo notaio e giudice Antonio figlio di Guglielmo Gallo da Trento ${ }^{187}$.

Fra gli altri clienti di ser Antonio possono inoltre essere ricordati Odorico del fu Federico da Povo, Giovanni di ser Luca e il figlio Luca de Lippi, Melchiorre ab Oleo, Bonadomano de Accerbis, il notaio Nicolò de Capris, ser Pietro Iacob e Gianpietro da Feltre. Nomi che ricorrono, iterati, non soltanto come auctores di negozi giuridici ma anche fra i testimoni di un largo numero di documenti redatti da Antonio e che furono fra loro legati anche da interessi di tipo economico. Una clientela che appare abbastanza stabile nel corso degli anni, anche se - come già evidenziato - mancano, almeno fino al 1423-1424, registrazioni utili a ricostruire la clientela privata nei primi anni di attività del professionista.

Nella documentazione redatta da ser Antonio per alcuni dei clienti citati sono, come visto, i matrimoni. Numerosi furono infatti i clienti che, fra il 1423 e il 1434 stipularono il proprio matrimonio davanti al notaio; molti fra questi sono nomi già noti perché membri della classe dirigente trentina di inizio Quattrocento, altri invece non compaiono così frequentemente fra le carte del notaio. Si tratta, complessivamente, di ventisette contratti matrimoniali con dote ${ }^{188}$ e di tredici doti ${ }^{189}$.

In ultimo, fra le tipologie documentarie redatte da Antonio si è riscontrato un certo numero di testamenti, diciannove per la precisione, che rinviano ad altrettanti clienti. Il primo testamento prodotto è datato 4 settembre $1390^{190}$ e trasmette le disposizioni testamentarie di Lola del fu ser Andrea dal Cantone di Trento che dettò le proprie ultime volontà al notaio mentre giaceva, malata, nel letto della sua abitazione nella contrada del Mercato. L'ultimo testamento è invece datato 12 ottobre $1422^{191}$ e fu dettato dal canonico, cappellano e pievano di Fiemme, nonché priore dell'ospedale di San Martino, Giovanni de Austria.

Si tratta di documentazione reperita esclusivamente in fondi pergamenacei di enti quali il Capitolo, la confraternita dei Battuti, il convento degli Eremitani di

183 Ivi, nn. 27, 142, 210, 242, 283, 393, 467, 507.

184 Ivi, n. 208 (la nomina a conte palatino è datata 10 novembre 1426).

185 Ivi, n. 210 (19 novembre 1426).

186 Ivi, n. 209 (8 maggio 1427).

187 Ivi, n. 467.

188 Ivi, nn. 16bis, 17, 23, 34, 41, 47, 49, 79, 85, 86, 88, 100, 158, 176, 211, 219, 247, 250, 258, $272,274,312,392,433,462,522,622 \mathrm{a}$.

189 Ivi, nn. 321a, 20, 83, 103, 192, 197, 264, 349, 388, 401, 539, 627, 640.

190 ADTn, ACap, capsa testamenti, rotoli corti/a, n. 14.

191 ADTn, ACap, capsa testamenti, rotoli medi/b, n. 15. 
San Marco, che conservarono con ottica tesaurizzante le ultime volontà di personaggi che disposero a loro favore dei lasciti, richiedendo spesso la contestuale celebrazione di messe o la sepoltura nel cimitero della chiesa di pertinenza. Per tale ragione, si deve presumere che la reale produzione di testamenti da parte di Antonio fu superiore a quella fino ad oggi conservata, tanto più che il notaio scelse di non registrare tale tipologia documentaria su Instrumenta capitularia 8bis, cosicché - in assenza di pergamene sciolte - dopo il 1422 non vi sono più testamenti redatti dal notaio.

A conclusione di questa rapida analisi sulla clientela privata di Antonio, si dedicherà un'ultima riflessione ai luoghi scelti per la rogazione. A differenza di quanto riscontrato per le istituzioni, caratterizzate da una certa stabilità in questo senso, le date topiche dei committenti privati si contraddistinguono, come è logico, per la spiccata eterogeneità. Se infatti un buon numero di documenti furono richiesti al notaio proprio presso la sua abitazione nel Borgonuovo, molti altri furono commissionati in altre zone della città. In particolare, i matrimoni furono spesso celebrati presso l'abitazione di uno fra gli sposi; nel 1424, ad esempio, il matrimonio fra Aldrighetto del fu Giovanni Mezaoveta e Lucia figlia di Guglielmo Gallo fu rogato «in contrata Sancte Marie Magdalene, in curtivo domus solite habitationis Guillelmi condam ser Delaiti Gali de Tridento» ${ }^{192}$. Nel 1430 1'unione nuziale fra Bartolomeo del fu Blanchus detto de Sangue de Can da Nomi e Giovannina fu invece celebrato «in contrata Merchati veteris, in domo habitationis Dominigacii laboratoris patrigni infrascripte sponse» ${ }^{193}$.

\subsection{Antonio da Borgonuovo e la vicenda dell'eredità di Antonio Belenzani}

A completamento di quanto finora detto sulla clientela privata di ser Antonio è utile menzionare un episodio che, sia per i protagonisti coinvolti sia per l'enorme mole documentaria scaturita costituisce un importante spaccato sull'attività scrittoria del notaio all'inizio degli anni Trenta del Quattrocento.

Gli instrumenta che, in forma di imbreviatura o di esteso sul registro Instrumenta capitularia 8bis o in forma di redactio in mundum, ser Antonio redasse fra il 1430 ed il 1432, rappresentano infatti una fetta per nulla trascurabile della sua attività in quello scorcio d'anni. Considerando, ad esempio, l'intera produzione documentaria relativa al periodo giugno-dicembre 1430 registrata su Instrumenta capitularia 8bis, si nota come dei 56 documenti ivi redatti, ben 30 siano relativi alla vicenda di cui ora si tratterà ${ }^{194}$.

Per tale ragione si è scelto di inserire questo excursus a chiusura del paragrafo inerente ai clienti privati del notaio ma, a ben vedere, i fatti di cui fra breve si parlerà risultano rilevanti non soltanto per la scelta di Antonio quale autenticatore e 'registratore' di negozi giuridici che da quegli stessi eventi scaturirono, ma soprattutto per la portata dei personaggi implicati: alcuni membri della nota famiglia Belenzani, il vescovo Alessandro di Masovia e, non ultimo per impor-

192 Cfr. Appendice, regesti nn. 5, 6, 7 (ADTn, ACap, Instrumenta capitularia 8bis, nn. 47a-b-c).

193 ADTn, ACap, Instrumenta capitularia 8bis, n. 392.

194 Il campione giugno-dicembre è stato scelto perché è dal giugno del 1430 (ADTn, ACap, Instrumenta capitularia 8bis, n. 395, datato 23 giugno 1430) che iniziano le registrazioni in originale relative alla vicenda. 
tanza, ser Pietro di Nanni da Siena, figura altrimenti poco nota alla storiografia trentina.

Gli eventi vanno collocati in un periodo cronologicamente piuttosto limitato, compreso fra la fine di giugno del 1430 e il gennaio del 1432; altrettanto importanti, per la ricostruzione dell'episodio, sono tuttavia gli antefatti, riconducibili a quasi cinquant'anni prima, esattamente al 1383.

Si inizierà a narrare questi fatti non dal principio, ma dal 23 giugno 1430, allorquando Alessandro di Masovia chiese a ser Antonio di registrare una compravendita $^{195}$ : il vescovo, infatti, vendette a ser Pietro di Nanni da Siena, civis Tridentinus ac civis et habitator Verone, lì rappresentato dal procuratore ser Pietro Iacob, una serie di affitti e redditi pagati annualmente al fu Antonio Belenzani e, dopo la morte di questi, ai suoi eredi, il tutto per il considerevole prezzo di 1.200 ducati d'oro. Come era entrato in possesso il vescovo di Trento dei beni che facevano parte dell'eredità di Antonio Belenzani?

Membro della nota famiglia Belenzani, Antonio, un lontano parente del più noto Rodolfo, era figlio di Giovanni e Todeschina del fu Guglielmo da Trento ${ }^{196}$. L'11 settembre del 1383, corpore infirmus, costretto a letto nella sua abitazione della contrada dei Belenzani, dettò testamento dinanzi a un nutrito gruppo di testimoni, fra cui la moglie Giacoma. Il giorno successivo alla morte del testatore ${ }^{197}$ si presentò dinanzi al vicario vescovile Giovanni de Pugnis da Parma il notaio ser Francesco da Molveno, in rappresentanza di tutti i legatari. Costui dichiarò che Antonio Belenzani aveva espresso le sue ultime volontà in punto di morte dinanzi a testimoni fededegni; si rendeva tuttavia necessaria la redazione in forma pubblica delle disposizioni del defunto. Al contempo ser Francesco chiese al vicario l'assegnazione di un curatore idoneo ai due figli di Antonio, Guglielmo e Giovanni, che erano minori di sette anni. Udite tali richieste, il vicario assegnò i due fanciulli alla cura del notaio Giacomo da Ravazzone, che prestò giuramento; per quest'ultimo si costituì al contempo fideiussore il notaio Marco del fu ser Odorico da Spormaggiore.

Volendo altresì dimostrare la veridicità delle ultime disposizioni del defunto, avvenute - si presume - in sola forma orale e temendo la morte dei testimoni propter pestem yminentem, ser Francesco e ser Giacomo chiesero al vicario la convocazione dei testes che il giorno precedente avevano presenziato al testamento, affinché potessero essere interrogati su una serie di capitula.

Fra i convocati figuravano i legatari ser Simone de Cambo, sindico dei Battuti di Trento, Biagio del fu ser Franchetto da Pomarolo, sindaco della chiesa di Santa Maria Maggiore di Trento, Giacoma, moglie del defunto Belenzani, la madre di costui Todeschina, e Ottolino sartor del fu ser Paolo cursor abitante a Trento. Il 15 settembre, interrogati uno dopo l'altro, i testimoni confermarono tutti i capitoli

195 Ibidem.

196 Sulla famiglia Belenzani cfr. Bettotti, La nobiltà trentina nel medioevo, cit., e, in particolare, l'albero genealogico (tavola n. 1) allegato alla pubblicazione.

197 La morte di Antonio Belenzani avvenne quello stesso giorno, 1'11 settembre 1383 (cfr. Appendice, regesti nn. 1, 2). Ne danno conferma, all'interrogatorio del vicario vescovile, gli stessi testimoni nel momento in cui furono chiamati a confermare le ultime volontà del Belenzani. Come si ricava da G. Tovazzi, Malographia Tridentina. Cronaca dei fatti calamitosi avvenuti nel Trentino e regioni adiacenti dai primi anni d.C. al 1803, Lions Club, Trento 1986, p. 49; nel settembre del 1383 a Trento scoppiò una grave pestilenza (è possibile che Tovazzi avesse ricavato tale informazione sulla pestilenza proprio dal documento poc'anzi citato). 
stilati da Francesco da Molveno e Giacomo da Ravazzone. Essi dichiarono cioè che Guglielmo e Giovanni, figli di Antonio, erano stati scelti come eredi universali; un affitto perpetuo di 10 lire di denari piccoli era stato lasciato alla chiesa di Santa Maria Maggiore di Trento perché vi si celebrasse il suo anniversario; un affitto perpetuo di 10 lire di denari era stato assegnato ai Battuti; a Giacoma sua moglie egli aveva lasciato invece 100 ducati come disposto nell'istrumento dotale; a sua madre Todeschina 300 lire di denari piccoli; al magister Ottolino 100 lire di denari piccoli; al notaio Francesco da Molveno, che era stato scelto quale fideicommissario, aveva invece assegnato 1.000 ducati d'oro, che avrebbe ottenuto dopo la morte di Guglielmo e Giovanni. Aveva disposto inoltre che, se i suoi figli fossero morti, l'intera eredità sarebbe andata ai pauperes Christi egenos. Dopo aver così espresso le sue ultime volontà ser Antonio era morto.

Tralasciando gli ulteriori dettagli espressi super capitulis dai testimoni, è interessante annotare quanto affermò il prete Pietro da Parma, pievano della chiesa di Santa Maria Maggiore il quale, interrogato super nono capitulo, disse:

Quod tempore et loco proxime suprascriptis in dicto primo capitulo contentis, dictus Antonius volebat et ordinabat et disponebat si contingeret dictos suos filios mori sine heredibus legitimis, deductis et extractis mille ducatis legatis dicto ser Francisco de Molveno notario, quod reliqua sua bona deberent dari et distribui inter pauperes Christi egenos per dictum ser Franciscum notarium de Molveno et hiis omnibus presens fuit et audivit ${ }^{198}$.

L'affermazione non è irrilevante, soprattutto alla luce degli eventi occorsi pochi anni più tardi.

Dopo questi fatti, le fonti disponibili non sembrano fornire ulteriori notizie, fin quando, il 9 giugno 1426, ser Bonadomano de Accerbis, ser Marco Belenzani e il notaio Iosio, in qualità di procuratori di Felicia, moglie del fu Giovanni Belenzani, di ser Michele a Plata e dei fratelli Erasmo e Guglielmo Thun, eredi del detto Giovanni, vendettero al notaio Antonio da Nogaredo un affitto perpetuo per il prezzo di 15 ducati d'oro che ad essi servivano per pagare le spese del funerale di Giovanni ${ }^{199}$. Meno di un mese dopo, il 3 luglio 1426, la stessa Felicia, insieme a ser Michele $a$ Plata ed Erasmo Thun, «causa solvendi et satisfaciendi funeralia et expensas factas ad sepeliendum ipsum Iohannem de Belenzanis», vendettero a Michele, figlio di ser Domenico da Cortesano, un ulteriore affitto perpetuo al prezzo di 36 ducati d'oro ${ }^{200}$. Pochi mesi più tardi, precisamente l'11 febbraio 1427, gli stessi Michele ed Erasmo, alla presenza della vedova Felicia, vendettero a Palamidesio, figlio del fu ser Giacomo di Palamidesio da Trento, un affitto perpetuo di 16 grossi carentani, al prezzo di 40 lire di denari trentini ${ }^{201}$. Alla morte di Giovanni Belenzani, dunque, gli eredi e la moglie sembravano aver ottenuto piena disponibilità sui suoi averi.

Non trascorse, tuttavia, molto tempo prima che, fra il giugno e l'ottobre del 1427, fosse intrapresa una causa contro gli eredi del defunto, i quali non avevano

198 Cfr. Appendice, regesto n. 2 (ADTn, ACap, Instrumenta capitularia 8 bis, cc. 188r-190r. n. $498[\mathrm{~b}])$.

199 ADTn, ACap, Instrumenta capitularia 8bis, n. 184.

200 Cfr. Appendice, regesto n. 31 (ADTn, ACap, Instrumenta capitularia 8bis, n. 190). Lo stesso giorno, essi vendono a magister Pietro a caminis un ulteriore affitto perpetuo di 15 soldi di denari trentini al prezzo di 3 ducati d'oro (ivi, n. 191). 
rispettato le ultime volontà di Antonio Belenzani: in caso di morte senza figli di Giovanni (Guglielmo era infatti già defunto), l'intera eredità doveva essere devoluta ai pauperes Christi egenos. E ad intraprendere la causa fu proprio il vescovo Alessandro di Masovia, per mezzo del suo procuratore Gioacchino Mezzasoma. Questi, infatti, si presentò dinanzi a Goffredo Friling, vicario in spiritualibus, e ad Antonio de Zivolis, vicario in temporalibus, giudici delegati per la causa, per agire contro i nobili Michele a Plata, Erasmo e Guglielmo Thun, e contro la vedova Felicia.

La narratio del documento informa che Guglielmo, figlio di Antonio Belenzani, era morto, privo di eredi, sedici anni dopo il padre. Nel 1426 anche Giovanni morì senza eredi, pertanto l'intera eredità sarebbe dovuta andare ai pauperes Christi. Poiché il vescovo agiva quale rappresentate dei pauperes Christi, de iure doveva incamerare l'intera eredità. Il 27 ottobre 1427 fu emessa la sentenza definitiva: i beni del fu Antonio Belenzani furono devoluti ai pauperes, ossia al vescovo di Trento; Michele a Plata, Erasmo e Gugliemo, eredi del fu Giovanni, dovettero invece restituire al presule trentino due terzi di tutti i beni che Giovanni aveva posseduto, trattenendo unicamente la terza parte dell'eredità corrispondente a quanto egli aveva ottenuto mentre era in vita. Gli eredi furono inoltre condannati al risarcimento completo di quanto il defunto Belenzani aveva venduto o alienato contro le disposizioni paterne, mentre tutti i beni mobili di cui era stato depredato tempore sachomani non andavano né conteggiati, né risarciti; né tantomeno andavano messi in conto i legati ad pias causas lasciati da Giovanni nel suo testamento ${ }^{202}$.

Il 4 settembre 1428, circa un anno più tardi, veniva emessa un'ulteriore sentenza; il vicario vescovile Antonio de Zivollis aveva incaricato ser Pietro Iacob, Battista da Bologna e i notai Antonio da Borgonuovo e Antonio de Castro di dividere i beni e l'eredità del fu Giovanni Belenzani, assegnandone due parti al vescovo Alessandro di Masovia e la parte rimanente agli eredi legittimi ${ }^{203}$. Fra le varie proprietà e affitti specificati nella sentenza, gli eredi del Belenzani poterono conservare la casa in cui abitavano situata nell'omonima contrada; molto più cospicua fu invece la quantità dei beni che finirono in mano al presule il quale, il 23 giugno 1430, in qualità di pater pauperum e legittimo amministratore e difensore dei poveri, «ut comodius dicta bona inter pauperes Christi valeat disponere et dispenssare», vendette l'intera eredità a ser Pietro di Nanni da Siena, lì rappresentato - come ricordato - dal procuratore ser Pietro Iacob ${ }^{204}$. Con la vendita dei due terzi dell'eredità Belenzani, il vescovo otteneva immediatamente una cospicua quantità di denaro, liberandosi al contempo di un'enorme numero di proprietà e beni sul possesso dei quali avrebbero potuto pesare in futuro ulteriori cause da parte degli eredi di Giovanni.

Con l'acquisto dell'eredità Belenzani da parte di ser Pietro di Nanni, si possono riannodare le fila del discorso con cui si è introdotta la vicenda; a partire da questo evento, infatti, subentra la figura di Antonio da Borgonuovo, quale notaio chiamato a rogare tutta una serie di negozi giuridici su richiesta dello stesso ser Pietro da Siena. Dopo aver acquistato per 1.200 ducati l'eredità Belenzani, il 9 settembre 1430, egli prese possesso di una casa situata nella contrada di San Benedetto; l'entrata in possesso della casa sanciva, simbolicamente, l'acquisizione

\footnotetext{
201 Ivi, n. 222.

202 Cfr. Appendice, regesto n. 34 (ADTn, ACap, Instrumenta capitularia 8bis, n. 404).

203 Cfr. Appendice, regesto n. 39 (ADTn, ACap, Instrumenta capitularia 8bis, n. 405).
} 


\section{Stefano Malfatti}

di diritti su tutte le altre proprietà acquistate dal vescovo Alessandro ${ }^{205}$. Le fonti trentine presentano ser Pietro di Nanni come civis Tridentinus ac civis et habitator Verone; non era dunque sempre presente nel capoluogo vescovile, tanto da dover nominare, proprio il 9 settembre 1430, un procuratore, nella persona di ser Pietro Iacob, per vendere, alienare e dare in affitto tutti i beni, i terreni e gli affitti acquistati dal vescovo Masovia ${ }^{206}$. Ser Pietro era un banchiere e mercante arricchitosi negli anni grazie a remunerative attività speculative ${ }^{207}$. Risulta che nel 1403 egli aveva già stabilito la propria residenza in Verona, e l'estimo del 1409 attesta che il senese abitava nella contrada di San Benedetto ${ }^{208}$.

Le attività economiche di Pietro a Trento sono attestate dal 1425 circa, allorquando, 1'11 ottobre di quell'anno, acquistò per ben 1.000 ducati d'oro la muta sive teloneo supra lignaminibus della porta di San Martino, che fino ad allora era stata concessa in feudo dall'episcopato a Francesco da San Martino e, prima ancora, al padre di costui Adelperio; Alessandro di Masovia ne aveva quindi investito lo stesso ser Pietro ${ }^{209}$. Pochi giorni più tardi, il 15 ottobre, lo si ritrova ancora a Trento in qualità di procuratore di Giovanni da Fondo, per il quale aveva venduto alla cifra di 70 ducati d'oro al notaio ser Giacomo a Ture da Trento il dominio utile su una casa nella contrada di San Benedetto, rimettendone poi ogni diritto nelle mani dei canonici $^{210}$. Pochi mesi più tardi, il 28 febbraio 1426, ser Pietro rilasciava quietanza di pagamento per un credito di 360 ducati che vantava nei confronti del decano del Capitolo, Giovanni da Isny, di alcuni canonici e di cittadini fideiussori ${ }^{211}$. Il 6 febbraio 1431 il banchiere rilasciava una nuova quietanza: Bonadomano de Accerbis, infatti, aveva saldato come procuratore del nobile Giacomo de Federicis de Arbano in Valcamonica 875 ducati d'oro che quest'ultimo doveva a ser Pietro di Nanni in virtù di un prestito ricevuto per recuperare il castello di Campo nelle Giudicarie ${ }^{212}$.

Più redditizio, almeno in apparenza, si dimostrò per il senese l'acquisto dell'eredità Belenzani da Alessandro di Masovia ${ }^{213}$. Come ricordato, infatti, il 9 settembre 1430 ser Pietro entrava in possesso dei beni acquistati dal vescovo; poche settimane

${ }^{204}$ Cfr. Appendice, regesto n. 43 (ADTn, ACap, Instrumenta capitularia 8bis, n. 395).

${ }^{205}$ Cfr. Appendice, regesto n. 44 (ADTn, ACap, Instrumenta capitularia 8bis, n. 395b).

${ }^{206}$ Cfr. Appendice, regesto n. 45 (ADTn, ACap, Instrumenta capitularia 8bis, n. 446).

207 Per dati biografici più precisi su Pietro di Nanni da Siena si rinvia a Malfatti, Toscani a Trento, cit., pp. 439-442, da cui sono tratte le informazioni qui esposte.

${ }^{208}$ Un verbale del Consiglio vicentino del 1403 lo definisce «bancherio in Verona» (BCB, Archivio Torre, reg. 777, c. 132v). Nell'estimo veronese del 1409 è allibrato per 3 lire e 13 soldi (ASVr, Antico archivio del Comune, Estimi, reg. 249). A partire dal 1409 è possibile seguire le attività economiche del senese a Verona: egli aveva certamente un banco nel quartiere di residenza, al quale si rivolsero anche cittadini veronesi di grande rilevanza, fra cui i Guarienti e i Dal Verme, per citare due delle famiglie più note. Non meno importanti furono le attività di Pietro nella città natale Siena, dove in due distinte occasioni (nel 1419 e nel 1427) fu nominato priore del Comune.

209 ASTn, APV, Sezione latina, capsa 3, n. 48; cfr. anche R. Stenico, Il dazio di Trento. Alcuni documenti dei secoli XII-XV, «Studi trentini di scienze storiche. Sezione prima», 66, 1987, pp. 159-161.

${ }^{210}$ ADTn, ACap, Instrumenta capitularia 8, n. 171a.

211 Cfr. Appendice, regesto n. 30 (ADTn, ACap, Instrumenta capitularia 8bis, n. 164).

212 Cfr. Appendice, regesto n. 46 (ADTn, ACap, Instrumenta capitularia 8, n. 458).

${ }^{213}$ La presenza, pressoché completa, degli atti di vendita delle proprietà di Antonio Belenzani sul registro di Antonio da Borgonuovo permette di stimare, approssimativamente, il guadagno di Pietro da Siena a fronte del prezzo d'acquisto di 1.200 ducati. La somma dei prezzi di vendita delle proprietà tuttavia, pari a quasi 900 ducati, non sembrerebbe aver permesso a ser Pietro di ottenere alcun guadagno dall'investimento fatto: la perdita sarebbe infatti ammontata a oltre 300 ducati. 
più tardi, precisamente dal primo ottobre 1430, egli, spesso per il tramite del suo procuratore ser Pietro Iacob, iniziò a vendere, pezzo per pezzo, tutte le proprietà e gli affitti da poco acquistati, ottenendone cospicui ricavi. Senza entrare nel dettaglio delle singole compravendite, che si svolsero prevalentemente in un limitato periodo compreso fra l'ottobre del 1430 e l'ottobre del 1431, è possibile fare un confronto fra l'elenco delle proprietà di cui ser Pietro di Nanni entrò in possesso nel settembre del 1430 e l'insieme delle emptiones concretizzate nei mesi successivi; ebbene, la quasi totalità delle registrazioni furono affidate al notaio Antonio da Borgonuovo, che certamente poté trarne un ottimo profitto sia per quanto concerne la messa per iscritto nel suo registro personale sia per quanto riguarda l'estrazione dei relativi munda.

Ser Pietro di Nanni rappresentò senza dubbio un'importante fetta della clientela del professionista nei primissimi anni Trenta del Quattrocento; dal canto suo, ser Antonio aveva esemplato sul suo registro anche le copie di quei documenti che sancivano il passaggio dell'eredità dei Belenzani ad Alessandro di Masovia. A cominciare dal testamento del fu Antonio, «hic per me transcriptus ad avisamentum et deffenssionem eorum qui emerunt de bonis suis ab antedicto ser Petro de Senis, qui emit a domino nostro executore dicti testamenti» ${ }^{214}$, fino ai verbali delle escussioni testimoniali svolte pochi giorni dopo la morte del testatario, alla sentenza con la quale si assegnavano i due terzi dell'eredità al vescovo trentino e, in ultimo, alla divisione della stessa eredità.

In conclusione, sarà utile verificare chi furono gli acquirenti di ser Pietro di Nanni. Si tratta di un numero piuttosto consistente di individui, prevalentemente riconducibili alla città di Trento o ai suoi dintorni (ma non mancano anche acquirenti provenienti fuori dell'episcopato) fra cui, ad esempio, ser Marco Belenzani ${ }^{215}$, Rambaldo del fu ser Clemente de Murlinis ${ }^{216}$ e suo fratello Matteo ${ }^{217}$, Adelpreto del fu ser Federico notaio da Povo $^{218}$, il notaio Gioacchino Mezzasoma ${ }^{219}$, il notaio Giacomo a Ture da Trento ${ }^{220}$, il notaio Antonio da Fai ${ }^{221}$, ser Pietro Iacob ${ }^{222}$, Giovanni del fu magister Cristoforo da Bologna ${ }^{223}$, Antonio a Dominabus da Trento ${ }^{224}$, Odorico del fu ser Federico notaio da Povo ${ }^{225}$, Marco da Lusiana ${ }^{226}$, il notaio Graziadeo da Terlago $^{227}$, il notaio Giovanni Conto de Fatis $^{228}$, per citare soltanto i nomi più noti. Il 9 ottobre 1430 anche la vedova di Giovanni Belenzani, Felicia, acquistò un terreno broylivo e coltivato ad orto nella contrada del Ponte dell'Adige, pagando a ser

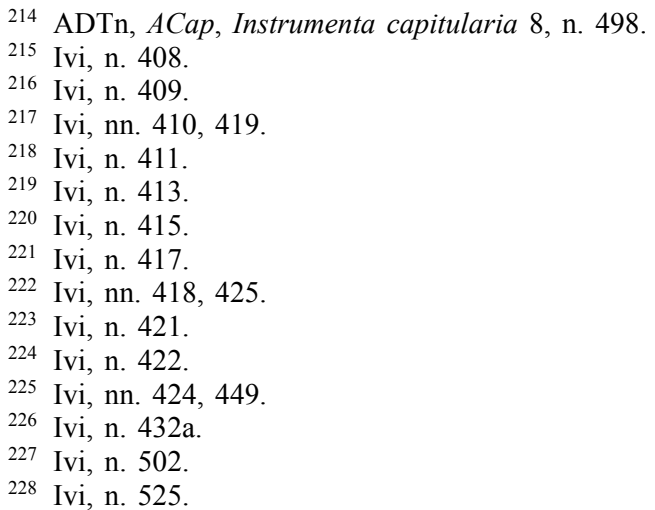


Pietro di Nanni ben 125 ducati d'oro ${ }^{229}$; costei era stata successivamente costretta a rivolgersi al notaio Giovanni da Giovo per ottenere in prestito 70 ducati d'oro così da pagare il debito contratto per acquistare il broilo ${ }^{230}$.

Una vicenda, quella appena narrata, che senza dubbio contribuisce a mettere bene in luce, ancora una volta, la fiducia che Antonio da Borgonuovo poté vantare anche nel campo della clientela privata, e il ruolo chiave da questi ricoperto, anche in episodi meno noti della storia della città e dell'Episcopato di Trento.

\section{Per un quadro di sintesi della clientela di Antonio da Borgonuovo}

Non è semplice determinare quale fu, complessivamente, l'impegno di Antonio nei confronti dei numerosissimi clienti nell'arco degli oltre cinquant'anni di attività documentata; se da un lato, infatti, si è sottolineata la straordinarietà del numero dei documenti da lui prodotti ancora oggi conservati, dall'altro si deve constatare la quasi assoluta mancanza di documentazione per una buona parte della sua carriera. Tentare una stima del rogato prodotto da Antonio risulta pertanto impraticabile, mentre pur con i limiti che l'operazione presenta - è possibile prendere a riferimento un periodo limitato, circa dieci anni, corrispondenti all'arco temporale in cui si ritiene che la documentazione conservata sia quantitativamente più rappresentativa: si tratta degli anni compresi fra il 1423, anno di inizio delle registrazioni su Instrumenta capitularia 8bis e il 1434, anno in cui si concludono le registrazioni sul volume Instrumenta capitularia 8. Per questo decennio si hanno dunque a disposizione, contemporaneamente, $\mathrm{i}$ rogiti prodotti per una clientela più variegata (grazie al volume n. 8bis e alle pergamene sciolte) e per il Capitolo; se si fosse optato per un periodo più ampio, precedente ad esempio al 1423, la preponderante presenza di documentazione capitolare trasmessa dal registro n. 8 avrebbe certamente distorto il quadro dell'impegno di Antonio per i vari clienti. Tenuto conto di questi fattori e dei limiti dell'operazione, si sono censiti per il periodo di riferimento 1.183 documenti, dunque oltre la metà dei circa 2.000 rogiti di mano del notaio oggi reperiti, di cui 615 rogati per clientela privata, 437 per conto del Capitolo della cattedrale, 21 per il Comune di Trento, 21 per l'Episcopato, 37 per la confraternita dei Battuti laici e 49 per conventi e monasteri cittadini (San Marco, Santa Chiara, San Lorenzo, San Francesco e Santa Croce) e per la Prepositura (grafico n. 1).

\section{I ritmi di lavoro}

Come ricordato, sono circa 2.000 i documenti redatti da Antonio da Borgonuovo rinvenuti fra $\mathrm{i}$ fondi archivistici contenenti documentazione tre-quattrocentesca. $\mathrm{Si}$ tratta di un numero certamente rilevante, soprattutto se messo a confronto con la parallela produzione documentaria di altri notai contemporanei dei quali, ad oggi, spesso non rimane altro che una traccia. A ben vedere, di questi circa 2.000 documenti, più di due terzi si trovano fra le carte dei registri Instrumenta capitularia 8 e 8 bis, mentre soltanto una minima parte è veicolata da pergamene sciolte. Se dunque si tolgono dal computo gli atti nei registri cartacei, ciò che rimane è un mo-

229 Ivi, n. 416.

230 Ivi, n. 465. 


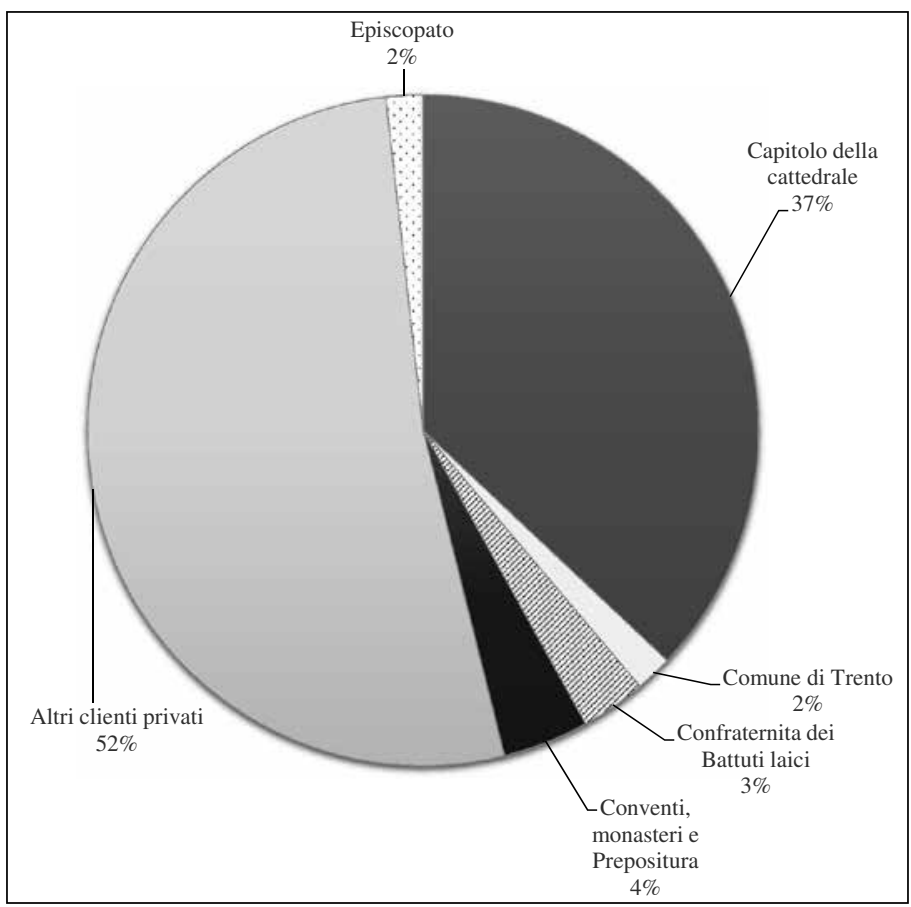

Grafico 1. La documentazione prodotta da Antonio per enti e privati fra il 1423 e il 1434 .

desto numero di instrumenta, per quantità di poco superiore rispetto alla produzione superstite di altri notai coevi. Con ciò non si vuole sminuire l'importante impegno scrittorio concretizzato nel corso della sua lunga carriera da Antonio, soprattutto per quanto concerne l'attività di notaio, quasi esclusivo, dei canonici del Capitolo per oltre trent'anni. Tuttavia, analizzando anno per anno la produzione documentaria residua, si nota come ciò che oggi rimane è probabilmente ben lontano da quella che dovette essere l'attività scrittoria complessiva del notaio in quel periodo, e ciò è vero soprattutto per i primi anni, quando il numero dei documenti conservati è tale da ritenere che la totalità degli atti in registro sia ormai deperdita. Il numero assolutamente irrilevante dei documenti prodotti tra gli anni Ottanta e Novanta del Trecento, infatti, è solo parzialmente imputabile alla presunta giovane età del professionista.

Fino agli anni 1400-1402 sono piuttosto radi i documenti conservati tanto che l'intero periodo 1386-1400 conta pochissimi instrumenta superstiti (tab. 2). Di seguito è possibile osservare come, in particolare a partire dal 1402, il numero dei documenti tenda ad aumentare gradualmente - pur attestandosi su quantità annue ancora poco rilevanti - e ciò è dovuto in buona misura all'inizio delle registrazioni su Instrumenta capitularia 8. Il numero massimo di documenti conservati si ha invece nel periodo 1424-1431, che corrisponde all'arco temporale in cui si hanno a disposizione contemporaneamente le registrazioni su Instrumenta capitularia 8 (1402-1434) e Instrumenta capitularia 8bis (1423-1437).

Ciò che attualmente si conserva, dunque, non è che una minima parte - soprattutto per alcuni periodi - dell'intera produzione documentaria di Antonio da Borgonuovo; si deve infatti considerare persa, o attualmente non reperibile, una buona parte della 
Stefano Malfatti

\begin{tabular}{|c|c|c|c|}
\hline anno & n. documenti & & \\
\hline 1386 & 1 & 1412 & 28 \\
\hline 1387 & 0 & 1413 & 26 \\
\hline 1388 & 3 & 1414 & 33 \\
\hline 1389 & 4 & 1415 & 65 \\
\hline 1390 & 2 & 1416 & 42 \\
\hline 1391 & 5 & 1417 & 30 \\
\hline 1392 & 2 & 1418 & 35 \\
\hline 1393 & 2 & 1419 & 23 \\
\hline 1394 & 13 & 1420 & 37 \\
\hline 1395 & 5 & 1421 & 58 \\
\hline 1396 & 0 & 1422 & 66 \\
\hline 1397 & 2 & 1423 & 51 \\
\hline 1398 & 5 & 1424 & 106 \\
\hline 1399 & 10 & 1425 & 146 \\
\hline 1400 & 20 & 1426 & 137 \\
\hline 1401 & 6 & 1427 & 133 \\
\hline 1402 & 19 & 1428 & 88 \\
\hline 1403 & 29 & 1429 & 110 \\
\hline 1404 & 53 & 1430 & 113 \\
\hline 1405 & 66 & 1431 & 139 \\
\hline 1406 & 58 & 1432 & 71 \\
\hline 1407 & 57 & 1433 & 83 \\
\hline 1408 & 63 & 1434 & 72 \\
\hline 1409 & 20 & 1435 & 27 \\
\hline 1410 & 10 & 1436 & 8 \\
\hline 1411 & 30 & 1437 & 2 \\
\hline
\end{tabular}

Tab. 2. Documentazione annua reperita.

documentazione prodotta per le istituzioni e, soprattutto, per i privati. Si dedicherà il capitolo successivo all'analisi del modus operandi del notaio; qui basterà per il momento accennare al fatto che certamente il professionista produsse più registri, di diverso formato, da utilizzare anche contemporaneamente a quelli oggi noti. Se ciò che rimane non è che un frammento dell'intera attività di ser Antonio, risulta alquanto complesso, se non addirittura fuorviante, tentare di spiegare diminuzioni o aumenti nella produzione documentaria durante i cinquant'anni di attività. Soltanto in parte, infatti, queste riduzioni possono essere messe in relazione con eventi paralleli all'attività notarile di Antonio, quali ad esempio l'impegno politico nel Comune di Trento o la sua assenza dalla città.

Fatte le dovute premesse, con cautela si cercherà di analizzare più nel dettaglio, anno per anno, quanto rimane dell'attività scrittoria del notaio. Si dividerà a tal scopo la lunga carriera del professionista in tre fasi; la prima coincide con gli anni compresi fra il 1386 e il 1401, gli esordi della sua attività, per i quali si hanno a disposizione pochi documenti, vergati integralmente su pergamene sciolte. La seconda fase prende avvio nel 1402 con la redazione del registro Instrumenta capitularia 8 per il Capitolo della cattedrale e prosegue almeno fino al 1433-1434. Si tratta, come già osservato, del periodo nel quale maggiore sembra essere la produzione del professionista in ragione della duplice presenza dei registri Instrumenta capitularia 8 e 8bis. Per concludere, gli ultimi anni, compresi fra il 1435 e il 1437, in 
cui la quantità della documentazione conservata si riduce considerevolmente, fino ad arrestarsi.

Per quanto concerne il primo periodo di attività (1386-1401), si hanno a disposizione soltanto 80 documenti, concentrati peraltro negli anni compresi fra il 1399 e il 1401. Un numero così ridotto di instrumenta in uno spazio temporale di quindici anni è spiegabile soltanto in parte; se infatti è probabile che nei primissimi anni di lavoro Antonio rogasse con meno frequenza, forse a motivo della sua giovane età, per gli anni seguenti la carenza di documentazione deve essere ricondotta alla perdita quasi completa di carte e registri. Fra i committenti che si rivolsero al professionista durante questo primo periodo si hanno, per quantità di documentazione richiesta, il vicario vescovile, le Clarisse di San Michele, il Capitolo della cattedrale, il Comune di Trento e, non ultimi per importanza, un certo numero di privati per i quali Antonio redasse prevalentemente atti negoziali e testamenti. Già all'inizio della sua carriera, dunque, egli sembra vantare una clientela notevole per prestigio e piuttosto diversificata. Il secondo periodo, compreso fra il 1402 e il 1433/1434, corrisponde agli anni per i quali si ha a disposizione la maggior parte dei documenti reperiti. Dal 1402 al 1414 la documentazione conservata è redatta quasi per intero per il Capitolo della cattedrale e ciò è dovuto alle registrazioni su Instrumenta capitularia 8 e al buon numero di pergamene sciolte conservate presso i fondi capitolari dell'Archivio diocesano tridentino e dell'Archivio di Stato di Trento. Durante questi dodici anni di attività, tuttavia, non mancano anche documenti redatti su richiesta di altri committenti, fra cui un buon numero di privati, la confraternita dei Battuti, il monastero di San Lorenzo, le Clarisse di San Michele e il vescovo, ad ulteriore dimostrazione che la documentazione conservata per questo periodo manca certamente di buona parte di ciò che Antonio redasse per piccoli enti e privati e che, con ogni probabilità, trovò concretizzazione scritta in registri oggi deperditi. Se, infatti, è vero che il Capitolo della cattedrale rappresentò per il notaio una delle maggiori committenze, se non addirittura la maggiore, la grande quantità di documentazione relativa a questo ente non deve indurre a ritenerlo cliente esclusivo di Antonio. Lo dimostrano gli atti conservati negli anni seguenti, per i quali si dispone del solo registro Instrumenta capitularia 8bis; in esso i rogiti redatti su richiesta del Capitolo sono in numero assai più contenuto rispetto a quelli vergati per una clientela più eterogenea. L'assenza di buona parte della documentazione prodotta per i piccoli enti e, soprattutto, per i privati da parte di molti notai trentini medievali e, in parallelo, una sovrabbondanza di atti rogati dalle maggiori istituzioni ecclesiastiche della diocesi (vescovo e Capitolo in prima posizione), rappresenta una sorta di 'specchio deformante' nello studio della società trentina medievale che spesso si può conoscere soltanto attraverso il filtro dei documenti conservati dall'uno o dall'altro ente.

Dal punto di vista dell'analisi quantitativa, una lievissima flessione sembra coincidere con il periodo 1409-1414, il che potrebbe forse essere spiegato, almeno in parte, con una sua temporanea assenza dalla città; risale infatti a quel periodo l'assegnazione della cittadinanza veronese al notaio, per cui è probabile che egli non fosse stabilmente presente nel capoluogo vescovile. Un secondo tentativo per spiegare il leggero calo nella produzione scrittoria del notaio può forse essere azzardato per gli anni 1409 e 1410. Osservando la tabella (tab. 2) si nota infatti come per quel biennio si siano conservati rispettivamente soltanto 20 e 10 rogiti, peraltro tutti rogati su commissione del Capitolo, mentre nei periodi precedente e successivo il numero 
dei documenti sembra essere più elevato. Pur riconoscendo che si tratta comunque di un'ipotesi, non si può fare a meno di collegare questa flessione anche agli eventi occorsi a Trento proprio in quel torno d'anni; da un lato le guerre successive alle rivolte contro il vescovo Georg Liechtenstein, dall'altra il sacco, perpetrato dalle truppe tirolesi proprio nel 1409, che depauperò, fra l'altro, anche gli archivi vescovile e capitolare. Tale decrescita nella documentazione di Antonio potrebbe dunque dipendere in parte da una minore richiesta di documentazione da parte di una città in preda agli eventi rivoltosi, in parte dalla razzia che gli archivi cittadini subirono proprio in quegli anni. A tal scopo, è utile notare come, proprio nel 1410, il notaio dopo aver elencato i pochi canonici presenti a un negozio giuridico ricordava: «nullis pluribus ad presens in dicta ecclesia residentibus propter gueram de presenti existentem ad civitatem Tridenti» ${ }^{231}$. La guerra citata in questa circostanza da Antonio non manca di essere menzionata in molteplici occasioni nella documentazione degli anni successivi. Alcuni privati si rivolgono infatti al notaio per registrare rinnovi di locazione, «quia in gueris proxime elapsis amiserunt omnia sua instrumenta» ${ }^{232}$; nel 1412 si ricorda come, dopo la morte del prete Antonio del fu Tura da Trento «in guera interfectus et intestatus defunctus a dicta guera sachemani in Tridento facti» ${ }^{233}$, un terreno vignato che era stato dato al suddetto Antonio in locazione dovette essere riconcesso in affitto dai canonici poiché nel frattempo era divenuto incolto. Ancora nel 1412 si menzionano numerose abitazioni e terreni distrutti «propter combustionem ... et devastationem ..., propter gueram superventam ${ }^{234}$.

Si è ripartito il lungo periodo 1402-1433/1434 in due sezioni, ponendo quale cesura periodizzante il 1415; a partire da quell'anno, infatti, si presenta fra i clienti con maggiore frequenza il Comune di Trento, istituzione che da allora aveva iniziato a registrare $\mathrm{i}$ verbali di elezione dei propri officiali. Si deve comunque attendere il 1423-1424 prima di veder annoverati quali committenti del notaio un buon numero di clienti che, in un certo senso, 'spezzano' l'apparente esclusività del Capitolo della cattedrale. Con l'inizio delle registrazioni su Instrumenta capitularia 8bis, fa la sua comparsa una serie variegata di clienti, spesso membri della classe dirigente trentina, e agli enti poc'anzi citati (Capitolo, Comune, Episcopato, confraternita dei Battuti, monastero di San Lorenzo, Clarisse di San Michele ecc.) si aggiungono i fatres dell'Ordine teutonico, il convento di San Francesco, l'ospedale di San Pietro, il convento di Santa Croce, l'ospedale di San Martino, l'ospedale di Santa Maria Maddalena, la Prepositura, l'ospedale di San Tommaso presso Romeno, la pieve di Santo Stefano a Mori. Insomma, una clientela quanto mai diversificata caratteristica di un notaio di un certo prestigio operante nell'ambito della città di Trento.

Il periodo compreso fra il 1424 e il 1431 è, come ricordato, quello più ricco di documentazione tanto che, per ciascuno degli otto anni, il numero dei documenti conservati supera le cento unità. Come anticipato, risulta alquanto problematico tentare di spiegare i ritmi di lavoro del notaio, soprattutto a ragione della parzialità del numero delle registrazioni che oggi si hanno a disposizione. Tuttavia, qualche lieve fluttuazione nella produzione scritta sembra coincidere proprio con gli anni in cui Antonio esercitò la carica consolare; ne è un caso esemplare il 1428, allorquando

231 ADTn, ACap, Instrumenta capitularia 8, c. $218 r$, n. 434 e ADTn, ACap, capsa anniversari, rotoli lunghi/c, n. 15 .

${ }^{232}$ ADTn, ACap, Instrumenta capitularia 8, c. $235 v$, n. 468.

233 Ivi, c. $284 r$, n. 578.

${ }^{234}$ Ivi, cc. $284 v-285 r$, n. 579. 
L'attività professionale di Antonio da Borgonuovo

si constata una diminuzione di ben 50 unità rispetto all'anno precedente (tab. 3). Il tracollo dell'attività scrittoria degli ultimi anni, in particolare dopo il 1435, può essere invece spiegato adducendo due motivazioni: il periodo coincide infatti con lo scoppio delle rivolte contro il vescovo Alessandro di Masovia, oltre al fatto che gli anni coincidono con gli ultimi anni di vita di Antonio; un calo del ritmo di lavoro che dipese, con ogni probabilità, sia da un rallentamento fisiologico della sua attività sia da motivazioni 'esterne' quali potevano essere le ribellioni cittadine.

I pochi dati fin qui ricavati dall'analisi quantitativa sui rogiti di ser Antonio confermano indubbiamente il prestigio del professionista in seno alla comunità cittadina, soprattutto in quella frangia dell'élite che ruotava intorno alle magistrature comunali. Da questo punto di vista, infatti, l'attività socio-politica di Antonio si interseca con quella professionale, dal momento che le relazioni con una buona parte dei maggiorenti della città poterono consolidare il suo ruolo di pubblico notaio, già favorito dal 'nome' e dalla fama di notaio 'esclusivo' del Capitolo.

\begin{tabular}{|c|c|c|c|}
\hline anno & n. documenti & attività politi & che di Antonio da Borgonuovo \\
\hline 1407 & 57 & 2-3 febbraio & scoppiano le rivolte contro vescovo Liechtenstein \\
\hline 1408 & 63 & 25 gennaio & ottiene la cittadinanza veronese \\
\hline 1409 & 20 & & \\
\hline 1410 & 10 & & \\
\hline 1411 & 30 & & \\
\hline 1412 & 28 & & \\
\hline 1413 & 26 & & \\
\hline 1414 & 33 & & \\
\hline 1415 & 65 & 5 ottobre & nomina a console \\
\hline 1416 & 42 & 4 ottobre & scadenza carica console \\
\hline 1417 & 30 & & \\
\hline 1418 & 35 & $\begin{array}{l}6 \text { febbraio } \\
5 \text { giugno } \\
9 \text { ottobre }\end{array}$ & $\begin{array}{l}\text { nomina a sindico } \\
\text { domenica, scadenza carica sindico - nomina a gastaldo } \\
\text { scadenza carica gastaldo - nomina a console }\end{array}$ \\
\hline 1419 & 23 & 8 ottobre & scadenza carica console \\
\hline 1420 & 37 & & \\
\hline 1421 & 58 & $\begin{array}{l}5 \text { giugno } \\
5 \text { ottobre }\end{array}$ & $\begin{array}{l}\text { nomina a giudice degli appelli } \\
\text { scadenza carica giudice degli appelli }\end{array}$ \\
\hline 1422 & 66 & 7 ottobre & nomina a console \\
\hline 1423 & 51 & 3 ottobre & scadenza carica console - nomina a giudice delle tutele \\
\hline 1424 & 106 & $\begin{array}{l}6 \text { febbraio } \\
4 \text { giugno } \\
8 \text { ottobre }\end{array}$ & $\begin{array}{l}\text { nomina a sindico } \\
\text { scadenza carica sindico - nomina a gastaldo } \\
\text { scadenza carica gastaldo }\end{array}$ \\
\hline 1425 & 146 & & \\
\hline 1426 & 137 & $\begin{array}{l}5 \text { febbraio } \\
2 \text { giugno } \\
13 \text { ottobre }\end{array}$ & $\begin{array}{l}\text { nomina a giudice delle tutele } \\
\text { scadenza carica giudice delle tutele } \\
\text { nomina a giudice degli appelli }\end{array}$ \\
\hline 1427 & 133 & $\begin{array}{l}9 \text { febbraio } \\
19 \text { ottobre }\end{array}$ & $\begin{array}{l}\text { scadenza carica giudice degli appelli } \\
\text { nomina a console }\end{array}$ \\
\hline
\end{tabular}




\begin{tabular}{|c|c|c|c|}
\hline anno & n. documenti & attività polit & che di Antonio da Borgonuovo \\
\hline 1428 & 88 & $\begin{array}{l}8 \text { febbraio } \\
10 \text { ottobre }\end{array}$ & $\begin{array}{l}\text { nomina a procurator ad causas } \\
\text { scadenza carica console - nomina a sindico e pro- } \\
\text { curator ad causas }\end{array}$ \\
\hline 1429 & 110 & $\begin{array}{l}10 \text { febbraio } \\
5 \text { giugno } \\
9 \text { ottobre }\end{array}$ & $\begin{array}{l}\text { scadenza carica sindico e procurator ad causas - } \\
\text { nomina a sindico } \\
\text { scadenza carica sindico - nomina a gastaldo } \\
\text { scadenza carica gastaldo - nomina a giudice delle } \\
\text { tutele }\end{array}$ \\
\hline 1430 & 113 & & \\
\hline 1431 & 139 & & \\
\hline 1432 & 71 & 9 giugno & nomina a giudice degli appelli \\
\hline 1433 & 83 & $\begin{array}{l}5 \text { febbraio } \\
7 \text { giugno } \\
25 \text { ottobre }\end{array}$ & $\begin{array}{l}\text { scadenza carica giudice degli appelli - nomina a } \\
\text { giudice delle tutele } \\
\text { scadenza carica giudice delle tutele - nomina a giu- } \\
\text { dice degli appelli } \\
\text { scadenza carica giudice degli appelli }\end{array}$ \\
\hline 1434 & 72 & $\begin{array}{l}17 \text { febbraio } \\
13 \text { giugno }\end{array}$ & $\begin{array}{l}\text { nomina a giudice delle tutele } \\
\text { scadenza carica giudice delle tutele }\end{array}$ \\
\hline 1435 & 27 & 15 febbraio & $\begin{array}{l}\text { iniziano le rivolte contro il vescovo Alessandro di } \\
\text { Masovia }\end{array}$ \\
\hline 1436 & 8 & & \\
\hline
\end{tabular}

Tab. 3. Attività politiche di Antonio.

Se le analisi sui ritmi di lavoro del professionista risultano poco fruttuosi, qualche dato in più - con le dovute cautele - si può ricavare dall'esame sull'attività giornaliera. A tal proposito si può osservare come fra gli oltre 2.000 atti reperiti, non manchino quelli rogati di domenica, mentre non si trovano documenti redatti nei giorni di Natale e di Pasqua, festività durante le quali Antonio sembra sospendere la propria attività. Per quanto concerne la festività pasquale, il notaio non pare accogliere rogiti nemmeno nei giorni immediatamente precedenti e successivi alla solennità. Se infatti si analizza nel dettaglio il periodo in cui si hanno a disposizione più documenti, ovvero quello compreso fra il 1424 e il 1431, si nota come nel primo anno del periodo la Pasqua cadde il 23 aprile e non si trovino atti redatti fra l' $11^{235}$ e il $28^{236}$ del mese. Nel 1425 la Pasqua cadde 1'8 aprile e non si riscontrano rogiti fra il $3^{237}$ e il $14^{238}$; nel 1427 non si trovano documenti fra il $19^{239}$ e il $25^{240}$ aprile, con la Pasqua festeggiata il giorno 20; nel 1428 l'attività sembra essere sospesa fra il 28 marzo $^{241}$ e il 9 aprile ${ }^{242}$, mentre la Pasqua cadde il 4. Questa apparente sospensione nella redazione dei rogiti da parte del notaio in corrispondenza del dies paschalis è regola costantemente seguita negli anni in

\footnotetext{
235 ADTn, ACap, Instrumenta capitularia 8bis, n. 37.

236 Ivi, n. 40.

237 BCTn, BCT3, capsa 28, mazzo 1, p. 56.

238 ADTn, ACap, Instrumenta capitularia 8bis, n. 82.

239 ASTn, $A C D$, n. 570.

240 ADTn, ACap, Instrumenta capitularia 8bis, n. 240

241 Ivi n. 196 e ADTn, ACap, Instrumenta capitularia 8, n. 199.

242 ADTn, ACap, Instrumenta capitularia 8bis, n. 284.
} 
questione. L'unica variabile è rappresentata dal 1426, anno in cui la Pasqua cadde il $31 \mathrm{marzo}$; in quell'occasione Antonio redasse una compravendita il giorno precedente, ossia nel Sabato Santo ${ }^{243}$, e una confessio, ovvero una dichiarazione fra privati, il primo aprile ${ }^{244}$, con una sospensione dell'attività ridotta al solo giorno festivo. Concentrando ora l'attenzione alla festività del Natale, il notaio sembra interrompere in genere il proprio lavoro il 23 dicembre, mentre in un solo caso, circoscrivibile all'anno 1415, registra un'immissione in possesso per il Capitolo nel giorno della vigilia ${ }^{245}$. Nessun documento rogato, invece, il 26 dicembre, mentre l'attività sembra riprendere spesso il 27 del mese.

Non avendo a disposizione norme precise sulle giornate in cui era prevista l'astensione dal lavoro da parte dei notai trentini, è difficile individuare altre date durante l'anno in cui probabilmente vigeva l'obbligo o la consuetudine di sospendere l'attività di rogazione. Altrove, ad esempio, gli statuti dell'Arte dei notai e dei giudici stabilivano un'interruzione nel giorno del Corpus Domini ${ }^{246}$. Verificando il rispetto di questa norma nella documentazione redatta da Antonio si nota come, anche a Trento, poteva valere analoga disposizione, considerato che non si trova alcun rogito redatto nelle date in cui cadde la festività. Nessuna pausa nell'attività del professionista, invece, nel giorno del patrono della città e della diocesi, san Vigilio, il 26 giugno, data che Antonio sembra considerare normale giorno lavorativo ${ }^{247}$.

\section{Il 'tariffario'}

Un tema d'interesse collegato alla clientela è quello relativo alle tariffe richieste dal professionista per il rilascio degli instrumenta. Anche per quanto concerne il costo dei documenti rogati, lo statuto dei notai e dei giudici trentini emanato alla fine degli anni Venti del Quattrocento sembra tacere; per trovare qualche informazione al riguardo si deve ricorrere agli statuta nova del 1340-1343, che aggiornavano gli antiqua di inizio Trecento, poi ripresi nella normativa roveretana del 1425 . In particolare, il capitolo 62 degli statuta nova, intitolato «De preciis instrumentorum debendis tabellionibus ${ }^{248}$, stabiliva specifiche tariffe per ciascuna tipologia docu-

243 Ivi, n. 173.

244 Ivi, n. 174.

245 ADTn, ACap, Instrumenta capitularia 8, n. 340d.

246 Cfr., ad esempio, per la città di Firenze, S. Calleri, L'Arte dei giudici e notai di Firenze nell'età comunale e nel suo statuto del 1344, Giuffrè, Milano 1966, pp. 93.

${ }^{247}$ Cfr., fra i molti casi, ADTn, ACap, Instrumenta capitularia 8bis, nn. 188, 213a, 293, 352, 621a-b; ASTn, APV, Sezione latina, capsa 66, n. 2. Nel giorno del patrono san Vigilio, così come nella festività dell'Assunzione di Maria, gli statuti del collegio dei notai del 1427 prevedevano la visita alla chiesa cattedrale: «Item in primis statuimus et ordinamus quod unusquisque advocatus et procurator et tabelio sotietatis et colegii notariorum Tridenti, circha reverentiam sanctissimi patroni nostri beati Vigilii in die festivitatis sue, et in die annuntiationis et assu(n)tionis Virginis gloriose et aliis festivitatibus sole(m)nibus que indicte fuerint per bidellum collegii de mandato prioris sive rectoris ipsius collegii et eius consciliariorum, se presentare et convenire debeant ad requisitionem dicti bidelli in loco deputato et una cum dicto rectore visitare predictam ecclesiam prelibatam patroni nostri predicti secundum quod per dictum dominum rectorem ordinatum et iniunctum fuerit, sub pena decem sol(idorum) denariorum pro quolibet et qualibet vice qua fuerit contrafactum».

248 Statuti di Rovereto del 1425, cit., pp. 178-179. 


\section{Stefano Malfatti}

mentaria prodotta dal notaio. Si definivano anzitutto gli emolumenti da versare al tabellione per la redazione degli atti di natura giudiziaria:

Item de contestacione litis XII denarios tantum, et hoc intelligatur de imbreviatura. Item de terminis post litem contestatam, VI denarios tantum de imbreviatura.

Item de sentencia interlocutoria, pro scriptura XXII denarios et non ultra, nisi de licencia et voluntate officialis predicti, et qui contrafecerit solvat $\mathrm{X}$ soldos Veronensium pro qualibet vice.

Item de sentencia difinitiva a $\mathrm{X}$ libris infra $\mathrm{V}$ soldi Veronensium; et a $\mathrm{X}$ libris supra usque ad L, et a L autem supra usque ad C libras accipiantur soldi XX Veronensium; a $C$ vero supra $X L$ soldi Veronensium parvorum et non plus, nisi de licentia et voluntate officialis predicti. Et si contrafecerit solvat episcopali camere XX soldos Veronensium pro qualibet vice; et quilibet sit accusator et habeat terciam partem ${ }^{249}$.

Successivamente gli statuti si premurano di indicare il tariffario dovuto ai notai per la redazione degli altri instrumenta; in particolare, 10 soldi veronesi per il contratto di compravendita (de contractu empcionis) se la vendita non eccedeva le 50 lire; in caso contrario il notaio avrebbe avuto 20 soldi veronesi; qualora il contratto avesse superato invece le 100 lire si sarebbero dovuti versare 30 soldi veronesi. Lo stesso vale per i testamenti, per i codicilli e le ultime volontà, così come per i contratti dotali, le locazioni, i compromessi, le societates, le permute e gli arbitrii. Per quanto concerne, invece, i mutui andavano pagati 22 denari per contratti fino a 10 lire, 2 grossi per i contratti compresi fra le 10 e le 50 lire, e 4 grossi per quelli superiori alle 50 lire.

In mancanza di normative diverse da quelle trecentesche e vista l'assenza di disposizioni in materia negli statuti masoviani, si deve ritenere plausibile che anche Antonio da Borgonuovo si fosse attenuto ai capitoli succitati. Per quanto è oggi noto, infatti, soltanto con gli statuti clesiani del 1528 (capitolo 148) venivano fissati i nuovi compensi per gli instrumenta prodotti dai notai trentini ${ }^{250}$.

Sembra utile, prima di verificare quanto veniva richiesto da ser Antonio per alcune tipologie documentarie, soffermarsi brevemente su un aspetto sottolineato nella prima parte del capitolo trecentesco relativo agli emolumenti per gli atti giudiziari; la norma, infatti, precisa che il documento relativo alla «contestacione litis» debba essere pagato 12 denari, ma subito dopo specifica «et hoc intelligatur de imbreviatura», come dire, si intende per la sola imbreviatura. Lo stesso vale per la redazione «de terminis post litem contestatam». Questa puntualizzazione introduce la possibilità, ovvia si dirà, di prezzi diversificati per la redazione della semplice imbreviatura, priva di elementi di formalità e conservata sui registri del notaio, $\mathrm{o}$ della più costosa pergamena in mundum. La questione non è secondaria, come si vedrà, anche nel tentativo di individuare il tariffario di Antonio da Borgonuovo. Allo stesso tempo, tuttavia, essa palesa l'impossibilità di fare un confronto fra la normativa e quanto si ricava dalla documentazione di ser Antonio, e ciò per due motivi sostanziali: anzitutto non è dato di sapere se i prezzi stabiliti nei capitoli roveretani del 1425 furono tratti, talquali, dagli statuti trentini di XIV secolo. In

${ }^{249}$ Si noti il riferimento alla camera episcopale (trentina) negli statuti del borgo, veneziano all'epoca, di Rovereto; ulteriore riprova della copiatura de litera ad literam dal testo statutario trentino da parte del cancelliere Giacomo da Persichello (sulle questioni relative alla genesi degli statuti roveretani cfr. Statuti di Rovereto del 1425, cit.)

${ }^{250}$ Casetti, Il notariato trentino, cit., p. 252. 
secondo luogo, mentre per gli atti giudiziari si chiarisce che i prezzi sono relativi alle sole imbreviature, per tutti gli altri instrumenta non si fornisce questa informazione. Non rimarrà, dunque, che indicare semplicemente i dati raccolti dalla documentazione del notaio, senza svolgere alcun raffronto.

Per quanto riguarda ser Antonio, ciò che resta per tentare di ricavare qualche informazione sul tema sono le brevi e rade annotazioni che egli - come i colleghi pose sul verso delle pergamene consegnate ai committenti, spesso privati. Se ne proporranno in questa sede alcuni esempi, iniziando con una nota posta in attergato ad una sentenza emanata il 6 febbraio 1391, in episcopali palatio, da Antonio da Trento, giudice in civilibus et criminalibus causis del duca d'Austria Alberto, e relativa alla causa vertente fra Andriota del fu Nicola da Trento e Romano, figlio di Giacomo da Padova. La pergamena, che oggi è conservata presso il fondo Thun dell'Archivio provinciale di Trento ${ }^{251}$, presenta un'annotazione di mano del notaio che recita: «Pro instrumento ducat(um) $1 \frac{1}{2}$ »); il prezzo richiesto per il rilascio della pergamena in mundum fu dunque di un ducato e mezzo.

Un'indicazione ancora più precisa in merito al costo per la prestazione effettuata da Antonio, nel caso in questione per la redazione in publicam formam di un testamento, si ricava dal testo di un documento sul registro Instrumenta capitularia $8^{252}$. Il notaio ricorda di aver redatto, il giorno 15 maggio 1400, le ultime volontà del canonico Morandino da Trento. Nel testo del documento egli menziona esplicitamente un debito contratto dal canonico, ormai defunto, nei suoi confronti: «Item octo ducatos quos debentur michi Antonio notario infrascripto pro mercede et factura testamenti predicti et quos ipse Guillelmus michi dare, solvere et satisfacere promisit». La testimonianza è di grande rilevanza poiché da essa si deduce che il prezzo richiesto da Antonio per confezionare e redigere il testamento del canonico Morandino fu di 8 ducati che Guglielmo del fu ser Delaito Gallo aveva promesso di pagare al professionista, avendo egli ricevuto in cessione dai canonici del Capitolo una serie di affitti del valore di 173 ducati d'oro, che un tempo venivano riscossi dal canonico defunto, in cambio del saldo completo di una serie di debiti insoluti fra cui, appunto, la redazione delle ultime volontà di Morandino.

Non si hanno a disposizione ulteriori testimonianze sul corrispettivo richiesto da Antonio ad altri clienti per la redazione di analoga tipologia documentaria; un confronto è tuttavia possibile con il notaio Antonio del fu Bongiovanni da Fai il quale, nel 1414, redasse il testamento di Francesca, figlia del fu ser Giacomo $\mathrm{Be}$ schapani da Trento ${ }^{253}$. Dopo aver redatto le ultime volontà della donna, il professionista pose sul verso della pergamena la seguente annotazione: «Recepi a Iohanne Alde pro presenti legati grossos XXIIII ${ }^{\text {or }}$ et in amore Dei». Si tratta, dunque, di una cifra considerevolmente più bassa rispetto agli 8 ducati richiesti da Antonio per la redazione del testamento del canonico Morandino.

Qualche dato ancora in merito al prezzo richiesto dal notaio per confezionare gli instrumenta si deduce da una nota posta in margine ad un documento sul registro Instrumenta capitularia $8^{254}$. Si tratta della locazione da parte del Capitolo in favore di Pasqua del fu Mercadento da Levico, moglie del fu Alberto Bonomi.

251 APTn, Thun, n. 1382.

252 ADTn, ACap, Instrumenta capitularia 8, n. $277 \mathrm{~b}$.

253 BCTn, BCT3, capsa 28, mazzo 1, n. 23.

254 ADTn, ACap, Instrumenta capitularia 8, n. 619. 
Nel margine sinistro dell'atto Antonio da Borgonuovo pose l'usuale nota relativa all'estrazione del mundum, «Facta extra duplex videlicet locatori et conductori», specificando però ulteriormente «Grossos 36 ex conventione pro locat(ori)». La breve annotazione è utile per due motivi; anzitutto informa del costo richiesto al locatore, ovvero al Capitolo della cattedrale, per il rilascio del mundum: 36 grossi. Inoltre si viene a conoscenza del fatto che questo prezzo fu richiesto ex conventione; con ogni probabilità, visto il remunerativo e costante impegno del notaio nei confronti dell'ente ecclesiastico e il grande numero di documenti da quest'ultimo richiesto, è probabile che fosse intercorso una sorta di accordo con il professionista, in ragione del quale Antonio applicò una tariffa meno onerosa.

Ancora relativamente al Capitolo: una nota posta dal notaio sul dorso di una locazione del $1419^{255}$ registra «pro mercede mea exhibita in processu facto contra dictum Iohannem, ducatum 1». Il notaio Giovanni, insieme al padre Pietro Longino, tenevano in enfiteusi dal Capitolo una casa situata nella contrada della Roggia Grande. Poiché, tuttavia, Giovanni non aveva pagato l'affitto per anni e, dopo la sua morte, la casa era stata totalmente abbandonata, tanto da risultare «discoperta et enormiter putrefacta», il Capitolo ne investì Odorico detto Pizolo, il quale si era presentato dinanzi ai canonici affermando di essere creditore del fu Giovanni e di sua madre Maddalena per ben 22 ducati d'oro, come sancito e dimostrato da una sentenza pronunciata da Giovanni da Isny il 20 dicembre 1415. L'annotazione posta da Antonio sul verso della locazione sembrerebbe dunque riguardare il costo richiesto dal notaio per il confezionamento della pergamena che fu presentata $a d$ banchum iuris nella causa contro Giovanni.

Poche, dunque, sono le informazioni che permettono di ricostruire un ipotetico 'tariffario' del notaio Antonio da Borgonuovo. Non si conosce, ad esempio, quando avvenisse il pagamento, se all'atto della rogazione o alla consegna dell'instrumentum. Le note poste sul verso delle pergamene farebbero propendere per questa seconda ipotesi; poteva comunque accadere che il versamento non avvenisse immediatamente e così, come nel caso del canonico Morandino, il notaio risultasse creditore degli eredi del defunto. Troppo scarni, inoltre, i dati relativi agli emolumenti percepiti a seconda della tipologia documentaria; peraltro è probabile che le tariffe variassero non soltanto in relazione alla tipologia del negozio trattato, ma anche al diverso grado di complessità nella redazione dello stesso. Il costo piuttosto elevato per la redazione del testamento del canonico Morandino porta inoltre a ritenere che la tariffa spettante ad Antonio (ma ciò probabilmente riguardava anche gli altri notai trentini) mutasse non soltanto da negozio a negozio, ma subisse variazioni anche all'interno della stessa tipologia documentaria in proporzione al valore dei beni oggetto di stipula. Del resto, un aumento progressivo del prezzo dell'instrumentum in ragione dell'incremento stesso del valore del negozio trattato era stabilito anche negli statuti. L'ipotesi sembra essere suffragata da un'annotazione che si trova sul verso di un testamento redatto dal notaio Nicolò del fu magister Ognibene dal Borgonuovo di Trento nel $1414^{256}$. Un non meglio identificato Guglielmo annotava infatti:

Ego Guilielmus consultor a superior(ibus) ad videndum, taxandum et limitandum labores factas per notarios in stipulatione et confectione dicti testamenti in [...]

255 ADTn, ACap, capsa 32, n. 102.2.

256 BCTn, BCT3, capsa 28, mazzo 1, n. 25 (1414 settembre 3). 


\section{L'attività professionale di Antonio da Borgonuovo}

veniant, viso dicto testamento, consciderato tempore pestis tunc (per)venientis, hereditate transeunte etiam ex(e)unte heredem, ac qualitate et quantitate hereditatis dic(te) tax(..) et limite pre(...) not(arii) pro labore et mercede suis ducatos quatuor auri $(\ldots)^{257}$.

Guglielmo informa dunque che, in qualità di consultor, fu chiamato a stimare il prezzo per la confezione del suddetto testamento; a tal fine egli considerò una serie di fattori, quali la peste appena trascorsa, la qualità e la quantità dell'eredità nonché il lavoro del notaio stesso, così da poter stimare in 4 ducati d'oro l'emolumento da richiedere per la redazione dell'instrumentum.

Un ulteriore, e per certi aspetti ancor più utile, esempio tratto dai registri del notaio è rappresentato dalla locazione, rogata il 28 novembre 1429, a Michael detto Rauschella da Appiano di metà di Giovanni pro indiviso di due mansi (el Mas dala Poza e el Mas dal Mont) da parte di Giovanni Zeiss da Bopfingen, canonico, priore e rettore della chiesa e dell'ospedale di San Martino di Trento. Ciò che più rileva ai fini di queste riflessioni non è tanto il contenuto del documento, quanto la nota che il notaio stese in calce all'esteso, la quale recita:

Notetur ad memoriam quod ipse Michael conductor in presentia dictorum testium promisit michi dare grossos XXXVI pro dicto tutore pretextu suorum instrumentorum non exactorum per dictum Iorium Chelum etc ad festum sancti Michaelis. Item et pro se pro dicta investitura ducatum unum ad dictum terminum ex intercessione dicti domini Iohannis Zeyss prioris etc quia me opportuit facere de necessitate virtutem etc ${ }^{258}$.

Il locatario Michele da Appiano aveva promesso ad Antonio di versare 36 grossi per un primo instrumentum che era la refuta del dominio utile dei due mansi fatta da Concio Sorn, quale tutore di Ulrico e Margherita, figli minorenni ed eredi dei defunti Giorgio Chelum e Barbara da Pressano, riconsegna che aveva redatto proprio Antonio da Borgonuovo. Oltre a ciò, Michele si era impegnato a versare un ducato d'oro al notaio per detta investitura alla scadenza del giorno di san Michele. Antonio specifica inoltre come ciò avvenne ex intercessione del canonico Giovanni Zeiss e, concludendo con una nota citazione da san Girolamo, aggiunge «me opportuit facere de necessitade virtutem», quasi a celare una leggera nota di malcontento, o per un prezzo che, per intervento del canonico, il professionista aveva dovuto calare o per il pagamento che era stato dilazionato al settembre successivo.

L'ultima parte di questo paragrafo sarà dedicata a tutta una serie di annotazioni che Antonio pose in margine ad alcuni documenti sui registri Instrumenta capitularia 8 e 8bis; si tratta delle usuali note relative all'estrazione del mundum. In alcune occasioni, tuttavia, il professionista specificò che trasse quell'instrumentum gratuitamente. L'interesse, in questo caso, sta non tanto nel fatto che il notaio non percepì alcun emolumento per la prestazione, ma piuttosto nei clienti cui rilasciò gratis la redactio in publicam formam. Scorrendo le carte dei due registri si sono reperiti almeno ventiquattro esempi, suddivisi fra Instrumenta capitularia 8 e 8 bis, cui si aggiunge un'annotazione dorsale ad una pergamena capitolare. Di questi venticinque documenti ben sedici furono rilasciati a notai colleghi di Antonio:

257 Risultano sbiadite molte parti del testo.

258 ADTn, ACap, Instrumenta capitularia 8bis, n. 368. 
Nicolò de Capris $^{259}$, Antonio da Nogaredo ${ }^{260}$, Leonardo da Sprè ${ }^{261}$, Nicolò a Sale e Leonardo suo genero ${ }^{262}$, Graziadeo da Terlago ${ }^{263}$, Giovanni Conto de Fatis da Terlago $^{264}$, Guglielmo Saraceno ${ }^{265}$ e Giovanni di Luca cirogicus $^{266}$. I restanti nove instrumenta concessi gratuitamente furono invece consegnati al nobile Baldessare Thun $^{267}$, a Michael Senftel ${ }^{268}$, a Giovanni di ser Domenico Tabarini da Brentoni$\mathrm{co}^{269}$, al nobile ser Michele da Coredo ${ }^{270} \mathrm{e}$, rispettivamente, ai canonici Prospero de Thomasiis da Cremona ${ }^{271}$, Giovanni dalla Carinzia ${ }^{272}$, Nicolò da Breslavia ${ }^{273}$ e Artuico da Passau ${ }^{274}$. Un'ultima nota si trova sul verso di una pergamena capitolare rogata nel $1402^{275}$. Si tratta di una locazione perpetua concessa dal Capitolo a Fignoclo piliparius; il notaio ritenne opportuno porre sul mundum consegnato ai canonici la seguente annotazione: «Nota quod simillem dedi Fignoclo cugnato meo gratis».

Si nota anzitutto una preferenza da parte di Antonio a non percepire alcun onorario da specifiche categorie di clienti: i colleghi notai anzitutto, alcuni canonici e personaggi a lui legati da qualche vincolo, quali ad esempio il daziere Michael Senftel o il cognato Fignoclo. È Antonio stesso che, rilasciando l'instrumentum ai notai Nicolò e Leonardo, scrisse: «Facta extra distincte ipsis emptoribus gratis propter professionem $\rangle^{276}$, riferendosi probabilmente proprio al fatto che tale gratuità derivava dalla professione che entrambi esercitavano. Non si hanno a disposizione altre informazioni che possano, in qualche modo, lasciar trapelare rapporti di collaborazione fra Antonio da Borgonuovo e alcuni di questi notai, con l'eccezione, ma si tratta di pura ipotesi, di Graziadeo da Terlago ${ }^{277}$. Non mancano, infatti, in alcune occasioni documenti recanti la doppia sottoscrizione di Antonio e di questo secondo professionista ${ }^{278}$, che, peraltro, rogò uno sparuto numero di pergamene per il Capitolo della cattedrale negli anni in cui il notaio del Borgonuovo sembrò esercitare una sorta di 'monopolio' per questo ente (1400-1434). Non si può inoltre fare a meno di notare come una buona parte dei documenti in cui ser Antonio risulta parte in causa (solitamente come auctor), per cui era prevista dagli statuti la

259 Ivi, nn. 82, 537.

260 Ivi, n. 184.

261 Ivi, n. 450.

262 Ivi, n. 464.

${ }^{263}$ Ivi, nn. 495b, 502, 639a-b.

${ }^{264}$ Ivi, n. 525 e ADTn, ACap, Instrumenta capitularia 8, n. 161.

265 ADTn, ACap, Instrumenta capitularia 8, n. 321a.

266 Ivi, nn. 477, 589, 602, 611a, 622.

267 ADTn, ACap, Instrumenta capitularia 8bis, n. 101bis.

${ }^{268}$ Ivi, n. 110.

269 Ivi, n. 443.

270 Ivi, n. 649.

271 Ivi, n. 656.

${ }^{272}$ ADTn, ACap, Instrumenta capitularia 8, n. 255.

273 Ivi, n. 513.

274 ADTn, ACap, Instrumenta capitularia 8bis, n. 111.

275 ADTn, ACap, capsa fabbrica, rotoli lunghi, n. 8.

276 ADTn, ACap, Instrumenta capitularia 8bis, n. 464.

277 Il notaio Graziadeo da Terlago era figlio di ser Antonio da Castel Terlago; aveva una figlia di nome Barbara che aveva sposato il notaio ser Cristoforo da Cadine: ADTn, ACap, Instrumenta capitularia 9, cc. $313 v-314 r$.

${ }^{278}$ Cfr., in particolare, in appendice, regesto n. 47 (ADTn, ACap, Instrumenta capitularia 8bis, cc. $218 v-219 r$, n. 579 ) e ASTn, $A P V$, Sezione latina, capsa 3, n. 52. 


\section{L'attività professionale di Antonio da Borgonuovo}

scrittura da parte d'altro professionista, fu redatta proprio da Graziadeo da Terlago, che dunque - se non un vero e proprio rapporto di collaborazione - sembrava quantomeno godere di fiducia da parte di ser Antonio ${ }^{279}$. Sulla questione poco altro si può dire, poiché mentre altrove sono ben attestati per tutto il Medioevo rapporti di collaborazione, spesso in botteghe comuni, fra notai, a Trento, almeno da quanto risulta nella documentazione del XIV e della prima metà del XV secolo presa in esame, non sembrano emergere associazionismi di questo genere. Ciò non significa, naturalmente, che siano esistiti rapporti di siffatta natura, che potevano condurre alla nascita di stationes specializzate nella redazione di particolari tipologie documentarie destinate a specifici enti (ecclesiastici ad esempio) ${ }^{280}$. Su questo tema, tuttavia, si rende necessario lo studio di un numero maggiore di fonti, diversificate sia per committenza sia per redazione; soltanto attraverso uno spoglio sistematico e dilatato negli anni sarà forse possibile trarre qualche maggiore informazione.

\section{Nomine di rettori di chiese, pievi e altari; nomine di canonici}

I capitoli cattedrali possedevano un certo numero di chiese, pievi e altari per le quali avevano il diritto di amministrare i sacramenti e gestire i beni immobili. L'amministrazione delle chiese era affidata dal collegio canonicale a un vicario, detto anche rettore, il quale otteneva in cambio una parte dei redditi riscossi, versando al Capitolo un canone annuo. Resasi vacante una pieve, una chiesa o un altare spettava al Capitolo nominare il nuovo vicario ${ }^{281}$. Fra la documentazione di Antonio si hanno le seguenti nomine:

\begin{tabular}{lll}
\hline nome del beneficiato & data del conferimento & tipologia e luogo del beneficio \\
\hline $\begin{array}{l}\text { Enrico, figlio del nobile Odo- } \\
\text { rico de Velden, canonico di }\end{array}$ & 17 ottobre 1401 & $\begin{array}{l}\text { pieve e chiesa di San Paolo in } \\
\text { Appiano }\end{array}$ \\
$\begin{array}{l}\text { Augusta } \\
\text { 282 }\end{array}$ & &
\end{tabular}

Tibaldo dalla Valsugana

19 settembre 1412

2 aprile 1424

Bertoldo Kol,
cesi di Trento

Federico del fu ser Andrea da 12 aprile 1427

Ferrara prete pieve di Meano 283

cappella di Sant'Andrea a Bolzano $^{284}$

altare dei Santi Pietro e Paolo nella cattedrale ${ }^{285}$

${ }^{279}$ Cfr., a titolo esemplificativo, ADTn, ACap, capsa 32, n. 224 e capsa 8, n. 24.

280 Cfr. per l'ambito senese G. Chironi, La mitra e il calamo. Il sistema documentario della Chiesa senese in età pretridentina (secoli XIV-XV), Accademia senese degli Intronati-Ministero per i Beni culturali e ambientali, Siena-Roma, 2005 (Monografie di storia e letteratura senese, 13; Saggi 85); per Milano C. Belloni, Dove mancano registri vescovili ma esistono fondi notarili: Milano tra Tre e Quattrocento, in A. Bartoli Langeli, A. Rigon (a cura di), I registri vescovili dell'Italia settentrionale (secoli XII-XV), Atti del convegno (Monselice 24-25 novembre 2000), Herder, Roma 2003 (Italia Sacra, 72), pp. 53-56; per Padova B. Pagnin, Note di diplomatica episcopale padovana, in P. Cancian (a cura di), La memoria delle chiese. Cancellerie vescovili e culture notarili nell'Italia centro-settentrionale (secoli X-XIII), Scriptorium, Torino 1995 (I florilegi, 4), p. 18.

${ }^{281}$ Curzel, I canonici e il Capitolo, cit., pp. 372 e ss.

282 ADTn, ACap, capsa 27, lunghe/a, n. [4]

283 Ivi, capsa 44, n. 12.

284 ADTn, ACap, Instrumenta capitularia 8bis, c. $12 r$, n. 36 (concessa dal conte del Tirolo Federico).

${ }^{285}$ Ivi, c. $84 v$, n. 239. 
Stefano Malfatti

\begin{tabular}{|c|c|c|}
\hline nome del beneficiato & data del conferimento & tipologia e luogo del beneficio \\
\hline $\begin{array}{l}\text { Tommaso pievano di Santa } \\
\text { Maria in Castelfondo }\end{array}$ & 2 luglio 1427 & $\begin{array}{l}\text { altare dei Santi Pietro e Paolo } \\
\text { nella cattedrale (risconsegnato ai } \\
\text { canonici da Federico del fu ser } \\
\text { Andrea da Ferrara) }{ }^{286}\end{array}$ \\
\hline $\begin{array}{l}\text { Pietro figlio di Ulmannus de } \\
\text { Huleym prete }\end{array}$ & 20 luglio 1430 & $\begin{array}{l}\text { altare di Santa Caterina in cattedra- } \\
\text { le (conferito in seguito alla morte } \\
\text { di Nicolò Saur, heri defunctus et } \\
\text { sepultus) })^{287}\end{array}$ \\
\hline Marquardo da Bamberga & 20 marzo 1431 & $\begin{array}{l}\text { altare di Santa Dorotea in catte- } \\
\text { drale }^{288}\end{array}$ \\
\hline Ulrico $^{289}$ & 9 giugno 1431 & $\begin{array}{l}\text { pieve di Meano, priva di rettore } \\
\text { per la morte di Nicolò dalla val } \\
\text { di Fassa }\end{array}$ \\
\hline $\begin{array}{l}\text { Giovanni Zeiss iuvenis, } \\
\text { procuratore del fratello, } \\
\text { il canonico Giovanni Zeiss } \\
\text { da Bopfingen }\end{array}$ & 11 dicembre 1431 & $\begin{array}{l}\text { altare di Sant'Agostino e la cappel- } \\
\text { la di San Giorgio presso Trento }{ }^{291}\end{array}$ \\
\hline Bricius prete & $1^{\circ}$ febbraio 1433 & $\begin{array}{l}\text { altare di Santa Massenza, attribuito } \\
\text { a Giovanni Ottenheim nel } 1431^{292} \\
\text { (conferimento da parte del canoni- } \\
\text { co e preposito Stanislao) }\end{array}$ \\
\hline Rugerio di Nicolò de Apulia & 25 luglio 1433 & $\begin{array}{l}\text { pieve di Santa Maria di Baselga } \\
\text { di Sopramonte }\end{array}$ \\
\hline Antonio da Palermo prete & 20 luglio 1434 & $\begin{array}{l}\text { altare di Santa Massenza, nella } \\
\text { cripta della cattedrale }{ }^{294}\end{array}$ \\
\hline $\begin{array}{l}\text { Ulrico Kuker, chierico della } \\
\text { diocesi di Augusta }\end{array}$ & 11 marzo 1435 & $\begin{array}{l}\text { altare di Santa Massenza, nella } \\
\text { cripta della cattedrale }{ }^{295}\end{array}$ \\
\hline Ulrico, pievano a Meano & $1^{\circ}$ maggio 1435 & $\begin{array}{l}\text { altare di San Pietro e Paolo, in } \\
\text { cattedrale }^{296}\end{array}$ \\
\hline Bertoldo da Trento prete & 12 agosto 1435 & $\begin{array}{l}\text { altare dei Santi Innocenti, in cat- } \\
\text { tedrale }\end{array}$ \\
\hline
\end{tabular}

Tab. 4. Nomine a rettore di pieve e collazioni d'altare registrate da Antonio da Borgonuovo.

${ }^{286}$ Ivi, c. $87 r-v$, n. 246.

287 Ivi, c. $153 r-v$, n. 397.

288 Ivi, c. $180 r$, n. 473.

${ }^{289}$ Null'altro è noto su questo personaggio viste le lacune dovute a guasto meccanico che hanno compromesso la lettura di parte del documento.

290 ADTn, ACap, Instrumenta capitularia 8, c. 70v, n. 152.

291 ADTn, ACap, Instrumenta capitularia 8bis, c. 198r, n. 517.

292 Ivi, c. $211 r$, n. 560.

293 ADTn, ACap, capsa 3, n. 11; 1'arcidiacono è in quest'occasione Antonio de Tosabech da Pavia. La pieve era fino ad allora detenuta dal prete Osvaldo, quel giorno stesso «sepultus in cimiterio ecclesie Tridentine».

294 ADTn, ACap, Instrumenta capitularia 8bis, c. 239v, n. 624.

295 Ivi, c. $248 r$, n. 645.

296 Ivi, c. $249 v$, n. 648.

297 Ivi, c. $252 v$, n. 657. 


\section{L'attività professionale di Antonio da Borgonuovo}

Fra le nomine di spettanza capitolare si hanno pure quelle dei nuovi canonici, di cui si trovano vari esempi fra i rogiti redatti da Antonio per questo ente.

\begin{tabular}{|c|c|}
\hline nome del canonico nominato & data del conferime \\
\hline $\begin{array}{l}\text { Nicolò Seldenhorn, chierico della } \\
\text { diocesi di Costanza e figlio di En- } \\
\text { rico, cancelliere del duca Federico } \\
\text { d'Austria }^{298}\end{array}$ & 28 dicembre 1411 \\
\hline $\begin{array}{l}\text { Corrado Czoppot, chierico della } \\
\text { diocesi di Bamberga e notaio di } \\
\text { Bertoldo, vescovo di Bressanone, } \\
\text { come rappresentante del canonico } \\
\text { Giorgio Hilprandi che ne era il } \\
\text { legittimo detentore }^{299}\end{array}$ & 11 aprile 1424 \\
\hline $\begin{array}{l}\text { Goffredo Friling, già canonico di } \\
\text { Bressanone }\end{array}$ & 11 febbraio 1425 \\
\hline $\begin{array}{l}\text { Artuico da Passau, già arciprete } \\
\text { in Rendena }\end{array}$ & 26 maggio 1425 \\
\hline $\begin{array}{l}\text { David Hayminger, prete della dio- } \\
\text { cesi di Varmia }\end{array}$ & 12 ottobre 1425 \\
\hline Zeno dalla Polonia ${ }^{303}$ & $1^{\circ}$ dicembre 1426 \\
\hline
\end{tabular}

Teobaldo di Michele Wolchestein, già canonico di Bressanone e di Zurigo ${ }^{304}$

Giovanni di Pietro Glasberger de Comitauu, diocesi di Praga ${ }^{305}$

Corrado Prehemer, pievano di Flavon e cappellano della cattedrale $^{306}$

29 aprile 1430

6 maggio 1430

21 marzo 1431
Preceduta dalla rinuncia al canonicato da parte del magister Giovanni di Luca, in qualità di procuratore del figlio Luca, canonico, della prebenda fino ad allora goduta

Prese il posto di Florio da Denno, hodie defunctus

Occupò lo stallo del defunto Giovanni da Isny, già decano del Capitolo

Canonicato e prebenda vacanti per la resignazione di Andrea di Corrado hospitalarius da Chiusa, cariche cedute ex causa permutationis allo stesso Corrado in cambio del beneficio dell'altare di Santa Massenza, che era stato a sua volta attribuito allo scolastico Giovanni Ottenheim, procuratore di Andrea.

\footnotetext{
298 ADTn, ACap, Instrumenta capitularia 8, c. $39 v$, n. 79.

299 Ivi, c. $12 r$, n. 37.

300 ADTn, ACap, Instrumenta capitularia 8bis, c. $28 r$, n. 81 .

301 Ivi, c. $40 r$, n. 111.

302 Ivi, c. $49 v$, n. 133.

303 Ivi, cc. $74 v-75 r$, n. 212.

304 Ivi, c. $146 v$, n. 382 .

305 Ivi, c. $147 r$, n. 384 .

306 Ivi, c. $180 v$, n. 475 e ivi, c. $182 r$, n. 480.
} 


\begin{tabular}{lll}
\hline nome del canonico nominato & data del conferimento & altre informazioni \\
\hline Nicolò Leonis da Venezia prete ${ }^{307}$ & 11 dicembre 1431 & Con la nomina acquisì anche la \\
& & prebenda vacante per la morte \\
di Giovanni de Empach detto & da Lana, hodie in ipsa ecclesia \\
& & sepultus
\end{tabular}

Francesco figlio del nobile Aldri- 27 febbraio 1432 ghetto da Castelbarco ${ }^{308}$

6 novembre 1432

Nicolò figlio del nobile Bongiacomo da Trissino ${ }^{309}$

Percivalle Weinecker, figlio del fu Ingenuino da Cortaccia, nobile ${ }^{310}$

26 marzo 1433

Rappresentato presso i canonici dai procuratori fra Giovanni dell'ordine dei Minori, vescovo di Tino e Micone e suffraganeo di Trento, e dal cancelliere del castello del Buonconsiglio Giovanni da Fraudental

Matteo, figlio di Ulrico da Monaco

22 febbraio 1435 di Baviera, già chierico della diocesi di Frisinga ${ }^{311}$

Giovanni Octhayn, già scolastico presso la cattedrale ${ }^{312}$

14 aprile 1435

$\mathrm{Fu}$ provvisto anche della prebenda canonicale che fu del defunto Osvaldo di Giovanni Keller

Costui fu inoltre provvisto della prebenda canonicale vacante per la morte di Giovanni Zeiss da Bopfingen, «nocte proxime precedente defunctus in Tridento et in dicta ecclesia Tridentina hodie sepultus»

Prospero de Thomasiis da Cre27 luglio 1435

Rappresentato dal suo procuratore Nicolò da Venezia all'Arena di Padova ${ }^{313}$

Tab. 5. Nomine a canonico registrate da Antonio da Borgonuovo.

307 Ivi, c. $198 r$, n. 517a.

308 Ivi, c. $202 v$, n. 531.

309 Ivi, c. $207 v$, n. 550.

310 Ivi, c. $213 v$, n. 569.

311 Ivi, c. $247 r$, n. 643.

312 Ivi, c. (foglio inserto) 248/1r-248/2r, n. 646.

313 ADTn, ACap, Instrumenta capitularia 8bis, c. $252 r$, n. 656. 



\section{Capitolo 4}

\section{Antonio da Borgonuovo al lavoro. Tracce di diplomatica notarile}

L'abbondante documentazione redatta da Antonio da Borgonuovo permette di svolgere una serie di considerazioni sul suo modus operandi o, per meglio dire, sui modi operandi, considerato che sembrano emergere schemi operativi alquanto diversificati e stratificati che, soltanto in parte, coincidono con lo schema, noto, della 'triplice redazione dell'instrumentum notarile delineato qualche decennio or sono da Giorgio Costamagna sulla base delle evidenze riscontrate nella documentazione notarile genovese di XIII secolo' ${ }^{1}$.

\section{Dalla 'scheda' all'instrumentum}

Il confronto e la collazione fra le diverse forme redazionali di uno stesso documento, nonché le numerose annotazioni marginali del notaio che si riferiscono a precedenti stesure dell'atto, contribuiscono, come si vedrà, a portare alla luce modalità di lavoro semplificate o ancor più complesse rispetto a quelle praticate dai notai genovesi. Si partirà, nell'analisi dei modi operandi di ser Antonio, dalla prima tappa, ossia dalla rogatio, atto attraverso il quale le parti in causa si rivolgevano al professionista per richiedere la messa per iscritto delle proprie volontà. Era questa l'occasione per il notaio di prendere nota, in maniera concisa, dei dati essenziali relativi al negozio giuridico: la data, i nomi dei testimoni e dei contraenti e gli

Referenze fotografiche. Le figg. 11-26 sono pubblicate con l'autorizzazione dell'Archivio diocesano tridentino.

1 G. Costamagna, La triplice redazione dell' instrumentum genovese. Con appendice di documenti, Società Ligure di Storia Patria, Genova 1961. Lo studio di alcuni cartolari e manuali di XIII e XIV secolo condusse infatti lo studioso a ricostruire l'iter redazionale dell'instrumentum publicum a partire dalla rogatio fino alla stesura del relativo mundum. Le pratiche redazionali messe in luce da Giorgio Costamagna per il notariato genovese bassomedievale, hanno trovato in seguito riscontro e conferma anche altrove, e ciò è vero in particolare fino a tutto il XIII secolo. Successivamente, anche se non ovunque e con sistematicità, la diminuita necessità di redigere il documento in mundum e il parallelo accrescimento del valore attribuito al documento su registro hanno condotto ad una semplificazione (o ad un aumento) dello schema valido per il documento genovese. In molti casi, ad esempio, la redazione sui protocolli, o su registri di formato ancora maggiore, ha finito con il divenire la stesura finale e definitiva dell'instrumentum, senza che vi fosse necessità di passare ad un'ulteriore redazione su pergamena (cfr., in proposito, M.I. Bossa (a cura di), Chiese e conventi degli ordini mendicanti in Umbria nei secoli XIII e XIV. Inventario delle fonti archivistiche e catalogo delle informazioni documentarie. La serie Protocolli dell'Archivio notarile di Perugia, Editrice Umbra cooperativa, Perugia 1987 (Archivi dell'Umbria. Inventari e ricerche, 12), in particolare A. Bartoli Langeli, Nota introduttiva, pp. IX-XXV). 
estremi contrattuali utili, in un secondo momento, all'intelligibilità e alla validità del documento stesso; in ultima analisi, un promemoria.

Non mancano esempi, fra le carte dei registri Instrumenta capitularia 8 e 8bis di lacerti cartacei, diversi per formato, in cui Antonio prese nota degli essentialia negotii, da cui successivamente avrebbe tratto il documento in forma di imbreviatura o esteso. Se ne prenderanno in esame alcuni casi.

Il 13 gennaio 1435 Giovanni di ser Bartolasio de Alemania, abitante in Borgonuovo, chiese a ser Antonio di registrare la dote ricevuta dalla moglie Onesta, pari a 150 lire di denari trentini: su un foglio di carta ${ }^{2}$ di modeste dimensioni (mm 156 $x$ 215), fortunatamente conservato ancora oggi nel suo registro Instrumenta capitularia 8bis, il notaio prese nota di alcuni dati essenziali, dai quali poté in seguito redigere il documento in forma più completa (fig. 11).

Si tratta di una 'scheda', prima tappa nell'iter redazionale dell'instrumentum notarile, in cui manca ancora quel valore giuridico che connoterà la redazione in registro e in pergamena sciolta. La seconda fase del 'processo' è rappresentata, verosimilmente, dalla redazione per esteso dello stesso documento, di cui si trova riscontro alla carta 245 del registro Instrumenta capitularia 8 bis $^{3}$. Il testo, affiancato dall'usuale rubrica che ha lo scopo di esporre brevemente il contenuto del documento, presenta tutti i dati necessari a conferire un pieno valore giuridico al negozio trattato, sebbene sia interrotto nella parte finale.

\section{'Scheda'}

\section{Maffeus}

Florianus die iovis XIII ianuarii in mea Faytelus stupa

Concerinus

dominus Antonius archidiaconus etc

Dos de centum et quinquaginta libris denariorum Tridentinorum in dictis rebus extimatis per testes suprascriptos, excepto domino archidiacono computatis in ipsa dote libr(is) XXV denariorum per eum Iohannem ser Bartholasii eidem domine Honeste donatis.

\section{Redazione 'in esteso'}

In Christi nomine, amen. Anno eiusdem nativitatis millesimo quatricentesimo trigesimo quinto, indictione terciadecima, die iovis terciodecimo mensis ianuarii, Tridenti, in contrata Sancti Vigilii, in stupa nova domus solite mei Antonii notarii infrascripti, presentibus venerabili in Christo viro domino Antonio de Tossabechis de Papia decretorum doctore et archidiacono et canonico Tridentino, Maffeo stazonerio condam ser Nigri de Brixia, Floriano Malicie condam Francisci de Tridento, Concerino laboratore de burgo Sancti Martini, Marcho dicto Faytelo caratore de Burgonovo de Tridento omnibus civibus et habitatoribus Tridenti testibus et aliis vocatis

2 ADTn, ACap, Instrumenta capitularia 8bis, foglio singolo fra le cc. 180-181, sul quale si trovavano già scritti degli appunti, fra cui una lista di beni affiancata dal valore in ducati e in lire, e una serie di conteggi sparsi nel margine inferiore. La mano che redasse la lista e i conteggi non è certamente quella di Antonio da Borgonuovo; pure l'inchiostro impiegato è visibilmente di tonalità più chiara rispetto a quello utilizzato dal notaio. Non si può affermare con certezza che questi appunti, peraltro vergati in lingua volgare, fossero già presenti sul foglio prima che Antonio vi redigesse la sua 'scheda', sebbene il fatto che il professionista abbia occupato con le sue annotazioni la sezione inferiore della carta faccia propendere per questa ipotesi. La mano anonima che redasse la lista è comunque databile alla prima metà del XV secolo.

3 ADTn, ACap, Instrumenta capitularia 8bis, c. $245 r$, n. 640 . La cedula cartacea con la 'scheda' del documento si trovava, fino al restauro del volume nel 1981, proprio fra le carte 244 e 245 del registro Instrumenta capitularia 8bis. 
et rogatis. Ibidem Iohannes ser Bartholasii de Burgonovo de Tridento, portitor in Tridento, solempniter fuit confessus et stetit contentus habuisse et recepisse a domina Honesta filia condam magistri Odorici cerdonis de Tridento eius uxore matrimonialiter copulata, ibidem presente et instante, et a domino presbitero Bertholdo eius domine Honeste fratre ibidem presente et dante et solvente in dote et nomine dotis dicte domine Honeste eius sororis et pro ea libras centum et quinquaginta denariorum Tridentinorum in rebus mobilibus extimatis per dictos testes excepto dicto domino archidiacono ut ipsi testes ibidem asserverunt, computatis tunc in dictis centum et quinquaginta libris denariorum, libr(as) viginti quinque denariorum Tridentinorum qu(a)s ipse Iohannes de suo donavit eidem sponse et uxori sue renuncians ...

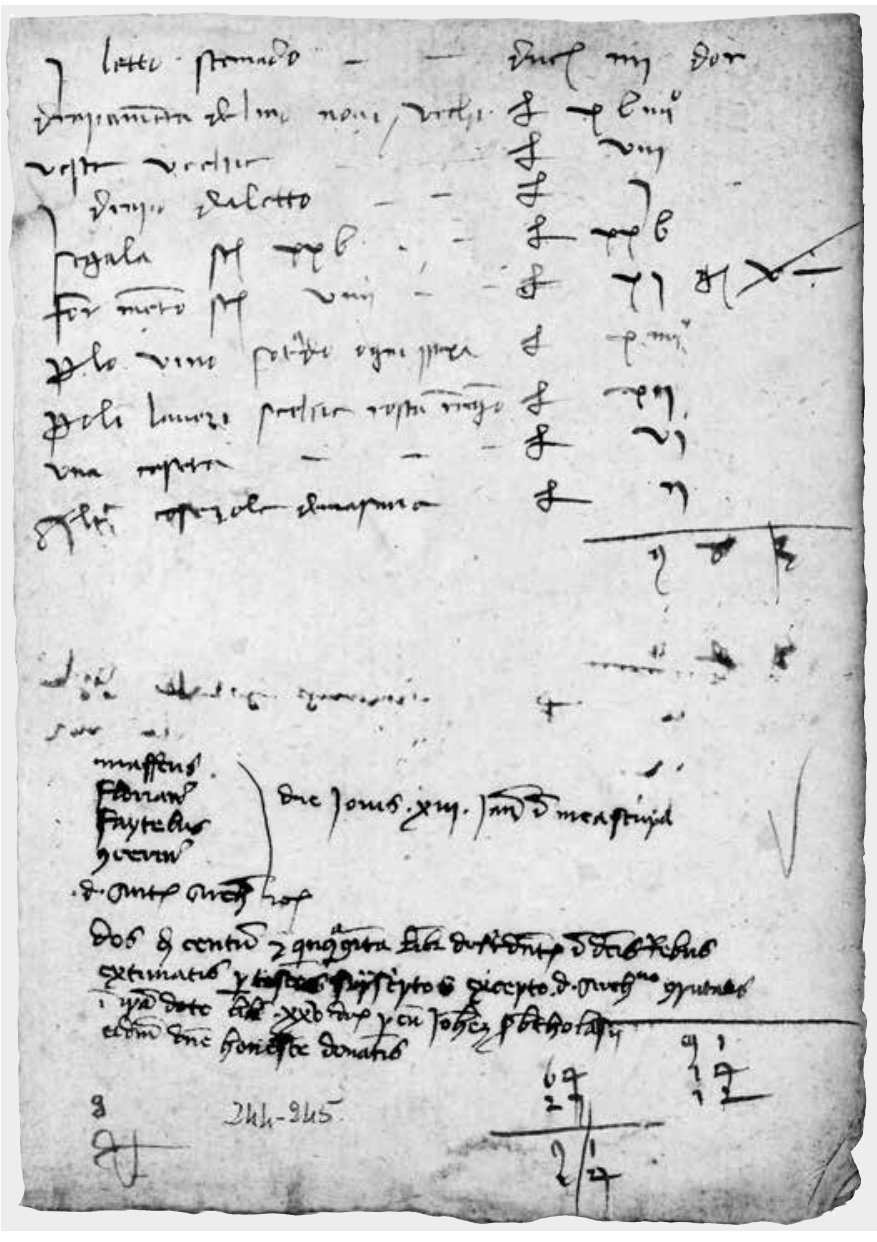

Fig. 11. ADTn, ACap, Instrumenta capitularia 8 bis, foglio singolo fra le cc. 244-245; ora posto nella tasca dei frammenti del registro $(\mathrm{mm} 156$ $x$ 215). In basso, con inchiostro di tonalità più scura, la 'scheda' redatta da Antonio da Borgonuovo relativa al documento n. 640 in Instrumenta capitularia 8bis. 
Nel caso suesposto, dunque, due soltanto sembrano essere le tappe seguite da Antonio per la redazione dell'instrumentum: la 'scheda' e la redazione 'in esteso' sul registro Instrumenta capitularia 8bis. Non sembra infatti essere stata tratta da questo documento alcuna redazione in mundum, vista l'assenza della nota relativa all'estrazione dell'atto in publicam formam, che solitamente il notaio apponeva in caso di successiva stesura su pergamena (fig. 12).

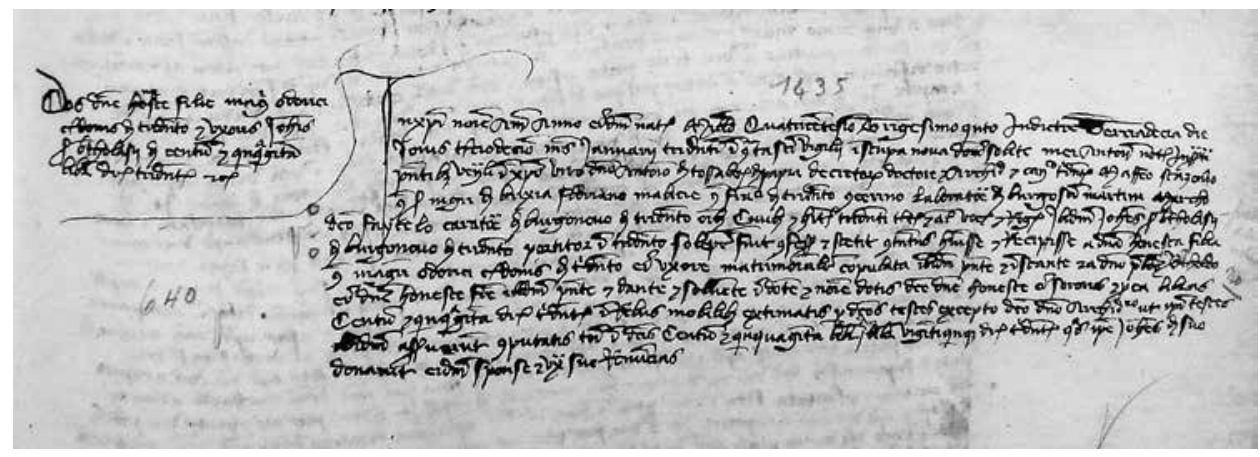

Fig. 12. ADTn, ACap, Instrumenta capitularia 8bis, c. $245 r$, n. 640. Redazione in esteso della 'scheda' alla fig. 11.

La maggior parte degli appunti su frammenti cartacei reperiti nei registri Instrumenta capitularia 8 e 8bis appartiene alla mano di Antonio da Borgonuovo, il quale talvolta utilizzò uno stesso foglio per annotare diversi negozi giuridici ${ }^{4}$ (fig. 13); tuttavia non mancano $\mathrm{i}$ casi in cui gli estremi contrattuali, o l'elenco dei terreni oggetto di un determinato negozio, vennero già predisposti su foglietti cartacei da una delle parti, mentre il notaio vi appose a completamento la sola registrazione della data (topica e cronica), dei nomi dei contraenti e dei testimoni.

$\mathrm{Ne}$ è un caso esemplare la cedula $(\mathrm{mm} 210 \mathrm{x} 131)$ oggi posta fra le carte 180 e 181 del registro Instrumenta capitularia 8bis. Il 19 marzo 1431 Giacomo detto del Fafaros, figlio di Giovanni de Alemania, contrasse una societas con il fratello uterino Francesco del fu Nicolino da Arco; si rivolsero pertanto al notaio Antonio affinché questi fissasse per iscritto il loro patto societario. La rogatio, come si ricava dalla data topica, avvenne nella piazza antistante il palazzo vescovile, «ad cambium domus stazionis appotece speciarie Melchioris ab Oleo». I fratelli, che evidentemente avevano già convenuto sui termini e sulle modalità della societas, predisposero a tal fine un piccolo foglio cartaceo sul quale Giacomo redasse in volgare le modalità e le tempistiche dell'accordo. Antonio completò quindi questo abbozzo di 'scheda' aggiungendo un'ulteriore specificazione relativa al patto, la lista dei testimoni presenti e le date topica e cronica (fig. 14).

Ricevuta la 'scheda' e completata con gli essentialia negotii e un'importante clausola, il notaio estrasse il documento in 'esteso': l'atto in questione si trova oggi in Instrumenta capitularia 8 bis $^{6}$.

4 Cfr, ad esempio, la c. 231/1, una cedula cartacea oggi posta fra le cc. 231 e 232 del registro Instrumenta capitularia 8 .

5 ADTn, ACap, Instrumenta capitularia 8bis, cedula cartacea fra le cc. 180 e 181.

6 Ivi, c. $179 v$, n. 471. 
Stefano Malfatti

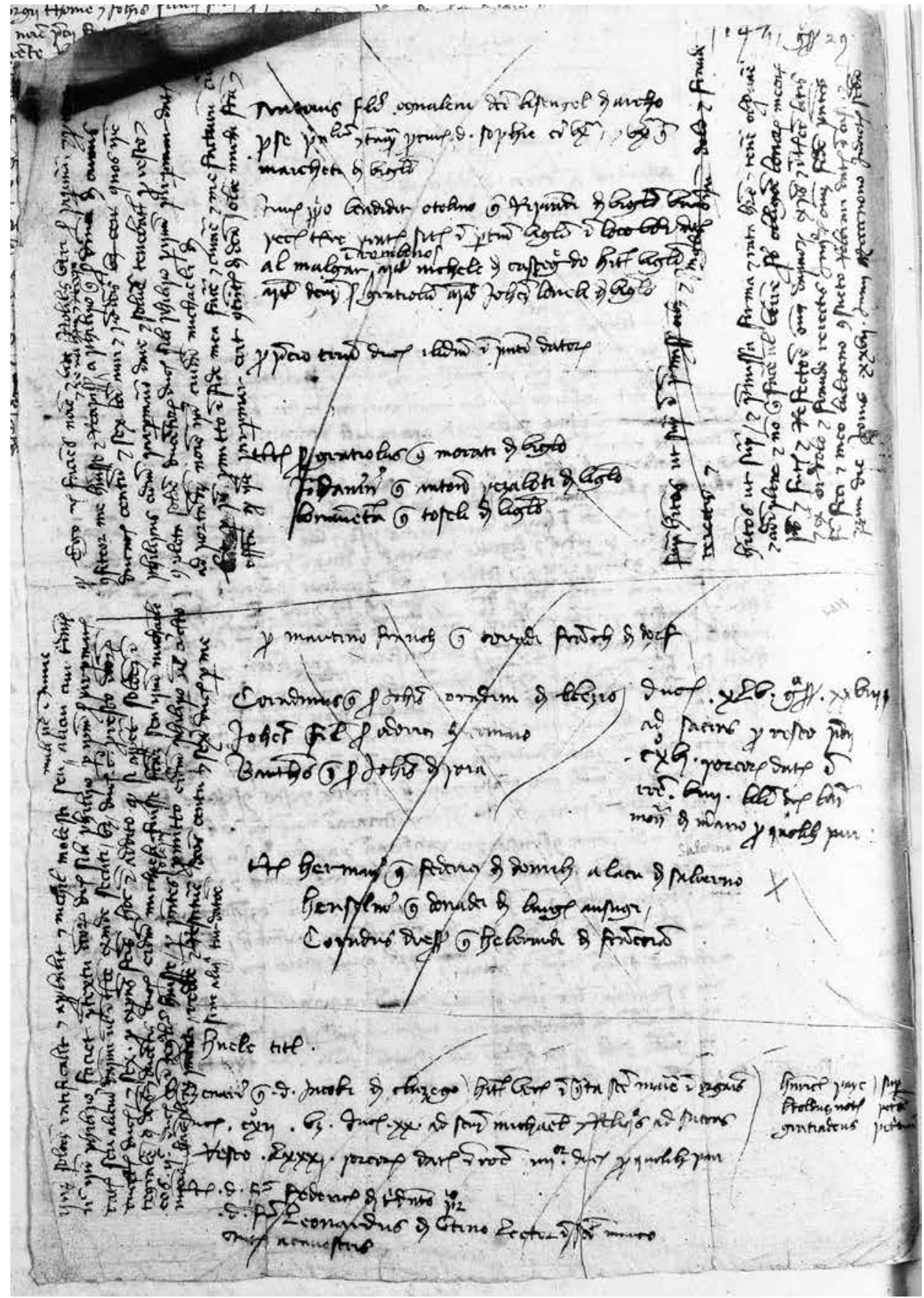

Fig. 13. ADTn, ACap, Instrumenta capitularia 8, c. 231/1v (mm 210 x 302): appunti per la redazione di instrumenta. 


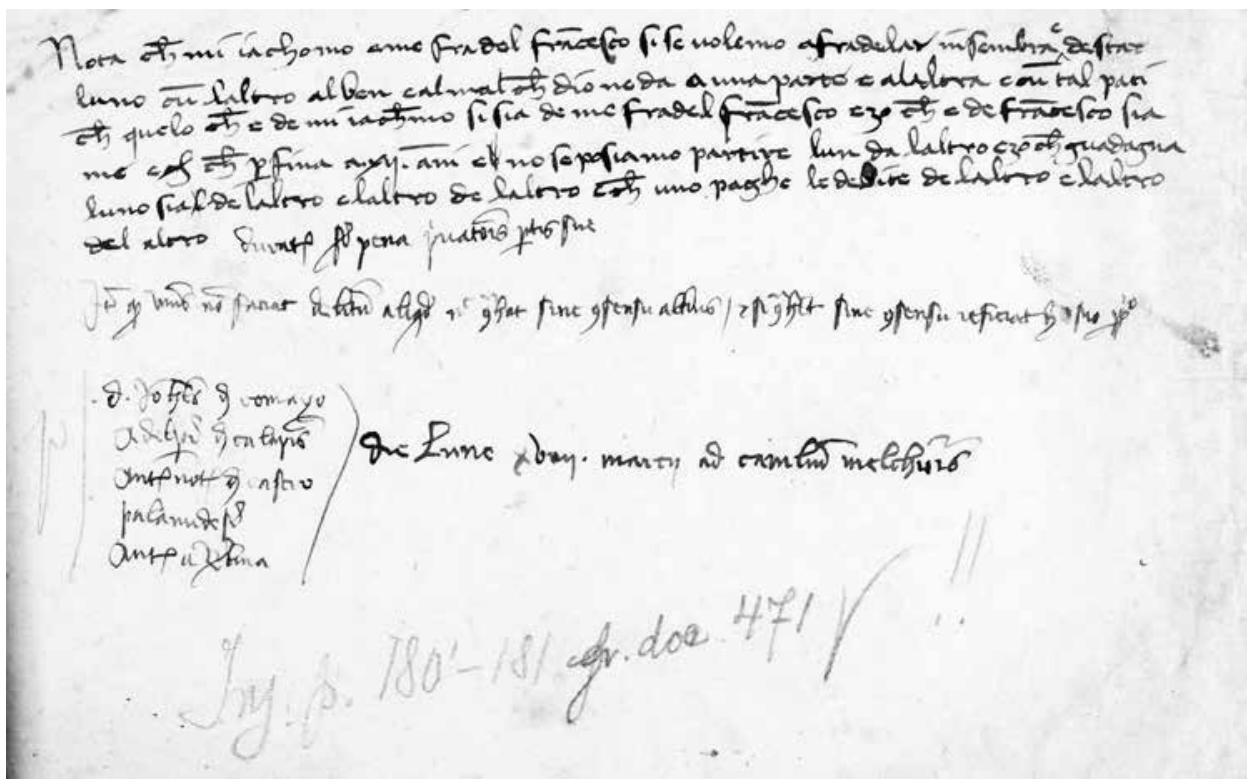

Fig. 14. ADTn, ACap, Instrumenta capitularia 8bis, foglio singolo fra le cc. 180 e 181. 'Scheda' relativa al documento n. 471 in Instrumenta capitularia 8bis. L'intervento di Antonio da Borgonuovo inizia dalla terza parola del sesto rigo.

Scheda

Nota che mi Iachomo e me fradel Francesco sì se volemo afradelar insembra a destar l'uno cum l'altro al ben e al mal che Dio ne dà a una parte e a l'altra, con tal pati: che quelo che è de mi Iachomo sì sia de me fradel Francesco e zò che è de Francesco sia meo, e che perfina a XII anni el no se posiamo partire l'un da l'altro, e che zo che guadagna l'uno sia de l'altro e l'altro de l'altro e che uno paga le debite de l'altro e l'altro del altro $\measuredangle d i$ mano del notaio> durat(ura) sub pena privationis partis sue.

Item quod unus non faciat debitum aliquod nec contrahat sine consensu alterius et si contrahet sine consensu reficiat de suo proprio.

d(ominus) Iohannes de Comayo

Adelperius de Calapinis

Antonius notarius de Castro

Palamidesius

Antonius a Berlina

die lune XVIII marcii ad cambium Melchioris «a destra della lista dei testimoni〉.
Redazione 'in esteso'

In Christi nomine, amen. Anno eiusdem nativitatis millesimo quatricentesimo tricesimo primo, indictione nona, die lune decimonono mensis marcii, Tridenti, ad plateam comunis, ad cambium domus stazionis appotece speciarie Melchioris ab Oleo, presentibus domino Iohanne de Comayo iurisperito, Adelperio condam ser Marchi de Calapinis de Tridento, Antonio notario condam ser Henrici de Castro, Palamidesio condam ser Iacobi de Tridento, Antonio a Berlina condam magistri Iacobi de Avolano, omnibus civibus et habitatoribus Tridenti testibus et aliis vocatis et rogatis. Ibidem Iacobus cerdo dictus del $\mathrm{Fa}-$ faros condam Iohannis de Alemania et Franciscus piliparius condam Nicholini de Archo fratres uteri, volentes fraternali et bono amore insimul ad locum focum 〈cosi nel testo〉 et in familia vivere et cohabitare, unionem et societatem de omnibus bonis suis mobilibus et immobilibus, iuribus et actionibus, fecerunt et contraxerunt duratam hinc ad duodecim annos proxime futuros et completos. Et promiserunt ipsi fratres sibi invicem solempnibus stipulationibus hinc inde intervenienti- 
bus fideliter, solicite et studiose et fraternaliter in dicta societate inter eos contracta se exercitare et ex licitis et honestis lucrari et de bono in melius se promovere et quicquid ad manus ipsorum pervenerit in ipsa societate et comodo isporum proponere et convertere et debita non contrahere, nec credentias facere unus sine scitu et consensu alterius. Et si alter ipsorum aliquod debitum vel credentiam faceret sine scitu et consensu alterius quod sic contrahens, de suo proprio resarcire et solvere teneatur et quod neutro ipsorum liceat nec requirat a dicta societate discedere usque ad dictum terminum duodecim annorum sub pena privationis partis sue applicande parti attendenti et ipsam societatem observare volenti. Et in fine dicti termini duodecim annorum omnia bona sua tam posita in dicta societate quam tunc reperta, acquisita et super lucrata per eos inter se fideliter dividere et partire et sibi invicem obedire et fraternaliter tractare. Que omnia et singula promiserunt ipsi fratres sibi invicem solempnibus stipulationibus hinc inde intervenientibus firma et rata habere et tenere, observare et adimplere et non contrafacere vel venire per se vel alios aliqua ratione vel causa de iure vel de facto, sub pena refectionis omnium dampnorum expensarum et interesse litis et extra, qua pena solepni stipulatione et expresse promissa soluta vel non nichilo rata maneant omnia et singula suprascripta, pro quibus omnibus et singulis suprascriptis firmiter observandis et plenius attendendis obligaverunt ipsi fratres sibi invicem omnia sua bona presentia et futura. Renunciantes exceptioni non facte et contracte dicte societatis et unionis fraternali amore et exceptioni doli, mali, pacti conventi, actioni in factum, condictioni indebiti et sine causa et omni alio suo iuri et legum auxilio.

Il documento 'in esteso' presenta dunque tutti gli elementi formali e formulari necessari a conferire piena validità giuridica al negozio; a partire dalle publicationes fino alle clausole finali e alle clausole di rinuncia alle eccezioni di legge che, come si deduce dalla diversa tonalità dell'inchiostro, il notaio aggiunse in calce al documento posteriormente alla prima stesura del testo. Il patto societario fra $\mathrm{i}$ fratelli Giacomo e Francesco ebbe tuttavia durata ben più breve rispetto ai dodici anni sanciti nell'atto visto che già il 28 ottobre successivo i due si recarono presso l'abitazione del notaio per rescindere il contratto, chiedendo contestualmente la cancellazione dell'instrumentum che lo attestava. Per tale ragione Antonio redasse l'atto relativo alla «Canzelatio et discessio a dicto contractu dicte societa- 
tis» ${ }^{7}$, ponendolo immediatamente sotto a quello della societas, nello spazio rimasto bianco in corrispondenza del margine inferiore della carta. Contemporaneamente il notaio provvide a cancellare il documento precedente annullandone così ogni valore giuridico (fig. 15). Anche in questo caso, dunque, due sembrano essere le tappe redazionali praticate da Antonio da Borgonuovo: la minuta e, successivamente, la redazione per esteso su registro. L'assenza di qualunque riferimento all'estrazione del mundum porta a ritenere che, anche in questo caso, $\mathrm{i}$ due fratelli si ritennero sufficientemente tutelati dal fatto che il documento fosse posto sul registro, pienamente valido sotto il profilo giuridico.

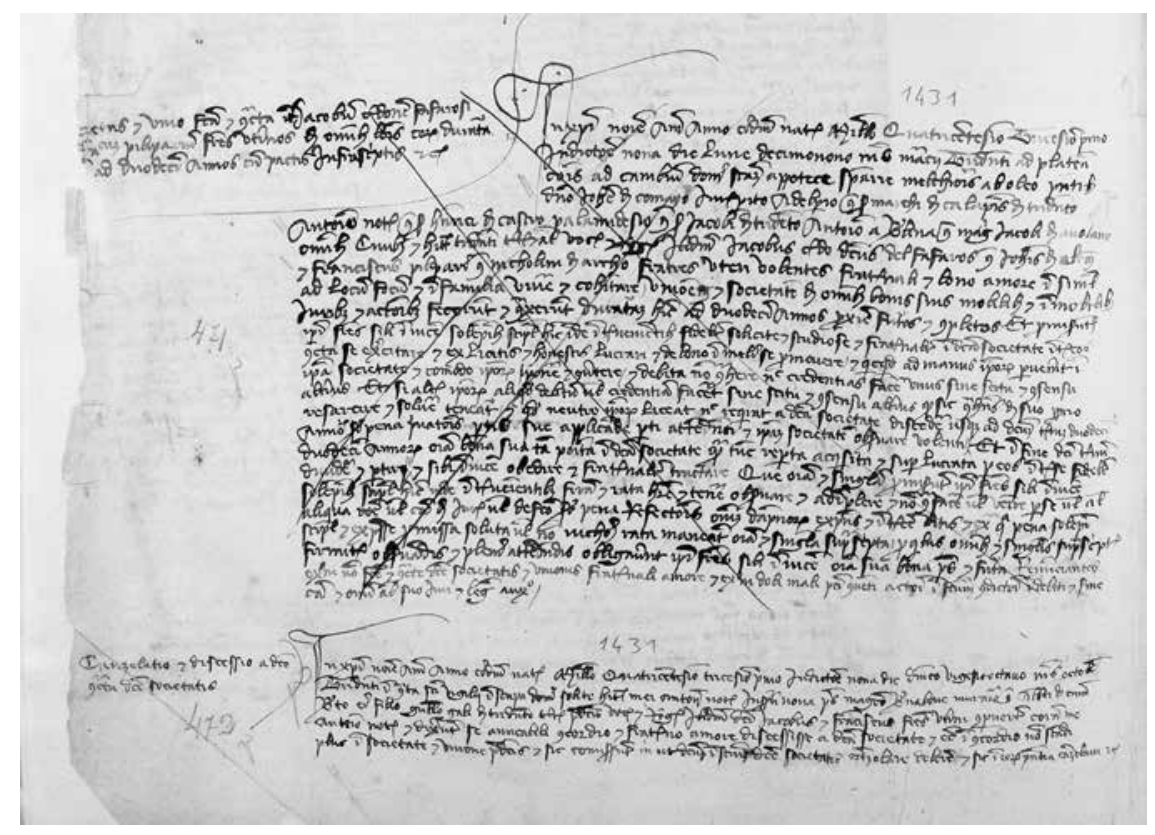

Fig. 15. ADTn, ACap, Instrumenta capitularia 8, c. 179v, nn. 471-472. Il doc. n. 471 è la redazione in esteso del patto societario riassunto nella 'scheda' rappresentata nella fig. 14; il doc. n. 472 è la rescissione del contratto fra i fratelli Giacomo e Francesco.

Ancora un esempio. Il 9 maggio 1427 il giurisperito Gioacchino Mezzasoma, fra i più importanti clienti privati di ser Antonio, consegnò al notaio una cedula cartacea manoscritta sulla quale, in forma oggettiva, dichiarava che Antonio da Molveno gli aveva venduto un affitto pagato annualmente per alcuni prati situati a Trento, fuori della porta di San Martino, al prezzo di 28 ducati d'oro ${ }^{8}$. Il foglietto, che misura $130 \times 210 \mathrm{~mm}$, ancora oggi trova spazio vicino all'esteso sul registro Instrumenta capitularia 8bis (fig. 16). Rispetto agli esempi finora esaminati, in questo caso ser Antonio non provvide a completare in alcun modo la 'scheda' con i dati mancanti. Il caso è interessante perché consente di ricostruire, ancor più nel dettaglio, le modalità di formazione dell'instrumentum.

Ivi, c. $179 v$, n. 472.

8 La cedula cartacea si trova ivi, foglio singolo fra le cc. 85 e 86 ; il documento in esteso si trova ivi, c. $85 v$, n. 242. 


\section{Inxpi non}

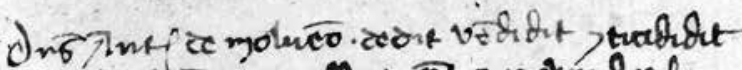

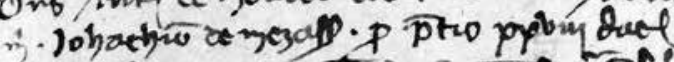

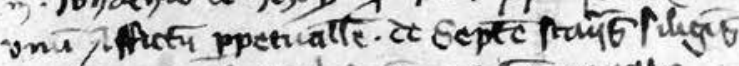

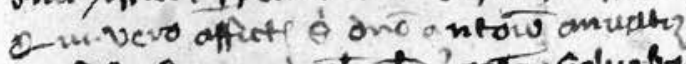

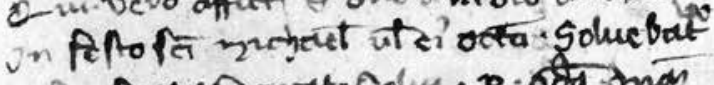
3 conpuetion evat-Golm-p.got onom

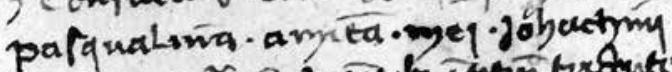

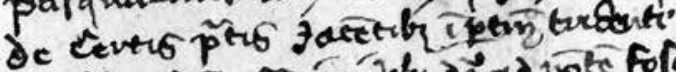

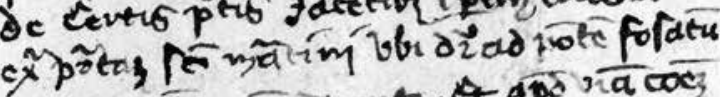
copd sturita cóz ec ente. et aps ra cos

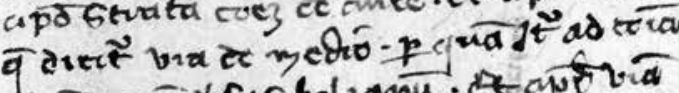

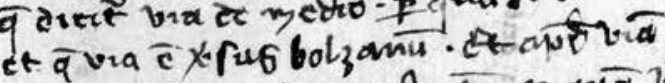

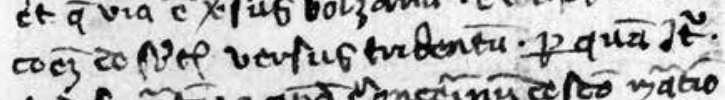

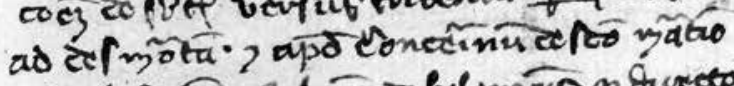

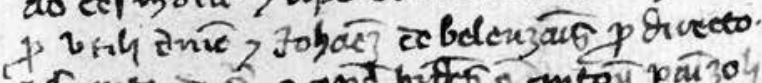
a capite is 6. , apd bites entoy panzol

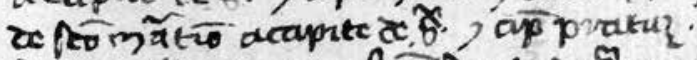

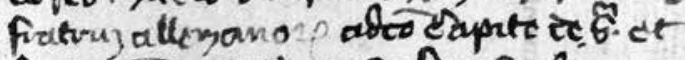

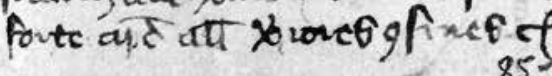

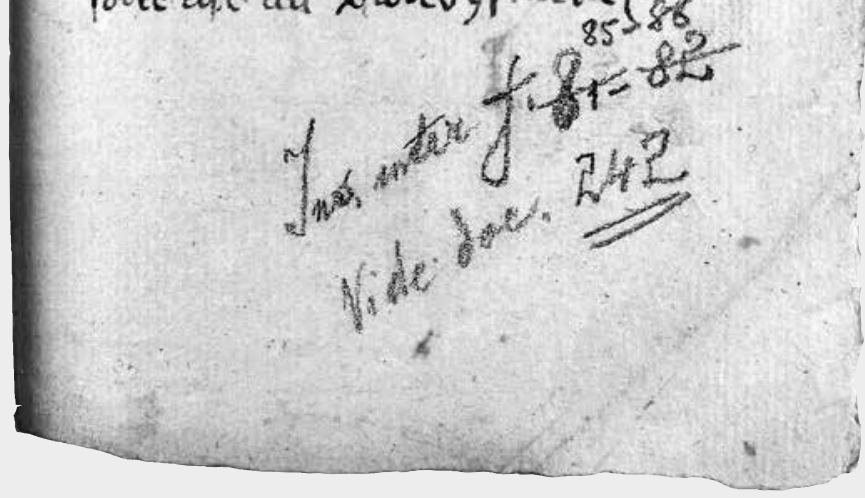

Fig. 16. ADTn, ACap, Instrumenta capitularia 8 bis, foglio singolo fra le cc. 85 e 86 . 'Scheda' relativa al documento n. 242 in Instrumenta capitularia 8 bis ( $\mathrm{mm} .130 \times 210)$.

Dal piccolo foglio cartaceo, infatti, si ricava che la compravendita, così come il pagamento, erano già avvenuti; in seguito, uno dei contraenti - in questo caso l'acquirente - si era recato dal notaio per la stesura del documento. Ricevuta la 'scheda' dalle mani di Gioacchino Mezzasoma, ser Antonio provvide quindi a redigere il documento per esteso sul proprio registro. Rispetto agli esempi sopra citati, tuttavia, in questo caso l'acquirente richiese anche l'estrazione del mundum come si ricava dalla nota posta dal notaio in corrispondenza del margine sinistro del registro: «Facta extra distincte dicto emptori etc». È utile, anche in questo caso, porre a confronto le due fasi redazionali, scheda ed esteso, per poterne valutare l'evoluzione. 
Antonio da Borgonuovo al lavoro

'Scheda'

In Christi nomine.

Dominus Antonius de Molveno dedit, vendidit et tradidit michi Iohachino de Mezassomis pro pretio XXVIII ducatis unum affictum perpetuallem de septem stariis siliginis qui vero affictus sibi domino Antonio annuatim in festo sancti Michaelis vel eius octava solvebatur et consuetus erat solvi per condam dominam Pasqualinam amitam mei Iohachini de certis pratis iacentibus in pertinenciis Tridenti, extra portam Sancti Martini ubi dicitur ad Pontem Fosatum, apud stratam comunem de ante. Et apud viam comunem que dicitur via de medio per quam itur ad comunia et que via ex versus Bolzanum. Et apud viam comunem de subtus versus Tridentum per quam itur ad Desmortam et apud Concerinum de Sancto Martino pro utili dominio et Iohannem de Belenzanis pro directo a capite de supra et apud heredes condam Antonii Panizoli de Sancto Martino a capite de supra et apud pratum fratrum Alemanorum a dicto capite de supra et forte apud allios veriores confines etc.
Redazione 'in esteso'

In Christi nomine, amen. Anno eiusdem nativitatis millesimo quatricentesimo vigesimo septimo, indictione quinta, die veneris nono mensis madii, Tridenti, in contrata Sancti Vigilii, in camino inferiori domus solite habitationis mei Antonii notarii infrascripti, presentibus Gratiadeo notario condam ser Antoni de Castello de Terlaco, Michaele condam ser Bartholomei Fenutoli de Sporo, Federico Polentono de burgo Sancti Martini de Tridento, Iohanne Rauter condam ser Pauli notarii de Tridento omnibus civibus et habitatoribus Tridenti testibus et aliis vocatis et rogatis.

Ibidem honorabilis et prudens vir dominus Antonius de Molveno iuris peritus filius condam ser Francisci notarii de Molveno civis Tridentinus, per se et suos heredes, iure proprio in perpetuum pro libero et expedito allodio, dedit, vendidit, cessit et tradidit honorabili viro domino Iohachino condam ser Petri notarii de Mezasomis de Tridento iurisperito civi Tridentino ibidem presenti pro se et suis heredibus ementi et recipienti unum affictum perpetualem septem stariorum siliginis ad mensuram Tridenti, cum directo dominio et proprietate unde solvitur ipse affictus, qui affictus solvitur solvi debet et consuetus est solvi perpetualiter omni anno in festo sancti Michaelis vel infra eius octavam secundum usum et consuetudinem locationum in perpetuum domorum merchati Tridenti et pro causa in eis consueta per heredes condam domine Pasqualine uxoris condam domini Marchi de Castro Campi et amitte olim dicti domini Iohachini emptoris de certis peciis terre prative sitis in pertinenciis Tridenti, extra portam Sancti Martini, in loco ubi dicitur ad Pontem Fossatum, apud stratam comunem de ante, apud viam comunem que dicitur via de medio per quam itur ad comunia et que via est versus Bulzanum et apud viam comunem de subtus versus Tridentum per qua itur ad Desmortam apud Concerinum de Sancto Martino pro utili dominio et Iohannem de Belenzanis pro directo, a capite de supra apud heredes condam Antonii Panizoli de dicto burgo Sancti Martini, a capite etiam de supra et apud iura fratrum Alemanorum a dicto capite etiam de supra, et forte alii sunt confines veriores, ad habendum, tenendum 
et possidendum et quicquid sibi domino Iohachino emptori et suis heredibus deinceps placuerit faciendum cum omnibus et singulis que infra predictos continetur confines vel alios si qui forent ulterius veriores accessibus et egressibus suis usque in viam publicam et cum omnibus et singulis que dicta res vendita habet supra se, infra se seu intra se in integrum omnique iure et actione, usu seu requisitione sibi venditori ex ea re vendita vel pro ipsa re aut ipsi rei vendite modo aliquo spectante dans, cedens et concedens ipse venditor dicto emptori presenti, requirenti et recipienti omnia iura et actiones reales et personales, utiles et directas, tacitas et expressas que et quas ipse venditor habebat et quoslibet habere poterat seu habere videbatur contra dictos heredes condam dicte domine Pasqualine affictaline perpetue de dicto affictu et contra quascumque alias personas et rem quacumque ratione vel causa et constituit eum dominum Iohachinum emptorem procuratorem ut in rem suam et posuit eum in locum suum, ita quod a modo ipse dominus Iohachinus emptor possit agere, petere, exigere, recipere, placitare, experiri, excipere et replicare, consequi et se tueri et omnia et singula facere et exercere quemadmodum et ipse venditor an(te) presentem contractum facere poterat. Et hoc nominatim pro precio et nomine precii et finiti merchati viginti octo ducatorum boni auri et iusti ponderis quod precium sive quos viginti octo ducatos auri nomine precii fuit ipse dominus Antonius venditor solempniter confessus et contentus habuisse et recipisse et sibi re vera et non sunt aliqua spem future numerationis dat(am) etc; renuncians exceptioni sibi non dati, non soluti etc. Et ipse venditor solempniter mandavit dictis heredibus condam dicte domine Pasqualine licet absent(ibus) quatenus de dicto affictu septem stariorum siliginis de cetero eidem domino Iohachino tamquam vero domino et proprietario dicti affictus respondere debeant et teneantur facere in omnibus et per omnia ut ipsi domino Antonio venditori ante presentem contractum facere tenebantur. Quam rem venditam ipse dominus venditor se nomine et vice dicti emptori et pro eo constituit possidere etc. Nec non et ipse venditor solempni stipulatione promisit dicto emptori presenti pro se et suis heredibus stipulantibus de evictione dicte rei vendite et de 
Antonio da Borgonuovo al lavoro

eius legitima deffensione et de ratificathione omnium et singulorum premissorum sub pena dupli precii predicti et refectionis dampnorum expensarum et interesse litis et extra et sub obligatione omnium suorum bonorum qua pena solepni stipulatione promissa etc ut in comuni forma.

Come si evince dal confronto fra i due testi, nel secondo caso il notaio ha scelto di redigere il documento per esteso, sciogliendo quasi tutte le formule, al pari di una redactio in mundum. Soltanto poche clausole, infatti, fra cui quelle finali, risultano ceterate; in quest'ultimo caso, il notaio interruppe la formula con la locuzione et cetera, richiamando, per il prosieguo, alla forma comune utilizzata per le compravendite: «ut in comuni forma». Per il resto, ciò che eventualmente distingue questo esteso da una redazione su pergamena è la sola mancanza del signum e della sottoscrizione di ser Antonio (fig. 17). Un'ultima considerazione va fatta sulla data del documento. Come ricordato, la nota di Gioacchino Mezzasoma non menziona né il tempo, né il luogo della compravendita; è noto, tuttavia, che l'azione giuridica, al momento della rogatio al notaio, era del tutto compiuta, come sembrerebbero indicare i verbi al perfetto. La data e il luogo indicati da ser Antonio si riferirebbero dunque, ma si tratta di un'ipotesi, non al momento della compravendita, ma all'atto della rogatio (coincidente con la consegna della 'scheda' da parte di Gioacchino) che avvenne, per l'appunto, il 9 maggio «in camino inferiori» della casa di Antonio da Borgonovo.

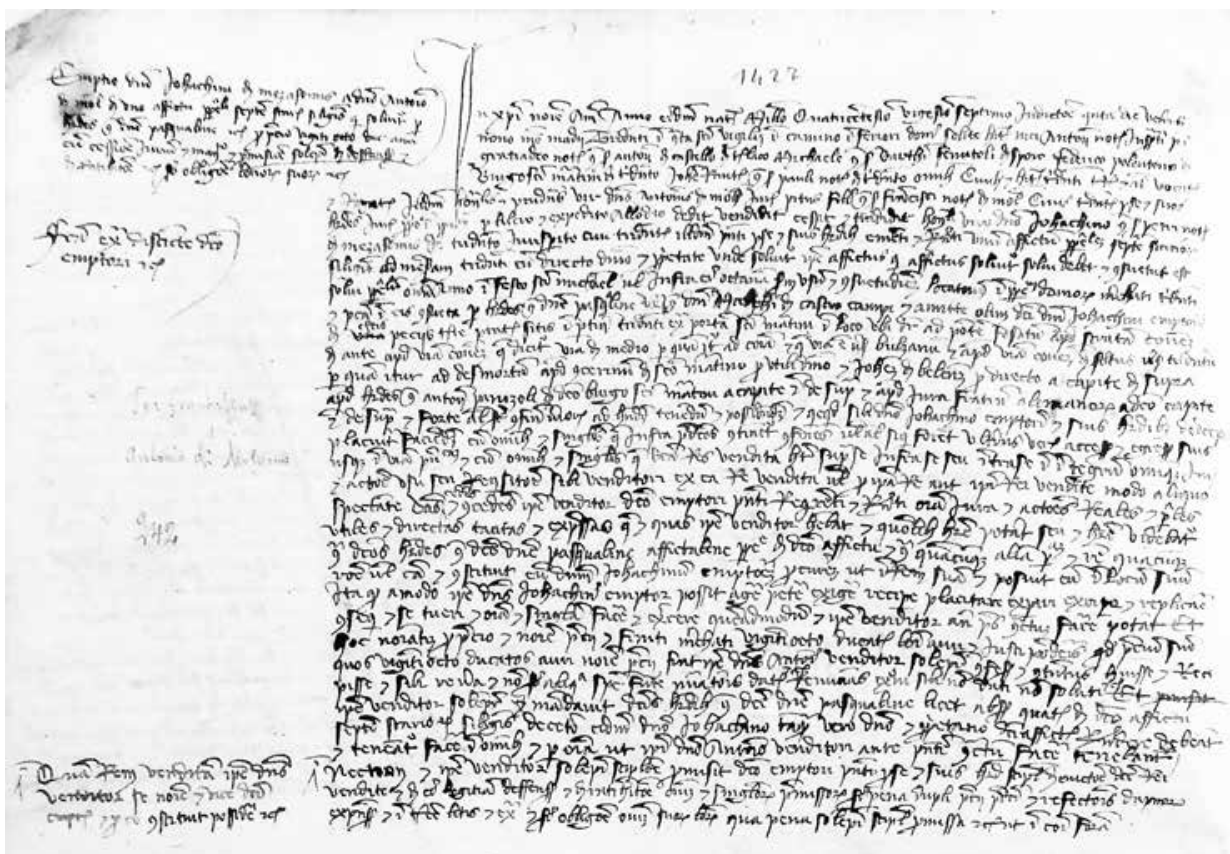

Fig. 17. ADTn, ACap, Instrumenta capitularia 8, c. $85 v$, n. 242. Redazione in esteso della 'scheda' alla fig. 16. 


\section{I quaternelli}

Finora si è fatto cenno unicamente a 'schede' redatte dal notaio, o da questi semplicemente completate, su foglietti volanti sparsi fra le carte dei registri Instrumenta capitularia 8 e 8bis. È noto, tuttavia, che i professionisti impiegavano abitualmente anche registri di piccolo formato, 'tascabili', più comodi rispetto ai protocolli e ai volumi in folio che essi tenevano invece presso il proprio studio. Costamagna li definisce 'manuali', perché il notaio li portava sempre con sé, per registrare in qualunque momento i negozi giuridici che i privati o le istituzioni gli chiedevano di mettere per iscritto. Anche lo statuto dei notai e giudici di Trento, compreso negli statuti masoviani del 1425-1427, ricorda l'obbligatorietà per i tabellioni trentini di avere sempre con sé il necessario per imbreviare e per annotare:

Item quod quilibet tabellio de collegio predicto ad offitium suum necessaria ad $\mathrm{i}(\mathrm{m})$ breviandum seu notandum, videlicet quaternelum, penarolum cum attramento et calamo ad minus, continuo secum portet et portare debeat, sub pena viginti sol(idorum) pro quolibet et qualibet vice ${ }^{9}$.

Piuttosto importante è la terminologia adottata. Il capitolo in questione parla infatti degli strumenti di lavoro che il notaio è tenuto a portare sempre (continuo) con sé quando non si trova presso la propria statio; si menzionano un quaternelus e un penarolus cum attramento et calamo. Nessun problema relativamente alla penna, al calamo e all'inchiostro necessari per scrivere; qualche incertezza in più, invece, sorge in merito al quaternelus che si deve probabilmente associare a un registro di formato ridotto, simile ai 'manuali' di cui parla Costamagna, in formato in ottavo o, addirittura, in sedicesimo. Il termine, dunque, non farebbe riferimento alla quantità delle carte che componevano il manufatto, ma piuttosto alle sue dimensioni ${ }^{10}$.

Rare sono le testimonianze relative a quaterneli di notai trentini medievali; fra questi si può ricordare ciò che rimane di un piccolo 'manuale' del notaio Antonio della Berlina (attivo fra il 1484 e il 1527) oggi conservato presso il fondo Manoscritti $(B C T 1)$ della Biblioteca comunale di Trento ${ }^{11}$, più tardo rispetto al periodo di cui ci si occupa, ma per formato e modalità di gestione del tutto simile a quelli descritti da Costamagna per l'area genovese. Per quanto concerne Antonio da Borgonuovo, invece, ciò che rimane - oltre ai lacerti cartacei sopra menzionati - sono le sole annotazioni poste dal professionista in margine ai documenti sui registri Instrumenta capitularia 8 e 8 bis. Se infatti il formato spesso eterogeneo delle cedule contenenti le annotazioni di ser Antonio porta ad escludere che queste provenissero in origine da manuali, i frequenti riferimenti del professionista ad un quaternelus da cui avrebbe relevato i documenti ${ }^{12}$ fa ipotizzare l'utilizzo, anche da parte sua, di piccoli quaterni per la prima registrazione dei negozi.

9 Liber I. De civilibus, capitolo 91 (cfr. Bortoli, Per un'edizione dei testi statutari del Comune di Trento, cit).

10 Si vedano analoghe considerazioni svolte da A. Romiti, L'armarium comunis della Camara Actorum di Bologna: l'inventariazione archivistica nel XIII secolo, Ministero per i Beni Culturali e Ambientali. Pubblicazioni degli Archivi di Stato, Roma 1994 (Fonti, 19), p. CXXI relativamente al termine quaternellus.

11 BCTn, BCT1, n. 1939/1-2-3.

12 Cfr., ad esempio, il riferimento posto in margine a ADTn, ACap, Instrumenta capitularia 8, n. 89 , «Relevata ex quaternelo». 
Dopo aver redatto in forma estesa una locazione perpetua concessa dai canonici a Giacomo, preposito del monastero di San Michele all'Adige ${ }^{13}$, ser Antonio pone in corrispondenza del margine sinistro della carta la nota Relevata ex quaternelo; in altra occasione, un'ulteriore locazione concessa sempre dal Capitolo al canonico Erardo da Eger $^{14}$ è affiancata da analoga annotazione: Relevata ex quaternelo. Ma il lessico di Antonio da Borgonuovo varia notevolmente e il termine quaternelus, che - pur mantenendo una certa cautela - è possibile associare al 'manuale', viene talvolta sostituito da locuzioni quale quaternelus prothocolli. In assenza di riscontro materiale che permetta di verificare la consistenza e il contenuto del quaternelus prothocolli ${ }^{15}$, non si può che formulare qualche ipotesi. Potrebbe, infatti, trattarsi di un piccolo registro 'tascabile', ovvero un manuale, per la registrazione dei primi dati raccolti all'atto della rogatio, come è probabile nel caso del semplice quaternelus citato dagli statuti e dallo stesso ser Antonio ma, al contempo, potrebbe trattarsi di una fattispecie documentaria più 'evoluta' rispetto a quella del 'manuale da tasca'. Il notaio utilizza infatti, insieme al termine quaternelus, anche l'espressione prothocollus, in genere impiegato dai notai per indicare un registro di formato in quarto (le cui misure medie sono solitamente di $300 \mathrm{~mm}$ in altezza e $220-225 \mathrm{~mm}$ in larghezza), su cui venivano posti i rogiti imbreviati o in esteso. Se questa seconda ipotesi si rivelasse corrispondente al vero, ci si troverebbe di fronte ad una ulteriore forma redazionale, quella in prothocollo, parallela o alternativa alla stesura su registri di formato ancora maggiore, quali ad esempio Instrumenta capitularia 8 (le cui carte misurano mediamente $417 \mathrm{~mm}$ in altezza e $337 \mathrm{~mm}$ in larghezza) e Instrumenta capitularia 8bis (che misura $440 \mathrm{~mm}$ in altezza e $345 \mathrm{~mm}$ in larghezza).

\section{I prothocolli}

Molto più numerosi sono i riferimenti di ser Antonio a documenti rilevati ex prothocollo ${ }^{16}$ o ex uno folio existenti in prothocollo meo ${ }^{17}$. Sulla natura dei prothocolli citati dal notaio si tornerà a breve. Si svolgerà ora qualche ulteriore riflessione in merito alle modalità operative seguite dal notaio per la redazione dell'instrumentum, e ciò andrà fatto prendendo in considerazione tutte le tipologie documentarie fin qui menzionate: a partire dalla 'scheda', passando per i prothocolli, fino a giungere ai registri di grande formato, oggi conservati nella serie degli Instrumenta capitularia. Oltre a ciò, si devono ricordare tutta una serie di fogli cartacei o bifolia che hanno la misura media di $305-310 \mathrm{~mm}$ in altezza per $210-215 \mathrm{~mm}$ in larghezza, oggi disseminati fra le carte dei registri di ser Antonio: essi trasmettono documentazione, inerente a diversi negozi giuridici, redatta in forma abbreviata, ma più frequentemente per esteso. Il notaio, secondo modalità che si trovano iterate anche nei registri Instrumenta capitularia 8 e 8bis, redigeva l'usuale rubrica nel margine in alto a sinistra e, di seguito, il documento in forma quasi completa, tralasciando solo in alcuni casi le formule ceterate. Tutt'al più si rilevano lievi differenze rispetto

13 Ivi, cc. $37 v-38 r$, n. 75.

14 Ivi, c. $44 r-v$, n. 89.

15 Se ne veda l'utilizzo quale nota in margine al documento in ADTn, ACap, Instrumenta capitularia 8bis, c. $132 r$, n. 353.

16 Per alcuni esempi cfr. ivi, nn. 115, 638.

17 Ivi, n. 626. 
alla redazione sui registri di formato maggiore per una minor cura redazionale - la scrittura diviene talvolta più fitta e più disordinata - o per una maggior presenza di correzioni; ma ciò è vero soltanto in alcuni casi, nel senso che l'aspetto generale della pagina scritta per alcuni di questi fogli e bifolia appare del tutto simile a quello che si riscontra nei registri. Non si rilevano, ad esempio, sostanziali differenze nella preparazione della pagina e nella successiva mise en page, ove, in entrambi i casi, il notaio sembra utilizzare analogo sistema: egli prevede cioè la piegatura del foglio in quattro sezioni parallele al lato lungo. Le pieghe che si vengono così a formare costituiscono le direttrici di giustificazione. Il margine sinistro è riservato all'usuale rubrica, alle eventuali note e alle integrazioni, la sezione centrale viene occupata dal testo del documento, mentre il margine destro, solitamente lasciato libero nei fogli e nei bifolia, viene invece sempre occupato dallo scritto nei registri Instrumenta capitularia 8 e 8bis. L'unica sostanziale differenza, dunque, sembra essere quella del formato, praticamente quasi la metà rispetto a quello dei registri (fig. 18).

Tornando alla natura dei fogli singoli o bifolia, come già ricordato, essi trovano attualmente spazio fra le carte dei registri o, in forma sciolta, nelle tasche dei frammenti poste nei volumi. Non se ne conosce l'esatta provenienza poiché essi potrebbero essere stati concepiti direttamente come singole unità su cui redigere i documenti, oppure provenire da veri e propri prothocolli, fatto tuttavia non dimostrabile per almeno un paio di motivi. Le nuove legature cui furono sottoposti questi bifolia in anni recenti hanno rimosso del tutto eventuali segni di fori lasciati da legature coeve in fascicoli e dunque in prothocolli. Oltre a ciò, si deve constatare l'assenza di qualsiasi traccia di cartulazione (sia coeva, sia moderna) sulle carte in questione, fatto alquanto inusuale se si trattasse veramente di carte estratte da qualche registro. Non si può inoltre fare a meno di notare che se detti bifolia fossero stati tratti da un fascicolo o da un registro legato, l'ordine progressivo e originario dei documenti sarebbe andato completamente perso, essendo venuta meno la consequenzialità delle carte in seguito alla sfascicolazione. L'ipotesi, dunque, che si tratti di fogli provenienti in origine da registri oggi deperditi appare alquanto debole, tanto più se si considera il fatto che sarebbe piuttosto inconsueto, da parte di un notaio, estrapolare parti di registro per allegarle ad altri volumi. Tutt'al più potrebbero provenire da 'aggregati' di carte fra loro non soggette a legatura ma, per quanto è oggi noto, non si trovano, per il periodo e per l'area in questione, esempi di organizzazione delle carte secondo questa modalità.

Fatte le dovute considerazioni, sarà ora utile esaminare le modalità con cui ser Antonio menziona questi fogli all'interno dei propri registri. Egli infatti, in più occasioni, si riferisce distintamente ai suoi prothocolli - oggi evidentemente non più conservati - dai quali avrebbe trascritto su registri come Instrumenta capitularia 8 e 8 bis alcuni documenti, mentre in altri casi dichiara esplicitamente una diversa origine dell'atto appena trasferito, e lo fa mediante locuzioni del tipo: relevata ex uno folio o ex uno foleo ubi erat notata e ancora, relevata ex foliis ubi primiter fuit notata. Da un lato, dunque, si hanno veri e propri registri, organizzati probabilmente come gli attuali Instrumenta capitularia 8 e 8 bis ma di formato più ridotto, presumibilmente in quarto, che il professionista chiama prothocolli e che certamente avevano già pieno valore giuridico, dall'altra fogli o bifolia sciolti su cui, con le stesse modalità utilizzate per i registri, ser Antonio metteva per iscritto i documenti.

Per quanto concerne le diverse fasi di redazione, si deve supporre che il notaio operasse secondo due diverse modalità. Anzitutto, in prima fase, egli redigeva 
Antonio da Borgonuovo al lavoro

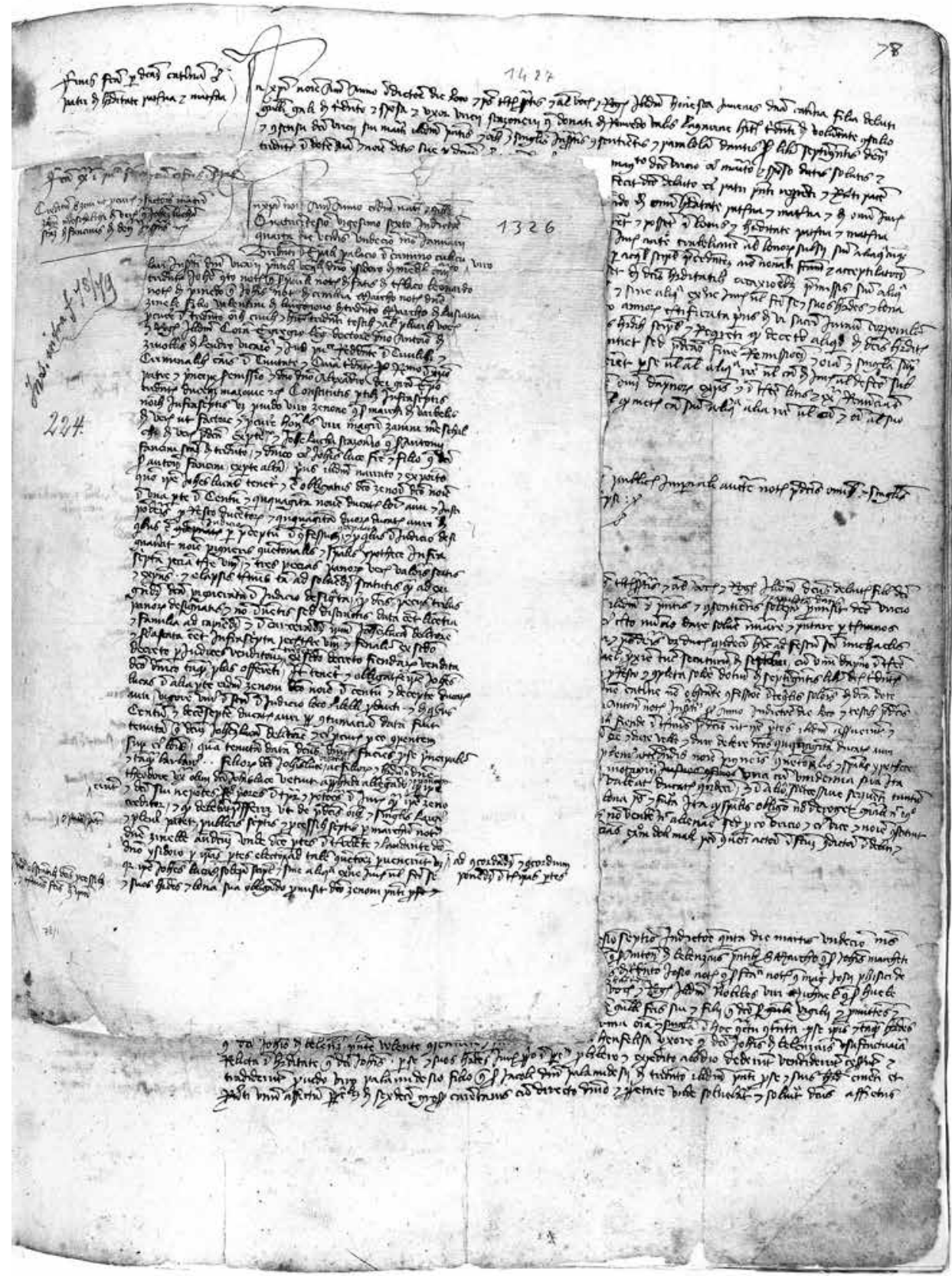

Fig. 18. ADTn, ACap, Instrumenta capitularia 8bis, bifolio fra le cc. 77 e 78 . 


\section{Stefano Malfatti}

la 'scheda' in fase di rogatio, tappa probabile ma forse non sempre praticata. Successivamente, da questa prima redazione nella forma di appunti, egli poteva passare a una forma redazionale ormai quasi completa, talvolta addirittura munita di sottoscrizione, che poteva concretizzarsi su fogli singoli o bifolia, oppure in quelli che egli definisce prothocolli. Si trattava di una 'tappa' non obbligata e non sempre praticata da Antonio, il quale, come sembra, poteva scegliere di passare direttamente o alla redazione su registri come Instrumenta capitularia 8 e 8 bis, o alla redazione di una forma abbreviata dell'atto - assimilabile a una vera e propria imbreviatura - sempre sulle carte dei suddetti volumi.

\section{I quaterni o libri}

Si svolgerà ora un rapido esame sulla terminologia adottata da ser Antonio per definire i registri che oggi si chiamano Instrumenta capitularia. Il termine impiegato dal notaio con maggiore frequenza è quaternus ${ }^{18}$, talvolta sostituito da liber $^{19}$. Così, ad esempio, nell'intestazione posta nella prima carta del registro Instrumenta capitularia 8 bis $^{20}$, dopo l'usuale invocazione alla divinità, In Christi nomine, amen, il notaio esordisce con le parole: "Quaternus instrumentorum notatorum et continuatorum per me Antonium notarium ser Bartholasii de Burgonovo de Tridento etc». I termini utilizzati dal notaio non sono casuali. Il registro 8bis, ma lo stesso vale anche per il volume del Capitolo, raccoglie instrumenta secondo due modalità: per annotazione (notatorum) e per esteso (continuatorum). Scorrendo le carte dei due volumi, infatti, si distinguono chiaramente documenti redatti in forma estesa, molto simili alle eventuali redactiones in mundum, e talvolta addirittura muniti di signum e sottoscrizione del notaio, da atti invece privi di qualunque elemento formulare, sostanzialmente abbreviati. Definire Instrumenta capitularia 8 e 8bis due registri di estesi sarebbe pertanto fuorviante, vista la duplice modalità di presentazione dei documenti. Non mancano, inoltre, riferimenti da parte di ser

18 Per l'uso del termine quaternus, che nel lessico medievale viene utilizzato per definire genericamente il fascicolo, senza tenere conto della sua reale consistenza (ossia se sia un ternione, un quinterno ecc.), cfr. S. Rizzo, Il lessico filologico degli umanisti, Edizioni di storia e letteratura, Roma 1984, p. 45; già nella tarda antichità il termine indica anche soltanto l'unione di più fogli, senza riferimento specifico al loro numero; nel medioevo, in particolare, ci sono esempi di quaternio per indicare un intero manoscritto o per un solo foglio; cfr. anche O. Weijers, Vocabulaire du livre et de l'écriture au moyen âge. Etudes sur le vocabulaire intellectuel du Moyen âge, Actes de la table tonde (Paris 24-26 septembre 1987), Brepol, Turnhout 1989, II, pp. 208-211. Ulteriore conferma a quanto detto sulla diversa consistenza del quaternus, ma applicata all'ambito archivistico, si riscontra in Romiti, L'armarium comunis della Camara actorum, cit., pp. CLXXXV-CXC; come è noto il quaternus dovrebbe consistere in otto carte, ma alla luce delle registrazioni in inventario questa affermazione non trova più riscontro materiale poiché talora il numero delle carte assegnate a questa unità archivistica è più basso di otto, talora è leggermente più elevato, talaltra si riferisce addirittura a numeri molto più alti. Secondo Romiti questa 'incoerenza' è dettata da una certa incertezza metodologica alla base dell'operare dei notai-archivisti della Camara Actorum di Bologna.

19 Secondo quanto afferma Romiti, $L$ 'armarium comunis della Camara actorum, cit., pp. CLXXXICLXXXV, il liber rappresentava un'entità già predisposta in tale forma prima del suo impiego, per il quale «non esistono tuttavia definite delimitazioni di consistenza». Ciò che emerge dall'analisi della terminologia impiegata da Antonio da Borgonuovo è che il termine quaternus e il termine liber per riferirsi a registri del tipo di Instrumenta capitularia 8 e 8 bis siano fra loro interscambiabili.

20 ADTn, ACap, Instrumenta capitularia 8bis, c. $117 r$; la carta in questione, fortemente danneggiata, è stata posta per errore a c. 117 anziché a c. 1 durante il restauro compiuto nel 1981. 
Antonio a volumi, definiti libri o quaterni, oggi deperditi, probabilmente simili nella loro facies ai registri 8 e 8bis. A tal proposito si può riportare un'annotazione che il professionista pose in corrispondenza di un'emptio su Instrumenta capitularia 8bis ${ }^{21}$. Facendo riferimento ad un atto dotale menzionato nella compravendita ivi redatta, Antonio ricorda: «Nota ad memoriam quod instrumentum huius dotis notatum est per me et continuatum super altero precedenti libro a carta 91», e certamente non può trattarsi di Instrumenta capitularia 8 che trasmette esclusivamente documenti capitolari. Ancor più esplicita della precedente è una nota posta in calce ad una refuta del 1416 sul registro del Capitolo della cattedrale:

Et nota ad memoriam quod dicta Beatrix ratificavit eadem die omnia suprascripta et aliam refutationem factam per dictum eius maritum de uno alio vineto continuatam per me super alio meo quaterno magno ut notavi in fine dicte refutationis, quam ratificationem debeo scribere in fine ambarum refutationum quas intendo facere super uno rodulo et in fine ipsius roduli postea eam ratificationem ponere etc ${ }^{22}$.

L'annotazione rivela dunque l'esistenza di un altro quaternus magnus, che si deve supporre essere stato simile, quantomeno nelle dimensioni, ad Instrumenta capitularia 8 e 8bis; il documento menzionato da ser Antonio venne redatto prima del 1416, dunque, in forma 'continuata' e non si trattava certo né dello stesso registro n. 8, che inizia sì nel 1402 ma non contiene questo atto, né tanto meno del n. 8bis che principia soltanto nel 1423. Difatti, che il notaio possedesse almeno un altro registro, per committenza forse simile al n. 8bis, pare verosimile, tenuto conto della totale assenza di registrazioni su carta per il periodo precedente al 1423 .

\section{Prothocolli e libri a confronto}

Quanto si è fin qui delineato serve a dimostrare un utilizzo parallelo, da parte del notaio, di più registri di formato diverso, prothocolli e quaterni instrumentorum; in alcuni casi ser Antonio scelse di redigere i documenti soltanto sui primi, in altri casi direttamente sui secondi, in altri ancora la scelta fu di dare concretizzazione scritta agli atti sia negli uni sia negli altri. Negli esempi che di seguito verranno esposti, si cercherà - ove possibile - di capire quali scelte furono sottese alle diverse modalità operative del notaio.

Si partirà dal già citato documento n. 353 su Instrumenta capitularia $8 b^{23}{ }^{23}$; si tratta di una semplice compravendita dove l'usuale rubrica è preceduta dalla nota relativa all'estrazione del mundum: «Facta extra distincte in uno rodulo cum solutione et cessione infrascriptis». L'annotazione marginale informa dunque che di tale documento il notaio estrasse la redactio in publicam formam, che ser Antonio definisce solitamente rodulus. Tale nota fu tuttavia affiancata da una ulteriore specificazione: «Relevata ex quaternelo prothocolli»; si deve dunque supporre che questo instrumentum rappresentasse una seconda forma redazionale rispetto a quella precedentemente approntata sul quaternelus prothocolli.

21 Ivi, n. 57.

22 Ivi, Instrumenta capitularia 8, n. 463.

23 Ivi, Instrumenta capitularia 8bis, n. 353. 


\section{Stefano Malfatti}

Le modalità redazionali di Antonio non si esauriscono con l'esempio appena citato; se infatti si prende il caso del documento n. 350 su Instrumenta capitularia $8^{24}$, lo schema suesposto sembra complicarsi. L'atto rappresenta una locazione perpetua in forma di esteso la cui rubrica recita: «Locatio perpetualis Floris de Barbarola de domo sua de Burgonovo cum revocatione cuiusdam locationis de dicta domo facte magistro Zampetro sartori». La nota relativa all'estrazione del mundum, «Facta extra duplex ipsi Saraceno», informa inoltre che di tale rogito vennero estratte ben due pergamene in mundum. L'interesse di questo documento deriva dal fatto che, dello stesso, si ha a disposizione anche un'ulteriore forma redazionale su bifolio cartaceo che misura $300 \mathrm{~mm}$ in altezza e $220 \mathrm{~mm}$ in larghezza, oggi ospitato in appendice al registro Instrumenta capitularia 3 del notaio Venturino de Trechis ${ }^{25}$. La rubrica ivi redatta da ser Antonio recita, diversamente dalla precedente, «Revocatio cuiusdam locationis perpetualis facte per dominos canonicos male informatos magistro Zampetro sartori et renovatio facta in Florem cuius primo erat domus etc». La collazione fra gli esemplari consentirà di rilevare le differenze fra $\mathrm{i}$ due documenti.

\section{Redazione su bifolio}

In Christi nomine, amen. Anno eiusdem nativitatis millesimo quatricentesimo decimo octavo, indictione undecima, die lune vigesimo primo mensis februarii, Tridenti, in contrata Burginovi, in stupa domus solite habitationis venerabilis viri domini Iohannis de Isnina bakalarii in decretis sbakalarii in decretis nel margine superiore con segno di inserzione sottostanter, decani et canonici infrascripti, presentibus honestis viris honestis viris nel margine superiore con segno di inserzione sottostante> magistro Hulrico de Isnina plebano plebis de Paho de supra Tridentum, Martino Denklinger de Augusta plebano plebis Sancti Sisini vallis Ananie diocesis Tridentine, Narcischo Taymel de Nurlinga familiare dicti domini decani, Hulrico de Isna familiare domini Iohannis de Flemis infrascripti testibus et aliis multis vocatis et rogatis.

Ibidem expressum fuit et dictum cum venerabilis vir dominus Henricus Milauner de Merano archidiaconus et canonicus Tridentinus de voluntate et consensu venerabilium virorum

\section{Redazione su Instrumenta capitularia 8}

In Christi nomine, amen. Anno eiusdem nativitatis millesimo quatricentesimo decimo octavo, indictione undecima, die lune vigesimo primo mensis februarii, Tridenti, in contrata Burginovi, in stupa domus solite habitationis venerabilis viri domini Iohannis de Isnina bakalarii in decretis, decani et canonici Tridentini inferius nominati,

presentibus honestis viris magistro Hulrico de Isnina plebano plebis de Paho de supra Tridentum, Martino Denklinger de Augusta plebano plebis Sancti Sisini de vale Anania diocesis Tridentine, Narcisco Thymel de Nurlinga familiare dicti domini decani, Hulrico de Isna familiare domini Iohannis de Flemis infrascripti testibus et aliis multis vocatis et rogatis.

Ibidem expressum fuit et dictum cum venerabilis vir dominus Henricus Milauner de Merano archidiaconus et canonicus Tridentinus de voluntate et consensu venerabilium virorum

24 Ivi, Instrumenta capitularia 8, n. 350.

25 Ivi, Instrumenta capitularia 3, n. 115; sulla presenza di questo bifolio di mano di ser Antonio fra le carte di Venturino de Trechis si può abbozzare soltanto qualche ipotesi; esso può infatti esservi finito casualmente nel corso dei secoli o in seguito al restauro del volume nel 1981, insieme ad altri fogli e bifolia di mani eterogenee; può altresì essere possibile che esso vi sia finito ancora all'epoca di Antonio da Borgonuovo considerato che nel 1430 il notaio ottenne l'autorizzazione ad estrarre documenti in originale dai rogiti di Venturino (cfr. ADTn, ACap, capsa 44, n. 5). 


\section{Antonio da Borgonuovo al lavoro}

dominorum Iohannis de Isnina decani et canonici Tridentini, Vigilii de Tridento, Florii de Eno, Zamboni de Tridento et Iohannis de Karinthia et (Iohannis de Karinthia et fuori dallo specchio di scrittura Iohannis Zeis de Bopfingen «Bopfingen corretto su termine precedente non decifrabile〉 canonicorum ecclesie Tridentine et ipsi domini canonici cum eo domino archidiacono in preiudicium Floris filie condam 〈segue Peti depennato〉 Dominici filii condam Petri filii condam ser Ordani de Barbarola habitatoris Tridenti in contrata Burginovi in domo infrascripta, minoris et in legitima etate non existentis et 〈segue habitantis tamquam depennato〉 orphane et private parentibus habitantis in vale Flemarum shabitantis in vale Flemarum nel margine destro con segno di richiamo> cum provido viro Guillelmo notario dicto Saraceno de Tridento qui tenebat et possidebat unam domum muris et lignamine edificatam sitam in civitate Tridenti in contrata Burginovi apud Nicholaum notarium condam ser Michaelis de Aproynis de Tridento ab una parte et de retro, apud dominum Petrum de Matarelo ab alia parte et apud viam comunis de ante et forte apud alios, de qua domo solvitur, solvi debet et consuetum est solvi perpetualiter omni anno in festo sancti Michaelis vel infra eius octavam archidiaconatui ecclesie Tridentine affictus perpetualis vigintiquinque solidorum denariorum Tridentinorum ob dictum affictum retentum et non solutum per triennium et ultra per eam in pupilari <-p-corretta su precedente -1-> etate relictam et privatam patre suo et ignorantem se teneri dictum affictum de dicta domo tamquam male informati a magistro Zampetro infrascripto «a «segue dicto depennato> Zampetro infrascripto aggiunto nel margine destro con inchiostro di tonalità più chiara de condictione et qualitate dicte Floris, eam Florem minorem et non requisitam inconsulte sinconsulte nel margine sinistro, preceduto da nullo actu iudiciali de contra eam facto depennatos, privando de dicta domo tenuta et possessa per eius Floris patrem «Floris nell'interlinea superiore con patrem aggiunto in seguito con inchiostro di tonalità più chiara), avum et bisavum predictos pluribus treginta annis continuis nulla actu iudiciali nec sactu iudiciali nec in interlinea superiore con inchiostro di tonalità dominorum Iohannis de Isnina decani et canonici Tridentini, Vigilii de Tridento, Florii de Enno, Zamboni de Tridento, Iohannis de Carinthia et Iohannis Zeis de Bophingen canonicorum dicte ecclesie Tridentine et ipsi domini canonici cum eo domino archidiacono in preiudicium Floris filie condam Dominici filii condam Petri filii condam ser Ordani de Barbarola habitatoris Tridenti in contrata Burginovi in domo infrascripta, minoris et in legitima etate non existentis et orphane et private parentibus habitantis in vale Flemarum cum provido viro Guillelmo notario dicto Saraceno de Tridento qui tenebat et possidebat unam domum muris et lignamine edificatam sitam in civitate Tridenti

in contrata Burginovi apud Nicholaum notarium condam ser Michaelis de Aproinis de Tridento ab una parte et de retro, apud dominum Petrum de Matarelo ab alia parte et apud viam comunis de ante et forte apud alios confines veriores, de qua domo solvitur, solvi debet et consuetum est solvi perpetualiter omni anno in festo sancti Michaelis vel infra eius octavam beneficio archidiaconatus ecclesie Tridentine predicte affictus perpetualis vigintiquinque solidorum denariorum Tridentinorum ob dictum affictum retentum et non solutum per triennium et ultra per eam in pupilari || etate relictam, patre suo «suo in interlinea superiore) privatam et ignorantem se teneri dictum affictum de dicta domo tamquam male et sinistre ret sinistre in interlinea superiores informati a magistro Zampetro infrascripto de condictione et qualitate dicte Floris, eam Florem minorem et non requisitam inconsulte, privando de dicta domo tenuta et possessa per eius Floris patrem, avum et bisavum predictos pluribus treginta annis continuis continuis in interlinea superiore nulla actu iudiciali nec processu facto nec agitato contra eam Florem minorem absentem et indeffenssam iure et nomine locationis et conductionis in perpetuum secundum usum et consuetudinem locationum in perpetuum dicte ecclesie Tridentine de dicta domo ssegue segno di richiamo che rimanda a integrazione mar- 


\section{Stefano Malfatti}

più chiara $>$ processu facto nec agitato contra eam Florem minorem 〈segue et depennato〉 absentem et indefenssam <et indefenssam aggiunto in seguito con inchiostro di tonalità più chiara iure et nomine locationis et conductionis in perpetuum secundum usum et consuetudinem locationum in perpetuum dicte ecclesie Tridentine de dicta domo investiverunt magistrum Zampetrum de Papia sartorem in Tridento cum certis pactis et condictionibus in ipsa locatione nominata per dominum presbiterum Perhengerium de Melango capelanum et sacristam in dicta ecclesia Tridentina descriptis.

Et cum post hec venerabiles viri dicti domini Iohannes de Isnina decanus, Vigilius, Florius, Iohannes de Karinthia et Iohannes capelanus plebanus in dicta vale Flemarum omnes canonici dicte ecclesie Tridentine ibidem capitulariter pro presenti actu et contractu fiendo eligentes sibi locum ibidem nullis pluribus de canonicis dicte ecclesie in civitate existentibus qui hinc actui et contractui comode interesse possint sed totum capitulum ipsius ecclesie representantes «venerabiles ... representantes nel margine sinistro con segno di richiamo> audita querela et iusta et iusta nel margine superiore con segno di inserzione sottostanter excusatione et supplicatione excusatione et supplicatione nel margine destro> ipsius Floris minoris seu dicti Guillelmi notarii Saraceni pro ea et eius vice et nomine exponentis, quod ipsa Flos in pupilari etate relicta, patre privata et a matre derelicta et nullum attinentem habens 〈habens nel margine superiore con segno di inserzione sottostante nec ex parte patris nec ex parte matris in Tridento et male tractata a dicto magistro Zampetro cum quo olim steterat se ab ipso separavit et ad habitandum ivit cum ipso Guillelmo notario et ignorabat se teneri aliquem affictum de domo sua prelibata, omnibus iure, via, modo et forma quibus melius potuerunt $\|$ accasaverunt et irritaverunt dictam locationem factam per eos de dicta domo magistro Zampetro prelibato et eam cassam et nullam et nullius valoris esse voluerunt et mandaverunt attendentes quod falsus precator deb(et) carere impetratis tamquam male informati ab ipso magistro Zampetro <attendentes ... Zampetro nel margine sinistro con inchiostro di tonalità più chiara> et ipsam Florem minorem et ginale depennata, di cui si legge: [...]ale et pessime informati> investiverunt magistrum Zampetrum de Papia sartorem in Tridento cum certis pactis et condictionibus in ipsa locatione nominata per dominum presbiterum Perhingerium de Melango capelanum et sacristam in dicta ecclesia Tridentina descriptis.

Et cum post hec venerabiles viri dicti domini Iohannes de Isnina decanus, Vigilius, Florius, Iohannes de Karinthia et Iohannes capelanus plebanus in dicta vale Flemarum omnes canonici dicte ecclesie Tridentine ibidem pro presenti actu et contractu fiendo locum eligentes capitulariter congregati nullis pluribus de canonicis dicte ecclesie in civitate existentibus qui huic actui et contractui comode «segue comode iterato) interesse possint sed totum capitulum ipsius ecclesie representantes, audita querela et iusta excusatione et supplicatione ipsius Floris minoris seu dicti Guillelmi Saraceni notarii pro ea et eius nomine et vice exponentis, quod ipsa Flos in pupilari etate relicta, patre privata et a matre maritata in Vesentina, derelicta et nullum attinente habens nec ex parte patris nec ex parte matris in Tridento et male tractata a dicto magistro Zampetro cum quo olim steterat decepta <decepta in interlinea superiore con segno di inserzione sottostanter se ab ipso magistro Zampetro separavit et ad habitandum ivit cum ipso Guillelmo et ignorabat se teneri aliquem affictum de dicta domo, omnibus iure, via, modo et forma quibus melius potuerunt accasaverunt et irritaverunt dictam locationem factam per eos de dicta domo ipsi magistro Zampetro et eam cassam et nullam et nullius valoris esse voluerunt et mandaverunt cum deliberatione prehabita et consciderato quod falsus precator carere debet impetratis; et ipsam Florem minorem et dictum Guillelmum notarium Saracenum eius procuratorem et procuratorio nomine pro ea et vice et nomine ipsius Floris facientem, petentem et supplicantem integrum restituerunt et ad ipsum statum tenute dicte domus reduxerunt 
dictum 〈d- corretto su lettera precedente〉 Guillelmum notarium Saracenum eius procuratorem et procuratorio nomine pro ea et vice et nomine ipius Floris petentem et supplicantem in integrum restituerunt et ad pristinum statum tenute stenute nel margine destro con segno di richiamo e con inchiostro di tonalità più chiara; segue, nel rigo successivo, restituerunt depennato> reduxerunt et locationem perpetualem de dicta domo in personam ipsius Floris renovando, iure et nomine renovationis «renovationis nell'interlinea superiore con segno di inserzione sottostanter locationis et conductionis in perpetuum secundum usum et consuetudinem locationum in perpetuum dicte ecclesie Tridentine et pro causa infrascripta pro uno ducato auri ibidem et in presenti per dictum Guillelmum notarium dato et soluto dicto domino Iohanni de Karinthia ut factori et 〈factori et nell'interlinea superiore), procuratori et procuratorio dicti domini archidiaconi ibidem presenti et recipienti in solutionem et pro solutione affictuum retentorum de dicta domo usque ad presentem diem, et asserenti habere in mandatis a dicto domino archidiacono faciendi revocari dictam locationem factam dicto magistro Zampetro et faciendi investiri ipsam Florem de domo sua predicta omnibus iure, via, modo et forma quibus melius potuerunt nunc plene de bona fama et honestate dicte Floris et de veritate informati «segue segno di richiamo a integrazione in margine depennata e di inchiostro di tonalità più chiara che recita: pietate moti et faventes minoribus taliter visis ut de supra tenentur> investiverunt dictam Florem absentem et dictum Guillelmum notarium Saracenum eius Floris procuratorem et procuratorio nomine pro ea et vice et nomine ipsius petentem et recipientem de dicta domo superius confinata sive de eius utili dominio, ad habendum, tenendum et possidendum et quicquid sibi Flori et suis heredibus deinceps placuerit perpetuo faciendum, salvo iure directi domini et salvis pactis et condictionibus infrascriptis cum omnibus et singulis que infra predictos continetur confines vel alios si qui forent ulterius veriores accessibus et egressibus suis usque in viam publicam et cum omnibus et singulis que dicta domus habent supra se, infra se seu intra se in integrum omnique iure et actione, usu seu requisitione «segue et restituerunt et locationem perpetualem de dicta domo in persona ipsius Floris renovando iure et nomine renovationis locationis et conductionis in perpetuum secundum usum et consuetudinem locationum in perpetuum dicte ecclesie Tridentine et pacta infrascripta pro uno ducato auri ibidem et in presenti per dictum Guillelmum notarium dato et soluto dicto domino Iohanni de Carinthia ut factori et procuratori et procuratorio nomine dicti domini archidiaconi ibidem presenti et recipienti in solutionem et pro solutione affictuum de dicta domo retentorum usque ad presentem diem et asserenti se habere in mandatis a dicto domino archidiacono faciendi revocari dictam locationem factam dicto magistro Zampetro et faciendi investiri ipsam Florem de domo sua predicta, et ipsi Flori minori taliter «segue lese espunto〉 inopinate providere volentes omnibus iure, via, modo et forma quibus melius potuerunt nunc de veritate, fama et honestate dicte Floris plene informati investiverunt ipsam Florem absentem et dictum Guillelmum notarium Saracenum eius Floris procuratorem et procuratorio nomine pro ea et vice et nomine ipse petentem et recipientem de dicta domo superius confinata sive de eius utili dominio, ad habendum, tenendum et possidendum et quicquid sibi Flori et suis heredibus deinceps placuerit perpetuo faciendum salvo iure directi dominii et salvis pactis et condictionibus infrascriptis cum omnibus et singulis que infra predictos continetur confines vel alios si qui forent ulterius veriores accessibus et egressibus suis usque in viam publicam et cum omnibus et singulis que dicta domus locata habet supra se, infra se seu intra se in integrum omnique iure et actione, usu seu requisitione ad ipsam domum locatam modo aliquo spectante et pertinente, cuius domus possessionem quam ipsa Flos et condam eius pater, avis et bisavis predicti habebant eidem Flori et dicto eius procuratori pro ea presenti et requirenti confirmaverunt ac dederunt 〈de- in interlinea superiore licentiam et plenam auctoritatem eidem Flori et dicto eius procuratori pro ea intrandi de novo in tenutam et corporalem possessionem dicte domus et eam domum tenendi et pacifice possidendi ut ante locationem factam dicto magistro solita erat tenere et possidere; et promiserunt ipsi domini locatores solempni 


\section{Stefano Malfatti}

sibi depennato> ad ipsam domum locatam modo aliquo spectante et pertinente, cuius domus possessionem quam ipsa Flos et condam eius pater, avis et bisavis predicti upredicti nel margine superiore con segno di inserzione sottostante> habebant eidem Flori et dicto eius procuratori pro ea recipienti confirmaverunt ac dederunt licentiam et plenam auctoritatem eidem Flori et dicto eius procuratori pro ea intrandi de novo in tenutam et corporalem possessionem dicte domus et eam domus tenendi et pacifice possidendi, ut ante locationem factam dicto magistro Zampetro solita erat tenere et possidere; et promiserunt ipsi domini locatores solempni stipulatione dicto Guillelmo Saraceno notario dicto nomine stipulanti et michi Antonio notario infrascripto ut publice persone stipulanti et recipienti dicte Floris minoris et pro ea de evictione 〈segue de depennato〉 dicte domus locate et de eius legitima deffensione secundum formam iuris et de ratificatione omnium et singulorum premissorum sub obligatione omnium bonorum dicti archidiaconatus presentium et futurorum et sub refectione omnium dampnorum expensarum et interesse litis et extra.

Et predicta fecerunt dicti domini locatores nominatim ex eo quia dictus Guillelmus Saracenus notarius dicto procuratorio habens plenum et specialem mandatum ad infrascripta et ad alia plura peragenda ut ibidem asseruit solempni stipulatione et sine aliqua exceptione iuris vel facti dictam Florem et eius heredes et bona obligando promisit dictis dominis canonicis presentibus pro se et suis successoribus pro archidiaconatu predicto stipulantibus dictam domum reparare et de bono in melius ipsam promovere et perpetualiter omni anno in festo sancti Michaelis vel infra eius octavam dare, solvere, numerare et presentare $\|$ domino archidiacono dicte ecclesie Tridentine qui nunc est et pro tempore fuerit ad domum sue solite habitationis in Tridento soldos viginti quinque denariorum Tridentinorum nomine affictus et censsus perpetuus dicte domus locate cum hiis pactis et condictionibus ibidem expressis et solepni stipulatione valatis videlicet quod primo anno quo ipsa Flos dictum affictum non solverit et presentaverit infra terminum predictum tunc duplum ipsius affictus dare et solvere teneatur ... stipulatione dicto Guillelmo notario Saraceno dicto nomine stipulante et michi Antonio notario infrascripto ut publice persone stipulanti et recipienti vice et nomine dicte Floris minoris et pro ea «segue stip(u)l(anti) depennato> de evictione dicte domus locate et de eius legitima deffensione secundum formam iuris et de ratificatione omnium et singulorum premissorum sub obligatione omnium bonorum beneficii dicti archidiaconatus presentium et futurorum et sub refectione omnium dampnorum, expensarum et interesse litis et extra.

Et predicta fecerunt dicti domini locatori nominatim ex eo quia dictus Guillelmus Saracenus notario dicto procuratorio nomine habens plenum et specialem mandatum ad infrascripta et ad alia plura peragenda ut ibidem asseruit solempni stipulatione et sine aliqua exceptione iuris vel facti dictam Florem et eius heredes et bona obligando promisit dictis dominis canonicis presentibus et pro se et successoribus suis quod beneficio archidiaconatus predicti stipulant(ibus) dictam domum reparare et de bono in melius ipsam promovere et perpetualiter omni anno in festo sancti Michaelis vel infra eius octavam dare, solvere, numerare et presentare domino archidiacono dicte ecclesie Tridentine qui nunc est vel pro tempore fuerit ad domum sue solite habitationis in Tridento soldos viginti quinque denariorum Tridentinorum nomine affictus et census perpetui dicte domus locate, cum hiis pactis et condictionibus ibidem expressis et solempni stipulatione valatis videlicet quod primo anno quo ipsa Flos dictum affictum integraliter non solverint et presentaverint infra terminum predictum tunc duplum ipsius affictus dare et solvere teneatur ... 
Il confronto fra i due esemplari palesa immediatamente affinità e divergenze; anzitutto si nota che il documento in bifolio (nella colonna di sinistra) rappresenta una fase redazionale precedente a quello in Instrumenta capitularia 8 (nella colonna di destra); sono infatti ben visibili nel primo le numerose integrazioni e aggiunte ${ }^{26}$ che il notaio, dopo aver redatto il testo in prima stesura, inserì in interlinea e nei margini delle carte; in seconda stesura, invece, tali aggiunte furono completamente integrate nel testo. Si tratta, dunque, di due distinti livelli redazionali dello stesso atto, per cui il confronto fra i due scritti evidenzia la perfetta corrispondenza fra $\mathrm{i}$ testi, salvo rarissime e circoscritte difformità, tali comunque da non mettere in dubbio l'ipotesi che, nel caso del documento redatto in Instrumenta capitularia 8, ci si trovi di fronte alla messa per iscritto 'in bella copia' del documento precedentemente steso su bifoglio sciolto e poi integrato in seconda battuta con aggiunte sostanziali. Ulteriore motivo d'interesse di questo documento è dato dal fatto che in margine al testo sul registro capitolare si trova una nota relativa all'estrazione del mundum, o, per meglio dire, dei munda: «Facta extra duplex videlicet locatori et conductori». Da ciò si deduce che di tale documento il notaio estrasse due redactiones su pergamena, una per il locatore, l'altra per il conduttore. Sebbene non si sia conservata alcuna scheda né minuta preparatoria, risulta alquanto improbabile che ser Antonio non abbia redatto, al momento della rogatio, i consueti appunti da cui avrebbe poi tratto la stesura più completa, magari con accanto un formulario ${ }^{27}$, rappresentata dalla redazione su bifolio. Nel qual caso, egli avrebbe 'percorso' ben tre tappe redazionali prima di arrivare alle due pergamene in mundum: la scheda o minuta, il documento su bifolio, la redazione sul registro del Capitolo.

$\mathrm{Ci}$ si deve ora interrogare su quali furono le motivazioni che spinsero il notaio, in talune occasioni e non in altre, a redigere l'instrumentum sia sui prothocolli (o, come nel caso appena citato, su bifolia) sia sui quaterni instrumentorum. Si può osservare, a tal proposito, come praticamente tutti i documenti registrati in libro e dichiarati dal notaio come relevati ex prothocollis o ex foliis vadano ad interrompere la successione cronologica dei documenti; sembrerebbe, dunque, che questi atti siano stati inseriti nel registro sebbene, in un primo tempo, non ne fosse prevista una loro registrazione in quella sede.

Un secondo interrogativo può essere posto sulle ragioni per cui soltanto alcuni documenti trovarono concretizzazione scritta, o in esteso o in forma abbreviata, direttamente nei quaterni mentre altri si fermarono alla redazione nei prothocolli e, soltanto in casi limitati, furono successivamente trascritti anche nei registri di formato maggiore. La risposta non è univoca, pertanto si elaboreranno una serie di ipotesi. Se si verifica, in un registro come Instrumenta capitularia 8bis, quali tipologie documentarie sono presenti, si noterà l'assenza dei testamenti per i quali, tuttavia, non mancano nel periodo di riferimento gli esempi su pergamena sciolta ${ }^{28}$.

$26 \mathrm{Si}$ è scelto di conservare nel testo, entro parentesi uncinate, le integrazioni e le correzioni che il notaio attuò sul documento, così da rendere palesi, anche nell'edizione, le difformità rispetto all'atto in Instrumenta capitularia 8.

27 Alcune ripetizioni nel formulario e alcune sviste del documento su bifolio (nella colonna di sinistra) lascerebbero intravvedere la possibilità che ser Antonio avesse redatto il documento tenendo accanto un formulario; ad esempio, quel «tunc ipse Flos», dove il nome proprio è stato corretto, in fase di revisione, su un precedente «conductor»; poche righe più sotto il notaio itera l'avverbio dummodo, il che talvolta accade nella copiatura, disattenta, da un formulario o da un testo.

${ }_{28}$ Cfr. P. Cammarosano, Italia medievale. Struttura e geografia delle fonti scritte, Nuova Italia Scientifica, Roma 1991, p. 274, dove si ricorda come i testamenti trovano sovente spazio in registri ad hoc. 


\section{Stefano Malfatti}

Per altre tipologie, come le procure, si rilevano pochissimi esempi e, ove esse sono presenti, sono sovente associate a determinati documenti, oppure - per citare le note del notaio - sono state «relevate ex prothocollo» o «ex uno folio ubi erant notate». In alcuni casi, dunque, è possibile che l'assenza di specifiche tipologie documentarie dai quaterni dipenda dall'oggetto trattato nei documenti stessi; tale spiegazione è tuttavia insufficiente poiché nei registri si trovano anche alcune compravendite, almeno una donazione e una locazione che ser Antonio dichiara di aver estratto ex prothocollo. Un'ulteriore motivazione che può in qualche modo giustificare la presenza di questi rogiti nei quaterni, fuori dal normale ordine cronologico, può essere ricondotta alla necessità da parte del notaio di redigerne la pergamena in mundum; la discordanza nell'ordine cronologico deriverebbe dunque dal fatto che ser Antonio vi trascrisse l'atto soltanto nel momento in cui redasse la pergamena su richiesta dei committenti; ciò potrebbe essere avvenuto qualche giorno dopo $\mathrm{o}$, addirittura, qualche mese o anno più tardi. Pure questa spiegazione, tuttavia, non chiarisce del tutto il problema, poiché in alcuni, limitati casi non c'è alcuna annotazione relativa all'estrazione del mundum sebbene il documento fosse posto sia sul prothocollo o sui bifolia, sia sul quaternus instrumentorum. In altri casi, inoltre, le note relative all'estrazione del mundum poste a margine dei documenti sui bifolia mostrano che ser Antonio, se necessario, trasse la redactio in publicam formam direttamente da essi. A tal proposito si può verificare quanto affermano le norme statutarie relative ai notai nel periodo in questione. Vista l'assenza di capitoli specificamente dedicati al tema della redazione dell'instrumentum notarile negli statuti masoviani del 1425-1427, ci si deve avvalere delle norme emanate nel 1425 a Rovereto ${ }^{29}$; il capitolo 61, intitolato «De tabellionibus debentibus scribere imbreviaturas suas in libro», ricorda a tal proposito:

Item statuimus et ordinamus quod quilibet tabellio debeat et teneatur in libris et non in cetulis suis ponere imbreviaturas suas; et si contrafecerint pro qualibet vice solvant XX soldos Veronensium ducali camere, et sub dicta pena et damnum et interesse partibus reficienda scribere debeant in libris imbreviaturarum actorum et contractuum antequam dent partibus instrumenta, et ponatur imbreviature instrumentorum in quaternis sub dicta pena ${ }^{30}$.

La disposizione è dunque molto chiara sulle modalità operative richieste ai notai sia a tutela delle parti, con la conservazione dei documenti in libris e non in cetulis, ossia in fogli sciolti, al fine di evitare facili dispersioni dei rogiti; sia riguardo alla registrazione degli atti in libris imbreviaturarum, actorum et contractuum prima della consegna alle parti dell'instrumentum, ovvero - si deve intendere - della redactio in mundum. Tale norma, dunque, sembrerebbe deporre a favore dell'ipotesi che l'inserimento dei documenti nei quaterni instrumentorum avvenne soltanto nel momento in cui ser Antonio estrasse la pergamena in mundum, portando così ad un'interruzione dell'ordine cronologico dei documenti.

Alle modalità fin qui descritte, tuttavia, va aggiunto un ulteriore modus operandi. Scorrendo le carte dei due registri, infatti, si notano intere pagine rimaste bianche in cui il notaio scelse di redigere la sola rubrica relativa al documento, rinviando per quanto concerne l'atto vero e proprio a fogli o bifolia allegati al volume; si

29 Statuti di Rovereto del 1425, cit.

30 Ivi, p. 178. 
trovano così annotazioni quali «ut in folio hic posito»o «ut in folio hic inserto». È il caso della rubrica posta alla carta $241 r$ del registro Instrumenta capitularia 8bis, la quale recita: «Conventio cum procura facta in Dominicum et Iohannem Zeschi Floris de Voltolina continuata hic in folio inserto ${ }^{31}$. La pagina è lasciata completamente bianca mentre il foglio inserto di cui parla Antonio è un bifolio e si trova attualmente fra le carte 240 e 241 dello stesso registro.

Alla carta $55 r$ del registro Instrumenta capitularia 8bis si trova un'ulteriore pagina, in cui il notaio si è premunito di redigere la rubrica e, con inchiostro di analoga tonalità, ulteriori due annotazioni ${ }^{32}$; si tratta di una «Conventio et promissio facta per dominam Constanciam de Nigris comunitati Tridenti de solvendo ducatos III $^{\mathrm{C}}$ heredibus condam domini Herasmi et absolutio facta comunitati per dominum nostrum cum cessione etc ut infra». Ser Antonio ricorda poi che questa promissio, della quale tuttavia non procede alla trascrizione, fu «Relevata ex uno folio ubi erat notata», foglio che ancora oggi si trova fra le carte 75 e 76 del registro. Il professionista aggiunge poi, sotto la rubrica, la nota relativa all'estrazione della pergamena che venne «Facta extra ex ipso folio», ossia direttamente dal bifolio sciolto.

Un ulteriore esempio, che sembra ancor più esplicativo del modus operandi del notaio, è quello del documento n. 646 del registro n. 8bis. Come nel caso precedente, ser Antonio appose sul quaternus instrumentorum la sola rubrica relativa all'atto - nel caso in questione una elezione a canonico - senza redigerne la forma 'continuata' ma apponendo la sola nota relativa all'estrazione del mundum, che recita: «Facta extra ut hic in folio inserto etc» (fig. 19).

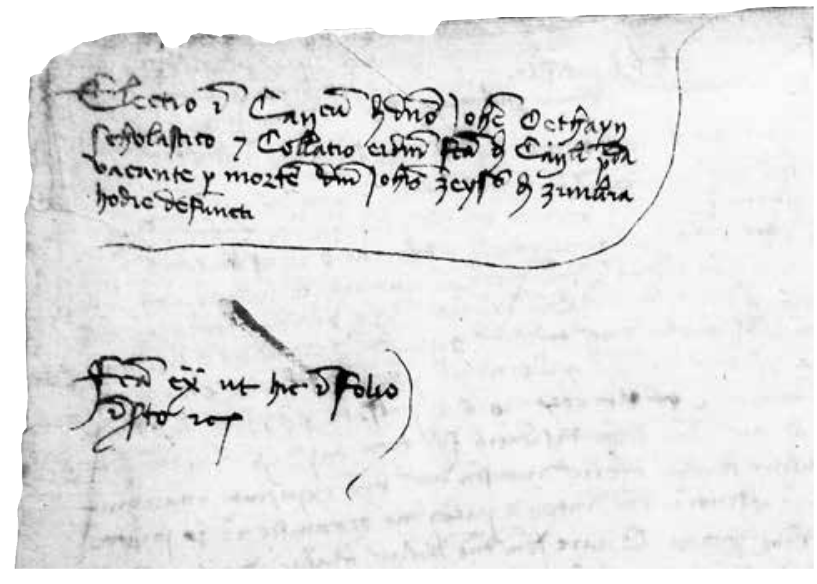

Fig. 19. ADTn, ACap, Instrumenta capitularia 8bis, c. $248 v$ (particolare). Rubrica e, sotto, la nota relativa all'estrazione del mundum. Bianco il resto della carta.

Il folius insertus di cui parla il notaio è pure un bifolio cartaceo che veicola il documento in forma pressoché completa, dotato addirittura di un 'abbozzo' di sottoscrizione, «Ego Antonius etc». Ma rilevante, in questo caso, è una seconda sottoscrizione, completa rispetto alla prima, che Antonio aggiunse all'inizio della carta successiva:

Ego Antonius condam ser Bartholasii de Burgonovo de Tridento civis Tridentinus, publicus imperiali auctoritate notarius predictis electioni, collationi, promissioni,

31 ADTn, ACap, Instrumenta capitularia 8bis, c. $241 r$.

32 Ivi, c. $55 r$. 
inductioni in tenutam captioni tenute et protestationi et omnibus aliis et singulis premissis dum sic agerentur et fierent ut premissus est, una cum dictis testibus interfui, vidi et sic audivi. Et quia ego rogatus et requisitus infirmitate oppressus predictam in formam publicam ad presens scribere non valui, alteri scribendum comissi et in fidem et verum testimonium premissorum me hic subscripsi et signum mei tabellionatus consuetum hic apposui et signavi ${ }^{33}$.

Da quanto si deduce, dunque, essendogli stata richiesta la redazione in mundum del documento e non essendo stato in grado di farlo perché infirmitate oppressus, egli appose sul quaternus instrumentorum la sola rubrica, tralasciando di copiarvi l'intero documento in esteso e dando ad altri il compito di redigerne il mundum. Questa modalità operativa di Antonio attesterebbe, in questo caso, la necessità da parte del notaio di redigere, o almeno annotare, sul registro i documenti di cui trasse la pergamena, così come previsto dal capitolo statutario cui si fatto cenno sopra (fig. 20).

\section{Documenti in forma abbreviata redatti direttamente sui quaterni}

L'ultima casistica che si prenderà in considerazione riguarda un certo numero di documenti posti sui registri Instrumenta capitularia 8 e 8 bis. Si tratta, come già ricordato in più occasioni, di quaterni instrumentorum notatorum et continuatorum. In molti casi, infatti, si tratta di vere e proprie imbreviature in cui il notaio, nella forma di una rubrica più ricca di dati rispetto alle usuali, e predisponendo una diversa mise en page (il testo è addossato al margine sinistro, mentre viene lasciato completamente libero il destro), redige un documento che - pur contenendo i dati essenziali alla sua validità giuridica - è presentato in forma sostanzialmente ridotta, abbreviata. Ciò è vero soprattutto per determinate tipologie documentarie, quali ad esempio le attestazioni di debito, le quietanze, le carte finis ecc., ovvero atti generalmente connessi per datazione e per altri elementi ad un documento precedente (una compravendita, una locazione, un matrimonio ecc.). In talune occasioni, tuttavia, ser Antonio scelse di redigere in forma abbreviata anche documenti quali le stesse compravendite, locazioni ecc., soprattutto nel caso in cui queste fossero poste consecutivamente dopo negozi dello stesso tipo, richiesti dagli stessi clienti e, talvolta, rogati nella stessa giornata; si tratta di casi in cui, vista la brevità del testo, è lecito supporre che il notaio avesse redatto i documenti prendendo i dati direttamente dalla 'scheda' o, addirittura, se la rogatio avvenne nella sua abitazione, senza alcun passaggio intermedio.

Un ulteriore esempio sarà dedicato ai documenti 86 e 87 nel registro Instrumenta capitularia 8bis ${ }^{34}$; si tratta, rispettivamente, del matrimonio fra Giovanni Rauter e Andriota figlia di Bonadomano de Accerbis. Esso è redatto per esteso e nelle forme consuete ed è fatto seguire dalla carta finis attraverso la quale Andriota dichiarò al padre, lì presente anche in rappresentanza della moglie Antonia, di non richiedere più nulla di quanto le sarebbe spettato dell'eredità, in ragione della dote ricevuta. Questo secondo documento sembra essere stato steso contestualmente a quello attestante il matrimonio, come dimostra la medesima tonalità dell'inchiostro; mentre, tuttavia, il primo venne redatto per esteso, il secondo è presentato in forma abbreviata, pur con

33 Ivi, c. $248 / 1$, n. 646.

34 Cfr., a tal proposito, i documenti ivi, nn. 86 e 87. 


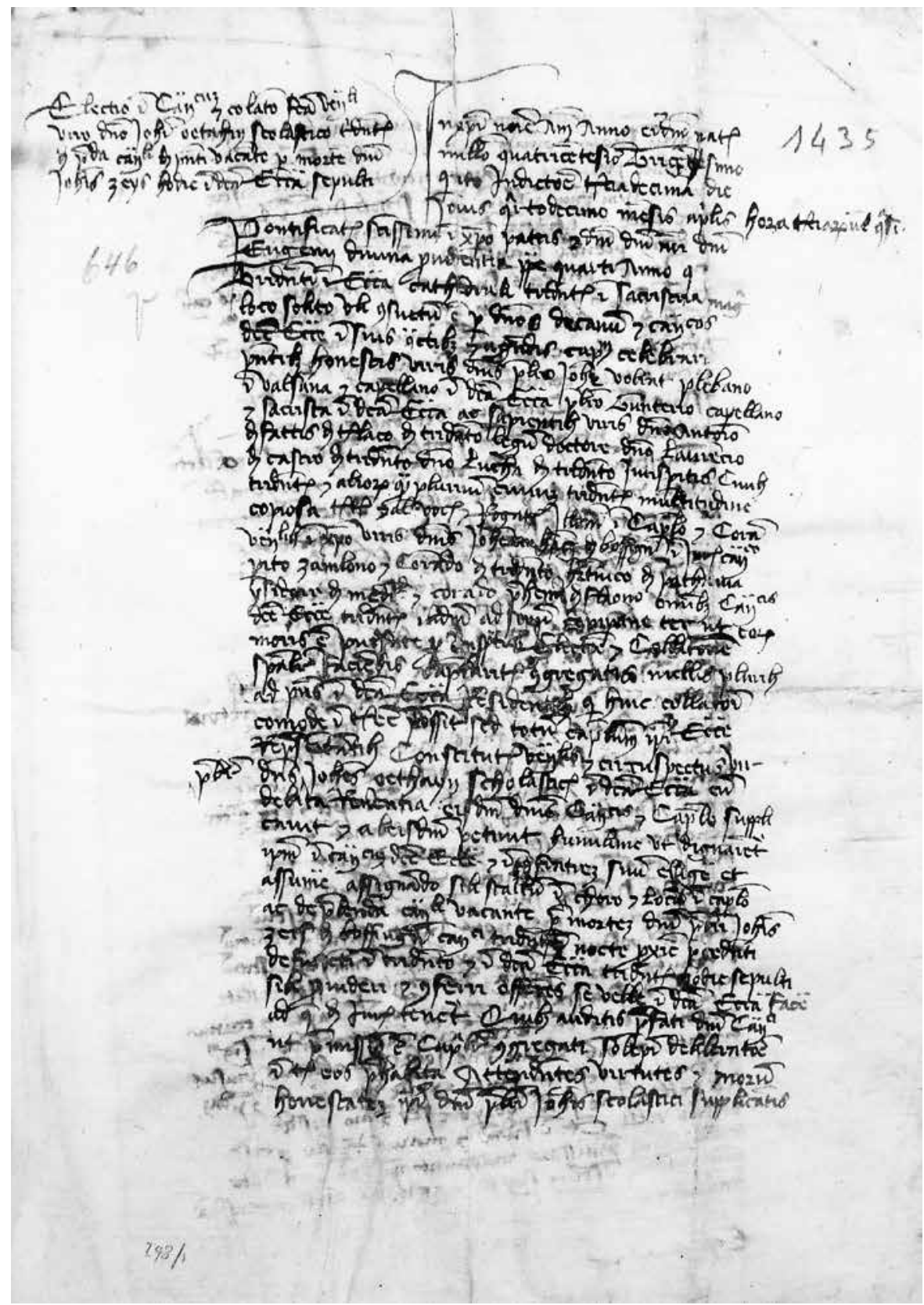

Fig. 20. ADTn, ACap, Instrumenta capitularia 8bis. c. $248 / 1 r$ (bifolio fra le carte 248 e 249). Documento in esteso cui fa riferimento la rubrica alla fig. 19.

un numero maggiore di dati rispetto alle solite rubriche. In un secondo momento, probabilmente su richiesta di Bonadomano de Accerbis, ser Antonio dovette estrarre per entrambi gli atti il documento in publicam formam; tuttavia, mentre nel caso del matrimonio con ogni probabilità egli non dovette fare altro che copiare quanto 
già c'era sul registro, eventualmente aggiungendo poche formule ceterate, nel caso della carta finis egli dovette, sullo stesso foglio, redigere analogo documento ma in forma estesa, ossia con l'aggiunta del formulario richiesto. Da questa seconda forma redazionale estrasse poi il relativo mundum. Che ciò fosse avvenuto nel momento in cui ser Bonadomano richiese ad Antonio la pergamena, lo dimostrerebbe la diversa tonalità dell'inchiostro con cui il professionista redasse la forma 'continuata' della carta finis e le relative note di estrazione del mundum (fig. 21).

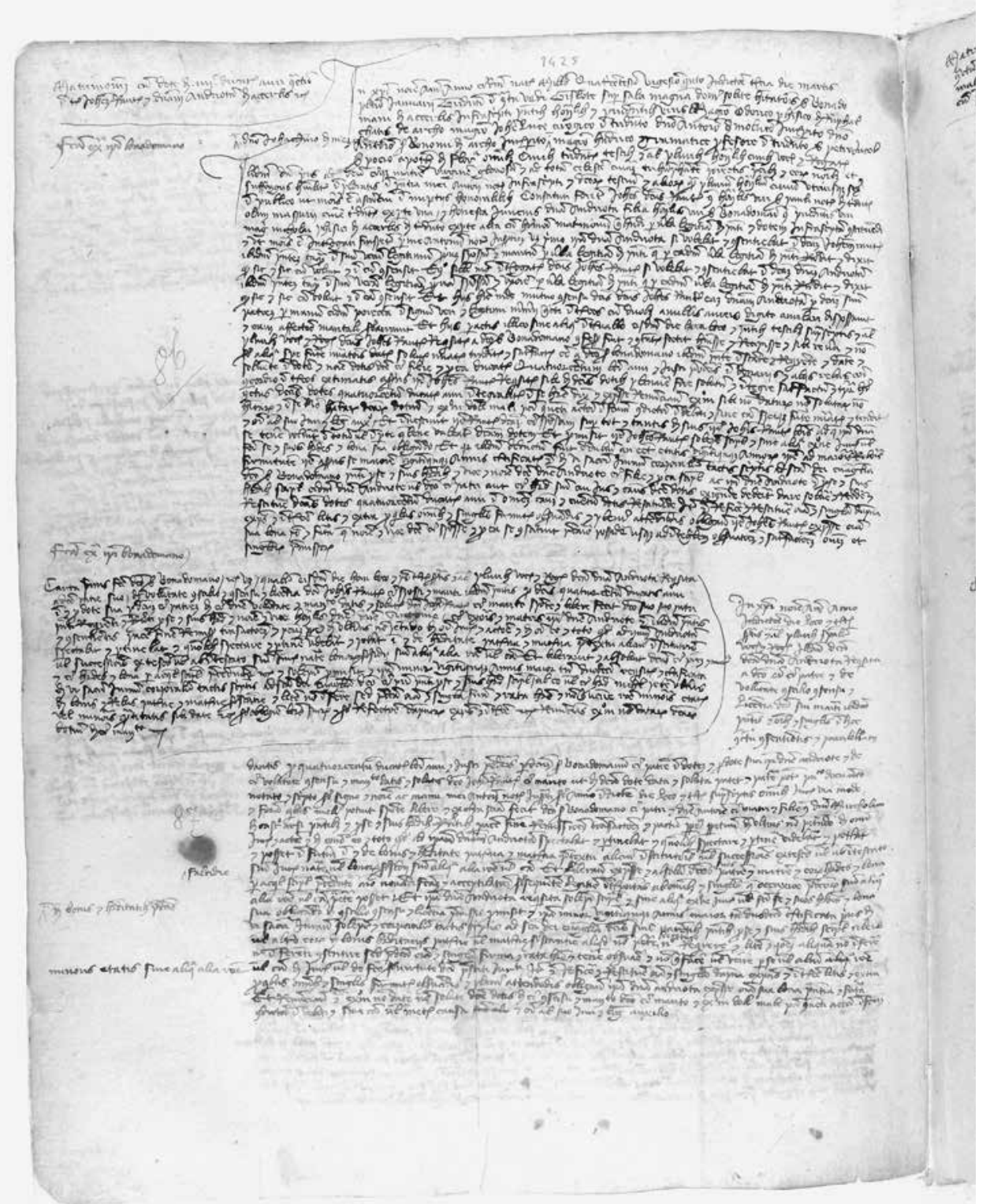

Fig. 21. ADTn, ACap, Instrumenta capitularia 8bis, c. 30v, docc. nn. 86 e 87 . 
Si hanno dunque, fortunatamente riprodotti sulla stessa carta, due diverse forme redazionali dello stesso documento. Si deve quindi immaginare che il notaio avesse anzitutto approntato una 'scheda', oggi perduta, al momento del matrimonio, presso l'abitazione di ser Bonadomano, con tutti i dati relativi al contratto matrimoniale e alla carta finis; in un secondo tempo, presso la sua abitazione in Borgonuovo, dove egli conservava probabilmente il quaternus di grandi dimensioni, redasse il documento che attestava il matrimonio in forma estesa, seguito da una forma abbreviata del secondo atto. È infatti improbabile che egli portasse con sé, fuori dalla propria statio, un registro di tale peso e grandezza.

Come fatto per alcuni esempi suesposti, anche in questo caso, si metteranno a confronto il testo della carta finis in forma abbreviata, con il relativo documento in esteso, in modo da evidenziarne gli elementi distintivi.

\section{Redazione in forma abbreviata}

Carta finis facte dicto ser Bonadomano etc videlicet qualiter eisdem die, hora, loco et presentibus testibus suprascriptis et aliis pluribus vocatis et rogatis dicta domina Andriota requisita a dicto patre suo, de voluntate, conscilio et consensu et licentia dicti Iohannis Rauter eius sponssi et mariti ibidem presentis pro dictis quatuor centum ducatis auri in et pro dote sua per dictum eius patrem de eius domine voluntate et mandato datis et solutis dict(o) Iohanni Rauter eius marito, sponte et libere fecit dicto suo <segue suo iterato> patri presenti, requirenti et recipienti pro se et suis heredibus et nomine et vice honorabilis domine domine Antonie eius uxoris et matris ipsius domine Andriote etiam ibidem presentis et consentientis pacem, finem, remissionem, transactionem et pactum perpetuum de ulterius non petendo de omni iure et actione et de omni eo et toto quod ad ipsam Andriotam spectabat et pertinebat et quoslibet spectare et pertinere videbatur et poterat in et de hereditate paterna et materna, pretextu alicuius institutionis vel successionis ex testamento vel ab intestato sive iure nature bonorum subsidii, sive aliqua alia ratione vel causa. Et liberavit et absolvit dictum eius patrem et matrem et eius heredes et bona per aliquam stipulationem precedentem etc et solempniter promisit et ipsa minor viginti quinque annis maior tamen duodecim requisita et certificata de vi sa(cri) iuravit corporaliter tactis scripturis ad sancta Dei evangelia dicto eius patri presenti pro se et suis heredibus stipulantibus ab eo vel eius heredibus nichil petere ulterius de bonis et rebus paterne et materne substantie et litem

\section{Redazione 'in esteso'}

In Christi nomine, amen. Anno, indictione, die, loco et testibus suprascriptis et aliis pluribus specialiter vocatis et rogatis, ibidem dicta 〈segue dicta iterato〉 domina Andriota requisita a dicto eius $\langle s e g u e$ eius iterato $\rangle$ patre et de voluntate, conscilio, consensu et licentia dicti sui mariti ibidem presentis et omnibus et singulis in hoc contractu consentientis et parabolam dantis pro quatuorcentum ducatis boni auri et iusti ponderis per dictum ser Bonadomanum eius patrem in dotem et pro dote sua ipsi domine Andriote et de eius voluntate, consensu et mandato datis et solutis dicto Iohanni Rauter eius marito ut de dicta dote data et soluta patet et patere potet publico documento notato et scripto sub signo et nomine ac manu mei Antonii notarii infrascripti sub anno, indictione, die, loco et testibus suprascriptis, omnibus iure, via, modo et forma quibus melius potuit, sponte, libere et ex certa scientia fecit dicto ser Bonadomano eius patri et domine Antonie eius matri et filie condam domini Marcholini de Castro Arsi, presentibus et pro se et suis heredibus recipientibus pacem, finem, remissionem, transactionem et pactum perpetuum de ulterius non petendo de omni iure et actione et de omni eo et toto quod ad ipsam dominam Andriotam spectabat et pertinebat et quoslibet spectare et pertinere videbatur et poterat et posset in futurum in et de bonis et hereditate paterna et materna pretextu alicuius institutionis vel successionis ex testamento vel ab intestato sive iure nature falcidie (falcidie nel margine sinistro con segno di richiamo> vel bonorum subsidii sive aliqua alia ratione vel causa. Et liberavit 
non inferre sed predicta omnia et singula firma et rata habere et non contravenire ratione minoris etatis vel minoris quantitatis sibi date etc sub obligatione bonorum suorum et sub refectione dampnorum, expensarum et interesse etc, renuncians exceptioni non datarum dictarum dotium de eius mandato etc expresse et absolvit dictos suos patrem et matrem et eorum heredes et bona per aliquam stipulationem precedentem animo novandi factam et acceptilationem subsequentem legitime interpositas ab omnibus et singulis, que occaxione predictorum sive aliqua alia ratione vel causa de bonis et hereditatibus predictis «de bonis ... predictis nel margine sinistro con segno di richiamo> petere posset. Et ipsa domina Andriota requisita solempni stipulatione et sine aliqua exceptione iure vel facti se et suos heredes et bona sua obligando de conscilio, consensu et licentia premissis promisit et ipsa minor viginti quinque annis maior tamen duodecim certificata prius de vi sa(cri) iuravit solempniter et corporaliter tactis scripturis ad sancta Dei evangelia dictis suis parentibus presentibus pro se et suis heredibus stipulantibus ab eis vel altero eorum de bonis hereditariis paterne vel materne substantie aliquod non petere de cetero de cetero in interlinea superiores nec requirere et litem et questionem aliquam non inferre nec inferrenti consentire sed predicta omnia et singula firma et rata habere et tenere, observare et non contrafacere vel venire per se vel alium aliqua ratione vel causa de iure vel de facto sub virtute dicti prestiti iuramenti. Item etiam reficere et restituere omnia et singula dampna, expensas et interesse litis et extra pro quibus omnibus et singulis firmiter observandis et plenius attendendis obligavit ipsa domina Andriota expresse omnia sua bona presentia et futura et renunciavit etiam exceptioni non date, non solute dicte dotis de eius consensu et mandato dicto eius marito et exceptioni doli, mali, pacti conventi, actioni in factum condictioni indebiti et sine causa vel met(...) causa et omni alio suo iuri et legum auxilio.

Nel primo documento il notaio elimina del tutto l'invocazione, esordendo immediatamente con l'oggetto del negozio, Carta finis, quasi come se si trattasse di una rubrica. Per le date topica e cronica e per l'elenco dei testimoni rinvia poi al documento precedente. Per quanto concerne il testo vero e proprio, le formule e le clausole vengono ridotte al minimo e spesso troncate dalla locuzione et cetera. L'esteso presenta invece quasi tutti gli elementi tipici di una redactio in mundum, a partire dall'invocazione a Cristo. Ser Antonio tralascia, tuttavia, anche in questo caso, l'elenco preciso dei testimoni nonché la datazione, per i quali rinvia pure al documento precedente. L'avverbio ibidem introduce di seguito il testo dell'atto, redatto per intero, senza mai ceterare alcuna parte del formulario. Inoltre in corrispondenza delle prime righe del testo, il notaio trascrisse per errore due volte lo 
stesso termine, il che potrebbe essere indizio della copiatura dal testo abbreviato posto lì accanto ${ }^{35}$.

Il caso non è il solo fra le carte dei registri di Antonio da Borgonuovo; alla carta $167 r$ di Instrumenta capitularia $8^{36}$ il notaio redasse un documento in forma abbreviata (si tratta di una promissio), aggiungendo successivamente, come si deduce dall'inchiostro di tonalità più chiara, la seguente annotazione: «Continuata supra I foleo causa faciendi eam in publicam formam petitam a massaro Capituli» (fig. 22). Essendogli, dunque, stata richiesta la redazione della relativa pergamena in mundum, il notaio dovette preventivamente redigere una forma continuata dello stesso documento su fogli sciolti. Evidentemente, come nel caso della carta finis

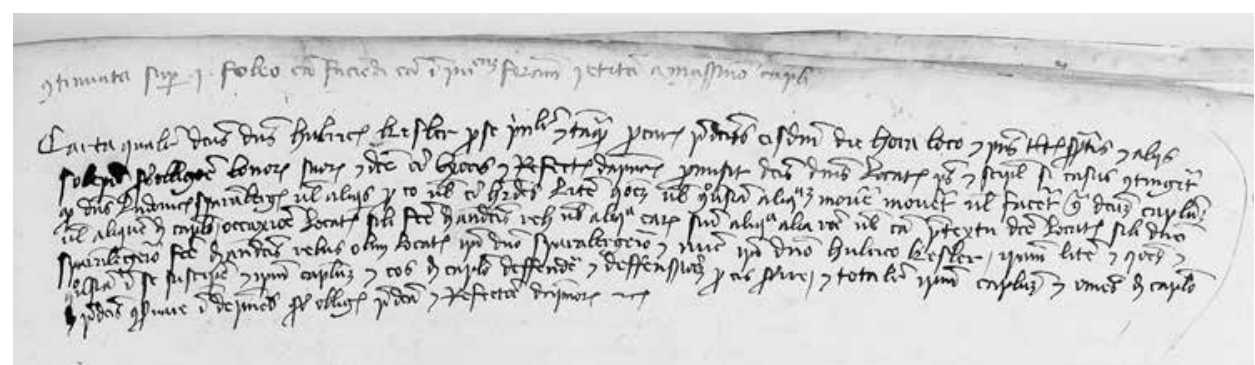

Fig. 22. ADTn, ACap, Instrumenta capitularia 8, c. 167r, n. 322b. Redazione in forma abbreviata del documento alla fig. 23. Nel margine superiore la nota, di mano del notaio Antonio da Borgonuovo: «Continuata supra I foleo causa faciendi eam in publicam formam petitam a massaro Capituli».

esaminata, egli aveva bisogno di redigere il documento in forma pressoché completa prima di stendere la pergamena in bella copia, dove errori, omissioni e integrazioni andavano evitate. L'esteso di cui parla ser Antonio è ancora oggi presente fra le carte del registro ${ }^{37}$ ed è trasmesso da un foglio sciolto dove la rubrica recita: «Promissio facta Capitulo ecclesie Tridentine per dominum Hulricum Kessler etc» (fig. 23). Il testo è vergato con un inchiostro del tutto simile a quello impiegato per redigere la nota alla carta $167 r^{38}$; probabilmente, mentre nel caso precedente egli ebbe a disposizione uno spazio sufficiente sul registro per redigere la forma 'continuata' dello stesso documento, in questo secondo esempio egli non poté stendere l'atto con tutte le formule al completo se non impiegando un foglio sciolto.

35 Ivi, n. 85, si riscontra un caso per certi aspetti analogo. Il notaio ha redatto in prima battuta l'atto relativo ad un matrimonio in forma pressoché completa. Subito dopo si trova, con inchiostro della medesima tonalità, un creditum vergato in forma abbreviata poi depennato da ser Antonio nel momento in cui, come si legge nel documento successivo, fu rilasciata relativa quietanza. Relativamente alla quietanza, pure redatta in forma abbreviata, il professionista scrisse: «Posita continua in hoc foleo hic inserto et facta extra ipsi Iohanni Rauter etc». Il foglio con l'esteso della quietanza è oggi deperdito, tuttavia l'annotazione dimostra che, prima di procedere alla redazione del mundum, venne stesa una redazione continuata, quale tappa intermedia fra l'imbreviatura e la bella copia in pergamena.

36 ADTn, ACap, Instrumenta capitularia 8, c. $167 \mathrm{r}$, n. $322 \mathrm{~b}$.

37 Ivi, c. $117 / 3$, n. 248 c.

38 Non mancano casi analoghi; in ADTn, ACap, Instrumenta capitularia 8bis, n. 551, ser Antonio redasse la forma abbreviata di un'opzione di prebenda. In un secondo momento, egli appose in margine la seguente annotazione: «Posita continua super folio hic inserto et facta extra ipsi domino Hertuico». In questo secondo caso, tuttavia, il foglio sciolto non si è conservato. 


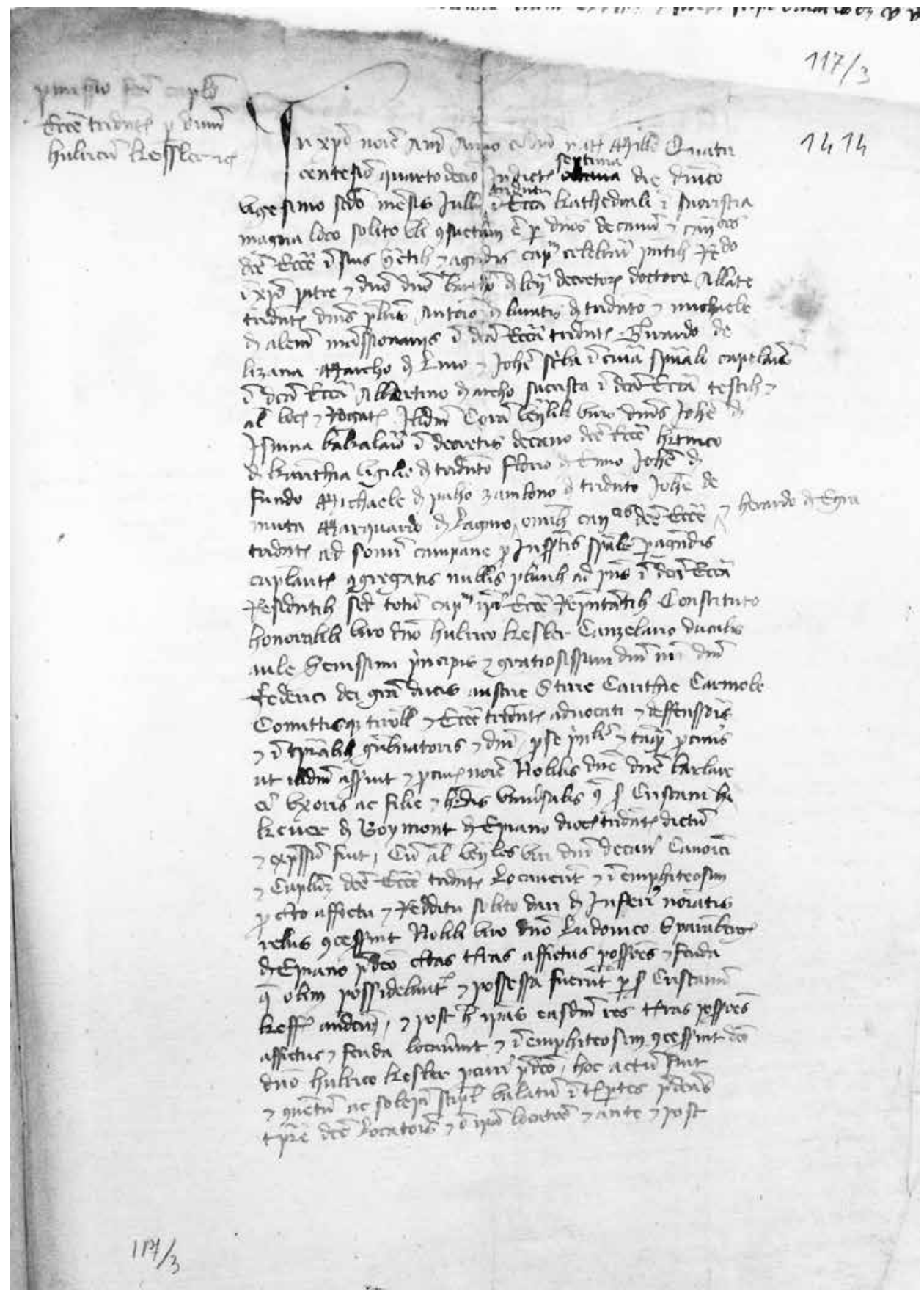

Fig. 23. ADTn, ACap, Instrumenta capitularia 8, c. 117/3r, n. 248c. Documento in esteso su bifolio relativo al documento in forma abbreviata alla fig. 22 . 
Sembra esplicativo di questo modus operandi anche l'esempio tratto dalle carte $323 v-324 r$ di Instrumenta capitularia 8; in prima battuta il notaio redasse una locazione perpetua in forma abbreviata, ovvero scegliendo di ricordare solo gli elementi essenziali del documento, senza alcuna formula. Scrisse infatti:

[Loca]tio perpetualis Iohannis condam Bertholini de Edolo de Valecamonica habitatoris super mansso [in]ferius nominato videlicet qualiter dicti domini prepositus et canonici eidem die, hora, loco [et presen]tibus testibus dictis Bartholomeo Cevoleta et Iohannes notario de Legatis nomine locationis perpetue [...] ade etc investiverunt dictum Iohannem presentem etc de uno mansso terre casalive, [vi]neate, prative etc quem tenebat a dicta ecclesia Leonardus Trivisanus nuperrime [defunctu]s pro affictu unius plaustri vini albi buliti debendi infrascriptis prebendis [...] de Tayo, trium brentarum prebende de Pinedo, unius brente cum dimidia [prebend]e prime de Sancto Petro, unius brente cum dimidia etc ${ }^{39}$.

Il notaio allegò contestualmente al registro un bifolio cartaceo, che recava l'elenco di tutte le proprietà oggetto di locazione, redatto con un inchiostro che per tonalità è simile, se non del tutto corrispondente, a quello con cui vergò il documento abbreviato. Successivamente, forse in vista di una redazione in mundum di cui però non c'è riscontro materiale, egli predispose subito sotto all'imbreviatura, ma con inchiostro di tonalità più scura, lo stesso documento ma in forma completa, con tutte le formule previste dalla locazione e con l'elenco di tutte le proprietà del manso in oggetto.

Le molteplici tappe redazionali praticate da Antonio per la stesura dei documenti sono rilevabili anche in alcuni rogiti redatti per la dignità capitolare della Prepositura; il registro Instrumenta capitularia 8bis, come noto, conserva infatti anche alcuni documenti redatti per questo ente. Si tratta, per la maggior parte, di locazioni o rinnovi di locazioni registrate in forma pressoché completa; due particolari esempi sembrano esplicativi delle procedure del notaio. Il documento n. 374 a carta $143 v$ è una refuta redatta per esteso ${ }^{40}$; il professionista ha infatti tralasciato le sole clausole finali del documento richiamando, come di consueto, alla forma comune («etc ut in comuni forma»). In margine, sotto la rubrica, si legge inoltre una sua annotazione che recita: «Continuata super quaterno instrumentorum dicte prepositure sub signo et nomine meis et etiam facta extra conductori». La nota, redatta con inchiostro di tonalità più chiara rispetto al testo del documento, informa dunque che di quella refuta ser Antonio trasse successivamente anche una ulteriore forma 'continuata', completa di ogni elemento formulare e, addirittura, munita del suo signum e della sua sottoscrizione.

Questa ulteriore redazione trova infatti posto fra le carte dell'ottavo registro della Prepositura ed è redatta in forma pressoché corrispondente a quella di un mundum $^{41}$. La stessa annotazione in Instrumenta capitularia 8bis ricorda che dell'atto venne anche stesa una redactio in mundum, oggi tuttavia deperdita, che fu consegnata al conduttore. Tre sono dunque le fasi percorse certamente da Antonio da Borgonuovo nella redazione di questo instrumentum; a queste si dovrebbe forse aggiungere una ulteriore tappa, allo stato attuale non disponibile, cioè quella della scheda o minu-

39 ADTn, ACap, Instrumenta capitularia 8, n. 671.

ADTn, ACap, Instrumenta capitularia 8bis, c. 143v, n. 374.

ADTn, $A P$, reg. VIII, c. $178 r-v$, n. 190. 
ta. Se, in fase di rogatio, il notaio avesse appuntato i principali dati del negozio giuridico così da poterne poi trarre una stesura in forma più completa sarebbero quattro le fasi compositive dell'instrumentum.

Un'analisi simile è applicabile ad un ulteriore documento in Instrumenta capitularia 8bis; si tratta di un rinnovo di locazione redatto, al pari del rogito precedente, in forma quasi completa cui, in un secondo momento, il notaio affiancò una nota del tutto corrispondente a quella poc'anzi citata ${ }^{42}$. Essa rimanda, infatti, ad un'altra redazione dell'atto sui registri pergamenacei della Prepositura ${ }^{43}$ e ad un mundum consegnato al conduttore che, diversamente dal caso precedente, ancora oggi è conservato nell'archivio dell'ente ${ }^{44}$.

La pergamena presenta inoltre una nota del notaio che ricorda come «simillem posui et continuavi super quaterno instrumentorum dicte [prepositure]»; il confronto fra la redactio in publicam formam sulla pergamena e il rogito sul registro membranaceo della Prepositura rivela infatti, salvo lievissime difformità, una corrispondenza quasi perfetta fra $\mathrm{i}$ due dettati. Anche in questo caso, pur non avendo a disposizione la prova materiale che in prima stesura ser Antonio appuntò i dati essenziali del negozio, è possibile ipotizzare che egli avesse redatto almeno una 'scheda'; nel qual caso sarebbero parimenti quattro le tappe redazionali percorse dal notaio.

I numerosi esempi esposti manifestano le diverse modalità di lavoro di Antonio da Borgonuovo; come ricordato all'inizio di questo paragrafo, infatti, pur rimanendo sostanzialmente valido lo schema della 'triplice redazione' proposto da Costamagna, emergono anche ulteriori modi operandi. Si è infatti potuto constatare come nei registri siano presenti diverse 'schede', testimonianza della prima fase di redazione dell'instrumentum notarile, quella che scaturisce dalla rogatio del cliente al notaio. Da essa il professionista può aver tratto un documento in forma di imbreviatura o di esteso da porre sui prothocolli o sui quaterni instrumentorum o, come rilevato, su entrambe le tipologie di registro.

L'ultima tappa di questo processo era rappresentata dalla redactio in rodulo, per utilizzare il lessico di ser Antonio. Si tratta, tuttavia, di una fase redazionale che, a seconda delle necessità e delle richieste dei clienti, egli poteva scegliere di non realizzare, cosicché il documento in registro rappresenta in certi casi la versione finale.

Probabilmente non c'è un'unica motivazione sottesa alle diverse modalità operative di ser Antonio, ma l'inserimento di un certo numero di documenti nei registri Instrumenta capitularia 8 e 8bis, dopo che questi erano già passati per una redazione su protocollo o su bifolia sciolti, non rappresenta la norma. Si tratta piuttosto di eccezioni all'interno di registri in cui il notaio scelse di redigere solitamente la documentazione in ordine cronologico, probabilmente poco tempo dopo la rogatio, se non addirittura contestualmente alla rogatio. Non può, infatti, passare inosservato il fatto che il professionista annotò, in margine a questi specifici rogiti, l'avvenuta relevatio da un precedente supporto, quasi a voler sottolineare e giustificare l'interruzione dell'usuale ordine cronologico e, allo stesso tempo, del suo tradizionale modus operandi. Per quanto riguarda le tempistiche di rogazione, il capitolo 63 degli statuti roveretani del 1425, intitolato «De tabellionibus facere et scribere debentibus instrumenta de quibus fuerint rogati infra certum tempus» è specificamente dedicato alle tempistiche:

42 ADTn, ACap, Instrumenta capitularia 8bis, cc. $143 v-144 r$, n. 375.

43 ADTn, $A P$, reg. VIII, cc. $178 v-179 r$, n. 191.

44 ADTn, $A P$, Tomo III, n. 4. 
Antonio da Borgonuovo al lavoro

Item statuimus et ordinamus quod tabelliones infra tres dies postquam fuerint requisiti, nisi infirmitate vel iusta absencia, debeant facere et scribere acta iudicii de quibus sunt rogati; alia vero instrumenta sive contractus infra octo dies postquam fuerint requisiti, nisi infirmitate vel iusta absencia fuerint impediti. Et si contrafecerint solvant XX soldos parvorum ducali camere, et damnum et interesse ei cui acta vel instrumenta fuerint teneantur solvere ${ }^{45}$.

La norma è quanto mai chiara sulle tempistiche; gli acta iudicii andavano scritti entro tre giorni dal momento della rogatio, mentre per tutte le altre tipologie di instrumenta il tempo a disposizione saliva ad otto giorni.

\section{Il registro Instrumenta capitularia 8 (1402-1434). Descrizione codicologica}

Instrumenta capitularia 8 è un codice costituito da 332 carte, della misura media di $417 \mathrm{~mm}$ in altezza per $337 \mathrm{~mm}$ in larghezza, distribuite in venti fascicoli $^{46}$. Dopo i due fogli di guardia applicati nel corso dell'ultimo restauro ${ }^{47}$ e un ulteriore bifolio cartaceo coevo ${ }^{48}$, ha inizio il primo fascicolo (A) che comprende le cc. 1-14; esso era in origine un ottonione completo cui però oggi mancano le prime due carte. Il primo fascicolo, così come i successivi, sono caratterizzati dalla presenza di danni da bruciatura che interessano soprattutto i margini esterni delle carte; non mancano inoltre macchie e gore sparse che hanno indebolito il supporto. Per tale ragione si è persa quasi del tutto la cartulazione coeva posta in corrispondenza del margine superiore destro del recto d'ogni carta, che è stata rimpiazzata da una nuova numerazione, realizzata con inchiostro di colore marrone scuro e attribuibile ad una mano più tardiva (forse del XV secolo exeunte-XVI ineunte); nei casi in cui questa seconda cartulazione non fosse più visibile, una mano moderna, attribuibile all'archivista Ivo Leonardi, ha integrato a matita i numeri mancanti. La numerazione applicata fra XV e XVI secolo è sostanzialmente corretta fino a

45 Statuti di Rovereto del 1425, cit., p. 179.

46 Per la descrizione dei manoscritti si è tenuto conto delle norme proposte da A. Petrucci, La descrizione del manoscritto: storia, problemi, modelli, La Nuova Italia scientifica, Roma 2008, E. Casamassima, Sulla descrizione dei codici, «Rassegna degli Archivi di Stato», 23, 1963, pp. 197-205 e V. Jemolo, M. Morelli (a cura di), Guida a una descrizione uniforme dei manoscritti e al loro censimento, Istituto centrale per il catalogo unico delle biblioteche italiane e per le informazioni bibliografiche, Roma 1990. Per quanto concerne la terminologia da impiegarsi nella descrizione dei codici cfr. M. Maniaci, Terminologia del libro manoscritto, préface di D. Mazurelle, Istituto centrale per la patologia del libro, Roma-Milano 1998 (Addenda, 3), e Ead., Archeologia del manoscritto: metodi, problemi, bibliografia recente, con contributi di C. Federici e di E. Ornato, Viella, Roma 2002 (I libri di Viella, 34). Si è scelto, per praticità, di distinguere ogni fascicolo mediante lettere dell'alfabeto maiuscolo; essi infatti non sembrano avere alcuna numerazione coeva.

47 Il restauro del codice risale al 1981 ed è stato eseguito presso il laboratorio di restauro del libro di Santa Maria di Rosano. Sul recto del primo foglio di guardia è redatta a matita la dicitura «Instrumenta capitularia 8 1402-1434»; sul recto del secondo foglio si legge invece: «Notaio Antonio di Borgonuovo (SN). Vedi questo segno ai fogli: $1,62,99 v, 110,111 v, 261,315,320,322 v, 324 v$, $325 v, 329$ ». Entrambe le note sono di mano dell'archivista Ivo Leonardi. Al primo foglio di guardia è stata allegata una cedula cartacea dattiloscritta che recita: «Microfilmato nel 1986 da Giuliano Baroni Tel 0464/35750 Microfilm system studio bi quattro Snc Via Tartarotti 1838068 Rovereto Tn a cura della Provincia Autonoma di Trento Assessorato ai Beni culturali».

${ }_{48}$ Sul recto della prima carta si legge: «INDICI Vedere a fogli 77bis |112bis | 192bis | $193 \ldots$ Anniversariorum | 263 ... Capellanorum et Fabrice | 216/1 | 279/1», di mano dell'archivista Ivo Leonardi. 
c. 86; in seguito viene saltata una carta (oggi numerata 87a) perdendo così un'unità. Dopo c. 93, si passa direttamente a c. 97, saltando così ben tre unità; la c. 142 è immediatamente seguita da c. 145. La numerazione prosegue regolare fino a c. 304, numero che viene iterato anche nella carta successiva (oggi chiamata 304bis). Il secondo (B) (cc. 15-30), il terzo (C) (cc. 31-46), il quarto (D) (cc. 47-62) e il sesto (F) (cc. 78-96) fascicolo sono costituiti da ottonioni completi. Il quinto (E) consta pure di otto bifolia cui è stata allegata una carta (c. 77bis) che rappresenta una tabula instrumentorum di mano di ser Antonio. Il settimo fascicolo (G) (cc. 97-112) è un ottonione regolare, mentre il seguente $(\mathrm{H})$ è costituito da soli sette bifolia; la mancanza di un bifolio che serviva a completare il fascicolo va forse fatta risalire al confezionamento originario: l'ultimo documento posto a c. $125 v$, infatti, prosegue nella prima carta $(126 r)$ del fascicolo successivo. Verificando la consistenza del nono fascicolo (I), si nota come esso sia costituito da un ottonione cui manca l'ultima carta. Sostanzialmente regolare è invece la struttura dei fascicoli dal 10 al $15(\mathrm{~J}-\mathrm{O})$, con la sola eccezione del tredicesimo $(\mathrm{M})$ cui è stata allegata una tabula instrumentorum di mano del notaio. Il fascicolo $16(\mathrm{P})$ ha una struttura diversificata rispetto ai precedenti: esso è infatti attualmente costituito da 22 carte; sono ottonioni regolari i fascicoli 17-19 (Q-S). L'ultimo (T) presenta 24 carte cui è stato allegato un frammento cartaceo. A chiusura del codice è presente un ulteriore bifolio di fattura moderna cui è stata annessa una «tasca dei frammenti»; in essa sono stati posti diversi lacerti cartacei reperiti probabilmente fra le carte del registro ${ }^{49}$. Un ultimo bifolio di guardia è stato posto a protezione delle carte interne in occasione del restauro del $1981^{50}$.

Per quanto concerne la legatura, essa è realizzata in piena pelle di colore marrone chiaro, liscia al tatto, su cartoncino semifloscio ${ }^{51}$; il piatto posteriore della coperta presenta un prolungamento, chiamato ribalta, che ha lo scopo di proteggere il taglio laterale delle carte; esso si ripiega sul piatto superiore coprendolo per la misura di circa $70 \mathrm{~mm}$. Il dorso mostra tre serie di cuciture in pelle allumata realizzate su altrettanti nervi doppi; esse hanno lo scopo di assicurare la coperta ai fascicoli. La chiusura del registro è caratterizzata da una stringa in cuoio allumato, solo parzialmente conservata, cucita direttamente sulla coperta; essa presenta, nella parte superiore, un fermaglio metallico che serviva al bloccaggio della cinghia. Sebbene non si possa datare con certezza, la legatura non sembra essere coeva e va ricondotta con ogni probabilità alla seconda metà del XVI secolo ${ }^{52}$.

In precedenza si è evidenziato il precario stato di conservazione di molte carte, spesso combuste in corrispondenza dei margini esterni, tali da renderne necessario, forse già all' epoca, la rifilatura; in fase di restauro, inoltre, le singole carte sono state

49 Si tratta in parte di lacerti cartacei con testi di mano del notaio Antonio, estremamente danneggiati e combusti in corrispondenza dei margini; in parte si tratta invece di fogli attribuibili alle mani di notai capitolari del XV secolo o ad altre mani anonime ancor più tarde.

50 L'ultimo foglio di guardia presenta un'etichetta cartacea del laboratorio di restauro di Santa Maria di Rosano (1981). Furono contestualmente inviati a restauro i registri Instrumenta capitularia 3, 5, 6 e 1'attuale 8bis (allora denominato Registrum Prepositurae 91).

51 Il contropiatto anteriore del codice presenta annotazioni di mano del tardo XV inerenti a investiture da ricercare fra le carte del registro. Esse sono vergate direttamente sul cartoncino che, nei due contropiatti, è libero dal rivestimento in pelle.

52 In particolare, una serie di annotazioni massariali poste da una mano anonima sul contropiatto anteriore della coperta, debordanti parzialmente anche sulla stessa pelle ribattuta, possono essere ricondotte alla seconda metà del Cinquecento. 
rinforzate mediante carta di fattura moderna. Tali danni non sono altrimenti rintracciabili sulla coperta esterna che attualmente risulta in discreto stato di conservazione. Evidentemente la coperta originaria venne rimpiazzata con quella attuale oppure, al tempo in cui il registro fu danneggiato, i fascicoli non presentavano ancora una legatura; a tal proposito si deve rilevare la presenza di consistenti danni, quali ad esempio lacerazioni e perdite di supporto, alle prime carte di taluni fascicoli, il che farebbe presupporre una loro conservazione sciolta almeno in una prima fase ${ }^{53}$. Sul piatto anteriore della coperta sono visibili due distinte etichette cartacee; la prima, più in alto, di forma rettangolare, è la più antica e rivela un breve testo non decifrabile. La seconda, al centro, venne presumibilmente applicata nel XVIII secolo, in occasione del riordino dell'archivio capitolare da parte del canonico Francesco Felice Alberti d'Enno; essa presenta la dicitura: «Instrumenta capitularia Antonii | Bertolasii ab anno 1402 usque ad | annum 1433. | N. 8». Più in basso, una mano recente, forse attribuibile all'archivista Ivo Leonardi ha scritto a matita «C. 53», in riferimento alla capsa in cui era collocato il registro. Per quanto concerne la coperta inferiore, essa presenta al centro la dicitura «Bartolas», che una mano anonima databile alla seconda metà del XV ha redatto direttamente sulla pelle con inchiostro di tonalità molto scura. Sul dorso si trovano invece ulteriori tre etichette; la prima, in alto, è databile al XIX secolo e recita: «Instrumenta | capitularia | ex notario | Antonio Bertolasio | ab anno 1402 ad annum 1433». La seconda appartiene alla mano dell'archivista Ivo Leonardi ed è stata con ogni probabilità applicata dopo il restauro del 1981: «Instrumenta | capitularia | notaio | Antonio fu Bertolasio | 1402-1434». In ultimo, più in basso, si trova un'etichetta di forma circolare, pure applicata nel corso degli anni Ottanta, con la segnatura «8». La carta impiegata per comporre i fascicoli del registro è uniforme per qualità, tuttavia si possono individuare due diverse tipologie di filigrana. La prima, riscontrabile su tutti i primi 19 fascicoli ${ }^{54}$, misura $91 \mathrm{~mm}$ in altezza per $45 \mathrm{~mm}$ in larghezza. Essa rappresenta una testa di cervo, privo di occhi, sormontato da un tratto 'stellato', ovvero una sorta di croce. Il disegno è una variante del $n^{\circ} 15505$ del repertorio di Moïse Briquet ${ }^{55}$ dal quale, tuttavia, si distingue per una leggera differenza nella forma delle corna. Il disegno è molto simile anche a quello che si riscontra al n. 230 del repertorio di Gerhard Piccard, il quale riporta alla città di Vicenza e all'anno $1403^{56}$, e al n. 2224 del repertorio di Mošin e Traljič, dal quale si ricava come questo tipo di raffigurazione sia riconducibile a produzioni italiane comprese fra il 1360 ed il $1437^{57}$.

La seconda filigrana ( $93 \mathrm{~mm}$ in altezza per $45 \mathrm{~mm}$ in larghezza) veicolata dalle sole carte impiegate per confezionare il ventesimo fascicolo $(\mathrm{T})$, rappresenta una testa di cervo con occhi sormontata da un tratto che termina con un fiore a cinque petali. Il disegno è una variante simile al n. 15507 del repertorio di Briquet ${ }^{58}$, dalla quale tuttavia si distingue per la posizione degli occhi che, nell'esempio di Briquet,

53 Questo dato è interessante alla luce di quanto si dirà, più avanti, in merito alla suddivisione in volumina (cioè gruppi di fascicoli), ciascuno dedicato ad un particolare cespite d'entrata, e preceduto da relative tabule istrumentorum, per mano del notaio Antonio da Borgonuovo.

54 Ben visibile ad esempio al centro della c. 334.

55 C.M. Briquet, Les Filigranes. Dictionnaire historique des marques du papier dès leur apparition vers 1282 jusqu'en 1600, Hiersemann, Leipzig 1923, IV, n. 15505, p. 777 (Verona, 1422-38).

56 G. Piccard, Wasserzeichen Hirsch, W. Kohlhammer, Stuttgart 1961, XV, 1, n. 230.

57 V.A. Mošin, S.M. Traljič, Vodeni Znakovi XIII i XIV vijeka; Filigranes des XIII et XIV ss., Jugoslavenska akademija znanosti i umjetnosti. Historijski institut, Zagreb 1957, I, n. 2224.

58 Briquet, Les Filigranes, cit., IV, n. 15507, p. 777. 
toccano la circonferenza del muso, appena sotto le orecchie, mentre nelle carte del registro sono al centro del muso stesso, con l'occhio destro leggermente più in alto rispetto al sinistro. Il disegno è molto simile anche a quello che si riscontra al n. 385 della raccolta di Gerhard Piccard ${ }^{59}$. È importante ricordare che questa tipologia di filigrana è quella che si ritrova nelle carte di tutti i fascicoli del registro Instrumenta capitularia 8bis.

\subsection{L'impostazione della pagina scritta}

Le carte non presentano segni di marginatura o di rigatura, tuttavia il professionista predispose la mise en page attraverso una preventiva piegatura di ciascuna carta in quattro sezioni parallele al lato lungo. Le pieghe che si venivano così a formare costituivano le direttrici di giustificazione. Nei documenti redatti per esteso egli solitamente principia il testo del documento (a partire dall'invocazione alla divinità) in corrispondenza della prima piega; lascia cioè in bianco l'intero margine sinistro cui è riservata la rubrica, delimitata a sua volta da un tratto di penna che la incornicia, e da eventuali annotazioni, quali ad esempio le note relative all'estrazione del mundum o eventuali integrazioni al testo. Le rubriche, come in passato è già stato più volte messo in luce, non avevano probabilmente alcun scopo giuridico; esse servivano piuttosto a reperire più facilmente $\mathrm{i}$ documenti all'interno dei registri ${ }^{60}$. Non è un caso, infatti, che il notaio utilizzasse per la loro redazione forme-tipo che egli ripeteva costantemente; così, ad esempio, nel caso delle refute il formulario di ser Antonio prevedeva un periodo così articolato: «Refutatio facta per [...] de [...] sito/a ad [...] pro [...] de qua investitus fuit [...] etc», con minime modifiche.

Il documento vero e proprio, come anticipato, viene solitamente introdotto dall'invocazione divina In Christi nomine, amen, ove $I$ - incipitaria è realizzata in dimensioni notevolmente potenziate e sovente con i tratti raddoppiati. Il testo del rogito occupa quindi le ulteriori tre sezioni della pagina sino a toccare il margine destro. Per quanto riguarda i documenti che ser Antonio scelse di redigere sul registro in forma abbreviata ${ }^{61}$, essi presentano - come si è del resto già avuto modo di ricordare - le caratteristiche di una rubrica più ricca di informazioni; il testo viene addossato direttamente al margine sinistro della carta e parzialmente incorniciato (sui margini destro e inferiore) da un tratto di penna. Da notare come, analogamente alle rubriche, nei casi di rogiti così presentati, il notaio scelse di far principiare il testo con termini o locuzioni che ne definivano immediatamente il contenuto: carta finis, creditum, carta resignationis etc. Qualora il professionista avesse dovuto aggiungere, anche a distanza di tempo, un documento che modificava quello redatto in prima battuta ${ }^{62}$, egli affiancava o faceva seguire il nuovo rogito a quello precedente, procedendo, se richiesto, anche alla cancellatura o alla semplice depennatura del vecchio documento. Fra le annotazioni di ser Antonio che maggior-

59 Piccard, Wasserzeichen Hirsch, cit., XV, 1, n. 385 (Colonia, 1406, 1407). Simile, ma non del tutto corrispondente, soprattutto nella forma delle corna, è anche l'esempio di testa di cervo riportato in Mošin, Traljič, Vodeni Znakovi, cit., I, n. 2234 (Monaco di Baviera, 1397).

60 Cfr., ad esempio, Costamagna, La triplice redazione dell'instrumentum genovese, cit., p. 33.

61 Si tratta, in molti casi, di documenti il cui contenuto si riallaccia dal punto di vista giuridico o contrattuale al testo del rogito precedente; nel tal caso il notaio scelse dunque di non ripetere alcune informazioni (come la data o i testimoni presenti) più facilmente ricavabili dal primo documento.

62 È il caso, ad esempio, delle quietanze che annullavano precedenti attestazioni di debito. 
mente si riscontrano sulle carte del registro ${ }^{63}$ si trovano, come detto, quelle relative all'estrazione del o dei munda; esse si risolvono in locuzioni del tipo: «Facta extra», oppure, «Facta extra duplex locatori et conductori», nel caso di rilascio del mundum sia al locatore sia al conduttore, «Facta extra in publicam formam», «Facta extra in publicam formam ut moris est» etc. Scorrendo le carte del registro si nota come in alcuni casi il notaio abbia scelto di redigere i documenti in forma pressoché completa, tanto da aggiungervi anche il proprio signum e la propria sottoscrizione; sebbene non esista una motivazione univoca per spiegare questo modus operandi, in molti casi i segni convalidatori individuavano la presenza di un rogito cui il notaio voleva conferire maggiore solennità, vuoi per il contenuto dello stesso vuoi per i personaggi implicati. In altri casi ancora, signum e sottoscrizione, seppur non con costanza, venivano posti in calce al primo documento di ciascun anno.

Un ulteriore aspetto su cui è utile riflettere è quello relativo alla progressione cronologica dei documenti. Come noto, infatti, i rogiti raccolti in Instrumenta capitularia 8 attestano l'attività di ser Antonio per un periodo di oltre trent'anni, che va dal settembre del $1402^{64}$ al gennaio del $1434^{65}$. Il registro non presenta, diversamente dal n. 8bis, alcuna intestazione di mano del notaio, sebbene diverse indicazioni ricavabili dai documenti in esso redatti definiscano il volume come liber o quaternus. Mentre solitamente con il primo termine si indica l'unità perfettamente compiuta, spesso destinata a raccogliere i rogiti nella loro stesura definitiva e più completa, si è soliti chiamare quaterni i singoli fascicoli costituenti il registro ${ }^{66}$. L'analisi sulla terminologia adottata da ser Antonio, tuttavia, porta a far corrispondere liber e quaternus; in molti casi, infatti, il notaio chiama il registro del Capitolo quaternus Capituli $^{67}$, mentre altrove la locuzione è sostituita da liber $^{68}$. Non è possibile sapere con certezza se i venti fascicoli costituenti il volume fossero già rilegati all'epoca in cui vi lavorava Antonio da Borgonuovo; tuttavia, osservando la scansione temporale dei rogiti, immediatamente ci si rende conto che essi non

63 Si è scelto di non menzionare le numerose altre mani che dal XV secolo in poi si alternarono nella redazione di annotazioni, appunti ecc., a margine dei documenti su Instrumenta capitularia 8. Si tratta, in larga misura, di note di tipo massariale redatte probabilmente da canonici o massari capitolari con finalità ricognitive. Si ricordano, invece, le segnature numeriche attribuite ai documenti redatte a matita da Vigilio Zanolini sul finire degli anni Trenta del secolo scorso. Ogni documento è inoltre preceduto dall'indicazione del millesimo in cui il rogito fu redatto; queste note sono attribuibili alla mano dell'archivista Ivo Leonardi.

64 ADTn, ACap, Instrumenta capitularia 8, n. 1.

65 Ivi, n. 695 b. In realtà esiste un ulteriore documento, per la precisione una solutio debiti, datata al 18 gennaio 1435 che è stata aggiunta successivamente. Si è tuttavia scelto di considerare concluso il registro nel 1434, allorquando il notaio registrò in esso l'ultimo documento su richiesta dei canonici.

66 Cfr., ad esempio, Imbreviature. I registro (1294-1296). Ser Matteo di Biliotto notaio, a cura di M. Soffici, F. Sznura, SISMEL, Firenze 2002 (Memoria scripturarum, 1), p. XX.

67 Cfr., ad esempio, ADTn, ACap, Instrumenta capitularia 8bis, n. 93 ove, riferendosi all'odierno Instrumenta capitularia 8, ser Antonio afferma: «Nota ad memoriam quod infrascriptum instrumentum supra quaterno Capituli continuare non potui quia dominus Iohannes Zeiss ipsum habuit et dimisit in castro». Il termine liber è impiegato dal notaio anche in riferimento ai registri pergamenacei della Prepositura, come si ricava da ADTn, ACap, Instrumenta capitularia 8bis, n. 230, dove nella nota relativa all'estrazione del mundum si legge: «Facta extra distincte ipsi conductori et posita continua cum (ipse) signo et subscriptione super libro auctentico instrumentorum continuatorum dicte prepositure etc».

68 Allo stesso modo il notaio chiama i registri redatti per il Capitolo dai notai attivi per l'ente nel corso del XIV secolo: l'attuale Instrumenta capitularia 7 (ASTn, $A C D$, n. 1398-01) è ad esempio definito «Quaternus instrumentorum dicti Capituli». 
sono disposti in semplice ordine cronologico; la datazione, infatti, sembra tornare indietro per ben cinque volte. Si hanno così, per le cc. $1 r-72 v$, i documenti relativi agli anni 1402-1431, per le cc. $73 r$-112/3r i documenti relativi agli anni 1423-1432, per le cc. $112 / 3 v-193 v$ quelli relativi al periodo $1402-1423$, per le cc. $194 r-263 v$ quelli inerenti agli anni $1402-1430$ e, in ultimo, per le cc. $264 r-335 r$ i rogiti inerenti agli anni 1402-1434. Cinque distinti gruppi di fascicoli trasmettono, dunque, documentazione disposta secondo blocchi cronologici non consecutivi così che ogni gruppo sembra principiare ogni volta dal 1402 (con la sola eccezione del secondo che presenta documenti redatti dal 1423).

Un'analisi più dettagliata del registro ha portato ad una spiegazione del modus operandi del notaio. Egli infatti, sin dall'inizio, concepì blocchi di fascicoli differenziati; su ciascuno di questi blocchi, che egli definisce volumina, redasse gli instrumenta i cui censi spettavano rispettivamente o all'intero Capitolo, ossia alla mensa (universitas) comune, o alle prebende individuali, o agli anniversari, o alle cappellanie e alla fabbrica della cattedrale. Ciascun volumen veniva poi fatto precedere da un apposito indice, denominato tabula instrumentorum, su cui il professionista redigeva i titoli dei documenti, corrispondenti alle relative rubriche, con il numero della carta su cui erano posti. Ciò avrebbe facilitato, in un secondo momento, il reperimento dei rogiti nel volumen. Mentre in alcuni casi ser Antonio provvide a lasciare un numero sufficiente di carte bianche all'inizio del fascicolo con cui principiò il volumen, così da redigervi a mano a mano l'elenco dei documenti cronologicamente disposti, in altri casi queste carte non furono sufficienti, tanto da obbligare il notaio ad allegare un certo numero di fogli sciolti o bifolia, spesso di dimensioni ridotte rispetto a quelle delle carte del registro, su cui proseguì le tabulae degli instrumenta.

L'analisi sulla scrittura e sull'inchiostro con cui furono vergati i testi dei documenti in Instrumenta capitularia 8 ha confermato questa modalità operativa; la documentazione relativa ai primi anni di attività (ad esempio quella redatta nel 1402) presenta, in ogni volumen, una tonalità d'inchiostro piuttosto chiara, mentre scrittura e mise en page risultano alquanto accurate, essendo quasi del tutto assenti correzioni ed integrazioni. Con il procedere degli anni, l'inchiostro tende a divenire più scuro, la scrittura si fa più trasandata, e aumentano visibilmente correzioni e integrazioni; sembra quasi che per i primi anni il notaio abbia riversato nei vari volumina tematici gruppi di documenti già pronti, frutto di un'attività di cernita e messa a libro già impostata e precostituita; col tempo, invece, il registro sembra essere divenuto sede di registrazione progressiva dei documenti che i canonici, di volta in volta, chiedevano al notaio di rogare. Per tale ragione, ad atti in esteso, perfettamente compiuti dal punto di vista formulare, si affiancano rogiti solo parzialmente conclusi o soltanto abbozzati; allo stesso tempo, come già evidenziato, aumentano il numero delle correzioni e degli interventi sui testi già scritti, frutto di una successiva attività di revisione. 


\subsection{L'organizzazione del registro}

La c. $1 r$ si apre con un documento in esteso redatto nel settembre del 1402 che, dal punto di vista contenutistico è una locazione perpetua il cui censo annuo si deve versare alla comunitas Capituli, ovvero alla mensa comune. Salvo qualche eccezione la documentazione redatta sull'intero fascicolo A (cc. 1-14, con i rogiti dal n. 1 al n. 26), e sui fascicoli B (cc. 16-29, con i rogiti dal n. 27 al n. 60), C (cc. 31-46, con i rogiti dal n. 61 al n. 94) e D (cc. 47-62, con i rogiti dal n. 95 al n. 133) riguarda dal punto di vista contenutistico la mensa comune; si tratta inoltre di documentazione redatta sostanzialmente, fatte salve alcune limitate eccezioni, in ordine cronologico e riguardanti l'arco temporale compreso fra il 1402 ed il 1429 . Il successivo fascicolo E (a c. 63) viene inaugurato con un documento di data 7 aprile 1429, ancora relativo alla comunitas Capituli (doc. n. 134); evidentemente il notaio non aveva potuto inserirlo nel fascicolo precedente, che era uniformemente dedicato a questo cespite d'entrata. Con l'atto successivo (n. 135), cronologicamente consecutivo rispetto al n. 134, si apre una serie di rogiti riguardanti redditi diversi; i censi annui delle locazioni ivi redatte, infatti, spettavano agli anniversari. Lo stesso vale per i documenti nn. 136-139 pure riguardanti gli anniversari e datati fra l'aprile 1430 e l'ottobre 1431. Ancora nel fascicolo che per convenzione si è denominato E trovano spazio, consecutivamente, tre atti relativi alla comunitas del Capitolo (datati al febbraio del 1431) e una serie di documenti inerenti alle prebende canonicali (docc. n. 140, che data al dicembre del 1430, e nn. 144-148 del maggio 1431). Fin qui il notaio, sebbene abbia mescolato, all'interno dello stesso fascicolo, una serie di instrumenta relativi a diversi cespiti d'entrata ha, tuttavia, mantenuto un sostanziale ordine cronologico, che prosegue inalterato fino al documento n. 157 a c. 72 , inerente agli anniversari. A partire dal rogito successivo (n. 158) a c. 73 l'ordine cronologico si perde, ritornando al febbraio del 1423. Con esso, tuttavia, si inaugura una serie continua di instrumenta le cui rendite spettano alle prebende individuali. Il notaio, infatti, non manca di aggiungere in corrispondenza del margine superiore della c. 73 l'annotazione «Instrumenta prebendarum». Sul perché egli abbia redatto questi documenti (fra l'altro principiandoli soltanto dal 1423) partendo a metà d'un fascicolo già avviato, anziché inaugurarne uno nuovo si tornerà più avanti. A questo punto egli prosegue la registrazione degli instrumenta prebendarum rispettando un ordine sostanzialmente cronologico fino alla fine del fascicolo E (c. 78, documento n. 172a), continuando lo stesso rogito sulla carta successiva (c. 79 r) e inaugurando così il nuovo fascicolo F. Nei fascicoli F (cc. 79-96, con i docc. nn. 172b-205) e G (cc. 97-112, con la continuazione del doc. n. 205 fino al doc. n. 237) non si rilevano particolari problemi: in essi continua infatti senza importanti anomalie la registrazione degli instrumenta relativi alle prebende individuali, con l'ultimo documento che data al maggio del 1432. Il fascicolo H (cc. 112/3-125, con i documenti nn. 238-262) è aperto da una tabula instrumentorum e prosegue dal punto di vista contenutistico con i rogiti delle prebende individuali, tuttavia la data fa un salto indietro al dicembre 1402 (doc. n. 238). Di seguito, l'ordine cronologico e l'argomento trattato continuano inalterati per l'intero fascicolo, proseguendo poi nei successivi I (cc. 126-143, con i documenti nn. 263-289), J (cc. 145-161, con i documenti nn. 290-315), K (cc. 161-176, con i documenti nn. 315-350) e L (cc. 177-192, con i documenti nn. 351-388). L'ultimo documento ivi redatto è datato al gennaio 1423. In corrispondenza della c. $192 v$ del fascicolo L, in parte rimasta 
bianca, il notaio pose un'annotazione interessante: «Continuando ad scribendum instrumenta locationum prebendarum require ad cartam 75 in precedenti etc».

Questa nota permette di comprendere le modalità operative di ser Antonio. Evidentemente egli aveva esaurito lo spazio da dedicare agli instrumenta prebendarum nel volumen (che iniziava col fascicolo $\mathrm{H}$ ), ovvero nel gruppo di fascicoli, predisposto per questi documenti. Fu dunque costretto a continuare la redazione dei rogiti successivi, quelli inerenti al periodo seguente al gennaio 1423, in altra sede, cioè nel volumen precedente, quello altrimenti dedicato alla communitas Capituli, dove evidentemente erano rimaste bianche molte carte. La carta 75 citata da ser Antonio corrisponde, nella nuova cartulazione, alla c. 73 del fascicolo che si è chiamato $\mathrm{E}$, dove infatti si trovano gli instrumenta relativi alle prebende, così come specificato dallo stesso notaio in principio alla carta. La datazione prosegue quella dell'ultimo documento nel fascicolo L che, si ricorda, era del gennaio 1423, continuando con un rogito del febbraio dello stesso anno. Il documento n. 389 all'inizio del fascicolo M (cc. 193-208) si trova dopo una nuova tabula instrumentorum che ricorda come quel volumen sia dedicato agli «instrumenta anniversariorum ecclesie Tridentine». Anche in questo caso, i documenti iniziano regolarmente con l'anno 1402 e proseguono rispettando l'ordine cronologico fino alla fine del fascicolo P, dove l'ultimo rogito (n. 538) è datato 28 aprile 1430. Tuttavia ser Antonio non lasciò all'inizio del primo fascicolo di questo volumen un numero sufficiente di carte per accogliere, a mano a mano, l'elenco degli instrumenta ivi redatti, cosicché fu costretto in corso d'opera ad allegare un bifolio di medie dimensioni, sul quale redasse l'indice relativo ai documenti posti attualmente fra le cc. 251 e 262 (ovvero $\mathrm{i}$ docc. nn. 507-538). A c. $262 v$, in calce al documento n. 538, datato all'aprile del 1430, il notaio pose un'annotazione che recita: «Require superius in capitulo locationum comunitatis cont[inuandu]m locationum dictorum anniversariorum incipiendo 65 carta». Si spiega così la presenza nel fascicolo $\mathrm{E}$ (per la precisione dal doc. n. 135) di un certo numero di instrumenta relativi agli anniversari che proseguono, infatti, la cronologia interrotta alla fine del fascicolo P, dove l'ultimo documento (n. 538) è datato 28 aprile 1430; il primo rogito relativo agli anniversari posto da ser Antonio nelle carte rimaste bianche del fascicolo E è infatti datato 29 aprile 1430 (doc. n. 135). L'ultimo blocco di fascicoli (Q-T) è più uniforme sia dal punto di vista contenutistico sia dal punto di vista cronologico: esso veicola infatti, come ricordato nelle due carte dedicate alla tabula (cc. 263r-v) gli «instrumenta capellanorum et fabrice antedicte ecclesie Tridentine» (fig. 24).

Scorrendo l'indice redatto dal notaio ci si rende tuttavia conto che esso si ferma ben prima della conclusione effettiva del volumen; l'ultimo documento registrato nella tabula, infatti, è una refuta (doc. n. 647) che, secondo l'attuale cartulazione del registro, si trova a c. $314 r$. Rimangono dunque esclusi un buon numero di documenti posti attualmente fra le cc. 314-335. Anche in questo caso il professionista proseguì con la redazione della tabula su un foglio allegato che oggi si trova fra le cc. 279 e 280 . Si tratta, come nel caso precedente, di un bifolio di medie dimensioni in cui ser Antonio registrò consecutivamente l'elenco degli instrumenta oggi posti dalla c. 314 (doc. n. 648) alla c. 335 (doc. n. 695).

L'ultimo rogito è redatto su un foglio fortemente danneggiato e corrisponde all'ultima 'rubrica' posta da ser Antonio in calce alla tabula; citando la carta in cui si trovava l'atto il notaio ricordava come si trattasse dell'ultima dell'intero volumen; questa informazione conferma che l'attuale conformazione del registro corrisponde 


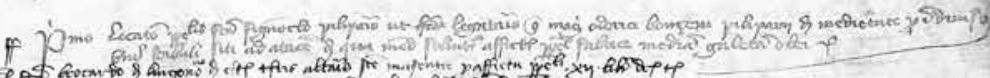

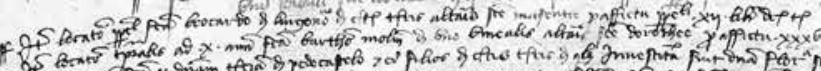

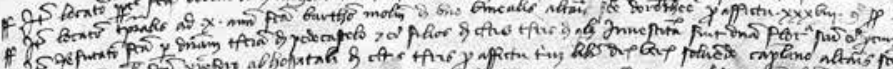

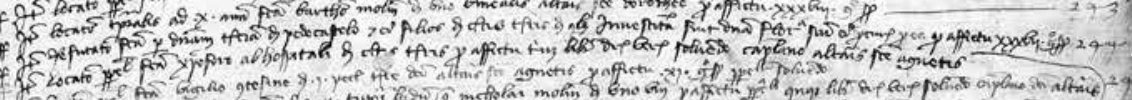

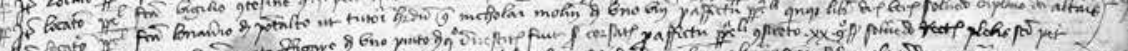

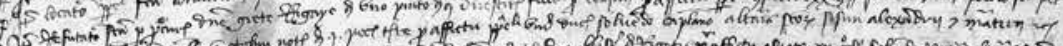

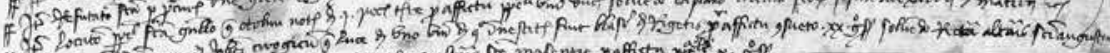

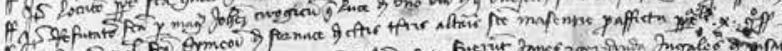

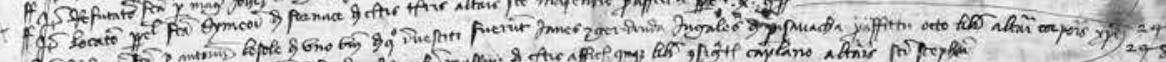

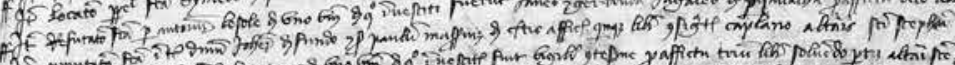

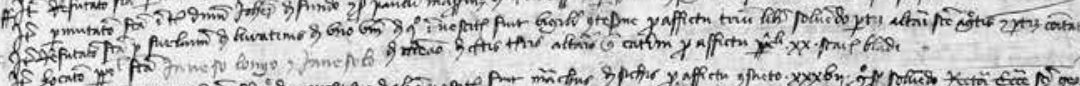

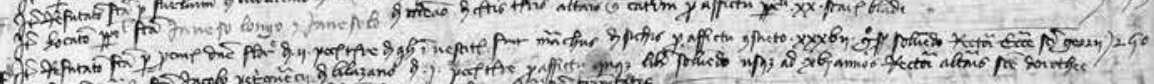

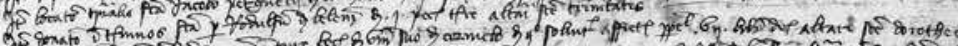

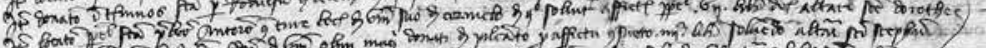

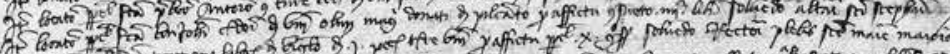

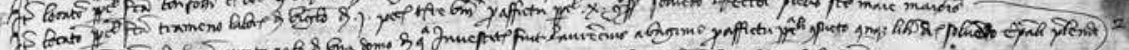

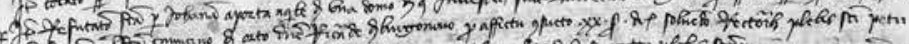

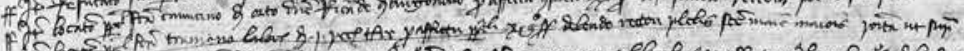

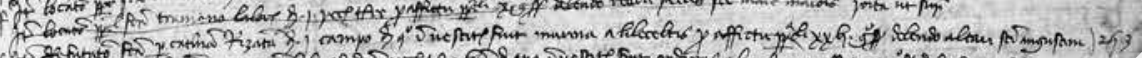

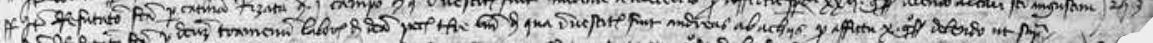

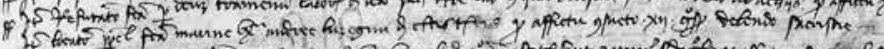

Len

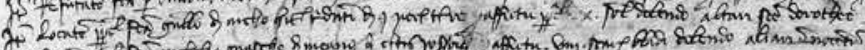

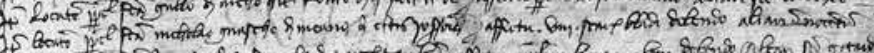

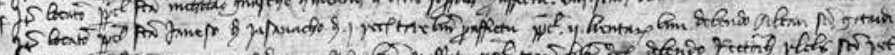

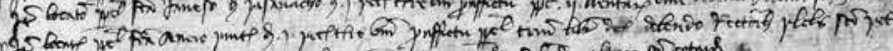

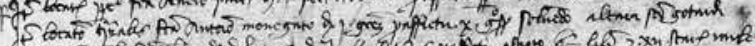

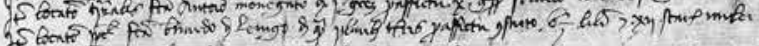

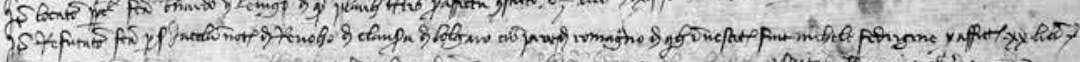

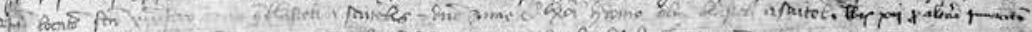

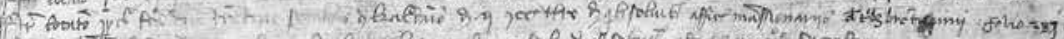

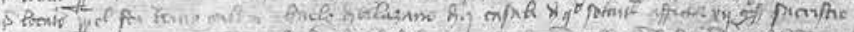

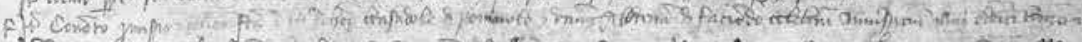

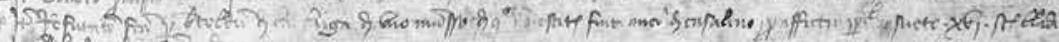

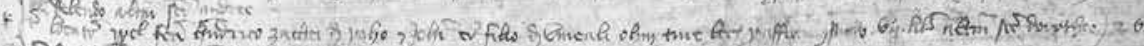

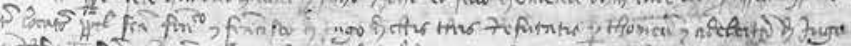

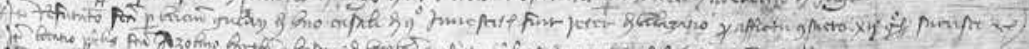

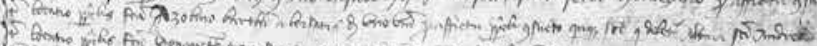

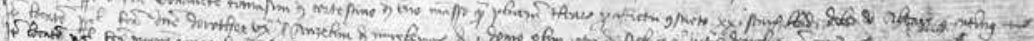

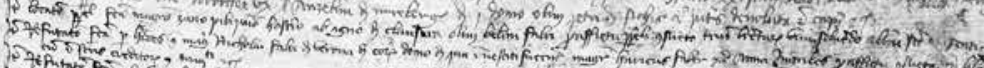

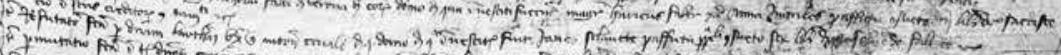

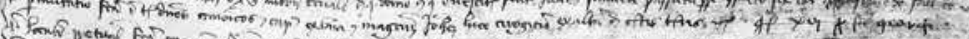

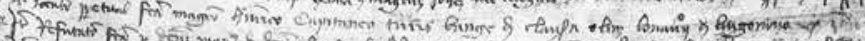

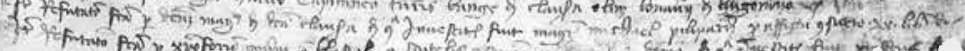

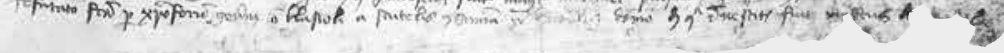

Fig. 24. ADTn, ACap, Instrumenta capitularia 8, c. 263r. Tabula instrumentorum relativa alle cappellanie e alla fabbrica della cattedrale. Si noti come, in corrispondenza del margine sinistro, a fianco di ogni rubrica, il notaio abbia redatto la sigla $f$ o $f f$. Un confronto con le note relative all'estrazione dei munda poste dallo stesso ser Antonio in margine a ciascun rogito porta a ricondurre la semplice $f$ alla redazione di una sola pergamena in mundum (o per il locatore o per il conduttore), la ff alla redazione di due distinti munda (Facta extra duplex locatori et conductori). 
a quella che all'epoca predispose il professionista; non sembrano dunque mancare carte alla fine del codice.

Un'analisi precisa su tutti i documenti dell'ultimo fascicolo del registro Instrumenta capitularia 8 permette di svolgere un'ulteriore considerazione. Se infatti le prime carte del fascicolo $\mathrm{T}$ sono occupate, come nei precedenti, da rogiti relativi all'amministrazione dei censi dovuti alle cappellanie e alla fabbrica, da c. $318 \mathrm{v}$, in particolare con il documento n. 658 datato 21 dicembre 1431, il notaio iniziò a registrare tutta una serie di instrumenta relativi sia agli anniversari sia alle prebende individuali; e così fino alla fine del registro a c. 335, ove il documento n. 695 riguarda infatti la prebenda canonicale di Meano. Evidentemente egli non aveva più spazio a disposizione per completare la messa a libro di quei rogiti nei volumina che aveva specificamente dedicato a quei cespiti d'entrata; e ciò sembra essere confermato da un'ulteriore annotazione che ser Antonio pose in calce alla c. 112v, alla fine del fascicolo G, ove si legge: «Require in parte capellanorum instrumenta de cetero notanda ubi sunt adhuc plura folia vacua, cum pluries et pluries et centies petiveram alium quaternum per capitulum emi et non fui exauditus etc».

\subsection{Le tabule instrumentorum}

Qualche parola andrà spesa ancora sulle tabule instrumentorum che si trovano sparse, talvolta in posizioni non adeguate, fra le carte del registro. Se ne seguirà l'organizzazione e la struttura per comprendere il modus operandi di ser Antonio. Tornando alla documentazione redatta nei primi fascicoli del registro, quelli dedicati alla mensa comune, si rileva l'assenza, all'inizio del fascicolo A, di una tabula dedicata ai rogiti seguenti. A c. 77 bis si trova il primo indice del volume, mutilo e gravemente danneggiato. Esso trasmette nel recto l'elenco dei documenti che attualmente occupano le cc. 47-71 del registro (corrispondenti ai documenti nn. 96-154), mentre sul verso appaiono gli atti stesi alle cc. 71-84 (corrispondenti ai documenti nn. 155-186).

Si tratta, con ogni probabilità, di quanto rimane dell'indice iniziale di Instrumenta capitularia 8 , la cui prima carta è andata, come ricordato, deperdita e doveva trasmettere la tabula per le cc. 1-46 (docc. nn. 1-94) ${ }^{69}$. Evidentemente questo lacerto, che avrebbe dovuto trovarsi all'inizio del registro, è stato erroneamente posto nel corso dell'ultimo restauro dopo la c. 77. Verificando l'argomento trattato negli instrumenta elencati in questo frammento di indice, si nota come i documenti nn. 96-154 siano relativi alla mensa comune; ad essi furono poi aggiunti, come sopra ricordato, alcuni rogiti relativi agli anniversari e alle prebende. I documenti nn. 155-186 riguardano invece interamente le prebende individuali. Tuttavia, come poc'anzi rilevato, gli instrumenta concernenti le prebende proseguono ancora; il notaio, infatti, aggiunge opportunamente in calce alla tabula di c. 77bis la seguente nota: «Require de subsequentis cartis in tabula subsequenti de prebendis». Questo indice è attualmente posto a c. 112/1 in posizione che, parimenti al caso precedente, sembra errata. Si tratta di un bifolio cartaceo di medie dimensioni su cui ser Antonio registrò l'elenco dei documenti 187-237 (relativi alle prebende), oggi posti alle

69 Descrivendo l'attuale struttura dei fascicoli di Instrumenta capitularia 8 si è in precedenza rilevato come al fascicolo $\mathrm{A}$, in origine un quaterno completo, manchino proprio le prime due carte. Mentre la prima, veicolante la prima parte della tabula instrumentorum della comunitas Capituli, è andata come detto perduta, la seconda è oggi posta erroneamente a c. 77bis. 
cc. 85-112. Manca, a questo punto, l'ultima parte della tabula relativa alle prebende, che si trova a c.192bis; si tratta di un frammento gravemente danneggiato, pure posto in posizione errata, costituendo esso la prosecuzione dell'indice che oggi è a c. 77bis. Questo lacerto cartaceo veicola l'elenco degli instrumenta nn. 238-354 alle cc. 112-178 del registro e probabilmente era posto in origine prima dell'attuale c. 112/3, ovvero all'inizio del fascicolo H. In effetti, all'inizio di codesto fascicolo, si trova la prosecuzione della tabula oggi a c. 192bis; qui il notaio ha continuato l'elenco dei documenti relativi alle prebende che oggi si trovano alle cc. 178-192 (docc. nn. 355-388) e, sempre sulla stessa carta, dopo aver tracciato una linea divisoria, ha posposto un'aggiunta di indice relativo ai documenti nn. 158-186, posti alle cc. 73-84; si tratta di quella documentazione relativa alle prebende, redatta dopo l'anno 1423, che ser Antonio dovette aggiungere dopo gli instrumenta della mensa comune e che in modo pressoché completo si trovano anche nella tabula oggi a c. 77 bis. Fra l'altro, redigendo questa aggiunta all'indice di c. 112/3, ser Antonio aggiunse una nota che recita «Quere retro» ovvero 'cerca indietro', riferendosi con essa ai documenti che si trovavano nelle carte anteriori e non, come nell'elenco posto sopra, nei fogli successivi.

\section{Il registro Instrumenta capitularia 8bis (1423-1437). Descrizione codicologica}

Molte delle considerazioni finora svolte per il registro del Capitolo in relazione al modus operandi del notaio sono valide anche per Instrumenta capitularia 8bis, su cui ser Antonio redasse quasi 700 documenti in un arco temporale compreso fra il $1423^{70}$ ed il $1437^{71}$. Il codice che si andrà ora a descrivere consta di 266 carte, della misura media di $440 \mathrm{~mm}$ in altezza per $345 \mathrm{~mm}$ in larghezza, distribuite in sedici fascicoli che non presentano numerazioni o segnature atte a identificarne l'inizio ${ }^{72}$. Il margine superiore-destro del recto di ogni carta, comprese quelle bianche, presenta una cartulazione realizzata con inchiostro di colore marrone chiaro attribuibile, ma si tratta di un'ipotesi, alla mano di ser Antonio. La stessa numerazione si trova infatti anche nella tabula instrumentorum che il notaio pose all'inizio del registro; diversamente da Instrumenta capitularia 8 , infatti, egli previde per questo codice un solo 'indice', predisponendo a tal scopo un certo numero di carte nel primo fascicolo del volume. A mano a mano che, con sequenza prevalentemente cronologica, inserì i documenti nel registro, egli aggiornò anche la tabula instrumentorum, aggiungendovi i nuovi rogiti con il numero della carta in cui furono redatti ${ }^{73}$. La numerazione delle carte appare sostanzialmene corretta, con la sola eccezione della c. 46, la cui cartulazione viene iterata anche nel foglio successivo; una mano anonima databile al XVII-XVIII secolo ha pertanto provveduto ad aggiungere la

70 Si hanno due documenti datati rispettivamente 1421 (ADTn, ACap, Instrumenta capitularia 8bis, n. 9) e 1422 (ivi, n. 38) che il notaio dichiara di aver rilevato ex quaternelo e ex uno folio; per il resto la registrazione progressiva dei rogiti sembra aver inizio dall'anno 1423 (ivi, n. 321a).

71 ADTn, ACap, Instrumenta capitularia 8bis, nn. 665a-665b; 1'ultimo documento in Instrumenta capitularia 8bis corrisponde all'ultimo documento redatto da ser Antonio di cui siamo a conoscenza.

72 Come per Instrumenta capitularia 8 si attribuisce per praticità ad ogni fascicolo una lettera dell'alfabeto maiuscola.

73 Probabilmente egli procedette a periodici aggiornamenti della tabula instrumentorum come si ricava dalle diverse tonalità dell'inchiostro impiegato. 
specificazione «primo» e «secundo» così da distinguere le due carte. Per il resto soltanto laddove, a causa di cadute parziali del supporto, i numeri originari sono andati perduti, due diverse mani hanno provveduto ad integrarle. Si tratta della già citata mano di età moderna (XVII-XVIII secolo), riscontrabile anche sui registri pergamenacei della Prepositura, e - in anni recenti - degli interventi dell'archivista Ivo Leonardi.

Il registro presenta inizialmente due carte di guardia moderne, applicate in occasione del restauro del 198174; fa seguito il primo fascicolo (A) (cc. 1-17) che consta di otto bifolia da cui, tuttavia, manca l'ultima carta. Il fascicolo seguente (B) è attualmente formato da sette bifolia e comprende le cc. 18-31. Analoga consistenza ha il quarto fascicolo (D) (cc. 51-64), mentre il precedente (C) consta di ben venti carte (cc. 32-50). Da c. 65 a c. 82 si ha il quinto fascicolo (E) che presenta nove bifolia, analogamente al settimo $(\mathrm{G})$, che va da c. 99 a c. 116. Più regolare in quanto a conformazione il sesto fascicolo $(\mathrm{F})$ che presenta sedici carte distribuite da c. 83 a c. 98 . L'ottavo fascicolo $(\mathrm{H})$ presenta una prima carta (c. 117) rilegata per errore in questa posizione in occasione dell'ultimo restauro; essa, sia per datazione sia per altri elementi su cui si dirà fra breve, andrebbe infatti posta all'inizio del primo fascicolo, probabilmente dopo le tabule instrumentorum. In seguito si ha un ottonione completo che occupa le cc. 118-132. Il nono fascicolo (I) è pure un ottonione, così come M (cc. 199-214), N (cc. 215-230) e P (cc. 249-264), dove sono rimaste bianche, seppur cartulate, le cc. 256-264. Il decimo fascicolo (J) presenta la seguente struttura: quattro bifolia completi + una carta aggiunta + ulteriori tre bifolia . Tale doveva essere, già al momento della cartulazione, la conformazione di questo fascicolo visto che non si rinvengono salti nella numerazione né sono riscontrabili mancanze o lacune. In analogia a quanto poc'anzi rilevato per il decimo fascicolo, il seguente (K) presenta un'organizzazione particolare. Anzitutto si hanno due bifolia, cui segue una carta e ulteriori sei bifolia completi. Come nel caso precedente non si riscontrano salti nella numerazione. Il dodicesimo fascicolo (L), da c. 181 a c. 198 e il quindicesimo $(\mathrm{O})$, da c. 231 a c. 248 , presentano 18 carte ciascuno. Il registro è chiuso da due ulteriori fogli di guardia in carta moderna, cui è stata allegata una tasca con i frammenti cartacei reperiti, in fase di restauro, fra le carte del codice ${ }^{75}$. Non si può sapere con certezza se il notaio conservò, già in origine, i vari fascicoli rilegati oppure li mantenne in forma sciolta, tuttavia $i$ frequenti rimandi a documenti redatti in precedenza con l'indicazione dell'esatto numero di carta fa ritenere che egli considerasse i fascicoli come parte di un intero ben definito. Basterà un solo esempio: a c. $81 r$ (documento n. 229) Antonio registrò la refuta di una proprietà della Prepositura; nel margine sinistro, oltre alla consueta rubrica, si leggono tre ulteriori annotazioni: la nota relativa all'estrazione del mundum, la registrazione del documento «in quaterno continuatorum» della Prepositura e, in riferimento a un mandato citato nel testo del rogito, «Nota ad memoriam quod ipsum mandatum notavi super presenti libro in carta decima». Se si verifica la c. 10 di Instrumenta

74 Sul primo foglio di guardia l'archivista Ivo Leonardi ha scritto: «ARCHIVIO DELLA PrEPOSITURA | Vol 91 | collocato per praticità | fra gli | "Instrumenta capitularia" | col n 8bis | 1423-1437». Nel secondo foglio di guardia, la stessa mano ha scritto: «Notaio | Antonio da Borgonuovo | (SN) | Vedi questo segno ai fogli: 119v, 129v, 150v, 170r, 199r, 210v,| 214r, 218v, 245r, 245v, 249r, 253v, 255r».

75 A carta $264 v$ del fascicolo P si trova una data («15.VIII.1939») vergata a matita probabilmente dalla mano di Vigilio Zanolini, il quale - come in Instrumenta capitularia 8 - ha numerato progressivamente $\mathrm{i}$ documenti del registro. 
capitularia 8bis, posta fra i documenti 30 e 31, si legge proprio la procura citata da ser Antonio, che egli aggiunse probabilmente in un secondo momento nello stretto spazio che separava $\mathrm{i}$ due rogiti ${ }^{76}$.

L'esempio suesposto permette di svolgere una considerazione relativamente ai termini impiegati da ser Antonio per definire questo registro. In analogia a quanto appurato per il volume del Capitolo, egli sembra, anche in questo caso, attribuire lo stesso significato ai termini quaternus e liber; se infatti, come nell'esempio a c. $81 r$, egli definisce Instrumenta capitularia 8 un liber, altrove impiega quaternus. Parlando del modus operandi si è verificata la definizione che egli stesso pone a principio del volume ove, lo si ricorda nuovamente, formula la seguente intestazione: "Quaternus instrumentorum notatorum et continuatorum» ${ }^{77}$.

Nulla si dirà in merito all'impostazione della pagina scritta, vista la corrispondenza con quanto già rilevato per Instrumenta capitularia 8 . Sarà invece necessario, dopo aver parlato della consistenza dei fascicoli, soffermarsi brevemente su alcuni aspetti estrinseci del registro, a partire dalla coperta. Essa è realizzata in cuoio di colore marrone scuro, liscio e uniforme al tatto; il piatto superiore presenta un prolungamento, detto ribalta, che sormonta il taglio laterale delle carte e copre per la misura di circa un terzo il piatto posteriore. In corrispondenza del dorso si notano quattro contrafforti, di materiale analogo alla coperta, che sono stati applicati mediante cuciture a vista; essi presentano una serie di intrecci a disegni geometrizzanti, realizzati mediante sottili strisce di pelle. La chiusura del registro avveniva, con ogni probabilità, mediante una stringa in cuoio cucita direttamente sulla coperta, come si deduce dai frammenti ancora rinvenibili ${ }^{78}$. La legatura così descritta è caratterizzata anche dalla presenza di due controguardie di forma rettangolare in pergamena, entrambe assicurate alla coperta mediante cuciture realizzate con stringhe di pelle allumata. La controguardia anteriore misura $470 \mathrm{~mm}$ in altezza per $410 \mathrm{~mm}$ in larghezza e ricalca, almeno nella forma la coperta di cuoio esterna. Si nota infatti la presenza di una ribalta applicata attraverso cuciture di colore azzurro al lato più lungo del foglio di pergamena, mentre sul lato carne si possono leggere alcune annotazioni riconducibili alla seconda metà del XV secolo ${ }^{79}$. In generale la coperta si presenta in buono stato di conservazione; più diversificata, invece, la condizione delle carte interne, dove in un contesto generale abbastanza buono, si devono tuttavia evidenziare consistenti perdite nel supporto, soprattutto in corrispondenza delle prime carte ${ }^{80}$, nonché gore, frequenti strappi e lacerazioni.

76 Leggermente più tenue è la tonalità dell'inchiostro e visibilmente più piccolo il corpo delle lettere.

77 Anche in margine al documento n. 582 a c. $221 v$ egli scrive: «Quere venditionem factam de predictis factam ipsi Francisco in hoc quaterno in carta $97^{\mathrm{a}} \gg ;$; per quaternus egli intende quindi l'intero registro. Se si verifica a c. 97 , sul recto si trova proprio la compravendita citata, molti fascicoli più avanti, da ser Antonio.

78 Si trattava probabilmente di una cintura in cuoio dipinta di colore rosso vivo, come sembra dimostrare un piccolo frammento della stessa in corrispondenza della sezione centrale della coperta.

$79 \mathrm{Al}$ centro si legge: «Nota ad memoriam quod hic in ista coperta est quedam scripture | ut ad predictum devenire non possint et ad melius cognoscendum et $\mid$ memoria adducendum in coperta exteriori suprascripti etc». Più in basso, un'altra mano anonima della metà del XV secolo, scrisse: «Desiderat(us)». La controguardia posteriore presenta invece una sola annotazione, pure attribuibile ad una mano anonima del XV secolo: «Iacob(us)».

80 Fortemente danneggiata risulta anche l'attuale c. 117 che, come poc'anzi ipotizzato, doveva trovarsi in origine all'inizio del registro. I tagli sulla carta e le cadute del supporto corrispondono infatti ai danni che si rilevano sulle prime carte del codice. 
Nulla si dirà, invece, in questa fase sulle etichette, ovvero le segnature, poste sulla coperta del registro; ad esse si dedicherà infatti specifica attenzione parlando della trasmissione documentaria di tale volume ${ }^{81}$.

La carta impiegata per confezionare il registro è uniforme per qualità; si individua infatti una sola tipologia di filigrana, ovvero la testa di cervo sormontata da un tratto con in apice un fiore a cinque petali già riscontrato e descritto nell'ultimo fascicolo di Instrumenta capitularia 8. Essa è posta all'incirca al centro della carta ed è compresa esattamente fra due filoni distanti $43 \mathrm{~mm}$.

\subsection{L'organizzazione del registro}

Il primo fascicolo del codice, come ricordato, inizia con una tabula instrumentorum $^{82}$ dove il notaio registrò progressivamente le rubriche dei documenti stesi. Essa, tuttavia, si presenta allo stato attuale mutila di almeno una carta, cosicché vi risultano registrati soltanto i documenti vergati dalla c. 183 compresa in poi. La struttura dell'indice non si discosta sostanzialmente da quella descritta per Instrumenta capitularia 8; in questo caso, tuttavia, ser Antonio non provvide a segnalare mediante le usuali sigle $f$ e $f f$, all'inizio di ciascuna rubrica, l'avvenuta estrazione della pergamena (o delle pergamene) in mundum.

Per quanto concerne invece la progressione cronologica dei documenti raccolti nel registro, l'analisi sulle date croniche dei rogiti mostra in sostanza il rispetto dell'ordine cronologico, con alcune eccezioni (fig. 25): anzitutto, si è già avuto modo di sottolinearlo, interrompono il consueto ordine cronologico della documentazione quei rogiti che ser Antonio dichiata di aver relevato dai suoi protocolli, da fogli sciolti o da bifolia. Sul motivo di questo modus operandi si è già ampiamente discusso e dunque non ci si soffermerà oltre. Non mancano poi i documenti in forma di copia semplice; si tratta di documentazione che, in larga misura, il notaio ritenne doveroso esemplare sul proprio registro in quanto utile a suffragare altri rogiti lì registrati. Sarà utile proporre almeno un esempio. Nel 1430 il vescovo Alessandro di Masovia vendette l'intera eredità del fu Antonio Belenzani a ser Pietro di Nanni da Siena ${ }^{83}$, il quale nei mesi immediatamente successivi procedette alla vendita dell'intero patrimonio a vari cittadini di Trento e dintorni. E interessante notare in proposito come ser Antonio ritenne opportuno copiare anche il testamento di Antonio Belenzani e ciò avvenne, per sua stessa ammissione, «ad avisamentum et deffenssionem eorum qui emerunt de bonis suis ab antedicto ser Petro de Senis qui emit a domino nostro executore dicti testamenti» ${ }^{84}$.

Un'altra serie di rogiti sembra interrompere il normale ordine cronologico; si tratta solitamente di quelle tipologie documentarie (quali, ad esempio, le quietanze (solutio debiti) o quelle che egli definisce «mandatum ad canzelandum»), che andavano a modificare o annullare il dispositivo di un documento precedente. A c. $161 v$ Antonio registrò il 9 ottobre $1430^{85}$ un creditum, ovvero la promessa da parte di Felicia, vedova di Giovanni Belenzani, di versare nei termini stabiliti (entro il Natale successivo) 125 ducati d'oro a ser Pietro di Nanni da Siena, come prezzo

81 Cfr. infra, capitolo 5.

82 Rispetto alle altre carte del registro la tabula non presenta cartulazione.

83 ADTn, ACap, Instrumenta capitularia 8bis, n. 395. Cfr. supra, § 10.1.

84 Cfr. Appendice, regesti nn. 1, 2 (ADTn, ACap, Instrumenta capitularia 8bis, n. 498).

85 ADTn, ACap, Instrumenta capitularia 8bis, n. $416 \mathrm{~b}$. 


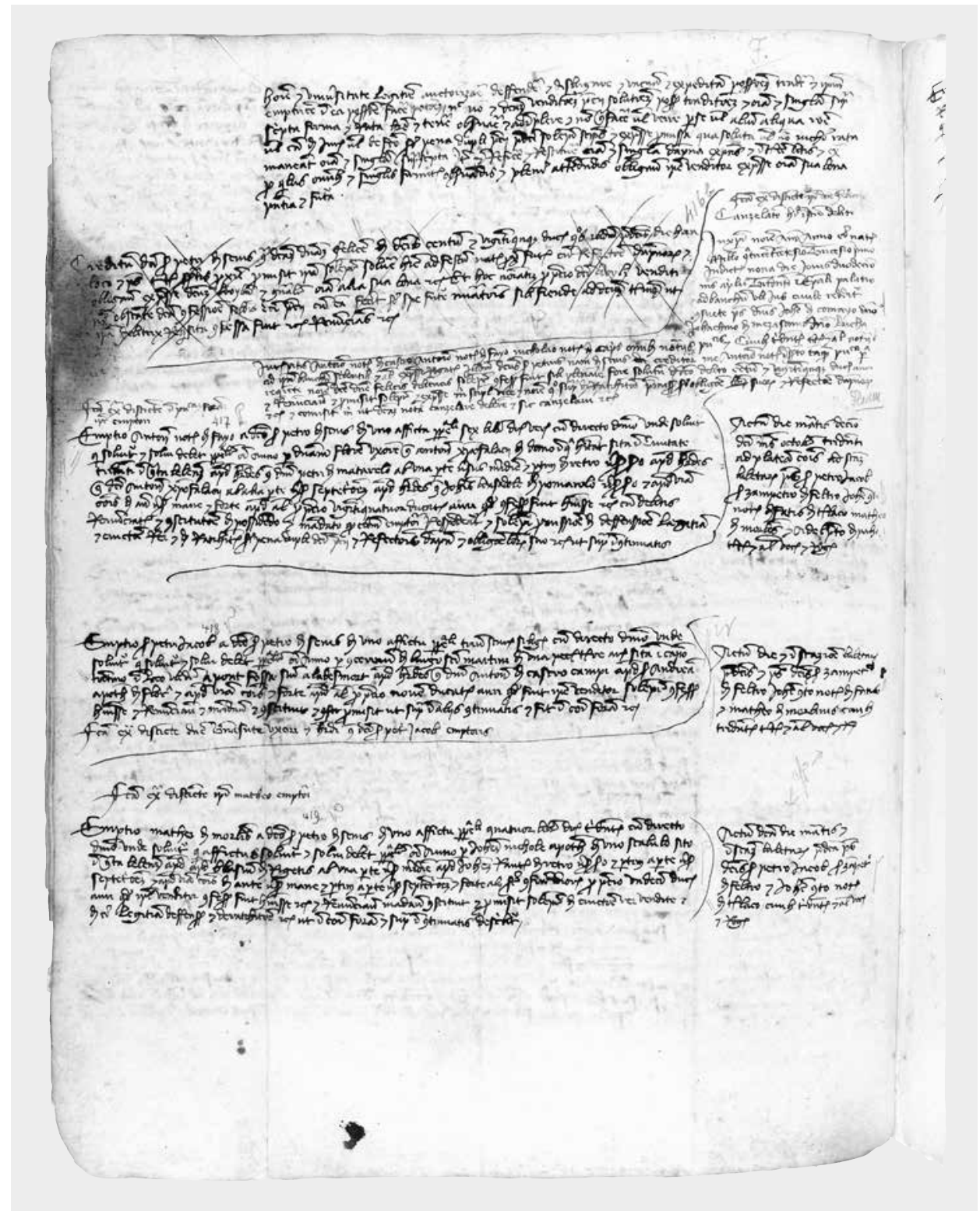

Fig. 25. ADTn, ACap, Instrumenta capitularia 8bis, sezione superiore della c. $161 v$. In alto, l'ultima parte del documento attestante la compravendita (n. 416). Segue il creditum cancellato (n. 416b) e, sulla destra nello spazio fra i due rogiti, con inchiostro di tonalità più chiara, la Canzelatio huius instrumenti debiti. In basso nuova compravendita redatta in forma abbreviata (n. 417) cui il notaio ha affiancato alcuni dati relativi alla data topica e ai testimoni presenti. 
dovuto per l'acquisto di un broilo. Il rogito in questione è posto immediatamente dopo la relativa compravendita e rispetta la progressione cronologica. Il documento seguente, infatti, è datato 10 ottobre $1430^{86}$. Ma in margine al creditum il notaio aggiunse il 12 aprile 1431 un ulteriore rogito denominato «Canzelatio huius instrumenti debiti». Esso rappresentava da un lato la quietanza di pagamento per il debito di 125 ducati rilasciata a Felicia da ser Pietro, dall'altro conferiva al notaio il compito di cancellare l'atto che attestava il debito. Il professionista non mancò infatti di cancellare quel documento annullandone così l'efficacia giuridica; in seguito egli rilasciò il relativo mundum a Felicia la quale avrebbe così potuto dimostrare in futuro di essere libera da qualunque debito nei confronti di ser Pietro.

Si tratta, dunque, di anomalie cronologiche perfettamente giustificabili derivanti in parte anche dal continuo aggiornamento del registro da parte del professionista ${ }^{87}$. In conclusione rimane un ulteriore aspetto da chiarire relativo al modus operandi di ser Antonio: la sua scrittura. Si tratta di una componente che travalica le caratteristiche proprie di ogni singolo registro e che, per tale ragione, si tratterà singolarmente.

\section{La scrittura di Antonio da Borgonuovo}

L'analisi degli esiti grafici di ser Antonio mette in rilievo l'utilizzo di una scrittura minuscola corsiva, comunemente impiegata dai notai, anche trentini, fra XIV e $\mathrm{XV}$ secolo, dalla quale tuttavia si discosta per alcuni elementi morfologici. Anzitutto l'impiego di $s$ diritta ed $f$ con aste fortemente rastremate e inclinate ottenute mediante un doppio tratteggio dall'alto verso il basso e viceversa. Si ottengono così due lettere dalla morfologia particolare, tipica di scritture transalpine più che cisalpine; ciò non è secondario e aggiunge senza dubbio nuovi elementi a quanto già evidenziato sulla formazione del notaio. Fra le lettere caratteristiche si notano anche una $a$ in forma corsiva, $d$ in forma onciale con l'occhiello superiore molto sviluppato, $g$ con l'occhiello superiore chiuso da un trattino orizzontale che talvolta lega con il primo tratto della lettera seguente, $i$ presenta solo raramente gli apici, $r$ è bimorfa, solitamente diritta in inizio o in corpo di parola, tonda in fine oppure dopo lettera con convessità a destra (ad esempio, -or-). Particolare pure la morfologia di $u / v$ dal primo tratto molto alto sul rigo che tende poi a ripiegarsi verso il basso conferendo alla lettera una tipica forma 'a cuore'. Più semplice ed usuale la $u$ in corpo $o$ in fine di parola. Numerose sono le legature, sebbene, rispetto agli esiti documentari d'altri notai del periodo, sia qui riscontrabile una minore corsività; ciò è dovuto anche all'utilizzo di uno strumento scrittorio dalla punta temperata obliquamente, come si deduce dalla presenza di leggeri chiaroscuri determinati dal contrasto fra tratti pieni e filetti sottili, ad esempio, nella realizzazione della nota tironiana et a forma di sette, con il tratto discendente visibilmente più sottile rispetto a quello orizzontale che lo sormonta. Allo stesso modo sembrano essere stati realizzati di frego i numerosi segni abbreviativi che, nella usuale forma del titulus ricurvo, risultano tanto sottili dall'essere talvolta appena percettibili.

86 Ivi, n. 417.

87 Ai casi suesposti si aggiungono i documenti nn. 321, 321a-b-c-d che datano al novembre del 1423 e si trovano fra rogiti del 1429. Essi infatti sono trasmessi da un foglio (c. 117) che, come si è già avuto modo di ricordare, è stato erroneamente rilegato in questa posizione, avendo invece dovuto trovare posto all'inizio del registro, subito dopo la tabula instrumentorum. 
Una menzione a parte meritano le lettere maiuscole che ser Antonio impiega con una certa frequenza ma senza rispettare alcun criterio; per quanto riguarda, anzitutto, la forma delle singole lettere, esse presentano spesso i tratti raddoppiati, come è tipico della gotica. Particolare è la morfologia di $A$ maiuscola che il notaio riprende al centro del proprio segno tabellionale, come pure di $R$ maiuscola che assume la forma di una $V$ alta sul rigo tagliata da un trattino trasversale che le permette di legare con la lettera seguente. Come ricordato, non è possibile individuare alcun criterio nell'utilizzo della lettera maiuscola; talvolta essa trova infatti impiego nei nomi propri, talaltra nei nomi comuni o, addirittura, negli attributi, mentre lemmi che ne richiederebbero l'uso vengono scritti con la lettera minuscola (fig. 26).

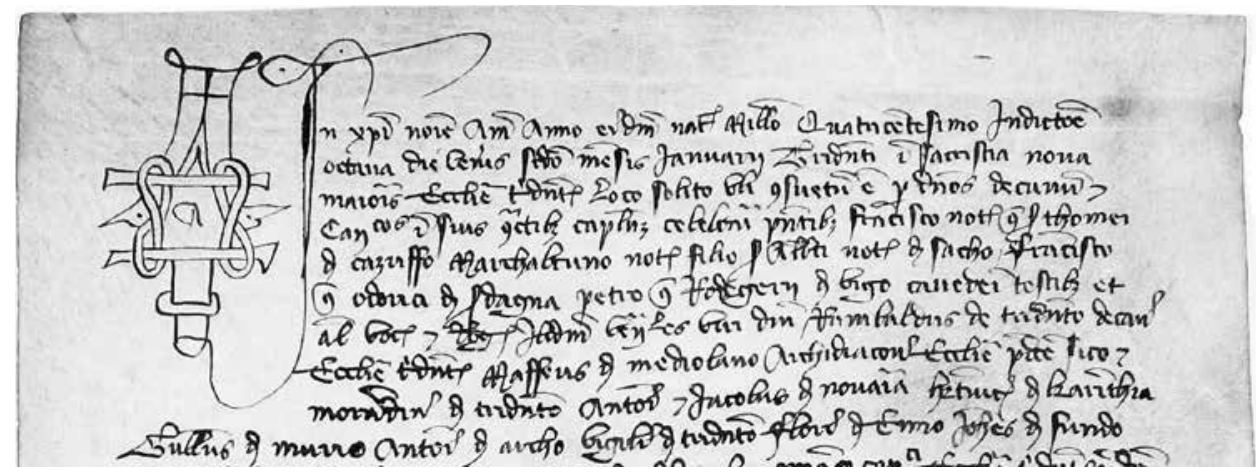

Fig. 26. ADTn, ACap, capsa 1, n. 8 (particolare). Dettaglio del signum e del protocollo in un instrumentum di Antonio da Borgonuovo. Si noti come la lettera $I$ - che apre l'invocatio sia raddoppiata e allungata, quasi ad incorniciare, in corrispondenza del margine sinistro, tutti gli elementi costitutivi del protocollo (invocazione, datazioni cronica e topica ed elenco dei testimoni). Nei documenti in mundum il notaio predispose prima di tutto il testo, delimitando lo specchio di scrittura con una marginatura a secco e lasciando bianco lo spazio in cui, in un secondo momento, disegnò il suo signum: da evidenziare, al centro del segno tabellionare, la presenza di due teste zoomorfe (a destra e a sinistra), simili a teste d'uccello, nelle quali si distinguono il becco e gli occhi. Al centro, la lettera $A$ maiuscola, iniziale del nome del notaio.

Se negli esiti grafici su pergamena sciolta e in buona parte della documentazione in esteso su registro ser Antonio tende a mantenere un modulo di scrittura piuttosto uniforme, con una tendenza tuttavia a inclinare le lettere verso destra procedendo negli anni ${ }^{88}$, l'esecuzione dei testi redatti su brogliacci o negli spazi rimasti bianchi fra le carte dei registri tende ad essere mediamente meno controllata; il ductus assume gradazioni diversificate, il modulo tende a rimpicciolirsi o ad ingrandirsi. Sono molti i casi in cui, non avendo lasciato uno spazio sufficiente per accogliere, prima di un nuovo rogito, un ulteriore documento, egli fu costretto a scrivere il testo con un modulo di scrittura visibilmente più ridotto.

Come è tipico delle scritture documentarie, molto numerose sono le abbreviazioni; il generico titulus di forma più o meno arcuata o ripiegata viene utilizzato per segnalare la caduta delle desinenze in $-m$ e $-n$, anche laddove le nasali siano in corpo di parola, oppure per indicare generiche abbreviazioni per contrazione.

88 Soprattutto negli ultimi anni di attività, corrispondenti al periodo $1435-1437$, la scrittura di ser Antonio tende a slargarsi e diviene meno 'controllata'. Ciò è rilevabile in buona misura soprattutto nella documentazione su registro ed è meno visibile negli esiti scritti delle pergamene in mundum. 
Un segno a forma di fiocco che si ripiega verso il basso individua invece, in fine di parola, alcune abbreviazioni per troncamento. Ser Antonio predilige poi l'uso di et tachigrafico a guisa di 7 in luogo di et e del $c$ converso per con/cum, anche in corpo di parola. Si tratta di modalità operative tipiche delle scritture documentarie, impiegate comunemente anche fra i notai trentini dell'epoca.

Praticamente sconosciuto al notaio sembra invece essere un qualsiasi sistema di punteggiatura; l'esame sui registri e sulle pergamene sciolte non rivela l'impiego d'alcun particolare segno di interpunzione; non sono separati da punti fermi nemmeno i diversi periodi che, all'interno del dettato, rappresentano le diverse partizioni del documento. Un solo segno compare nei rogiti di ser Antonio: si tratta di due punti affiancati da una sorta di doppia s allungata che ha probabilmente lo scopo, posta a chiusura del testo e della sottoscrizione, di non permettere alcuna aggiunta al dettato.

Se piuttosto 'pulito' è l'aspetto dei testi redatti su pergamena sciolta, maggiormente diversificata è la situazione riscontrabile nei documenti in registro; più vicini ai munda, per qualità del lavoro sono, ad esempio, una parte dei rogiti redatti per esteso in Instrumenta capitularia 8 e 8 bis. Una porzione di questi, tuttavia, mostra evidenti interventi di correzione e integrazione attuati in seconda battuta dal notaio; probabilmente in fase di rilettura (come spesso si ricava dall'utilizzo di un inchiostro di diversa tonalità), ma talvolta anche in corso di stesura. Ser Antonio provvide infatti a depennare parti del testo o singoli lemmi integrandoli con i termini corretti in interlinea o in margine. Qualora le parti di testo da emendare fossero state tali da obbligare il notaio ad aggiungerle nel margine o in calce al documento, egli utilizzò coppie di segni di richiamo, mentre per la semplice inserzione di termini posti in interlinea impiegò l'usuale $\mathrm{V}$ capovolta. Numerose sono le occasioni in cui, forse a causa di una copiatura disattenta da precedenti stesure o da specifici formulari, egli iterò lo stesso termine. Meno praticata, quale tecnica di correzione, l'espunzione o, nel caso delle pergamene, la raschiatura mediante apposito strumento del termine errato e la sua sostituzione. Non mancano, infine, i casi in cui, non avendo a disposizione tutti i dati necessari a completare il rogito, Antonio lasciò intenzionalmente in bianco alcuni brevi spazi nel testo. Taluni di quegli spazi, che avrebbero dovuto essere completati, ad esempio, con informazioni relative al patronimico o al luogo di provenienza, non furono però colmati dal notaio il quale non aveva verosimilmente ricevuto in seguito le informazioni necessarie. 
Antonio da Borgonuovo al lavoro

Struttura dei fascicoli del registro "Instrumenta capitularia” 8
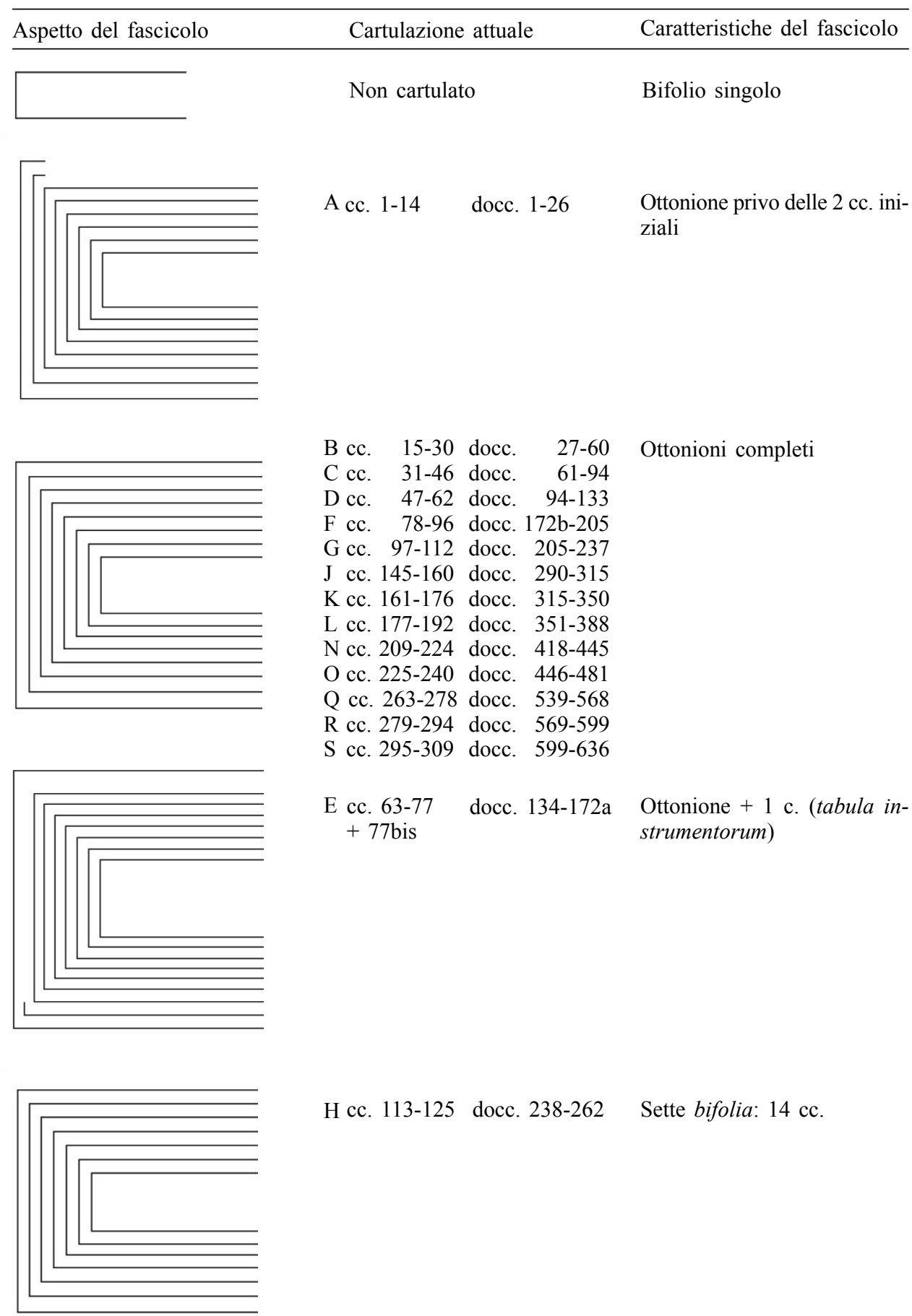

H cc. 113-125 docc. 238-262 Sette bifolia: 14 cc. 
Stefano Malfatti

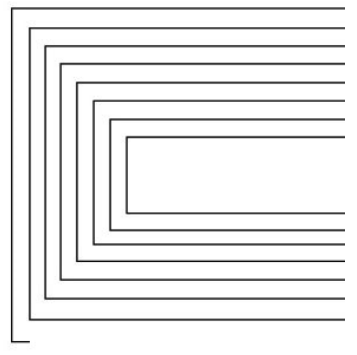

I cc. 126-142 docc. 262-289 Ottonione privo dell'ultima carta

M cc. 192 bis docc. 389-417 1 c. (tabula instrumentorum) (tabula instrumentorum ) + cc. $193-208$ + ottonione
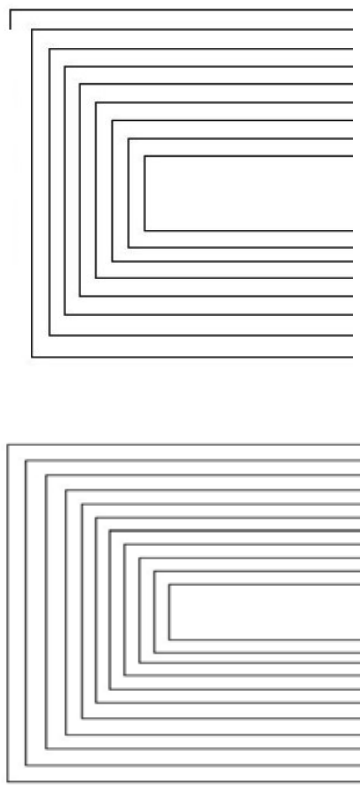

P cc. 241-262 docc. 482-538b 11 bifolia: 22 cc.

T cc. 310-335 docc. 638-695 12 bifolia: 24 cc. 
Antonio da Borgonuovo al lavoro

Struttura dei fascicoli del registro "Instrumenta capitularia” 8bis

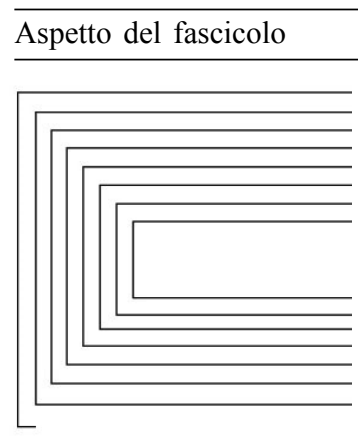

Cartulazione attuale

A cc. $1-17$ docc. $1 \mathrm{a}-50$

A

B cc. $18-31$ docc. $51-89$

D cc. $51-64$ docc. $135-179$

C cc. $32-50$ docc. $90-134[\mathrm{~b}]$

10 bifolia: $20 \mathrm{cc}$.
Ottonione privo dell'ultima carta; le prime 2 cc. sono occupate dalla tabula instrumentorum

7 bifolia: 14 cc.

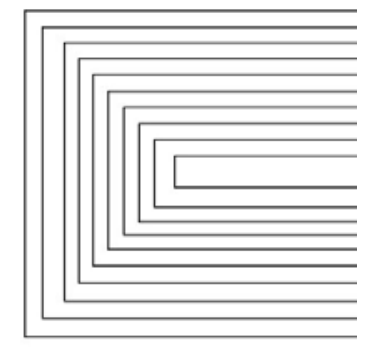

E cc. $65-82$ docc. 180-232[b] 9 bifolia: 18 cc.

G cc. $99-116$ docc. $272-321$

L cc. $181-198$ docc. $476-518$

O cc. $231-248$ docc. $605-645$ 


\section{Stefano Malfatti}

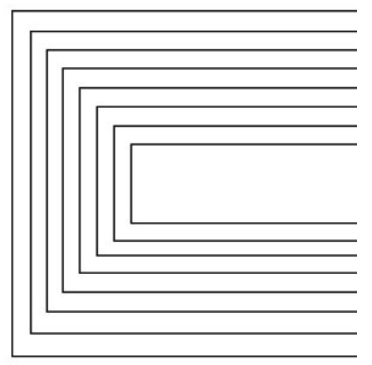

F cc. $\quad 83-98$ docc. $233-2718$ bifolia: 16 cc.

I cc. $133-148$ docc. $356-388$

M cc. $199-214$ docc. $518-572[\mathrm{~b}]$

$\mathrm{N}$ cc. $215-230$ docc. $573-604[\mathrm{~b}]$

$P$ cc. $249-264$ docc. 647-665

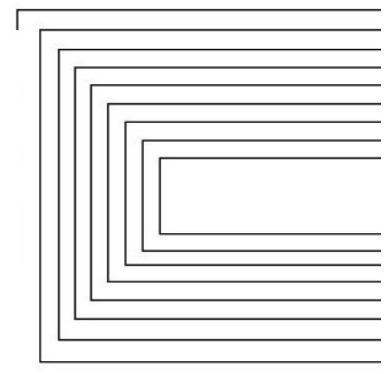

H cc. 117-132 docc. 321-355 1 c.(117)+8bifolia (cc. 118132)

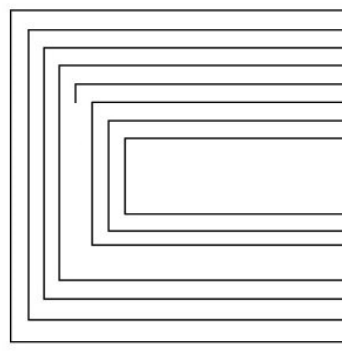

J cc. $149-163$ docc. $389-428$

Ottonione mancante della dodicesima carta

(struttura: 4 bifolia $+1 \mathrm{c}$.

+3 bifolia)

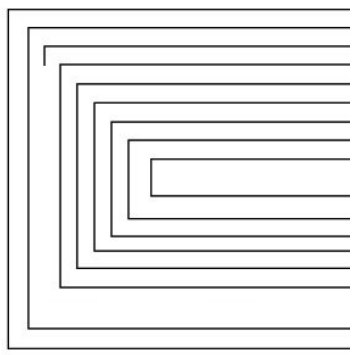

K cc. $164-180$ docc. $429-475$ Struttura: 2 bifolia +1 c. +6 bifolia 



\section{Capitolo 5 \\ Il 'destino' delle carte}

\section{Tracce di trasmissione di documentazione notarile fra XIV e XV secolo}

Carte, protocolli e registri prodotti dai notai nel corso della loro carriera rappresentavano non soltanto gli strumenti per la garanzia degli interessi di enti o privati che si erano rivolti ai professionisti per la registrazione dei propri negozi giuridici, ma anche un'importante fonte di reddito per quanti avevano redatto e conservato quelle registrazioni; ciò è vero sia per il notaio che aveva originariamente registrato le volontà dei contraenti sia per quanti - anche a distanza d'anni - furono autorizzati a trarre da quelle carte documenti in mundum pienamente validi sotto il profilo giuridico.

Anche a Trento, come altrove nell'Italia medievale, i volumi di imbreviature e di estesi erano affidati alla gestione e alla cura dei notai che li avevano prodotti, dopo la morte dei quali essi passavano quindi agli eredi, qualora questi avessero esercitato la medesima professione, o ad altri colleghi. Poiché, tuttavia, l'importanza e l'interesse della documentazione redatta da un notaio andava diminuendo col tempo, calava progressivamente anche il vantaggio, per gli eredi o per altri notai, di conservare per più di un paio di generazioni gli atti del professionista defunto; accadde così, con una certa frequenza, che molti registri - prevalentemente con documentazione redatta per privati - andarono inesorabilmente persi, perché scartati dopo pochi decenni per volontà degli stessi notai che li avevano ereditati. Vennero sottratti a questo destino soltanto registri e carte che, per via del contenuto, furono ritenuti d'interesse da parte di enti laici e religiosi, tanto da essere ricondotti ai loro rispettivi archivi. Tali modalità operative si riflettono, oggi, nella scarsità se non quasi totale assenza in alcuni periodi - di registri notarili almeno fino al Quattrocento avanzato'.

È quanto si constata a Trento, dove il problema della salvaguardia e trasmissione delle carte dei notai defunti venne risolto soltanto in piena età moderna, con l'istituzione, dal 1595, di un 'archivio dei notai morti' e un 'archivio dei notai vivi'2.

Referenze fotografiche. Le figg. 27-32 sono pubblicate con 1'autorizzazione dell'Archivio diocesano tridentino.

1 Sulla trasmissione delle scritture dei notai trentini nei secoli XII e XIII cfr. Codex Wangianus, cit., in particolare le pp. 78-79 e Cagol, Il ruolo dei notai, cit., pp. 148-153. Come osserva Franco Cagol, «se oggi abbiamo a disposizione documentazione prodotta per committenti privati, lo dobbiamo spesso a operazioni di recupero messe in atto da alcuni notai attivi al servizio di episcopati e comuni al fine di avere a disposizione documentazione prodotta in ambito pubblico dai loro colleghi defunti» (ivi, p. 152).

${ }_{2}$ Cfr. Casetti, Il notariato trentino, cit. 


\section{Il 'destino' delle carte}

Ad inizio Quattrocento, Antonio da Borgonuovo opera dunque in una fase di piena transizione, con un collegio notarile ancora incapace di esercitare il pieno controllo sulla produzione documentaria dei propri notai e, di riflesso, sui procedimenti di trasmissione delle carte dei notai defunti.

La fortunata conservazione di due documenti relativi alla trasmissione di atti di notai sul registro Instrumenta capitularia 8bis consente di ricostruire almeno parzialmente le pratiche di affidamento delle carte ad un nuovo professionista. Il 10 dicembre $1425^{3}$ Cristina, moglie del defunto notaio Giacomino da Posina ${ }^{4}$, fu chiamata in episcopali palatio, ad banchum iuris civilis, alla presenza del vicario per le cause civili e criminali Antonio de Zivolis da Ledro e dei notai Antonio de Castro, Antonio da Nogaredo, Antonio da Fai, Guglielmo Saraceno, Nicolò de Capris e Vito da Dambel. Costei, rappresentata dal notaio Antonio da Molveno, giurisperito e, soprattutto, priore e rettore del collegio notarile di Trento ${ }^{5}$, fu invitata ad esibire a un membro del collegio «imbreviaturas et prothocola» lasciati dal marito defunto, affinché potessero essere rilevati da un professionista ritenuto «ydoneum et sufficientem». Ricevuta, dunque, «ad instantiam et petitionem quam plurium» la disponiblità di «rogationes et imbreviaturas» del defunto Giacomino, il rettore scelse quale notaio relevator Nicolò del fu ser Pietro Baldovini de Capris, il quale ricevette dal vicario vescovile

licentiam ... et plenam auctoritatem relevandi, transcribendi et in publicam formam reddigendi rogationes, imbreviaturas et prothocolla dicti condam Iacobini notarii ... mandans et decernes ipse dominus vicarius rogationes et imbreviaturas quas ipse Nicholaus relevabit ex imbreviaturis et rogationibus ipsius condam Iacobini notarii illam vim habere et obtinere qua(s) habuissent si ipse Iacobinus notarius dum viveret perficisset ${ }^{6}$.

Mentre nell'esempio qui preso in considerazione si è messo in luce uno dei casi forse più usuali, ovvero la comissio delle carte di un notaio defunto, il documento che si andrà ora ad esaminare, cronologicamente di poco successivo, mostra una circostanza meno attestata nelle fonti; è infatti il caso di un notaio, Vigilio del fu ser Enrico Rigaie da Trento, ancora vivente ma, «gravi infirmitate oppressus», e dunque inabile a proseguire la propria attività.

Il 29 aprile 1426 ser Vigilio si presenta infatti dinanzi al già citato vicario vescovile Antonio de Zivolis da Ledro. Sede dell'atto, in quest'occasione, non è il

3 ADTn, ACap, Instrumenta capitularia 8bis, n. 142.

4 Cfr. Stenico, Notai che operarono nel Trentino, cit., sub voce Giacomino da Posina. Il notaio Giacomino era figlio di Albertino, un tabernarius proveniente da Posina, nel vicentino, e fu attivo a Trento fra la fine del Trecento e la metà degli anni Venti del Quattrocento. All'inizio del XV secolo fu in più occasioni procuratore con il compito di refutare proprietà immobiliari al Capitolo della cattedrale di Trento: ADTn, ACap, capsa 22, n. 2; capsa 22, nn. 3 e 3.1; capsa anniversari, rotoli lunghi/b, n. 15; di mano di Giacomino si conservano due pergamene presso l'archivio della Prepositura: ADTn, $A P$, Tomo III, nn. 5 e 9.

5 Per quanto oggi è noto, il rettore o priore del collegio esercitava funzioni di rappresentanza dinanzi ad autorità esterne e coordinava le attività del collegio; veniva eletto dalla maggioranza dei votanti e restava in carica per un anno. Coadiuvavano il rettore quattro consiglieri, eletti secondo analoghe modalità, un massaro, che amministrava il patrimonio, un notaio e due bidelli che mantenevano la carica per la durata di quattro mesi. Poco altro si conosce, ad inizio XV secolo, relativamente al collegio e alla sua struttura (cfr. Varanini, Il Collegio notarile di Trento, cit.).

6 ADTn, ACap, Instrumenta capitularia 8bis, n. 142. 
palazzo vescovile, ma la stazione delle bollette, poco distante; presenziano in qualità di testimoni i notai Antonio de Castro, Iosio del fu ser Francesco da Trento e Marco domine Zinele dal Borgonuovo. In quell'occasione il notaio Vigilio:

asseruit et proposuit et dixit quod plurima et plurima instrumenta diversarum manierarum et rogationes notavit et scripsit in suis prothocollis, que et quas tamen in publicam formam non redegit nec redigere potet propter eius gravem infirmitatem, ex quo supplicavit et petivit ab ipso domino vicario ut dignaretur concedere licentiam et plenam auctoritatem Gasparino notario filio Antonii notarii de Nogaredo civi Tridentino relevandi et in publicam formam redigendi eius Vigilii notas et rogationes et imbreviaturas ${ }^{7}$.

Di fronte alla necessità di estrarre da questi protocolli numerosi documenti su pergamena, vista la grave infermità, ser Vigilio fu dunque costretto ad affidare le proprie carte al notaio Gasparino, figlio del notaio Antonio da Nogaredo. La petizione fu rivolta direttamente al vicario vescovile ${ }^{8}$, senza alcuna apparente 'mediazione' da parte del collegio notarile. Udita la richiesta e ritenuto Gasparino adeguato al compito, il vicario concesse a quest'ultimo la licenza e la piena autorità di rilevare, trascrivere e redigere in forma pubblica «rogationes et imbreviaturas». L'atto si concluse con l'usuale giuramento prestato dal notaio relevator dinanzi al vicario, al notaio rogante e a ser Vigilio. L'autorizzazione concessa a Gasparino diede a quest'ultimo la possibilità di rilevare le carte del notaio Vigilio, dalle quali - su richiesta dei committenti - poté trascrivere pergamene in mundum ${ }^{9}$.

\section{Antonio da Borgonuovo relevator di documentazione capitolare}

Lo spoglio sistematico dei fondi pergamenacei e l'analisi sulle note relative all'estrazione di redactiones in mundum sui registri notarili del Trecento hanno permesso di individuare alcuni notai di cui ser Antonio rilevò la documentazione. Si tratta, in larga parte, di professionisti attivi nel corso del XIV secolo per il Capitolo della cattedrale: Venturino de Trechis, Pietro de Stanchariis, Federico di Enrico da Albiano e Giacomo da Ravazzone, unico fra i notai citati a non essere qualificato come notaio capitolare.

Vista la quasi totale corrispondenza fra la documentazione rilevata da Antonio e gli esiti documentari di gran parte dei notai capitolari attivi nel corso del Tre-

7 ADTn, ACap, Instrumenta capitularia 8bis, n. 179. Particolare sembra la locuzione impiegata dal notaio per descrivere e qualificare le modalità di registrazione dei numerosi documenti sui suoi protocolli: «instrumenta diversarum manierarum ... notavit et scripsit»; si potrebbero ricondurre queste 'diverse maniere', alle molteplici modalità di redazione dell'instrumentum notarile. In alcuni casi si sarà forse trattato di vere e proprie imbreviature, in altri di estesi molto simili ad eventuali redazioni in mundum.

8 Se per la città di Trento l'autorità competente per il rilascio delle licentie relevandi era il vicario/podestà vescovile, per i restanti territori della diocesi tale funzione fu attribuita all'autorità giudiziaria del relativo comitato o giurisdizione; ciò avvenne, ad esempio, quando ser Osvaldo detto Sengel da San Michele all'Adige, vicario generale e ius publice redens in civilibus et criminalibus causis in toto comitatu Cunespergi nec non in Cimbrie iurisdictionis per il conte del Tirolo, concesse a Nicolò, figlio di ser Giovanni de Ligatis da Serso, cittadino di Trento ma residente a Cembra, di rilevare i rogiti del fu ser Giovanni da Giovo (BCTn, BCT3, capsa 32, mazzo 1, n. 1).

9 È il caso del testamento della domina Elica del fu ser Gabriele dal Borgonuovo di Trento, redatto il 25 agosto 1407 da ser Vigilio (ADTn, ACap, capsa testamenti, rotoli medi/a, n. 14). 


\section{Il 'destino' delle carte}

cento, l'analisi sulle comissiones del professionista non potrà che intrecciarsi con analoghe licentie relevandi concesse nei decenni precedenti, per le stesse carte, agli altri notai dell'ente.

Si inizierà con l'autorizzazione ad estrarre dalle «rogationes, prothocolla, instrumenta et imbreviaturas» redatte fra il 1354 e il 1392 dal canonico e notaio Pietro del fu Stefano de Stanchariis da Teglie di Brescia ottenuta da ser Antonio il 14 luglio $1411^{10}$ per concessione del vicario vescovile Antonio Schichignoli da Trento. Ciò che distingue nella sostanza le licenze esaminate in precedenza da quelle relative a documentazione capitolare è il fatto che queste ultime avvennero, praticamente sempre, su richiesta, ovvero «ab instanciam et petitionem», degli stessi canonici. Così, ad esempio, l'estrazione di redactiones in mundum dalle rogazioni, dai protocolli, dagli instrumenta e dalle imbreviature di Pietro de Stanchariis fu concessa ad Antonio da Borgonuovo su richiesta di Florio da Denno, sindaco e massaro del Capitolo della cattedrale ${ }^{11}$. La licenza conferita ad Antonio è oggi leggibile sia in calce ad una locazione su pergamena posta presso il fondo capitolare dell'Archivio diocesano tridentino, sia alla c. $606 r$ del volume del de Stanchariis Instrumenta capitularia 7.

Numerosi rogiti contenuti nel suddetto registro recano annotazioni relative all'estrazione di munda da parte dei notai che, in epoche diverse, furono autorizzati a lavorarvi: in primis Alberto del fu ser Negratus da $\mathrm{Sacco}^{12}$, del quale si hanno ben trentadue annotazioni in margine a rogiti estratti ${ }^{13}$; in seconda posizione, Antonio da Borgonuovo, del quale si trovano dodici note ${ }^{14}$ del tipo Relevata est, oltre a un intero documento rogato da ser Antonio l'11 maggio 1412 e posto in uno spazio rimasto bianco della c. $31 v^{15}$. Il professionista si premurò, in talune occasioni, di specificare per quale ragione, oppure su istanza di quale autorità, dovette estrarre il mundum. Si hanno così annotazioni del tipo: «Relevata per me Antonium notarium ser Bartholasii pro Capitulo etc ${ }^{16}$, «Relevata per me Antonium notarium ser Bartolasii ad instanciam Otolini conductoris infrascripti et sibi data» ${ }^{17}$ oppure "Relevata per me Antonium notarium ser Bartholasii ex licentia michi data et data ipsi Bartolo ex eo quia in sachemano amisit sibi factam et datam etc» ${ }^{18}$ e ancora «Relevata per

10 Cfr. ADTn, $A C a p$, capsa 32, n. 224 e ASTn, $A C D$, n. 1398-01, c. $306 r$ (ex Instrumenta capitularia 7).

${ }_{11}$ Su Florio da Denno cfr. la scheda biografica in Curzel, I canonici e il Capitolo, cit., pp. 529-531.

12 Cfr. le sottoscrizioni con la licentia relevandi di Alberto da Sacco in calce ai documenti in ADTn, ACap, capsa 3, n. 8 e BCTn, BCT3, capsa 3, mazzo 1, n. 22. L'autorizzazione fu concessa da Bartolomeo da Bologna, decretorum doctor e abate di San Lorenzo, vicario generale in spiritualibus per il vescovo di Trento Georg Liechtenstein e da Nicolò de Tanuciis da Levico legum doctor, come attestato dal documento rogato da Antonio di ser Paolo Pevrada notaio cittadino di Trento.

13 Le note relative all'estrazione di munda di Alberto da Sacco si trovano in margine ai documenti posti alle carte: $50 r-v, 51 r, 67 v-68 r, 89 r-v, 89 v-90 r, 90 r-v, 93 v, 98 v-99 r, 108 r-v, 133 v-134 v, 148 v-149 r$, $153 r-v, 167 r-v, 169 v-170 r, 171 v-172 r, 177 v-178 r, 206 v-207 v, 209 v-210 r, 210 r-v, 220 r-221 r, 243 r-v$, $245 v-246 r, 265 r-v, 273 r-274 r, 283 r-v, 292 v-293 v, 295 v, 298 r-v, 300 v-301 r, 301 r-v, 304 v, 305 r-v$. I documenti sul registro non sono numerati.

14 Le note relative all'estrazione di munda di Antonio da Borgonuovo si trovano in margine ai documenti posti alle carte: $12 r-v, 30 v-31 v, 34 v-35 v, 35 v-36 r, 48 r-v, 57 r, 102 r, 142 r-v, 204 r-205 r$, $240 r-v, 257 v, 281 r-v$.

15 ASTn, $A C D$, n. $1398-01$, c. $31 v$; si tratta di una dichiarazione collegata al documento posto immediatamente sopra e rogato da Pietro de Stanchariis il 3 dicembre 1380.

16 Ivi, c. $12 r-v$.

17 Ivi, cc. $30 v-31 v$.

18 Ivi, cc. $34 v-35 v$. 
me Antonium ser Bartholasii ad petitionem domini Marquardi in causa quam movit contra heredes condam ser Federici de Gardulis pretextu affictus non soluti etc ${ }^{19}$.

L'analisi sui Relevata e sulle sottoscrizioni di Antonio da Borgonuovo e di Alberto da Sacco forniscono ulteriori informazioni: anzitutto si nota come gli interventi dei notai sul registro di Pietro de Stanchariis siano piuttosto frequenti negli anni immediatamente successivi alla sua morte, mentre si facciano sempre più radi con il trascorrere degli anni, fino a divenire quasi del tutto assenti alla metà del Quattrocento, quando è Odorico da Brez a lavorare sul quel registro. Ciò è assolutamente naturale poiché, come osservava Marino Berengo relativamente alle fonti notarili piemontesi e nel Ducato di Milano fra Trecento e Quattrocento,

il valore venale degli atti diminuiva in proporzione diretta col loro grado d'invecchiamento poiché l'unico utile che potevano offrire, quello delle copie, scemava progressivamente col trascorrere degli anni. A distanza di una generazione, o al massimo di due, dal decesso del notaio che li aveva rogati, veniva a mancare ogni convenienza non solo al loro acquisto, ma anche alla loro ingombrante conservazione $^{20}$.

Un secondo tema di riflessione che si ricava dalla sottoscrizione di ser Antonio in calce ai documenti rilevati dai registri del suo predecessore de Stanchariis è relativo alla presunta proprietà di questi registri veicolanti unicamente documentazione capitolare. Il notaio, riferendosi proprio ai registri di Pietro de Stanchariis, parla infatti di:

rogationes, prothocolla, instrumenta et imbreviaturas Petri nati condam Stephani de Stanchariis de Telliis Brixiensis diocesis habitatoris Tridenti publici imperiali auctoritate notarii ex quaterno auctentico instrumentorum dicti Capituli scripto sub signo et nomine et manu dicti Petri notarii ut de dicta licentia et comissione michi datis et concessis patet publico documento scripto sub signo et nomine Gratiadei filii ser Antonii de Castello publici imperiali auctoritate notarii die martis quartodecimo mensis iullii $\mathrm{M}^{\circ} \mathrm{CCCC}^{\circ} \mathrm{XI}^{\circ}$, indictione quarta, suprascriptam rogationem sive instrumentum ex dicto quaterno instrumentorum dicti Capituli scripto sub signo et nomine et manu dicti Petri notarii ${ }^{21}$.

I volumi redatti dai notai nell'esercizio delle loro funzioni di pubblici autenticatori della memoria documentaria di enti e privati erano di piena proprietà del professionista che li aveva posti in essere, il quale - come spesso veniva ribadito negli statuti delle matricole - aveva l'obbligo di conservarli con cura affinché in futuro fosse possibile reperire un documento su richiesta di qualche cliente. Ma nel caso qui preso in esame, il notaio parla esplicitamente di «quaternus auctenticus instrumentorum dicti Capituli», ribadendolo poi poche righe dopo, «ex dicto quaterno instrumentorum dicti Capituli». L'affermazione di Antonio, dunque, sembrerebbe attribuire la proprietà del registro, scritto «sub signo et nomine» da Pietro de Stan-

19 Ivi, c. $57 r$.

20 M. Berengo, Lo studio degli atti notarili dal XIV al XVI secolo, in Fonti medioevali e problematica storiografica, Atti del congresso internazionale per il $90^{\circ}$ anniversario della fondazione dell'Istituto Storico Italiano per il Medioevo (Roma 22-27 ottobre 1973), Istituto Storico Italiano per il Medioevo, Roma 1976, I, pp. 149-172.

21 ADTn, ACap, capsa 32, n. 224. 


\section{Il 'destino' delle carte}

chariis, allo stesso Capitolo che, in quanto proprietario, poteva disporne pienamente, affidandone la gestione, dopo la morte del rogatario, ad altri notai di propria fiducia. In questo caso, come rilevato, fu il canonico Florio da Denno, sindaco e massaro del Capitolo fra il 1411 e il 1420, a richiedere al vicario vescovile la licenza per Antonio da Borgonuovo.

Il 30 giugno 1430 Antonio ottenne la licentia per rilevare le carte di Venturino del fu Antonio de Trechis da Mantova, notaio attivo per il Capitolo fra il 1324 ed il $1348^{22}$. Di costui si conservano ancora oggi una serie di fascicoli, di formato in ottavo, che probabilmente fra gli anni Venti e Trenta del Quattrocento furono rilegati a formare quel registro che oggi si conserva nella serie Instrumenta capitularia con il numero $3^{23}$. Se non è dimostrabile la presenza presso l'archivio capitolare dei rogiti di Venturino sin dall'epoca in cui tali documenti furono prodotti, pare invece certa una loro collocazione in quella sede a breve distanza temporale dalla sua morte nel 1348. La cartulazione e una serie di annotazioni poste in margine ai documenti nei fascicoli, così come lacerti cartacei e bifolia sciolti fra le carte del registro, dimostrano infatti che tale manufatto trovò posto, forse ancora nel corso del Trecento, fra le carte dell'archivio capitolare. Fu inoltre, con ogni probabilità, all'inizio del Quattrocento, forse su iniziativa del canonico Giovanni Zeiss da Bopfingen $^{24}$ che $\mathrm{i}$ fascicoli, rimasti presumibilmente fino ad allora in forma sciolta, vennero rilegati a formare un registro unitario. Che il volume di Venturino fosse presso l'archivio capitolare sembra essere confermato dalla sottoscrizione di Antonio da Borgonuovo in calce a un documento estratto proprio da Instrumenta capitularia $3^{25}$. La collazione fra $i$ testi dei due esemplari ha permesso di constatare l'assoluta corrispondenza fra il documento in registro e la redactio in mundum, che ser Antonio esemplò copiando, de verbo ad verbum, la stesura del prete-notaio di inizio Trecento. Questo documento e un bifolio cartaceo con due atti stesi da Antonio posto fra le carte di Instrumenta capitularia 3 contribuirebbero poi a confermare il fatto che quel volume fu in mano al professionista all'inizio degli anni Trenta del Quattrocento ${ }^{26}$.

$22 \mathrm{Su}$ Venturino de Trechis cfr. Malfatti, Il registro del notaio Venturino de Trechis, cit.

23 ADTn, ACap, Instrumenta capitularia 3.

24 Giovanni Zeiss da Bopfingen fa la sua comparsa tra i canonici trentini per la prima volta il 3 maggio del 1415. Operò più volte come procuratore del Capitolo, non solo a Trento ma anche a Caldaro, Appiano e Lavis, tra il 1423 e il 1433; nel 1431 fu massaro degli anniversari. L'ultima sua attestazione risale all'11 marzo 1435; morì nella notte fra il 13 e il 14 aprile di quell'anno e fu sepolto nella cattedrale: Curzel, I canonici e il Capitolo, cit., pp. 578-579.

25 Il documento su registro è in ADTn, ACap, Instrumenta capitularia 3, n. 177; la redactio in mundum di Antonio da Borgonuovo in ADTn, ACap, capsa 44, n. 5. Per la collazione dei due testi cfr. Malfatti, Il registro del notaio Venturino de Trechis, cit., doc. n. VII.

${ }^{26}$ ADTn, ACap, Instrumenta capitularia 3, nn. 115-116. Al pari di Pietro de Stanchariis, anche per Venturino de Trechis non mancarono altre comissiones che, su volere dei canonici, furono concesse prima di ser Antonio - ad almeno altri due notai: Alberto del fu Martino de Floriis da Mantova e, pochi anni più tardi, al già citato Pietro de Stanchariis. Il notaio e canonico di origini mantovane ottenne la licenza ad estrarre redactiones in mundum «ex imbreviaturis» del defunto Venturino de Trechis già il 2 maggio 1349, dunque a meno di un anno dalla morte del mansionario, su autorizzazione concessa da Giacomo Bonsenni, canonico di Padova e vicario generale per il vescovo di Trento (ADTn, ACap, capsa 30, n. 147). Dopo Alberto da Mantova fu il già noto Pietro de Stanchariis a ottenere tale facoltà, come sembra dimostrare un lacerto cartaceo che, sulla base di un confronto grafico, si può attribuire proprio alla mano del canonico e notaio Pietro (il documento è datato 28 marzo 1340. Il notaio Pietro afferma: "extraxi de quodam quaterno pergameni instrumentorum Venturini...» [ADTn, ACap, capsa 39 , n. 173]). Non si tratta di un atto rogato su pergamena, ma certamente l'affermazione del notaio 


\section{Stefano Malfatti}

Il terzo notaio di cui Antonio da Borgonuovo ottenne la licenza ad estrarre redactiones in mundum è Federico di Enrico da Albiano, attivo a Trento fra gli anni Cinquanta e l'inizio degli anni Settanta del Trecento. Poche sono le notizie relative a questo personaggio. Uno spoglio sistematico della documentazione capitolare trecentesca lo vede impegnato a rogare un buon numero di pergamene per $\mathrm{i}$ canonici del Capitolo ${ }^{27}$, oltre a pochi altri rogiti trasmessi da un foglio oggi posto a c. 2 del registro Instrumenta capitularia 6, volume altrimenti redatto per intero da Pietro de Stanchariis ${ }^{28}$. La sottoscrizione di Antonio in calce a un documento esemplato dai rogiti di Federico da Albiano ${ }^{29}$ informa che la licenza venne concessa il 4 dicembre $1431 \mathrm{su}$ petizione e istanza del canonico Artuico da Passau ${ }^{30}$ che ricopriva in quell'occasione la carica di massaro e sindaco del Capitolo. Della redactio in mundum esemplata da ser Antonio si ricorda poi che fu tratta «ex quodam quaternelo prothocollorum sive rogationum», probabilmente un registro di piccolo formato su cui Federico da Albiano predispose la forma abbreviata dei rogiti; Antonio da Borgonuovo ricopiò infatti il documento tale e quale, «nil addens vel minuens», tanto che la refuta riprodotta sulla pergamena risulta fortemente compendiata nelle forme ${ }^{31}$.

Fra i notai che furono certamente oggetto di licenza per Antonio da Borgonuovo si annovera anche Giacomo del fu ser Bartolomeo da Ravazzone ${ }^{32}$. Questi, a differenza dei professionisti citati finora, non sembra aver esercitato per il Capitolo alcuna mansione notarile; egli figura, infatti, in alcune occasioni, quale testimone a negozi giuridici stipulati fra canonici e privati cittadini ${ }^{33}$ e, altrove, quale notaio rogatario di testamenti ${ }^{34}$. Eppure, non si può del tutto escludere l'esistenza di un qualche rapporto di fiducia fra Giacomo da Ravazzone e il Capitolo della cattedrale, come peraltro sembrerebbe dimostrare il ruolo di sindaco e procuratore ricoperto per i canonici ${ }^{35}$. Tuttavia, mentre negli esempi testé menzionati sembra che le li-

de Stanchariis secondo cui il documento fu estratto «de quodam quaterno pergameni instrumentorum Venturini» porta a ritenere che egli poté prendere visione e lavorare sui rogiti del mansionario della cattedrale. Oltre a ciò è importante osservare come la matrice di questo documento si trovi proprio nell'odierno Instrumenta capitularia 3 (ADTn, ACap, Instrumenta capitularia 3, n. 178).

27 Si tratta di circa settanta redactiones su pergamena.

28 ADTn, ACap, Instrumenta capitularia 6, c. $2 r-v$, che trasmette quattro locazioni.

29 ADTn, ACap, capsa 8, n. 24. 478.

$30 \mathrm{Su}$ Artuico da Passau cfr. la scheda biografica in Curzel, I canonici e il Capitolo, cit., pp. 477-

31 Il testo inizia direttamente con «Item die decimo mensis ianuarii, in ecclesia Tridentina, ...», secondo la forma solita degli atti, con data cronica analoga, posti consecutivamente in un registro di imbreviature. Prima di Antonio da Borgonuovo la documentazione redatta per il Capitolo da Federico da Albiano era passata nelle mani di Pietro de Stanchariis e di Alberto da Sacco. Il primo ottenne la licenza fra il 9 e il 29 settembre 1390 (ADTn, ACap, capsa 30, n. 152) da Bartolomeo da Bologna, vicario generale in spiritualibus per il Capitolo; il secondo pochi anni più tardi, considerato che l'autorizzazione venne concessa dallo stesso Bartolomeo da Bologna, insieme a Nicolò de Tanuciis da Levico, vicario in temporalibus per il neo vescovo Georg Liechtenstein (ADTn, ACap, capsa anniversari, rotoli medi/b, n. 8).

32 Ravazzone è una frazione della pieve di Mori.

33 Cfr., ad esempio, ADTn, ACap, capsa 20, n. 8; capsa 23, nn. 103.1.1 e 113.

34 Cfr., ad esempio, ADTn, ACap, capsa testamenti, rotoli medi/b, n. 10; rotoli medi/a, n. 10.

35 Cfr., ad esempio, ADTn, ACap, capsa 23, n. 54 (1390 dicembre 28). Il decretorum doctor Bartolomeo da Bologna, abate trentino e vicario in spiritualibus generale del Capitolo, su richiesta di Giacomo da Ravazzone, agente in qualità di sindaco del Capitolo, aveva ordinato al prete Perengerio da Castelfondo, pievano di Malé, di ammonire bonos homines antiquos che avevano occupato certi beni posti in val di Sole per i quali si doveva invece pagare un affitto annuo al Capitolo. 
cenze siano state rilasciate relativamente alla sola documentazione prodotta per il Capitolo, trattandosi con ogni probabilità di carte e registri in buona parte posti da tempo nell'archivio capitolare, nel caso degli atti di Giacomo da Ravazzone, l'autorizzazione riguardò «rogationes et imbreviaturas» prodotte dal notaio defunto durante la sua carriera, senza alcun specifico legame con il Capitolo ${ }^{36}$. Ne è prova il fatto che la sottoscrizione di ser Antonio recante la licentia relevandi è posta in calce al testamento del magister Antonio detto a zopellis di Trento; si tratta di un instrumentum di carattere pienamente privato, che il notaio trascrisse, talquale, «ex quodam quaterno bonbacino instrumentorum continuatorum per ipsum ser Iacobum, qui quaternus est quinquaginta cartarum ...», copiandone addirittura la rubrica posta nel margine superiore sinistro ${ }^{37}$.

\section{La trasmissione della documentazione capitolare prodotta da Antonio da Borgonuovo}

Sul finire degli anni Trenta del Quattrocento, con l'esaurirsi dell'attività di Antonio, il Capitolo affidò la registrazione della propria documentazione a tre notai che, uno dopo l'altro, furono impegnati nella redazione di pergamene sciolte e di un registro di instrumenta, oggi noto con il nome di Instrumenta capitularia $9^{38}$. Si tratta, rispettivamente, di Luca de Lippi, Antonio da Brez e Nicolò de Capris. Il primo $^{39}$ è noto per essere stato eletto canonico del Capitolo di Trento nel 1421; tre anni più tardi, tuttavia, fu dichiarato intrusus, cosicché, nel 1436, quando intraprese l'attività di notaio al servizio dell'ente (iniziando la redazione del registro Instrumenta capitularia 9), non ricopriva ormai più la carica canonicale. Una seconda sezione del nono volume capitolare è invece occupata dalla documentazione redatta da Antonio, figlio del fu Gotmanino da Brez. Questi proseguì l'opera del de Lippi con documentazione datata fra il 1455 ed il 1458, continuando con le registrazioni fino al $1461 \mathrm{ma}$ in un nuovo volume, oggi denominato Instrumenta capitularia $10^{40}$. Una terza partizione del registro n. 9 è invece occupata dai documenti capitolari redatti da Nicolò de Capris fra il 1451 e il 1454.

Come era avvenuto per i notai trecenteschi anche per costoro furono messe in atto dai canonici analoghe modalità operative: il notaio scelto dal Capitolo per sostituire il collega da poco defunto ricevette l'autorizzazione a lavorare, e dunque ad estrarre documentazione dalle carte redatte per l'ente dal professionista o dai

36 La licentia relevandi si trova in calce a un testamento in BCTn, BCT3, capsa 21 , mazzo 1 , n. 16 e in calce alla sentenza arbitramentale in ASTn, $A P V$, Sezione latina, capsa 30, n. 60 (la sentenza fu tratta «ex quodam quaterno vivo et non canzelato processus dicte questionis mote inter dictas comunitates ut supra scriptus manu dicti ser Iacobi notarii de Ravazoni sumpsi et fideliter prout in dicto quaterno dicti processus in tribus ultimis cartis scriptis inveni, transcripsi nichil addens vel minuens quod sensum vel formam [variet] et in hanc publicam formam redegi et me in fidem et verum testimonium premissorum subscripsi et signum mei tabelionatus apposui consuetum»).

37 BCTn, BCT3, capsa 21, mazzo 1, n. 16.

$38 \mathrm{Su}$ Instrumenta capitularia 9 si vedano storia e caratteristiche tracciate in E. Curzel, Ricerche sul Capitolo della cattedrale di Trento alla metà del Quattrocento, tesi di laurea, Università degli Studi di Trento, a.a. 1989-1990, rel. G.M. Varanini, in particolare le pp. 7-21. Cfr. anche Curzel, Per la storia del Capitolo, cit., pp. 242-245.

39 Su Luca de Lippi cfr. la scheda biografica in Curzel, I canonici e il Capitolo, cit., p. 617.

40 Su Instrumenta capitularia $10 \mathrm{cfr}$. Curzel, Per la storia del Capitolo, cit., pp. 243-245 e 248251. 
professionisti che lo avevano preceduto. Ciò significa che, conclusasi alla metà degli anni Trenta del Quattrocento l'opera di ser Antonio per i canonici, i nuovi notai ottennero la licentia relevandi dalle carte da lui rogate per il Capitolo; ma tale autorizzazione riguardò probabilmente soltanto la documentazione redatta per i canonici, il che comprendeva certamente anche il registro Instrumenta capitularia 8 , ma non il registro 8bis che, come si vedrà, seguì un percorso diverso.

Nel 1450, Antonio da Brez ottenne dal vicario vescovile Bonamente Aliprandi da Mantova la licenza a trarre redactiones in mundum da «protocola et imbreviaturas scriptas et notatas» di Luca de Lippi ${ }^{41}$; analoga autorizzazione ottenne, nella seconda metà del Quattrocento, Odorico del fu Guglielmo da Brez, che era subentrato ad Antonio da Brez quale nuovo notaio del Capitolo nel $1461^{42}$. Lo stesso Odorico, poi, aveva ottenuto, in analogo scorcio d'anni, la licentia relevandi dalle carte capitolari di Antonio da Borgonuovo ${ }^{43}$ e, su concessione di Girolamo Mussato da Padova, podestà di Trento nel 1462, l'autorizzazione a rilevare ed esemplare in publicam formam «rogationes et imbreviaturas» del suo predecessore Antonio del fu Gotmanino da $\mathrm{Brez}^{44}$. Ancora sul finire del XV secolo, anche il notaio capitolare Balzano Balzanini poté servirsi del registro del Capitolo redatto da Antonio da Borgonuovo, come sembra dimostrare il Facta extra in margine ad una investitura di feudo:

[Fact]a extra per me Balzanum de [Balza]ninis notarium Capituli vener[abilis] de iussu dominorum et mandato [et] istantia Udalrici Hamer [...]h de Alia Saline vigesimo [...]no mensis iulii 1488 indictione [...] et data Adam monetario [...]o suo, presentibus Iohanne [...] etio curie Tridentine et domini [Vig]ilio ser Iohannis Laurentii de [...]reto ac Alexandro Copa[...]si de Tueno valis Ananie [d]iocesis Tridentine et etiam scit [... domin] us Albertus Confeller canonicus Tridentinus qui hoc supra $[\ldots]$ olicitavit ${ }^{45}$.

Qualche anno più tardi, nel 1511, una mano anonima aggiunse: «Factum extra iterum anni $1511 »^{46}$. L'analisi sulla documentazione capitolare tre-quattrocentesca fa emergere l'immagine di un'istituzione capace di esercitare una forte azione di tutela dei propri diritti patrimoniali, il che avviene anche attraverso il recupero di documentazione d'interesse, ma in mano ai notai (o ai loro eredi) che l'avevano posta in essere. Può essere il caso, ad esempio, del registro, fin qui non menzionato, del notaio Antonio da Pomarolo, oggi noto come Instrumenta capitularia $4^{47}$

41 Cfr. BCTn, BCT3, capsa 4, mazzo 1, n. 76 e capsa 28, mazzo 1, n. 102. Il documento attestante la licenza venne registrato dal notaio ser Francesco da Fondo.

42 Odorico del fu Guglielmo da Brez roga il suo primo documento nel 1461 in Instrumenta capitularia 10, per poi proseguire la sua attività di registrazione in Instrumenta capitularia 11, con rogiti compresi fra il 29 gennaio 1463 e il 5 aprile 1474: cfr. Curzel, Per la storia del Capitolo, cit., pp. 248250. L'autorizzazione ad estrarre dalle carte capitolari di Luca de Lippi fu ottenuta da Odorico da Brez direttamente per mandato dei canonici (ADTn, ACap, capsa 38, n. 4.9): «(SN) Et ego Odoricus condam Guillelmi de Brezio civis et habitator Tridenti publicus imperiali auctoritate notarius, de mandato et commissione et ex licentia et auctoritate michi facte et concesse per reverendos dominos canonicos et venerabilem Capitulum ecclesie Tridentine relevandi, conscribendi et in publicam formam reddigendi ...».

43 Cfr. ADTn, ACap, capsa 38, n. 4.4.

44 Cfr. BCTn, BCT3, capsa 4, mazzo 2, n. 7.

45 ADTn, ACap, Instrumenta capitularia 8, n. 205.

46 Ibidem. 321.

47 Il registro è oggetto di studio in Mattivi, Il registro del notaio Antonio da Pomarolo, cit., pp. 295- 
Il 'destino' delle carte

che, pur trasmettendo solo in minima parte documentazione d'interesse capitolare, forse già nella seconda metà del Trecento, entrò a far parte dell'archivio dell'ente.

\section{La trasmissione di altra documentazione prodotta da Antonio da Borgonuovo}

Le note relative all'estrazione di documenti in publicam formam, le formule di autenticazione in calce a documenti estratti dai registri e dai protocolli di Antonio da Borgonuovo, altri elementi formali, nonché considerazioni di natura archivistica e codicologica, consentono di ricostruire, almeno in parte, i passaggi che le carte del professionista subirono dopo la sua morte.

La prima testimonianza proviene da una pergamena in mundum con un testamento che il notaio Iohannes Staufer ${ }^{48}$ estrasse da un «liber continuatorum dicti condam ser Antonii notarii» ${ }^{49}$ (fig. 27).

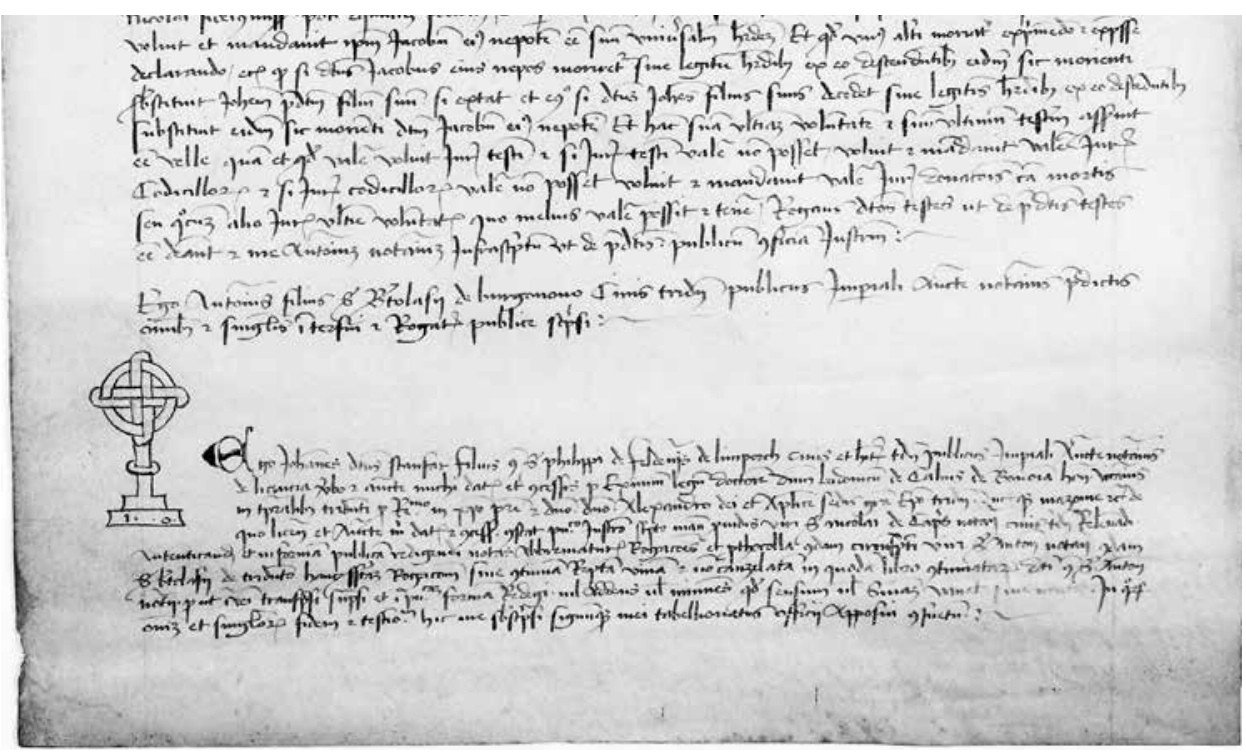

Fig. 27. ADTn, ACap, capsa testamenti, rotoli lunghi/b, n. 15 (particolare). Sottoscrizione del notaio Iohannes Staufer in calce al testamento estratto da un liber continuatorum del defunto Antonio da Borgonuovo.

Fra il 1437, presunto anno della morte di Antonio da Borgonuovo, e il 1441, il notaio di origini transalpine aveva infatti ottenuto dal vicario vescovile Ludovico de Calvis da Bologna, la licenza «relevandi, autenticandi et in formam publicam redigendi notas, abbreviaturas, rogationes et prothocolla condam circumspecti viri ser Antonii notarii condam ser Bartholasii de Tridento»; è dunque assai probabile che Iohannes Staufer sia stato il primo, dopo la morte di ser Antonio, a ottenere la licenza di rilevare ed estrarre documenti dalle carte del collega defunto ${ }^{50}$.

48 Talvolta trascritto come Stanfar o Stanfer o Stamfar.

49 Cfr. ADTn, ACap, capsa testamenti, rotoli lunghi/b, n. 15.

50 Di Staufer si conserva un limitato numero di documenti: un testamento nel fondo capitolare dell'Archivio diocesano e due ulteriori pergamene con disposizioni testamentarie nel miscellaneo 


\section{Stefano Malfatti}

Considerata la biografia del notaio di origini transalpine, «qui fuit notarius doctus et praticus $\rangle^{51}$, è lecito supporre che egli acquisì la documentazione di Antonio anche in virtù della sua posizione politica e delle sue qualità come notaio. L'ultima notizia nota su Iohannes Staufer risale al 1455, quando venne eletto per la decima volta «nodar dy sindicy». Con la sua morte la documentazione da lui prodotta, e probabilmente anche quella di ser Antonio, furono rilevate da un collega più giovane: il notaio e giurisperito Antonio Facini ${ }^{52}$ (fig. 28).

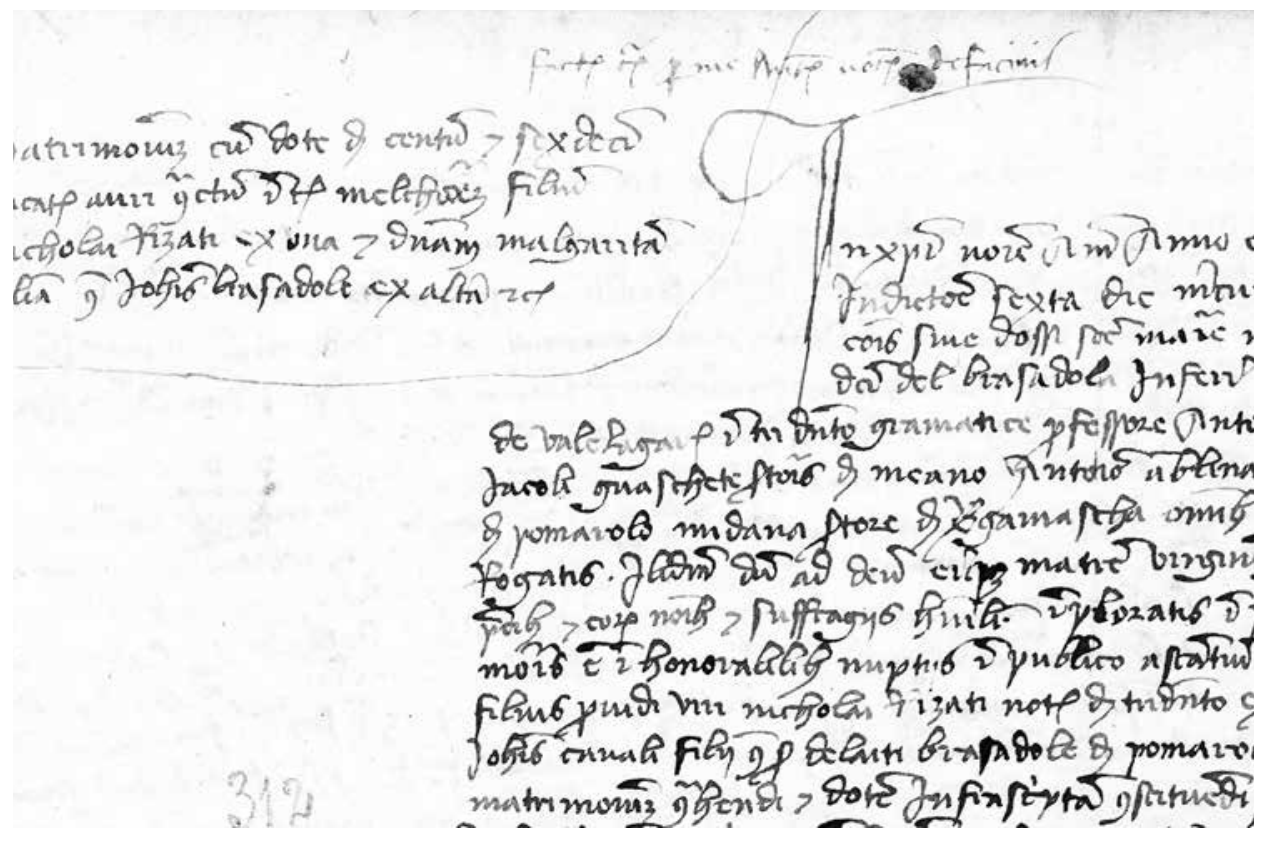

Fig. 28. ADTn, ACap, Instrumenta capitularia 8bis, c. 113 v, n. 312 (particolare). Facta extra di Antonio Facini.

BCT3. Cfr. BCTn, BCT3, capsa 4, mazzo 1, n. 79 e capsa 4, mazzo 2, n. 10. In BCTn, BCT3, capsa 4, mazzo 1, n. 76 compare quale autore di una compravendita. Il notaio ricoprì una serie di ruoli di grande rilevanza nel Comune di Trento fra il 1434 ed il 1455. Nel febbraio del 1437 fu eletto una prima volta insieme al notaio Pietro da Sporo alla carica di giudice delle tutele, ruolo che ricoprì una seconda occasione nel febbraio del 1441, insieme a un certo Sandrino chaliar. Nel 1434, nell'ottobre del 1437, nel febbraio e ancora nell'ottobre del 1440, nel novembre del 1441, nel 1447, nel giugno del 1449 , nel novembre del 1452 , nello stesso mese dell'anno 1454 ed un'ultima volta nel giugno del 1455, Staufer fu eletto alla carica di notaio dei sindici. Nel 1443, nel 1446, nel 1447 e nel 1454 ricoprì l'incarico di massaro dei sindici. Nel 1443 e nel 1450 fu scelto quale giudice degli appelli. In ultimo, fu console nel 1453, insieme a Giacomo Fanzini, Matteo a Vaginis, ser Giovanni de Maleferatis, ser Giacomo da Roccabruna, ser Giacomo Perozo e ser Luchino de Gragnano. Nel 1446 viene inoltre menzionato un certo Ianes laborator definito «olim famulus Stanfer notarii», eletto alla carica di mensurator salis.

51 Varanini, Il Collegio notarile di Trento, cit., p. 499.

52 Antonio era il figlio del noto giureconsulto padovano Melchiorre; come il padre esercitò ruoli di rilievo nel Comune: fu infatti eletto console per due volte nel 1494 e nel 1498 . Nel 1462 ricoprì la duplice mansione di notaio dei sindici e massaro del Comune. Nel 1477 era stato eletto amministratore dei beni vescovili insieme ad Antonio Migazzi. Tuttavia, i gravami prodotti dai rappresentanti delle valli in un documento del febbraio 1477 accusarono i due amministratori di appropriazione indebita, blasfemia e azioni fraudolente tali da far scoppiare, di lì a pochi mesi, una vera e propria insurrezione 


\section{Il 'destino' delle carte}

Un'unica nota relativa all'estrazione del mundum posta nel margine superiore del matrimonio, registrato il 24 novembre 1428, fra Melchiorre figlio del notaio Nicolò Rizato da Trento e Margherita figlia del fu Giovanni Brasadole da Pomarolo ${ }^{53}$, sembra infatti attestare un'attività di Antonio Facini sul registro 8bis.

Figura politica di primo livello nella Trento della seconda metà del Quattrocento il Facini svolse la sua attività di notaio fra gli anni Sessanta e la fine degli anni Novanta del Quattrocento. Da quanto è noto, oltre alle carte di Antonio da Borgonuovo, il giurisperito rilevò sul finire del XV secolo anche «rogationes, instrumenta, imbreviaturas, scriptas et scripta» del fu Balzano Balzanini ${ }^{54}$. I suoi rogiti furono invece relevati ad inizio Cinquecento dal notaio Giovanni Giacomo, figlio di ser Giovanni de Gaisis da Calavino, che era stato autorizzato ad estrarre dalle carte di Antonio Facini dal podestà Ercole Tassoni da Modena ${ }^{55}$.

Non è noto come la documentazione di Antonio da Borgonuovo, il registro Instrumenta capitularia 8bis in particolare, passarono nella seconda metà del Quattrocento dalle mani del notaio Iohannes Staufer al giurisperito Antonio Facini; l'annotazione marginale di quest'ultimo non fornisce infatti informazioni in merito. È possibile che, vista la morte senza eredi del primo, il 'passaggio' delle carte di Antonio fosse avvenuto nell'ambito del collegio notarile, il quale scelse di volta in volta i membri più adatti a conservare tale documentazione.

Diverse note relative all'estrazione di documenti in publicam formam in margine a rogiti redatti da Antonio da Borgonuovo sul registro 8bis furono redatte, a fine Quattrocento, da Andrea Gallo ${ }^{56}$. Scorrendo le carte di cui si compone il registro

contadina. Il vescovo mise in piedi un processo nei loro confronti, affidandone l'istruttoria a Calepino Calepini e Antonio Schrattenberg. Il Facini, spesso accusato di collericità, fu così ben presto allontanato dalla carica, ma la sua carriera non subì battute d'arresto, considerato che, nel 1482, ottenne l'officio di notaius maleficiorum insieme a Battista da Campo detto Gratiadeus (cfr., sulla vicenda, F. Chiarotti, L'insurrezione contadina del 1525 nell'analisi degli avvenimenti dell'Anaunia, in Storia del Trentino, IV: L'età moderna, a cura di M. Bellabarba, G. Olmi, Il Mulino, Bologna 2000, pp. 157-192 e M. Bellabarba, La giustizia ai confini: il principato vescovile di Trento nella prima età moderna, Il Mulino, Bologna 1996 [Monografie dell'Istituto storico italo-germanico in Trento], p. 331; per la carica di notaius maleficiorum cfr. ivi, p. 298, in particolare la nota 93 e ASTn, APV, Sezione latina, capsa 85, n. 6, c. $99 r$, noto come Liber denunciatiorum et querelarum coram domino potestate). Nel 1497 Antonio fu scelto quale nuovo massaro dei canonici del Capitolo della cattedrale nonché amministratore della fabbrica di San Vigilio (ASTn, $A P V$, Sezione latina, capsa 74, n. 3, c. 23r; cfr. per queste informazioni, M. Bellabarba, La giustizia ai confini, cit., p. 332). Oltre a ciò si deve menzionare l'ufficio di notaio del Comune, che il Facini ricoprì ininterrottamente fra il 1495 e il 1497.

53 ADTn, ACap, Instrumenta capitularia 8bis, n. 312.

54 Cfr. BCTn, BCT3, capsa 29, mazzo 2, nn. 8, 14. La licenza fu concessa da Giambattista Lambertini da Bologna, podestà di Trento nel 1490.

55 Cfr. ASTn, $A P V$, Sezione latina, capsa 64, n. 321; BCTn, BCT3, capsa 4, mazzo 2, n. 65 e $\mathrm{BCTn}, B C T 1$, n. 1057, cc. 52-54, n. 19. Ercole Tassoni è podestà di Trento nel 1501. L'instrumentum attestante la comissio fu redatto dal notaio Antonio da Vigolo. Giovanni Giacomo da Calavino aveva anche ottenuto la licentia relevandi delle carte del padre Giovanni (cfr. BCTn, BCT3, capsa 29, mazzo 2 , nn. 2, 57; capsa 31 , mazzo 1 , n. 13; capsa 29, mazzo 2, nn. 10, 17; capsa 5, mazzo 1, n. 43; capsa 29, mazzo 2, n. 29), come risulta dal documento redatto da Antonio da Vigolo. L'autorizzazione fu concessa da Nicolò Basileus da Trieste, podestà di Trento nel 1506.

56 Andrea, figlio di ser Giovanni Gallo da Trento, era un notaio e giureconsulto attivo a Trento nella seconda metà del Quattrocento (cfr. BCTn, BCT2, n. 2478 [1500 gennaio 25] dove è attestato come giureconsulto). Andrea Gallo abitava nella contrada di Santa Maria Maggiore (cfr. la scheda biografica in Stenico, Notai che operarono nel Trentino, cit., sub voce Gallo Andrea). Come si ricava dai verbali di ammissione al Collegio notarile di Trento, Andrea, insieme ad Antonio da Grigno e Giovanni da Calavino, fecero domanda di ammissione alla matricola il 26 gennaio 1470 ma furono in prima battuta respinti; per un anno vennero infatti invitati a «scholas visitare et audire Notariam saltim per 


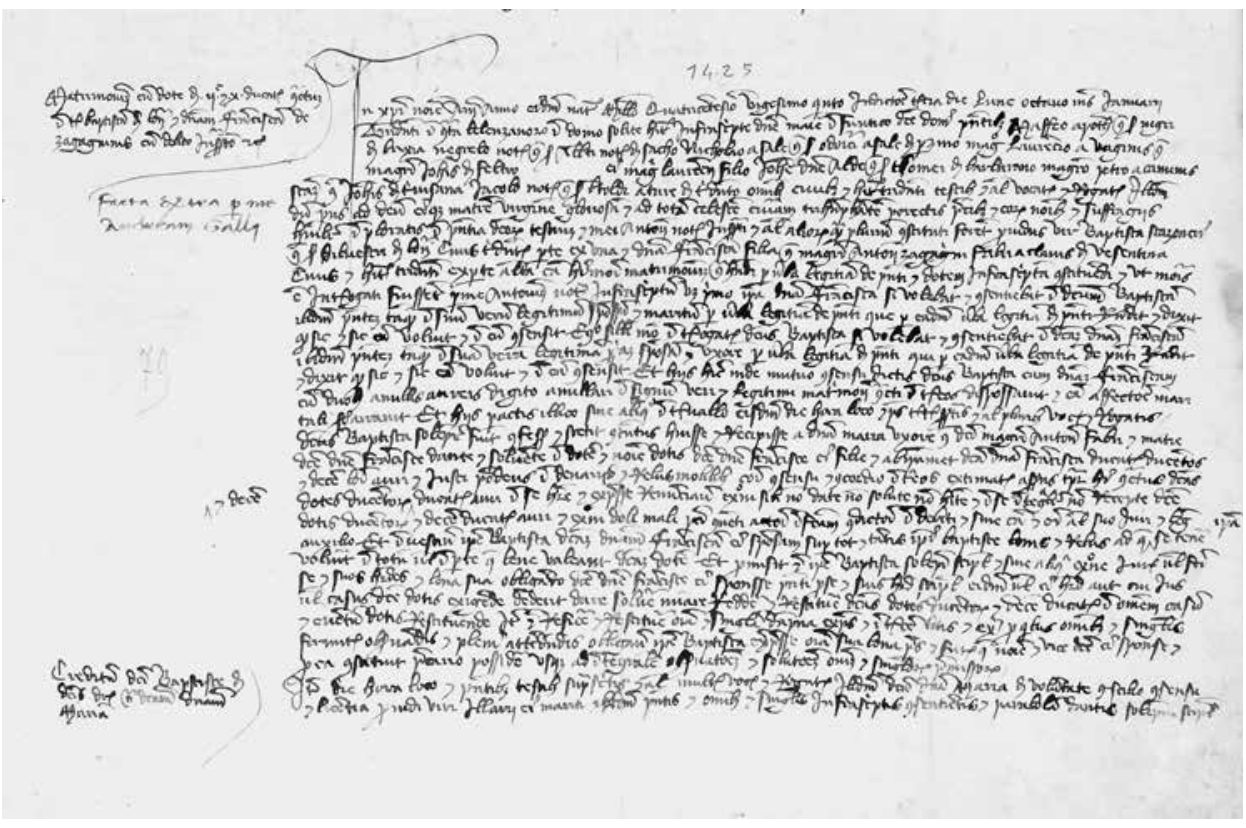

Fig. 29 ADTn, ACap, Instrumenta capitularia 8bis, c. $27 r$, n. 79 (particolare). Facta extra di Andrea Gallo.

Instrumenta capitularia 8bis, infatti, si trovano ben diciannove note relative all'estrazione di munda ${ }^{57}$ (fig. 29). In particolare, fra queste annotazioni, merita speciale attenzione quella posta in margine ad una compravendita rogata da ser Antonio il 14 novembre 1427. 'Copiando' l'instrumentum Andrea annotò: «Nota qualiter ego Andreas Galli, de mandato domini Iohannis Victoris de Feltro pot(estatis) Tridenti, aliud instrumentum in publicam formam etc exemplavi et dedi Bartolameo et Nicholao de Margono» ${ }^{58}$. La nota informa dunque che Andrea Gallo ottenne la licenza per estrarre documenti dal registro di Antonio da Gianvittore di Burgasio da Feltre, il quale fu podestà di Trento sotto il vescovo Iohannes Hinderbach nell'anno $1481^{59}$; da ciò si deduce che, certamente prima dell'anno 1500 , ovvero prima della sua

annum vel Institutam a domino potestate vel ab alio lectore iuxta posse suum» (cfr. ASCTn, Comune di Trento, Antico regime, sezione antica, ACT1-4272, c. 15r, edito in Ceraolo, Il collegio notarile di Trento, cit.). L'attività di Andrea quale notaio proseguì almeno fino alla fine del Quattrocento, la morte è infatti attestata il 5 dicembre 1500 (Stenico, Notai che operarono nel Trentino, cit., sub voce Gallo Andrea ). Di lui si conserva un registro di instrumenta contenente documentazione in esteso redatta fra il 1495 e il 1500 insieme al figlio Guglielmo (ASTn, Atti dei notai, Giudizio di Trento, Gallo Andrea e Guglielmo ).

57 ADTn, ACap, Instrumenta capitularia 8bis, docc. nn. 29, 41, 79, 115b, 116, 158, 256, 305, 321, 453, 462, 469, 516, 540, 607, 620a, 625, 626b, 651 .

58 ADTn, ACap, Instrumenta capitularia 8bis, n. 256; Adelperio del fu ser Marco Calepini da Trento, in qualità di procuratore di Calepino suo fratello, vende ad Andriota del fu Francesco detto Tibay da Terlago un affitto perpetuo di 2 staia e mezzo di frumento secondo la misura di Trento che venivano pagati annualmente prima da Terlaco Marele da Terlago, dopo di lui da Flordela sua moglie e in seguito da Andriota per alcuni terreni situati nelle vicinanze di Terlago, al prezzo di 15 ducati d'oro che Andriota paga immediatamente al venditore.

59 Ambrosi, Commentari della storia trentina, cit., p. 492. Non è noto se Gianvittore da Feltre ricoprì la carica podestarile in altre occasioni. 
morte, Andrea Gallo fu autorizzato a lavorare su questo registro e, probabilmente, anche sulle altre carte (non capitolari) di Antonio da Borgonuovo. La nota si riferisce inoltre all'estrazione da lui effettuata di un 'altro' instrumentum a partire dalla stessa matrice, ovvero la registrazione originale nel registro di Antonio.

Null'altro si può aggiungere su questo notaio, se non che la sua documentazione finì in seguito nelle mani del figlio Guglielmo ${ }^{60}$. Questi, infatti, ottenne dal podestà di Trento Ercole Tassoni da Modena la licenza «relevandi, conscribendi et exemplandi scripturas, abreviaturas, notas et continuas» di suo padre Andrea ${ }^{61}$.

Per quanto concerne la sua attività come notaio, egli fu al servizio della Prepositura, per la quale, fra il 1500 ed il 1514, produsse un registro di estesi ${ }^{62}$. La c. $1 r$ del volume prepositurale reca infatti la seguente annotazione: «Hic est liber instrumentorum Prepositurae ecclesiae Tridentine continuatum et rogatum per me Guglielmum Gallum notarium, civem et habitatorem Tridenti inceptus a me notaio infrascripto $[\ldots]\rangle\rangle^{63}$. Il registro di instrumenta è seguito da un ulteriore volume, pure di mano di Guglielmo Gallo, che riporta sulla coperta la seguente dicitura: «Investiture libro C dall'anno 1522 fino all'anno 1542» ${ }^{64}$.

Sul finire del Quattrocento, dunque, la documentazione di ser Antonio sembra essere a disposizione di Andrea e probabilmente, dopo la morte di questi, del figlio Guglielmo $^{65}$. È forse in questa fase che il registro oggi noto come Instrumenta capitularia 8bis subì una sorte diversa rispetto al resto delle carte di ser Antonio, entrando a far parte dell'archivio della Prepositura.

\subsection{Il registro Instrumenta capitularia 8bis nell'Archivio della Prepositura}

Alcune considerazioni di natura archivistica e codicologica permettono di collocare il registro Instrumenta capitularia 8bis nell'archivio della Prepositura ${ }^{66}$. Il volume, già descritto nella sua facies esterna, presenta infatti una legatura e una

60 Guglielmo, notaio e giurisperito, ricoprì importanti cariche pubbliche nel Comune di Trento, fra cui, nel 1506, nel 1521 e nel 1526, quella di console (Ambrosi, Commentari della storia trentina, cit., p. 474). Ricoprì inoltre l'ufficio di procuratore della città il 5 dicembre 1521 (Stenico, Notai che operarono nel Trentino, cit., sub voce Gallo Guglielmo ).

61 Cfr. BCTn, BCT3, capsa 5, mazzo 1, n. 72; capsa 29, mazzo 2, nn. 20, 31, 53, 69, 70, 74. Ercole Tassoni fu podestà a Trento nell'anno 1501; l'instrumentum attestante la licenza fu redatto da Bonaventura da Povo.

62 ADTn, $A P$, Registri, n. 18. Il registro reca sulla coperta la segnatura $A$.

63 Cfr. anche Ioppi, I registri del monastero di San Lorenzo, cit., p. 63 e ss.

64 ADTn, AP, Registri, n. 19. I documenti sono tuttavia datati fra il 1521 e il 1549 , con una lacuna relativa agli anni Trenta.

65 La documentazione di Andrea, alla morte di Guglielmo, viene ereditata da Girolamo Gallo, nipote del primo e figlio del secondo, che ottiene da Giovanni Francesco Bebio da Reggio, pretore per il vescovo Bernardo Cles, la licenza «conscribendi et in publicam formam redigendi abbreviaturas, scripturas et instrumenta rogatas et scriptas» del nonno Andrea (cfr. BCTn, BCT3, capsa 29, mazzo 2, n. 41). Giovanni Francesco da Reggio è pretore di Trento nel 1525. Il notaio che redige relativo instrumentum è Francesco da Calavino. La documentazione di Girolamo Gallo viene invece rilevata da Leonardo del fu Giovanni Antonio Colombini da Terlago, su licenza di Giovanni Battista Moragium da Piazenza, doctor e pretore di Trento nel 1565 (cfr. ADTn, ACap, capsa anniversari, rotoli medi/f, n. 21). La documentazione prodotta da Guglielmo Gallo per la Prepositura viene invece rilevata da Giovanni del fu Antonio Malfer su licenza di Giulio Locholum (o Tocolum) da Parma, pretore di Trento nel 1577 (cfr. ADTn, AP, Tomo III, n. 30).

66 Le considerazioni che qui si esporranno sono già state in gran parte svolte in Ioppi, I registri del monastero di San Lorenzo, cit. 


\section{Stefano Malfatti}

serie di segnature progressive che ne testimoniano le vicissitudini archivistiche di età moderna e contemporanea. L'etichetta posta sulla coperta anteriore del volume mostra chiaramente la stratificazione delle segnature prepositurali; si hanno infatti cinque numerazioni riconducibili ad altrettanti inventari. La prima, e più antica, è quella indicata con il «N. 37» ed è ricollegabile all'inventario prodotto nel 1794 . L'inventario in questione venne redatto alla morte del preposito Carlo Giuseppe Sebastiano conte Trapp, in funzione del suo successore, Giovanni Giacomo Pizzini de Thürberg ${ }^{67}$. Fra la documentazione più antica in esso descritta si trova anche un «Libro vecchio in folio grande segnato n. 37», che coincide con il registro di Antonio da Borgonuovo (fig. 30).

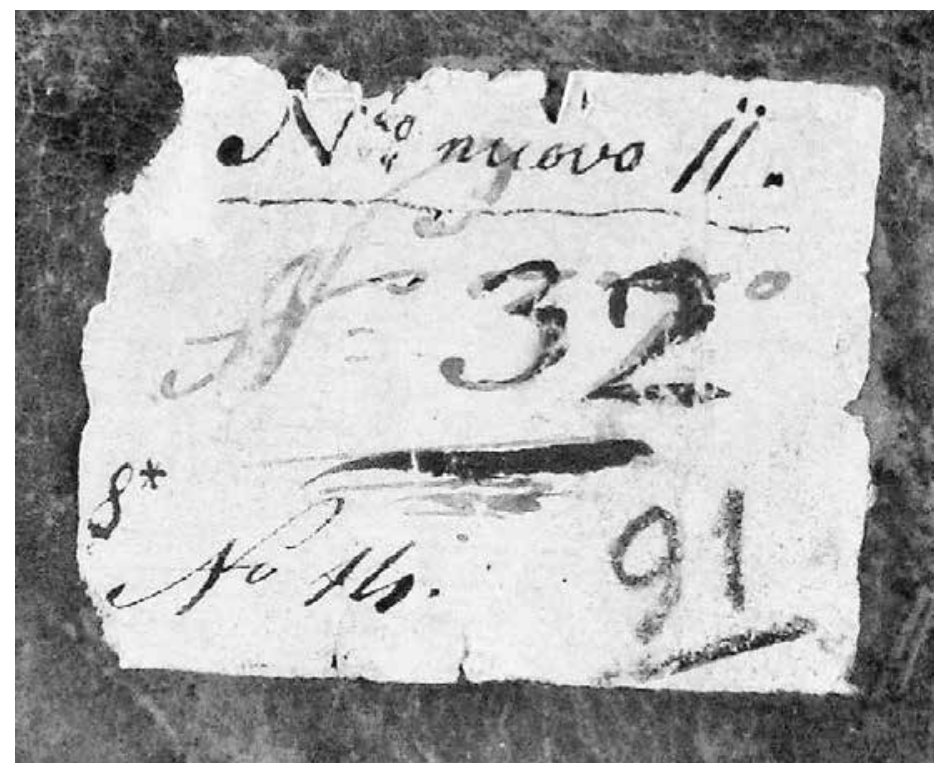

Fig. 30. ADTn, ACap, Instrumenta capitularia 8bis. Particolare delle segnature poste sull'etichetta della coperta del volume.

Nell'angolo inferiore, a sinistra, è posta invece una numerazione, «N. 14», vergata con penna di colore rosso scuro; essa corrisponde alla segnatura posta nell'inventario prodotto dopo la nomina del nuovo preposito Giuseppe Urbano Buffa 1'8 ottobre 1826. Il nuovo ordinamento cui fu sottoposto l'archivio prepositurale modificò sostanzialmente le segnature pregresse, tanto da rendere necessaria una sorta di «tavola di raffronto» ${ }^{68}$ fra la vecchia numerazione dell'inventario Pizzini e la nuova numerazione adottata. Fra la documentazione più antica spiccano due registri del monastero di San Lorenzo, il volume di Antonio da Borgonuovo e i registri di investiture della Prepositura dall'anno 1472.

Proseguendo diacronicamente nel censimento delle segnature archivistiche del registro di Antonio, si rileva il «Numero nuovo 11», redatto in occasione dell'inven-

67 Si tratta dell' «Inventario delle scritture e documenti esistenti nell'Archivio della reverendissima Prepositura di Trento [...]», di cui si conserva un esemplare di mano di Pietro Carlo Ducati. Cfr. ivi, pp. $72-73$.

68 Ibidem. 
tario del 1833, steso in seguito alla morte del preposito Tomaso Giuseppe Powondra per il subentrante Luigi Dal Piaz ${ }^{69}$.

Un'ultima segnatura numerica, «91», vergata a matita da una mano moderna ${ }^{70}$ si riferisce all'inventario fatto compilare, nel 1897, dal preposito Giovanni Battista Zambelli ${ }^{71}$. La numerazione 91, che il registro assunse in occasione del riordino fatto eseguire dal preposito Zambelli nel 1897, lo pone come ultima unità della Sezione Prima dell'inventario, quella contenente i volumi legati e le pergamene della Prepositura. Sul Catalogo del 1897 il volume di Antonio da Borgonuovo è menzionato infatti con il nome «Rogiti di Antonio fu ser Bartolasio notaio di Borgonuovo di Trento. Contiene 665 documenti dal 1423 al 1437 codice cartaceo di centimetri 47 × 37 ».

Dalla fine dell'Ottocento fino al 1980 la struttura dell'archivio prepositurale non sembrò subire variazioni di rilevo; all'inizio degli anni Ottanta la documentazione capitolare, e con essa quella della Prepositura, fu affidata all'archivista don Ivo Leonardi, il quale scelse di spostare il registro di Antonio da Borgonuovo nella serie Instrumenta capitularia del fondo capitolare dove, posto dopo il registro $\mathrm{n}$. 8 , assunse la nuova segnatura n. 8 bis $^{72}$.

Valutando le segnature d'archivio del registro di Antonio si è potuti risalire fino al XVIII secolo; manca, tuttavia, qualsiasi notizia sul volume per i secoli precedenti. Come già ricordato, almeno fino alla fine del Quattrocento-inizi del Cinquecento, carte e registri (non capitolari) di Antonio da Borgonuovo furono probabilmente nelle mani del notaio Andrea Gallo, prima, e del figlio di costui Guglielmo poi. Un'ipotesi certamente plausibile per tentare di spiegare 'l'ingresso' del registro nell'archivio dell'ente è la presenza in esso dell'atto fondativo, avvenuto il 10 ottobre 1425, della dignità capitolare della Prepositura ${ }^{73}$. Del resto, si è potuta constatare l'estrema varietà degli atti trasmessi da Instrumenta capitularia 8bis, eterogenei sia per committenza sia per argomento trattato. La documentazione redatta da ser Antonio per il monastero di San Lorenzo e per la Prepositura è numericamente esigua, pertanto è la sola presenza di quell'atto, d'importanza capitale per l'ente, che potrebbe giustificarne per quest'ultimo l'acquisizione. Peraltro, come si deduce dalla nota relativa all'estrazione del relativo mundum redatta dallo stesso Antonio, di quel documento fu prodotta una sola pergamena: «Facta extra per manum Marchi Zinele et per me et Antonium notarium de Castro et Nicholaum de Capris subscriptam et ad curiam Romanam per dominum Stanslaum portatam etc»: un'unica redactio in mundum e, per giunta, consegnata al nuovo preposito Stanislao Sobnowski perché la portasse «ad curiam Romanam».

69 Ibidem; il riordinamento e la nuova descrizione del fondo avvenne per opera del segretario magistratuale Giulio Sardagna.

70 Potrebbe trattarsi della mano di Vigilio Zanolini che numerò anche i documenti all'interno del volume negli anni Trenta del Novecento, ma, più probabilmente, dell'archivista Ivo Leonardi (anni Ottanta del Novecento).

71 Ioppi, I registri del monastero di San Lorenzo, cit., p. 77.

72 Lo spostamento dall'archivio della Prepositura all'archivio capitolare avvenne probabilmente nel corso del 1981, anno in cui il volume fu restaurato; in precedenza presentava ancora la segnatura «91». L'archivista Ivo Leonardi, ricevuto il registro dopo il restauro, pose a matita sulla prima carta di guardia la seguente nota: «Archivio della Prepositura vol. 91 collocato per praticità fra gli "Instrumenta capitularia" col n 8 bis 1423-1437»

73 Cfr. Appendice, regesto n. 28 (ADTn, ACap, Instrumenta capitularia 8bis, n. 131). 


\section{Stefano Malfatti}

Se non è accertabile un ingresso del volume di Antonio nell'archivio della Prepositura nel corso del Quattrocento, durante il quale, come appurato, diversi notai poterono estrarvi documenti, tale 'passaggio' potrebbe essere avvenuto sul finire del secolo o, tutt'al più, all'inizio del Cinquecento, grazie al notaio Guglielmo Gallo (erede della documentazione del padre Andrea e dunque, presumibilmente, anche delle carte di ser Antonio), il quale, come ricordato, fu al servizio della Prepositura fra il 1500 ed il $1549^{74}$. Non mancano inoltre elementi di somiglianza fra la legatura e la coperta attuale di Instrumenta capitularia 8bis, databile proprio alla fine del XV-inizio del XVI secolo, e un volume notarile dell'archivio prepositurale che porta la segnatura n. $17^{75}$ (fig. 31).

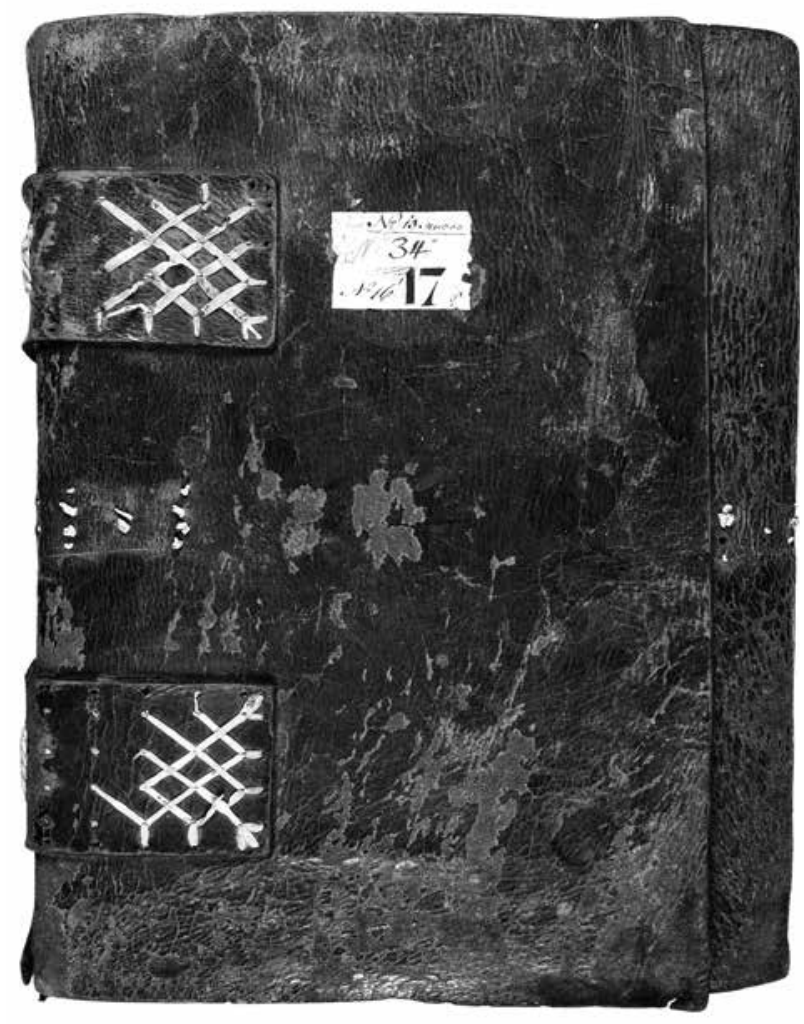

Fig. 31. ADTn, AP, Registri, n. 17. Coperta superiore del registro.

Quest'ultimo presenta infatti una coperta in cuoio marrone scuro che riveste piatti in cartone floscio. Il piatto superiore è provvisto, analogamente ad Instrumenta capitularia 8bis, di una ribalta che ha lo scopo di proteggere il taglio anteriore del registro. Il dorso e parte dei piatti sono fasciati da contrafforti in cuoio dello

74 ADTn, AP, Registri, nn. 18 e 19.

75 Ivi, n. 17 (1472-1500); si tratta di un registro di estesi con i rogiti del notaio Francesco del fu Domenico Gelfo. Il fratello di questi, Bernardino, che ne aveva ricevuto relativa licentia relevandi dal podestà Ercole Tassoni da Modena, incaricò uno scriptor di trascrivere i rogiti di Francesco (le licentie relevandi si trovano in calce a molti documenti, fra cui: BCTn, BCT3, capsa 4, mazzo 2, n. 28; capsa 5, mazzo 1, nn. 8, 24, 25, 41; capsa 29, mazzo 1, n. 34; ADTn, ACap, capsa anniversari, rotoli corti/a, n. 16.1) 
stesso colore, che hanno la caratteristica di essere abbelliti da cuciture intrecciate in pelle allumata. Chiudeva in origine il volume una cinghia, probabilmente del tipo di quella che stava sul registro di Antonio, cucita direttamente sulla coperta ${ }^{76}$. La legatura, forse coeva alla stesura del registro, al pari di quella di Instrumenta capitularia 8bis, è databile fra la fine del XV e l'inizio del XVI secolo (fig. 32).

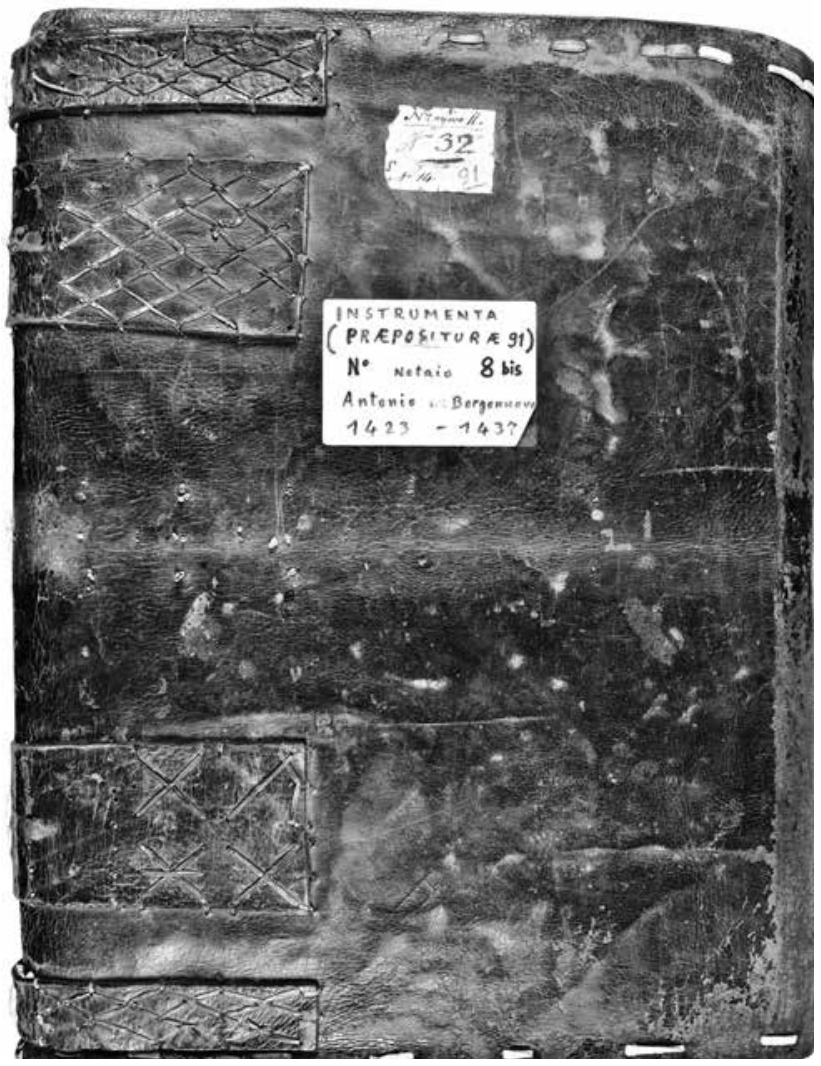

Fig. 32. ADTn, ACap, Instrumenta capitularia 8bis. Coperta superiore del registro.

Nessuna somiglianza mostrano invece, rispetto alle legature di questi due codici cartacei, i volumi prepositurali prodotti dal XVI secolo in poi ${ }^{77}$. Come ha evidenziato Rossella Ioppi studiando la storia dell'archivio prepositurale, pare che

anche nella fattura materiale dei volumi sia riconoscibile una cesura tra quanto prodotto sino al XV secolo e la documentazione di epoca posteriore. Se un primo intervento di revisione del materiale monastico/prepositurale pervenuto all'archivio del nuovo ente nella seconda metà del secolo XV è ravvisabile a cavallo dei secoli XV-XVI, un cambiamento sembra essersi verificato nel trattamento e gestione di quanto, pertinente alla Prepositura, fu prodotto dopo il $1500^{78}$.

76 Cfr. Ioppi, I registri del monastero di San Lorenzo, cit., pp. 93-94.

77 Il volume notarile contrassegnato con la segnatura n. 18, ovvero il già citato registro di Guglielmo Gallo (Libro A), contenente documentazione rogata fra il 1500 e il 1514, presenta una legatura completamente diversa databile al secolo XVIII: ivi, p. 96.

78 Ibidem. 


\section{Conclusioni}

All'inizio di questo volume si è segnalato il punto d'arrivo di un lungo percorso evolutivo che, al principio dell'età moderna (dal periodo clesiano in poi), portò i notai trentini a definire con maggior precisione i propri ruoli e il proprio funzionamento; sotto questo profilo il notariato trentino del Quattrocento, in particolare della prima metà, appare debole, incerto e, soprattutto, ancora privo di una coscienza professionale e di gruppo.

Pur attestato e normato negli statuti masoviani della metà degli anni Venti del XV secolo, un Collegio notarile in grado di esercitare un solido controllo sull'accesso alla professione, sulla gestione della documentazione da parte dei suoi iscritti e sulla conservazione e trasmissione delle carte dei notai defunti non sembra essere pienamente operativo prima della metà del Quattrocento.

Nei decenni precedenti le fonti mostrano le tracce dei tentativi di scostarsi dall'ombra ingombrante del potere vescovile, come stava facendo, in analogo scorcio d'anni, il Comune di Trento. Si è ben lontani - per fare un esempio - dalla situazione della vicina città di Verona, dove, con l'inizio della dominazione veneziana, nel 1408 fu prescritta la registrazione dei rogiti notarili in appositi volumi presso l'Ufficio del Registro, l'istituzione (di lì a poco creata anche a Vicenza) che aveva il compito di conservare le registrazioni in copia autentica dei rogiti notarili; a Trento, invece, soltanto sul finire del secolo furono intrapresi provvedimenti per salvare i protocolli superstiti da sicura distruzione.

Gli esiti dell'assenza di una sistematica politica di conservazione sono ben visibili oggigiorno negli archivi trentini dove, fatti salvi $\mathrm{i}$ registri prodotti per $\mathrm{i}$ grandi enti del Principato (Vescovado e Capitolo della cattedrale), che pure non sono numerosissimi, i volumi notarili con documentazione varia (prodotta cioè per piccoli enti e privati cittadini) si contano sulle dita di una mano: fra questi c'è Instrumenta capitularia 8bis, che deve tuttavia la sua sopravvivenza all'interesse che rivestì per la dignità capitolare della Prepositura, nell'archivio della quale confluì fra fine Quattrocento e inizio Cinquecento; in caso contrario avrebbe forse subito la stessa sorte di tutta l'altra documentazione (ad esclusione di quella capitolare) prodotta da Antonio e dagli altri notai trentini di inizio Quattrocento, scartata dagli eredi dei professionisti una volta venuto meno l'interesse economico a conservarla.

Gli studi sui notai trentini tardo medievali si sono concentrati prevalentemente sul XIII e sul XIV secolo, mentre sono rimasti spesso nell'ombra taluni aspetti del notariato di inizio Quattrocento. La biografia di Antonio da Borgonuovo tracciata nelle pagine di questo volume ha permesso di 'fotografare' in un arco cronologico di circa cinquant'anni, compreso fra gli anni Ottanta del Trecento e la fine degli anni Trenta del Quattrocento, le caratteristiche del notariato trentino in una fase di piena transizione. 


\section{Conclusioni}

'Transizione' è forse la parola chiave che permette di leggere e comprendere l'intera vicenda personale e professionale di Antonio. Figlio di un parvenu di origini lagarine che a Trento svolgeva la remunerativa - ma non eccelsa - professione di portitor vini, Antonio era riuscito sin da giovanissimo a fare carriera nel centro politico del Principato. L'ascesa sociale e politica fu forse un riflesso, ma non si può affermare con assoluta certezza, del suo ruolo di primo piano come professionista della scrittura, come sembrano dimostrare le molteplici cariche politiche, il consolato in primis, ricoperte dagli anni Dieci del Quattrocento nel Magistrato consolare di Trento.

Da questo punto di vista Antonio da Borgonuovo può essere collocato fra i maggiorenti della città che, dopo le rivolte del Belenzani negli anni Dieci, avevano iniziato a ricoprire cariche pubbliche nel Comune: famiglie come $\mathrm{i}$ da Molveno, $\mathrm{i}$ Mezzasoma, i Belenzani, i de Murlinis, i Mercadenti, gli ab Oleo, i Calepini, per citare soltanto alcune fra le più note, si spartirono il potere per buona parte del Quattrocento, spesso imparentandosi fra loro e garantendo in tal modo un saldo controllo sugli uffici del Comune.

L'apice dell'ascesa sociale di Antonio può essere forse rappresentato dai matrimoni di due sue figlie con Adelperio e Bonaventura, membri di spicco della potente famiglia Calepini. D'altra parte, nella documentazione prodotta da Antonio nei primi tre decenni del Quattrocento emerge la fitta rete di clienti, anche di un certo prestigio, che a lui si rivolgevano con frequenza per registrare i propri negozi giuridici; oltre ai Calepini, si trovano molti fra i membri della classe dirigente sopra citati.

Allo scoppio delle rivolte cittadine contro Alessandro di Masovia (1435-1437), diversi fra questi personaggi - Antonio da Borgonuovo compreso - furono fra le fila del partito anti-vescovile, additati poi dall'anonimo autore delle Rime filo-vescovili come proditores. Un certo numero di questi cittadini, fra i molti Adelperio Calepini, Gioacchino Mezzasoma, Guglielmo Saraceno, Luca di Giovanni de Cirogicis ecc., erano inoltre giurisperiti, con una solida cultura giuridica formatisi fuori dal capoluogo vescovile, dove forse avevano avuto occasione di osservare il funzionamento dei grandi comuni dell'Italia centro-settentrionale.

Stando alla documentazione rimasta, l'attività di Antonio da Borgonuovo era iniziata nel 1386 presso il palatium episcopi, al servizio del vicario vescovile. Ma nell'arco di pochi anni compaiono fra i suoi clienti, oltre ai privati, anche i vescovi, il Comune di Trento e diversi fra conventi e monasteri. Gli anni Ottanta e Novanta del XIV secolo costituiscono dunque per il notaio una fase di apprendistato di primissimo livello, preparatoria all'intensa attività svolta soprattutto fra il 1402 e il 1434 per il Capitolo della cattedrale, per il quale egli produsse un intero registro di imbreviature ed estesi, monumento dell'attività patrimoniale dei canonici e testimonianza di un rapporto continuativo - e potremmo pensare 'di fiducia' - fra il professionista e il secondo ente del Principato.

All'interno di questo contesto Antonio si configura come 1' 'erede' di Pietro de Stanchariis da Teglie di Brescia, notaio capitolare operante nella seconda metà del Trecento e, soprattutto, canonico della cattedrale. Sotto il profilo dell'organizzazione della produzione documentaria capitolare pare essere proprio il canonico de Stanchariis ad introdurre elementi di novità, tanto da poterlo considerare, per certi aspetti, il 'maestro' di Antonio; da questo punto di vista il registro Instrumenta capitularia 8 presenta una struttura del tutto sovrapponibile al precedente n. 7 (oggi conservato presso l'Archivio di Stato di Trento con altra segnatura), con un'orga- 
nizzazione e partizioni interne dedicate ai diversi cespiti d'entrata del Capitolo: ser Antonio non sembra inventare nulla, ma prosegue semplicemente la linea di uno dei suoi predecessori.

L'analisi sul modus operandi di Antonio, svolta a partire dal registro capitolare e da Instrumenta capitularia 8bis, ha inoltre portato alla luce le molteplici modalità operative del notaio per la redazione dell'instrumentum notarile: dalla rogatio, in cui il notaio appuntava in maniera concisa i dati essenziali del negozio giuridico su un foglietto di carta ('scheda') o su una sorta di 'manuale' di dimensioni molto contenute, scegliendo per la redazione di una forma più completa del documento registri di medie dimensioni (in formato in quarto) che solitamente egli definisce prothocolli, o fogli o bifolia sciolti. In taluni casi egli non procedette alla stesura di ulteriori redazioni di un certo documento, cosicché la versione su protocollo sembra essere seconda e ultima tappa di questo procedimento; in altre occasioni ser Antonio trasse invece da quei registri una stesura completa e definitiva del documento, il mundum, su pergamena sciolta. La particolarità è rappresentata da una quarta forma redazionale dello stesso atto: egli infatti, in alcune occasioni, redasse oltre alla 'scheda' e al documento in protocollo (o su fogli sciolti) una terza stesura dello stesso rogito, spesso in forma pressoché completa e munita addirittura di signum e sottoscrizione, su registri di grande formato (in folio) come Instrumenta capitularia 8 e 8bis, che egli definisce quaterni o libri. Essi sono, a seconda delle necessità, la sede di registrazione di documenti in forma completa, del tutto sovrapponibili alle redactiones in mundum su pergamena e talvolta già preventivamente stesi sui prothocolli, e, al contempo, luogo deputato alla redazione di rogiti in forma di imbreviatura, privi di qualsiasi elemento formulare, esito di una stesura immediata dopo la rogatio ricevuta dai committenti. I grandi quaterni di Antonio sono dunque testimonianza delle molteplici modalità operative del notaio: due, tre e, nel caso gli fosse stato richiesto il mundum, addirittura quattro stesure dello stesso documento.

Si tratta di modalità operative diversificate e stratificate che trovano riscontro in altri notai tardo medievali, ma che spesso sfuggono a causa della perdita pressoché totale di forme redazionali dei documenti che i notai consideravano 'transitorie', e dunque non meritevoli di essere conservate.

La figura di Antonio da Borgonuovo sembra ben impersonare quel 'mondo nuovo' che fa la sua comparsa a Trento fra Trecento e Quattrocento, e che permette a individui anche di modesta estrazione, ma capaci professionalmente, certamente ambiziosi e attenti alle relazioni sociali, di emergere dall'anonimato e di affermarsi all'interno della società in cui vivono.

\section{Ringraziamenti}

Giunto al termine di questo lavoro desidero ringraziare coloro che mi hanno aiutato: Stefano Moscadelli che è stato mio tutor durante i tre anni di corso dottorale e mi ha seguito durante le ricerche e la stesura della tesi da cui ho tratto questo libro; Andrea Giorgi ed Emanuele Curzel che per primi hanno segnalato l'interesse della figura del notaio Antonio da Borgonuovo e hanno messo a disposizione le loro competenze durante le varie fasi della ricerca; Andrea Zorzi, coordinatore del XXIX ciclo del dottorato in «Studi storici» delle Università di Firenze e Siena e i membri del Consiglio di curriculum in «Scienze del libro, istituzioni e archivi»; 


\section{Conclusioni}

Donatella Frioli, Marco Stenico e Francesca Santoni per i proficui suggerimenti. La mia gratitudine va inoltre a Gian Maria Varanini che ha avuto la gentilezza di leggere la prima stesura di questo libro fornendomi preziose indicazioni e utilissimi consigli, e ad Anna Zangarini per la collaborazione nella compilazione degli indici. La ricerca negli archivi è stata agevolata dalla disponibilità di Katia Pizzini, Claudio Andreolli e Renato Giacomelli dell'Archivio diocesano tridentino, Franco Cagol dell'Archivio storico del Comune di Trento, Paolo Giovannini e Luciana Chini dell'Archivio di Stato di Trento, e del personale della Sala Trentina della Biblioteca comunale di Trento, della biblioteca dell'Istituto storico italo-germanico della Fondazione Bruno Kessler, dell'Archivio provinciale di Trento e dell'Archivio di Stato di Verona. Sono inoltre grato ad Adalberta Bragagna che ha curato l'impaginazione del volume e a Gabriele Weber per l'elaborazione grafica delle mappe. 
Appendice

Stefano Malfatti, Antonio da Borgonuovo. L'ascesa di un notaio a Trento fra Trecento e Quattrocento, ISBN 978-88-6453-820-4 (print), ISBN 978-88-6453-821-1 (online) CC BY 4.0, 2018, Firenze University Press 
Appendice

Monete e unità di misura

I dati si riferiscono al sistema in uso a Trento.

Monete lira (libra) veronese, divisa in 20 soldi e corrispondente a 240 denari $(1$ soldo $=12$ denari) marca (d'argento), corrispondente a 10 lire grosso tirolese (o carentano), corrispondente a 20 denari ( 1 lira $=12$ grossi) ducato (d'oro), corrispondente a 38 carentani o 3 lire e 2 grossi

Superficie piovo (plodium) a Trento corrispondente a $3382 \mathrm{mq}$ staio $($ starium $)$ pari a $845,7 \mathrm{mq}(4$ staia $=1$ piovo $)$

Capacità carro (plaustrum), pari a 6 brente $(1$ brenta $=104,6$ litri $)$, per il vino staio (starium) pari a circa 21.3 litri, per i cereali

Peso libbra, a Trento corrispondente a $0,336 \mathrm{~kg}$ 


\section{Regesti}

1.

1383 settembre 12 e 15 , Trento

(a) [settembre 12] Ser Francesco del fu ser Bonaventura da Molveno notaio, a titolo personale e a nome di tutti gli eredi del fu Antonio del fu Giovanni Belenzani da Trento, chiede a Giovanni de Pugnis da Parma, vicario vescovile, che le ultime volontà del defunto Antonio vengano considerate fededegne, che ne venga redatta attestazione scritta e che venga scelto un curatore per i figli del fu Antonio, Guglielmo e Giovanni. Udite le richieste, il vicario stabilisce che ser Giacomo da Ravazzone, notaio, cittadino e abitante di Trento, è idoneo come curatore di Guglielmo e Giovanni; lo nomina quindi tutore e questi presta giuramento, costituendo come proprio fideiussore Marco del fu ser Odorico da Spormaggiore, notaio, cittadino e abitante di Trento. Ser Francesco notaio da Molveno e ser Giacomo notaio da Ravazzone, curatore, volendo conoscere la buona fede e provare le ultime volontà del defunto Antonio, producono in giudizio una petizione e una serie di capitoli da sottoporre ai testimoni citati; chiedono altresì al vicario di stabilire un termine nel giorno del martedì successivo (15 settembre) per far comparire in giudizio i legatari del fu Antonio. Udite tali richieste il vicario stabilisce che i legatari del fu Antonio debbano comparire il martedì successivo nel palazzo vescovile per ascoltare i testimoni sui suddetti capitoli e assistere alla pubblicazione del testamento.

(b) [settembre 12] Giacomo da Ravazzone, notaio, cittadino di Trento, in quanto curatore di Guglielmo e Giovanni, figli del fu Antonio Belenzani, e Francesco da Molveno, notaio, a nome dei legatari, considerato che Antonio aveva stabilito le sue ultime volontà e temendo che $\mathrm{i}$ testimoni presenti possano morire per la pestilenza in corso, chiedono che $\mathrm{i}$ testimoni chiamati esaminino $\mathrm{i}$ capitoli di seguito prodotti e che delle loro attestazioni venga redatto un documento: che il giorno 11 settembre Antonio, nella sua abitazione nella contrada dei Belenzani, aveva disposto le sue ultime volontà; che aveva stabilito che Guglielmo e Giovanni, suoi figli, fossero eredi universali; che aveva lasciato un affitto perpetuo di 10 lire per celebrare il suo anniversario nella chiesa di Santa Maria Maggiore in Trento; che aveva lasciato alla casa dei Battuti di Trento un affitto perpetuo di 10 lire; che aveva lasciato a Giacoma sua moglie 100 ducati che le aveva promesso nel caso fosse morto prima di lei; che aveva lasciato a Todeschina, moglie del suo defunto padre, 300 lire di denari piccoli dei suoi beni; che aveva lasciato a magister Ottolino sarto 100 lire; che aveva lasciato a ser Francesco da Molveno notaio 1.000 ducati da ricevere dopo la morte dei figli di Antonio stesso; che nel caso in cui i suoi figli muoiano senza eredi, i suoi beni debbano essere distribuiti fra i pauperes Christi egenos; che aveva nominato quale fidecommissario e tutore dei suoi figli ser Francesco da 
Appendice

Molveno notaio; che i testimoni presenti alle ultime volontà erano stati Pietro da San Bonaventura prete, Odorico notaio, Andrea orefice, Ottolino sarto, Giovanni detto Mosca, Odorico da Molveno cerdo, magister Antonio tornerius, domine Todeschina, Lena, Flora, Sibilia, Giuliana; che dopo ciò detto Antonio era morto; che di quanto sopra esiste publica vox et fama.

(c) [settembre 15] Benassuto, viator della curia tridentina, dichiara al notaio di aver citato i testimoni e i legatari. I nomi dei legatari citati sono: ser Simone da Campo sindaco dei Battuti, Biagio da Pomarolo, sindaco della pieve di Santa Maria Maggiore in Trento, domina Giacoma moglie del fu Antonio, domina Todeschina moglie del fu Giovanni Belenzani, Ottolino sarto del fu Paolo cursor. I nomi dei testimoni citati sono: dominus Pietro da Parma vicario nella chiesa di Santa Maria Maggiore in Trento, Bonaventura monaco del fu Pasquale da Verona, Odorico notaio del fu Rosso becarius da Trento, Andrea orefice del fu ser Ottolino dal Borgonuovo di Trento, Ottolino sarto del fu ser Paolo cursor, magister Giovanni detto Mosca $a$ Balistis del fu Bartolomeo Mastelus da Fai, Odorico cerdo da Molveno del fu ser Sicherio abitante in Trento, magister Antonio tornerius del fu ser Pietro da Verona, domina Giuliana del fu ser Adelperio da Denno in val di Non, domina Sibilia del fu Chierico da Montecchio Precalcino, diocesi di Vicenza, domina Lena del fu Bono da Nomi, moglie di Pancera da Sardagna, domina Todeschina del fu ser Guglielmo di Giustiniano, Flora di magister Tura a Porta. Coloro che sono chiamati a testimoniare prestano quindi giuramento.

Testimoni (12 settembre): ser Giacomo notaio da Ravazzone del fu ser Bartolomeo, Marco notaio del fu ser Odorico da Spormaggiore in val di Non, Ottolino notaio del fu ser Trentino da Trento, Vigilio notaio del fu ser Enrico Rigaie, magister Nicolò Schichignolus del fu ser Alberto da Trento, ser Guglielmo notaio detto ab Equabus et aliis.

Testimoni (15 settembre): Vigilio Rigaie, Ottolino notaio, Luca *** da Firenze oste in Trento et aliis.

Notaio: Alberto del fu ser Negratus da Sacco, diocesi di Trento, abitante di Trento, notaio pubblico per autorità imperiale.

Copia semplice; ADTn, ACap, Instrumenta capitularia 8bis, cc. 186v-187v, n. 498[a] [B]. Nel margine superiore, di mano del notaio rogatario del testo: Copia testamenti condam Antonii de Belenzanis de Tridento hic per me transcripti ad avisamentum et deffenssionem eorum qui emerunt de bonis suis ab antedicto ser Petro de Senis qui emit a domino nostro executore dicti testamenti etc. Nel margine sinistro, della stessa mano, la rubrica: Actus et processus factus in causa reductionis ad perpetuam rei memoriam dispositionem et voluntatem olim Antonii condam domini Iohannis de Belenzanis de Tridento prout infra sequitur.

2.

1383 settembre 16 e 17 , Trento

Domina Flora, figlia del magister Tura sarto detto a Porta da Trento, domina Giuliana del fu ser Adelperio notaio da Denno in val di Non, abitante di Trento, domina Lena del fu ser Bono, già abitante a Nomi e ora abitante a Trento, domina Sibilia del fu Chierico da Montecchio Precalcino, in diocesi di Vicenza, ora abitante a Trento, magister Giovanni detto Mosca a Balistis del fu Bartolomeo detto Mastelus da Fai, magister Andrea orefice del fu ser Ottolino da Trento, ser Bonaventura del 
fu ser Pasqualino da Verona da borgo San Nazaro, monachus della chiesa di Santa Maria Maggiore in Trento, Odorico da Molveno cerdo del fu Sicherio abitante a Trento nella contrada dei Belenzani, Antonio tornerius abitante a Trento nella contrada di Santa Maria del fu ser Pietro da Bologna, magister Ottolino sarto del fu ser Paolo cursor abitante a Trento, domina Todeschina figlia del fu dominus Guglielmo del dominus Giustiniano da Trento e moglie del fu dominus Giovanni Belenzani da Trento, dominus Pietro da Parma, prete pievano della pieve di Santa Maria Maggiore di Trento, interrogati su istanza di ser Francesco da Molveno e ser Giacomo da Ravazzone, notai, confermano ogni singolo capitolo; Pietro da Parma, super nono capitulo, afferma che il suddetto Antonio Belenzani aveva disposto che qualora i suoi figli fossero morti senza eredi legittimi tutti i suoi beni, con l'eccezione dei 1.000 ducati spettanti a ser Francesco da Molveno notaio, avrebbero dovuto essere distribuiti dallo stesso ser Francesco ai pauperes Christi egenos.

Notaio: Alberto del fu ser Negratus da Sacco, diocesi di Trento, abitante di Trento, notaio pubblico per autorità imperiale.

Copia semplice; ADTn, ACap, Instrumenta capitularia 8bis, cc. 188r-190r, n. 498[b] [B].

3.

1386 febbraio 13 , Trento

Vigilio da Seregnano del fu Guglielmo da Roccabruna notaio, Alberto del fu ser Negratus da Sacco notaio e Federico figlio di ser Ognibene da Povo notaio, cittadini di Trento, arbitri scelti in comune tra Morandino da Trento canonico e Loisio del fu ser Cristoforo notaio detto dal Dosso da Trento per esaminare l'amministrazione fatta da Morandino come tutore di Loisio per un periodo di dieci anni, visti tutti i libri, i quaderni e gli scritti, condannano Morandino a pagare a Loisio le quantità di cereali non riscosse per dieci anni, come appare nel quaternus receptorum di Morandino, cioè 116 staia di frumento, 30 di siligine, 85 di miglio, panico e spelta, entro la prossima Pasqua; come resto degli affitti non esatti, Morandino deve pagare a Loisio 8 ducati, restituendogli tutti i libri, le carte, gli instrumenta. Morandino dichiara di non acconsentire alla sentenza.

Testimoni: ser Simone notaio del fu $d$. [Azzone] Tuynus da Campo, ser Mercadento del fu ser Francesco de Lost Mercadenti da Trento, ser Giacomo figlio del d. Enrico da Roccabruna, Vigilio notaio del fu ser Enrico detto Rigaie da Trento, Iorio notaio del fu ser Desiderato notaio da Ala, Bonaventura detto Turolo del fu Trentino detto Madurence da Trento, tutti cittadini e abitanti di Trento, Donato del fu Delaíto da Civezzano.

Notaio: Antonio figlio di ser Bartolasio da Borgonuovo di Trento cittadino di Trento, notaio pubblico per autorità imperiale.

Originale; ASTn, APV, Sezione latina, capsa miscellanea I, n. 126 [A]. Nel verso: in corrispondenza del margine superiore, nota di contenuto in tedesco di mano del XV secolo di cui si legge: urlt Morandin contra Lud noder e la nota vacat di mano del XV secolo; in corrispondenza del margine sinistro, il millesimo 1386, di mano del XVI secolo.

Regesto: Ippoliti, Zatelli, Archivi Principatus Tridentini regesta, vol. Misc. I e II, pp. 76-77. 
Appendice

4.

1401 marzo 18, Trento

Giovanni detto Zibechino del fu ser Riprando a Navibus, cerdo abitante a Trento, in quanto erede di Giacomo suo nipote, vende per 50 ducati ad Antonio notaio figlio di ser Bartolasio dal Borgonuovo l'utile dominio su una casa situata a Trento nella contrada di San Marco, rimettendone ogni diritto nelle mani dei canonici; questi ne investono quindi detto Antonio per il canone d'affitto annuo di 40 denari.

Testimoni: domini preti Gerardo da Verona pievano in Flavon, Donato da Verona sacrista nella cattedrale, Giovanni del fu Antonio Belenzani da Trento.

Canonici presenti: domini Rambaldo da Trento decano, Morandino, Sicco e Vigilio da Trento, Artuico dalla Carinzia, Antonio e Giacomo da Novara, Antonio da Arco, Florio da Denno, Nicolò da Trento.

Notaio: Alberto del fu ser Negratus da Sacco, notaio pubblico per autorità imperiale.

Originale; BCTn, BCT1, n. 1868, cc. $119 v-120 r$ [A].

Regesto: Zamboni, Economia e società in una piccola città alpina, n. 66.

5.

1408 febbraio 23, Trento

Wilhelm conte di Matsch, luogotenente e capitano di Trento per Federico duca d'Austria, conte del Tirolo e avvocato della Chiesa di Trento, Ianes di Liechtenstein e Christoph Fuchs, consiglieri del duca, dichiarano che il castello di Stenico appartenente all'Episcopato di Trento fu consegnato in custodia dai cittadini di Trento nelle mani di Negro da San Pietro di Trento durante la recente rivolta che vide coinvolto il vescovo di Trento. Poiché Negro e i suoi seguaci si rifiutano di riconsegnare il castello al duca, questi ha inviato a Stenico alcuni cittadini di Trento, prima imprigionati in una rocca di dominio tirolese, con il mandato di provvedere al recupero del castello: il capitano e i consiglieri del duca fissano agli inviati il termine della domenica delle Palme ( 8 aprile) per portare a compimento il mandato; in caso di esito positivo, saranno posti in libertà, in caso di fallimento, dovranno riconsegnarsi prigionieri nelle mani del duca. A favore di ciascuno dei cinque cittadini di Trento, prigionieri del duca e destinatari del mandato di trattativa per il recupero del castello di Stenico, si costituiscono fideiussori diverse persone.

Testimoni: domini Giovanni da Fondo, Giovanni da Lana, Michele da Povo canonici della cattedrale di San Vigilio in Trento, magister Nicolò phisicus de Accerbis da Trento, Bonadomano suo figlio, ser Paolo notaio del fu ser Martino da Trento, Buratinus notaio figlio di ser Giovanni de Buratinis da Trento, Marco del fu Franceschino notaio de Sichis da Trento.

Notaio: Guglielmo di ser Paolo cittadino e abitante di Trento, notaio pubblico per autorità imperiale.

Originale; ASTn, $A P V$, Sezione latina, capsa 8, n. 11 [A]. Nel verso: in alto stemma Unitas del principe vescovo Bernardo Cles (XVI sec.) e, sulla destra, nota in lingua tedesca e millesimo 1408. Sotto, a destra, croce greca inchiostrata.

Regesto: scheda SIAS (a cura di M. Stenico). 
Appendice

6.

1418 dicembre 7 , Trento

Ser Bartolasio del fu ser Cristiano da Mori cittadino e abitante a Trento, padre del notaio sottoscritto, cede a Domenica sua figlia e a Desiderato lanarolus del fu ser Francesco da Magrè dal Vicentino, suo genero, il dominio utile su un appezzamento di terra arativa e vignata, in parte piantata a filari, di circa 3 piovi, situato nelle pertinenze di Trento a San Bartolomeo presso il cimitero, rimettendone nelle mani dei canonici ogni diritto; questi ne investono dunque Domenica e Desiderato per il canone d'affitto annuo di 1 carro di vino bianco per tutto il Capitolo, da portare a Trento alla canipa dell'ente. Nonostante quanto stabilito, Bartolasio dovrà avere finché vivrà la metà del vino e pagare la metà dell'affitto.

Testimoni: domini preti Antonio de Buratinis da Trento mansionario nella cattedrale, Marco pievano in Livo e cappellano nella cattedrale, dominus Lorenzo de Gauslinis legum doctor da Feltre, Bartolomeo Toschanelus stazonerius in Trento del fu Alberto da Volano.

Canonici presenti: domini Giovanni cappellano dall'Austria, Florio da Denno, Giovanni da Fondo, Giovanni dalla Carinzia, magister Ulrico da Isny, Gaspare de Murlinis da Trento.

Notaio: [Antonio figlio di ser Bartolasio dal Borgonuovo di Trento, notaio pubblico per autorità imperiale].

Originale; ADTn, ACap, Instrumenta capitularia 8, c. $47 r$-v, n. 95 [A]. In corrispondenza del margine sinistro, di mano del notaio rogatario del testo, la rubrica: Refutatio facta per ser Bartholasium patrem mei Antonii notarii infrascripti de eius vineali de Sancto Bartholomeo de quo investiti fuerunt Desideratus et Dominica eius filia per dominos canonicos etc; sotto, in corrispondenza del censo annuo, la nota di mano del XVI secolo: Capitulo, che integra una precedente nota di mano del tardo XV secolo: vini brentas 6 ad Sanctum Bartholomeum.

7.

1420 luglio 15 , Trento

Antonio di ser Bartolasio dal Borgonuovo di Trento, notaio, vende per 18 ducati e $1 / 2$ a Ognibene del fu Azzio da Calliano abitante in Borgonuovo il dominio utile su uno stabulum edificato in muratura e legno, situato in contrada di Borgonuovo, rimettendone ogni diritto nelle mani dei canonici; questi ne investono quindi detto Ognibene per il canone d'affitto annuo di 40 soldi di denari trentini - ossia 16 grossi carentani - per la prebenda canonicale di Brentonico, attualmente spettante a Giovanni de Muta dalla Carinzia, nunc infirmatus.

Testimoni: domini preti Antonio de Buratinis da Trento mansionario nella cattedrale, Pietro de Drageto de Terra Laboris vicario nella pieve di Denno, Giovanni Conto notaio del fu ser Paolo notaio de Fatis da Terlago.

Canonici presenti: domini Ulrico da Isny, arcidiacono nella cattedrale, Giovanni da Fondo, Zambono da Trento, Florio da Denno, Giovanni Zeiss e Giovanni Anhang da Bopfingen, Corrado da Trento e Gaspare de Murlinis da Trento.

Notaio: [Antonio figlio di ser Bartolasio dal Borgonuovo di Trento, notaio pubblico per autorità imperiale]. 
Appendice

Originale; ADTn, ACap, Instrumenta capitularia 8, c. 181v, n. 362 [A]. In corrispondenza del margine sinistro, di mano del notaio rogatario del testo, la rubrica: Refutatio facta per me Antonium notarium infrascriptum de uno stabulo de quo feci investiri Ognabenum Azzii de Caliano pro affictu perpetuali consueto sexdecim grossorum carentanorum canonicali prebende de «segue de iterato〉 Brentonico; sotto, della stessa mano la nota relativa all'estrazione del mundum: Facta extra duplex videlicet locatori et conductori.

8.

1422 ottobre 10 , Trento

Wilhelm Matsch, capitano generale all'Adige e della città di Trento, nella vertenza tra il Comune di Trento, rappresentato dai procuratori Antonio da Molveno e Antonio di Bartolasio notai da una parte, e ser Negro de Negri da San Pietro di Trento, rappresentato dal suo procuratore Giovanni da Comano dall'altra, stabilisce che Negro de Negri e il fratello Leone contribuiscano alle spese pubbliche (factiones) come tutti gli altri cittadini, intimando ai procuratori del Comune di Trento di restituire loro il paiolo e le altre sostanze pignorate.

Testimoni: dominus Bartolomeo da Bologna decretorum doctor abate dell'abbazia di San Lorenzo fuori dalle mura di Trento, dominus Pietro de la Ture da Bergamo iuris utriusque doctor vicario in temporalibus di Trento.

Notaio: Antonio del fu ser Giovanni da Fai, diocesi di Trento, cittadino e abitante di Trento, notaio pubblico per autorità imperiale.

Originale; ASCTn, Comune di Trento, Antico regime, Sezione antica, ACT1-3263 [A]. Nel verso: a destra in senso opposto al recto, la nota di contenuto di mano del XVI secolo: Instrumentum sententie familia illorum de Nigris contribuat cum [al]iis; a destra, nel senso del recto, la segnatura archivistica della pergamena: $N 3263$.

9.

1424 giugno 18, Trento

Aldrighetto del fu Giovanni Mezaoveta da Pomarolo contrae matrimonio con Lucia, figlia di Guglielmo Gallo da Trento. Aldrighetto riceve dal suddetto Guglielmo 200 ducati come dote per la figlia Lucia, insieme ad altri beni mobili, promettendo solennemente che, nel caso di morte di Lucia senza figli legittimi e naturali, restituirà tutto l'ammontare della dote ricevuta.

Testimoni: domini Antonio da Molveno, Antonio di ser Bonomo da Arco giurisperiti, dominus Gioacchino Mezzasoma da Trento notaio, ser Gianpietro del fu ser Corradino da Feltre, ser Andrea apothecarius del fu Bernardo da Firenze, Adelperio e Odorico fratelli e figli del fu ser Marco Calepini, Nicolò del fu ser Mercadento Mercadenti, Antonio notaio de Castro, Odorico a fecibus tutti cittadini e abitanti di Trento.

Notaio: [Antonio del fu ser Bartolasio dal Borgonuovo di Trento, notaio pubblico per autorità imperiale]. 
Appendice

Originale; ADTn, ACap, Instrumenta capitularia 8bis, c. 16r, n. 47a [A]. In corrispondenza del margine sinistro, di mano del notaio rogatario del testo, la rubrica: Matrimonium cum dote de ducentis ducatis contractum inter Aldrigetum Mezaoveta ex una et dominam Luciam filiam Guillelmi Gali ex alia et cetera cum fine etiam facta etc. Sotto, della stessa mano, la nota relativa all'estrazione del mundum: Facta extra in publicam formam.

Edizione: Berlanda, Il matrimonio nel tardo medioevo, n. 5.

10.

[1424 giugno 18, Trento]

Lucia, per volontà del marito Aldrighetto, dichiara a Guglielmo Gallo, suo padre, di essere interamente soddisfatta della dote per lei versata e di non richiedere altro di quanto le spetterà dell'eredità paterna e materna.

Testimoni: come sopra (regesto n. 9).

Notaio: [Antonio del fu ser Bartolasio dal Borgonuovo di Trento, notaio pubblico per autorità imperiale].

Originale; ADTn, ACap, Instrumenta capitularia 8bis, c. 16r, n. 47b [A].

11.

1424 luglio 2, Trento

Guglielmo Gallo promette al genero Aldrighetto di versare 105 ducati come saldo e completo pagamento della dote per la figlia secondo i termini prescritti, ossia 50 ducati entro la festa di san Michele e, in seguito, secondo le richieste di Aldrighetto.

Testimoni: Calepino e Nascimbene fratelli e figli del fu ser Marco Calepini, Filippo del fu Bonaventura del fu ser Antonio da Volano, Vigilio detto Barbustelo macellaio in Trento, Francesco Pona murarius in Trento.

Notaio: [Antonio del fu ser Bartolasio dal Borgonuovo di Trento, notaio pubblico per autorità imperiale].

Originale; ADTn, ACap, Instrumenta capitularia 8bis, c. 16r, n. 47c [A]. In corrispondenza del margine sinistro, di mano del notaio rogatario del testo, la rubrica: Creditum dicti Aldrigeti contra dictum Guillelmum Gali de centum et quinque ducatis auri pro resto et completa solutione dicte dotis etc.

12.

1425 gennaio 8 , Trento

Battista del fu Silvestro da Bologna, stazonerius, contrae matrimonio con Francesca del fu Antonio Zagagnini; subito dopo, Battista dichiara di ricevere da Maria, moglie del fu Antonio e madre di Francesca, 210 ducati a titolo di dote, insieme ad alcuni beni mobili.

Testimoni: Maffeo apothecarius del fu ser Nigro da Brescia, Negrello notaio del fu ser Alberto notaio da Sacco, Nicolò a Sale del fu ser Odorico a Sale da Pergine, 
Appendice

magister Lorenzo a Vaginis del fu magister Giovanni da Feltre, ******* figlio di magister Lorenzo, Giovanni domine Alde del fu ser Tommaso da Barbarano, magister Pietro a caminis stazonerius del fu Giovanni dal Trevigiano, Giacomo notaio del fu ser Bertoldo a Ture da Trento, tutti cittadini e abitanti di Trento.

Notaio: [Antonio del fu ser Bartolasio dal Borgonuovo di Trento, notaio pubblico per autorità imperiale].

Originale; ADTn, ACap, Instrumenta capitularia 8bis, c. 27r, n. 79[a] [A]. In corrispondenza del margine sinistro, di mano del notaio rogatario del testo, la rubrica Matrimonium cum dote de II et $X$ ducatis contractum inter Baptistam de Bononia et dominam Franciscam de Zagagninis cum debito infrascripto etc. Sotto di mano del notaio Andrea Gallo (fine XV sec.), la nota Facta extra per me Andream Galli.

Edizione: Berlanda, Il matrimonio nel tardo medioevo, n. 7.

13.

[1425 gennaio 8, Trento]

Maria, col consenso del marito Ilario, promette al genero Battista, figlio del fu ser Silvestro da Bologna, di versargli entro quattro anni 200 ducati a titolo di dote per la figlia Francesca, nonostante la dichiarazione di pagamento effettuato che risulta dall'istrumento rogato dal notaio Antonio da Borgonuovo, ipotecando allo scopo un mulino con una casa edificata in muratura e legno de cupis coperta et cum scalis et ortis, canalibus, rotis, molis, aqueductibus et aliis fulcimentis ad ipsum molendinum et folognum spectantibus, situata nelle vicinanze di Trento, nella località dei Molini di San Francesco, fuori della città.

Testimoni: come sopra (regesto n. 12).

Notaio: [Antonio del fu ser Bartolasio dal Borgonuovo di Trento, notaio pubblico per autorità imperiale].

Originale; ADTn, ACap, Instrumenta capitularia 8bis, c. 27r-v, n. 79[b] [A]. Nel margine sinistro, in corrispondenza dei primi due righi, nota di contenuto di mano del notaio rogatario del testo: Creditum dicti Baptiste de dictis denariis contra dictam dominam Mariam.

14.

1425 gennaio 8 , Trento

Adelperio del fu nobile ser Marco Calepini da Trento vende a ser Michael daziere (mutarius), cognato del suddetto Adelperio e figlio del fu Ludovico da Monaco di Baviera, una serie di affitti, al prezzo di 167 ducati.

Testimoni: Nicolò del fu dominus Mercadento Mercadenti, Nicolò del fu ser Michele Approvini, magister Lorenzo a Vaginis del fu magister Giovanni da Feltre, Giacomo Batedelus del fu Nicolò Batedelus da Terlago, tutti cittadini e abitanti di Trento.

Notaio: [Antonio del fu ser Bartolasio dal Borgonuovo di Trento, notaio pubblico per autorità imperiale]. 
Appendice

Originale; ADTn, ACap, Instrumenta capitularia 8bis, c. 40v, n. 112 [A]. In corrispondenza del margine sinistro, note di mano del notaio rogatario del testo: in alto, la nota Relevata ex nota in uno foleo posita etc. Sotto, la nota di contenuto, Emptio ser Michaelis mutarii de affictibus infrascriptis Odorici de Calapinis sibi venditis per Adelperium eius fratrem etc. Sotto ancora, la nota relativa all'estrazione del mundum: Facta extra cum confessionibus affictalinorum usque ad affictum XVIIII 1/1 librarum denariorum Tridentinorum inclusive in uno rodulo etc. Sotto ancora, della stessa mano, la nota: Nota ad memoriam quod Odoricus de Calapinis hos affictus in partem suam perventos vendidit ser Zampetro et ser Zampetrus vendidit dicto Adelperio ut de ipsis duabus venditionibus notavit instrumenta dominus Iohachinus de Mezasomis etc. I documenti segnati sul registro come 113 a-g (regesti nn. $15-18,24,26,27)$ rappresentano le confessiones (dichiarazioni) degli affittuari relative alle proprietà oggetto della vendita di cui al doc. n. 112 (regesto n. 14).

15.

1425 gennaio 10 , Trento

Michele da Zell, sopra Trento, in quanto erede del fu suo genero Giovanni Briche, dichiara di tenere in enfiteusi da ser Michael daziere (mutarius) un appezzamento di terra aratoria e vignata, di circa mezzo piovo, in località Maderno, nel luogo detto in Brayda, per un affitto perpetuo di 16 grossi carentani.

Testimoni: Antonio notaio del fu ser Enrico de Castro da Trento, Francesco del fu ser Adelperio de Sichis da Trento, cittadini di Trento.

Notaio: [Antonio del fu ser Bartolasio dal Borgonuovo di Trento, notaio pubblico per autorità imperiale].

Originale; ADTn, ACap, Instrumenta capitularia 8bis, cc. 40v-41r, nn. 113-113a [A]. In corrispondenza del margine sinistro del documento n. 113, di mano del notaio rogatario del testo, la rubrica: Confessiones affictalinorum infrascriptorum.

16.

[1425] gennaio 13, Trento

Federico Polentone da borgo San Martino del fu Giovanni Pazolus da Levico dichiara di tenere in enfiteusi da ser Michael daziere (mutarius) un appezzamento di terra vignata di circa 2 piovi, situato nelle vicinanze di Trento, in località Martignano, per un affitto di 30 grossi carentani.

Testimoni: Bonaventura notaio sociorum, Antonio notaio de Castro, ser Francesco del fu Matteo da Chiusole cittadini di Trento.

Notaio: [Antonio del fu ser Bartolasio dal Borgonuovo di Trento, notaio pubblico per autorità imperiale].

Originale; ADTn, ACap, Instrumenta capitularia 8bis, c. 41r, n. 113b [A]. In corrispondenza del margine sinistro, di mano del notaio rogatario del testo, la rubrica: Confessio Federici Polentoni. 
Appendice

17.

[1425] gennaio 13, Trento

Francesco del fu ser Vincenzo da Gabbiolo da Vigolo Baselga dichiara di tenere in enfiteusi da ser Michael daziere (mutarius) una casa con orto, edificata in muratura e legno, situata a Vigolo Baselga in località ala Fontana, un appezzamento di terra arativa della misura di circa $3 / 4$ di piovo, situato nelle pertinenze di Vigolo in località ala Madom, e un appezzamento di terra vignata della misura di circa 1/3 di piovo situato a Terlago in località al Cere, per un affitto di 16 grossi carentani e 1 staio di frumento.

Testimoni: Rigo apothecarius del fu Domenico da Valeggio di Verona, Vito notaio di ser Pietro da Amblar, Giovanni Chelote da Gabbiolo.

Notaio: [Antonio del fu ser Bartolasio dal Borgonuovo di Trento, notaio pubblico per autorità imperiale].

Originale; ADTn, ACap, Instrumenta capitularia 8bis, c. 41r, n. 113d [A]. In corrispondenza del margine sinistro, di mano del notaio rogatario del testo, la rubrica: Confessio Francisci Vincencii de Vigulo Supramontis.

18.

[1425] gennaio 18, Trento

Giovannino del fu Giacomo Bruno dalla porta di San Martino di Trento dichiara di tenere in enfiteusi da ser Michael daziere (mutarius) una casa edificata in muratura e legno, situata a Trento in borgo San Martino, per un affitto di 10 soldi trentini.

Testimoni: Rigo apothecarius, Antonio Gans del fu ser Giovanni Gans da Trento, Serafino del fu Antonio Secadinari da Mezzolombardo.

Notaio: [Antonio del fu ser Bartolasio dal Borgonuovo di Trento, notaio pubblico per autorità imperiale].

Originale; ADTn, ACap, Instrumenta capitularia 8bis, c. 41r, n. 113c [A]. In corrispondenza del margine sinistro, la rubrica: Confessio Iohanini Bruni.

19.

1425 gennaio 30 , Trento

Gioacchino del fu ser Pietro Mezzasoma notaio da Trento contrae matrimonio con Speranza, figlia del fu ser Paolo notaio da Trento, già massaro della curia tridentina; il suddetto Gioacchino dichiara di ricevere da Giovanni Rauter del fu detto ser Paolo, fratello di Speranza, 350 ducati e beni mobili a titolo di dote per la sorella.

Testimoni: ser Pietro Iacob del fu ser Rigo da Rovereto, ser Bonadomano del fu magister Nicolò phisicus de Accerbis da Trento, ser Gianpietro del fu ser 
Appendice

Corradino da Feltre, ser Odorico Stratenperger del fu dominus Ottone dall'Austria, ser Guglielmo detto Saraceno notaio del fu ser Paolo da Trento, Adelpreto del fu ser Federico da Povo, Odorico del fu ser Marco Calepini, Palamidesio del fu ser Giacomo da Trento, tutti cittadini e abitanti di Trento.

Notaio: [Antonio del fu ser Bartolasio dal Borgonuovo di Trento, notaio pubblico per autorità imperiale].

Originale; ADTn, ACap, Instrumenta capitularia 8bis, c. 30r, n. 85a [A]. In corrispondenza del margine sinistro, di mano del notaio rogatario del testo, la rubrica: Matrimonium cum dote de trecentis et quinquaginta ducatis contractum inter dominum Iohachinum de Mezasomis et dominam Speranzam condam ser Pauli massari etc. Sotto, della stessa mano, la nota relativa all'estrazione del mundum: Facta extra ipsi Rauter(i) et ei data in questionem quam habebat cum domino Zambono eius fratre de 1429 ad monstrandum quod ipse solus solvit dotes ipsas.

20.

[1425 gennaio 30, Trento]

Giovanni Rauter promette a Gioacchino di versare i 350 ducati a lui dovuti come dote per la sorella Speranza.

Testimoni: come sopra (regesto n. 19).

Notaio: [Antonio del fu ser Bartolasio dal Borgonuovo di Trento, notaio pubblico per autorità imperiale].

Originale; ADTn, ACap, Instrumenta capitularia 8bis, c. 30r, n. 85b [A]. Il documento, posto in corrispondenza del margine sinistro della carta, è stato depennato, con inchiostro di tonalità più scura, probabilmente in occasione del saldo del debito da parte di Gioacchino. Sopra, nota di mano del notaio rogatario e con inchiostro di tonalità più scura: Facta extra in publicam formam distincte et data ipsi domino Iohachino etc.

21.

[1425 gennaio 30, Trento]

Speranza, col consenso del marito Gioacchino, dichiara a Giovanni Rauter e a Zambono suoi fratelli di non chiedere altro di quanto le sarebbe spettato dell'eredità.

Testimoni: come sopra (regesto n. 19).

Notaio: [Antonio del fu ser Bartolasio dal Borgonuovo di Trento, notaio pubblico per autorità imperiale].

Originale; ADTn, ACap, Instrumenta capitularia 8bis, c. 30r, n. 85c [A]. Il documento, posto in corrispondenza del margine sinistro della carta, è preceduto dalla nota, di mano del notaio rogatario, relativa all'estrazione del mundum vergata con inchiostro di tonalità più scura: Posita continua in hoc foleo hic inserto et facta extra ipsi Iohanni Rauter etc. 
Appendice

22.

1425 gennaio 30 , Trento

Giovanni detto Rauter contrae matrimonio con Andriota, figlia di ser Bonadomano del fu magister Nicolò phisicus de Accerbis da Trento; Giovanni dichiara di ricevere da ser Bonadomano 400 ducati e altri oggetti a titolo di dote per la figlia.

Testimoni: magister Odorico phisicus de Ruphalchatis da Arco, magister Giovanni di Luca cirogicus di Trento, dominus Antonio da Molveno giurisperito, dominus Antonio di ser Bonomo da Arco giurisperito, dominus Gioacchino Mezzasoma, magister Enrico professore in grammatica di Trento, ser Pietro Iacob, ser Perozzo apothecarius da Firenze, tutti cittadini di Trento, et alii plures honorabiles cives.

Notaio: [Antonio del fu ser Bartolasio dal Borgonuovo di Trento, notaio pubblico per autorità imperiale].

Originale; ADTn, ACap, Instrumenta capitularia 8bis, c. $30 v$, n. 86a, [A]. In corrispondenza del margine sinistro, di mano del notaio rogatario del testo, la rubrica: Matrimonium cum dote de IIII ${ }^{c}$ ducatis auri contractum inter Iohannem Rauter et dominam Andriotam de Accerbis etc; sotto, della stessa mano ma con inchiostro di tonalità più chiara, la nota relativa all'estrazione del mundum: Facta extra ipsi Bonadomano.

Edizione: Berlanda, Il matrimonio nel tardo medioevo, n. 7.

23.

[1425 gennaio 30, Trento]

Andriota, col consenso del marito Giovanni Rauter, dichiara al padre Bonadomano, lì presente anche in rappresentanza della moglie Antonia, di non chiedere altro di quanto le spetterà dell'eredità paterna e materna e dei fratelli, giurando di fronte al notaio in ragione della sua minore età.

Testimoni: come sopra (regesto n. 22).

Notaio: [Antonio del fu ser Bartolasio dal Borgonuovo di Trento, notaio pubblico per autorità imperiale].

Originale; ADTn, ACap, Instrumenta capitularia 8bis, c. 30v, n. 86b [A]. Il documento, in forma abbreviata, è posto in corrispondenza del margine sinistro.

Originale; ADTn, ACap, Instrumenta capitularia 8bis, c. 30v, n. 86c [A']. Il documento, in forma di esteso, corrisponde al n. 86b, che affianca, ed è redatto con inchiostro di tonalità più chiara, così come la nota relativa all'estrazione del mundum, di mano del notaio rogatario del testo: Facta extra ipsi Bonadomano.

24.

[1425] febbraio 12, Trento

Di fronte ad Alberto da Marostica vicario, Giovanni del fu Stefano, marito di Oliva, figlio del fu Benvenuto, del fu Pellegrino, del fu Gilberto da Stravino di 
Appendice

Cavedine dichiara di tenere in enfiteusi da ser Michael daziere (mutarius) un appezzamento di terra arativa di circa 1 piovo, situato nelle vicinanze di Cavedine, nel luogo detto in Arzill, per un affitto di 1 staio di frumento.

Testimoni: Antonio notaio de Castro, Antonio notaio da Fai, Graziadeo notaio da Terlago.

Notaio: [Antonio del fu ser Bartolasio dal Borgonuovo di Trento, notaio pubblico per autorità imperiale].

Originale; ADTn, ACap, Instrumenta capitularia 8bis, c. 41r, n. 113e [A]. In corrispondenza del margine sinistro, di mano del notaio rogatario del testo, la rubrica: Confessio Iohannis Olive de Stravino etc.

25.

1425 aprile 18 , Trento

Alessandro di Masovia, vescovo di Trento, su richiesta del nobile Ianes Thun, che agisce quale erede dei beni di Erasmo Thun anche a nome dei fratelli, ordina a Vigilio da San Vigilio, procuratore del Comune di Trento, e a Guglielmo notaio detto Saraceno, a Giustiniano, a Odorico Schratemperger, a Matteo de Murlinis, ad Adelperio Calepini e a Nicolò Fridele, consoli, che la città di Trento e i suoi districtuales paghino a Ianes 700 ducati, quale rimanenza degli 800 ducati che il Comune deve ancora sborsare per le spese di fortificazione di Castel Stenico sostenute da suo padre Erasmo. La somma dovrà essere pagata dalla città e da Negro de Negri, a suo tempo capitano di Castel Stenico, sia per le spese di fortificazione sia per risarcimento del danno e degli interessi fin lì maturati, ovvero ulteriori 100 ducati da corrispondere entro quindici giorni, pena il pagamento di 1.000 ducati da versare alla camera episcopale in caso di inadempienza. Se il detto Negro de Negri non si accorderà con il Comune entro quattro giorni, trascorso un mese, il Comune sarà messo in possesso dei beni del de Negri, reclamando la somma pagata dal Comune nonostante l'appello interposto dal de Negri presso la Sede apostolica e fatti salvi i diritti dotali della moglie. Infine, poiché alcuni cittadini chiedono che tutte le abitazioni del de Negri siano distrutte ad perpetuam rei memoriam quale azione dimostrativa per il reato di lesa maestà contro il vescovo, stabilisce, su richiesta dello stesso de Negri, che nessuno possa distruggerle senza il suo permesso.

Testimoni: domini Gioacchino a Mami, ser Vivamento da Caldes, ser Michael a Muta, ser Michele da Coredo et alii pluribus.

Notaio: Artuico di Enrico chierico da Passau, notaio pubblico per autorità imperiale (estensore del documento); Antonio del fu ser Bartolasio dal Borgonuovo di Trento, notaio pubblico per autorità imperiale (estensore della rubrica in registro).

Originale, ASCTn, Comune di Trento, Antico regime, Sezione antica, ACT1-3328 [A]. Rubrica relativa a questo documento in ADTn, ACap, Instrumenta capitularia 8bis, c. $37 r$ : Mandatum factum per dominum nostrum procuratori et consulibus civitatis que infra certos terminos solvatur sub certa pena domino Ianeso de Castro Thoni ducatos VII auri pro munitionibus dat(is) Nigro etc. Il notaio Antonio da Borgonuovo non ha steso sul registro il documento lasciando in bianco il resto della carta. 
Appendice

26.

[1425] maggio 6, Trento

Pellegrino del fu Bertoldo detto Scherp, Antonio del fu ser Beloto e Giovanni Chelotus del fu Chelotus da Povo, in quanto eredi dei fu Giovannetto e Giovannino, fratelli e figli del fu Paramusius da Povo, dichiarano di tenere in enfiteusi da Michael daziere (mutarius) un appezzamento di terra vignata di circa 1 piovo situato nelle pertinenze di Graffiano, in località al Dosso di Calonegi, un appezzamento di terra vignata di circa mezzo piovo situato nelle pertinenze di Graffiano in località al Rivozzo, un prato cum antanis e un casale nelle pertinenze di Graffiano, un appezzamento di terra arativa di circa $1 / 3$ di piovo situato nelle pertinenze di Graffiano in località in Clesura, un appezzamento di terra arativa e vignata con un pero al suo interno, situato nelle pertinenze di Povo in località a Fontana, un prato nelle pertinenze di Povo, a Gabbiolo, in località a Pra Mazor, un appezzamento di terra arativa di circa 1 piovo situato in località detta in Gabium sive Agilon, un casale fractu(s) con un orto situato nelle medesime pertinenze in località a Fulan e un appezzamento di terra di circa 1 piovo in località a Redundol, per un affitto di 19 lire trentine e mezzo.

Testimoni: Giovannino del fu Chous, Bartolomeo detto Sachart del fu Rolando, Giovanni di ser Bartolomeo Bayli e Nichele Bragalda tutti da Povo et alii.

Notaio: [Antonio del fu ser Bartolasio dal Borgonuovo di Trento, notaio pubblico per autorità imperiale].

Originale; ADTn, ACap, Instrumenta capitularia 8bis, c. 41r, n. 113f [A]. In corrispondenza del margine sinistro, di mano del notaio rogatario del testo, la rubrica: Confessio Pelegrini Antonii Beloti et Iohannis Chelote heredes ut infra.

27.

[1425] giugno 16, Trento

Margherita detta Stanfferina, figlia ed erede del fu Nichele a labro scisso dalla contrada di San Marco, dichiara di tenere in enfiteusi da Michael daziere (mutarius) una casa edificata in muratura e legno, con un orto nel retro, situata nella contrada di San Marco, per un affitto di 3 lire trentine.

Testimoni: Giacomo del fu Nichele a Silva, Abrianus del fu Nicolò Nichi mulattiere, Pietro del fu Guglielmo Merino tutti da Levico, Giovanni del fu ser Adelpreto da Covelo del Pedegazza et alii.

Notaio: [Antonio del fu ser Bartolasio dal Borgonuovo di Trento, notaio pubblico per autorità imperiale].

Originale; ADTn, ACap, Instrumenta capitularia 8bis, c. $41 v$, n. 113g [A]. In corrispondenza del margine sinistro, di mano del notaio rogatario del testo, la rubrica: Confessio domine Malgarite Stanfferine etc. 
Appendice

28.

1425 ottobre 10 , Trento

Essendo morto Bartolomeo da Bologna, ultimo abate di San Lorenzo, che per più di quarant'anni ha governato l'abbazia con non più di uno o due monaci, celebrando raramente la messa nella cappella di Sant'Apollinare, Alessandro vescovo e principe, con il consenso del Capitolo, erige nella Chiesa trentina una nuova prelatura, detta dignità della Prepositura, disponendo che sia la seconda in ordine d'importanza dopo quella pontificale, ossia il decanato; vi assegna tutti i redditi dell'abbazia, con le chiese parrocchiali di Sant'Apollinare e di San Pietro in Ora, volendo che il nome stesso dell'abbazia sia soppresso. Inoltre, affinché il culto divino non diminuisca, istituisce nella Prepositura due cappellani presbiterali perché celebrino la messa in Sant'Apollinare; istituisce inoltre nella cattedrale due diaconi, uno dei quali leggerà il vangelo e l'altro l'epistola, che tutti i giorni devono essere presenti alla Messa del $\mathrm{Re}$, la cui istituzione e ordinazione spetta al Capitolo con il consenso del vescovo; il preposito dovrà dare ad essi 15 ducati il giorno di Pasqua e 15 a san Michele e, nelle distribuzioni, a ciascuno di loro spetterà quanto un cappellano. Il preposito dovrà inoltre giurare di osservare gli statuti, sarà eletto dal vescovo e dovrà essere scelto fra i canonici del Capitolo. Essendo dunque vacante tale dignità, il vescovo elegge preposito Stanislao di Giovanni Sobniowski artium liberalium magister, canonico di Trento e suo protocancelliere, che prende possesso della carica.

Testimoni: domini Riprando da Cles, Baldessarre Thun, magister Giacomo phisicus da Castel Romano, domini preti Antonio a Dominabus da Trento arciprete nel Banale, Nicolò da Venezia pievano in Sanzeno, Nicolò da Trento arciprete in Tignale, Giovanni di Brunetto cantore da Treviso sacrista e cappellano in cattedrale, Bonadomano de Accerbis da Trento, Antonio notaio del fu ser Enrico de Castro, Iosio notaio del fu ser Francesco notaio di magister Iosio phisicus da Trento, Nicolò notaio del fu ser Pietro Balduino de Capris da Maderno, Bartolomeo notaio figlio di Giacomino da Torchio cittadini di Trento, Giovanni notaio da Bondo, Paolo notaio de Levi dalle Giudicarie.

Notaio: [Antonio del fu ser Bartolasio dal Borgonuovo di Trento, notaio pubblico per autorità imperiale].

Originale; ADTn, ACap, Instrumenta capitularia 8bis, cc. 47v-48r, n. 131 [A]. Nel margine sinistro, di mano del notaio rogatario del testo, la rubrica: Prepositura et unio facta per dominum nostrum de abbatia Tridentina etc ut infra. Sotto, della stessa mano, ma con inchiostro di tonalità più chiara, la nota relativa all'estrazione del mundum: Facta extra per manum Marchi Zinele et per me et Antonium notarium de Castro et Nicholaum de Capris subscriptum et ad curiam Romanam per dominum Stanslaum portatam etc.

Ed. parziale: Santifaller (a cura di), Urkunden und Forschungen, n. 392.

29.

1425 ottobre 11 , Trento

Il Comune di Trento ha un debito nei confronti del prestatore di denaro ebreo Ganzele pari a 80 ducati, chiesti per pagare un dono fatto ad Alessandro di 
Masovia, vescovo di Trento; numerose tassazioni furono imposte alla città, sia pro satisfaciendo stipendiariis transmissis, sia per espugnare e prendere il castello di Breguzzo dalle mani di Paride Lodron, ma anche per saldare il debito di 350 ducati nei confronti degli eredi di Erasmo Thun in seguito a una sentenza emanata dal vescovo per risarcire $i$ danni a Castel Stenico che era stato dato in mano a Negro de Negri da San Pietro dallo stesso Erasmo. Considerando che un'ulteriore tassazione avrebbe causato dei moti in città a causa del già pesante carico di tasse, considerando inoltre che il Comune di Trento possiede un terreno grezzo, spinoso e boschivo di circa 300 piovi, situato nelle vicinanze di Trento in località Lavis, vicino al fiume Avisio, e che lo stesso Comune non possiede più denaro per soddisfare detto prestatore, Vigilio cerdo dalla contrada di San Vigilio di Trento, in qualità di procuratore del Comune di Trento, investe Nicolò del fu Mercadento Mercadenti da Trento per la somma di 40 ducati che riceve a titolo di intratica di una parte del suddetto appezzamento, ossia di 60 piovi, per il canone di 12 lire e mezza di denari trentini a 8 grossi carentani per lira.

Testimoni: Giacomo notaio e stazonerius del fu ser Bertoldo a Ture da Trento, Antonio Abrianus oste in Trento del fu Benvenuto da Tierno, Aldrighetto del fu ser Giovanni Mezaoveta da Pomarolo, Domenico del dominus arcidiacono già speziale, magister Bernabono murarius del fu Alberto da Como, Giacomo murarius nipote di Bernabono, Marco detto Barozzo radarolo del fu Orso da Lizzana, tutti cittadini e abitanti di Trento, Perisonus del fu ser Francesco Pisoni da Riva del Garda di Trento, Lutterio del fu Bonaventura Zanzarelus da Sopramonte et alii pluribus.

Notaio: [Antonio del fu ser Bartolasio dal Borgonuovo di Trento, notaio pubblico per autorità imperiale].

Originale; ADTn, ACap, Instrumenta capitularia 8bis, cc. 48v-49r, n. 132 [A]. Nel margine sinistro, di mano del notaio rogatario del testo, la rubrica: Locatio perpetualis Nicholai de Merchadentis sibi factam per procuratorem Comunitatis de plodiis LX terreni buschivi et spinosi siti ad Avisium pro intratica XL ducatorum auri et affictum perpetuali XII 1/1 librarum denariorum Tridentinorum et cum pactis ut infra. Sotto, della stessa mano, la nota relativa all'estrazione del mundum: Facta extra conductori.

30.

\section{6 febbraio 28 , Trento}

〈Giovanni da Isny〉, decano del Capitolo, al tempo in cui era stato eletto vescovo di Trento, mancava del denaro necessario per farsi rilasciare dalla cancelleria apostolica le bolle papali per la conferma; ser Perozzo apothecarius del fu Angelo da Firenze cittadino di Trento, su richiesta dell'eletto e di molti canonici allora residenti e dei cittadini stessi, aveva destinato le sue lettere di cambio alla curia romana per avere 3.000 ducati ad extrahendum le suddette bolle. Ser Perozzo, in forza di un accordo arbitrale fatto da Alessandro vescovo di Trento e duca di Masovia, deve avere dal decano, dai canonici e dai cittadini fideiussori 360 ducati. Perozzo ha ceduto a Pietro di Nanni da Siena suo creditore tutti i diritti in merito. Il decano, i canonici e i cittadini pagano quanto dovuto a Pietro di ser Nanni.

Testimoni: domini preti Toma pievano a Thaur, diocesi di Bressanone, Nicolò da Trento pievano in Tignale e cappellano nella cattedrale, Vigilio Turcheti da Trento 
Appendice

vicecappellano in cattedrale, Corrado de Alemania pievano in Civezzano e vicario in Cembra et alii plures.

Canonici presenti: domini Giovanni da Isny bakalarius in decretis, decano del Capitolo, Giovanni de Cavalis da Venezia, Giovanni dalla Carinzia, Corrado da Trento, Giovanni Anhang e Giovanni Zeiss da Bopfingen, Goffredo Friling da Bressanone, Isidoro da Milano.

Notaio: [Antonio del fu ser Bartolasio dal Borgonuovo di Trento, notaio pubblico per autorità imperiale].

Originale; ADTn, ACap, Instrumenta capitularia 8bis, c. 60v, n. 164 [A]. In corrispondenza del margine sinistro, di mano del notaio rogatario del testo, la rubrica: Finis facta per ser Petrum de Senis dominis canonicis et civibus infrascriptis etc ut infra.

31.

1426 luglio 3 , Trento

Felicia, figlia del fu Francesco notaio da Trento e moglie del fu Giovanni Belenzani, ser Michele del fu ser Huele a Plata ed Erasmo del fu ser Vigilio Thun, per sé e a nome di suo fratello Guglielmo, in quanto eredi del fu Giovanni, al fine di poter pagare i funerali e le spese fatte per seppellire il suddetto Giovanni Belenzani, vendono a Michele figlio di ser Domenico da Cortesano un affitto di 10 lire trentine che lo stesso Michele soleva pagare annualmente al fu Giovanni per una casa, terreni e possessi situati a Cortesano al prezzo di 36 ducati.

Testimoni: ser Bonadomano del fu magister Nicolò phisicus de Accerbis da Trento; Iosio notaio del fu ser Francesco notaio di magister Iosio phisicus da Trento, Antonio notaio del fu ser Bonifacio da Nogaredo, magister Pietro a caminis stazonerius del fu Giovanni dal Trevigiano, tutti cittadini e abitanti di Trento, Simeone del fu Tommasino da Vervò et alii plures.

Notaio: [Antonio del fu ser Bartolasio dal Borgonuovo di Trento, notaio pubblico per autorità imperiale].

Originale; ADTn, ACap, Instrumenta capitularia 8bis, c. 68r, n. 190 [A]. In corrispondenza del margine superiore, a sinistra, di mano del notaio rogatario del testo, la nota relativa all'estrazione del mundum: Facta extra in publicam formam. Sotto, della stessa mano, la rubrica: Emptio Michaelis filii ser Dominici de Cortesano de uno affictu perpetuali decem librarum denariorum Tridentinorum ab heredibus condam Iohannis de Bellenzanis pro precio XXXVI ducatorum auri.

32.

1426 ottobre 16 , Trento

Adelperio del fu ser Marco Calepini da Trento presta a Morandino figlio del magister Giovanni Bono da Trento, giurisperito e studente in diritto civile, che gliene aveva fatto richiesta, alcuni libri legali, fra cui un bel Digestum Novum e un Digestum Vetus, i Tres Libri, le Institutiones e le Decretali; Morandino promette inoltre ad Adelperio di conservare al meglio i suddetti libri che vengono stimati di comune accordo 125 ducati. 
Appendice

Testimoni: ser Pietro Iacob, ser Gianpietro da Feltre, Bartolomeo del dominus Sicco, magister Tomeo fisicus dal Tesino, Adelpreto da Povo, Cristoforo Capuzio, magister Pietro a caminis, tutti cittadini e abitanti di Trento, Iosio notaio de Iosiis cittadino di Trento pro secundo notario et alii plures.

Notai: [Antonio del fu ser Bartolasio dal Borgonuovo di Trento, notaio pubblico per autorità imperiale] (notaio redattore del documento); Iosio di Iosio cittadino di Trento (pro secundo notario).

Originale; ADTn, ACap, Instrumenta capitularia 8bis, c. 71v, n. 203 [A]. In corrispondenza del margine sinistro, di mano del notaio rogatario del testo, la rubrica: Carta accomodati facta domino Morandino de infrascriptis libris per Adelperium de Calapinis etc, che risulta depennata; sotto, della stessa mano, la nota relativa all'estrazione del mundum: Facta extra ut subscribatur a Iosio.

33.

[1425] ottobre 17, Trento

Matteo del fu ser Clemente de Murlinis da Trento, uno fra i consoli e provvisori di Trento, acconsente e ratifica la locazione (doc. 24).

Testimoni: Martino notaio del fu Francesco notaio da Volano, Giacomo Fanzini notaio del fu ser Bertoldo a Ture da Trento, Marco notaio domine Zinele figlio di Valentino dal Borgonuovo, Adelperio e Odorico Calepini, Guglielmo notaio Saraceno, tutti cittadini e abitanti di Trento et alii plures.

Notaio: [Antonio del fu ser Bartolasio dal Borgonuovo di Trento, notaio pubblico per autorità imperiale].

Originale; ADTn, ACap, Instrumenta capitularia 8bis, c. 49r, n. 132b [A]. Nel margine sinistro, di mano del notaio rogatario del testo, la rubrica: Consensus et ratificatio facta per Matheum de Murlinis.

34.

1427 giugno 11 , ottobre 7, Trento

Goffredo, in iure canonico peritus, canonico trentino, e Antonio de Zevolis da Ledro legum doctor, emanano la sentenza della causa che intercorre fra Alessandro di Masovia (ovvero Nicolò Saur beneficiato nella cattedrale e Gioacchino Mezzasoma da Trento, procuratori del vescovo) e i nobili Michele a Plata da Cortaccia ed Erasmo del fu Vigilio Thun, presente e agente per sé e per il fratello Guglielmo, assente, in merito all'eredità del fu Giovanni Belenzani da Trento: i beni del fu Antonio Belenzani saranno devoluti ai pauperes Christi, ossia al vescovo di Trento; condannano Michele a Plata, Erasmo e Guglielmo fratelli ed eredi del fu Giovanni Belenzani a restituire al vescovo Alessandro due terzi di tutti i beni che il fu Giovanni possedette e dell'eredità del fu Antonio Belenzani; potranno invece trattenere la terza parte dei beni cui il suddetto fu Giovanni pervenne de iure e di cui legittimamente poté disporre durante la sua vita; condannano altresì i suddetti Michele, Erasmo e Guglielmo al risarcimento di tutti quei beni e diritti che il fu Giovanni 


\section{Appendice}

in vita vendette e alienò contro le disposizioni del testamento del padre Antonio; tutti i beni mobili che furono depredati al fu Giovanni al tempo del saccheggio non devono invece essere conteggiati e risarciti, né devono essere conteggiati i legati ad pias causas lasciati dal fu Giovanni nel suo testamento; stabiliscono inoltre che il vescovo Alessandro sia tenuto per le altre due parti di eredità al pagamento e all'esecuzione dei legati ad pias causas rimanenti. Tutti gli altri redditi dell'eredità del fu Antonio goduti da Giovanni durante la sua vita, sia i beni tenuti in enfiteusi sia quelli derivanti dai frutti dell'eredità del padre, non possono in alcun modo essere computati; inoltre stabiliscono che i 100 ducati che Antonio lasciò in eredità al fu Giovanni debbano essere pagati e sottratti dalla somma totale dell'eredità; qualora, inoltre, venga trovato l'instrumentum dotale di Giacoma, già moglie del fu Antonio, questa dote dovrà essere sottratta dal totale dell'eredità e dovrà essere data agli eredi del fu Giovanni, figlio legittimo della suddetta Giacoma.

Testimoni: dominus Odorico monachus nella Prepositura, Giovanni del fu Giovanni de Alemania capitano alla porta del ponte sull'Adige, Giovanni figlio del fu ser Antonio notaio de Piperatis da Trento, Pederzolo e Andrea familiares del vicario vescovile et alii.

Notaio: Antonio del fu ser Giovanni da Fai, notaio pubblico per autorità apostolica e imperiale.

Copia semplice; ADTn, ACap, Instrumenta capitularia 8bis, cc. 146r-147r, n. 404 [B]. In corrispondenza del margine sinistro, di mano del notaio rogatario del testo, la rubrica: Copia sententie late inter dominum nostrum dominum Alexandrum etc et heredes condam Iohannis de Belenzanis de bonis et hereditate condam Antonii de Belenzanis olim patris dicti Iohannis vigore test(ament)i per ipsum Antonium facti ad pauperes Christi etc.

35.

1427 luglio 16, 21 e agosto 2, Trento

(a) [luglio 16] Vigilio cerdo da San Vigilio, in qualità di procuratore del Comune di Trento, aveva investito Nicolò Mercadenti del fu Mercadento Mercadenti da Trento di un terreno spinoso, sterile e boschivo di 60 piovi per un certo affitto perpetuo, ottenendo 40 ducati come intratica che servivano al Comune per pagare Ganzele, ebreo prestatore di denaro in Trento, creditore del Comune per molti ducati; non essendo detto Nicolò stato in grado di riportare allo stato di prato detto terreno, chiese di riavere i 40 ducati che aveva versato; pertanto i consoli e i procuratori del Comune diedero a Nicolò la licenza di vendere l'utile dominio del terreno di 60 piovi. Michele Fenutoli oste a Trento e Federico Polentone da borgo San Martino in qualità di procuratori del Comune, su licenza dei consoli e provvisori del Comune, investono quindi Adelperio del fu ser Marco Calepini da Trento del terreno spinoso, boschivo, grezzo e sterile di 20 piovi situato nelle vicinanze di Trento, a Lavis, con l'impegno che questi versi l'ammontare dell'intratica dovuta al Comune, ossia 20 ducati, al suddetto prestatore di denaro Ganzele, e versi ai procuratori del Comune a titolo di canone annuo 10 lire trentine.

(b) [luglio 21] Antonio da Molveno, uno fra i consoli e decurioni sopra mezionati, approva il contratto di locazione perpetua.

(c) [agosto 2] Ser Francesco Leonardo da Mattarello acconsente. 
Appendice

Testimoni alla locazione (16 luglio): ser Francesco del fu ser Adelperio de Sichis da Trento, Cristoforo Capuzio del fu ser Tommaso Capuzio da Como, Aldrighetto del fu Giovanni Mezaoveta da Pomarolo, Antonio Abrianus oste dell'osteria al Falcone del fu Benvenuto da Tierno, Giacomo da Gardumo stazonerius in Trento e Antonio notaio del fu ser Enrico de Castro, tutti cittadini e abitanti di Trento et alii plures.

Testimoni alla ratifica (21 luglio): Maffeo del fu Negro da Brescia apothecarius in Trento, Marco domine Zinele notaio e buletarius in Trento, entrambi cittadini di Trento, et alii.

Testimoni alla ratifica (2 agosto): Francesco del fu ser Adelperio de Sichis, Giovanni Luca Fanzini, entrambi cittadini di Trento, et alii.

Notai: [Antonio del fu ser Bartolasio dal Borgonuovo di Trento, notaio pubblico per autorità imperiale] (notaio redattore del documento); Antonio notaio del fu ser Enrico de Castro (rogatus una mecum ad scribendum hoc instrumentum).

Originale, ADTn, ACap, Instrumenta capitularia 8bis, cc. 89v-90r, nn. 253a-b-c [A]. Nel margine sinistro, di mano del notaio rogatario del testo, la rubrica: Locatio perpetualis Adelperii de Calapinis sibi facta uper> procuratores et consules civitatis Tridenti de quadam pecia terre sterili et spinosa viginti plodiorum terreni pro affictu perpetuali decem librarum denariorum Tridentinorum, preceduta dalla nota della stessa mano: Relevata ex quodam foleo.

36.

1427 luglio 22, Trento

Giovanni domine Alde figlio del fu ser Tommaso da Barbarano contrae matrimonio con Beatrice figlia del fu ***, che conviveva con lui, e che gli versa 200 lire di denari trentini a titolo di dote.

Testimoni: magister Lorenzo a Vaginis del fu magister Giovanni da Feltre, magister Zeschele cerdo de fossato cerdonum da Trento del fu Federico da Rovereto in Vallagarina, magister Pietro murarius a caminis stazonerius da Trento del fu Giovanni dal Trevigiano, cittadini e abitanti a Trento, Simeone da Ravina del fu Federico da Terragnolo in Vallagarina, Odorico del fu Bartolomeo Gatabriga da Vigolo Vattaro.

Notaio: [Antonio del fu ser Bartolasio dal Borgonuovo di Trento, notaio pubblico per autorità imperiale].

Originale; ADTn, ACap, Instrumenta capitularia 8bis, c. 88v, n. 250 [A]. In corrispondenza del margine sinistro, di mano del notaio rogatario del testo, la rubrica: Matrimonium cum dote II $^{c}$ libris denariorum Tridentinorum contractum inter Iohannem domine Alde et Beatricem suam concubinam etc. Sotto, della stessa mano, la nota relativa all'estrazione del mundum: Facta extra ipsi domine.

Edizione: Berlanda, Il matrimonio nel tardo medioevo, n. 15. 
Appendice

37.

1428 gennaio 28 , Trento

Rigo da Francoforte, oste dell'osteria alla Corona a Trento, riceve da ser Giovanni Layner del fu Ulrico da Termeno 100 marche di buona moneta di Merano a titolo di dote per la figlia Clara, moglie di detto Rigo.

Testimoni: Osvald Gerart, Achazius Margarayder, Ulrich Truus, Bartolomeo lanaiolo, Ulrich Sbarzpniger, Giacomo notaio da Egna (Novo Foro), Giovannino del fu Giacomo Bruno a Porta da Trento, Stasio apothecarius figlio di ser Andrea apothecarius da Firenze, magister Pietro sarto del fu Ambrogio da Milano, Ianes capitano della Torre Vanga e della porta di Ponte Adige in Trento, gli ultimi quattro cittadini e abitanti di Trento, et alii plures.

Notaio: [Antonio del fu ser Bartolasio dal Borgonuovo di Trento, notaio pubblico per autorità imperiale].

Originale; ADTn, ACap, Instrumenta capitularia 8bis, c. 100r, n. 276 [A]. In corrispondenza del margine sinistro, di mano del notaio rogatario del testo, la rubrica: Dos domine Clare filie ser Iohannis Layner de Tremeno et uxoris Rigi de Franchfort hosterii a Corona in Tridento de centum marchis denariorum bone monete de Merano etc. Sotto, della stessa mano, la nota relativa all'estrazione del mundum: Facta extra de 1436 de mensis ianuario et data dicto ser Iohanni Layner. In calce al testo la seguente nota del notaio rogatario: Et nota tamen ad memoriam quod non debeo dare dictum instrumentum extra dicto ser Iohanni Layner [si]ne licentia dicti Rigi et quod ipse ser Iohannes Layner non dedit nisi sexaginta marchas et ipse Rigus de suo proprio donavit quadraginta.

38.

1428 giugno 28 , Trento

Battista da Bologna, Giovanni domine Alde, magister Michele piliparius e Antonio di ser Bartolasio notaio, in qualità di consoli della città, insieme a Nicolò Mercadenti come procuratore della città stessa, nominano Antonio da Molveno, ser Odorico detto Stratenperger e ser Francesco del fu ser Adelperio de Sichis ambasciatori, sindaci e procuratori per comparire dinanzi al consiglio generale a Bolzano per ascoltare e udire quanto verrà deciso per parte dell'imperatore e dei duchi d'Austria in merito alle tasse da imporre per debellare l'eresia ussita.

Testimoni: Giovanni figlio del magister Bartolomeo da Lucca, Meo del fu Giovanni da Pistoia, familiares del vicario, Baldessarre detto Bruschino cerdo da Vigolo Vattaro abitante di Trento et alii.

Notaio: [Antonio del fu ser Bartolasio dal Borgonuovo di Trento, notaio pubblico per autorità imperiale].

Originale; ADTn, ACap, Instrumenta capitularia 8bis, c. 106r-v, n. 294 [A]. Nel margine sinistro, di mano del notaio rogatario del testo, la rubrica: Sindicatus comunitatis Tridenti in dominos Antonium de Molveno, Franciscum de Sichis et Stratenperger ad comparendum ad conscilium in Bulzano fiendum contra Usos «sic〉 etc, preceduta dalla nota, della stessa mano, relativa all'estrazione del mundum: Facta extra statim etc. 
Appendice

39.

1428 settembre 4 , Trento

Pietro Iacob, Antonio di ser Bartolasio notaio, Antonio de Castro notaio e Battista da Bologna, eletti da Antonio de Zivollis da Ledro, già vicario di Trento, e incaricati di dividere $i$ beni e l'eredità del fu Giovanni figlio del fu ser Antonio Belenzani da Trento fra il vescovo, da una parte, e Michele del fu Huele a Plata da Cortaccia, Erasmo e Guglielmo, fratelli e figli del fu Vigilio Thun, come eredi del fu detto Giovanni, dall'altra, secondo la forma della sentenza emanata da Goffredo canonico e vicario in spiritualibus e da Antonio de Zivollis già vicario in temporalibus, assegnano agli eredi del fu Giovanni Belenzani tutti i beni che il fu Giovanni teneva in enfiteusi dalla Chiesa trentina e da altri enti; assegnano inoltre agli eredi del fu Giovanni tutti i beni e gli affitti che egli acquisì in vita; assegnano agli eredi del fu Giovanni la casa di abitazione con tutte le altre case circostanti che egli possedeva; assegnano alla domina Felicia, vedova del fu Giovanni Belenzani, i beni, gli affitti che il defunto le assegnò in testamento; le assegnano inoltre tutti i beni mobili e i suppellettili del fu Giovanni. Tutti gli altri beni immobili, i diritti, i redditi e gli affitti dell'eredità del fu Antonio Belenzani vengono assegnati al vescovo in locum pauperum Christi.

Testimoni: Leonardo figlio di ser Perozzo apothecarius da Firenze cittadino di Trento, Marco da Lusiana e Nicolò Rizato cittadini di Trento et alii.

Notaio: Antonio del fu ser Giovanni da Fai, notaio pubblico per autorità apostolica e imperiale.

Copia semplice; ADTn, ACap, Instrumenta capitularia 8bis, cc. 147v-148r, n. 405 [B]. In corrispondenza del margine sinistro della c. $157 v$, di mano del notaio rogatario del testo, la rubrica: Copia sententie divisionis sive assignationis de bonis et hereditate condam Antonii de Belenzanis facte per infrascriptos ellectos domino nostro domino episcopo Tridentino et heredibus condam Iohannis de Belenzanis et eius uxori domine Felici etc.

40.

1429 luglio 25, Trento

Domenica, vedova del fu magister Bonomo da Arco, vende ad Antonio detto Prevedonus figlio del fu magister Giacomo a rotis da Arco, fratello del fu magister Bonomo, un terreno vignato situato nelle pertinenze di Trento, a Mesiano, nel luogo detto a Gozador, al prezzo di 53 ducati.

Testimoni: Adelperio e Bonaventura fratelli e figli del fu ser Marco Calepini, Giorgio del fu Guglielmo del fu ser Iorio notaio da Vigolo Vattaro, Bartolomeo cerdo del fu magister [...] di Tommaso cerdo de fossato cerdonum di Trento, Baldessarre laborator dal Borgonuovo del fu Guglielmo de Mura da Piné, tutti cittadini e abitanti di Trento et alii.

Notaio: [Antonio del fu ser Bartolasio dal Borgonuovo di Trento, notaio pubblico per autorità imperiale]. 


\section{Appendice}

Originale; ADTn, ACap, Instrumenta capitularia 8bis, c. 132r-v, n. 354 [A]. In corrispondenza del margine sinistro, di mano del notaio rogatario del testo, la rubrica: Emptio Antonii dicti Prevedoni de uno vineali a domina Dominica pro precio quinquaginta trium ducatorum auri, preceduto da una nota, della stessa mano, relativa all'estrazione del mundum: Facta extra ipsi emptori.

41.

[1429 luglio 25, Trento]

Antonio Prevedonus promette ad Adelperio Calepini di versargli entro i termini previsti 53 ducati, poiché Domenica, vedova del fu magister Bonomo da Arco, in qualità di madre ed erede di Antonio da Arco, era tenuta a pagare al suddetto Adelperio 53 ducati per restituirgli l'aiuto che ella aveva ricevuto; per tale motivo aveva venduto, su consiglio dello stesso Adelperio, un terreno vignato (doc. 35) in modo che Antonio si costituisse principale debitore nei confronti di Adelperio.

Testimoni: Giorgio [del fu Guglielmo del fu ser Iorio notaio da Vigolo Vattaro], Bartolomeo cerdo e Baldessarre [laborator dal Borgonuovo del fu Guglielmo de Mura da Piné].

Notaio: [Antonio del fu ser Bartolasio dal Borgonuovo di Trento, notaio pubblico per autorità imperiale].

Originale; ADTn, ACap, Instrumenta capitularia 8bis, c. $132 v$, n. 355 [A]. In corrispondenza del margine sinistro, di mano del notaio rogatario del testo, la rubrica: Creditum dicti Adelperii de Calapinis contra dictum Antonium Prevedonum de dictis quinquaginta tribus ducatis [...] quos principaliter constituit ad solvedum pro ea domina Dominica que ipsa tamquam mater et heres condam domini Antonii legum doctoris de Angulo tenebatur ex causa mutui facti ipsi domino Antonio per ip [su]m Adelperium ut ipsa domina Dominica ibidem asseruit etc.

42.

[1430]

Antonio da Borgonuovo notaio attesta che Adelperio del fu ser Marco Calepini suo genero, insieme al fratello Bonaventura, pure suo genero, hanno ricevuto dal notaio stesso 400 ducati come dote per la figlia Maddalena, moglie di detto Bonaventura; Adelperio, insieme a Bonaventura, hanno inoltre promesso di restituire la suddetta dote nei casi previsti. Attesta inoltre che Adelperio aveva venduto al notaio metà pro indiviso di un affitto perpetuo di 24 staia di cereali, ossia 12 di frumento e 12 di siligine, che veniva pagato da Antonio Sumptag e Federico, fratelli e figli del fu Sumptag da Gardolo di Mezzo per un manso di 20 piovi.

Notaio: [Antonio del fu ser Bartolasio dal Borgonuovo di Trento, notaio pubblico per autorità imperiale].

Scrittura semplice; ADTn, ACap, Instrumenta capitularia 8bis, c. $145 v$, n. 380. In corrispondenza del margine sinistro, di mano del notaio rogatario del testo, la rubrica: Carta sive littera traditionis facte per me Antonium notarium ser Bartholasii Adelperio de Calapinis generi meo de rebus infrascriptis ex causis infrascriptis etc. 
Appendice

43.

1430 giugno 23, Trento

Antonio del fu Giovanni Belenzani da Trento aveva nominato eredi universali i figli legittimi Guglielmo e Giovanni, stabilendo altresì che se costoro fossero morti senza figli, eredi sarebbero stati i pauperes Christi; i beni del fu Antonio pervennero ai pauperes Christi ovvero al vescovo Alessandro di Masovia, in qualità di pater pauperum e legittimo amministratore e difensore dei poveri. Essendo sorta una lite fra il vescovo e il nobile Michele a Plata da Cortaccia e i fratelli Erasmo e Guglielmo, figli del fu Vigilio Thun, in quanto eredi del fu Giovanni Belenzani, per un'altra parte dei detti beni del fu Antonio Belenzani, fu emessa una sentenza secondo la quale due terzi dei beni del fu Antonio dovevano andare ai pauperes Christi ossia al vescovo in qualità di loro rappresentante. In seguito furono eletti dei boni homines per dividere i beni ereditari in tre parti. Dopo ciò, il vescovo, volendo distribuire detti beni fra i pauperes più agevolmente, vende a ser Pietro di Nanni da Siena, cittadino di Trento e abitante a Verona, assente, e a ser Pietro Iacob procuratore di detto Pietro, presente, gli affitti, i redditi e tutti i beni ereditati dal vescovo per i pauperes, e ciò al prezzo di 1.200 ducati.

Testimoni: domini Isidoro da Milano canonico di Trento, prete Federico Halr cancelliere presso il castello [del Buonconsiglio], Giovanni de Alemania cappellano nella cattedrale, notaio e scriba presso il castello [del Buonconsiglio].

Notaio: [Antonio del fu ser Bartolasio dal Borgonuovo, notaio pubblico per autorità imperiale].

Originale; ADTn, ACap, Instrumenta capitularia 8bis, c. 151v, n. 395 [A]. In corrispondenza del margine sinistro, di mano del notaio rogatario del testo, la rubrica: Emptio ser Petri Nani de Senis a domino nostro executore ut infra de infrascriptis rebus et bonis pro precio mille et ducentorum «segue decentorum iterato〉 auri etc ut hic in folio instrumento notatum est postea distinuenda quando res vendite michi erunt date etc; sotto, della stessa mano, la nota relativa all'estrazione del mundum: Facta extra domino nostro de comissione et mandato mihi factis per dominum Antonium de Tosabech de Papia vicarium Tridentinum ex parte domini nostri, ex relatione sibi facta per dominum Ysidorum etc ut mihi retulit. In calce al documento, la nota della stessa mano: Et nota ad memoriam quod ego rogatus notavi instrumentum procure facte per suprascriptum de Senis in personam dicti ser Petri Iacob ad emendum dicta bona a domino nostro super prothocollo die mercuri vigesimo primo suprascripti mensis iunii 1430 etc. In corrispondenza del margine sinistro della c. $152 v$ il notaio rogatario annota: Et nota quod istos tres ultimos affictus non posui in instrumento quia non fuerunt venditi sed tamen confessati quia ser Petrus Iacob dixit mihi quod facta sint (ideo) de venditis non conputatis ipsis tribus in ea ratione. Et dimisi bene spacium possendi scribere in eo si michi mandabitur per dominum.

44.

1430 settembre 9, Trento

Ser Pietro di Nanni da Siena, approvando e ratificando la compravendita fatta da ser Pietro Iacob, suo procuratore, prende possesso tanto della casa edificata in muratura e legno situata nella contrada di San Benedetto quanto di tutte le altre proprietà acquisite. 
Appendice

Testimoni: dominus Isidoro, ser Gianpietro del fu ser Corradino da Feltre, Antonio notaio del fu ser Enrico de Castro da Trento, Rigo suo figlio, Odorico del fu ser Federico notaio da Povo, tutti cittadini e abitanti di Trento, et alii.

Notaio: [Antonio del fu ser Bartolasio dal Borgonuovo, notaio pubblico per autorità imperiale].

Originale; ADTn, ACap, Instrumenta capitularia 8bis, c. 152r-v, n. 395b [A]. In corrispondenza del margine sinistro, di mano del notaio rogatario del testo, la rubrica: Intratio possessionis facta per ipsummet ser Petrum Nani.

45.

1430 settembre 9 , Trento

Ser Pietro di Nanni da Siena, cittadino di Trento e cittadino e abitante a Verona, nomina ser Pietro Iacob del fu ser Rigo da Rovereto proprio procuratore per vendere, alienare e dare in affitto tutti i beni, le terre e gli affitti che egli aveva acquistato da Alessandro di Masovia, il quale li aveva ricevuti come eredità del fu Giovanni del fu Antonio Belenzani da Trento in quanto pater pauperum ed esecutore del testamento del fu detto Antonio.

Testimoni: dominus Isidoro da Milano canonico di Trento, ser Gianpietro del fu ser Corradino da Feltre, Odorico del fu ser Federico notaio da Povo, Antonio notaio del fu ser Enrico de Castro, Rigo suo figlio, tutti cittadini e abitanti di Trento et alii.

Notaio: [Antonio del fu ser Bartolasio dal Borgonuovo, notaio pubblico per autorità imperiale].

Originale; ADTn, ACap, Instrumenta capitularia 8bis, c. 171r, n. 446 [A]. In corrispondenza del margine sinistro, di mano del notaio rogatario, la rubrica: Procuratorium factum per ser Petrum Nani de Senis in ser Petrum Iacob ad vendendum omnia bona sibi vendita per dominum nostrum de bonis condam Iohannis de Belenzanis; il documento è preceduto dalla nota, della stessa mano, Relevata ex uno folio super quo notata fuit vendicio sibi ser Petro Nani facta per dominum nostrum.

46.

1431 febbraio 6, Trento

Ser Pietro di Nanni da Siena dichiara di aver ricevuto da ser Bonadomano del fu magister Nicolò phisicus de Accerbis da Trento, in qualità di procuratore del nobile Giacomino de Federicis de Arbano in val Camonica, diocesi di Brescia, suocero del suddetto ser Bonadomano, 875 ducati che detto Giacomino era tenuto a restituire a ser Pietro in virtù di un prestito da lui ricevuto per recuperare Castel Campo nelle Giudicarie.

Testimoni: dominus Isidiro da Milano canonico di Trento, Odorico e Bonaventura, fratelli e figli del fu ser Marco Calepini, Rodolfo di ser Gianpietro da Feltre, Giovanni del fu Antonio Bommartino da Trento, tutti cittadini e abitanti di Trento, et alii. 
Appendice

Notaio: [Antonio del fu ser Bartolasio dal Borgonuovo, notaio pubblico per autorità imperiale].

Originale; ADTn, ACap, Instrumenta capitularia 8bis, c. 175r, n. 458 [A]. In corrispondenza del margine sinistro, di mano del notaio rogatario del testo, la rubrica: Confessio solutionis facte per ser Petro Nani de Senis de ducatis octigentis et septuaginta quinque per dominum Iacobinum de Fedricis et finis et absolutionis hinc inde generaliter facte de omnibus qui sibi invicem petere possent usque ad presentem diem etc ut infra.

47.

1433 luglio 9, Trento

Dinanzi a Giacomo de Ursulinis da Tossignano di Bologna vicario, Bartolomeo del fu Sicco da Trento dona ad Adelperio del fu ser Marco Calepini da Trento una serie di proprietà sottoposte ad affitto perpetuo e non, con il patto che il donatore potrà godere di tutti i beni donati, con l'eccezione della casa con cantina. Inoltre Adelperio sarà tenuto a versare a Elena e Francesca, figlie di Bartolomeo, 100 ducati, 50 a ciascuna, quando esse si sposeranno, e a Giovanni Sich e a Maria sua nipote 100 ducati, sempre 50 ciascuno, da versare al primo quando raggiungerà la maggiore età e alla seconda all'atto del matrimonio.

Testimoni: dominus Antonio de Tosabech da Pavia decretorum doctor, arcidiacono e canonico tridentino, Melchiorre del fu ser Domenico ab Oleo da Trento, Benedetto del fu ser Paolo Peurade da Trento, Pietro stazonerius in Trento del fu Paolino da Arco, Odorico del fu Giacomo da Amblar in val di Non e Graziadeo notaio, cittadini e abitanti di Trento, et alii.

Notai: [Antonio del fu ser Bartolasio dal Borgonuovo di Trento, notaio pubblico per autorità imperiale] (notaio redattore del documento); Graziadeo [da Terlago] notaio pubblico per autorità imperiale (rogatus una mecum ad scribendum et se subscribendum huic instrumento).

Originale; ADTn, ACap, Instrumenta capitularia 8bis, cc. $218 v-219 r$, n. 579 [A]. In corrispondenza del margine sinistro, di mano del notaio rogatario del testo, la rubrica: Donatio Adelperii de Calapinis sibi facta per Bartholomeum domini Siconis de infrascriptis bonis et cum pactis infrascriptis; sotto, della stessa mano, la nota relativa all'estrazione del mundum: Facta extra dicto Adelperio cum subscriptione Gratiadei; il notaio appone anche il suo signum e la sua sottoscrizione.

Originale; ASTn, APV, Sezione latina, capsa 3, n. 52 [A].

48.

[1434 febbraio 24, Trento]

Adelpreto da Povo dichiara di ricevere dalla moglie Antonia, figlia del fu ser Pietro Iacob stazonerius in Trento, 127 ducati come aumento della dote, ricavati dalla vendita di una serie di beni.

Testimoni: [Matteo del fu ser Clemente de Murlinis da Trento, Cristoforo figlio di Matteo, Odorico del fu ser Federico notaio da Povo, Desiderato lanarolus del fu 
Appendice

Francesco da Magré dal Vicentino cittadini e abitanti di Trento], ser Pietro Iacob et alii.

Notaio: [Antonio del fu ser Bartolasio dal Borgonuovo di Trento, notaio pubblico per autorità imperiale].

Originale; ADTn, ACap, Instrumenta capitularia 8bis, cc. 227v-228r, n. 598a [A]. In corrispondenza del margine sinistro, di mano del notaio rogatario, la rubrica: Augmentum dotis dicte domine Antonie de centum et XXVII «-V- corretto su precedente -X-〉 ducatis auri perventis ex precio infrascriptarum rerum etc ultra dotes suas de IIIC ducatis constitutis.

49.

[1434 febbraio 24, Trento]

Matteo de Murlinis, alla presenza del figlio Cristoforo e della moglie Bartolomea, figlia del fu ser Iacob speziale in Trento, dichiara di aver ricevuto in occasione del contratto di matrimonio da ser Pietro Iacob 300 ducati come dote per la nipote.

Testimoni: Odorico e Adelpreto fratelli da Povo, Desiderato lanarolus, cittadini di Trento, Leonardo del fu Endrico da Roncogno di Pergine, Nicolino del fu Morzanto da Sopramonte.

Notaio: [Antonio del fu ser Bartolasio dal Borgonuovo di Trento, notaio pubblico per autorità imperiale].

Originale; ADTn, ACap, Instrumenta capitularia 8bis, c. 228r, n. 598b [A]. In corrispondenza del margine sinistro, di mano del notaio rogatario, la rubrica: Carta dotis domine Bartholomee filie condam dicti ser Iacob et uxoris Mathei de Murlinis contra ipsum Matheum de trecentis ducatis auri.

50.

1434 marzo 14 , Trento

Bonadomano de Accerbis, Marco Belenzani, Battista da Bologna, Melchiorre $a b$ Oleo, Matteo de Murlinis, Cristoforo Capuzio, Rambaldo de Murlinis e Giovanni Rauter, consoli e provisores del Comune di Trento, insieme con i più saggi fra $\mathrm{i}$ cittadini della città, nominano Luca iurisperitus figlio di magister Giovanni di Luca e Adelperio Calepini propri procuratori per presentarsi dinanzi al vescovo trentino a Basilea, dinanzi al sacro concilio di Basilea, all'imperatore e a qualunque altro delegato o subdelegato, per esporre le volontà del Comune e per chiedere il rilascio di grazie, lettere, privilegi e immunità utili al Comune.

Testimoni: Trentino di Nicolò Chechi sindico da Meano, Baldino di ser Andrea a Poza da Povo sindico da Povo, Bartolomeo Carnessarii sindico da Vigolo, Michele de Orzano sindico da Civezzano, Simeone di Guglielmo sindico da Vattaro, Domenico Fraschete sindico da Cadine, Giovannino Rizius sindico da Calavino.

Honorabiliores de savioribus populi: magister Giovanni di Luca, Michael a Muta, Giacomo da Arco, Gianpietro da Feltre, Adelperio Calepini, dominus Giovanni da Comano, Antonio notaio de Castro, dominus Luca giurisperito, Francesco 
Appendice

di Adelperio de Sichis, ser Antonio notaio di ser Bartolasio, Leonardo notaio da Piné, Cristoforo speziale a Libeceltis, Nicolò Mercadenti, Giacomo Fanzini, Odorico a fecibus, Pietro notaio da Spormaggiore, Franceschino Prandi de Luero, Antonio speziale da Feltre, Maffeo da Brescia, Odorico Calepini, Odorico Stratenperger, Vricius da Rovereto, Adelpreto da Povo, Lorenzo Cazuff, Guglielmo Gallo, Bartolomeo da Arco, Giovanni Zilie, magister Antonio parolarius, Palamidesio de Palamidesiis, Vigilio speziale Gardeline, Biagio da Telve, Stasio apothecarius, Tomeo Mezaoveta, Midana a caliginis, magister Zeno murarius, magister Zeschele cerdo, Giacomo Passera, Leonardo da Gardolo, Antonio ab ancha stazonerius, Michele Fenutoli, Odorico di Nicolò Zilie, Giovanni Pecini, Martino murarius, Domenico Facini, Sandrino cerdo, Vigilio notaio da Seregnano, Nicolò barberius.

Notaio: [Antonio del fu ser Bartolasio dal Borgonuovo di Trento, notaio pubblico per autorità imperiale].

Originale; ADTn, ACap, Instrumenta capitularia 8bis, c. 230v, n. 604a [A]; in corrispondenza del margine sinistro, di mano del notaio rogatario, la rubrica: Sindicatus factus per cives Tridenti in dominum Lucam et Adelperium de Calapinis ad eundum ad conscilium Basilee pro tota comunitate Tridenti.

51.

[1434 marzo 14, Trento]

I consoli e i provisores, insieme con i procuratori del Comune, a nome del Comune, promettono ai suddetti sindaci e procuratori (cfr. doc. n. 50) di mantenerli in sicurezza da ogni pericolo e ostacolo in cui potrebbero incappare mentre stanno trattando i negoziati per l'utilità del Comune, in qualunque luogo: mentre vanno a Basilea, mentre sono colà presenti e mentre fanno ritorno; promettono inoltre di difenderli anche nel caso al loro arrivo fossero angariati dal vescovo.

Testimoni: come sopra (n. 50).

Notaio: [Antonio del fu ser Bartolasio dal Borgonuovo di Trento, notaio pubblico per autorità imperiale]

Originale; ADTn, ACap, Instrumenta capitularia 8bis, cc. 230v-231r, n. 604b [A]; in corrispondenza del margine sinistro, di mano del notaio rogatario, la rubrica: Promissio facta dictis sindicis de conservando eos indempnes etc. 


\section{Abbreviazioni}

ADTn Archivio diocesano tridentino, Trento

ACap Archivio del Capitolo della cattedrale

AP Archivio della Prepositura

APTn Archivio provinciale, Trento

ASCTn Archivio storico del Comune, Trento

ASTn Archivio di Stato, Trento

ACD Archivio del Capitolo del Duomo

APV Archivio del Principato Vescovile

ASVr Archivio di Stato, Verona

BCB Biblioteca Civica Bertoliana, Vicenza

BCTn Biblioteca comunale, Trento

BCT1 (ex fondo Manoscritti)

BCT2 (ex fondo Diplomatico)

BCT3 (ex fondo Congregazione di Carità)

BFSB Biblioteca Fondazione San Bernardino, Trento

TLA Tiroler Landesarchiv, Innsbruck

Urk. I Urkundenreihe I 



\section{Bibliografia}

\section{Opere manoscritte}

ms. 1

ms. 6

ms. $12-13$

ms. 14

ms. 26

ms. 30

ms. 42
G. Tovazzi, Compendium diplomatum sive tabularum veterum loci, temporis, et argumenti multiplicis servata earumdem primigenia fhrasi, et orthographia diphthongis tantum exceptis digestum a frate Iohanne Chrysostomo de Avolano ss. Domini Iesu Christi Crucifixi Servo ac Minorita Reformato Provinciae Vigilianae, Trento 1787. Disponibile online la trascrizione del manoscritto all'indirizzo http://www.fondazionebibliotecasanbernardino.it/ index.php/it/strumenti-e-testi/category/4-giangrisostomo-tovazzi\#.

G. Tovazzi, Collectio diplomatum, aliorumque monumentorum veterum, quae vel ex Codice Wangiano Ecclesiae tridentinae, vel ex originalibus, aut autenticis tabulis fideliter descripsi Ego Frater Iohannes Chrysostomus de Avolano Ordinis Minorum Reformatorum, Trento, 1765.

G. Tovazzi, Monumenta orphanotrophii Tridentini sive hospitalis et fraternitatis sanctae Mariae de Misericordia descripta, 2 voll., Trento 1802. Disponibile online la trascrizione del manoscritto all'indirizzo http:// www.fondazionebibliotecasanbernardino.it/index.php/it/strumenti-e-testi/ category/4-giangrisostomo-tovazzi?start=20.

G. Tovazzi, Spicilegium archivii orphanotrophii Tridentini Sanctae Mariae de Misericordia scriptum anno 1802, Trento 1802. Disponibile online la trascrizione del manoscritto all'indirizzo http://www.db.ofmtn.pcn.net/ ofmtn/files/biblioteca/Spicilegium\%20Archivi\%20Orphanotrophii\%20 Tridentini.pdf.

G. Tovazzi, Topographia Lagarina seu brevis notitia oppidorum, terrarum, pagorum, vicorum, montium, silvarum, fluviorum, rivorum, lacuum, locorumque omnium totius Vallis Lagarinae, historico-diplomatico-literario-sacro-profanis monumentis instructa et illustrata. Abbozzo primo, Trento 1776. Disponibile online la trascrizione del manoscritto all'indirizzo http:// www.fondazionebibliotecasanbernardino.it/index.php/it/strumenti-e-testi/ category/4-giangrisostomo-tovazzi?limitstart $=0$.

G. Tovazzi, Familiarium Tridentinum, Trento 1790-1805. Disponibile online la trascrizione del manoscritto all'indirizzo http://www.fondazionebibliotecasanbernardino.it/index.php/it/strumenti-e-testi/category/4-giangrisostomotovazzi?limitstart $=0$.

G. Tovazzi, Chronologia Calapina seu nobilium Calapinorum Tridenti ab anno 1256 usque ad annum 1606, deducta et ab ipsismet veterum monumentorum verbis contexta, Trento 1795. 


\section{Bibliografia}

\section{Opere a stampa}

Alberti G., L'antica corporazione dei portatori di vino, «Tridentum», 2, 1899, pp. 49-90, 149-165.

Albertoni G., Varanini G.M. (a cura di), Il territorio trentino nella storia europea, II: L'età medievale, FBK Press, Trento 2011.

Ambrosi F., Commentari della storia trentina, Artigianelli, Trento 1985 (prima ed. 1887).

Antonelli Q., Storia della scuola trentina, dall'umanesimo al fascismo, Il Margine, Trento 2013.

Ausserer C., Il castello di Stenico nelle Giudicarie coi suoi signori e capitani, Scotoni e Vitti, Trento 1911 (ed. orig. Schloss Stenico in Judicarien [Süd Tirol], seine Herren und seine Hauptleute, «Jahrbuch der heraldischen Gesellschaft 'Adler' in Wien», 18, 1908, pp. 93-147).

Azzolini G., Vocabolario vernacolo-italiano pei distretti roveretano e trentino, Manfrini, Trento 1976 (edizione del manoscritto, Lizzana 1836).

Bartoli Langeli A., Nota introduttiva, in M.I. Bossa (a cura di), Chiese e conventi degli ordini mendicanti in Umbria nei secoli XIII e XIV. Inventario delle fonti archivistiche e catalogo delle informazioni documentarie. La serie Protocolli dell'Archivio notarile di Perugia, Editrice Umbra cooperativa, Perugia 1987 (Archivi dell'Umbria. Inventari e ricerche, 12), pp. IX-XXV.

- D'Acunto N., I documenti degli Ordini mendicanti, in G. Avarucci, R.M. Borraccini Verducci, G. Borri (a cura di), Libro, scrittura, documento della civiltà monastica e conventuale nel basso Medioevo, Atti del convegno della Associazione Italiana dei Paleografi e dei Diplomatisti (Fermo, settembre 1997), CISAM, Spoleto 1999 (Studi e ricerche. Centro italiano di studi sull'Alto Medioevo, 1), pp. 381-415.

- Irace E., Gli archivi, in G. Pugliese Carratelli (a cura di), La città e la parola scritta, Scheiwiller, Milano 1997 («Civitas europaea»), pp. 401-428.

- Rigon A. (a cura di), I registri vescovili dell'Italia settentrionale (secoli XII-XV), Atti del convegno (Monselice 24-25 novembre 2000), Herder, Roma 2003 (Italia Sacra, 72).

Bellabarba M., Legislazione statutaria cittadina e rurale nel Principato vescovile di Trento (sec. XV), in P. Schiera (a cura di), 1948-1988. L'autonomia trentina. Origini ed evoluzioni fra storia e diritto, Consiglio della Provincia autonoma di Trento, Trento 1988, pp. 17-37.

- Rovereto castrobarcense, veneziana, asburgica: identità ed equilibri istituzionali, in Statuti di Rovereto del 1425 con le aggiunte del 1434 e del 1538, a cura di F. Parcianello, introduzione di M. Bellabarba, G. Ortalli, D. Quaglioni, Comune di Rovereto, Biblioteca Civica - Accademia roveretana degli Agiati, Venezia 1991 (Corpus statutario delle Venezie, 9), pp. 9-29.

- Tra la città e l'impero. Il principato vescovile di Trento nella prima età moderna, in G. Coppola, P. Schiera (a cura di), Lo spazio alpino: area di civiltà, regione cerniera, Liguori, Napoli 1991 (Europa Mediterranea. Quaderni, 5), pp. 147-164.

- La giustizia ai confini: il principato vescovile di Trento nella prima età moderna, Il Mulino, Bologna 1996 (Annali dell'Istituto storico italo-germanico in Trento. Monografie, 28).

Belloni C., Dove mancano registri vescovili ma esistono fondi notarili: Milano tra Tre e Quattrocento, in A. Bartoli Langeli, A. Rigon (a cura di), I registri vescovili dell'Italia settentrionale (secoli XII-XV), Atti del convegno (Monselice 24-25 novembre 2000), Herder, Roma 2003 (Italia Sacra, 72), pp. 43-84. 


\section{Bibliografia}

Berengo M., Lo studio degli atti notarili dal XIV al XVI secolo, in Fonti medioevali e problematica storiografica, Atti del congresso internazionale per il $90^{\circ}$ anniversario della fondazione dell'Istituto Storico Italiano per il Medioevo (Roma 22-27 ottobre 1973), Istituto Storico Italiano per il Medioevo, Roma 1976, I, pp. 149-172.

- L'Europa delle città. Il volto della società urbana europea tra Medioevo ed Età moderna, Einaudi, Torino 1999 (Biblioteca di cultura storica, 224).

Berlanda M., Il matrimonio nel tardo medioevo: studio preliminare ed edizione di documentazione notarile trentina (1424-1428), tesi di laurea, Università degli Studi di Trento, a.a. 2012-2013, rel. E. Curzel.

Bertoluzza A. (a cura di), Studenti trentini all'università di Bologna (dal 1200 al 1700), Centro culturale "Fratelli Bronzetti", Trento 1989.

Bettotti M., La nobiltà trentina nel Medioevo (metà XII-metà XV secolo), Il Mulino, Bologna 2002 (Annali dell'Istituto storico italo-germanico. Monografie, 36).

Bocchi R., Analisi dell'evoluzione della struttura urbana di Trento fino al secolo XVI, «Studi trentini di scienze storiche. Sezione seconda», 58, 1979, pp. 209-270.

- Oradini C., Immagine e struttura della città. Materiali per la storia urbana di Trento, Laterza, Roma-Bari 1983.

— Oradini C., Trento, Laterza, Roma-Bari 1983 (Le città nella storia d'Italia).

Borrelli L., Lo stemma dei Calepini, in L. de Finis, L. Borrelli, M. Lupo (a cura di), Palazzo Calepini a Trento in cinque secoli di storia, Fondazione Cassa di Risparmio di Trento e Rovereto, Trento 2010, pp. 63-69.

Bortoli C., Per un'edizione dei testi statutari del Comune di Trento dei secoli XIV-XV, tesi di laurea, Università degli Studi di Trento, a.a. 2009-2010, rel. A. Giorgi.

Bossa M.I. (a cura di), Chiese e conventi degli ordini mendicanti in Umbria nei secoli XIII e $X I V$. Inventario delle fonti archivistiche e catalogo delle informazioni documentarie. La serie Protocolli dell'Archivio notarile di Perugia, Editrice Umbra cooperativa, Perugia 1987 (Archivi dell'Umbria. Inventari e ricerche, 12).

Brandstätter K., Vescovi, città e signori. Rivolte cittadine a Trento 1435-1437, Società di Studi trentini di scienze storiche, Trento 1995 (Monografie, 51).

Briquet C.M., Les Filigranes. Dictionnaire historique des marques du papier dès leur apparition vers 1282 jusqu'en 1600, Hiersemann, Leipzig 1923.

Brunelli B., Cagol F. (a cura di), Rodolfo Belenzani e la rivolta cittadina del 1407, Comune di Trento, Trento 2009 (Quaderni per la storia di Trento, 1).

Bullough D.A., Le scuole cattedrali e la cultura dell'Italia settentrionale prima dei comuni, in Vescovi e diocesi in Italia nel medioevo (sec. IX-XIII), Atti del II convegno di storia della Chiesa in Italia (Roma, 5-9 settembre 1961), Antenore, Padova 1964, pp. 111-143.

Cagol F., L'Archivio del comune di Trento di antico regime: ordinamenti e strumenti repertoriali, «Studi trentini di scienze storiche. Sezione prima», 79, 2000, pp. 749-827.

- Il Comune di Trento in antico regime, in M. Hausberger (a cura di), "Volendo questo illustrissimo magistrato consolare": trecento anni di editoria pubblica a Trento, Provincia autonoma di Trento, Soprintendenza per i beni librari e archivistici, Trento 2005 (Quaderni, 6), pp. IX-XLVII.

- Il ruolo dei notai nella produzione e conservazione degli atti delle cancellerie giudiziarie della città di Trento (secoli XIII-XVI), in A. Giorgi, S. Moscadelli, C. Zarrilli (a cura di), La documentazione degli organi giudiziari nell'Italia tardo-medievale e moderna, Atti 


\section{Bibliografia}

del convegno (Siena, 15-17 settembre 2008), Ministero per i beni culturali e ambientali, Roma 2012 (Pubblicazioni degli Archivi di Stato. Saggi, 109), I, pp. 139-190.

- Dal palatium episcopatus al palatium comunis. Spazi dell'identità comunale tra XIII e XVI secolo, in F. Cagol, S. Groff, S. Luzzi (a cura di), La torre di piazza nella storia di Trento, Atti della giornata di studio (Trento, 27 febbraio 2012), Società di Studi trentini di scienze storiche, Trento 2014 (Monografie. Nuova serie, 3), pp. 205-223.

- Brunelli B., Archivio pretorio o archivi notarili? Primi risultati di un'indagine archivistica sulla documentazione giudiziaria della città di Trento, «Annali dell'Istituto storico italo-germanico in Trento», 28, 2002, pp. 691-700.

- Mura A., Trento e Bolzano: due città a confronto. Modi e forme di produzione documentaria nei due Comuni tra Quattro e Cinquecento, in L. de Finis (a cura di), La proclamazione imperiale di Massimiliano I d'Asburgo (4 febbraio 1508), Atti del convegno (Trento, 9 maggio 2008), supplemento a «Studi trentini di scienze storiche», 87, 2008, pp. 855-886.

Calleri S., L'Arte dei giudici e notai di Firenze nell'età comunale e nel suo statuto del 1344, Giuffrè, Milano 1966.

Cammarosano P., Italia medievale. Struttura e geografia delle fonti scritte, Nuova Italia Scientifica, Roma 1991.

Casagrande Mazzoli M.A. et alii (a cura di), I manoscritti datati della provincia di Trento, Sismel, Firenze 1996 (Manoscritti datati d'Italia, 1).

Casamassima E., Sulla descrizione dei codici, «Rassegna degli Archivi di Stato», 23, 1963, pp. 197-205.

Casetti A., Il notariato trentino e l'istituzione dei più antichi archivi notarili in Trento: l'archivio (vecchio) dei morti e l'archivio (nuovo) dei vivi (1595-1607), "Studi trentini di scienze storiche», 31, 1952, pp. 242-286.

Ceraolo M.V., Il collegio notarile di Trento nella seconda metà del Quattrocento, tesi di laurea, Università degli Studi di Trento, a.a. 2001-2002, rel. G.M. Varanini.

Chemotti B., La legislazione statutaria nel Principato vescovile di Trento: gli Statuti alessandrini (1425), tesi di laurea, Università degli Studi di Trento, a.a. 1989-1990, rel. D. Quaglioni.

Chiarotti F., L'insurrezione contadina del 1525 nell'analisi degli avvenimenti dell'Anaunia, in Storia del Trentino, IV: L'età moderna, a cura di M. Bellabarba, G. Olmi, Il Mulino, Bologna 2000, pp. 157-192.

Chironi G., La mitra e il calamo. Il sistema documentario della Chiesa senese in età pretridentina (secoli $X I V-X V$ ), Accademia senese degli Intronati - Ministero per i Beni culturali e ambientali, Siena-Roma 2005 (Monografie di storia e letteratura senese, 13; Saggi, 85).

Chittolini G., "Episcopalis curiae notarius". Cenni sui notai di curie vescovili nell'Italia centrosettentrionale alla fine del Medioevo, in Società, istituzioni, spiritualità. Studi in onore di Cinzio Violante, CISAM, Spoleto 1994, pp. 221-232.

Codex Wangianus. I cartulari della Chiesa trentina (secoli XIII-XIV), a cura di E. Curzel, G.M. Varanini, con la collaborazione di D. Frioli, Il Mulino, Bologna 2007 (Annali dell'Istituto storico italo-germanico in Trento. Fonti, 5).

Codicis Clesiani archivi episcopalis Tridenti regesta, a cura di M. Morizzo, D. Reich, Comitato diocesano tridentino, Trento [s.d.], e in «Rivista Tridentina», 7, 1907, pp. 193-226; 8, 1908, pp. 97-128, 185-199, 249-280, 345-360; 9, 1909, pp. 49-64, 113-128, 193-208, 


\section{Bibliografia}

269-288; 10, 1910, pp. 49-64, 129-144, 191-207, 261-276; 11, 1911, pp. 49-64, 113-128, $177-192,257-288 ; 12,1912$, pp.49-78, 127-158, 199-222, 271-318; 13, 1913, pp. 183198, 271-286, 343-358; 14, 1914, pp. 359-454.

Costa A., I vescovi di Trento. Notizie, profili, Àncora, Milano 2017.

Costamagna G., La triplice redazione dell'instrumentum genovese. Con appendice di documenti, Società Ligure di Storia Patria, Genova 1961.

Costisella G., Rasmo N., Il palazzo Calepini a Trento, Istituto di Credito Fondiario della Regione Tridentina, Trento 1996.

Curzel E., Ricerche sul Capitolo della cattedrale di Trento alla metà del Quattrocento, tesi di laurea, Università degli Studi di Trento, a.a. 1989-1990, rel. G.M. Varanini.

- Per la storia del Capitolo della Cattedrale di Trento nel Trecento e nel Quattrocento: la serie degli Instrumenta Capitularia, «Studi trentini di scienze storiche. Sezione prima», 71, 1992, pp. 223-260.

- La croce del vescovo Giorgio Liechtenstein, in E. Curzel (a cura di), In factis mysterium legere: miscellanea di studi in onore di Iginio Rogger in occasione del suo ottantesimo compleanno, Edizioni Dehoniane, Bologna 1999 (Istituto trentino di cultura. Pubblicazioni dell'Istituto di Scienze Religiose in Trento. Series maior, 6).

- I documenti del Capitolo della cattedrale di Trento. Regesti 1147-1303, Società di Studi trentini di scienze storiche, Trento 2000 (Rerum Tridentinarum Fontes, 6).

- I canonici e il Capitolo della cattedrale di Trento dal XII al XV secolo, Edizioni Dehoniane, Bologna 2001 (Istituto per le Scienze Religiose in Trento. Series maior, 8).

- Scolastici e scolares nella cattedrale di Trento (secoli XII-XV), «Annali di storia dell'educazione e delle istituzioni scolastiche», 9, 2002, pp. 191-204.

- I vescovi di Trento nel basso medioevo: profili personali, scelte di governo temporale e spirituale, in Storia del Trentino, III: L'età medievale, a cura di A. Castagnetti, G.M. Varanini, Il Mulino, Bologna 2004.

- Delaito da Noarna, notaio e "civis Tridentinus" († 1323), in F. Leonardelli, G. Rossi (a cura di), Officina humanitatis. Studi in onore di Lia de Finis, Società di studi trentini di scienze storiche, Trento 2010, pp. 345-356.

- Trento, CISAM, Spoleto 2013 (Il medioevo nelle città italiane, 5).

- Notai di nomina vescovile a Trento tra XII e XIII secolo, in A. Giorgi, S. Moscadelli, D. Quaglioni, G.M. Varanini (a cura di), Il notariato nell'arco alpino: produzione e conservazione delle carte notarili tra medioevo ed età moderna, Atti del convegno di studi (Trento, 24-26 febbraio 2011), Giuffrè, Milano 2014 (Studi storici sul notariato italiano, XVI), pp. 461-482.

- Garbellotti M., Rossi M.C. (a cura di), Confraternite in Trentino e a Riva del Garda, Cierre edizioni, Caselle di Sommacampagna (Vr) 2017 (Biblioteca dei Quaderni di storia religiosa, IX).

- Gentilini S., Varanini G.M. (a cura di), Le pergamene dell'archivio della Prepositura di Trento (1154-1297), Il Mulino, Bologna 2004 (Annali dell'Istituto storico italo-germanico in Trento. Fonti, 2).

- Pamato L., Varanini G.M., Giovanni da Parma, canonico della cattedrale di Trento, e la sua cronaca (1348-1377), «Studi trentini di scienze storiche. Sezione prima», 80, 2001, pp. 211-239.

Dal Piaz I., La confraternita dei battuti laici nella città di Trento fra il 1340 e il 1450, tesi di laurea, Università degli Studi di Verona, a.a. 1985-1986, rel. G. De Sandre Gasparini. 


\section{Bibliografia}

- Il movimento francescano e la confraternita trentina dei Battuti in un documento del 1452, «Le Venezie francescane», 4, 1987, 1, pp. 105-117.

De Festi C., Studenti trentini alle università italiane, «Archivio storico per Trieste, l'Istria e il Trentino», 4, 1885, pp. 36-63.

- Memorie genealogiche sulla nobil famiglia de’ Belenzani, Pozzati, Verona 1896.

de Finis L., Dai maestri di grammatica al liceo di via S. Trinità in Trento, Società di Studi trentini di scienze storiche, Trento 1987 (Monografie, 44).

- Palazzo Calepini a Trento in cinque secoli di storia, in L. de Finis, L. Borrelli, M. Lupo (a cura di), Palazzo Calepini a Trento in cinque secoli di storia, Fondazione Cassa di Risparmio di Trento e Rovereto, Trento 2010, pp. 9-61.

- Borrelli L., Lupo M. (a cura di), Palazzo Calepini a Trento in cinque secoli di storia, Fondazione Cassa di risparmio di Trento e Rovereto, Trento 2010.

De Sandre Gasparini G., Statuti di confraternite religiose di Padova nel Medioevo. Testi, studio introduttivo e cenni storici, Istituto per la storia ecclesiastica padovana, Padova 1974.

Fabbri L., Alleanza matrimoniale e patriziato nella Firenze del '400: studio sulla famiglia Strozzi, Olschki, Firenze 1991.

Fossali R., Il più antico Liber actorum del Comune di Trento. Prime considerazioni per l'edizione, «Studi trentini. Storia», 91, 2012, pp. 323-364.

Frioli D., La "costruzione" di un registro vescovile: Nicolò da Brno, vescovo di Trento (1338-1347) e il Codex Wangianus Maior, in G.G. Merlo (a cura di), Vescovi Medievali, Biblioteca francescana, Milano 2003, pp. 207-266.

Giorgi A., Moscadelli S., Ut ipsa acta illesa serventur. Produzione documentaria e archivi di comunità nell'alta e media Italia tra medioevo ed età moderna, in A. Bartoli Langeli, A. Giorgi, S. Moscadelli (a cura di), Archivi e comunità tra medioevo ed età moderna, Ministero per i beni culturali e ambientali - Università degli Studi di Trento, Roma-Trento 2009 (Pubblicazioni degli Archivi di Stato, Saggi, 92; Labirinti, 114), pp. 1-110.

- Moscadelli S., Quaglioni D., Varanini G.M. (a cura di), Il notariato nell'arco alpino: produzione e conservazione delle carte notarili tra medioevo ed età moderna, Atti del convegno di studi (Trento, 24-26 febbraio 2011), Giuffrè, Milano 2014 (Studi storici sul notariato italiano, XVI).

- Moscadelli S., Zarrilli C. (a cura di), La documentazione degli organi giudiziari nell'Italia tardo-medievale e moderna, Atti del convegno (Siena, 15-17 settembre 2008), Ministero per i beni culturali e ambientali, Roma 2012 (Pubblicazioni degli Archivi di Stato, Saggi, 109).

Imbreviature. I registro (1294-1296). Ser Matteo di Biliotto notaio, a cura di M. Soffici, F. Sznura, SISMEL, Firenze 2002 (Memoria scripturarum, 1).

Inama V., Una scuola di grammatica in Cles nel secolo XIV, «Archivio trentino», 13, 18961897, pp. 231-234.

Ioppi R., I registri del monastero di San Lorenzo di Trento. Regesto degli atti (1369-1430), tesi di laurea, Università degli Studi di Trento, a.a. 2013-2014, rel. A. Giorgi.

Ippoliti G., Zatelli A.M., Archivi Principatus Tridentini regesta. Sectio latina (1027-1777), a cura di F. Ghetta, R. Stenico, 2 voll., Nuove Arti Grafiche, Trento 2001.

Jemolo V., Morelli M., (a cura di), Guida a una descrizione uniforme dei manoscritti e al loro censimento, Istituto centrale per il catalogo unico delle biblioteche italiane e per le informazioni bibliografiche, Roma 1990. 


\section{Bibliografia}

Landi W., Il palatium episcopatus di Trento fra XI e XIII secolo. Dato documentario ed evidenze architettoniche, in F. Cagol, S. Groff, S. Luzzi (a cura di), La torre di piazza nella storia di Trento, Atti della giornata di studio (Trento, 27 febbraio 2012), Società di Studi trentini di scienze storiche, Trento 2014 (Monografie. Nuova serie, 3), pp. 141-203.

Little L.K., Indispensable Immigrants. The Wine Porters of Northern Italy and their Saints, 1200-1800, Manchester University Press, Manchester 2015.

Lupo M., scheda n. 7 (Monumento funebre di Calepino Calepini), in E. Castelnuovo et alii (a cura di), Il duomo di Trento, Temi, Trento 1992, pp. 100-101.

Luzzi S., Stranieri in città. Presenza tedesca e società urbana a Trento (secoli XV-XVIII), Il Mulino, Bologna 2003 (Annali dell'Istituto storico italo-germanico. Monografie, 38).

Maino L., 50 testamenti medievali nell'Archivio Capitolare di Trento, Liberty House, Ferrara 2001.

Malfatti S., Il registro del notaio Venturino de Trechis nell'archivio del Capitolo della cattedrale di Trento - Instrumenta capitularia 3 (1324-1347). Edizione e commento, tesi di laurea, Università degli Studi di Trento, a.a. 2011-2012, rel. A. Giorgi.

- Toscani a Trento nel tardo medioevo, «Studi trentini. Storia», 97, 2018, 2, pp. 409-448.

Mangini M.L., Il notariato a Como: «Liber matricule notariorum civitatis et episcopatus Cumarum»(1427-1605), Insubria University Press, Varese 2007.

Maniaci M., Terminologia del libro manoscritto, préface di D. Mazurelle, Istituto centrale per la patologia del libro, Roma-Milano 1998 (Addenda, 3).

- Archeologia del manoscritto: metodi, problemi, bibliografia recente, con contributi di C. Federici e di E. Ornato, Viella, Roma 2002 (I libri di Viella, 34).

Mattivi S., Il registro del notaio Antonio da Pomarolo (1351-1357). Economia e società a Trento alla metà del Trecento, tesi di laurea, Università degli Studi di Trento, a.a. 2009-2010, rel. E. Curzel.

Mira G., Primi sondaggi su taluni aspetti economico-finanziari delle confraternite dei disciplinati, in Risultati e prospettive della ricerca sul Movimento dei Disciplinati, Atti del convegno (Perugia, 5-7 dicembre 1969), Deputazione di storia patria per l'Umbria, Perugia 1972, pp. 229-260.

Mošin V.A., Traljič S.M., Vodeni Znakovi XIII i XIV vijeka; Filigranes des XIII et XIV ss., Jugoslavenska akademija znanosti i umjetnosti. Historijski institut, Zagreb 1957.

Orioli L., Le confraternite medievali e il problema della povertà: lo statuto della Compagnia di Santa Maria Vergine e di San Zenobio di Firenze, Edizioni di storia e letteratura, Roma 1985.

Ortalli G., Percorsi statutari trentini, in Statuti di Rovereto del 1425 con le aggiunte del 1434 e del 1538, a cura di F. Parcianello, introduzione di M. Bellabarba, G. Ortalli, D. Quaglioni, Comune di Rovereto, Biblioteca Civica - Accademia roveretana degli Agiati, Venezia 1991 (Corpus statutario delle Venezie, 9), pp. 31-47.

- Scuole e maestri fra medioevo e rinascimento: il caso veneziano, Il Mulino, Bologna 1996.

Pagnin B., Note di diplomatica episcopale padovana, in P. Cancian (a cura di), La memoria delle chiese. Cancellerie vescovili e culture notarili nell'Italia centro-settentrionale (secoli X-XIII), Scriptorium, Torino 1995 (I florilegi, 4).

Pamato L., Le confraternite medievali. Studi e tendenze storiografiche, in G. De Sandre Gasparini, G.G. Merlo, A. Rigon (a cura di), Il buon fedele. Le confraternite tra me- 


\section{Bibliografia}

dioevo e prima età moderna, Cierre, Sommacampagna (Vr) 1998 («Quaderni di storia religiosa», V), pp. 9-51.

Papaleoni G., Rime di anonimo sulla sollevazione di Trento nel 1435, «Archivio Trentino», 8, 1889, pp. 167-207.

Pegoretti C., Le rime sulla sollevazione di Trento del 1435. Esame linguistico del manoscritto della biblioteca di San Bernardino in Trento, tesi di laurea, Università degli Studi di Trento, a.a. 1992-1993, rel. P. Cordin.

- "Rime" di anonimo sulla sollevazione del 1435 a Trento, in E. Banfi, G. Bonfadini, P. Cordin, M. Iliescu (a cura di), Italia settentrionale: crocevia di idiomi romanzi, Atti del convegno internazionale di studi (Trento, 21-23 ottobre 1993), Max Niemeyer Verlag, Tübingen 1995, pp. 299-310.

Petrucci A., La descrizione del manoscritto: storia, problemi, modelli, La Nuova Italia scientifica, Roma 2008.

Piccard G., Wasserzeichen Hirsch, W. Kohlhammer, Stuttgart 1961.

Polli G., Le Clarisse di San Michele a Trento. Ricostruzione dell'archivio ed edizione dei documenti (1193-1500), Società di Studi trentini di scienze storiche, Trento 2014 (Monografie. Nuova serie, 4).

Puncuh D., La diplomatica comunale in Italia dal saggio del Torelli ai nostri giorni, in W. Prevenier, Th. De Hemptinne (a cura di), La diplomatique urbaine en Europe au moyen âge, Actes du congrès de la Commission internationale de Diplomatique (Gand, 25-29 août 1998), Garant, Leuvein-Apeldoom 2000, pp. 383-406.

Rando D., Motter M., Il «Quaternus rogacionum» del notaio Bongiovanni di Bonandrea (1308-1320), Il Mulino, Bologna 1997 (Storia del Trentino. Serie II. Fonti e testi, 1).

Reich D., I castelli di Sporo e Belforte, Scotoni e Vitti, Trento 1901 (rist. anast. Regione Autonoma Trentino-Alto Adige 2011).

- Rodolfo de' Belenzani e le rivoluzioni trentine (1407-1409). Tradizione e storia, "Tridentum», 10, 1907, pp. 1-38.

Ressegotti D., «Spala de portadoro»: una nota quattrocentesca in volgare trentino, «Studi trentini. Storia», 91, 2012, pp. 191-202.

- Gli antichi statuti della confraternita dei Battuti, «Studi trentini. Storia», 92, 2013, pp. 65-96.

Ricci I., Aspetti della società e della chiesa trentina nella seconda metà del Trecento, dal protocollo del notaio Pietro Paolo (1376), tesi di laurea, Università degli Studi di Trento, a.a. 1991-1992, rel. G.M. Varanini.

Rizzo S., Il lessico filologico degli umanisti, Edizioni di storia e letteratura, Roma 1984.

Rolandini Passaggerii Summa totius artis notariae, Forni, Sala Bolognese (Bo) 1977 (rist. anastatica a cura del Consiglio nazionale del notariato).

Romiti A., L'armarium comunis della Camara Actorum di Bologna: l'inventariazione archivistica nel XIII secolo, Ministero per i Beni Culturali e Ambientali. Pubblicazioni degli Archivi di Stato, Roma 1994 (Fonti, 19).

Sancassani G., L'archivio dell'Antico Ufficio del Registro di Verona, «Vita veronese», 10, 1957, pp. 481-490.

- Il collegio dei notai di Verona, in G. Sancassani, M. Carrara, L. Magagnato (a cura di), Il notariato veronese attraverso i secoli. Catalogo della mostra in Castelvecchio, Collegio Notarile di Verona, Verona 1966. 


\section{Bibliografia}

Santarelli L., Un giurista nel Quattrocento trentino: Calepino de Calepini, «Studi trentini di scienze storiche. Sezione prima», 75, 1996, pp. 245-265.

Santifaller L. (a cura di), Urkunden und Forschungen zur Geschichte des Trientner Domkapitels im Mittelalter, I: (1147-1500), Universum, Wien 1948 (Veröffentlichungen des Instituts für Österreichischer Geschichtforschung, 6).

Savigny F.C. von, Geschichte des Römischen Rechts im Mittelalter, Scientia, Aalen 1986.

Sinisi L., Formulari e cultura giuridica notarile nell'età moderna: l'esperienza genovese, Giuffrè, Milano 1997 (Fonti e strumenti per la storia del notariato italiano, 8).

Statuti di Rovereto del 1425 con le aggiunte del 1434 e del 1538, a cura di F. Parcianello, introduzione di M. Bellabarba, G. Ortalli, D. Quaglioni, Comune di Rovereto, Biblioteca Civica - Accademia roveretana degli Agiati, Venezia 1991 (Corpus statutario delle Venezie, 9).

Stenico R., Il dazio di Trento. Alcuni documenti dei secoli XII-XV, «Studi trentini di scienze storiche. Sezione prima», 66, 1987, pp. 159-161.

- Notai che operarono nel Trentino dall'anno 845 ricavati soprattutto dal Notariale Tridentinum del P. Giangrisostomo Tovazzi, Trento 1999 (http://www.db.ofmtn.pcn.net/ ofmtn/files/biblioteca/Notai.pdf).

Tagliaferri A., L'economia veronese secondo gli estimi dal 1409 al 1635, Giuffrè, Milano 1966.

Tamba G., (a cura di), Rolandino e l'ars notaria da Bologna all'Europa, Atti del convegno internazionale di studi storici sulla figura e l'opera di Rolandino (Bologna, 9-10 ottobre 2000), Giuffrè, Milano 2002 (Per una storia del notariato nella civiltà europea, 5).

- Formazione professionale del notaio, Relazione tenuta presso il Centro G. Costamagna (Genova, 18 aprile 2007) (http://www.centrostudicostamagna.it/testi/GiorgioTAMBAGenova163KB.pdf).

- Tavilla E. (a cura di), Nella città e per la città. I notai a Modena dal IX al XX secolo, Atti del convegno di studi (Modena, 16 ottobre 2010), Giuffrè, Milano 2013 (Collana del dipartimento di scienze giuridiche e della facoltà di giurisprudenza dell’Università di Modena e Reggio Emilia. Nuova serie, 89).

Tomasi B., Le pergamene della capsa Fabricae dell'Archivio del Capitolo della cattedrale di Trento (1267-1674): edizione e commento, tesi di laurea, Università degli Studi di Trento, a.a. 2008-2009, rel. A. Giorgi.

- L'archivio del Capitolo della Cattedrale di Trento: produzione e conservazione documentaria (secoli XIII-XX). Con un'edizione delle più antiche pergamene (1147-1250), tesi di dottorato di ricerca, Università degli Studi di Trento, XXV ciclo, a.a. 2011-2012.

Torelli P., Studi e Ricerche di Diplomatica Comunale, Consiglio Nazionale del Notariato, Roma 1980 (Studi storici sul notariato italiano, 5).

Tovazzi G., Malographia Tridentina. Cronaca dei fatti calamitosi avvenuti nel Trentino e regioni adiacenti dai primi anni d.C. al 1803, Lions Club, Trento 1986.

- Variae inscriptiones Tridentinae, a cura di R. Stenico, con saggi di L. Borrelli e F. Leonardelli, Biblioteca Padri Francescani, Trento 1994.

Valenti E., Il «liber electionum officialium magnificae communitatis Tridenti» (1415-1462): edizione e studio introduttivo, tesi di laurea, Università degli Studi di Trento, a.a. 20032004, rel. G.M. Varanini.

Varanini G.M., La famiglia Del Bene di Rovereto nel Quattrocento: l'affermazione sociale e le attività economiche, in G.M. Varanini (a cura di), Atti della giornata di studio. La 


\section{Bibliografia}

famiglia Del Bene di Verona e Rovereto e la villa Del Bene di Volargne (Rovereto-Volargne, 30 settembre 1995), Accademia roveretana degli Agiati, Rovereto (Tn) 1996, pp. 9-34.

- L'economia. Aspetti e problemi (XIII-XV secolo), in Storia del Trentino, III: L'età medievale, a cura di A. Castagnetti, G.M. Varanini, Il Mulino, Bologna 2004, pp. 461-515.

- Le annotazioni cronistiche del notaio Bartolomeo Lando sul Liber dierum iuridicorum del comune di Verona (1405-1412), in A. Castagnetti, A. Ciaralli, G.M. Varanini, Medioevo. Studi e documenti, Libreria Universitaria Editrice, Verona 2007, II, pp. 551-604.

- Rodolfo Belenzani e il comune di Trento agli inizi del Quattrocento, in B. Brunelli, F. Cagol (a cura di), Rodolfo Belenzani e la rivolta cittadina del 1407, Comune di Trento, Trento 2009 (Quaderni per la storia di Trento, 1), pp. 9-20.

- Il Collegio notarile di Trento nella seconda metà del Quattrocento, in A. Giorgi, S. Moscadelli, D. Quaglioni, G.M. Varanini (a cura di), Il notariato nell'arco alpino: produzione e conservazione delle carte notarili tra medioevo ed età moderna, Atti del convegno di studi (Trento, 24-26 febbraio 2011), Giuffrè, Milano 2014 (Studi storici sul notariato italiano, XVI), pp. 483-514.

- Gli uffici del Comune di Trento nel Quattrocento: spunti comparativi, in F. Cagol, S. Groff, S. Luzzi (a cura di), La torre di piazza nella storia di Trento, Atti della giornata di studio (Trento, 27 febbraio 2012), Società di Studi trentini di scienze storiche, Trento 2014 (Monografie. Nuova serie, 3), pp. 225-237.

Vareschi S., Profili biografici dei principi vescovi di Trento dal 1338 al 1444, «Studi trentini di scienze storiche. Sezione prima», 76, 1997, pp. 257-265.

Vitaliani A., L'organizzazione e il funzionamento dell'Ufficio del Registro in Verona nei primi decenni del sec. $X V$, "Atti dell'Accademia di agricoltura, scienze e lettere di Verona», s. V, 16, 1938, pp. 199-218.

Voltelini H. von, Gli antichi statuti di Trento, Accademia Roveretana degli Agiati, Rovereto 1989 (ed. orig. Die ältesten Statuten von Trient und ihre Überlieferung, Wien 1902).

Weber S., La corporazione dei portitori, «L'Amico delle Famiglie», 22, 1914, pp. 110, 122-123.

- I maestri di grammatica a Trento fino alla venuta dei pp. Gesuiti, «Studi trentini di scienze storiche», 1, 1920, pp. 193-200.

Weijers O., Vocabulaire du livre et de l'écriture au moyen âge. Etudes sur le vocabulaire intellectuel $d u$ Moyen âge, Actes de la table tonde (Paris, 24-26 septembre 1987), Brepol, Turnhout 1989.

Welber M., Stenico M., Gli statuti dei sindici nella tradizione trentina, UTC, Trento 1997 (Collana di Storia).

Woś J.W., Alessandro di Masovia vescovo di Trento (1423-1444). Un profilo introduttivo, Trento 1990 («Civis». Supplemento, 6).

Zamboni L., Economia e società in una piccola città alpina: Trento negli atti del notaio Alberto Negrati da Sacco (1399-1402). Con l'edizione o il regesto di 109 documenti, tesi di laurea, Università degli Studi di Trento, a.a. 1995-1996, rel. G.M. Varanini.

Zanella G.B., S. Maria di Trento: cenni storici, G.B. Monauni, Trento 1879. 


\section{Indice dei nomi di persona}

Per l'individuazione delle forme cognominali attestate si è fatto prevalentemente riferimento a Bettotti, La nobiltà trentina, cit.; Curzel, I canonici e il Capitolo, cit.; ove manchi una forma cognominale il nome della persona è accompagnato da un'indicazione relativa alla carica, allo stato o alla condizione ricavabile dal materiale utilizzato per questo lavoro. Si rinvia da una voce ad altra voce di questo stesso indice qualora risulti utile segnalare la relazione esistente fra diverse persone.

Il rinvio è al numero di pagina e per l'Appendice dei regesti al numero del documento.

*** figlio di magister Lorenzo Appendice, reg. 12

Abrianus di Nicolò Nichi da Levico, mulattiere Appendice, reg. 27

Accerbis (de) Andriota di Bonadomano di Nicolò phisicus, moglie di Giovanni Rauter 28, 105, 157, 160, 161; Appendice, regg. 22, 23

Accerbis (de) Bonadomano di Nicolò 25, 27, 32, 46, 58, 106, 109, 111, 158, 159, 160; Appendice, regg. 5, 19, 22, 23, 28, 31, 46, 50 e vedi Antonia, moglie

Accerbis (de) Nicolò, phisicus Appendice, reg. 5

Adam, monetarius 197

Adelperio da San Martino, mutarius $111 e$ vedi Francesco, figlio

Adelpreto, vescovo di Trento 57

Adelpreto di Federico notaio da Povo, proditor episcopi 24, 27, 96, 112; Appendice, regg. 19, 32, 48, 49, 50 e vedi Antonia, moglie

Agostino di Corradino da Feltre [ $m a$ Grigno, dioc. Feltre] 58

Alberti d'Enno Francesco Felice 168

Alberti G. 16, 18

Alberto, duca d'Austria 23, 24, 90, 122

Alberto Bonomi 122 e vedi Pasqua, moglie

Alberto da Marostica, vicario vescovile Appendice, reg. 24

Alberto di Negratus da Sacco, notaio 15, 22, 23, 27, 36, 76, 88, 89, 90, 100, 192, 193, 195; Appendice, regg. 1, 2, 3, 4
Alberto di Ortenburg, vescovo di Trento 20, $25,39,72,74,76,78,81,83,88$

Alessandro di Masovia, vescovo di Trento 7, $17,24,25,31,34,40,41,47,48,72,78$, $79,80,81,82,83,84,95,96,97,102$, 107, 108, 110, 111, 112, 118, 179, 208; Appendice, regg. 25, 28, 29, 30, 34, 39, $43,45,50$

Alexander Copa[...] de Tueno 197

Aliprandi Bonamente 197

Ambrosi F. 25, 27, 28, 29, 32, 33, 201, 202

Andrea, familiaris del vicario vescovile Appendice, reg. 34

Andrea, rettore della confraternita dei Battuti 33

Andrea di Bernardo da Firenze, apothecarius Appendice, reg. 9

Andrea di Ottolino dal Borgonuovo di Trento, orefice Appendice, regg. 1, 2

Andrea di Pace maestro di grammatica, notaio 59

Andriota di Francesco detto Tibay da Terlago, acquirente di un affitto perpetuo 201

Andriota di Nicola da Trento, contendente in una lite 122

Anhang Giovanni da Bopfingen, canonico della cattedrale 82, 83; Appendice, regg. 7, 30

Antonelli Q. 59

Antonia di Cristiano da Mori, famulla di Marco di Hendricus da Molina di Mori 13

Antonia di Pietro Iacob stazonerius, moglie di Adelpreto da Povo Appendice, reg. 48 
Indice dei nomi di persona

Antonio ab ancha, stazonerius Appendice, reg. 50

Antonio parolarius, rettore della confraternita dei Battuti 33; Appendice, reg. 50

Antonio Abrianus di Benvenuto da Tierno, oste dell'osteria al Falcone Appendice, regg. 29,35

Antonio da Arco, canonico della cattedrale Appendice, reg. 4

Antonio di Beloto da Povo, enfiteuta Appendice, reg. 26

Antonio di Bommartino da Trento, notaio 96

Antonio di Bonaventura da Ravina, notaio 11,12

Antonio di Bongiovanni da Fai, notaio 99, 112, 122, 190; Appendice, regg. 8, 24, 34, 39

Antonio di Bonifacio da Nogaredo, notaio 94, 99, 101, 109, 125, 190; Appendice, reg. 31

Antonio di Bonomo da Arco, giurisperito 28; Appendice, reg. 22 e vedi Domenica, madre

Antonio da Feltre, speziale Appendice, reg. 50

Antonio di Filippo da Mori, notaio 74

Antonio di Gotmanino da Brez, notaio 65, 176, 196, 197

Antonio da Grigno, notaio 64, 200

Antonio di Michele da Ranzo, notaio 74

Antonio da Novara, canonico della cattedrale Appendice, reg. 4

Antonio da Palermo, prete 127

Antonio di Paolo Peverada/Pevrada da Milano, notaio 76, 192

Antonio di Pietro da Bologna, tornerius Appendice, reg. 2

Antonio di Pietro da Verona, tornerius Appendice, reg. 1

Antonio da Pomarolo, notaio 12, 13, 22, 74, $86,87,197$

Antonio Prevedonus di Giacomo a rotis da Arco 31; Appendice, regg. 40, 41

Antonio da Seregnano 61 e vedi Gasperina, moglie

Antonio Schichignoli, vicario vescovile 192

Antonio da Trento, giudice in civilibus et criminalibus causis del duca d'Austria Alberto 122

Antonio di Tura, prete 117

Antonio da Venezia, priore del convento di Santa Croce 103

Antonio da Vigolo, notaio 200

Antonio detto a zopellis da Trento, testatore 196
Antoniolus a Turi/de dona Mocina, satelles proditorum episcopi 24

Antonius Nicholai sartoris de Nogaredo, notaio 11

Antonius Panizoli de burgo Sancti Martini 140

Antonius de Pederxano, magister sartor 39

Approvini Approvino 65

Approvini Michele 19 e vedi Ysabeta, moglie

Approvini Nicolò di Michele 19, 25, 150; Appendice, reg. 14

Approvini Stefano 65

Arsio (d') Antonia di Marcholinus, moglie di Bonadomano de Accerbis 157, 160

Artuico dalla Carinzia, canonico Appendice, reg. 4

Artuico/Hertuicus/Hertwicus di Enrico da Passau, canonico e notaio 84, 125, 128, 162, 195; Appendice, reg. 25

Ausserer C. 42

Avarucci G. 100

Azzolini G. 49

Baldessarre dal Borgonuovo di Guglielmo de Mura da Piné, laborator Appendice, regg. 40, 41

Baldessarre detto Bruschino da Vigolo Vattaro, cerdo Appendice, reg. 38

Baldino di Andrea a Poza da Povo, sindico Appendice, reg. 50

Balistis (a) Giovanni detto Mosca di Bartolomeo detto Mastelus da Fai Appendice, regg. 1,2

Balistis (a) Ungarus, satelles proditorum episcopi 24

Balzanini, fam. 29

Balzanini Balzano 197, 200

Balzanini Guglielmo di Guglielmo da Verona, notaio $25,46,94,99$

Banfi E. 23

Barbara di Graziadeo di Antonio di Biagio da Castel Terlago, moglie di Cristoforo da Cadine 125

Barbara da Pressano, moglie di Giorgio Chelum 124

Bartolasio di Cristiano da Piazzo/da Mori, padre del notaio Antonio da Borgonuovo $11,12,13,14,15,16,18,19,20,36,38$, 49, 101, 103; Appendice, reg. 6

Bartoli Langeli A. 85, 100, 126, 131

Bartolomea di Iacob speziale, moglie di Matteo de Murlinis 28; Appendice, reg. 49

Bartolomeo, lanaiolo Appendice, reg. 37 


\section{Indice dei nomi di persona}

Bartolomeo di Anselmo da Borgonuovo, testimone a una compravendita 14

Bartolomeo da Arco, honorabilioris de savioribus populi Appendice, reg. 50

Bartolomeo di Belino da Bosentino, portitor 15,16

Bartolomeo di Blanchus detto de Sangue de Can da Nomi 107 e vedi Giovannina, moglie

Bartolomeo da Bologna, abate dell'abbazia di San Lorenzo 46, 58, 192, 195; Appendice, regg. 8,28

Bartolomeo da Camerino, priore dell'ospedale di Santa Croce in Trento 90

Bartolomeo Carnessarii da Vigolo, sindico Appendice, reg. 50

Bartolomeo Cevoleta, testimone 27, 46, 164

Bartolomeo di Giacomino da Torchio, notaio Appendice, reg. 28

Bartolomeo di Ognibene da Fadano di Brentonico, portitor 14

Bartolomeo detto Sachart di Rolando da Povo Appendice, reg. 26

Bartolomeo di Sicco olim canonico di Trento 26, 31, 46, 48, 83; Appendice, regg. 32, 47

Bartolomeo detto Tomeo da Tuenno, notaio 60,61

Bartolomeo di *** di Tommaso de fossato cerdonum di Trento, cerdo Appendice, regg. 40,41

Bartolomeo Toschanelus/detto Toscanelo di Alberto da Volano, stazonerius 42; Appendice, reg. 6

Bartolomeus de Margono 201

Bartolus 192

Basilio da Treviso, priore del convento di San Marco 90

Battista di Silvestro da Bologna, stazonerius 28, 40, 46, 110; Appendice, regg. 12, 13, 38, 39, 50 e vedi Zagagnini Francesca, moglie

Beatrice di ***, moglie di Giovanni domine Alde 28, 148; Appendice, reg. 36

Beatrix [di Antonio Crescimbene da Madruzzo], ratificante una refuta 148

Bebio Giovanni Francesco, pretore del vescovo Bernardo Cles 202

Belenzani, fam. 25, 107, 108, 110, 111, 112, 208

Belenzani Antonio di Giovanni 19, 108, 109, 110, 111, 112, 119, 179; Appendice, regg. 1, 2, 34, 43, 45 e vedi Giacoma, moglie; Todeschina, madre
Belenzani Giovanni 19, 27 e vedi Todeschina, moglie

Belenzani Giovanni di Antonio 108, 109, 110, 112, 140, 179; Appendice, regg. 1, 4, 31, 34, 39, 43, 45 e vedi Felicia, moglie

Belenzani Guglielmo di Antonio 19, 108, 109, 110; Appendice, regg. 1, 43

Belenzani Marco 109, 112; Appendice, reg. 50

Belenzani Rodolfo di Francesco di Belenzano 18, 22, 23, 25, 30, 42, 61, 108, 208

Bella, moglie di Pietro da Sant'Orsola, detentrice del dominio utile su una casa 36

Bellabarba M. 16, 20, 41, 55, 65, 200

Belloni C. 126

Benassuto, viator della curia tridentina Appendice, reg. 1

Benedetto di Paolo Peurade Appendice, reg. 47

Benevenutus quondam Degeleguardi de Dresio 60

Berengo M. 40, 193

Berlanda M. 26, 28; Appendice, regg. 9, 12, 22,36

Berlina (della) Antonio di Giacomo magister sarto da Volano, notaio, magister bullettarum 32, 136, 143

Bernabono di Alberto da Como, murarius Appendice, reg. 29 e vedi Giacomo, nipote

Bernardis (de) Bernardo 46

Berto detto Cadella, laborator 15

Bertoldo, vescovo di Bressanone 128

Bertoldo di Bonomo da Dro, testimone 15

Bertoldo magistri Odorici cerdonis da Trento, prete 92, 127, 133 e vedi Onesta, sorella

Bertoluzza A. 66

Beseno (da) Corrado 57

Bettotti M. 20, 25, 28, 108

Biagio di Franchetto da Pomarolo, sindaco di Santa Maria Maggiore in Trento 108; Appendice, reg. 1

Biagio da Telve, honorabilioris de savioribus populi Appendice, reg. 50

Biasio fornaser, rettore della confraternita dei Battuti 33

Bocchi R. 13

Bonacordo di Maffeo da Brescia, magister bullettarum 32

Bonafede di Giovanni da Lendinara, abitante a Pergine, portitor 15

Bonalinis (de) Giacomo da Verona 43

Bonaventura, notaio sociorum Appendice, reg. 16

Bonaventura di Pasquale/Pasqualino da Verona da borgo San Nazaro, monachus di Santa 
Indice dei nomi di persona

Maria Maggiore in Trento Appendice, regg. 1, 2

Bonaventura di Pellegrino da Riva, notaio 71

Bonaventura detto Turolo di Trentino detto Madurence Appendice, reg. 3

Bonaverio di Antonio da Isera abitante in Borgonuovo, portitor 15, 16

Bonazonta aurifex, satelles proditorum episcopi 24

Bonetti Bartolomeo 36

Bonfadini G. 23

Bongiovanni di Bonandrea, notaio 71

Bonifacio IX, papa 90

Bonifacio da Chiusole 36, 42

Bonifacio di Pace maestro di grammatica, apothecarius 59

Bonomo da Arco, garante 42

Bonsenni Giacomo 194

Borraccini Verducci R.M. 100

Borrelli L. 30, 33, 34

Borri G. 100

Bortoli C. 18, 56, 143

Bossa M.O. 131

Brandstätter K. 20, 24, 25, 26, 27, 28, 31, 32, $40,41,46,48$

Bricius, prete 127

Briquet C.M. 168

Brunelli B. 8, 23, 27, 28, 42

Buffa Giuseppe Urbano 203

Bullough D.A. 62

Buonfine, fratello di Giovanni Rauter 27

Buratinis (de) Antonio Appendice, regg. 6, 7

Buratinis (de) Buratinus Appendice, reg. 5

Cagol F. 7, 8, 22, 23, 24, 27, 28, 36, 42, 46, $72,80,81,94,97,98,189$

Calepini, fam. 7, 25, 29, 34, 208

Calepini Adelperio di Marco di Bonaventura $24,26,28,29,30,31,34,38,49,58,95$, 97, 105, 136, 201; 208; Appendice, regg. $9,14,25,32,33,35,40,41,42,47,50$

Calepini Bonaventura di Adelperio 30, 32

Calepini Bonaventura di Marco di Bonaventura 19, 26, 29, 30, 32, 33, 38, 40, 49, 105; 208; Appendice, regg. 40, 42, 46 e vedi Maddalena di Antonio da Borgonuovo, moglie

Calepini Calepino di Bonaventura di Marco 19, 33, 64, 66, 200

Calepini Calepino di Marco di Bonaventura 26, 30, 34, 46, 201; Appendice, reg. 11

Calepini Donato di Bonaventura di Marco 19,33
Calepini Giovanni di Bonaventura di Marco 19,33

Calepini Gottardo di Bonaventura di Marco $19,33,63,66$

Calepini Marcadento di Calepino di Marco 26

Calepini Marco di Bonaventura di Adelperio $27,30,32,42,43$

Calepini Nascimbene di Marco di Bonaventura 26, 30, 32; Appendice, reg. 11

Calepini Odorico di Marco di Bonaventura 24, 26, 27, 29, 30, 32, 34, 46, 96, 105; Appendice, regg. 9, 19, 33, 46, 50 e vedi Sofia di Pietro da Isera, moglie

Calepini Onesta di Marco di Bonaventura, moglie di Michael Senftel 26

Calepini Pietro di Calepino di Marco 26

Calepini Tommaso di Calepino di Marco 26

Calleri S. 120

Calvis (de) Ludovico 96, 198

Cammarosano P. 154

Campo (da) Alberto 57

Campo (da) Battista detto Gratiadeus 200

Campo (da) Francesco 42

Campo (da) Marco vedi Pasqualina, moglie

Campo (da) Riprando 71

Campo (da) Simone di [Azzone detto] Tuynus 90; Appendice, regg. 1, 3

Cancian P. 126

Capris (de) Nicolò di Pietro Balduino/Baldovino da Maderno 25, 38, 40, 94, 99, 101, 106, 125, 190, 196, 204; Appendice, reg. 28

Capris (de) Pietro 95

Capuzio Cristoforo di Tommaso da Como 32; Appendice, regg. 32, 35, 50

[Carioli] Giacomo di Cariolo dalle Giudicarie, notaio e conte palatino 32,80

Carlo IV, imperatore 71

Carrara M. 43, 65

Casagrande Mazzoli M.A. 53

Casamassima E. 166

Casetti A. 8, 58, 121, 189

Castagnetti A. 17, 22, 43

Castelbarco (da) Francesco di Aldrighetto 129

Castelnuovo E. 34

Castro (de) Antonio di Enrico 28, 94, 96, 110, 136, 190, 191, 204; Appendice, regg. 9, $15,16,24,28,35,39,44,45,50$

Castro (de) Lorenzo di Antonio 59, 106

Castro (de) Rigo di Enrico Appendice, regg. 44,45

Cavalis (de) Giovanni da Venezia Appendice, reg. 30 


\section{Indice dei nomi di persona}

Cazuffi Lorenzo Appendice, reg. 50

Ceraolo M.V. 55, 63, 201

Ceris (de) Antonio da Pergine 25, 46

Ceris (de) Federico di Giovanni di Michele da Pergine, notaio 64

Chemotti B. 56, 63

Chironi G. 126

Chittolini G. 72

Christofalus ab hospitali, satelles proditorum episcopi 24

Ciaralli A. 43

Cicerone 54

Civolis vedi Zevolis

Cles Bernardo 8, 202; Appendice, reg. 5

Cles (da) Riprando Appendice, reg. 28

Colombini Leonardo di Giovanni Antonio da Terlago 202

Concerinus de burgo Sancti Martini, laborator 132,140

Concio Sorn, tutore di Ulrico e Margherita figli di Giorgio Chelum 124

Confeller Alberto 197

Constantia, moglie di Negro de Negri 156

Coppola G. 20

Cordin P. 23, 30

Coredo (da) Michele 125; Appendice, reg. 25

Corrado de Alemania, pievano in Civezzano e vicario in Cembra Appendice, reg. 30

Corrado da Chiusa, hospitalarius 128

Corrado Czoppot, chierico della diocesi di Bamberga 128

Corrado Friderici Greusseri de Monte Kuctis, notaio 71,72

Corrado da Trento, canonico della cattedrale Appendice, regg. 7, 30

Costa A. 17

Costamagna G. 131, 143, 165, 169

Costisella G. 30, 33, 37

Cristiano quondam ser Bertrami de villa Platii, nonno del notaio Antonio da Borgonuovo $11,12,13,20,24,41$

Cristina, vedova del notaio Giacomino da Posina 190

Cristoforo da Cadine, notaio 125 e vedi Barbara di Graziadeo di Antonio di Biagio da Castel Terlago, moglie

Curzel E. 8, 12, 13, 15, 17, 20, 27, 31, 32, 36, $38,39,57,62,71,78,80,85,86,87,88$, $90,93,98,126,192,194,195,196,197$

D'Acunto N. 100

Dal Piaz I. 27, 32, 33, 39, 40, 48

Dal Piaz Luigi 204
Dal Verme, fam. 111

De Festi C. 27, 66

De Finis L. 30, 34, 59, 60, 61, 94

De Hemptinne Th. 57

De Sandre Gasparini G. 15, 27, 39

Delaito di Benasuto a rotis da Trento, portitor 16

[Della Torre] Pietro de la Ture da Bergamo Appendice, reg. 8

Denklinger Martinus de Augusta, pievano di Sanzeno 149

Desiderato di Francesco da Magré nel Vicentino, lanarolus, cognato di Antonio da Borgonuovo 19, 38; Appendice, regg. 6, 48, 49 e vedi Domenica di Bartolasio, moglie

Desideratus 178

Dionisio di Pietro becarius, notaio 99

Domenica di Bartolasio di Cristiano da Mori, sorella di Antonio da Borgonuovo, moglie di Desiderato di Francesco da Magré 19, 38; Appendice, reg. 6

Domenica vedova di Bonomo da Arco 31; Appendice, regg. 40, 41

Domenico del dominus arcidiacono, speziale Appendice, reg. 29

Domenico Fraschete da Cadine, sindico Appendice, reg. 50

Dominabus (a) Antonio da Trento 112; Appendice, reg. 28

Dominicus de Voltolina, procuratore 156

Dominigacius laborator, patrignus di Giovannina, moglie di Bartolomeo di Blanchus detto de Sangue de Can da Nomi 107

Donato di Delaito da Civezzano, testimone a una sentenza Appendice, reg. 3

Donato da Verona, sacrista nella cattedrale Appendice, reg. 4

Ducati Pietro Carlo 203

Elena di Bartolomeo di Sicco Appendice, reg. 47

Elica di Gabriele dal Borgonuovo, testatrice 191

Enrico da Landsberg, canonico e notaio 71

Enrico di Metz, vescovo di Trento 83, 85

Enrico di Odorico de Velden, canonico di Augusta 126

Enrico, professore di grammatica in Trento Appendice, reg. 22

Equabus ( $a b$ ) Giovanni di Guglielmo 99

Equabus ( $a b$ ) Guglielmo di Antonio 74, 99; Appendice, reg. 1

Erardo da Eger, canonico della cattedrale 144 
Fabbri L. 29

Facini Antonio 199, 200

Facini Domenico Appendice, reg. 50

Facinis (de) Guglielmo detto Saraceno di Paolo, notaio 27, 58, 81, 96, 125, 149, 150, 151, 152, 153, 168, 190, 205, 208; Appendice, regg. 5, 19, 25, 33

Fanzini Giacomo di Bertoldo a Ture 46, 95, 111, 112, 199; Appendice, reg. 12, 29, 33,50

Fanzini Giovanni Luca Appendice, reg. 35

Fatis (de), fam. 7

Fatis (de) Antonio 65

Fatis (de) Fato da Terlago 78

Fatis (de) Giovanni Conto di Paolo da Terlago 25, 32, 74, 94, 112, 125; Appendice, reg. 7

Fatis (de) Paolo di Giovanni da Terlago 42, 78

Faustinus filius Mafei de Brixia, proditor episcopi 24

Federici C. 166

Federicis (de) Giacomino de Arbano in val Camonica 111; Appendice, reg. 46

Federico duca d'Austria, conte del Tirolo e avvocato della Chiesa di Trento 22, 23, 25, 41, 42, 30, 32, 34, 42, 49, 72, 96, 126; Appendice, reg. 5

Federico di Andrea da Ferrara, prete 126

Federico di Brandeburgo, principe elettore 46

Federico di Enrico da Albiano, notaio 191, 195

Federico di Ognibene da Povo, notaio 36, 80; Appendice, reg. 3

Federico Polentone da borgo San Martino in Trento di Giovanni Pazolus da Levico, enfiteuta 140; Appendice, regg. 16, 35

Federicus de Gardulis 193

Felicia di Francesco notaio, moglie di Giovanni Belenzani 109, 110, 112, 113, 179, 181; Appendice, regg. 31, 39

Fignoclus/Phignonclus di Pietro da Rovereto, piliparius, cognato di Antonio da Borgonuovo 19,125

Filippo di Bonaventura di Antonio da Volano, testimone a una promessa di pagamento Appendice, reg. 11

Fina da Folgaria, badessa di San Michele 100

Flora di Tura sarto detto a Porta da Trento, testimone Appendice, regg. 1, 2

Flordela, moglie di Terlaco Marele da Terlago 201

Floriano Malicie di Francesco da Trento, sindaco e provisor dell'ospedale di San Pietro 104, 132
Floridia di Tommaso da Folgaria, moglie di Antonio da Molveno 27, 89

Floriis (de) Alberto di Martino da Mantova $62,86,87,89,194$

Florio di Alberto da Pilcante, portitor 14

Florio da Denno, canonico della cattedrale 128, 150, 151, 192, 194; Appendice, regg. $4,6,7$

Flos Dominici Petri Ordani de Barbarola, locataria 149, 150, 151, 152, 154

Fossali R. 24, 93, 94

Francesca di Antonio Zagagnini a clavis, moglie di Battista di Silvestro da Bologna 28; Appendice, regg. 12, 13 e vedi Maria, madre

Francesca di Bartolomeo di Sicco da Trento Appendice, reg. 47

Francesca di Giacomo Beschapani, testatrice 122

Franceschino a candelis, garante 42

Franceschinus aurifex quondam ser Pancerie de Levigo, proprietario di una casa a Trento 13

Francesco magister a scutelis da Padova 103

Francesco di Bartolomeo da borgo San Martino, detto Polenton 61

Francesco da Calavino, notaio 202

Francesco di Cristiano detto Meiatus da Piazzo 13

Francesco di Endrico da Albiano, detentore di un dominio utile 37

Francesco da Ferrara, priore del monastero di San Lorenzo 90

Francesco da Fondo, notaio 197

Francesco di Giovanni detto dela Massa, detentore di un dominio utile 38

Francesco di Marco da Borgonuovo, venditore di un terreno 14

Francesco di Martino da Volano, notaio e sindaco del Capitolo 74, 82, 90, 98, 99, 101

Francesco di Matteo da Chiusole, testimone a una confessio Appendice, reg. 16

Francesco di Nicolino da Arco, socio di Giacomo detto del Fafaros figlio di Giovanni de Alemania 134, 136, 137

Francesco di Pietro da Isera, notaio 89

Francesco di Vincenzo da Gabbiolo da Vigolo Baselga, enfiteuta Appendice, reg. 17

Francesco di Vittore da Grigno, notaio 66

Francesco Leonardo da Mattarello, ratificante una locazione Appendice, reg. 35

Francischus magister barberius 39 


\section{Indice dei nomi di persona}

Friling Goffredo [de Oyta] da Bressanone 110, 128; Appendice, regg. 30, 34, 39

Frioli D. 38, 71

Fuchs Christoph Appendice, reg. 5

Gabriele di Ivano, apotecarius 42

Gaisis (de) Giovanni da Calavino 200

Gaisis (de) Giovanni Giacomo di Giovanni da Calavino 200

Gallo Andrea di Giovanni 19, 64, 200, 201, 202, 204, 205; Appendice, reg. 12

Gallo Antonio di Guglielmo 101, 106

Gallo Francesco 24, 48

Gallo Giovanni 19, 24, 48

Gallo Girolamo 202

Gallo Guglielmo di Andrea 201, 202, 204, 205, 206; Appendice, regg. 10, 11, 50

Gallo Guglielmo di Delaito 101, 107, 122, 201, 202

Gallo Lucia di Guglielmo, moglie di Aldrighetto di Giovanni Mezaoveta 28, 107; Appendice, regg. 9, 10

Gans Antonio di Giovanni Appendice, reg. 18

Ganzele, prestatore di denaro ebreo Appendice, reg. 29,35

Garbellotti M. 15

Gardumo (da) Antonio di Filudussio 12

Gardumo (da) Giacomo Appendice, reg. 35

Gasparino di Antonio notaio da Nogaredo, notaio 191

Gasperina di Stefano da Cles, moglie di Antonio da Seregnano 61

Gauslinis (de) Lorenzo da Feltre 95; Appendice, reg. 6

Gelfo Bernardino di Domenico 205

Gelfo Francesco di Domenico 205

Gentilini S. 98

Georgius de Romagnano, proprietario di un terreno 11

Gerardo da Verona, pievano in Flavon Appendice, reg. 4

Gerart Osvald Appendice, reg. 37

Giacoma, moglie di Antonio di Giovanni Belenzani 108, 109; Appendice, regg. 1,34

Giacomino detto a Cantono di Pace maestro di grammatica, notaio 59

Giacomino di Albertino tabernarius da Posina, notaio 76, 190 e vedi Cristina, moglie

Giacomo, preposito del monastero di San Michele all'Adige 144

Giacomo, fratello di Antonio di Bonaventura da Ravina 12
Giacomo da Arco, notaio, honorabilioris de savioribus populi 42, 47; Appendice, reg. 50

Giacomo di Bartolomeo da Ravazzone, notaio 19, 74, 108, 109, 191, 195, 196; Appendice, regg. 1, 2

Giacomo nipote di Bernabono, murarius Appendice, reg. 29

Giacomo da Cadore, scolaris 62

Giacomo da Castel Romano, phisicus Appendice, reg. 28

Giacomo da Egna (Novo Foro), notaio Appendice, reg. 37

Giacomo detto del Fafaros di Giovanni de Alemania, socio di Francesco di Nicolino da Arco 134, 136, 137

Giacomo di Federico de Rupprechtstorff, scriba episcopi 76

Giacomo di Nichele a Silva da Levico, testimone a una confessio Appendice, reg. 27

Giacomo da Novara, canonico della cattedrale Appendice, reg. 4

Giacomo da Persichello, cancelliere del Comune di Rovereto 121

Giacomo Perozo, console del Comune 199

Giacomo di Pietro da Revò, notaio 103, 104

Giacomo da Rumo, investito di feudi 93

Giacomo Batedelus di Nicolò Batedelus da Terlago, testimone a una vendita Appendice, reg. 14

Gianpietro di Corradino da Feltre [ $m a$ Grigno, dioc. Feltre] 27, 31, 46, 106; Appendice, regg. $9,14,19,32,44,45,58,50$

Gianvittore di Burgasio da Feltre, podestà di Trento 201

Gioacchino a Mami Appendice, reg. 25

Giorgi A. 7, 18, 19, 54, 57, 72, 76, 85

Giorgio Chelum 124 e vedi Barbara da Pressano, moglie; Concio Sorn, tutore di Ulrico e Margherita di Giorgio Chelum

Giorgio di Guglielmo di Iorio da Vigolo Vattaro, notaio Appendice, regg. 40, 41

Giorgio Hilprandi, canonico della cattedrale 128

Giovanazzo di Giacomo da Terlago 31

Giovannetto di Paramusius da Povo, enfiteuta Appendice, reg. 26 e vedi Giovannino fratello

Giovanni, frate dell'ordine dei Minori, vescovo di Tino e Micone, vescovo suffraganeo di Trento 129

Giovanni, magister repetitor in gramaticalibus puerorum 61

Giovanni, speziale 42 


\section{Indice dei nomi di persona}

Giovanni di Adelpreto da Covelo del Pedegaz$\mathrm{za}$, testimone a una confessio Appendice, reg. 27

Giovanni domine Alde di Tommaso da Barbarano 28, 40, 46, 122; Appendice, regg. 12, 36, 38 e vedi Beatrice, moglie

Giovanni de Alemania, cappellano nella cattedrale, notaio e scriba presso il castello del Buonconsiglio Appendice, reg. 43

Giovanni di Antonio Bommartino, testimone a una confessio solutionis Appendice, reg. 46

Giovanni de Austria canonico, cappellano della cattedrale, pievano di Fiemme, priore dell'ospedale di San Martino 106, 151; Appendice, reg. 6

Giovanni di Bartolasio di Giovanni de Alemania, portitor 16, 132, 133 e vedi Onesta, moglie

Giovanni di Bartolomeo Bayli da Povo, testimone a una confessio Appendice, reg. 26

Giovanni di Bartolomeo da Lucca, familiaris del vicario vescovile Appendice, reg. 38

Giovanni da Bondo, notaio Appendice, reg. 28

Giovanni Briche, enfiteuta Appendice, reg. 15 $e$ vedi Michele da Zell, genero

Giovanni di Brunetto da Treviso, cantore, sacrista e cappellano in cattedrale Appendice, reg. 28

Giovanni da Calavino, notaio 79

Giovanni Caligaroti, notaio 66

Giovanni da Capistrano 33

Giovanni di Chelotus da Gabbiolo, enfiteuta Appendice, regg. 17, 26

Giovanni da Comano, iurisperitus 136; Appendice, regg. 8,50

Giovanni di Corrado da Novacella, notaio 76

Giovanni di Cristoforo da Bologna 112

Giovanni detto Darvino da Como, magister 36

Giovanni di Domenico Tabarini da Brentonico 125

Giovanni de Empach da Lana, canonico della cattedrale 129; Appendice, reg. 5

Giovanni di Enrico da Viarago, notaio 74

Giovanni di Ermanno da Mori, notaio 58, 59, 106

Giovanni detto Feragu di Stefano da Cles 38,61

Giovanni da Fondo, canonico della cattedrale 111; Appendice, regg. 5, 6, 7

Giovanni de Fraudental, cappellano della cattedrale, cancelliere del castello del Buonconsiglio, notaio 59, 106, 129
Giovanni/Ianes di Giovanni de Alemania, capitano della Torre Vanga e della porta del ponte sull'Adige Appendice, regg. 34, 37

Giovanni da Isny, decano del Capitolo 81, 111, 123, 128, 149, 150, 151; Appendice, reg. 30

Giovanni Malicie 19

Giovanni da Mori, notaio 59

Giovanni de Muta/de Carinthia/Karinthia, canonico della cattedrale 125,150, 151, 152; Appendice, regg. 6, 7, 30

Giovanni da Ottenheim/Octhayn, scolastico e canonico della cattedrale 127, 128, 129

Giovanni Pecini, honorabilioris de savioribus populi Appendice, reg. 50

Giovanni di Pietro Longino, notaio 123

Giovanni detto Rancagno di Nicolò da Sant'Ilario, portitor 16

Giovanni Rauter di Paolo notaio 27, 28, 32, 105, 140, 157, 160, 162; Appendice, regg. 19, 20, 21, 22, 50 e vedi Accerbis (de) Andriota, moglie; Buonfine, fratello; Speranza, sorella; Zambono, fratello

Giovanni di Stefano di Benvenuto di Pellegrino di Gilberto da Stravino di Cavedine, marito di Oliva, enfiteuta Appendice, reg. 24

Giovanni da Verona, rettore del convento di San Francesco 103

Giovanni Zilie, honorabilioris de savioribus populi Appendice, reg. 50

Giovanni Battista Moragium da Piacenza, pretore di Trento 202

Giovanni Enrico di Lussemburgo, conte del Tirolo 71

Giovannina, moglie di Bartolomeo di Blanchus detto de Sangue de Can da Nomi 107

Giovannino di Chous da Povo, testimone a una confessio Appendice, reg. 26

Giovannino di Giacomo Bruno di Porta San Martino in Trento, enfiteuta e testimone a un matrimonio Appendice, regg. 18, 37

Giovannino Girardi, ministro e rettore dei Battuti 39, 104

Giovannino di Marchetto detto de la Porta da Trento, notaio 101

Giovannino di Paramusius da Povo, enfiteuta Appendice, reg. 26 e vedi Giovannetto, fratello

Giovannino Rizius da Calavino, sindico Appendice, reg. 50

Giovo (da) Giovanni 112, 113, 191

Girolamo, santo 124 
Indice dei nomi di persona

Gislemberto di Cristiano detto Meiatus da Piazzo 13

Giuliana di Adelperio notaio da Denno, testimone a un testamento Appendice, regg. 1, 2

Giustiniano, imperatore 65

Giustiniano, console del Comune Appendice, reg. 25

Glasberger Giovanni di Pietro de Comitauu, diocesi di Praga, canonico della cattedrale 128

Gratatulla 19

Graziadeo di Antonio di Biagio da Castel Terlago, notaio 26, 27, 31, 79, 83, 99, 100, 101, 112, 125, 126, 140, 193; Appendice, regg. 24, 47 e vedi Barbara, figlia

Graziadeo di Nicolò Galego, notaio 64

Groff S. 7, 24, 36

Guarienti, fam. 111

Guarinoni Alessandro 11

Guglielma di Nicolò Sardagnole 82

Guglielmo, consultor 123, 124

Guglielmo da Roncegno, magister 98

Guillelmus quondam ser Otonis notarii de Clex, notaio 60

Guillielmus Ropreti, confinante 37

Gulielmus de Pomarollo, proprietario di un terreno 11

Halr Federico Appendice, reg. 43

Hamer Udalricus de Alia Saline 197

Hausberger M. 94

Hayminger David, prete della diocesi di Varmia, canonico della cattedrale 128

Hendricus da Molina di Mori, testatore 13

Hendricus da Mori, magister a scolis 61

Henricus quondam domini Petri de castro Clex 60

Hinderbach Iohannes 33, 33, 67, 201

Hulricus de Isnina, plebanus de Paho 149

Hulricus Kessler 162

Iacob di Pietro Iacob, speziale 28

Iacobus 178

Iacobus magistri Odorici fisici de Archo, satelles proditorum episcopi 24

Iacobus de la Villa, satelles proditorum episcopi 24

Ianes, laborator, famulus di Staufer notaio 199 Ianes, magister sarto 46

Iesamantus de Archo, notaio 65

Ilario, consenziente a una promessa di pagamento Appendice, reg. 13 e vedi Maria, moglie
Iliescu M. 23

Inama V. 60

Iohannes magister barberius de Arimino habitator Tridenti 38

Iohannes condam Bertholini de Edolo, affittuario di un manso 164

Iohannes Zeschi Floris de Voltolina, procuratore 156

Iohannes Benedictus de Vesentina, proditor episcopi 24

Ioppi R. 72, 76, 99, 102, 202, 204, 206

Iorio di Desiderato da Ala, notaio Appendice, reg. 3

Iorius magistri Iohanis Luce, satelles proditorum episcopi 24

Iosiis (de) Iosio 96

[Iosiis (de)] Iosio di Francesco notaio di Iosio phisicus 109, 191; Appendice, regg. 28, 31,32

[Iosiis (de)] Iosio di Giordano phisicus da Trento 12

[Iosiis (de)] Tommaso di Giordano phisicus da Trento 12

Ippoliti G. 82; Appendice, reg. 3

Isidoro da Milano, canonico Appendice, regg. $30,43,44,45,46$

Jemolo V. 166

Kabriel laborator 18

Keller Osvaldo di Giovanni, detentore di prebenda 129

Kol Bertoldo, prete 126

Lambertini Giambattista 200

Landi W. 36

Lando Bartolomeo 43

Layner Clara di Giovanni da Termeno, moglie di Rigo da Francoforte 28; Appendice, reg. 37

Layner Giovanni di Ulrico da Termeno Appendice, reg. 37

Legatis (de) Iohannes 164

Lena di Bono da Nomi, moglie di Pancera da Sardagna Appendice, regg. 1, 2

Leonardelli F. 33, 39

Leonardi Ivo 166, 168, 170, 177, 204

Leonardo di Endrigo da Roncogno di Pergine, testimone a carta dotale Appendice, reg. 49

Leonardo da Gardolo, honorabilioris de savioribus populi Appendice, reg. 50

Leonardo, genero di Nicolò a Sale 125 


\section{Indice dei nomi di persona}

Leonardo di Perozzo apothecarius da Firenze, testimone a una sentenza Appendice, reg. 39

Leonardo da Piné, notaio Appendice, reg. 50

Leonardo da Sprè, notaio 125

Leonardus Trivisanus, affittuario 164

Libeceltis (a) Cristoforo, satelles proditorum episcopi 24, 97; Appendice, reg. 50

Liechtenstein Georg 7, 17, 20, 25, 27, 31, $39,41,46,56,76,78,81,83,84,93$, $117,192,195$

Liechtenstein Ianes, consigliere di Federico duca d'Austria Appendice, reg. 5

Lippi (de) Giovanni di Luca da Trento 24, 25, 28, 42, 58, 97, 106, 125, 128; Appendice, regg. 22,50

Lippi (de)/de Cirogicis Luca di Giovanni di Luca, canonico della cattedrale, notaio, proditor episcopi, iurisperitus 24, 31, 95, 106, 128, 196, 197, 208; Appendice, reg. 50

Little L.K. 14, 18

Locholum/Tocolum Giulio da Parma, pretore di Trento 202

Lodron Paride 82; Appendice, reg. 29

Loisio di Cristoforo detto dal Dosso, notaio 36, 80; Appendice, reg. 3

Lola di Andrea dal Cantone di Trento, testatrice 106

Luca di ${ }^{* * *}$ da Firenze, oste in Trento Appendice, reg. 1

Luca di Matteo da Bolzano, portitor 16

Luchino de Gragnano, console del Comune 199

Lupo M. 30, 33, 34

Lutterio di Bonaventura Zanzarelus da Sopramonte, testimone a una locazione Appendice, reg. 29

Luzzi S. 7, 24, 29, 36

Maddalena di Antonio da Borgonuovo, moglie di Bonaventura Calepini 29, 33, 37, 38, 46 Appendice, reg. 42

Maddalena, moglie del notaio Pietro Longino 123

Maffeo di Negro da Brescia, apothecarius 32, 46, 132; Appendice, regg. 12, 35, 50

Magagnato L. 43, 65

Magna carner, satelles proditorum episcopi 24

Maino L. 15, 16

Maleferatis (de) Giovanni, console del Comune 199

Malfatti S. 18, 54, 86, 111, 194
Malfer Giovanni di Antonio 202

Manci Sigismondo Antonio 23

Manfredo da Verona, maestro di grammatica 59

Mangini M.L. 61

Maniaci M. 166

Mansatoribus (de) Iohannes dictus Zoanella quondam Nicolai 13

Marcabruno di Belloto da Santorso, portitor 15

Marco, cappellano della cattedrale, pievano di Livo, priore e rettore ospedale di San Tommaso a Romeno 104; Appendice, reg. 6

Marco detto Barozzo di Orso da Lizzana, radarolo Appendice, reg. 29

Marco di Hendricus da Molina di Mori 13

Marco da Lusiana, tutore di Guglielma, figlia di Nicolò Sardagnole 82, 112; Appendice, reg. 39

Marco di Odorico di Giacomo da Spormaggiore, notaio 39, 74, 76, 88, 108; Appendice, reg. 1

Marco di Odorico da Trento, notaio 56

Marco domine Zinele/dela Zinella di Valentino dal Borgonuovo, notaio e buletarius 13, 19, 32, 191, 204; Appendice, regg. 33, 35,95

Marcus dictus Faytelus de Burgonovo de Tridento, carator 132

Margarayder Achazius Appendice, reg. 37

Margherita di Giovanni Brasadole da Pomarolo, moglie di Melchiorre di Nicolò Rizato 200

Margherita detta Stanfferina di Nichele a labro scisso dalla contrada di San Marco Appendice, reg. 27

Maria vedova di Antonio Zagagnini, moglie di Ilario e madre di Francesca, debitrice a una promessa di pagamento Appendice, regg. 12,13

Marquardo da Bamberga, beneficiato 127

Marquardus 193

Martino murarius, honorabilioris de savioribus populi Appendice, reg. 50

Martino di Francesco da Volano, notaio 58, 74, 82, 90, 98, 101; Appendice, reg. 33

Martino di Giovanni Ceraduce dal Tesino, notaio 56

Martino da Novara, mansionario 86

Matsch (von) Wilhelm 42, 47; Appendice, regg. 5,8

Matteo di Filippo da Sfruz, portitor 16

Matteo da Riva, professor artis gramatice 61 
Indice dei nomi di persona

Matteo di Ulrico da Monaco di Baviera, canonico della cattedrale 129

Mattivi S. 12, 14, 22, 86, 197

Mazurelle D. 166

Melchiorre di Domenico da Sasso di Vigolo Baselga 45

Melchiorre di Leonardo sarto da Trento, notaio 38

Melchiorre di Nicolò Rizato 200 e vedi Margherita moglie

Meo di Giovanni da Pistoia, console di Trento Appendice, reg. 38

Mercadenti, fam. 25, 208

Mercadenti Mercadento di Francesco de Lost Appendice, reg. 3

Mercadenti Nicolò 28, 46, 78

Merlo G.G. 39, 71

Metallis (de) Giacomo di magister Maffeo cirogicus di Gargnano 58

Mezaoveta Aldrighetto di Giovanni da Pomarolo 28, 107; Appendice, regg. 9, 10, 11, 29, 35, 107 e vedi Gallo Lucia, moglie

Mezaoveta Giovanni 28, 42, 46

Mezaoveta Tomeo Appendice, reg. 50

Mezzasoma, fam. 25, 29, 94, 208

Mezzasoma Agnese di Gioacchino 27

Mezzasoma Giacoma di Gioacchino 27

Mezzasoma Gioacchino di Pietro da Trento $25,27,28,29,37,54,58,72,82,94$, $97,105,110,112,138,139,140,141$, 142, 208; Appendice, regg. 9, 19, 20, 22, 34 e vedi Speranza di Paolo [di Martino] notaio, moglie

[Mezzasoma] Pasqualina [di Pietro] uxor Marchi de Castro Campi 140, 141

Mezzasoma Pietro di Gioacchino 74, 87, 95, 101

Michael detto Rauschella da Appiano, locatario 124

Michele di Andrea da Termeno, portitor 16

Michele di Domenico da Cortesano, emptor 109; Appendice, reg. 31

Michele Fenutoli di Bartolomeo da Sporminore, oste 36, 140; Appendice, regg. 35,50

Michele de Orzano da Civezzano, sindico Appendice, reg. 50

Michele da Povo, canonico della cattedrale Appendice, reg. 5

Michele da Trento, piliparius, 46; Appendice, reg. 38

Michele da Zell, enfiteuta Appendice, reg. 15 $e$ vedi Giovanni Briche, suocero
Midana a caliginis, honorabilioris de savioribus populi Appendice, reg. 50

Migazzi Antonio 199

Migliorati Cosma 90

Milauner Henricus de Merano, arcidiacono e canonico della cattedrale 149

Mira G. 15

Molveno (da), fam. 25, 27, 29, 30, 208

Molveno (da) Antonio 23, 25, 27, 40, 42, 46, 47, 46, 57, 58, 59, 74, 76, 81, 89, 100, 106, 108, 138, 140, 141, 190; Appendice, regg. 8, 9, 22, 35, 38 e vedi Floridia di Tommaso da Folgaria, moglie

Molveno (da) Cristoforo di Antonio 63, 65, 100,101

Molveno (da) Francesco di Bonaventura 74, 76, 108, 109; Appendice, regg. 1, 2

Molveno (da) Giovanni di Antonio 109

Molveno (da) Guglielmo di Antonio 109

Molveno (da) Marcabruno di Francesco 76

Molveno (da) Odorico di Sicherio Appendice, regg. 1,2

Morandino di Giovanni Bono da Trento, iurisperitus 31, 105; Appendice, reg. 32

Morandino da Trento, canonico della cattedrale 19, 36, 80, 122, 123; Appendice, regg. 3, 4

Morelli M. 166

Morizzo M. 39

Moscadelli S. 7, 57, 72, 85

Mošin V.A. 168, 169

Motter M. 71

Mucius baraterius de Tridento 36

Mura A. 94

Murlinis (de), fam. 25, 208

Murlinis (de) Cristoforo di Matteo Appendice, regg. 48,49

Murlinis (de) Gaspare Appendice, regg. 6, 7

Murlinis (de) Giovanni Battista 66

Murlinis (de) Matteo di Clemente 28, 79, 112; Appendice, regg. 25, 33, 48, 49, 50 e vedi Bartolomea, moglie

Murlinis (de) Nicolò Appendice, reg. 4

Murlinis (de) Rambaldo di Clemente 112; Appendice, reg. 50

Mussato Girolamo 197

Navibus (a) Giacomo Appendice, reg. 4

Navibus (a) Giovanni detto Zibechino di Riprando 38; Appendice, reg. 4

Negrello di Alberto notaio da Sacco, notaio Appendice, reg. 12

Negri (de), fam. 7; Appendice, reg. 8

Negri (de) Leone 24; Appendice, reg. 8 
Indice dei nomi di persona

Negri (de) Negro 41, 42, 46, 47, 84; Appendice, regg. 5, 8, 25, 29 e vedi Constantia, moglie

Nichele Bragalda da Povo, testimone a una confessio Appendice, reg. 26

Nicholaus de Margono 201

Nicolaus ad Cantonum, satelles proditorum episcopi 24

Nicolaus de Fundo, proditor episcopi 24

Nicolaus Galefi, satelles proditorum episcopi 24

Nicolaus quondam ser Otonis notarii de Clex, notaio 60

Nicolino di Morzanto da Sopramonte, testimone a una carta dotale Appendice, reg. 49

Nicolò barberius Appendice, reg. 50

Nicolò a torcularibus dal Borgonuovo di Trento, di Antonio da Isera, testatore 16

Nicolò di Antonio da Isera, portitor 15

Nicolò da Arco, notaio 82

Nicolò Basileus da Trieste, podestà di Trento 200

Nicolò di Benvenuto da Coredo, speziale 42

Nicolò di Bonaventura da Volano, notaio 71, 75,86

Nicolò da Breslavia, canonico della cattedrale 125

Nicolò da Brno, vescovo di Trento 71

Nicolò dalla val di Fassa, vicario e rettore della pieve di Meano 127

Nicolò Fridele, console del Comune Appendice, reg. 25

Nicolò di Giovanni de Ligatis da Serso 191

Nicolò Goseto dal Borgonuovo, suocero(?) di Antonio da Borgonuovo 37

Nicolò Leonis da Venezia, canonico della cattedrale 129

Nicolò da Mantova, guardiano del convento di San Francesco 90

Nicolò/Nicolino da Mechel, notaio e rector schollarum 60

Nicolò di Ognibene sarto da Borgonuovo, notaio $95,99,101,123$

Nicolò Rizato, testimone a una sentenza Appendice, reg. 39

Nicolò detto Rubeus di Giacomo da Folgaria, laborator 15

Nicolò Schichignolus di Alberto, testimone a un testamento Appendice, reg. 1

Nicolò da Trento, cappellano della cattedrale e pievano in Tignale Appendice, regg. 28,30

Nicolò da Trento, notaio 71, 75, 86
Nicolò da Trento, phisicus 27

Nicolò da Venezia, pievano in Sanzeno 129; Appendice, reg. 28

Odorico a fecibus/de le fesse 24, 28, 32, 42; Appendice, regg. 9, 50

Odorico, monachus nella Prepositura Appendice, reg. 34

Odorico, phisicus 46

Odorico di Bartolomeo Gatabriga da Vigolo Vattaro, testimone a un matrimonio Appendice, reg. 36

Odorico di Federico da Povo, notaio 24, 46, 106, 112; Appendice, regg. 44, 45, 48, 49

Odorico di Giacomo da Amblar, testimone a una donazione Appendice, reg. 47

Odorico di Guglielmo da Brez, notaio 193, 197

Odorico di Nicolò Zilie, honorabilioris de savioribus populi Appendice, reg. 50

Odorico detto Pizolo, notaio 123

Odorico da Pomarolo, magister 19

Odorico di Rosso, becarius, notaio Appendice, reg. 1

Ognibene di Adelperio da Trento, notaio 13

Ognibene di Azzio da Calliano, emptor 36, 38; Appendice, reg. 7

Ognibene di Pellegrino da Castellano, portitor 15

Oleo (ab), fam. 29, 208

Oleo $(a b)$ Bertoldo 19

Oleo (ab) Melchiorre di Domenico 24, 25, 33, 43, 46, 104, 106, 134; Appendice, regg. 47,50

Olmi G. 200

Onesta magistri Odorici cerdonis de Tridento, moglie di Giovanni di Bartolasio di Giovanni de Alemania 132, 133 e vedi Bertoldo fratello

Oradini C. 13

Orioli L. 15

Ornato E. 166

Ortalli G. 16, 55, 60

Osvaldo, pievano di Santa Maria di Baselga di Sopramonte 127

Osvaldo detto Sengel da San Michele all'Adige, vicario generale del conte del Tirolo e ius publice redens in civilibus et criminalibus causis in toto comitatu Cunespergi nec non in Cimbrie iurisdictionis 191

Otolinus, conductor 192

Ottolino di Paolo cursor, sarto 108, 109, 213; Appendice, regg. 1, 2

Ottolino di Trentino, notaio Appendice, reg. 1 
Pace, maestro di grammatica 59

Pagnin B. 126

Palamidesiis (de) Palamidesio di Giacomo di Palamidesio 27, 109; Appendice, regg. 19,50

Pamato L. 20, 39

Paolo di Aldrighetto da Brentonico, portitor 16

Paolo de Levi dalle Giudicarie Appendice, reg. 28

Paolo di Martino da Trento, notaio 27, 78; Appendice, reg. 5

Papaleoni G. 23, 24, 30, 48

Parcianello F. 16

Pasqua di Mercadento da Levico, moglie di Alberto Bonomi, locataria 122

Passera Giacomo Appendice, reg. 50

Passera Isepo, rettore della confraternita dei Battuti 33

Patheriis (de) Francesco da Parma 66

Pederzolo, familiaris del vicario vescovile Appendice, reg. 34

Pegoretti C. 23, 30, 31

Pellegrina, moglie di Stefano da Cles 61

Pellegrino di Bertoldo detto Scherp, enfiteuta Appendice, reg. 26

Perengerio da Castelfondo/de Melango, cappellano e sacrista della cattedrale, pievano di Malé 151, 195

Perozzo di Angelo da Firenze, apothecarius Appendice, regg. 22, 30

Petrucci A. 166

Petrus de Burmo, confinante con la casa di Antonio da Borgonuovo 38

Petrus de Matarelo, confinante con una proprietà 150

Phignonclus vedi Fignoclus

Piccard G. 168, 169

Pietro di Ambrogio da Milano, sarto Appendice, reg. 37

Pietro a caminis di Giovanni dal Trevigiano, murarius a caminis, stazonerius 109; Appendice, regg. 12, 31, 32, 36

Pietro detto a Caseo di Martino da Campo Lomaso, testimone a una compravendita 15

Pietro de Drageto de Terra Laboris, vicario della pieve di Denno Appendice, reg. 7

Pietro a Fossis dal Borgonuovo, testatore 18 Pietro di Guglielmo Merino da Levico, testimone a confessio Appendice, reg. 27

Pietro di Nanni da Siena, mercante e banchiere 108, 110, 111, 112, 113, 179, 181; Appendice, regg. 1, 30, 43, 44, 45, 46
Pietro di Paolino da Arco, stazonerius Appendice, reg. 47

Pietro da Parma, pievano di Santa Maria Maggiore in Trento 109; Appendice, regg. 1, 2

Pietro da San Bonaventura, prete Appendice, reg. 1

Pietro da Spormaggiore, notaio 199; Appendice, reg. 50

Pietro di Ulmannus de Huleym, prete 127

Pietro Iacob di Rigo da Rovereto, procuratore $19,27,28,40,46,58,103,106,108,110$, 111, 112; Appendice, regg. 19, 22, 32, 39, 43, 44, 45, 48, 49 e vedi Antonia, figlia

Pietro Longino, notaio 123 e vedi Maddalena, moglie

Pietro Paolo di Giovanni macellaio detto Ianes, notaio $76,98,99$

Piperatis (de) Giovanni di Antonio Appendice, reg. 34

Pisoni Perisonus di Francesco da Riva del Garda Appendice, reg. 29

Pizzini de Thürberg Giovanni Giacomo 203

Plata (a) Michele di Huele da Cortaccia 109, 110; Appendice, regg. 31, 34, 39, 43

Polli G. 11, 12, 13, 100

Pona Francesco Appendice, reg. 11

Pona Giovanni Antonio 58

Powondra Tomaso Giuseppe 204

Prandi Franceschino de Luero Appendice, reg. 50

Prehemer Corrado, canonico della cattedrale e pievano di Flavon 128

Prevenier W. 57

Pugliese Carratelli G. 85

Pugnis (de) Giovanni da Parma 108; Appendice, reg. 1

Puncuh D. 57

Quaglioni D. 7, 16, 56, 57

Rainaldo, maestro di grammatica 59

Rambaldo da Trento, decano del Capitolo della cattedrale 64, 84; Appendice, reg. 4

Rando D. 71, 74

Rasmo N. 30, 33, 34, 37

Reich D. 27, 39, 61

Ressegotti D. 39, 48, 49

Ricci I. 76

Rigo di Domenico da Valeggio di Verona, apothecarius Appendice, regg. 17, 18

Rigo da Francoforte, oste dell'osteria alla Corona a Trento 25, 28; Appendice, reg. 37 e vedi Layner Clara moglie 


\section{Indice dei nomi di persona}

Rigon A. 39, 126

Rizzo S. 147

Roccabruna, fam. 25

Roccabruna (da) Antonio 46

Roccabruna (da) Bartolomeo 46

Roccabruna (da) Giacomo di Enrico 42, 199; Appendice, reg. 3

Roccabruna (da) Vigilio di Guglielmo da Seregnano, notaio 36, 80; Appendice, regg. 3,50

Rodolfo di Basino da Trento, notaio 12, 74

Rodolfo di Gianpietro da Feltre [ $m a$ da Grigno, diocesi di Feltre] Appendice, reg. 46

Rolandino Passeggeri 53, 54, 65, 66

Romano di Giacomo da Padova, contendente in una lite 122

Romiti A. 143, 147

Ropretus quondam Venturini de Clex 60

Rossi G. 39

Rossi M.C. 15

Rugerio di Nicolò de Apulia, prete 127

Ruphalcatis (de) Giacomo da Arco 96

Ruphalcatis/Ruphalchatis (de) Odorico da Arco 28, 42; Appendice, reg. 22

Sale (a), fam. 29

Sale (a) Leonardus 65

Sale (a) Nicolò di Odorico da Pergine 40, 96, 125; Appendice, reg. 12 e vedi Leonardo, genero

Sale (a) Odorico di Nicolò da Ischia di Pergine 13

Salomone, vescovo di Trento 57

Sancassani G. 43, 65

Sandrino cerdo/chaliar, giudice delle tutele 199; Appendice, reg. 50

Santarelli L. 30, 33

Santifaller L. 83, 85, 86; Appendice, reg. 28

Sardagna Giulio 204

Saur Nicolò Appendice, reg. 34

Savigny (von) F.C. 53

Sbarzpniger Ulrich Appendice, reg. 37

Schiera P. 20, 41

Schratemperger Odorico Appendice, reg. 25

Schrattenberg Antonio 200

Seldenhorn Nicolò di Enrico, chierico della diocesi di Costanza, canonico della cattedrale 128

Senftel/dala Muda/a Muta Michael di Ludovico da Monaco di Baviera 24, 25, 26, 29, 125; Appendice, regg. 14, 15, 16, 17, $18,24,25,26,27,50$ e vedi Calepini Onesta, moglie
Serafino di Antonio Secadinari da Mezzolombardo, testimone a una confessio Appendice, reg. 18

Sibilia di Chierico da Montecchio Precalcino, testimone a un testamento Appendice, regg. 1,2

Sicco da Trento, canonico della cattedrale Appendice, reg. 4

Sicco Polenton 61

Sich Giovanni Appendice, reg. 47

Sich Maria Appendice, reg. 47

Sicherio di Michele da Vezzano, notaio 72

Sichis (de) Francesco di Adelperio da San Martino 25, 40, 46, 58, 96, 111, 178; Appendice, regg. 15, 35, 38, 50

Sichis (de) Marco di Franceschino Appendice, reg. 5

Sigismondo di Lussemburgo, imperatore 57, 78,106

Simeone di Guglielmo da Vattaro, sindico Appendice, reg. 50

Simeone da Ravina di Federico da Terragnolo, testimone a un matrimonio Appendice, reg. 36

Simeone di Tommasino da Vervò, testimone a una compravendita Appendice, reg. 31

Simone de Cambo, sindico dei Battuti 108

Sinisi L. 54, 65

Sobniowski Stanislao di Giovanni 78, 84, 102, 127, 204; Appendice, reg. 28

Soffici M. 170

Sofia di Pietro da Isera, moglie di Odorico Calepini 32

Speranza di Paolo [di Martino] notaio da Trento, sorella di Giovanni Rauter, moglie di Gioacchino Mezzasoma 27; Appendice, regg. 19, 20, 21

Sporo (da) Petrus Brusius, proditor episcopi 24

Stanchariis (de) Alberto 62

Stanchariis (de) Pietro di Stefano da Teglie di Brescia, notaio $86,87,88,89,191,192$, 193, 194, 195, 208

Stasio di Andrea da Firenze, apothecarius Appendice, regg. 37, 50

Staufer/Stanfar/Stanfer/Stamfar Iohannes 198, 199, 200

Stefano da Cles di magister Trentino, doctor artis gramatice 60, 61 e vedi Gasperina di; Giovanni detto Feragu di; Pellegrina, moglie

Stenico M. 16, 18, 27, 33, 39, 41; Appendice, reg. 5

Stenico R. 27, 33, 111, 190, 201 
[Sratinperger] Odorico detto Stratenperger di Ottone 27, 46; Appendice, regg. 19, 38, 50

Sumptag Antonio di Sumptag da Gardolo di Mezzo 38; Appendice, reg. 42

Sumptag Federico di Sumptag da Gardolo di Mezzo 38; Appendice, reg. 42

Sznura F. 170

Tagliaferri A. 43

Tamba G. 56, 57, 64

Tanuciis (de) Nicolò da Levico, legum doctor e vicario vescovile 192, 195

Tassoni Ercole 200, 202, 205

Tavilla E. 64

Terlaco Marele da Terlago, detentore di un affitto perpetuo 201 e vedi Flordela, moglie

Thomas da Friesach detto Vasthel, notaio 78

Thomasiis (de) Prospero da Cremona, preposito di Santa Maria all'Arena di Padova 125,129

Thun Baldessarre 125; Appendice, reg. 28

Thun Erasmo di Vigilio 41, 84, 109, 110, 156; Appendice, regg. 25, 29, 31, 34, 39, 43

Thun Guglielmo di Vigilio 109, 110; Appendice, regg. 31, 34, 39, 43

Thun Ianes di Erasmo Appendice, reg. 25

Thymel/Taymel Narciscus/Narcischus de Nurlinga, familiaris del decano del Capitolo della cattedrale 149

Tibaldo dalla Valsugana, pievano a Meano 126

Tocolum vedi Locholum

Todeschina di Guglielmo di Giustiniano [di Gardolo], moglie di Giovanni Belenzani 108, 109; Appendice, regg. 1, 2

Toma, pievano a Thaur Appendice, reg. 30

Tomasi B. $19,85,87$

Tomeo dal Tesino, magister fisicus 58; Appendice, reg. 32

Tommaso, pievano di Santa Maria in Castelfondo 127

Torelli P. 57

Tosabech (de) Antonio da Pavia 127, 132; Appendice, regg. 43, 47

Tovazzi Giangrisostomo 11, 12, 33, 34, 101

Trahugis (de) Vigilio da Povo, giudice 82

Traljič S.M. 168, 169

Trapp Carlo Giuseppe Sebastiano 203

Trechis (de) Venturino di Antonio da Mantova, notaio $54,62,86,89,101,149$, 191, 194, 210

Trentino detto a Sale di Bonaventura da Mori, notaio 8
Trentino di Nicolò Chechi da Meano, sindico Appendice, reg. 50

Trentino di Zuccolino da Tuenno, notaio 71, 75,86

Trissino (da) Nicolò di Bongiacomo 129

Truus Ulrich Appendice, reg. 37

Tura detto Turata di Trentino a Ranto(?) da Trento, portitor 14

Uborch, familiaris del vescovo Alessandro di Masovia 32

Ulrico Kuker, chierico della diocesi di Augusta 127

Ulrico da Isny, canonico e arcidiacono della cattedrale 149; Appendice, regg. 6, 7

Ulrico, pievano a Meano 127

Ulricus de Isna, familiaris Iohannis de Flemis 149

Ursulinis (de) Giacomo da Tossignano di Bologna Appendice, reg. 47

Vaginis (a) Lorenzo di Giovanni da Feltre 19; Appendice, regg. 12, 14, 36

Vaginis (a) Matteo 199

Vaginis (a) Vricius 33

Valenti E. 19, 24, 26, 32, 46, 96

Vanga Federico 71, 72

Varanini G.M. 7, 8, 17, 19, 20, 22, 24, 25, $27,38,43,46,55,57,63,64,65,66,76$, 93, 94, 98, 190, 196, 199

Vareschi S. 17

vescovi di Trento vedi Adelpreto; Alberto di Ortenburg; Alessandro di Masovia; Beseno (da) Corrado; Campo (da) Alberto; Enrico di Metz; Giovanni, frate dell'ordine dei Minori, vescovo di Tino e Micone, vescovo suffraganeo di; Hinderbach Iohannes; Liechtenstein Georg; Nicolò da Brno; Salomone

Vigilio detto Barbustelo da Trento, macellaio Appendice, reg. 11

Vigilio di Enrico detto Rigaie, notaio 190, 191; Appendice, regg. 1, 3

Vigilio Gardeline, speziale Appendice, reg. 50 Vigilio Morzati da Pergine 46

Vigilio da San Vigilio, cerdo Appendice, regg. $25,29,35$

Vigilio Saraceno, notaio 47

Vigilio da Trento, canonico della cattedrale 150, 151; Appendice, reg. 4

Vigilio Turcheti, vicecappellano della cattedrale Appendice, reg. 30

Vigilius Iohannis Laurentii de [...] 197 
Indice dei nomi di persona

Vitaliani A. 43

Vito da Dambel, notaio 190

Vito di Pietro da Amblar, notaio Appendice, reg. 17

Vivamento da Caldes, testimone a un mandato vescovile Appendice, reg. 25

Volek Giovanni, vescovo di Olmütz 71

Voltelini (von) H. 41

Vricius da Rovereto, honorabilioris de savioribus populi Appendice, reg. 50

Weber S. 16, 59, 60, 61

Weijers O. 147

Weinecker Percivalle di Ingenuino da Cortaccia, canonico della cattedrale 129

Welber M. 16, 18, 41

Wolchestein Teobaldo di Michele, canonico di Bressanone e Zurigo 128

Woś J.W. 17

Ysabeta, moglie di Michele Approvini da Borgonuovo 19
Zambelli Giovanni Battista 204

Zamboni L. 22, 27, 38, 54, 88, 89; Appendice, reg. 4

Zambono [di Paolo] da Trento, canonico della cattedrale, fratello di Giovanni Rauter 27, 92, 150; Appendice, regg. 7, 19, 21

Zampetrus de Papia, sarto 149, 150, 151, 152

Zanella G.B. 16, 17, 33

Zanolini Vigilio 170, 177, 204

Zatelli A.M. 82; Appendice, reg. 3

Zeiss Giovanni da Bopfingen 104, 124, 129, 150, 170, 194; Appendice, regg. 7, 30

Zeiss Giovanni iuvenis 127

Zeno murarius Appendice, reg. 50

Zeno dalla Polonia, canonico della cattedrale 128

Zeschele di Federico da Rovereto, cerdo Appendice, regg. 36,50

Zevolis (de)/Zivolis/Zivollis/Civolis Antonio da Ledro 81, 110, 190; Appendice, regg. 34,39 


\section{Indice dei nomi di luogo}

L'indicizzazione è stata fatta sulla base della denominazione attuale del luogo con eventuale indicazione della forma che figura nel documento, posta dopo il nome della persona, solo nel caso di sostanziali difformità, senza rinvio (ad esempio: Kutná-Hora (Rep. Ceca) vedi Corrado Friderici Greusseri de Monte Kuctis).

Il rinvio è al numero di pagina e per l'Appendice dei regesti al numero del documento.

Ala vedi Iorio di Desiderato da

Albiano vedi Federico di Enrico da; Francesco di Endrico da

Alemania vedi Corrado de; Giacomo detto del Fafaros, figlio di Giovanni de; Giovanni de; Giovanni di Bartolasio di Giovanni de; Giovanni/Ianes di Giovanni de

Amblar vedi Odorico di Giacomo da; Vito di Pietro da

Appiano/Eppan (Bz) 194 e vedi Michael detto Rauschella da; chiesa di San Paolo 126; Mas dal Mont 124; Mas dala Poza 124

Apulia vedi Puglia

Arbano vedi Erbanno

Arco 65 e vedi Antonio da; Antonio di Bonomo da; Antonio Prevedonus di Giacomo a rotis da; Bartolomeo da; Bonomo da; Domenica vedova di Bonomo da; Francesco di Nicolino da; Giacomo da; Iacobus magistri Odorici fisici de; Iesamantus de; Nicolò da; Pietro di Paolino da; Ruphalcatis (de) Giacomo da; Ruphalcathis/ Ruphalchatis (de) Odorico da

Arimino vedi Rimini

Arzill vedi Cavedine, loc.

Augusta (Germania) vedi Denklinger Martinus de; Enrico di Odorico de Velden, canonico di; Ulrico Kuker, chierico della diocesi di

Austria vedi Giovanni de

Avisio, fiume Appendice, reg. 29

Bamberga (Germania) vedi Marquardo da; Czoppot Corrado, chierico della diocesi di
Barbarano (Vi) vedi Giovanni domine Alde di Tommaso da

Basilea (Svizzera) Appendice, regg. 50, 51; concilio 95

Bergamo vedi [Della Torre] Pietro de la Ture da

Bologna 66 e vedi Antonio di Pietro da; Bartolomeo da; Battista di Silvestro da; Giovanni di Cristoforo da

Bolzano 46, 71, 140; Appendice, reg. 38 e vedi Luca di Matteo da; cappella di Sant'Andrea 126

Bondo vedi Giovanni da

Bondone, monte 81

Bopfingen (Germania) vedi Anhang Giovanni da; Zeiss Giovanni da

Bosentino vedi Bartolomeo di Belino da

Brandeburgo vedi Federico di

Breguzzo, castello Appendice, reg. 29

Brentonico Appendice, reg. 7 e vedi Giovanni di Domenico Tabarini da; Paolo di Aldrighetto da

Brescia vedi Bonacordo di Maffeo da; Faustinus filius Mafei de; Maffeo di Negro da

Breslavia vedi Wrocław

Bressanone/Brixen (Bz) vedi Bertoldo, vescovo di; Friling Goffredo [de Oyta] da; Wolchestein Teobaldo di Michele, canonico di Zurigo e di

Brez 65 e vedi Antonio di Gotmanino da; Odorico di Guglielmo da

Brno (Rep. Ceca) vedi Nicolò da

Cadore, territorio 62 e vedi Giacomo da 
Indice dei nomi di luogo

Calavino vedi Francesco da; Gaisis (de) Giovanni Giacomo di Giovanni da; Gaisis (de) Giovanni da; Giovanni da; Giovannino Rizius da

Caldaro/Kaltern (Bz) 194

Caldes vedi Vivamento da

Calliano 22 e vedi Ognibene di Azzio da

Camerino (Mc) vedi Bartolomeo da

Campo Lomaso vedi Pietro detto a Caseo di Martino da

Campo Trentino (Gardolo) 95

Campo, castello nelle Giudicarie 111, Appendice, reg. 46

Carinzia 78 e vedi Artuico dalla; Giovanni de

Castel Beseno 27

Castel Romano vedi Giacomo da

Castel Terlago vedi Barbara di Graziadeo di Antonio di Biagio da; Graziadeo di Antonio di Biagio da

Castel Thun 60

Castelfondo vedi Perengerio da Castelfondo/ de Melango, cappellano e sacrista della cattedrale, pievano di Malé; pieve di Santa Maria vedi Tommaso, pievano di

Castellano vedi Ognibene di Pellegrino da

Cavedine, loc. Arzill Appendice, reg. 24

Cembra 191 e vedi Corrado de Alemania, pievano in Civezzano e vicario in; Osvaldo detto Sengel da San Michele all'Adige, vicario generale del conte del Tirolo e ius publice redens in civilibus et criminalibus causis in toto comitatu Cunespergi nec non in

Cere (al) vedi Terlago

Chiusa/Klausen (Bz) vedi Corrado da

Chiusole (Pomarolo) vedi Bonifacio da; Francesco di Matteo da

Civezzano 100 e vedi Corrado de Alemania, pievano in; Donato di Delaito da; Michele de Orzano da

Cles 60 e vedi Gasperina di Stefano da; Giovanni detto Feragu del professor gramatice Stefano da; Guillelmus quondam ser Otonis notarii de; Nicolaus quondam ser Otonis notarii de; Pellegrina, moglie di Stefano da; Ropretus quondam Venturini de; Stefano da

Clesura vedi Trento, località extraurbane, Graffiano; Povo

Comano vedi Giovanni da

Chomutow (Rep. Ceca) vedi Glasberger Giovanni di Pietro de Comitauu

Como vedi Bernabono di Alberto da; Capuzio
Cristoforo di Tommaso da; Giovanni detto Darvino da

Coredo vedi Nicolò di Benvenuto da

Cortaccia/Kurtatsch (Bz) vedi Plata (a) Michele di Huele da; Weinecker Percivalle di Ingenuino da

Cortesano vedi Michele di Domenico da

Costanza (Germania) vedi Seldenhorn Nicolò di Enrico, chierico della diocesi di

Covelo del Pedegazza (Terlago) vedi Giovanni di Adelpreto da

Cremona vedi Thomasiis (de) Prospero da

Dambel vedi Vito da

Děčín (Rep. Ceca) 60

Denno vedi Florio da; Giuliana di Adelperio notaio da; Pietro de Drageto de Terra Laboris, vicario della pieve di

Dosso di Calonegi (al) vedi Trento, località extraurbane, Graffiano

Dresium (Dresio, Vb?) 60 e vedi Benevenutus quondam Degeleguardi de

Dro vedi Bertoldo di Bonomo da

Edolo (Bs) vedi Iohannes condam Bertholini de

Eger (Ungheria) vedi Erardo da

Egna/Neumarkt $(\mathrm{Bz})$ vedi Giacomo da Novo Foro

Erbanno (Bs) vedi Federicis (de) Giacomino de Arbano

Fadano (Brentonico) vedi Bartolomeo di Ognibene da

Fai vedi Antonio di Bongiovanni da; Balistis (a) Giovanni detto Mosca di Bartolomeo detto Mastelus da

Fassa, valle vedi Nicolò dalla

Feltre vedi Agostino di Corradino da; Antonio da; Gauslinis (de) Lorenzo da; Gianpietro di Corradino da; Gianvittore di Burgasio da; Rodolfo di Gianpietro da; Vaginis (a) Lorenzo di Giovanni da

Ferrara vedi Federico di Andrea da; Francesco da

Fersina, torrente 13

Fiavé 30

Fiemme, valle 150 e vedi Ulricus de Isna, familiaris Iohannis de

Firenze vedi Andrea di Bernardo da; Leonardo di Perozzo apothecarius da; Luca di *** da; Perozzo di Angelo da; Stasio di Andrea da 
Indice dei nomi di luogo

Flavon vedi Gerardo da Verona, pievano in; Prehemer Corrado, canonico della cattedrale e pievano di

Folàs (Isera) 22

Folgaria vedi Fina da; Floridia di Tommaso da; Nicolò detto Rubeus di Giacomo da

Fondo vedi Francesco da; Giovanni da; Nicolaus de

Fontana (a la) vedi Trento, località extraurbane, Vigolo Baselga

Francoforte (Germania) vedi Rigo da

Freudental (Germania) vedi Giovanni de Fraudental

Friesach (Austria) vedi Thomas da

Fulan (a) vedi Trento, località extraurbane, Gabbiolo

Gabium sive Agilon vedi Trento, località extraurbane, Gabbiolo

Gardas (ad) vedi Trento, località extraurbane, Romagnano

Gargnano (Bs) Luchino de Gragnano; Metallis (de) Giacomo di magister Maffeo cirogicus di

Giudicarie, valle vedi [Carioli] Giacomo di Cariolo dalle; Paolo de Levi dalle

Gozador (a) vedi Trento, località extraurbane, Mesiano

Grigno 66 e vedi Agostino di Corradino da; Antonio da; Gianpietro di Corradino da; Rodolfo di Gianpietro da; Francesco di Vittore da

Hall in Tirol (Austria) vedi Hamer Udalricus de Alia Saline

Ischia (Pergine Valsugana) 13 e vedi Sale (a) Odorico di Nicolò da

Isera 22 e vedi Bonaverio di Antonio da; Francesco di Pietro da; Nicolò a torcularibus dal Borgonuovo di Trento, di Antonio da; Nicolò di Antonio da; Sofia di Pietro da

Isny (Germania) vedi Giovanni da; Hulricus de Isnina, plebanus de Paho; Ulrico da; Ulricus de Isna, familiaris Iohannis de Flemis

Königsberg, comitatus Cunespergi vedi Osvaldo detto Sengel da San Michele all'Adige, vicario generale del conte del Tirolo e ius publice redens in civilibus et criminalibus causis in toto comitatu Cunespergi nec non in Cimbrie iurisdictionis
Kutná-Hora (Rep. Ceca) vedi Corrado Friderici Greusseri de Monte Kuctis

Lana $(\mathrm{Bz})$ vedi Giovanni de Empach da

Lavis 29, 194; Appendice, regg. 29, 35

Ledro vedi Zevolis (de) Antonio da

Lendinara (Ro) vedi Bonafede di Giovanni da

Levico 13 e vedi Abrianus del fu Nicolò Nichi da; Federico Polentone da borgo San Martino in Trento di Giovanni Pazolus da; Franceschinus aurifix quondam ser Pancerie de; Giovanni Pazolus da; Giacomo di Nichele a Silva da; Pasqua di Mercadento da; Pietro di Guglielmo Merino da; Tanuciis (de) Nicolò da

Lidorno, lago 26

Livo vedi Marco, pievano in

Lizzana (Rovereto) 22 e vedi Marco detto Barozzo di Orso da

Lucca vedi Giovanni di Bartolomeo da

Lusiana (Vi) vedi Marco da

Lussemburgo vedi Giovanni Enrico di; Sigismondo di

Maderno (Bs) vedi Capris (de) Nicolò di Pietro Balduino da; loc. Brayda Appendice, reg. 15

Madom vedi Trento, località extraurbane, Vigolo Baselga

Madruzzo vedi Beatrix [di Antonio Crescimbene da]

Magré (Vi) 19 e vedi Desiderato di Francesco da

Malé vedi Perengerio da Castelfondo/de Melango, cappellano e sacrista della cattedrale, pievano di

Mantova vedi Floriis (de) Alberto di Martino da; Nicolò da; Trechis (de) Venturino di Antonio da

Margone (Vezzano) vedi Bartolomeus de Margono; Nicholaus de Margono

Marostica (Vi) vedi Alberto da

Masovia, territorio (Polonia) vedi Alessandro di

Meano 126, 127, 175 e vedi Nicolò dalla val di Fassa, vicario e rettore della pieve di; Tibaldo dalla Valsugana, pievano a; Trentino di Nicolò Chechi da; Ulrico, pievano a

Mechel vedi Nicolò/Nicolino da

Melango vedi Castelfondo

Merano/Meran (Bz) Appendice, reg. 37 e vedi Milauner Henricus de

Metz (Francia) vedi Enrico di 
Mezzolombardo vedi Serafino di Antonio Secadinari da

Milano vedi Antonio di Paolo Peverada/Pevrada da; Isidoro da; Pietro di Ambrogio da; ducato di Milano 193

Molina (Mori) vedi Hendricus da; Marco di Hendricus da

Monaco di Baviera (Germania) vedi Matteo di Ulrico da; Senftel Michael di Ludovico da

Montecchio Precalcino (Vi) vedi Sibilia di Chierico da

Mori 11, 61; pieve di Santo Stefano $117 e$ vedi Antonia di Cristiano da; Antonio di Filippo da; Bartolasio di Cristiano da; Domenica di Bartolasio di Cristiano da; Giovanni da; Giovanni di Ermanno da; Hendricus da; Trentino detto a Sale di Bonaventura da

Mauthen (Austria) vedi Giovanni de Muta

Nogaredo 22 e vedi Antonio di Bonifacio da; Antonius Nicholai sartoris da; Gasparino di Antonio notaio da;

Nomi vedi Bartolomeo di Blanchus detto de Sangue de Can da; Giovannina, moglie di Bartolomeo di Blanchus detto de Sangue de Can da; Lena di Bono da

Noriglio vedi Flos Dominici Petri Ordani de Barbarola

Novacella/Neustift (Bz) Giovanni di Corrado da

Novara vedi Antonio da; Giacomo da; Martino da

Novo Foro vedi Egna

Nurlinga (Nürtingen, Germania?) vedi Thymel/Taymel Narciscus/Narcischus de Nurlinga

Oyten (Germania) vedi Friling Goffredo [de Oyta] da Bressanone

Olmütz [Olomouc, Rep. Ceca] vedi Volek Giovanni, vescovo di

Ora/Auer (Bz), chiesa di San Pietro Appendice, reg. 28

Ortenburg (Germania) vedi Alberto di

Ottenheim (Germania) vedi Giovanni da Ottenheim/Octhayn

Padova 66, 122 e vedi Francesco a scutelis da; Romano di Giacomo da; Santa Maria all'Arena 129 e vedi Thomasiis (de) Prospero da Cremona, preposito di

Palermo vedi Antonio da
Parma 57, 66 e vedi Locholum/Tocolum Giulio da; Patheriis (de) Francesco da; Pietro da; Pugnis (de) Giovanni da

Passau (Germania) vedi Artuico/Hertuicus/ Hertwicus di Enrico da

Pavia vedi Tosabech (de) Antonio da; Zampetrus de

Pergine 64 e vedi Bonafede di Giovanni da Lendinara, abitante a; Ceris (de) Antonio da; Ceris (de) Federico di Giovanni di Michele da; Sale (a) Nicolò di Odorico da; Vigilio Morzati da

Persichello (Cr) vedi Giacomo da

Piacenza vedi Giovanni Battista Moragium da

Piazzo (Villa Lagarina) 11, 13 e vedi Bartolasio di Cristiano da; Cristiano quondam ser Bertrami de; Francesco di Cristiano detto Meiatus da; Gislemberto di Cristiano detto Meiatus da

Pilcante (Ala) vedi Florio di Alberto da

Piné 100, 164 e vedi Baldessarre dal Borgonuovo di Guglielmo de Mura da; Leonardo da

Pistoia vedi Meo di Giovanni da

Planum (ad) vedi Trento, località extraurbane, San Bartolomeo

Polonia vedi Zeno dalla

Pomarolo 11 e vedi Antonio da; Biagio di Franchetto da; Gulielmus de; Margherita di Giovanni Brasadole da; Mezaoveta Aldrighetto di Giovanni da; Odorico da

Posina (Vi) vedi Cristina, vedova del notaio Giacomino da; Giacomino di Albertino tabernarius da

Pra Mazor (a) vedi Trento, località extraurbane, Gabbiolo

Pressano vedi Barbara da

Puglia vedi Rugerio di Nicolò de Apulia

Ranzo vedi Antonio di Michele da

Ravazzone (Mori) 195 e vedi Giacomo di Bartolomeo da

Redundol (a) vedi Trento, località extraurbane, Gabbiolo

Revò vedi Giacomo di Pietro da

Riga (Lettonia) 46

Rimini vedi Iohannes magister barberius de Arimino

Riva del Garda 46 e vedi Bonaventura di Pellegrino da; Matteo da; Pisoni Perisonus di Francesco da

Rivozzo (al) vedi Trento, località extraurbane, Graffiano 
Romeno, ospedale di San Tommaso 104, 117

Roncegno vedi Guglielmo da

Roncogno (Pergine Valsugana) vedi Leonardo di Endrigo da

Rovereto 65, 121, 155 e vedi Fignoclus/Phignonclus di Pietro da; Giacomo da Persichello, cancelliere del comune di; Pietro Iacob di Rigo da; Vricius da; Zeschele di Federico da; distretto di Rovereto 56

Rumo vedi Giacomo da

Rupprechtstorff [Altruppersdorf, Austria] vedi Giacomo di Federico de

Sacco (Rovereto) 22 e vedi Alberto di Negratus da; Negrello di Alberto notaio da

Sale (al)/ultra l Sale vedi Trento, località extraurbane, Povo

San Michele all'Adige vedi Giacomo, preposito del monastero di; Osvaldo detto Sengel da

Sant'Ilario (Rovereto) vedi Giovanni detto Rancagno di Nicolò da

Santorso (Vi) vedi Marcabruno di Belloto da

Sant'Orsola vedi Bella, moglie di Pietro da

San Bonaventura (?) vedi Pietro da

Sanzeno vedi Denklinger Martinus de Augu$s t a$, pievano di; Nicolò da Venezia, pievano in

Sasso vedi Trento, località extraurbane, Vigolo Baselga

Selva, castello 41

Seregnano vedi Antonio da; Roccabruna (da) Vigilio di Guglielmo da

Serso vedi Nicolò di Giovanni de Ligatis da

Sfruz vedi Matteo di Filippo da

Siena 111 e vedi Pietro di Nanni da

Sole, valle 81, 82, 195

Spormaggiore vedi Marco di Odorico da; Pietro da

Sporminore vedi Michele Fenutoli di Bartolomeo da

Stenico 72, Appendice, reg. 5; castello 41, 42; Appendice, regg. 5, 25, 29

Stravino (Cavedine) vedi Giovanni di Stefano di Benvenuto di Pellegrino di Gilberto da

Taio 164

Teglie (Vobarno, Bs) 87 e vedi Stanchariis (de) Pietro di Stefano da

Telve vedi Biagio da

Tenno 46; castello 41, 71

Terlago 34, Appendice, reg. 17 e vedi Andriota di Francesco detto Tibay da; Colombini Leonardo di Giovanni Antonio da; Fatis (de) Fato da; Fatis (de) Giovanni Conto di Paolo da; Fatis (de) Paolo di Giovanni da; Flordela, moglie di Terlaco Marele da; Giacomo Batedelus di Nicolò Batedelus da; Giovanazzo di Giacomo da; Graziadeo da; Terlaco Marele da; loc. al Cere Appendice, reg. 17

Termeno/Tramin $(\mathrm{Bz})$ vedi Layner Clara di Giovanni da; Layner Giovanni di Ulrico da; Michele di Andrea da

Terra di Lavoro, territorio vedi Pietro de Drageto de Terra Laboris

Terragnolo vedi Simeone da Ravina di Federico da

Tesino, territorio vedi Martino di Giovanni Ceraduce dal; Tomeo dal

Thaur (Austria) vedi Toma, pievano a

Tierno vedi Antonio Abrianus di Benvenuto da

Tignale (Bs) vedi Nicolò da Trento, pievano in

Torchio (Civezzano) vedi Bartolomeo di Giacomino da

Tossignano (Bo) vedi Ursulinis (de) Giacomo da

Toul (Francia) 46

Trento si veda dopo l'ultima voce di questo indice

Trevigiano, territorio vedi Pietro a caminis di Giovanni dal

Treviso vedi Basilio da; Giovanni di Brunetto da; Leonardus Trivisanus

Trieste vedi Nicolò Basileus da

Tuenno 60 e vedi Alexander Copa[...] de; Bartolameo detto Tomeo da; Trentino di Zuccolino da

Valeggio (Vr) vedi Rigo di Domenico da

Vallagarina 20, 22

Valsugana vedi Tibaldo dalla

Valtellina vedi Dominicus de Voltolina; Iohannes Zeschi Floris de Voltolina

Vattaro vedi Simeone di Guglielmo da

Venezia vedi Antonio da; Cavalis (de) Giovanni da; Nicolò da; Nicolò Leonis da

Verona 42, 64, 111, 207 e vedi Antonio di Pietro da; Balzanini Guglielmo di Guglielmo da; Bonalinis (de) Giacomo di; Donato da; Gerardo da; Giovanni da; Manfredo da; contrada di San Benedetto 111; contrada di San Giovanni in Foro 43, 45; contrada di San Nazaro vedi Bonaventura di Pasquale/Pasqualino da

Vervò vedi Simeone di Tommasino da

Vezzano vedi Sicherio di Michele da 
Indice dei nomi di luogo

Viarago vedi Giovanni di Enrico da

Vicentino, territorio vedi Iohannes Benedictus de

Vicenza 168, 207

Vigolo vedi Antonio da; Bartolomeo Carnessarii da; Simeone di Guglielmo da

Vigolo Vattaro vedi Baldessarre detto Bruschino da; Giorgio di Guglielmo di Iorio da; Odorico di Bartolomeo Gatabriga da

Villa Lagarina 11

Volano 22 e vedi Bartolomeo Toschanelus di Alberto da; Berlina (della) Antonio di Giacomo magister sarto da; Filippo di Bonaventura di Antonio da; Francesco di Martino da; Martino di Francesco da; Nicolò di Bonaventura da

Warmia (Polonia) vedi Hayminger David, prete della diocesi di Varmia

Welden (Germania) vedi Enrico di Odorico de Velden

Wrocław (Polonia) vedi Nicolò da Breslavia

Zurigo (Svizzera) vedi Wolchestein Teobaldo di Michele, canonico di Bressanone e di

Trento vedi Adelpreto, vescovo di; Andriota di Nicola da; Antonio da; Antonio detto $a$ Zopellis da; Antonio di Bommartino da; Bertoldo magistri Odorici cerdonis da; Corrado da; Delaito di Benasuto a Rotis da; Dominabus (a) Antonio da; Enrico, professore di grammatica in; Flora di Tura sarto detto a Porta da; Floriano Malicie di Francesco da; Francesca di Bartolomeo di Sicco da Trento; Gianvittore di Burgasio da Feltre, podestà di; Giovanni, vescovo suffraganeo di; Giovanni Battista Moragium da Piacenza, pretore di; Giovannino di Marchetto detto de la Porta da; [Iosiis (de)] Iosio di Giordano phisicus da; [Iosiis (de)] Tommaso di Giordano phisicus da; Lippi (de) Giovanni di Luca da; Locholum/Tocolum Giulio da Parma, pretore di; Luca di $* * *$ da Firenze, oste in; Marco di Odorico da; Melchiorre di Leonardo sarto da; Meo di Giovanni da Pistoia, console di; Mezzasoma Gioacchino di Pietro da; Michele da; Morandino da; Morandino di Giovanni Bono da; Mucius baraterius de; Nicolò notaio da; Nicolò phisicus da; Nicolò Basileus da Trieste, podestà di; Ogni- bene di Adelperio da; Onesta magistri Odorici cerdonis de; Paolo di Martino da; Rambaldo da; Rodolfo di Basino da; Salomone, vescovo di; Sicco da; Speranza di Paolo [di Martino] notaio da; Tura detto Turata di Trentino a Ranto[?] da; Vigilio da; Vigilio detto Barbustelo da; Zambono [di Paolo] da

banchum iuris maleficiorum nel palazzo vescovile 81,123

borghi, contrade, località urbane

Belenzani 108, Appendice, reg. 1

Borgonuovo 12, 13, 14, 30, 33, 36, 149 , 150, 160; Appendice, reg. 7 e vedi Andrea di Ottolino dal; Baldessarre dal; Bartolomeo di Anselmo da; Bonaverio di Antonio da Isera abitante in; Elica di Gabriele dal; Francesco di Marco da; Maddalena figlia del notaio Antonio da; Marco domine $\mathrm{Zi}$ nele di Valentino dal; Marcus dictus Faytelus de; Nicolò a torcularibus dal; Nicolò di Ognibene sarto da; Nicolò Goseto dal; Pietro a Fossis dal; Ysabeta, moglie di Michele de Aproinis da

Campo Marzio (piazza Fiera) 95

Cantone vedi Giacomino detto a Cantono; Lola di Andrea dal; Nicolaus ad Cantonum

ad Desmortam 140

fossatum cerdonum vedi Bartolomeo di *** di Tommaso de

del Mercato 106

Merchati veteris 107

platea comunis 136

ponte dell'Adige 38

ad Pontem Fossatum 140

della Roggia Grande 123

San Benedetto 33, 110, 111; Appendice, reg. 44

Sancte Marie Magdalene 107

Santa Maria Maggiore 200

San Marco 38; Appendice, reg. 27 e vedi Margherita detta Stanfferina di Nichele a labro scisso da

San Martino Appendice, reg. 18 e vedi Adelperio mutarius da; Antonius Panizoli de; Concerinus de; Federico Polentone da; Francesco di Bartolomeo da; Sichis (de) Francesco di Adelperio da

Sancti Petri 27

Santa Croce 18 
Indice dei nomi di luogo

San Vigilio 36, 37, 140, 142 e vedi Vigilio da

Vadum Gislote 19

Casa della Misericordia 101

Castelletto 36

castello del Buonconsiglio 17, 72, 78, 81, $84 e$ vedi Giovanni de Fraudental, cancelliere

chiese, conventi e monasteri

cattedrale di San Vigilio 27, 36, 84, 93, 126, 127, 128 e vedi Buratinis (de) Antonio, prete mansionario della; Donato da Verona, sacrista nella; Giovanni de Alemania, cappellano nella; Giovanni de Austria canonico, cappellano della; Giovanni di Brunetto da Treviso, cantore, sacrista e cappellano in; Giovanni de Fraudental, cappellano della; Marco, cappellano della; Nicolò da Trento, pievano in Tignale e cappellano della; Perengerio da Castelfondo/de Melango, cappellano e sacrista della; Vigilio Turcheti, vicecappellano della

Sant'Apollinare Appendice, reg. 28

San Bernardino 33, 34

Santa Chiara 113

Santa Croce 101, 103, 113, 117

Santa Elisabetta 104

San Francesco 33, 101, 103, 113, 117

San Lorenzo 38, 76, 84, 88, 100, 101, 102, 113, 116, 203, 204 e vedi Bartolomeo da Bologna, abate dell'abbazia di; Francesco da Ferrara, priore del monastero di

San Marco 26, 98, 99, 101, 102, 113

Santa Maria Maggiore 15, 27, 36, 108, 109; Appendice, regg. 1, 2 e vedi Biagio da Pomarolo, sindaco di; Bonaventura di Pasquale/Pasqualino da Verona da borgo San Nazaro, monachus di; Pietro da Parma, pievano di

San Michele 98, 100, 101, 102, 116

cimiteri di Santa Maria 26; di San Vigilio 14, 36

località extraurbane

Baselga di Sopramonte, pieve di Santa Maria 127 e vedi Osvaldo, pievano

a Brusa Laste 38

Bus de Vela 47

Cadine vedi Cristoforo da; Domenico Fraschete da

Cognola 26

Cortesano Appendice, reg. 31

Gabbiolo Appendice, reg. 26 e vedi Fran- cesco di Vincenzo da; Giovanni di Chelotus da; loc. a Fulan Appendice, reg. 26; Gabium sive Agilon Appendice, reg. 26; a Pra Mazor Appendice, reg. 26; a Redundol Appendice, reg. 26

Gardolo vedi Federicus de; Leonardo da

Gardolo di Mezzo vedi Sumptag Antonio di Sumptag da; Sumptag Federico di Sumptag da

Graffiano Appendice, reg. 26; loc. Clesura Appendice, reg. 26; al Dosso di Calonegi Appendice, reg. 26; al Rivozzo Appendice, reg. 26

Laste 26

Man 13

Martignano 26; Appendice, reg. 16

Mattarello vedi Francesco Leonardo da; Petrus de

Meano vedi Nicolò dalla val di Fassa, vicario e rettore della pieve di; Tibaldo dalla Valsugana, pievano a; Trentino di Nicolò Chechi da; Ulrico, pievano di

Mesiano Appendice, reg. 40, loc. a Gozador Appendice, reg. 40

Molini di San Francesco Appendice, reg. 13

Pietrapiana, castello 25, 26

Povo Appendice, reg. 26 e vedi Adelpreto di Federico da; Antonio di Beloto da; Baldino di Andrea a Poza da; Bartolomeo detto Sachart di Rolando da; Federico di Ognibene da; Giovannetto di Paramusius da; Giovanni di Bartolomeo Bayli da; Giovannetto di Paramusius da; Giovannino di Chous da; Giovannino di Paramusius da; Hulricus de Isnina, plebanus de; Michele da; Nichele Bragalda da; Odorico di Federico notaio da; Trahugis (de) Vigilio da; loc. Clesura Appendice, reg. 26; loc. al Sale/ultra l Sale 26

Preda Streta 26

Ravina vedi Antonio di Bonaventura da; Giacomo, fratello di Antonio di Bonaventura da; Simeone da

ad Rovredum 14

Romagnano, loc. ad Gardas 11

San Bartolomeo 19, 38; Appendice, reg. 6; loc. ad Planum 37

Sardagna vedi Lena di Bono da Nomi, moglie di Pancera da

Sopramonte vedi Lutterio di Bonaventura Zanzarelus da; Nicolino di Morzanto da 
Indice dei nomi di luogo

Vigolo Baselga, loc. a la Fontana Appendice, reg. 17; Madom Appendice, reg. 17 e vedi Francesco di Vincenzo da Gabbiolo da; Sasso e vedi Melchiorre di Domenico da

Zell vedi Michele da

ospedali di San Martino 104, 106, 117, 124;

di San Nicolò 98; di San Pietro 104, 117;

di Santa Croce 103; di Santa Maria della

Misericordia 33; di Santa Maria Maddalena 104,117

osterie alla Corona Appendice, reg. 37 e vedi Rigo da Francoforte, oste; al Falcone Appendice, reg. 35 e vedi Antonio Abrianus di Benvenuto da Tierno, oste

palazzi Calepini 37; vescovile 63, 80, 208; Appendice reg. 1 piazza d'Arogno 14

porte di Ponte Adige vedi Giovanni/Ianes di Giovanni de Alemania, capitano della Torre Vanga e della porta di; di San Martino $111,138,140$ e vedi Giovannino di Giacomo Bruno di; di Santa Croce 13, 26, 95

stazio speciarie/appotece speciarie Melchioris ab Oleo 104, 136

Prepositura vedi Odorico, monachus nella

Roggia Grande 37, 95

statere communitatis 96

stazione delle bollette $84,97,98$

strade via Carlo Dordi 37; via regalis, per quam itur ad portam Sancte Crucis 19

Torre Vanga 26 e vedi Giovanni/Ianes di Giovanni de Alemania, capitano della 


\section{PREMIO RICERCA CITTÀ DI FIRENZE}

Titoli pubblicati

\section{ANNO 2011}

Cisterna D.M., I testimoni del XIV secolo del Pluto di Aristofane

Gramigni T., Iscrizioni medievali nel territorio fiorentino fino al XIII secolo

Lucchesi F., Contratti a lungo termine e rimedi correttivi

Miniagio G., Soggetto trascendentale, mondo della vita, naturalizzazione. Uno sguardo attraverso la fenomenologia di Edmund Husserl

Nutini C., Tra sperimentalismo scapigliato ed espressivismo primonovecentesco poemetto in prosa, prosa lirica e frammento

Ottonelli O., Gino Arias (1879-1940). Dalla storia delle istituzioni al corporativismo fascista

Pagano M., La filosofia del dialogo di Guido Calogero

Pagni E., Corpo Vivente Mondo. Aristotele e Merleau-Ponty a confronto

Piras A., La rappresentazione del paesaggio toscano nel Trecento

Radicchi A., Sull'immagine sonora della città

Ricciuti V., Matrici romano-milanesi nella poetica architettonica di Luigi Moretti. 1948-1960

Romolini M., Commento a La bufera e altro di Montale

Salvatore M., La stereotomia scientifica in Amédée François Frézier. Prodromi della geometria descrittiva nella scienza del taglio delle pietre

Sarracino F., Social capital, economic growth and well-being

Venturini F., Profili di contrattualizzazione a finalità successoria

\section{ANNO 2012}

Barbuscia D., Le prime opere narrative di Don Delillo. Rappresentazione del tempo e poetica beckettiana dell'istante

Brandigi E., L'archeologia del Graphic Novel. Il romanzo al naturale e l'effetto Töpffer

Burzi I., Nuovi paesaggi e aree minerarie dismesse

Cora S., Un poetico sonnambulismo e una folle passione per la follia. La romanizzazione della medicina nell'opera di E.T.A. Hoffmann

Degl'Innocenti F., Rischio di impresa e responsabilità civile. La tutela dell'ambiente tra prevenzione e riparazione dei danni

Di Bari C., Dopo gli apocalittici. Per una Media Education "integrata”

Fastelli F., Il nuovo romanzo. La narrativa d'avanguardia nella prima fase della postmodernità (1953-1973)

Fierro A., Ibridazioni balzachiane. «Meditazioni eclettiche» su romanzo, teatro, illustrazione

Francini S., Progetto di paesaggio. Arte e città. Il rapporto tra interventi artistici e trasformazione dei luoghi urbani

Manigrasso L., Capitoli autobiografici. Poeti che traducono poeti dagli ermetici a Luciano Erba

Marsico C., Per l'edizione delle Elegantie di Lorenzo Valla. Studio sul V libro

Piccolino G., Peacekeepers and Patriots. Nationalisms and Peacemaking in Côte D'Ivoire (2002-2011)

Pieri G., Educazione, cittadinanza, volontariato. Frontiere pedagogiche 
Polverini S., Letteratura e memoria bellica nella Spagna del XX secolo. José María Gironella e Juan Benet

Romani G., Fear Appeal e Message Framing. Strategie persuasive in interazione per la promozione della salute

Sogos G., Le biografie di Stefan Zweig tra Geschichte e Psychologie: Triumph und Tragik des Erasmus von Rotterdam, Marie Antoinette, Maria Stuart

Terigi E., Yvan Goll ed il crollo del mito d'Europa

Zinzi M., Dal greco classico al greco moderno. Alcuni aspetti dell'evoluzione morfosintattica

\section{ANNO 2013}

Bartolini F., Antonio Rinaldi. Un intellettuale nella cultura del Novecento

Cigliuti K., Cosa sono questi «appunti alla buona dall'aria innocente»? La costruzione delle note etnografiche

Corica G., Sindaci e professionismo politico. Uno studio di caso sui primi cittadini toscani

Iurilli S., Trasformazioni geometriche e figure dell'architettura. L'Architectura Obliqua di Juan Caramuel de Lobkowitz

Pierini I., Carlo Marsuppini. Carmi latini. Edizione critica, traduzione e commento

Stolfi G., Dall'amministrare all'amministrazione. Le aziende nell'organizzazione statuale del Regno di Sardegna (1717-1853)

Valbonesi C., Evoluzione della scienza e giudizio di rimproverabilità per colpa. Verso una nuova tipicità del crimen culposum

Zamperini V., Uno più uno può fare tre, se il partito lo vuole! La Repubblica Democratica Tedesca tra Mosca e Bonn, 1971-1985

ANNO 2014

Del Giovane B., Seneca, la diatriba e la ricerca di una morale austera. Caratteristiche, influenze, mediazioni di un rapporto complesso

Gjata A., Il grande eclettico. Renato Simoni nel teatro italiano del primo Novecento

Podestà E., Le egloghe elegantissimamente composte. La Buccolica di Girolamo Benivieni edizione critica e commento

Sofritti F., Medici in transizione. Etica e identità professionale nella sanità aziendalizzata

Stefani G., Sebastiano Ricci impresario d'opera nel primo Settecento

Voli S., Soggettività dissonanti. Di rivoluzione, femminismi e violenza politica nella memoria di un gruppo di ex militanti di Lotta continua

ANNO 2015

Betti M., La costruzione sociale della finanziarizzazione: verso la convergenza dei sistemi bancari?

Chini C., Ai confini d'Europa. Italia ed Irlanda tra le due guerre

Galletti L., Lo spettacolo senza riforma. La compagnia del San Samuele di Venezia (1726-1749)

Lenzi S., La policromia dei Monochromata. La ricerca del colore su dipinti su lastre di marmo di età romana

Nencioni F., La prosa dell'ermetismo: caratteri e esemplari. Per una semantica generazionale

Puleri M., Narrazioni ibride post-sovietiche. Per una letteratura ucraina di lingua russa 
Chella A., Giovanni Raboni poeta e lettore di poesia (1953-1966)

Frilli G., Ragione desiderio, artificio. Hegel e Hobbes a confronto

Pieroni A., Attori italiani alla corte della zarina Anna Ioannovna (1731-1738)

Ponzù Donato P., Pier Candido Decembrio. Volgarizzamento del Corpus Caesarianum. Edizione critica

Rekut-Liberatore O., Metastasi cartacee. Intrecci tra neoplasia e letteratura

Schepis C., Carlo Cecchi. Funambolo della scena italiana: l'apprendistato e il magistero

In memoria di Lucrezia Borghi, Valentina Gallo ed Elena Maestrini

Franza T., Costituzionalizzare la Costituzione. Una prospettiva pleromatica

\section{ANNO 2017}

Bosco M., Ragion di stato e salvezza dell'anima. Il riscatto dei cristiani captivi in Maghreb attraverso le redenzioni mercedarie (1575-1725)

Malfatti S., Antonio da Borgonuovo. L'ascesa di un notaio a Trento fra Trecento e Quattrocento

Masciotta C., Costituzione e CEDU nell'evoluzione giurisprudenziale della sfera familiare

Matraini C., Lettere e Rime. Introduzione e commento a cura di Cristina Acucella

Pesini L., La paraipotassi in italiano antico

Valentini C., L'evoluzione della codifica del genitivo dal tipo sintetico al tipo analitico nelle carte del Codice diplomatico longobardo

In memoria di Lucrezia Borghi, Valentina Gallo ed Elena Maestrini

Fersini M.P., Diritto e violenza. Un'analisi giusletteraria 
